\title{
1995 Solid Waste 30-Year Volume Summary
}

\author{
O. J. Valero
}

Westinghouse Hanford Company

\author{
T. J. DeForest \\ K. J. Templeton \\ Pacific Northwest Laboratory
}

\begin{abstract}
This report was prepared as an account of work sponsored by an agency of the United States Government. Neither the United States Government nor any agency thereof, nor any of their employees, makes any warranty, express or implied, or assumes any legal liability or responsibility for the accuracy, completeness, or usefulness of any information, apparatus, product, or process disclosed, or represents that its use would not infringe privately owned rights. Reference herein to any specific commercial product, process, or service by trade name, trademark, manufacturer, or otherwise does not necessarily constitute or imply its endorsement, recommendation, or favoring by the United States Government or any agency thereof. The views and opinions of authors expressed herein do not necessarily state or reflect those of the United States Government or any agency thereof.
\end{abstract}

Prepared for the U.S. Department of Energy Office of Environmental Restoration and Waste Management

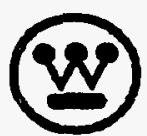
Hanford Company Richland, Washington

Hanford Operations and Engineering Contractor for the

U.S. Department of Energy under Contract DE-ACO6-87RL10930 


\section{RELEASE AUTHORIZATION}

Document Number: WHC-EP-0865

Document Title: $\quad 1995$ Solid Waste 30-Year Volume Summary

Release Date: $\quad 6 / 5 / 95$

This document was reviewed following the procedures described in WHC-CM-3-4 and is:

APPROVED FOR PUBLIC RELEASE

WHC Information Release Administration Specialist:

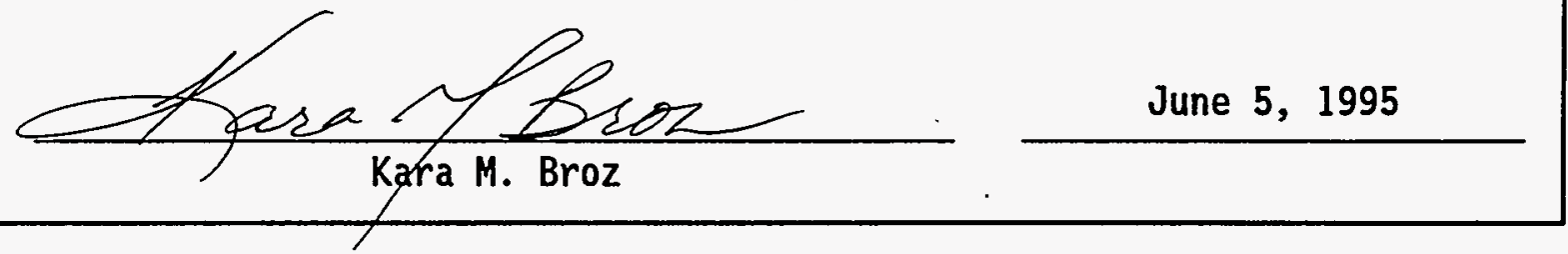




\section{DISCLAIMER}

Portions of this document may be illegible in electronic image products. Images are produced from the best available original document. 
Solid waste forecast volumes to be generated or received at the U.S. Department of Energy Hanford Site over the next 30 years are described in this report. The volumes described in detail are low-level mixed waste (LLMW) and transuranic/transuranic-mixed (TRU TRUM) waste. Summary level information pertaining to low-level waste (LLW) is described in Appendix B. Emphasis is placed on LLMW and TRU TRUM waste because it will require processing and storage at Hanford Solīd Waste's Central Waste Complex (CWC) prior to final disposal. The LLW will generally be sent directly to disposal. The volume data was collected from onsite and offsite waste generators who currently ship or plan to ship solid waste to the Hanford Site.

The total baseline volume of LLMW and TRU TRUM waste expected to be received at the CWC over the next 30 years is forecasted to be over 235,560 cubic meters. Based on ranges provided by the waste generators, this baseline volume could fluctuate between a minimum of 203,690 cubic meters and a maximum of 260,500 cubic meters. The range is primarily due to uncertainties associated with the Tank Waste Remediation System (TWRS) program. Uncertainties include the retrieval of the long-length equipment, scheduling, and tank retrieval technologies.

The Environmental Restoration and TWRS programs are expected to generate the largest portions of solid waste, generating $45 \%$ and $42 \%$ of the total expected waste volumes, respectively. The Environmental Restoration program waste volumes inciude decontamination and decommissioning (D\&D) of surplus facilities waste, D\&D of non-surplus facilities waste, and remediation waste from past practice sites. The TWRS program's waste stream is primarily longlength contaminated equipment (over 80,000 cubic meters). The remaining $13 \%$ of the waste expected at the Hanford Site is generated by the Facility Transitions Program, Other Programs (i.e., Spent Nuclear Fuel, Liquid Effluents, PNL, Analytical Services, and Solid Waste) Non-Programmatic Waste Generators (i.e., Buried Equipment, 100 Area Construction Services, Geotechnical

Engineering Laboratory, etc.), and Offsite Waste Generators. (a)

The data reported in this document establish.a starting point for solid waste management and $\mathrm{planning.} \mathrm{It} \mathrm{is} \mathrm{recognized} \mathrm{that} \mathrm{forecast} \mathrm{estimates} \mathrm{will}$ vary as facility planning and missions are more clearly defined. However, the

(a) Beginning in Fiscal Year 1996, offsite waste generators must have an approved Site Treatment Plan to ship waste to Hanford. The volumes presented in this report for offsite waste do not necessarily reflect the information contained in the Site Treatment Plans. 
information provided is useful in obtaining an insight into Hanford's future solid waste management requirements. Efforts such as onsite program integration meetings which were established to develop consistency between the individual waste generators and program volume forecasts, and the collection of minimum and maximum ranges are continually being implemented in order to improve the accuracy of the 30-year solid waste forecasts. 


\section{ACRONYMS AND ABBREVIATIONS}

$\begin{array}{ll}\text { CH } & \text { contact-handled (waste) } \\ \text { CWC } & \text { Central Waste Complex } \\ \text { CY } & \text { calendar year } \\ \text { D\&D } & \text { decontamination and decommissioning } \\ \text { EIS } & \text { environmental impact statement } \\ \text { ER } & \text { environmental restoration } \\ \text { ERD } & \text { Environmental Restoration Division } \\ \text { ETF } & \text { Effluent Treatment Facility } \\ \text { FFTF } & \text { Fast Flux Test Facility } \\ \text { FUSRAP } & \text { Formerly Utilized Sites Remedial Action Program } \\ \text { FY } & \text { fiscal year } \\ \text { HLVP } & \text { High-Level Waste Vitrification Plant } \\ \text { HLW } & \text { high-level (radioactive) waste } \\ \text { LERF } & \text { Liquid Effluent Retention Facility } \\ \text { LLMW } & \text { low-level (radioactive) mixed waste } \\ \text { LLVP } & \text { Low-Level Vitrification Project } \\ \text { LLW } & \text { low-level (radioactive) waste } \\ \text { nCi } & \text { nanocurie } \\ \text { PFP } & \text { Plutonium Finishing Plant } \\ \text { PNL } & \text { Pacific Northwest Laboratory } \\ \text { PSTP } & \text { Proposed Site Treatment Plans } \\ \text { PUREX } & \text { Plutonium-Uranium Extraction (process) } \\ \text { RA } & \text { remedial action } \\ \text { RH } & \text { remote-handled (radioactive waste) } \\ \text { SNF } & \text { Spent Nuclear Fuel } \\ \text { SWD } & \text { Solid Waste Disposal } \\ \text { TEDF } & \text { Treated Effluent Disposal Facility } \\ \text { TRU } & \text { transuranic (waste) } \\ \text { TRUM } & \text { transuranic mixed waste } \\ \text { TRUSAF } & \text { transuranic Storage and Assay Facility } \\ \text { TSD } & \text { treatment, storage, and disposal } \\ \text { TWRS } & \text { Tank Waste Remediation System } \\ \text { UST } & \text { underground storage tank } \\ \text { WATS } & \text { Waste Acid Treatment System } \\ \text { WESF } & \text { Waste Encapsulation and Storage Facility } \\ \text { WHC } & \text { Westinghouse Hanford Company } \\ \text { WSCF } & \text { Waste Sampling and Characterization Facility } \\ & \end{array}$




\section{WHC-EP-0865}

Intentionally Left Blank 


\section{CONTENTS}

EXECUTIVE SUMMARY ................... $\ldots$ i ...

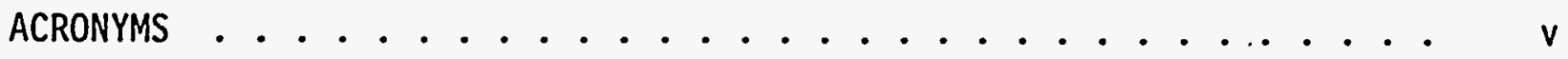

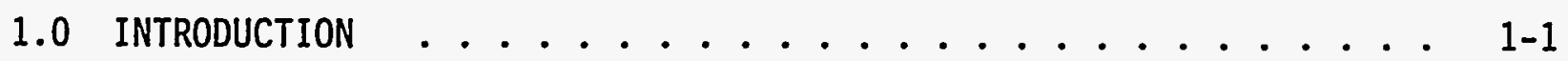

2.0 OVERVIEW OF 30 -YEAR WASTE VOLUMES ............ 2-1

2.1 MAJOR HANFORD PROGRAM AREAS ............ 2-1

2.2 WASTE CATEGORIES ...................... 2-2

2.3 WASTE GENERATOR LIFE-CYCLE PHASES .......... . . 2-3

2.4 PROJECTED WASTE VOLUMES .................... 2-4

2.4.1 Waste Volumes by Major Hanford Programs . . . . 2-4 2-4

2.4.2 Waste Volumes by Waste Category ........ 2-9

2.5 COMPARISON WITH 1994 FORECAST VOLUMES . . . . . . . 2-13

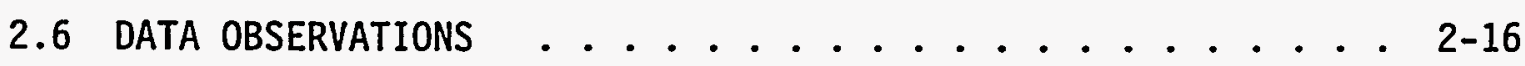

3.0 TANK WASTE REMEDIATION SYSTEM .............. . . . . . .

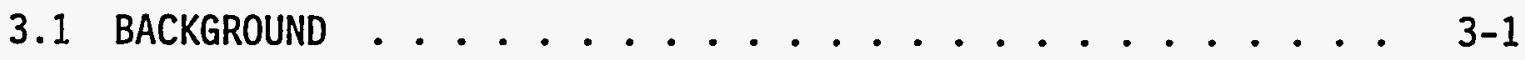

3.2 WASTE GENERATORS ......................... $3-1$

3.3 PROJECTED VOLUMES ........................ $3-4$

3.3.1 Projected Volumes for TWRS Waste
Generator Group . . . . . . . . . . 3-4

3.3.2 Projected Volumes by Waste Generator ...... 3-5

3.4 DATA OBSERVATIONS ........................ 3-6

4.0 ENVIRONMENTAL RESTORATION .............. . . . . . 4-1

4.1 BACKGROUND ........................... 4-1

4.2 WASTE GENERATORS ....................... 4-2 
4.3 PROJECTED VOLUMES ..................... 4-3

4.3.1 Forecasted Volumes for Environmental

Restoration Program ........... 4-3

4.3.2 Forecasted Volumes by Waste Generator . . . . . 4-5 4

4.4 DATA OBSERVATIONS .................... 4-7

5.0 TRANSITION FACILITIES .................... 5-1

5.1 BACKGROUND .......................... 5-1

5.2 WASTE GENERATORS ..................... 5-1

5.3 FORECASTED VOLUMES .................. 5-3

5.3.1 Forecasted Volumes for Transition
Facilities Program . . . . . . . . . . 5-3

5.3.2 Forecasted Volumes by Waste Generator . . . . . 5-4

5.4 DATA OBSERVATIONS ....................... 5-6

6.0 OTHER PROGRAMS ........................ $6-1$

6.1 BACKGROUND ....................... $6-1$

6.2 OTHER PROGRAMS ..................... 6-2

6.3 FORECASTED VOLUMES ................... 6-.

6.3.1 Forecasted Volumes for Other Programs . . . . . 6-4

6.3.2 Forecasted Volume by Program and Waste Generator . . 6-5

6.4 DATA OBSERVATIONS ................... 6-6

7.0 NON-PROGRAMMATIC ....................... 7-1

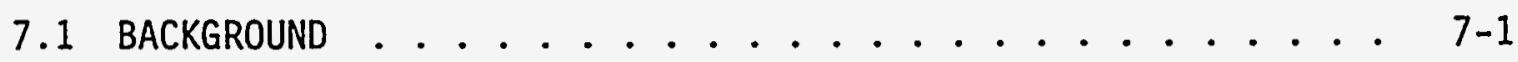

7.2 NON-PROGRAMMATIC ....................... $7-1$

7.3 FORECASTED VOLUMES .................... 7-3

7.3.1 Forecasted Volumes for Non-Programmatic . . . . 7-3

7.3.2 Forecasted Volumes by Waste Generator . . . . . . 7-4 
7.4 DATA OBSERVATIONS ................... 7-4

8.0 OFFSITE WASTE GENERATORS . . . . . . . . . . . 8-1

8.1 BACKGROUND .......................... 8-1

8.2 WASTE GENERATORS .................... 8- . . .

8.3 FORECASTED VOLUMES ................. 8- . . . .

8.3.1 Forecasted Volumes for Offsite Waste Generators . . 8-2

8.3.2 Forecasted Volumes by Program and Waste Generator . . 8-4

8.4 DATA OBSERVATIONS ......................... 8-4

9.0 REFERENCES ......................... $9-1$

APPENDIX A - ASSUMPTIONS FOR SUPPLEMENTARY SOURCES . . . . . . . A-1

APPENDIX B - LOW-LEVEL WASTE VOLUMES ............. B-I

APPENDIX C - ANNUAL WASTE GENERATOR, WASTE CLASS VOLUMES ......

APPENDIX D - ANNUAL WASTE CLASS, WASTE GENERATOR VOLUMES . . . . . D-1

APPENDIX E - WASTE GENERATORS BY HANFORD PROGRAM AREA ....... E-1 
FIGURES

2-1 Total 30-Year Solid Waste Volumes by Program Areas . . . . . 2-4

2-2 Annual 30-Year Solid Waste Volumes . . . . . . . . 2-5

2-3 TWRS Annual 30-Year Solid Waste Volumes . . . . . . . . 2-7

2-4 Environmental Restoration Annual 30-Year Solid Waste Volumes . . 2-8

2-5 Facility Transitions Annual 30-Year Solid Waste Volumes . . . 2-9

2-6 Other Programs Annual 30-Year Solid Waste Volumes . . . . . 2-10

2-7 Non-Programnatic Annual 30-Year Solid Waste Volumes . . . . . 2-11

2-8 Offsite Annual 30-Year Solid Waste Volumes . . . . . . . 2-12

2-9 Total 30-Year Solid Waste Volumes by Waste Category . . . . 2-13

2-10 CH_LLMW Annual 30-Year Solid Waste Volumes . . . . . . . 2-14

2-11 RH_LLMW Annual 30-Year Solid Waste Volumes . . . . . . . . 2-15

2-12 CH_TRU-TRUM Annual 30-Year Solid Waste Volumes . . . . . 2-16

2-13 RH_TRU_TRUM Annual 30-Year Solid Waste Volumes . . . . . 2-17

3-1 TWRS LLMW and TRU_TRUM Waste Generators . . . . . . . 3-2

3-2 TWRS Total 30-Year Solid Waste Volumes by Waste Category . . . 3-4

3-3 TWRS Annual Solid Waste Volumes by Waste Category . . . . . 3-5

3-4 Forecast Completeness for Primary Activities and Associated Life Cycle TWRS - Manage Tank Waste ........... 3-7

3-5 Forecast Completeness for Primary Activities and Associated Life Cycle TWRS - Process Waste ........... 3-8

3-6 Forecast Completeness for Primary Activities and Associated Life Cycle TWRS - Manage System-Generated Waste ....... 3-8 
4-1 Environmental Restoration LLMW and TRU_TRUM Waste Generators . . 4-2

4-2 Environmental Restoration Total 30-Year Solid Waste Volumes by Waste Category ... . . . . . . . . . 4-4

4-3 Environmental Restoration Annual Solid Waste Volumes by Waste Category ................ 4-5

5-1 Facility Transitions LLMW and TRU_TRUM Waste Generators . . . 5-2

5-2 Facility Transitions Total 30-Year Solid Waste Volumes by Waste Category. ............. 5-4

5-3 Facility Transitions Annual Solid Waste Volumes by Waste Category .............. 5-5

5-4 Forecast Completeness for Primary Activities and Associated Life Cycle Plutonium Finishing Plant Transition Projects . . . 5-7

5-5 Forecast Completeness for Primary Activities and Associated Life Cyčle PUREX Transition Projects .......... 5-8

5-6 Forecast Completeness for Primary Activities and Associated Life Cycle B-Plant Transition Projects . . . . . . . . . 5-9

5-7 Forecast Completeness for Primary Activities and Associated Life Cycle 300 Area Fuel Supply Transition Projects . . . . . 5-10

6-1 Other Programs LLMW and TRU_TRUM Waste Generators . . . . . 6-2

6-2 Other Programs Total 30-Year Solid Waste Volumes by Waste Category .............. 6- 6-5

6-3 Other Programs Annual Solid Waste Volumes by Waste Category . . 6-6

6-4 Forecast Completeness for Primary Activities and Associated Life Cycle Liquid Effluents ... . . . . . . 6-8

6-5 Forecast Completeness for Primary Activities and Associated Life Cycle Analytical Services . . . . . . . . 6-9

6-6 Forecast Completeness for Primary Activities and Associated Life Cycle Spent Nuclear Fuel . . . . . . . . 6-10

7-1 Non-Programmatic LLMW and TRU_TRUM Waste Generators . . . 7-1.

7-2 Non-Programmatic Total 30-Year Solid Waste Volumes by Waste Category 
7-3 Non-Programmatic Annual Solid Waste Volumes by Waste Category . • 7-4

8-1 Offsite LLMW and TRU TRUM Waste Generators . . . . . . . . . 8-1

8-2 Offsite Total 30-Year Solid Waste Volumes by Waste Category . . . 8-3

8-3 Offsite Annual Solid waste Volumes by Waste Category . . . . . 8-4 


\section{WHC-EP-0865}

\section{TABLES}

3-1 TWRS Waste Generator Volumes . . . . . . . . . . 3-6

4-1 Environmental Restoration Waste Generator Volumes . . . . . 4-6

5-1 Transition Facilities Waste Generator Volumes . . . . . . 5-5

6-1 Other Programs Waste Generator Volumes . . . . . . . . 6-7

7-1 Non-Programmatic Waste Generator Volumes . . . . . . 7-5

8-1 Offsi.te Waste Generator Volumes . . . . . . . . 8-5 
WHC-EP-0865

Intentionaliy Left $B$ lank 


\subsection{INTRODUCTION}

This document, prepared by Pacific Northwest Laboratory (PNL) ${ }^{(a)}$ under the direction of Westinghouse Hanford Company (WHC), provides a description of the annual low-level mixed waste (LLMW) and transuranic/transuranic mixed solid waste (TRU TRUM) volumes expected to be managed by Hanford's Solid Waste Central Waste Complex (CWC) over the next 30 years. The waste generation sources and waste categories are also described.

This document is intended to be used as a reference for short- and longterm planning of the Hanford treatment, storage, and disposal (TSD) activities over the next several decades. By estimating the waste volumes that will be generated in the future, facility planners can determine the timing of key waste management activities, evaluate alternative treatment strategies, and plan storage and disposal capacities. In addition, this document can be used by other waste sites and the general public to gain a better understanding of the types and volumes of waste that will be managed at Hanford.

For the past five years, a 30-year waste volume forecast has been collected annually from onsite and offsite generators that are planning to ship or currently are shipping waste to the Hanford Site. The waste is generated from ongoing operations and maintenance activities, deactivation activities, decontamination and decommissioning (D\&D) of facilities, and environmental restoration (ER) activities. The generators provide details about the amount of waste to be generated each year, the containers that will be used to ship the waste, and the specific waste characteristics that help determine the proper TSD requirements. This document, which is primarily based upon the 1994 Solid Waste Forecast, oniy provides information on the annual LLMW and TRU TRUM waste volumes; a summary of the expected low-level waste (LLW) volumes has been provided as Appendix B. Additional reports will follow that describe the waste characteristics and container types associated with each waste category.

As described in the following list, an additional effort was made this year to enhance the forecast information to improve data quality and provide the data to the users in a more timely manner.

- Optional Forecasting: For those waste generators that generate less than $1 \%$ of the total waste expected at the Hanford Site, the option to authorize the use of the 1993 forecast instead of completing a new 1994 forecast was provided if changes had not occurred since the last forecast was submitted. In these cases, those waste generator volumes were carried over from the previous year. This allowed for the data to be available in a more timely manner, because volumes were already reviewed

(a) Pacific Northwest Laboratory is operated by Battelle Memorial Institute for the U.S. Department of Energy under Contract DE-AC06-76RLO 1830. 
and did not require additional database input. However, some assumptions were used to account for changes in the new forecast request (i.e., maximum and minimum ranges were assumed to be $100 \%$ each; therefore, a range was not included).

- Data Collection Improvements: The data collection methods were revised slightly from previous years. The addition of maximum and minimum volume ranges was requested to assess the relative certainty of the baseline volume (i.e., best estimate). The baseline volume is the focus of this report; however, the maximum and minimum volumes are reported in the overview. section (Section 2.0). In addition, Appendixes C and D display the annual baseline, maximum, and minimum volumes for each waste generator. The maximum and minimum ranges were not available for waste generators that were carried over from previous years and for waste generator information that was obtained from supplementary sources.

- Onsite Program Integration: An extensive programmatic document review was conducted to identify programmatic primary activities that are not forecasted completely or accurately. The document review focused on the complete life cycle of each primary activity within a program. The document review findings are currently being resolved in onsite program integration meetings that could potentially provide waste information for those unforecasted activities (i.e., transition of B Plant,. Spent Nuclear Fuel disposition activities, etc.). These unforecasted activities are identified in this report; however, waste volume information is currently not available.

- Additional References: Several additional references have been used to supplement the forecast information and complete this document. Two sources, which are summarized in Appendix $A$, were used to further define the potential D\&D volumes. An interface control document (Casbon 1993) was used to determine the Remedial Action (RA) volumes; and a PUREX study, WHC-IP-0977 (WHC 1994), was used to provide surveillance and maintenance for that period between transition and D\&D.

Because this document must serve several audiences, the information is described at various levels of detail. Section 2.0 of this report provides an overview of the LLMW and TRU_TRUM waste volumes. The information is described by major Hanford program area and waste category:

- The major Hanford program areas, which have been established to provide consistency with Site-wide planning efforts, consist of the Tank Waste Remediation System (TWRS), Facility Transitions, Environmental Restoration, Other Programs (i.e., Pacific Northwest Laboratory, Liquid Effluent, Spent Nuclear Fuel, Solid Waste, and Analytical Services), Non-Programmatic (i.e., Buried Equipment, 100 Area Construction Services, Geotechnical Engineering Laboratory, etc.), and Offsite. Based on the document review conducted by the onsite program integration effort, the waste generators have been grouped according to their appropriate Hanford program area. 
- The waste categories that have been used to describe the waste are contact-handled (CH) LLMW, remote-handled (RH) LLMW, CH_TRU_TRUM, and RH_TRU_TRUM.

Sections 3 through 7 provide more detailed descriptions of the six Hanford program areas: TWRS, Environmental Restoration, Facility Transition, Other Programs, Non-Programmatic, and Offsite, respectively. Each section provides a description of the program area, profiles of the primary waste generators, and total and annual waste category volumes for both the program area and specific waste generators. In addition, identification of unforecasted activities is provided as a result of the onsite program integration effort. Appendix $A$ describes the assumptions used with supplementary sources of solid waste information. Low-level waste (LLW) volumes are reported in Appendix B. Appendixes $C$ and $D$ describe the annual waste generator volumes by waste class and annual waste class volumes by waste generator, respectively. Appendix E provides the Waste Generator List by Hanford program area. 
WHC-EP-0865

Intentionally Left Blank 


\subsection{OVERVIEW OF 30-YEAR WASTE VOLUMES}

This section provides an overview of the solid waste volumes expected to be shipped to the Hanford Site over the next 30 years by onsite and offsite waste generators. These waste volumes are being sent or are planned to be shipped to Hanford for TSD and range from LLMW that can be treated and sent to the Hanford burial grounds to TRU TRUM waste that will require repackaging and certification prior to being shipped offsite to the Waste Isolation Pilot Plant.

\subsection{MAJOR HANFORD PROGRAM AREAS}

The waste volumes described in this document are subdivided into six major program areas that were derived from the Hanford Site Multi-Year Program Plans. For the purposes of this document, several programs that generate minimal amounts of solid waste have been grouped into Other Programs. Waste generators that could not be assigned to specific program areas were included under Non-Programmatic. Offsite waste generators were grouped under the program area Offsite Waste Generators. See Sections 3.0 through 7.0 for specific information for each program (e.g., primary waste generators, assumptions).

- TWRS - Solid waste from this program is generated by current tank farm operations, retrieval of existing tank farm long-length contaminated equipment, retrieval pumps, miscellaneous equipment that will be used for retrieval of tank waste, and future tank waste treatment projects.

- Environmental Restoration (ER) - This program includes both the D\&D of Hanford facilities and remediation of past practice sites. The facilities currentiy listed on the surplus facilities list have been included in this report, as have several facilities that have been identified as potentially requiring D\&D in the future. In addition, waste that is generated during ER activities, such as excavated soil, will be included within this program description.

- Facility Transitions - This waste results from terminal cleanout and stabilization activities (deactivation) that are occurring at four Hanford Site facilities: PUREX, Plutonium Finishing Plant (PFP), 300 Area Fuel Supply, and B-P1ant. In addition, surveillance and maintenance waste is assumed to be handled by this program. 
WHC-EP-0865

- Other Programs. Several Hanford programs have forecasted minimal solid waste volumes, and these programs have been grouped into 0ther Programs. Analytical Services, Liquid Effluent, Spent Nuclear Fuel (only that stored at K-Basins), Pacific Northwest Laboratory, and Solid Waste (secondary solid waste generated during operations of the TSD facilities) have been included in 0ther Programs.

- Non-Programmatic. Several waste generators that forecast future solid waste are not within a Hanford program (i.e., they are not discussed specifically within any of the Hanford Multi-Year Program Plans). These include facilities such as the Large Sodium Fire Facility, the Fuel Fabrication Building, and the Geotechnical Engineering Laboratory.

- Offsite Facilities. Several offsite facilities have been certified to ship waste to the Hanford Site. Most offsite waste generators are national laboratories, universities, or gaseous diffusion plants conducting activities such as ER, D\&D, and operations/maintenance.

\subsection{WASTE CATEGORIES}

Solid waste can be characterized into four broad categories that will be used to define the waste volumes throughout the document. They are briefiy described below:

- Contact-handled low-level mixed waste(CH_LLMW) - This waste has a dose rate equal to or less than $200 \mathrm{mrem} / \mathrm{h}$ at contact with the waste container that contains radioactivity not classified as transuranic waste or spent nuclear fuel (concentrations of transuranic radionuclides less than or equal to $100 \mathrm{nCi} / \mathrm{g}$ of the waste matrix) and is also defined as dangerous waste in the Washington Administrative Code (WAC) 173-303.

- Remote-handled low-level mixed waste (RH LLMW) - This waste has a dose rate greater than $200 \mathrm{mrem} / \mathrm{h}$ at contact with the waste container that meets the definition for LLW and is also defined as dangerous waste in WAC 173-303.

- Contact-handled transuranic/transuranic mixed waste(CH TRU TRUM): This radioactive waste has a dose rate equal to or less than $20 \overline{0} \mathrm{mrem} / \mathrm{h}$ at contact with the waste container. At the time of assay, this waste contains more than $100 \mathrm{nCi} / \mathrm{g}$ of alpha-emitting isotopes with atomic numbers greater than 92 and half-lives greater than 20 years. TRUM waste is TRU waste that is also dangerous waste as defined in WAC 173-303. 
- Remote-handled transuranic/transuranic mixed waste (RH TRU TRUM): This waste has a dose rate greater than $200 \mathrm{mrem} / \mathrm{h}$ at contact with the waste container. At the time of assay, this waste contains more than $100 \mathrm{nCi} / \mathrm{g}$ of alpha-emitting isotopes with atomic numbers greater than 92 and half-lives greater than 20 years. TRUM waste is TRU waste that is also dangerous waste as defined in WAC 173-303.

- Held waste: Waste that is expected to be shipped to the CWC over the next 30 years; however, a shipping schedule has not been determined.

\subsection{WASTE GENERATOR LIFE-CYCLE PHASES}

Most waste generators within the major hanford programs undergo four life-cycle phases. The phases are described below:

- Operational - This phase is defined as that period of time in which a waste generator's activities are targeted toward a certain plan, project, end product, or service. Often the type of operational activity can categorize a waste generator into a specific program area.

- Transition (deactivation) - This phase is distinguished by specific deactivation activities to eventually turn over the facility to the D\&D program. Facilities in the deactivation phase are generally the responsibility of the Transition Facilities program.

- Surveillance and Maintenance - This phase is defined as that period of time between the completion of transition (deactivation) and D\&D. Programmatic responsibility for waste generated in this phase is currentiy undefined; however, for the purposes of this report, it has been assumed to be the responsibility of Transition Facilities.

- Decontamination and Decommissioning (D\&D) - This phase is defined as that period in which the facility/environment is completely remediated after deactivation has occurred. Waste generators in this phase are the responsibility of the Environmental Restoration program (Bechtel).

The four life-cycle phases are important in that each phase may determine the program that is responsible for the waste generator. For example, if a facility within Liquid Effluents phases into a deactivation mode, then responsibility for the facility would generally be transferred from Liquid Effluents to the Transition Facilities program. 


\subsection{PROJECTED WASTE VOLUMES}

The total volume of LLMW and TRU TRUM waste expected at the Hanford Site over the next 30 years is approximately 235,560 cubic meters. The volume information by major Hanford program and by the four waste categories is provided in the following sections.

\subsubsection{Waste Volumes by Major Hanford Programs}

Figure 2-1 shows the cumulative baseline, minimum, and maximum waste volumes that are expected to be generated by the six major program areas. The baseline total of 235,560 cubic meters displays approximately $45 \%$ of the waste to be generated by Environmental Restoration, $42 \%$ by TWRS, $6 \%$ by Offsite Waste Generators, $3 \%$ by Non-Programmatic Waste Generators, $2 \%$ by Other Programs, and $2 \%$ by Transition Facilities. The minimum total of 203,680 cubic meters shown is due to a decrease in the baseline for TWRS and Facility Transitions. The TWRS minimum estimate is driven by possible variations of the volume of longlength contaminated equipment being sent to the CWC. Facility Transitions' minimum estimate is driven by the uncertainty of the Plutonium Finishing Plant (PFP) being closed down permanently without completing deactivation. The

Figure 2-1. Total 30-Year Solid Waste Volumes by Program Areas.

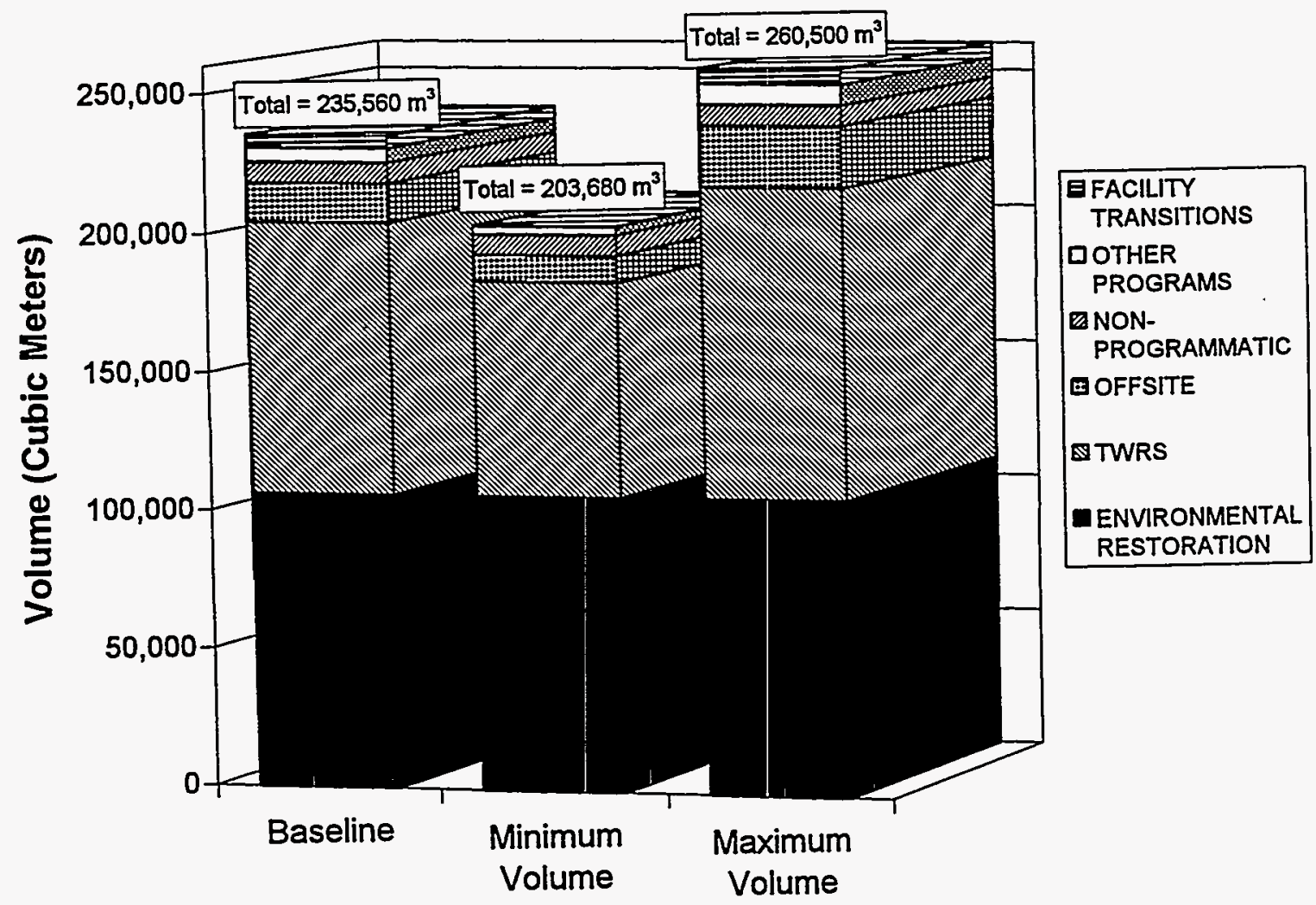


maximum total of 260,500 cubic meters displays a slight increase in TWRS solid waste generation due to uncertainties associated with future waste generation. Al1 other programs essentially majntain their baseline with slight fluctuations in the minimum and maximum estimates to account for any uncertainties commonty associated with future forecasts such as schedule delays or slight mission modifications.

It is important to note that the maximum and minimum. volumes reported here reflect only ranges provided by generators that completed the 1994 Solid Waste Forecast Request. For all other generators, the minimum and maximum volumes were assumed to be the same as the baseline. This-is especially significant for the Environmental Restoration program area. The majority of the waste generators in this program area did not complete a forecast in 1994 , which accounts for the consistency between baseline, minimum, and maximum volumes.

Figure 2-2 illustrates the expected timing of the baseline, minimum, and maximum waste volumes. The years shown are from 1995 through 2024, with a

Figure 2-2. Annual 30-Year Solid Waste Volumes.

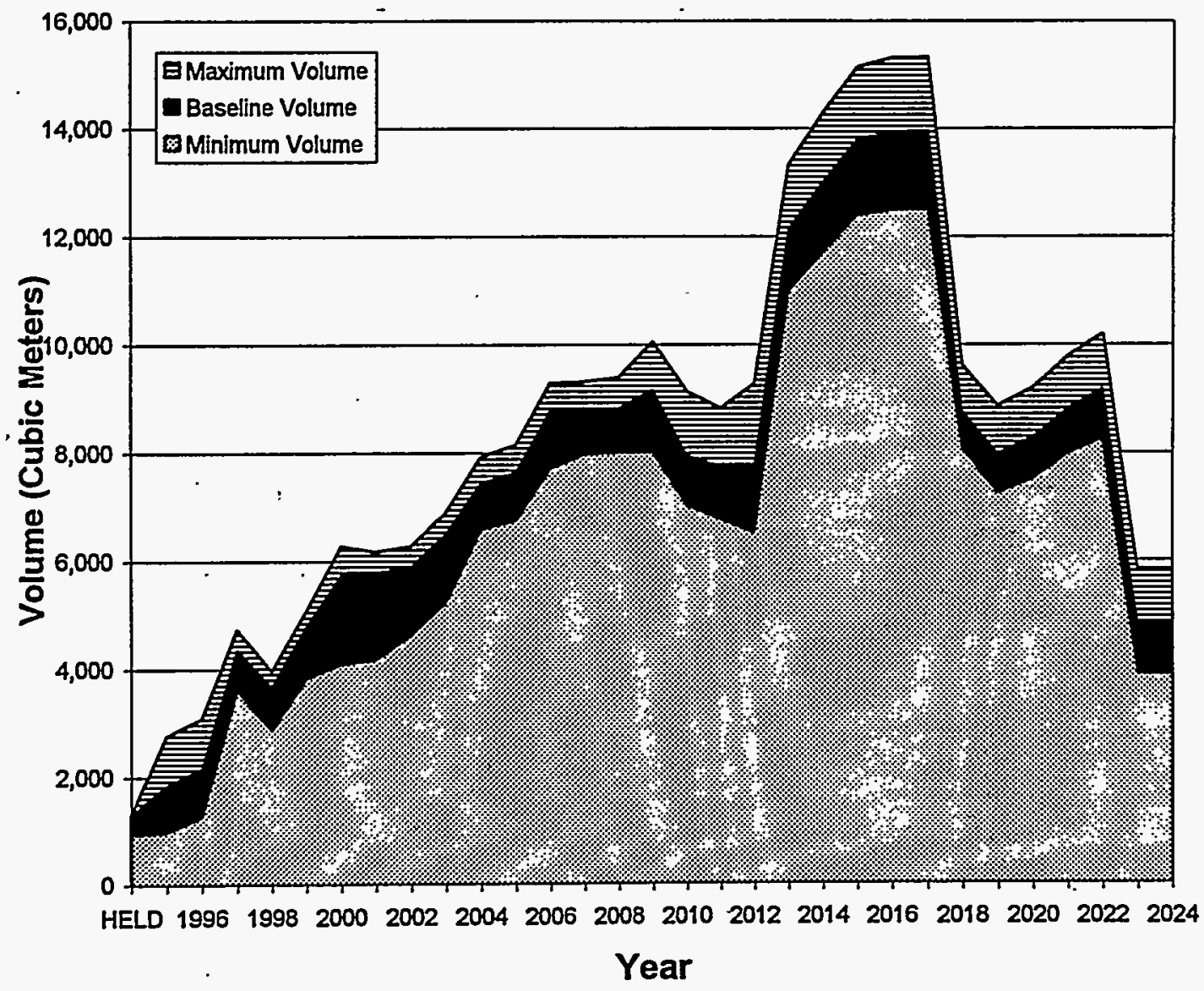


special designation for "HELD" waste. Figure 2-2 shows that the overall baseline volumes peak at approximately 14,000 cubic meters from 2013 through 2017. The two programs contributing significant volumes during these time periods are TWRS and Environmental Restoration. The TWRS program plans on increasing the pieces of long-length contaminated equipment retrieved each year from 2013 through 2017, which accounts for much of this increase. 'In addition, Environmental Restoration volumes from 2013 through 2022 are based upon an assumption that D\&D of onsite facilities not currently identified on the surplus facility list will begin in 2013 and be completed in 2022. The timing of this waste is currently not known, and, as better data become available for these facilities, refinements will be made. The minimum and maximum estimates shown in Figure 2-2 display the same trends reflected in the baseline volumes. Slight variations occur, due primarily to uncertainties associated with forecasting future waste volumes.

Figures 2-3, 2-4, 2-5, 2-6, 2-7, and 2-8 display the annual baseline, minimum and maximum waste volumes for TWRS, Environmental Restoration, Facility Transitions, Other Programs, Non-Programmatic, and Offsite, respectively. In Figure 2-3, there is a gradual increase in waste volumes from 1995 through 2017. This increase corresponds to the time period when the majority of the long-length contaminated equipment in the underground storage tanks is expected to be retrieved. The large decrease in 2018 indicates that all the single-shell tanks will have been retrieved; however, some residual wastes generated during retrieval will continue to be sent to the CWC for TSD purposes. The minimum and maximum volumes in Figure 2-3 indicate slight variations in the baseline due to volume generation uncertainties for the future.

In Figure 2-4, the minimum and maximum volume ranges are not noticeable since ER did not provide ranges; therefore, only the annual baseline volume is displayed. The volumes are expected to increase steadily from slightly less than 3,000 cubic meters in 2002 to over 5,000 cubic meters in 2006, driven primarily by Past Practice Remediation waste volumes. As shown in the figure, the volumes remain fairly steady from 2006 through 2009 and then drop sharply through 2012. In 2013, there is a substantial volume increase that continues through 2022, which reflects the D\&D of facilities. not currently listed as surplus. No volumes have been forecasted for 2023 or 2024 since the supplementary references did not provide estimates for these years.

Figure 2-5 displays the baseline, minimum, and maximum waste volumes for Facility Transitions. Most of the waste will be generated through 2009, which reflects the majority of PFP's deactivation waste. A peak of approximately 500 cubic meters occurs from 2005 through 2006, which is driven by PFP deactivation activities. The decrease in waste volumes from 2010 through 2024 is a result of the expected completion of most LLMW and TRU_TRUM waste deactivation 
activities for PFP. The maximum range varies most from 2010 through 2024 due to uncertainties associated with future missions and waste generation rates. The minimum range is reflective of the possibility of PFP being completely shut down without further deactivation activities occurring.

The baseline, minimum, and maximum waste volumes for Other Programs is displayed in Figure 2-6. Waste generation is fairly constant at 150 to 200 cubic meters for the baseline volume, approximately 250 cubic meters for the maximum volume, and 100 cubic meters for the minimum volume.

Figure 2-7 essentially displays only the baseline volume since the maximum and minimum ranges are not significant for Non-Programmatic waste generators. As seen in the figure, there are five 350-cubic meter peaks that are driven primarily by Buried Equipment's shipping schedule.

Figure 2-3. TWRS Annual 30-Year Solid Waste Volumes.

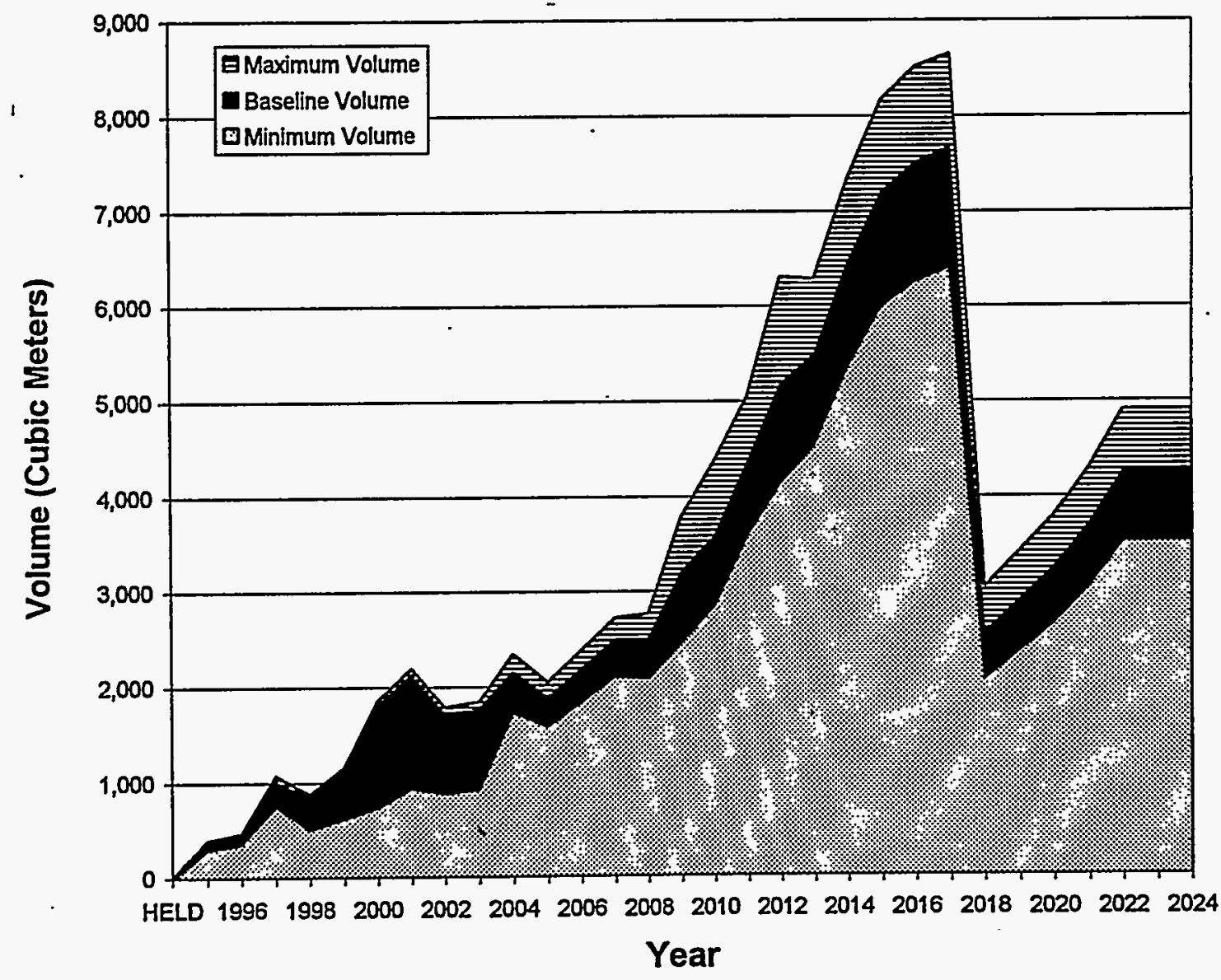


Figure 2-4. Environmental Restoration Annual 30-Year Solid Waste Volumes.

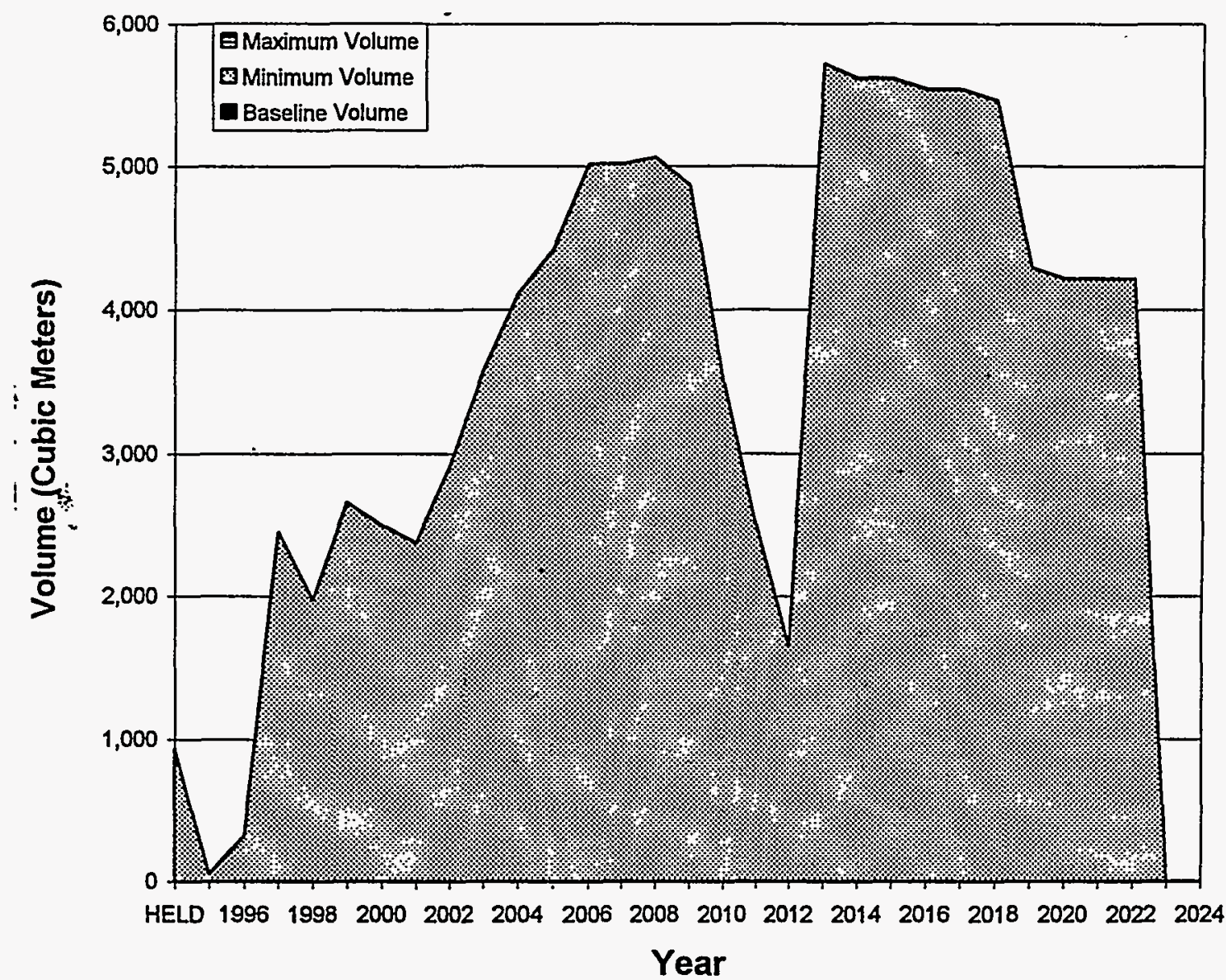

Figure 2-8 displays the baseline, minimum, and maximum waste volumes for offsite waste generators. The baseline volume shows a 600 -cubic-meter peak in 1995 and 1996 that is driven by Argonne National Laboratory waste shipments. The next peak is in 2000 at approximately 600 cubic meters, which reflects Portsmouth Gaseous Diffusion Plant - Energy Systems shipping schedule. The maximum volumes show a significant peak in 1995 and 1996 at approximately 1,400 cubic meters. This peak reflects the possibility that Argonne National Laboratory may ship three times the baseline due to underestimation and improved characterization. The minimum volumes are considerably less in 1995 and 1996 due to the potential for Argonne National Laboratory to not send any waste because of shipping schedule uncertainties. The remaining years, for both the maximum and minimum volumes, follow the same trends as the baseline with slight variations to account for future waste generation uncertainties. 
Figure 2-5. Facility Transitions Annual 30-Year Solid Waste Volumes.

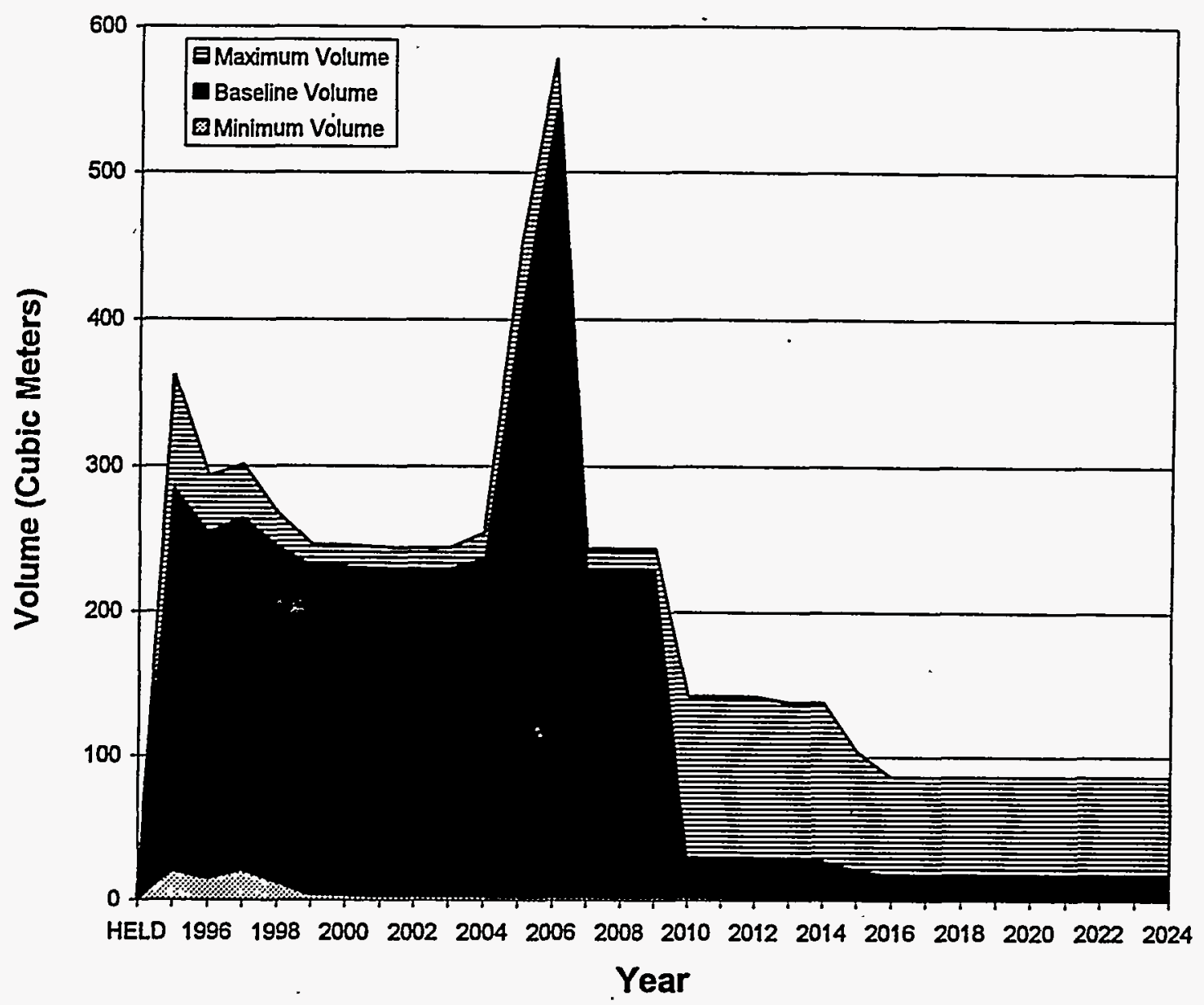

\subsubsection{Waste Volumes by Waste Category}

Figure 2-9 has been provided to illustrate the overall waste category volumes expected to be managed at the CWC. According to the baseline volume, the majority of the waste will be CH_LLMW, which is expected to exceed 180,000 cubic meters over the next $3 \overline{0}$ years. The-minimum estimates display CH_LLMW generation to be approximately 156,000 cubic meters, while the maximum estimate displays 202,000 cubic meters to be generated. In all scenarios, CH_LLMW is the primary waste category that will be received at the CWC.

Figures 2-10, 2-11, 2-12, and 2-13 117 ustrate the annual baseline, minimum, and maximum volumes for each waste category. The following is a summary of the waste volumes and the primary contributors. 
Figure 2-6. Other Programs Annual 30-Year Solid Waste Volumes.

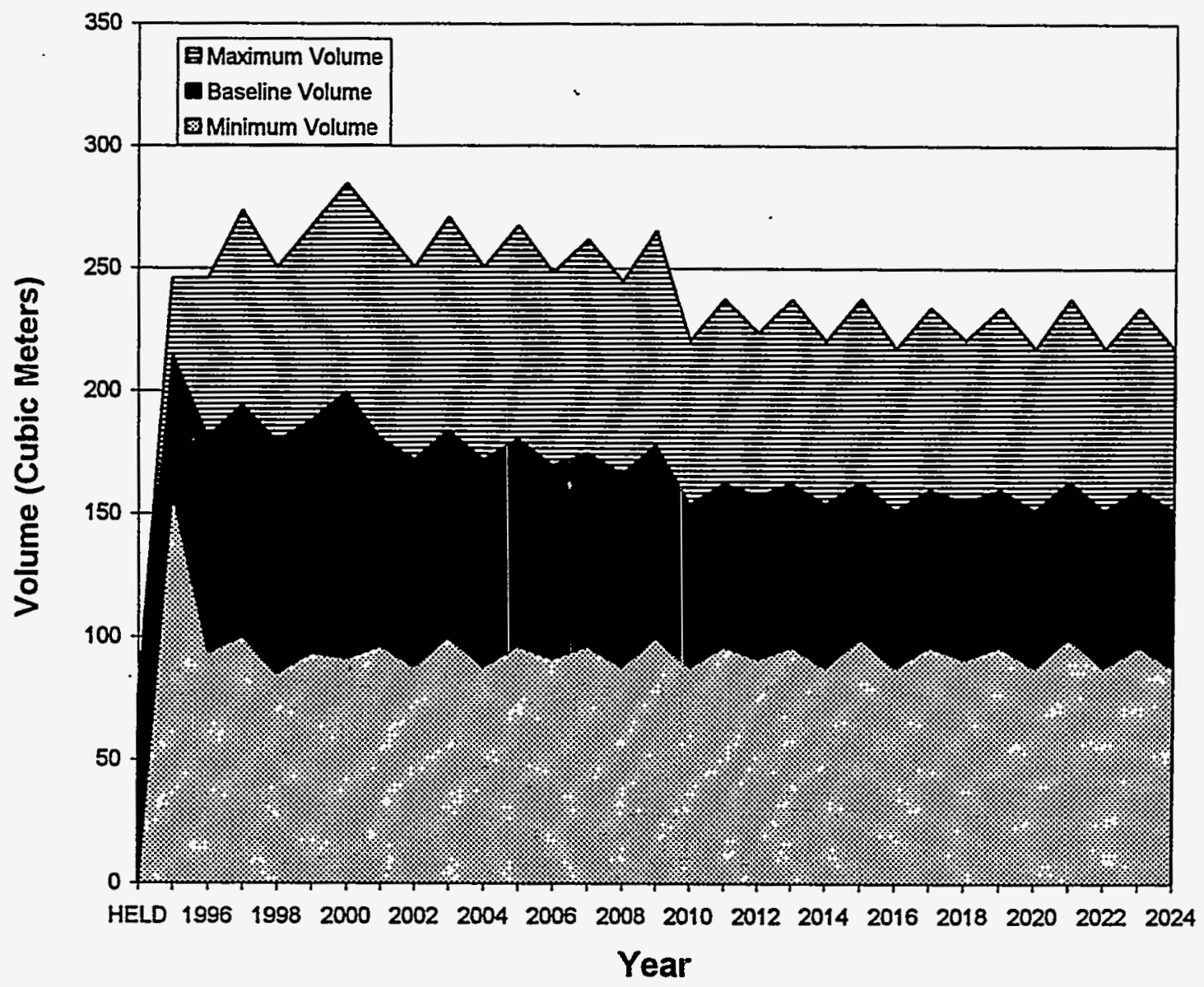

- CH_LLMW: Figure 2-10 displays CH_LLMW baseline volumes gradually increasing from 1995 through 2017, peaking at approximately 10,000 cubic meters from 2015 through 2017 . The waste volumes increase significantly during this time period due to Environmental Restoration and TWRS activities. The minimum volume shows $\mathrm{CH}_{-}$LLMW peaking at 8,500 cubic meters from 2015 through 2017. The peak ${ }^{-}$is driven by Environmental Restoration and TWRS. The decrease is due to the scenario that a decrease in retrieval of TWRS long-length contaminated equipment may occur. The maximum volume peaks at approximately 11,000 cubic meters.

- RH LLMW: Figure 2-11 displays approximately 200 cubic meters of "HELD" RH LLMW. RH LLMW generation gradually increases from 1999 through 2008, where it peāks at approximately 500 cubic meters. The next significant peak is in 2012 at 500 cubic meters for the baseline, 900 cubic meters for the maximum, and 200 cubic meters for the minimum. This peak is driven by Double-Shell Tank Retrieval within the TWRS program. 
Figure 2-7. Non-Programmatic Annual 30-Year Solid Waste Volumes.

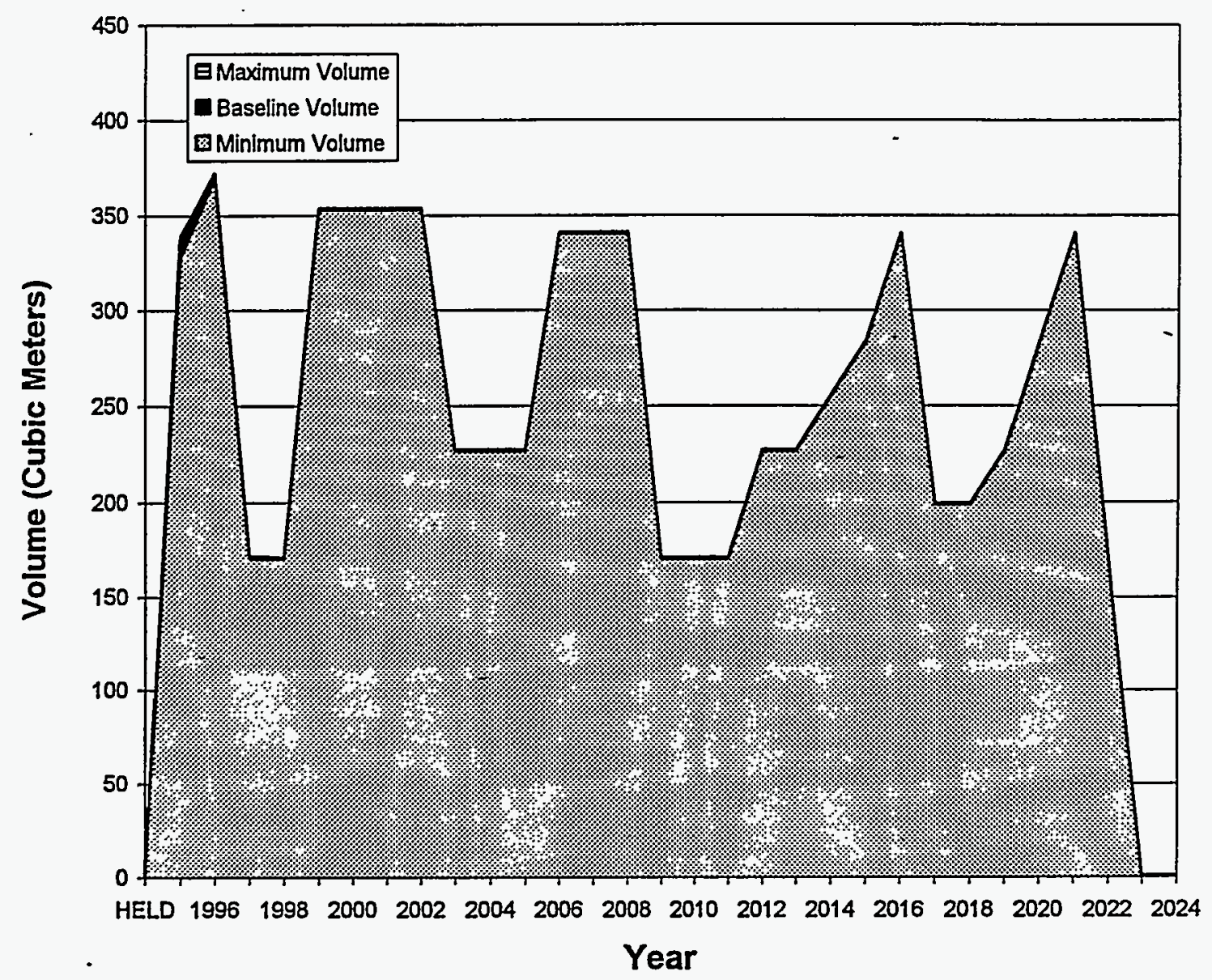

- CH TRU TRUM: Figure 2-12 displays CH_TRU TRUM waste volumes at less thān $9 \overline{0} 0$ cubic meters until 2012 , when the volumes increase to approximately 2,500 cubic meters per year for 10 years. This volume is from the D\&D of non-surplus facilities expected within the Environmental Restoration program. The minimum and maximum volumes vary slightiy from the baseline since the Environmental Restoration program did not provide volume ranges. 
WHC-EP-0865

Figure 2-8. Offsite Annual 30-Year Solid Waste Volumes.

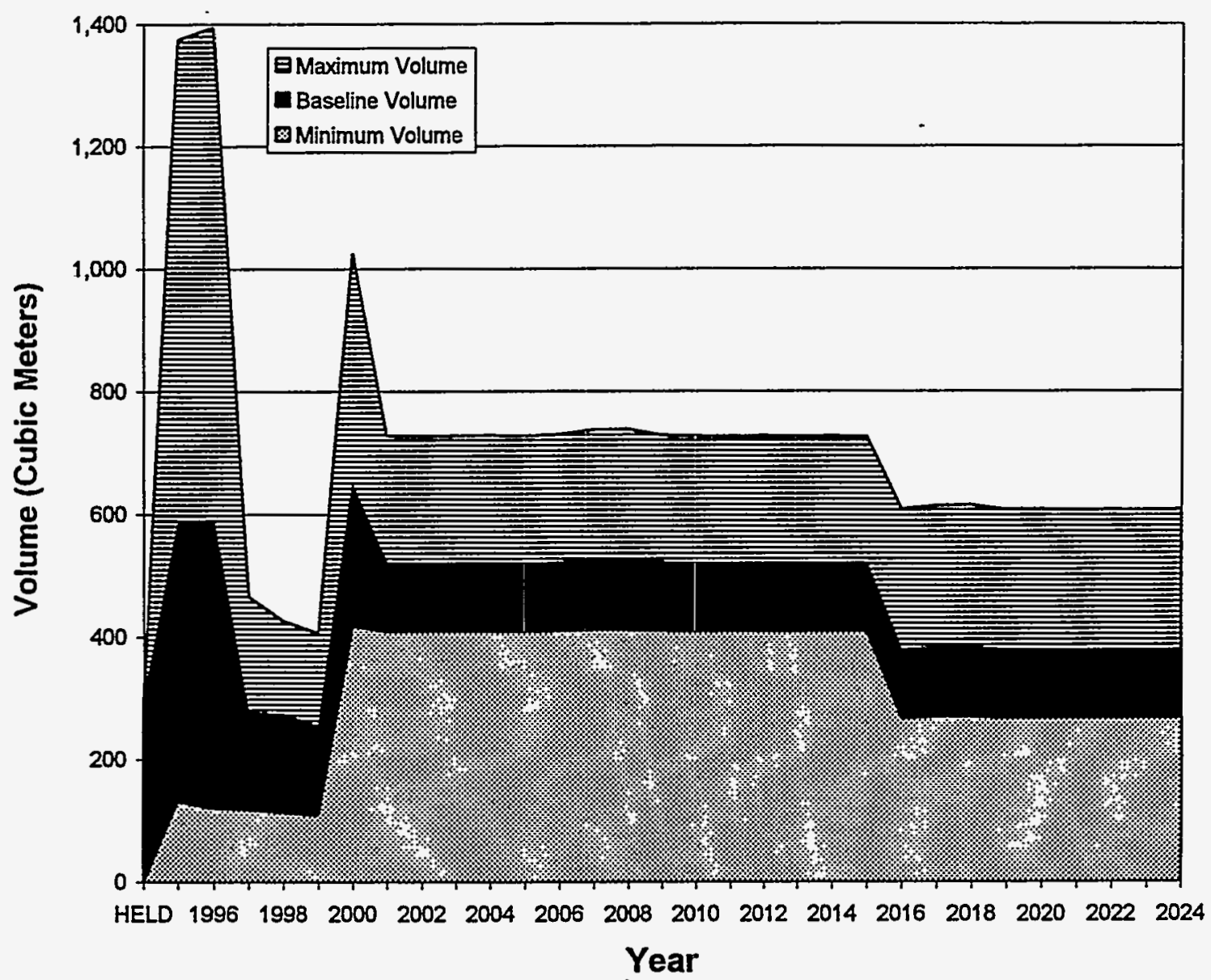

- RH TRU TRUM: Figure 2-13 displays Held waste volumes at 700 cubic meters. The RH TRU TRUM baseline waste volumes remain insignificant until 2010, with an increase of approximately 300 cubic meters for the baseline, 200 cubic meters for the minimum, and 650 cubic meters for the maximum. This peak is driven by the High-Level Vitrification Project and Double-Shel1 Tank Retrieval. The major peak in 2013 through 2022 at approximateiy 1,000 cubic meters is due to the expected D\&D of nonsurplus facilities. The same trend is noticeable for the maximum and minimum volumes. 


\section{WHC-EP-0865}

Figure 2-9. Total 30-Year Solid Waste Volumes by Waste Category.

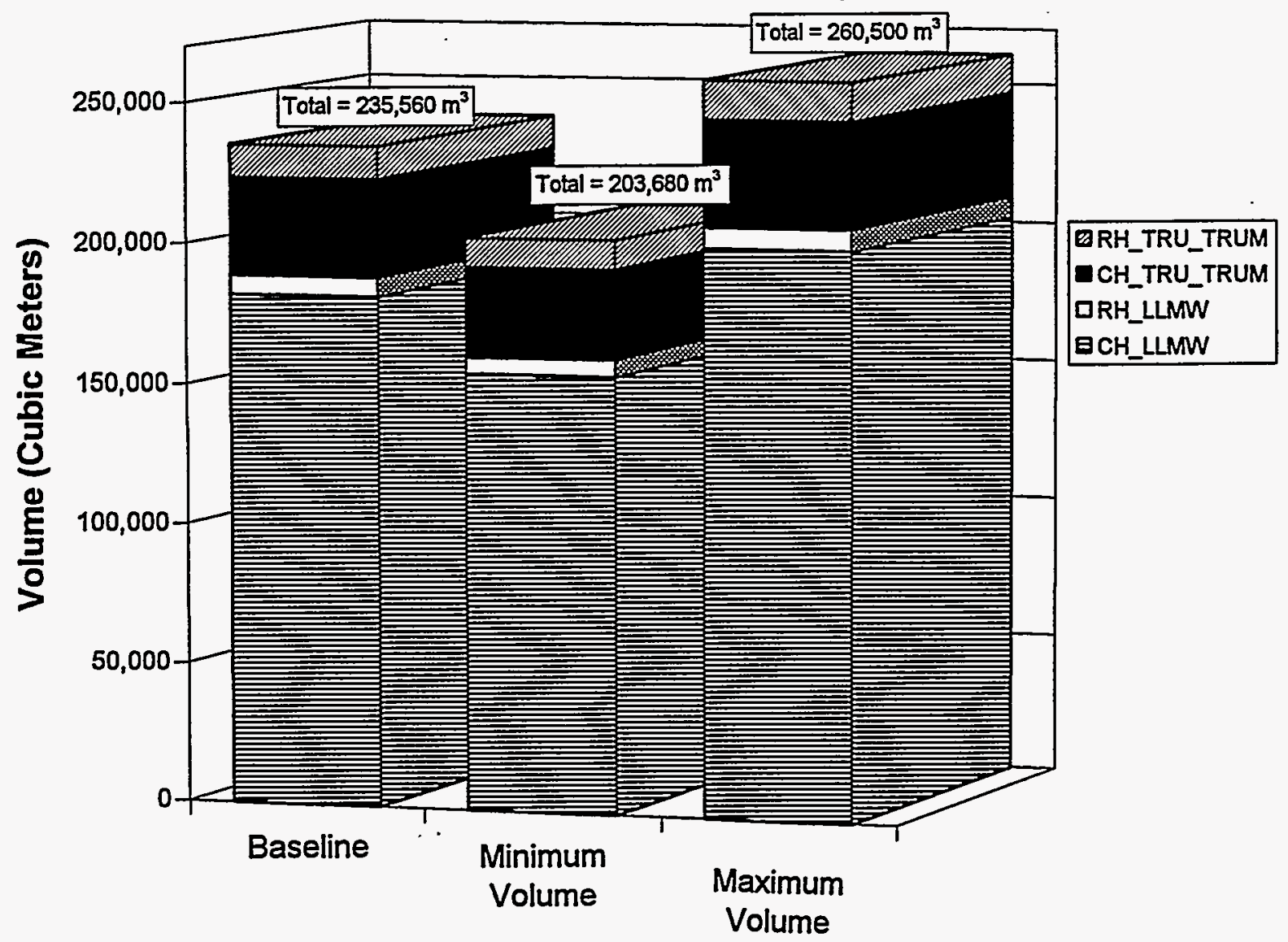

\subsection{COMPARISON WITH 1994 FORECAST VOLUMES}

The Sol.id Waste 30-Year Volume Summary, WHC-EP-0768, (1994) reports that the total 1993 Solid Waste Forecast data for LLMW and TRU TRUM solid waste for the time period of 1995 through 2023 was expected to be approximately 307,150 cubic meters. The 1994 Solid Waste Forecasts report 235,560 cubic meters. as the volume of LLMW and TRU TRUM solid waste to be shipped to the CWC from 1996 through 2024. This is a difference of approximately 71,590 cubic meters from the 1993 Solid Waste Forecasts. The causes of the decrease in forecasted volumes are explained below:

- Cancellation of the Double-Shell Tank Process Tests. This accounts for approximately 20,000 cubic meters. 
Figure 2-10. CH_LLMW Annual 30-Year Solid Waste Volumes.

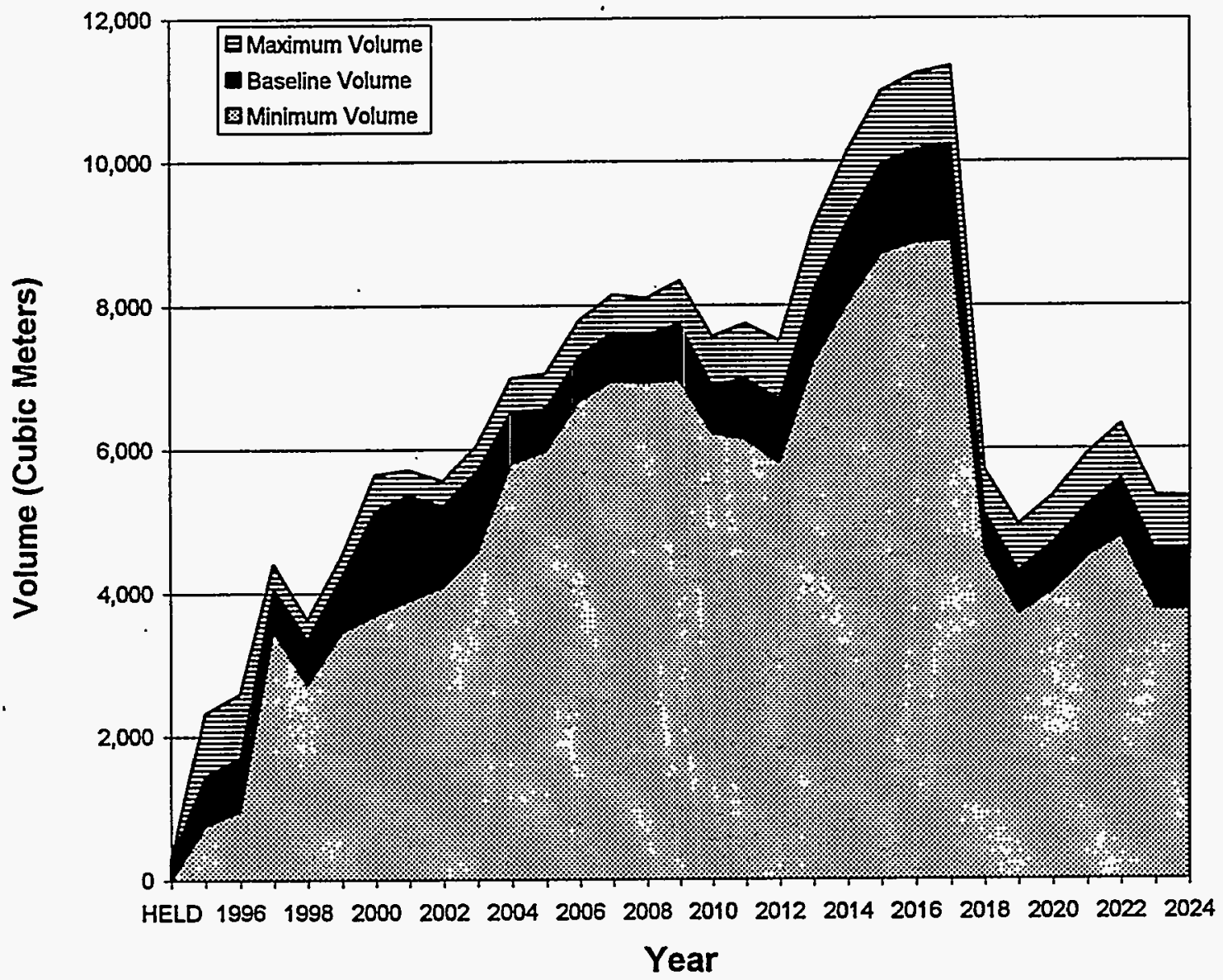

- Elimination of the D\&D volumes for the High-Level Vitrification Plant since these D\&D volumes are expected to occur after the 30-year forecasting period. This accounts for approximately 7,500 cubic meters.

- A decrease in expected Portsmouth Gaseous Diffusion Plant solid waste shipments of approximate7y 15,000 cubic meters. Portsmouth has developed a more defined ER program; therefore, they revised last year's forecast volumes.

- A decrease in the LLW treatment facility (tank farms) solid waste volumes of approximately 5,500 cubic meters. This is due to a change in the treatment technology from a grout to a vitrification treatment. 
Figure 2-11. RH_LLMW Annual 30-Year Solid Waste Volumes.

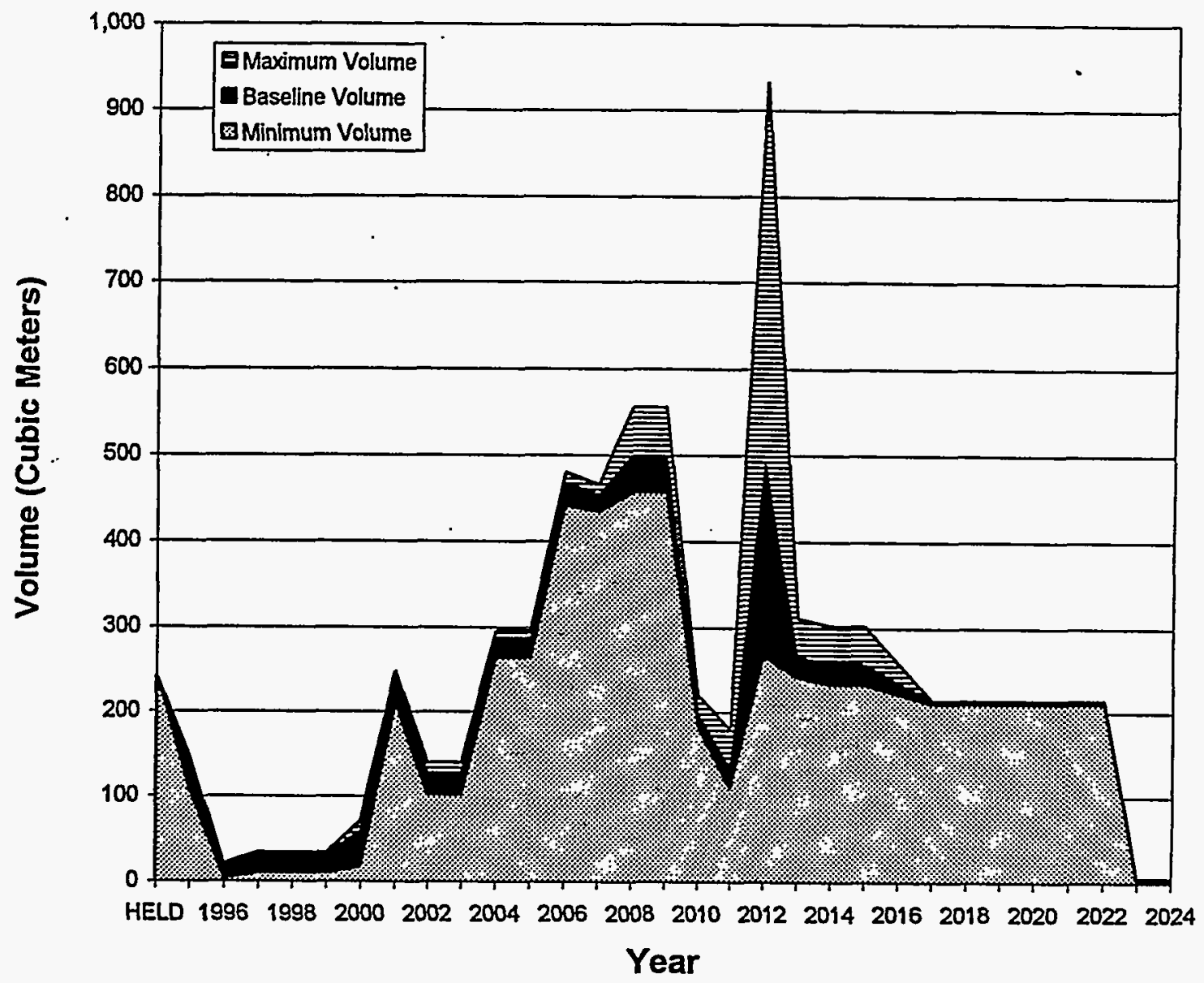

- A decrease in the 200 Area Effluent Treatment Facility (ETF) solid waste volumes of approximately 3,400 cubic meters. The ETF has refined its facility plans and expects to generate less solid waste.

- 222-S Analytical Laboratory and Argonne National Laboratory expect a decrease of approximately 1,000 and 1,500 cubic meters, respectively. The decrease is due to a better understanding of future solid waste generation rates.

- The remaining difference of 20,000 cubic meters between the 1993 and 1994 Solid Waste Forecasts is due to minor waste fluctuations in a number of waste generator forecasts. 
Figure 2-12. CH_TRU_TRUM Annual 30-Year Solid Waste Voiumes.

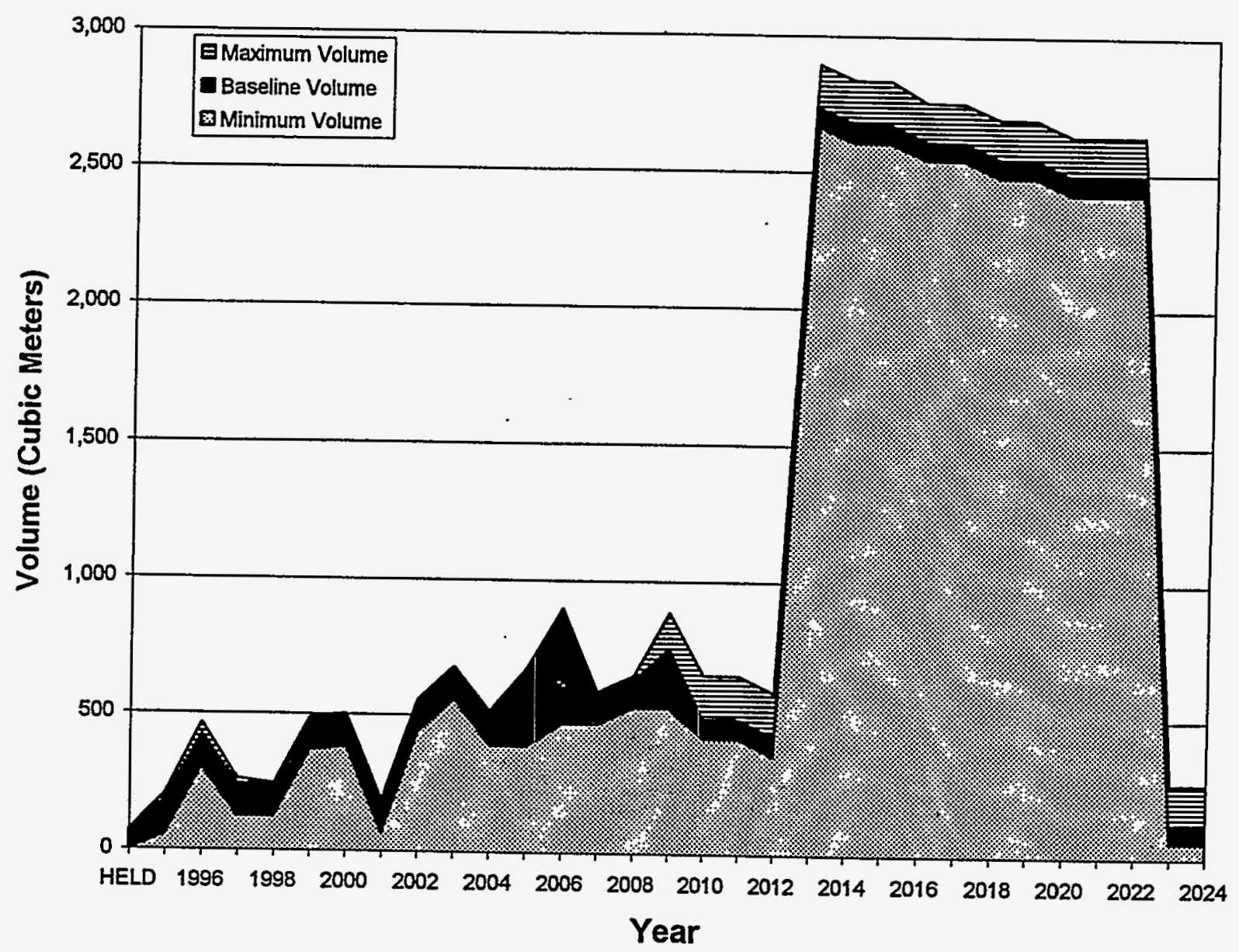

\subsection{DATA OBSERVATIONS}

Data presented in this report establish a starting point from which solid waste management plans can be developed, alternative planning scenarios can be evaluated, and full-scale design for specific TSD facilities can be initiated. The volumes in this report are believed to provide a reasonable estimate of the waste that will be managed at the CWC, and although missions and programs will continue to change, analysis of the primary waste sources indicates that the volumes have a strong potential to increase over time. However, several scenarios are being considered within TWRS and Facility Transitions that could cause a decrease in waste volumes. The factors that could cause significant volume increases or decreases are summarized below: 
Figure 2-13. RH_TRU_TRUM Annual 30-Year Solid Waste Volumes.

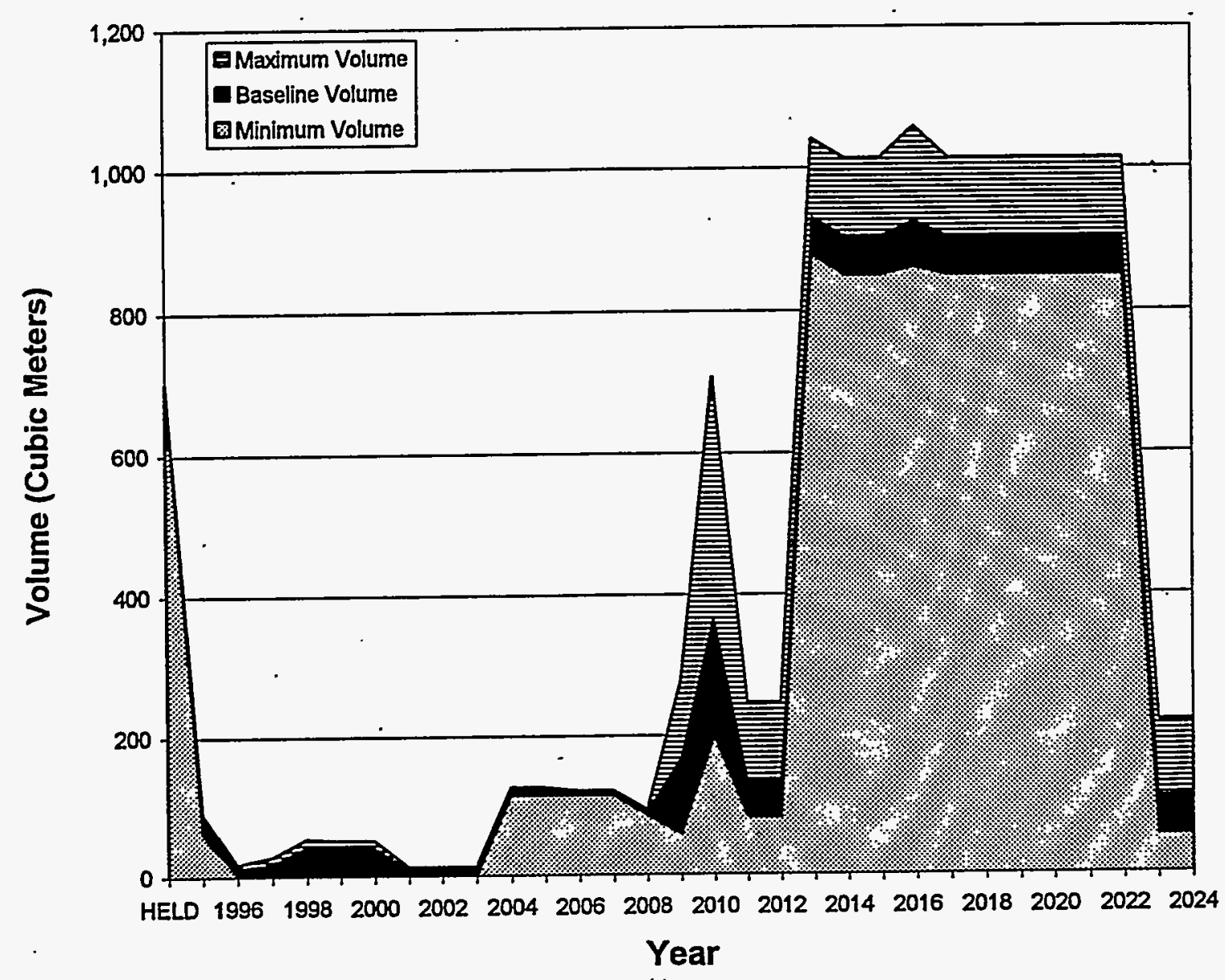

- TWRS - No solid waste estimation has been made for Waste Tank Safety or Waste Characterization activities (i.e., the mitigation pump recently installed in Tank 241-SY-101); therefore, if solid waste is generated from these activities, the waste volumes will increase. In addition, solid waste generated during the storage and transportation of the highlevel vitrified waste has not been forecasted. Although these activities could increase waste volumes, the potential scenario that assumes that the long-length contaminated equipment will not be sent to the CWC could significantly decrease current forecasted waste volumes.

- Environmental Restoration - Oniy those facilities currentiy identified on the 1993 surplus facilities list and 24 other Hanford facilities have been included in this program. As additional facilities are identified for D\&D, it is expected that this waste volume will increase. Fuel 
Supply's 18 facilities, Spent Nuclear Fuel disposition facilities, Liquid Effluent facilities, Analytical Services facilities, and the Fast Flux Test Facility (FFTF) currently have not provided D\&D waste information. The schedule for the non-surplus facilities has been assumed to occur from 2013 through 2022, since surplus facilities are estimated to be completed by 2013. As better data become available, refinements to the schedule can be made.

- Offsite Waste Generators - Additional waste volumes may be sent by offsite waste generators, causing a slight increase in forecasted waste volumes. Portsmouth Gaseous Diffusion Plant - Energy Systems waste volumes could increase if D\&D waste is sent to the Hanford Site. Additional waste could be sent from Paducah Energy Systems if they ship their TRU waste to the CWC.

- Facility Transitions - The four facilities within this program all have potential waste volumes that are not reported in the forecasts. PFP has not included waste information for plutonium stabilization activities or for surveillance and maintenance generated waste. The PUREX tunnels have not reported waste volumes for operations, deactivation, or surveillance and maintenance. The 300 Area Fuel Supply also has not provided waste volumes during surveillance and maintenance. In addition, B-Plant has not provided any solid waste volumes for deactivation or surveillance and maintenance, which could prove to be quite significant. However, if PFP is closed without further deactivation or D\&D, waste volumes for this program could decrease significantly, as shown in Figure 2-1.

- Other Programs - Several activities within the Other Programs designation are not currently forecasted. These activities include all Spent Nuclear Fuel disposition activities, several Liquid Effluent activities (i.e., LERF, 200 Area TEDF, and 300 Area process trenches), and the Waste Sampling and Characterization Facility (WSCF).

- Non-Programmatic - The potential for these waste generators to significantly impact the solid waste system is minimal. 
WHC-EP-0865

\subsection{TANK WASTE REMEDIATION SYSTEM}

This section provides volumes and waste generator descriptions for LLMW and TRU_TRUM solid waste expected from the tank farms. The waste results from current tank farm operations, retrieval of existing tank farm long-length equipment, retrieval pumps and miscellaneous equipment that will be used for retrieval of tank waste, and future tank waste treatment projects.

\subsection{BACKGROUND:}

The TWRS program mission is to store, treat, immobilize, and dispose or prepare for disposal the Hanford radioactive tank waste in an environmentally sound, safe, and cost-effective manner. Tank waste includes current tank waste, future tank waste, and the cesium and strontium capsules.

The TWRS program has three primary activities identified in the Hanford Site Tank Waste Remediation System Technical Strategy (1994) and the MultiYear Program Plan (1994) that could generate solid waste: managing tank waste, processing waste, and managing system-generated waste. The tasks included under each of these primary activities are listed below. It is expected that each of these primary activities will generate solid waste that could potentially be managed at the CWC.

- Manage Tank Waste: This activity includes tank farm operations and upgrades, waste tank safety and characterization, and waste retrieval.

- Process Waste: This activity includes tank waste pretreatment, HLW/TRU waste immobilization, and storage/transfer of immobilized HLW/TRU waste.

- Manage System Generated Waste: The main task associated with this primary activity is immobilization and disposal of LLW.

A11 of the data reported in this section were obtained from the 1994 Solid Waste Forecasts. As discussed in Section 3.4, the forecasts include estimates only for the operational life-cycle phase of several facilities and do not include estimates for tank safety or characterization issues. Supplemental data were either not available or not applicable to the 30-year forecast (i.e., occur after 2024) and therefore have not been included.

\subsection{WASTE GENERATORS}

The total volume of TWRS' LLMW and TRU TRUM solid waste is expected to be 98,090 cubic meters or $42 \%$ of the solid waste at the Hanford Site. Figure 3-1 shows that the top five generators account for $97 \%$ of this waste. 
Figure 3-1. TWRS LLMW and TRU_TRUM Waste Generators.

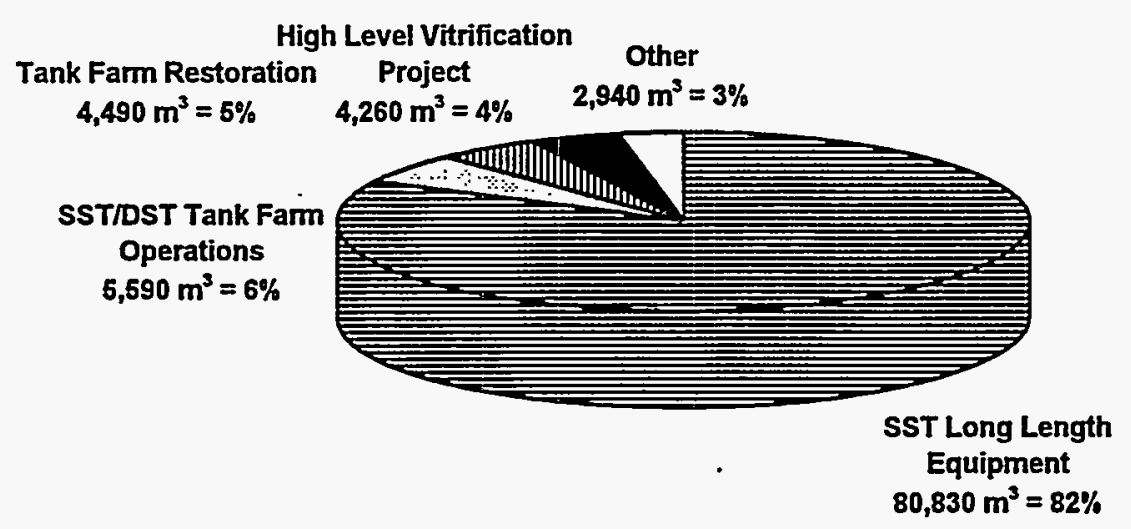

Single-She11 Tank (SST) Long-Length Contaminated Equipment is by far the largest generator of LLMW and TRU_TRUM waste from the TWRS program, generating $82 \%$ of the total volume. Other generators contributing significant volumes of waste include Single-She1l/Double-Shell Tank Farm Operations, Tank Farm Restoration, and the High-Level Vitrification Project. The following information briefly describes these major waste generators. The waste generator's mission, assumptions, and possible waste volume fluctuations are included.

- SST Long-Length Contaminated Equipment (HHC SSTLLE) : Current planning during tank farm cleanup is to remove the long-length pieces of contaminated equipment from the tanks and send this equipment to the CWC for treatment, storage, and disposal. The long-length equipment has been installed in the tanks and is greater than 12 feet in length, with some pieces in excess of 60 feet. Because of the unusual configuration of these wastes, special storage and.handling requirements will be necessary. In addition to the equipment currently in the tanks, estimates have been included for pumps and other miscellaneous equipment that will be used for retrieval of tank waste. The retrieval pumps and equipment are also expected to be sent to the CWC for processing and will have many of the same storage and handling requirements as the long-length equipment currently in the tanks. Equipment used to resolve tank waste safety issues (e.g.; the mixer pump in Tank 241-SY-101) have not been included in these estimates.

This generator forecasted all of its waste to be CH LLMW III. There is a high possibility, however, that much or all of the waste will be remote-handled. In addition, some of this waste may also be TRUM depending on the degree of decontamination at the time of removal from the waste tank. Both of these issues could significantly affect the TSD options being considered for the long-length contaminated equipment. In addition, there is a possibility that the long-length contaminated 
equipment may not be sent to the CWC for processing. This scenario would dramatically reduce the expected volume of waste at the CWC.

The minimum range reported for this waste generator is $85 \%$, and the maximum range is $110 \%$, indicating that the waste generator is fairly confident in the waste volumes forecasted. However, this waste generator assumed that the long-length equipment would be retrieved and sent to the CWC. The ranges do not reflect the possible scenario of the equipment not being shipped to the CWC.

- SST/DST Tank Farm Operations (WHC_TF_OPER): The waste generated from this project includes day-to-day operational and maintenance waste from the 200E and 200W tank farm facilities and old tank farm excess equipment. If the generator continues with daily operations and maintenance, the actual waste volumes should be close to the forecasted volumes. Waste characterization activities are not included within this forecast. The minimum range reported for this waste generator is $80 \%$, and the maximum range is $100 \%$, indicating that the reported baseline volume may decrease slightiy; however, an increase in waste volumes is not expected.

- Tank Farm Restoration (WHC TWP W314): This project will perform several upgrades to tank farm infrästrücture. Waste generated by this project will generally be contaminated soil, debris from construction activities, and removal of outdated or unused tank farm equipment. A detailed scope of work for this project is still being planned, and it is likely that forecasted volumes, characterization, and schedules will be updated with future analysis and planning. The maximum range reported by this waste generator is $100 \%$, which indicates that waste volumes should not be greater than the reported baseline. However, the minimum range reported is $10 \%$. This range is reported in case little dirt excavation is needed.

- High Level Vitrification Project (HHC HLVP): HLVP is a planned treatment/processing facility for high-activity tank waste. Waste volumes associated with the HLVP are based on preconceptual design criteria. Secondary waste will be generated from activities such as spill cleanup and decontamination, general maintenance and housekeeping, and laboratory activities. The waste will generally consist of contaminated failed equipment, metals, paper, plastic, rubber, and other miscellaneous debris. The minimum range for this waste generator is $0 \%$ in the first year of expected operations (2009) and 50\% for the remaining years of operation; the maximum range is $200 \%$ for all years of operation. This indicates that the waste generator is uncertain regarding waste generation volumes because the facility is not operational yet. 


\subsection{PROJECTED VOLUMES}

A description of the waste category volumes for the TWRS program and for the specific TWRS waste generators is provided in the following sections.

\subsubsection{Projected Volumes for TWRS Waste Generator Group}

Figure 3-2 graphically displays the distribution of the waste between waste categories. As shown in the figure, the majority of the waste is assumed to be contact-handled. This is primarily because the long-length equipment from the single-shell tanks has been categorized as contact-handled. But it is likely much of this equipment will actually be remote-handled.

Figure 3-3 shows the annual volumes by waste category. It is evident from Figure 3-3 that there will be a steady increase in the volume of waste generated from 2008 to 2017 . This increase corresponds to the time period when the majority of the long-length contaminated equipment in the underground storage tanks (USTs) are expected to be retrieved. The large decrease in 2018

Figure 3-2. TWRS Total 30-Year Solid Waste Volumes by Waste Category.

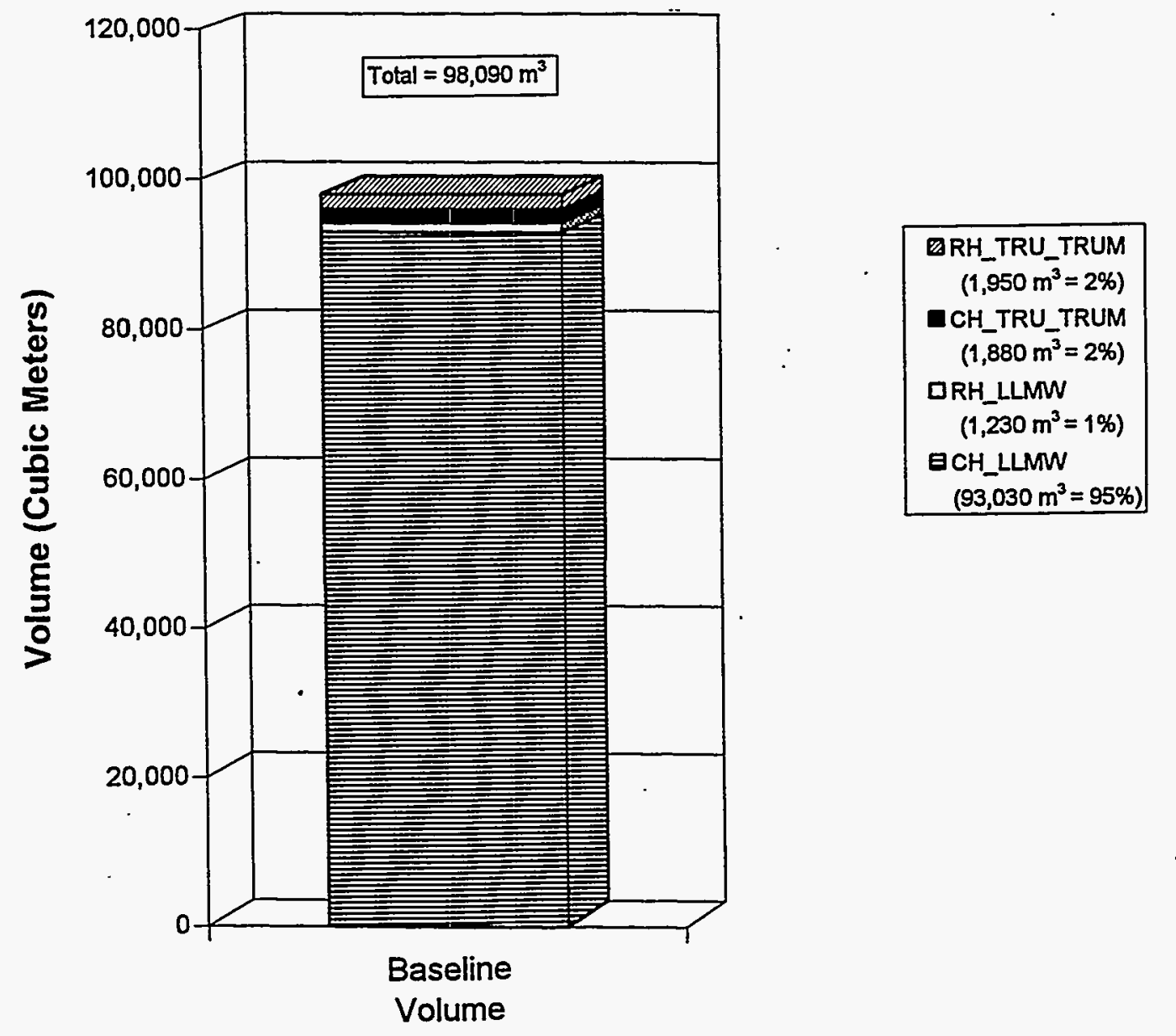


Figure 3-3. TWRS Annual Solid Waste Volumes by Category.

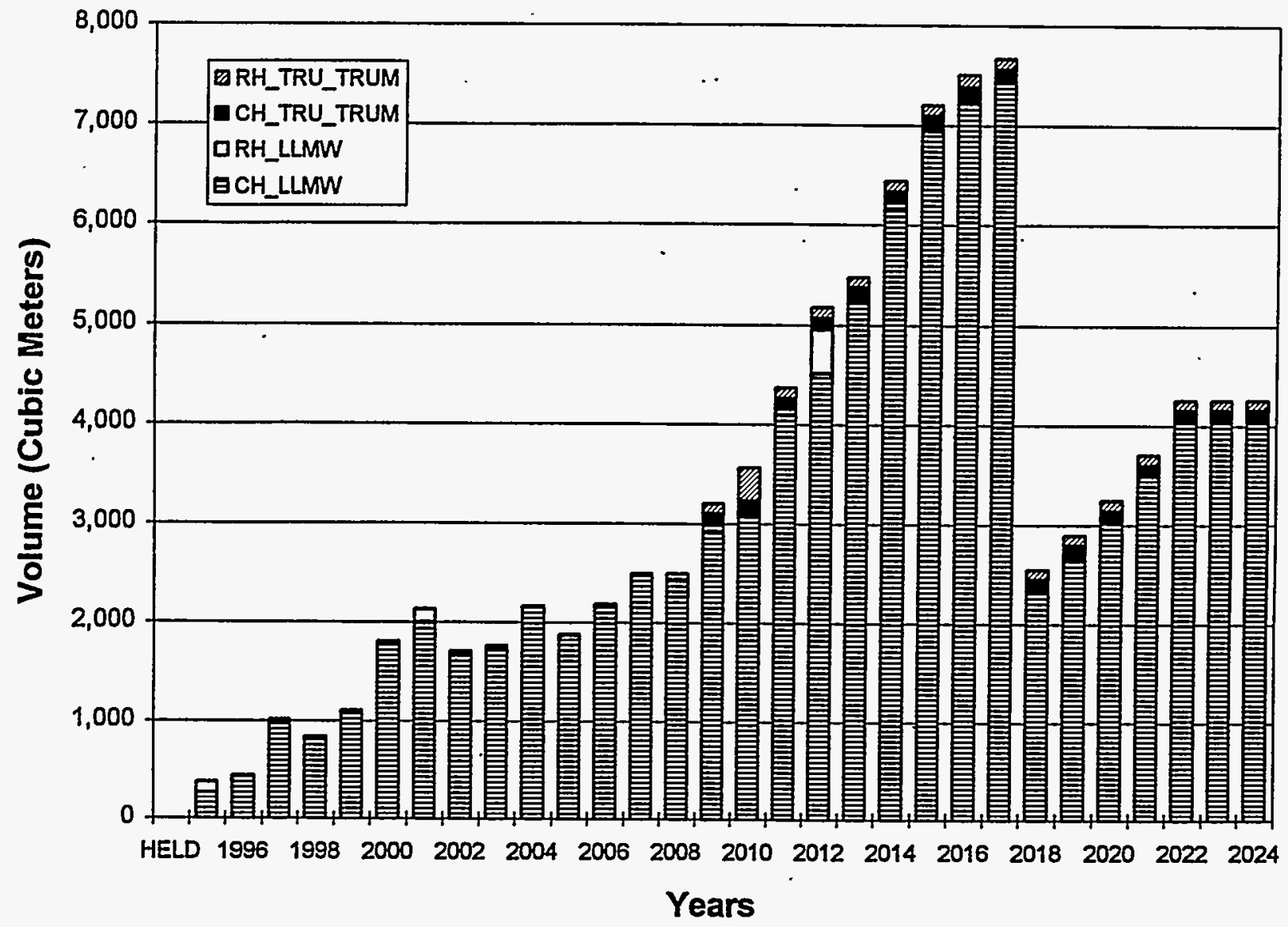

indicates that all the long-length contaminated equipment from the tanks will have been retrieved; however, some residual wastes generated during retrieval will continue to be sent to the CWC for TSD purposes. The remote-handled waste volumes shown in Figure 3-3 result from retrieval of double-shell tank long-length equipment and from the HLVP.

\subsubsection{Projected Volumes by Waste Generator}

There are 16 waste generators in the TWRS program; 11 of these forecast LLMW or TRU TRUM solid waste. Table 3-1 shows the waste volumes by waste category generated by the TWRS waste generators. This table also shows that the top three generators forecast only CH_LLMW. HLVP is the largest generator of TRU_TRUM waste. 
Table 3-1. TWRS Waste Generator Volumes. (a)

\begin{tabular}{|c|c|c|c|c|c|}
\hline Waste Generator & $\underset{\left(\mathrm{m}^{3}\right)}{\mathrm{CH} \text { LLMW }}$ & $\underset{\substack{\text { RH LLMW } \\
\left(\mathrm{m}^{3}\right)}}{2}$ & $\begin{array}{c}\text { CH_TRU TRUM } \\
\left(\mathrm{m}^{\mathrm{J}}\right)\end{array}$ & $\begin{array}{c}\text { RH_TRU TRUM } \\
\left(\mathrm{m}^{3}\right)\end{array}$ & $\begin{array}{c}\text { Total } \\
\left(\mathrm{m}^{3}\right)\end{array}$ \\
\hline $\begin{array}{l}\text { SST Long-Length } \\
\text { Equipment }\end{array}$ & 80,800 & 0 & 0 & 0 & 80,800 \\
\hline $\begin{array}{l}\text { SST/DST Tank } \\
\text { Farm Operations }\end{array}$ & 5,590 & 0 & 0 & 0 & 5,590 \\
\hline $\begin{array}{l}\text { Tank Farm } \\
\text { Restoration }\end{array}$ & 4,490 & 0 & 0 & 0 & 4,490 \\
\hline HLVP & 690 & 0 & 1,880 & 1,700 & 4,260 \\
\hline SST Retrieval & 1,080 & 0 & 0 & 0 & 1,080 \\
\hline DST Retrieval & 0 & 760 & 0 & 250 & 1,010 \\
\hline $\begin{array}{l}\text { SST 106C } \\
\text { STuicing }\end{array}$ & 290 & 0 & 0 & 0 & 290 \\
\hline $\begin{array}{l}\text { DST Retrieval } \\
\text { Systems }\end{array}$ & 0 & 260 & 0 & 0 & 260 \\
\hline $\begin{array}{l}\text { DST 101AZ } \\
\text { Retrieval }\end{array}$ & 0 & 220 & 0 & 0 & 220 \\
\hline LLVVP & 50 & 0 & 0 & 0 & 50 \\
\hline $\begin{array}{l}\text { Tank Waste } \\
\text { Pretreatment } \\
\text { Facility }\end{array}$ & 30 & 0 & 0 & 0 & 30 \\
\hline Total & 93,030 & 1,230 & 1,880 & 1,950 & 98,090 \\
\hline
\end{tabular}

\subsection{DATA OBSERVATIONS}

Figures 3-4, 3-5, and 3-6 represent the completeness of the forecasts in estimating future waste volumes from the TWRS program by the tasks performed under each primary activity. As shown in Figure 3-4, it has been assumed that only the operations phase of the life cycle is applicable for the Manage Tank Waste activity. This figure also shows that wastes associated with waste tank safety and waste characterization have not been forecasted. Volumes have been provided for the other tasks under this activity. 
Figure 3-4. Forecast Completeness for Primary Activities and Associated Life Cycle TWRS - Manage Tank Waste.

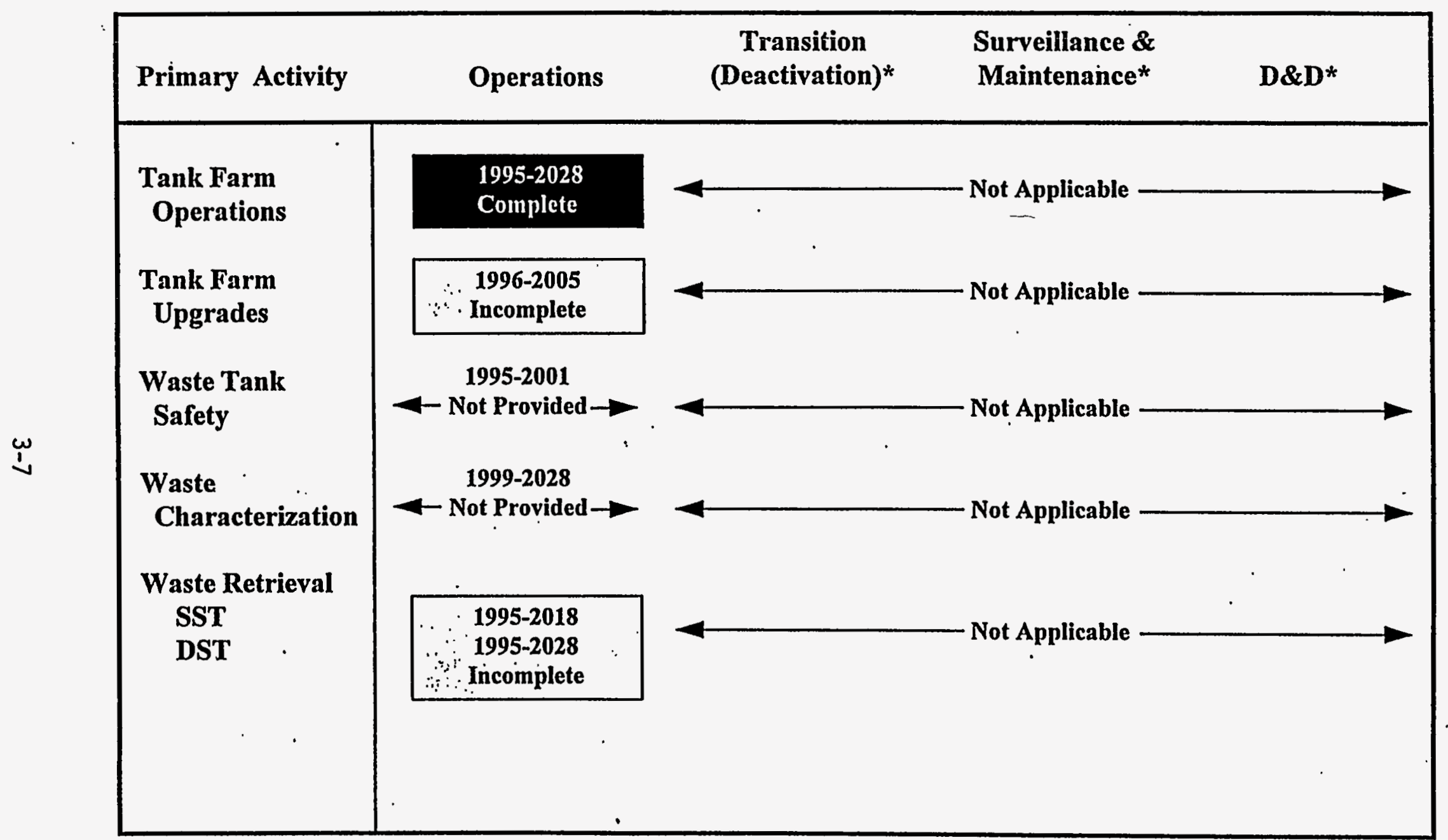

*Assumed by reviewer that these life-cycle elements are not applicable. 
Figure 3-5. Forecast Completeness for Primary Activities and Associated Life Cycle TWRS - Process Waste.

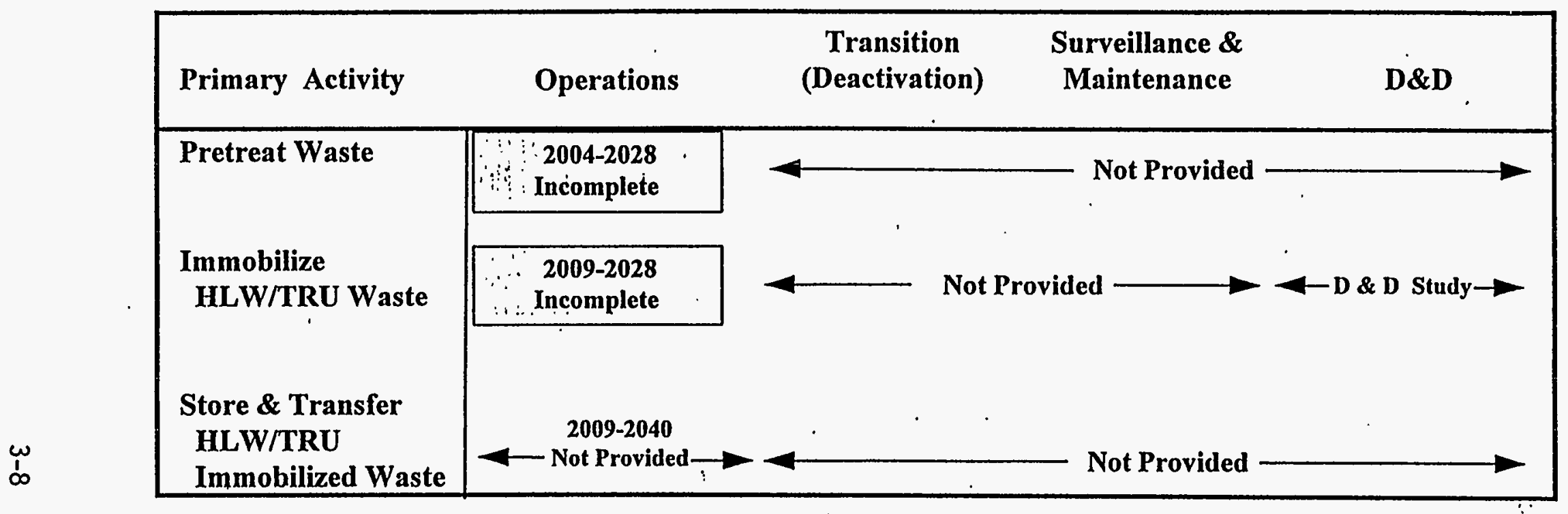

Figure 3-6. Forecast Completeness for Primary Activities and Associated Life Cycle TWRS - Manage System Generated Waste.

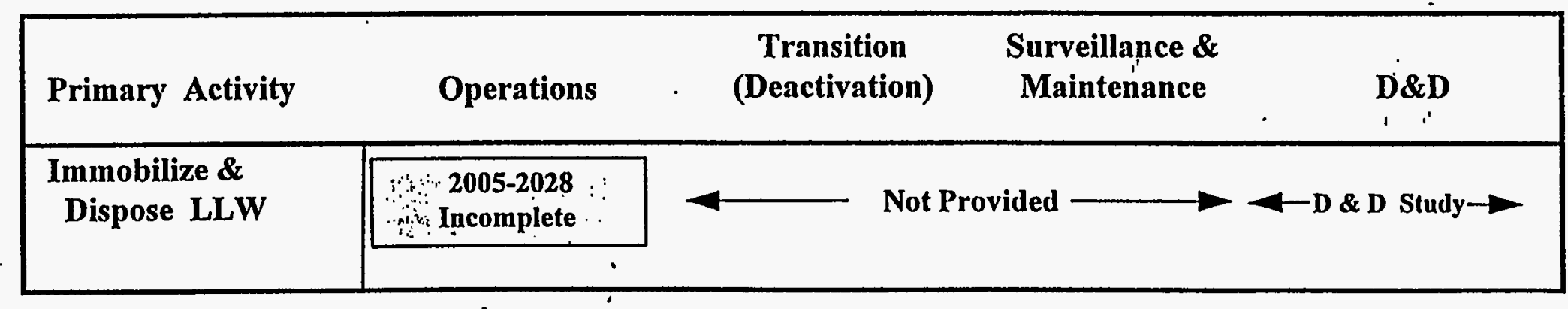


Figure 3-5 shows that only the operational phases for tank waste pretreatment and HLW/TRU waste immobilization have been forecasted. In addition, no estimates have been provided for storage and transfer of the immobilized HLW/TRU. It is expected, however, that the three life-cycle phases that have not been forecasted will occur after the 30-year forecast period and would therefore not be included in the estimates even if they were available. It is not known whether the storage and transfer of the immobilized HLW/TRU waste will generate significant volumes of solid waste.

As displayed in Figure 3-6, only "Operations" has been forecasted for immobilization and disposal of LLW. As with pretreatment and HLW/TRU immobilization, the other three life-cycle phases are expected to occur after the 30 -year forecast period.

Many of the generators in the TWRS program are forecasting waste for projects that are currently in the planning stages and could potentially undergo changes in scope. In addition, changes in the baseline strategy for the TWRS program could significantly impact the amount of waste sent to the CWC over the next 30 years. Leaving the long-length contaminated equipment in the tanks would dramatically decrease the amount of waste generated, while retrieving the single-shell tanks themselves has the potential to substantially increase the amount of waste. Under the current baseline, it is anticipated that the actual volumes might actualiy be greater than the forecasted volumes. The estimates are believed to be conservative, because several activities have not been forecasted (i.e., Waste Tank Safety, Waste Characterization, and Storage and Transfer of Immobilized HLW/TRU waste). 
WHC-EP-0865

Intentionally Left BTank 
WHC-EP-0865

\subsection{ENVIRONMENTAL RESTORATION}

This section summarizes waste volumes from environmental restoration activities on the Hanford Site. These wastes include both D\&D wastes from facilities and remedial action waste from past practice sites. D\&D will be required to some level for all existing and future facilities on the Hanford Site. This document addresses the facilities listed as surplus, which are the responsibility of the Environmental Restoration program, as well as several facilities not currently listed as surplus. The remedial action waste includes waste generated during operations geared toward remediation of contaminated sites, risk mitigation, and regulatory compliance.

\subsection{BACKGROUND}

The mission of the Environmental Restoration program is to decontaminate and remediate facilities and the environment to a designated end-use state. The end-use state may range from unrestricted use to controlled access and is generally determined on a case-by-case basis.

Information in the $1994^{\circ}$ forecasts was used for D\&D and remedial action waste volumes; however, this information is limited. Therefore, several additional sources were used to obtain a more complete representation of these waste streams. Additional D\&D data were obtained from four data sources:

- Fiscal Year (FY) 1993 Solid Waste Forecasts - Includes solid waste volumes and characteristics for D\&D waste of the facilities listed on the surplus facilities list. Forecast volumes from 1993 were used for these facilities, because completion of the 1994 forecast was waived and replaced with an Interface Control Document.

- Draft Report: Waste Stream Evaluation for Lonq-Term Decontamination Study (D\&D Study) - Includes data on the potential D\&D waste from 26 Hanford facilities not currently listed as surplus. Waste volumes from this report were not used for PUREX; however, the PUREX shipping schedule was used. In addition, waste volumes for the LLW Grout Facility and Hanford Waste Vitrification Plant were not used since these projects have been cancelled, and the projects that will replace them are not expected to undergo D\&D during the 30-year forecasting period.

- Estimation of PUREX Equipment and Materials that are Candidates for Removal and Waste Processing During PUREX Plant Closure, WHC-IP-0977 Includes detailed volume and characteristic information of PUREX D\&D waste. In general, volumes from this document were used in place of those contained in the D\&D study for PUREX. 
- Additional remedial action volumes were taken from Remediated Waste Projects, WHC-SD-ER-002 Rev.0 (Casbon 1993). This document describes the responsibilities of the Solid Waste Disposal (SWD) and the Environmental Restoration Division (ERD) programs in the remediation of Hanford's past practice sites. The document also provides waste volume estimates and indicates each program's responsibility. The ERD's' responsibilities include planning; field operations; environmental remediation; and site cleanup of unplanned release sites, inactive waste sites, and other areas contaminated with radioactive or chemical hazards. The SWD is responsible for management of centralized facilities for the receipt, containment, treatment, storage, and disposal of solid waste at Hanford. The SWD will provide storage of LLMW and TRU_TRUM waste; burial of LLW; treatment and disposal of LLMW; and characterization, size reduction, packaging, and shipment of TRU waste to the Waste Isolation Pilot Plant.

At this time, TRU TRUM waste will not be handled by ERD; therefore, the SWD Program will receive this waste. Any waste that the SWD Program has treatment technologies for will also be sent to this program rather than ERD. A detailed description of information included in these sources and the assumptions made when using these sources are found in Appendix A.

\subsection{WASTE GENERATORS}

Approximately 106,310 cubic meters of LLMW and TRU TRUM solid waste is expected from the Environmental Restoration program over the next 30 years. This waste will be generated by 27 waste generators. Other waste generators have been included in the ER program; however, these generators are not expected to generate mixed or transuranic waste. Figure 4-1 shows the primary ER waste generators and the percent of the total waste volume expected from

Figure 4-1. Environmental Restoration LLMW and TRU_TRUM Waste Generators.

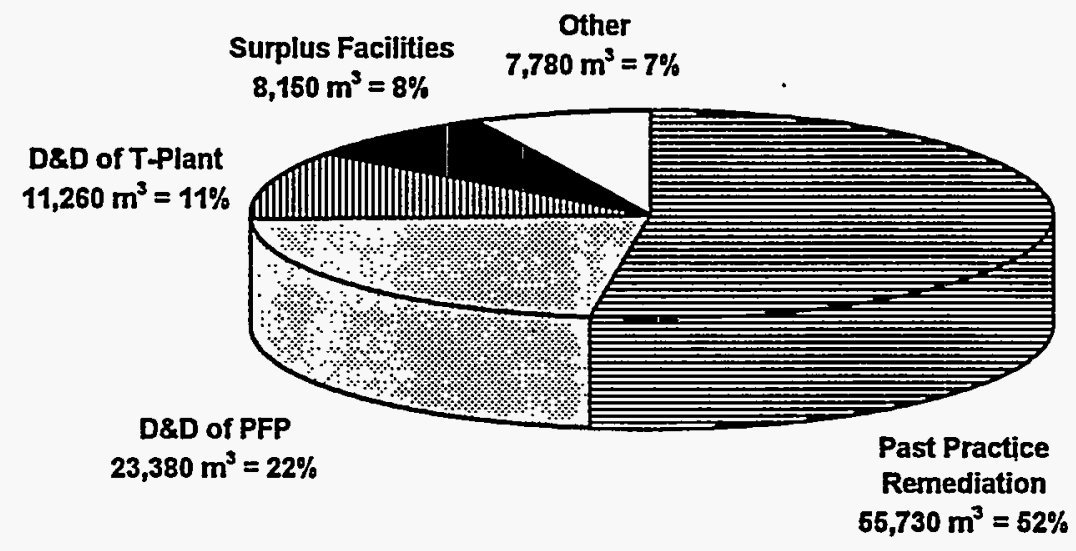


each. Past Practice Remediation is the largest, generating $52 \%$ of the waste. Other primary ER waste generators include D\&D of the PFP, D\&D of T-PTant, and Surplus Facilities. These generators are expected to send $22 \%, 11 \%$, and $8 \%$, respectively, of the ER waste to the CWC. All other ER generators are expected to contribute approximately $7 \%$ of the waste.

The primary ER waste generators are described below. The description includes each generator's past or present mission and the assumptions associated with its estimates:

- Past Practice Remediation Projects (PAST PRAC REM): It is assumed that the SWD program will be responsible for the TRUU TRUM waste and LLMW from past practice sites because SWD will have treatment technologies for this waste. The ERD is responsible for other remediation wastes. The wastes included for this generator are $10 \%$ of the LLMW total for treatment, all expected TRU TRUM waste, and greater-than-Category-3 waste. WHC-IP-0977 did not distinguish between LLW and LLMW for greater-than-Category-3 waste; therefore, for the purpose of this document, this waste was assumed to be LLMW.

- D\&D of Plutonium Finishing Plant (TWHC_PFP): In operational mode, the PFP processed plutonium-based chemical solutions and converted them to metals and oxides. The D\&D volume estimate includes the canyon building $2345-Z$ and its associated buildings 241-Z, 242-Z, and 236-Z. Contaminated materials included in the volume estimate are gloveboxes, pedestal storage, ventilation systems, pencil tanks; and ventilation fans.

- D\&D of T-Plant (TWHC TPLANT): T-Plant, a canyon building, is used to decontaminate equipment and sort and package waste from other facilities. Solid waste from the D\&D of T-Plant includes primarily contaminated equipment such as pumps, tanks, jumpers, and silver reactors.

- Surplus Facilities (HHC SURPLS FAC): Surplus Facilities includes surplus listed facilities and projects. This generator has indicated that waste minimization, recycling, or reduction technologies could reduce forecasted volumes by $30 \%$.

\subsection{PROJECTED VOLUMES}

A description of the waste category volumes for the ER program area and for the individual waste generators is provided in the following sections.

\subsubsection{Forecasted Volumes for Environmental Restoration Program}

As stated above, a total of approximately 106,300 cubic meters of LLMW and TRU TRUM waste is expected to be sent to the CWC by the Environmental Restoration program area. Figure 4-2 displays this waste volume by waste 
category. The majority of this waste, approximately 61,100 cubic meters, is expected to be CH_LLMW. Another 30,800 cubic meters is expected to be CH_TRU_TRUM waste. There is also a significant amount of remote-handled waste expectéd from this program. Roughly 9,400 cubic meters of RH TRU TRUM waste and 5,100 cubic meters of RH_LLMW are expected.

Figure 4-3 shows annual volumes by waste category for the ER program's solid waste. The volumes are expected to increase steadily from slightly less than 3,000 cubic meters in 2002 to over 5,000 cubic meters in 2006, driven

Figure 4-2. Environmental Restoration Total 30-Year Solid Waste Volumes by Waste Category.

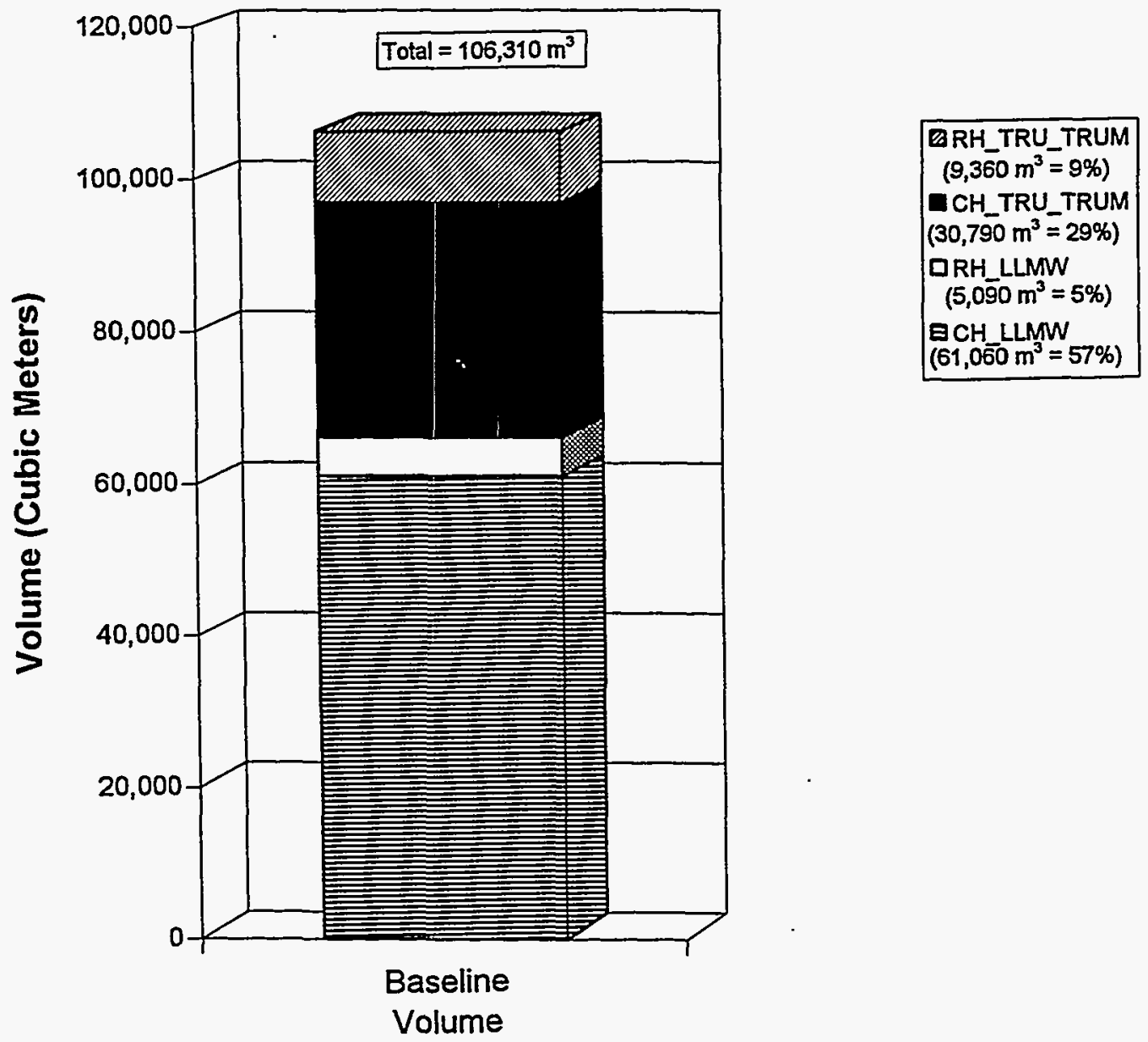


Figure 4-3. Environmental Restoration Annual Solid Waste Volumes by Waste Category.

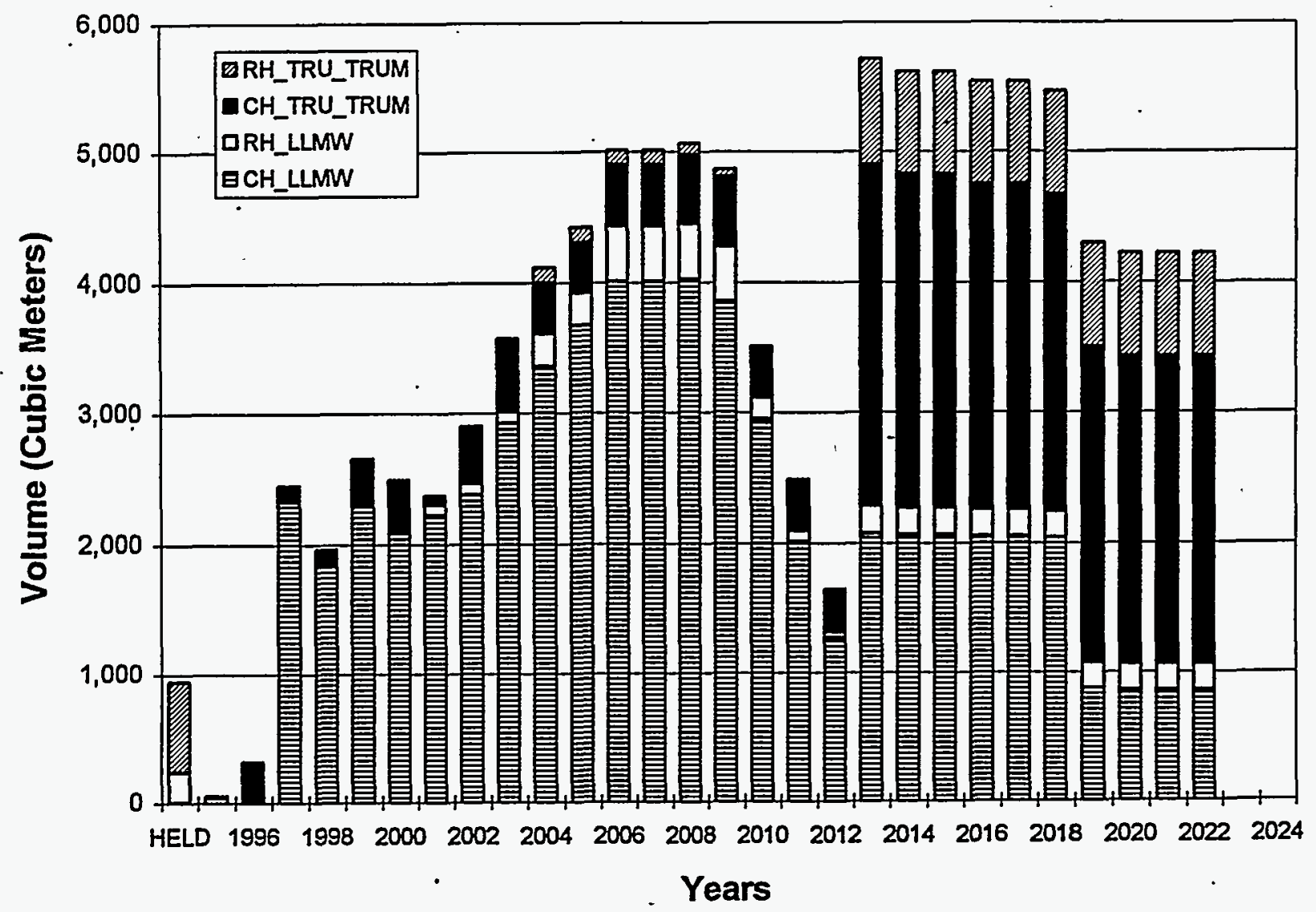

primarily by the CH LLMW volumes from Past Practice Remediation. As shown in the figure, the volumes remain fairly steady from 2006 through 2009 and then drop sharpiy through 2012. In 2013; there is a substantial increase in $\mathrm{CH}$ TRU TRUM and RH TRU TRUM wastes. These increased volumes continue through $20 \overline{2} 2$ and result from the D\&D of facilities not currently listed as surplus. No volumes have been forecasted for 2023 or 2024 since the supplementary references did not provide estimates for these years.

\subsubsection{Forecasted Volumes by Waste Generator}

Table 4-1 lists the waste volumes from each waste generator in the ER program area. Past Practice Remediation contributes the vast majority of the CH LLMW and aiso contributes a significant portion of the CH_TRU_TRUM waste. The D\&D of PFP will generate the majority of the CH TRU TRUM waste and half of

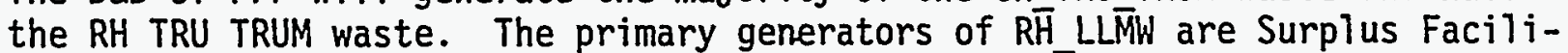
ties and the D\&D of T-Plant. 


\section{WHC-EP-0865}

Table 4-1. Environmental Restoration Waste Generator Volumes. (a)

\begin{tabular}{|c|c|c|c|c|c|}
\hline Waste Generator & $\begin{array}{l}\text { CH LLMW } \\
\left(\mathrm{m}^{3}\right)\end{array}$ & $\begin{array}{l}\text { RH LLMW } \\
\frac{\left(\mathrm{m}^{3}\right)}{}\end{array}$ & $\begin{array}{c}\mathrm{CH}_{-} \text {TRU TRUM } \\
\left(\mathrm{m}^{3}\right)\end{array}$ & $\begin{array}{c}\text { RH_TRU TRUM } \\
\left(m^{\top}\right)\end{array}$ & $\begin{array}{c}\text { Total } \\
\left(\mathrm{m}^{3}\right)\end{array}$ \\
\hline Past Practice Remediation & 49,790 & 0 & 5,940 & 0 & 55,730 \\
\hline D\&D of PFP & 0 & 0 & 18,700 & 4,680 & 23,380 \\
\hline D\&D of T-Plant & 5,440 & $\cdot 1,360$ & 3,570 & 890 & 11,260 \\
\hline Surplus Facilities & 2,850 & 2,770 & 1,830 & 710 & 8,150 \\
\hline $\begin{array}{l}\text { D\&D of } 200 \text { Area Effluent } \\
\text { Treatment Facility } \\
\text { (Project CO18H) }\end{array}$ & 1,600 & 0 & 0 & 0 & 1,600 \\
\hline D\&D of $N$ Reactor & 0 & 0 & 0 & 1,190 & 1,190 \\
\hline D\&D of PUREX & 240 & 60 & 600 & 150 & 1,050 \\
\hline D\&D of PNL 327 Building & 0 & 0 & 30 & 1,020 & 1,050 \\
\hline PUREX Tunnel Waste & 0 & 240 & 0 & 700 & 940 \\
\hline D\&D of PNL 324 Building & 3 & 420 & $\overline{0}$ & 0 & 430 \\
\hline D\&D of 242-A Evaporator & 330 & 20 & $\overline{0}$ & 0 & 350 \\
\hline D\&D of 242-S Evaporator & 330 & 20 & $\overline{0}$ & 0 & 350 \\
\hline D\&D of B Plant & 280 & 70 & 0 & 0 & 350 \\
\hline $\begin{array}{l}\text { D\&D of } 222-S \text { Analytical } \\
\text { Laboratory }\end{array}$ & 40 & 110 & 0 & 0 & 150 \\
\hline $\begin{array}{l}\text { D\&D of } 308 \text { Fuels } \\
\text { Development Laboratory }\end{array}$ & 0 & 0 & 90 & 0 & 90 \\
\hline D\&D of 242-T Evaporator & 60 & 10 & 0 & 0 & 80 \\
\hline $\begin{array}{l}100 \text { Area Transition } \\
\text { (Bechtel) }\end{array}$ & 60 & 0 & 0 & 0 & 60 \\
\hline D\&D of PNL 231-Z Building & 0 & 0 & 30 & 0 & 30 \\
\hline D\&D of PNL 326 Building & 20 & 10 & 0 & 0 & 20 \\
\hline $\begin{array}{l}\text { Well Drilling } \\
\text { Environmental Projects }\end{array}$ & 20 & 0 & 0 & 0 & 20 \\
\hline $\begin{array}{l}\text { D\&D of } 340 \text { Waste } \\
\text { Neutralization Facility }\end{array}$ & 0 & 0 & 0 & 20 & 20 \\
\hline D\&D of TRUSAF & 10 & 0 & 0 & 0 & 10 \\
\hline Total & 61,060 & 5,090 & 30,790 & 9,360 & 106,310 \\
\hline
\end{tabular}




\subsection{DATA OBSERVATIONS}

The ER program area deals primarily with the final phase (D\&D) of the life cycle of a waste generator. Therefore, the other three phases (operations, transition, and surveillance and maintenance) do not apply to these waste generators, and additional waste is not expected due to unforecasted phases. In addition, many of the generators in this section can be used to account for unforecasted D\&D phases of generators in other sections of this document (e.g., D\&D of the TWRS HLVP).

Only 24 facilities at Hanford, except those included in Surplus Facilities, SURPLUS FAC, have been included in these D\&D estimates. The volumes reported here provide a reasonable basis for a D\&D volume estimate, but it should be noted that other potentially large volumes may have been omitted. Specifically, SNF disposition facilities, Liquid Effluent facilities, Analytical Services facilities, Fuel Supply's 18 facilities, and the Fast Flux Test Facility (FFTF) have not provided D\&D waste information. These facilities may represent a significant portion of waste and could dramatically increase the amount of ER waste sent to the CWC.

Significant increases in Remedial Action waste volumes are not anticipated, since it is expected that most of the waste generated from past practice sites will be the responsibility of the ER program. For the purpose of this document, it was assumed that $10 \%$ of the LLMW for treatment would be the responsibility of the SWD program. The percentage could increase as program responsibilities and waste characteristics are better defined. 
WHC-EP-0865

Intentionally Left $\mathrm{Blank}$ 


\subsection{TRANSITION FACILITIES}

This section provides waste volumes and waste generator profiles for the Transition Facilities program. This program currently consists of four facilities: the Plutonium Finishing Plant (PFP), B-Plant, PUREX, and the 300 . Area Fuel Supply.

\subsection{BACKGROUND}

The Transition Facilities' mission is to manage the deactivation activities of those facilities that are no longer in the operational phase. As stored material and wastes are removed, the facilities will be deactivated and transferred to the D\&D program. The types of waste generated within this program can be operational waste, deactivation waste, and surveillance and maintenance waste. The responsibility of the surveillance and maintenance waste has not been clearly defined, but for this document it has been assumed to be the responsibility of the Transition Facilities program area.

The volumes reported for this program are from the 1994. Solid Waste Forecasts and a document estimating waste generation during PUREX plant closure, WHC-IP-0977 (WHC 1994, pp. 2-6). The PUREX document states that surveillance and maintenance waste will peak at $20 \%$ of the current waste generation rate. In addition, waste. volumes for the D\&D of PUREX were provided in this document (which was discussed in Section 4.0). The schedule was not provided in the PUREX document; therefore, it was assumed that surveillance and maintenance would occur from 1999 through 2012, and D\&D would be from 2013 through 2022.

\subsection{WASTE GENERATORS}

LLMW and TRU TRUM waste generation for the next 30 years is estimated to be approximately $\overline{4}, 400$ cubic meters for the Transition Facilities program. This waste volume is approximately $2 \%$ of the waste expected at the Site for the next 30 years. However, as facilities transition into a deactivation phase, the expected waste volumes associated with this program should increase. Figure 5-1 displays the waste volumes associated with each facility that is currentiy within the Transition Facilities program. As seen in the figure, $96 \%$ of the waste is forecasted by the PFP, $3 \%$ by PUREX, and 7 ess than $1 \%$ by both B-Plant and the 300 Area Fuel Supply.

Descriptions for each of the four facilities within the Transition Facilities program are provided below. The waste generator's mission, assumptions, and potential waste volume fluctuations are included. 
Figure 5-1. Facility Transitions LLMW and TRU_TRUM Waste Generators.

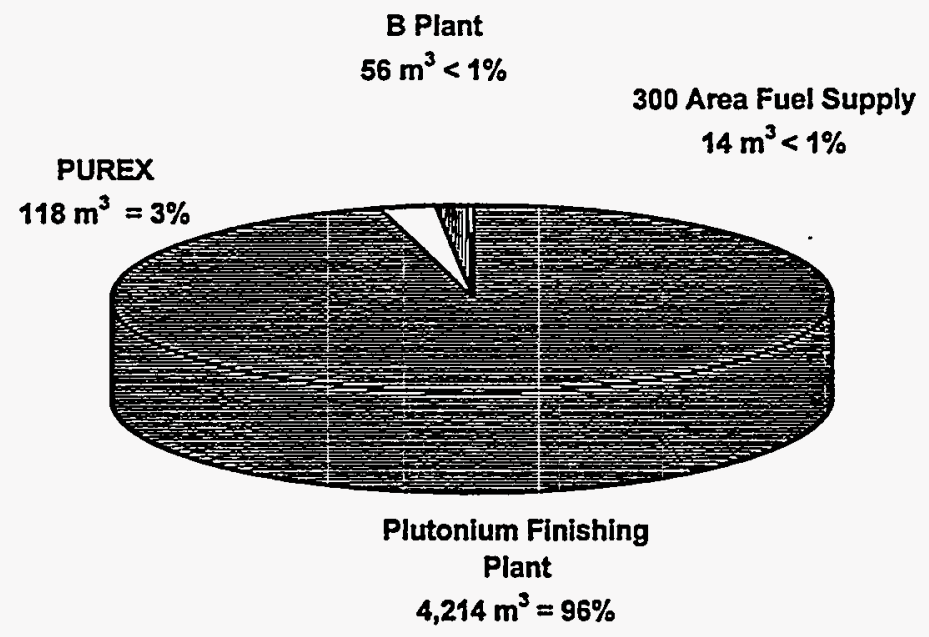

- Plutonium Finishing Plant (WHC PFP): The PFP consists of 14 facilities that generate solid waste. Currently, these facilities define operations as general maintenance activities, which. will continue until transition is complete in 2015. Surveillance and maintenance waste is only included in the forecast for two facilities, 2736-Z and 2736-ZB. It is assumed these facilities will store the stabilized plutonium during the surveillance and maintenance life-cycle phase; therefore, waste volumes are forecasted through 2024. In addition, it is assumed that stabilization of the plutonium will occur in 234-5Z and 236-Z. The plutonium stabilization activities are not forecasted and could result in additional solid waste generation.

The minimum volume range for PFP is $0 \%$ due to the possibility of PFP closure, although this scenario is highly unlikely. The maximum range for PFP varies from $100 \%$ to $500 \%$ due to uncertainties. The $100 \%$ scenario is indicated for the near term (1995 through 2009), and $500 \%$ is indicated for the out years.

- PUREX (WHC PUREX and WHC PUREX SM): Three facilities are included within this forecast: PÜREX, ŪO ${ }_{3}$, and the PUREX tunnels. The operational phase currently consists of general maintenance and surveillance. Transition (deactivation) will be complete in July 1998 for PUREX and in December 1995 for $\mathrm{UO}_{3}$. The tunnels do not currently have a planned mission for remediation. In fact, solid waste generated by the tunnels during this entire life cycle is not forecasted, with the exception of D\&D tunnel volumes abstracted from WHC-IP-0977. Lastly, surveillance and maintenance waste volumes are assumed to be $20 \%$ of the volumes forecasted for 1998 based upon the assumption in WHC-IP-0977.

The minimum range for PUREX varies from $50 \%$ to $100 \%$ due to shipping schedule uncertainties. The maximum range reported is $150 \%$ for 1995 and $200 \%$ for the remaining years due to waste volume uncertainties associated with deactivation and zone reduction activities. 
- B Plant (WHC BPLANT): Three facilities are included within this forecast: 221-B, 271-B, and 225-B (Waste Encapsulation and Storage Facility - WESF). The forecast accounts for operational waste only through 2023; however, transitional activities are planned to be initiated in 1997 with completion in 2001 (Bowen et al. 1992). Completion of operations is expected to gradually phase out as transition is completed in 2001. However, the initiation of the transition schedule is uncertain, explaining the lack of this waste type in the forecast.

The minimum range for B-Plant is $75 \%$, and the maximum range is $125 \%$, which indicates that the baseline volumes are fairly stable.

- 300 Area Fuel Supply Transition (WHC FUEL TRANS): Three facilities are included within this waste generator forecast: the 304 Building, the 303-K Building, and the 313 Building (Waste Acid Ireatment System WATS). The 300 Area Fuel Supply is no longer in its operational lifecycle phase but is now in a transitional life-cycle phase. The schedule for completion of deactivation is currently unknown. While in this life-cycle phase, 313 Building will be demolished due to an unstable roof, all radioactive and hazardous wastes will be removed, and the spent nuclear fuel stored there will be dispositioned. Surveillance and maintenance waste has not been provided.

The minimum and maximum ranges for this generator are both $100 \%$, which indicates that the baseline volumes should not fluctuate.

\subsection{FORECASTED VOLUMES}

A description of the waste category volumes for the Transition Facilities program and for the specific waste generators within the program is provided in the following sections.

\subsubsection{Forecasted Volumes for Transition Facilities Program}

Figure 5-2 displays the overall solid waste volumes for this program. Fifty-three percent of the waste is CH_LLMW, $47 \%$ is CH_TRU_TRUM, and less than $1 \%$ is RH_LLMW. No RH_TRU_TRUM waste is forecasted for this program. 


\section{WHC-EP-0865}

Figure 5-2. Facility Transitions Total 30-Year Solid Waste Volumes by Waste Category.

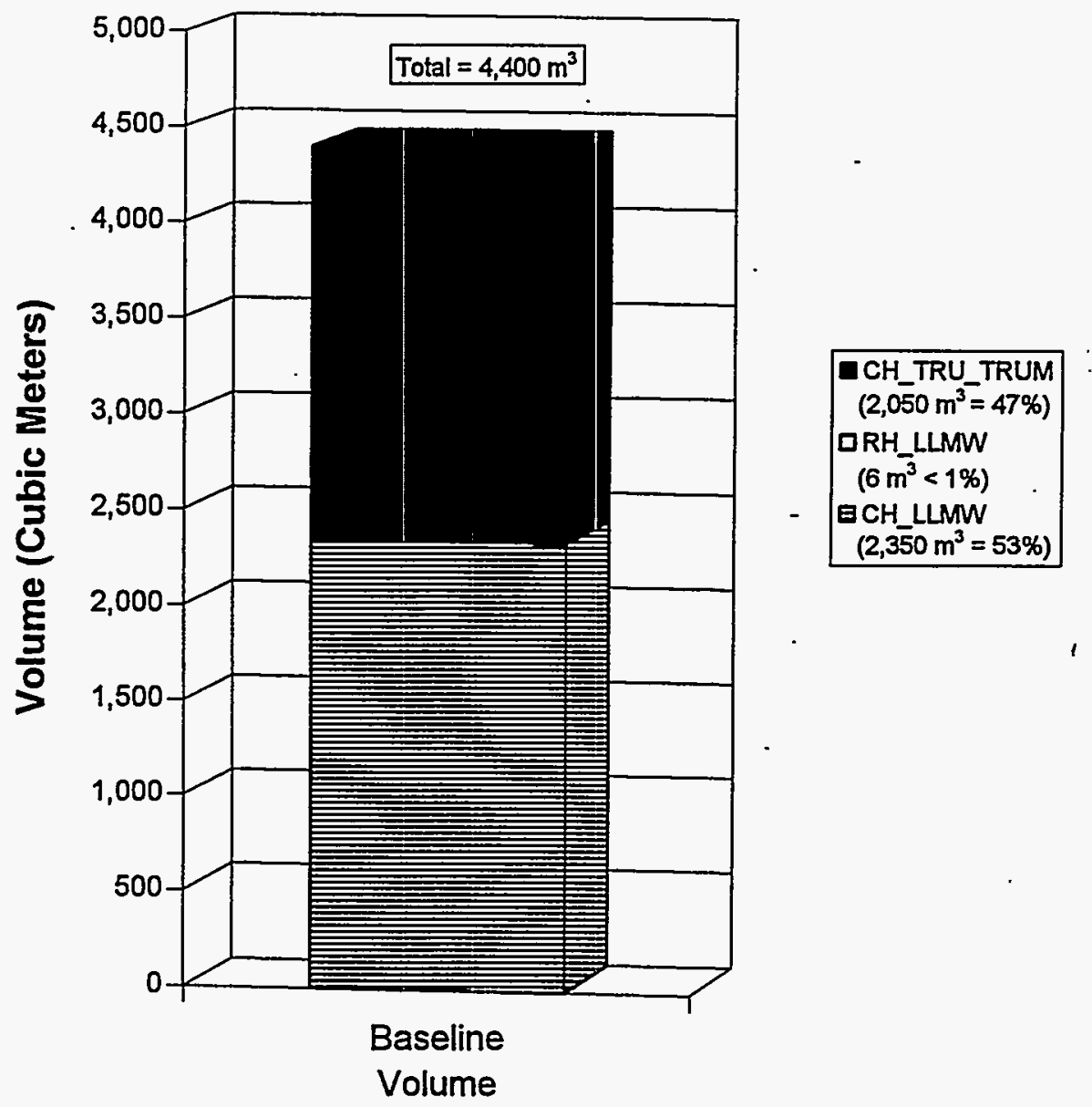

Figure 5-3 shows the annual volumes by waste category. As seen in the figure, most of the waste is forecasted through 2009, which reflects the majority of PFP's deactivation waste. A peak of approximately 500 cubic meters occurs in 2005 through 2006 and is driven by PFP deactivation activities. The decrease in waste volume from 2010 to 2024 is a result of the expected completion of most LLMW and TRU TRUM waste deactivation activities for PFP.

\subsubsection{Forecasted Volumes by Waste Generator}

Table 5-1 displays the amount of waste generated for each waste category and waste generator. The majority of the CH LLMW and CH TRU TRUM waste is generated by PFP. RH LLMW is the only other waste category and is generated 
Figure 5-3. Facility Transitions Annual Solid Waste. Volumes by Waste Category.

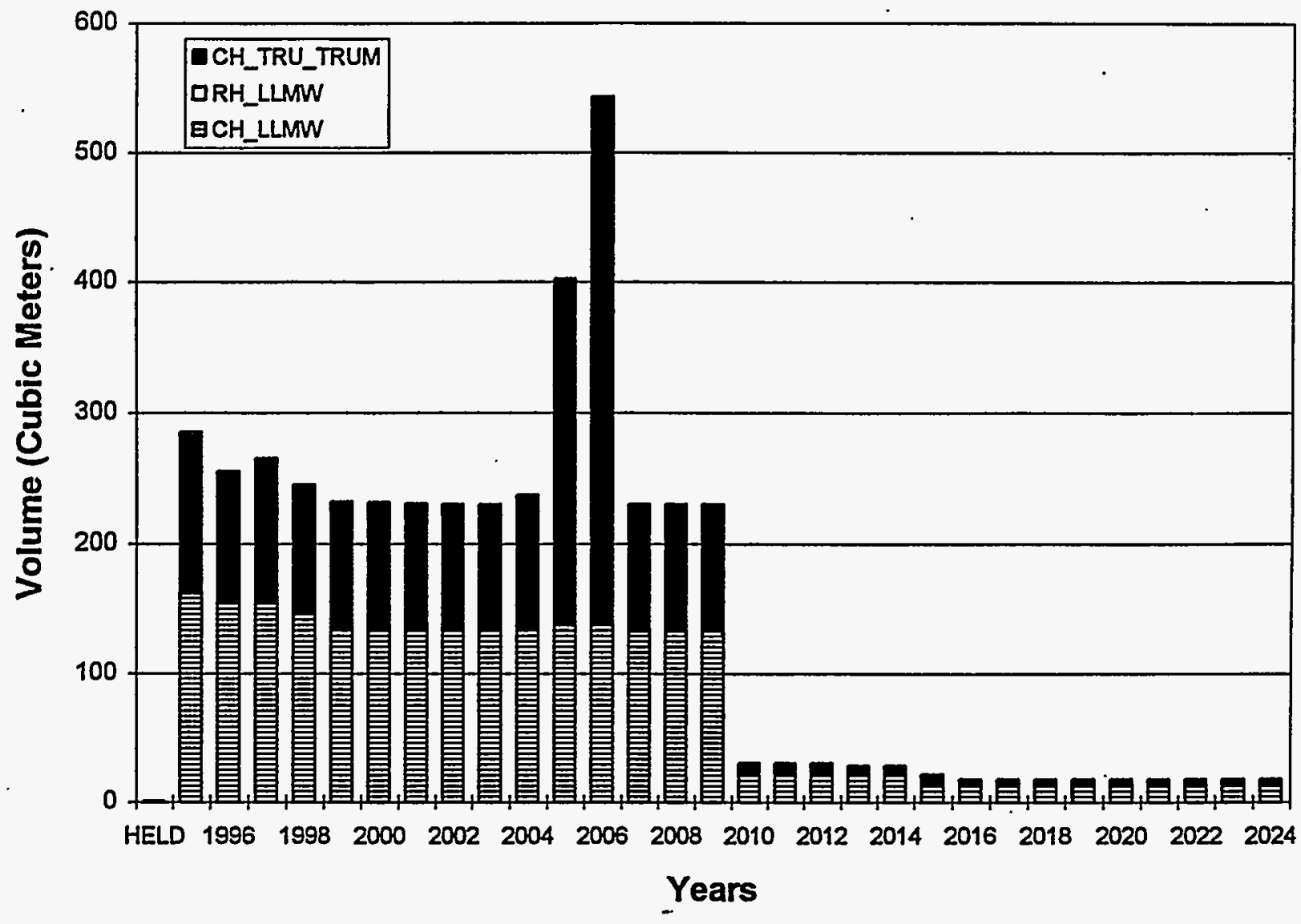

Table 5-1. Transition Facilities Waste Generator Volumes. ${ }^{\text {(a) }}$

\begin{tabular}{|c|c|c|c|c|c|}
\hline Waste Generator & $\underset{\left(\mathrm{m}^{3}\right)}{\mathrm{CH} \text { LLW }}$ & $\begin{array}{c}\text { RH LLMW } \\
\left(\mathrm{m}^{3}\right)\end{array}$ & $\begin{array}{c}\text { CH_TRU TRUM } \\
\left(m^{3}\right)\end{array}$ & $\begin{array}{c}\text { RH_TRU TRUM } \\
\left(\mathrm{m}^{3}\right)\end{array}$ & $\begin{array}{c}\text { Total } \\
\left(\mathrm{m}^{3}\right)\end{array}$ \\
\hline $\begin{array}{l}\text { Plutonium } \\
\text { Finishing Plant }\end{array}$ & 2,190 & 0 & 2,030 & 0 & 4,210 \\
\hline $\begin{array}{l}\text { PUREX } \\
\text { PUREX - SUR/MAIN }\end{array}$ & $\begin{array}{l}70 \\
30\end{array}$ & $\begin{array}{l}0 \\
0\end{array}$ & $\begin{array}{r}20 \\
0\end{array}$ & $\begin{array}{l}0 \\
0\end{array}$ & $\begin{array}{l}90 \\
30\end{array}$ \\
\hline B-Plant & 50 & 10 & 0 & 0 & 60 \\
\hline $\begin{array}{l}300 \text { Area FueT } \\
\text { Supply }\end{array}$ & 10 & 0 & 0 & 0 & 10 \\
\hline Tot'al & 2,350 & 10 & 2,050 & 0 & 4,400 \\
\hline
\end{tabular}


exclusively by B-Plant. As shown in the table, PUREX has two separate waste generator denotations to account for the additional surveillance and maintenance waste information obtained from the PUREX study (WHC 1994). The information obtained from this study is denoted as PUREX - SUR/MAIN.

\subsection{DATA OBSERVATIONS}

Figures 5-4, 5-5, 5-6, and 5-7 display the forecast completeness for PFP, PUREX, B-Plant, and 300 Area Fuel Supply, respectively. Each life-cycle phase is listed on the horizontal axis with the facilities along the vertical axis. Figure 5-4 displays PFP's forecast completeness for each PFP facility. The D\&D life-cycle phase has the D\&D Study listed for each facility. This study is currently a draft report on facilities that are not on the Surplus Facilities List and their associated waste volumes entitled, Waste Stream Evaluation for Long-Term Decontamination Study. Thus the report provides information for these unforecasted activities (these volumes are reported in Section 4.0).

As seen in Figure 5-4, additional solid waste volumes associated with surveillance and maintenance can be expected for PFP. Operational and transitional activities within 234-5Z and $236-Z$ are incomplete, because plutonium stabilization activities are not forecasted and could increase waste volumes. PFP waste volumes also have the potential to decrease if the plant is completely shutdown as a result of the Environmental Impact Statement (EIS) that is being prepared.

Figure 5-5 displays PUREX's forecast completeness for each PUREX facility. The PUREX forecast is complete in capturing operations and transitional waste for PUREX and $\mathrm{UO}_{3}$; however, surveillance and maintenance and D\&D waste is not forecasted for PUREX and $\mathrm{UO}_{3}$. Document WHC-IP-0977 (see Section 5.1) is listed in Figure 5-5 for the surveillance and maintenance and D\&D 1ifecycle phases (D\&D volumes are discussed in Section 4.0). As seen in the figure, additional waste volumes can be expected for the PUREX tunnels. Operational, transitional, and surveillance and maintenance wastes are not forecasted for the tunnels.

Figure 5-6 displays B-Plant's forecast completeness for each B-Plant facility. The figure shows that the forecast reports only operations of these facilities and does not include transitional, surveillance and maintenance, or $D \& D$ solid waste information. The D\&D waste volumes are supplemented with the D\&D Study and discussed in Section 4.0. However, the other life-cycle phase waste volumes are not forecasted, which indicates that a significant volume of waste can be expected in the near future if deactivation activities are initiated in 1997 as p]anned. 
Figure 5-4. Forecast Completeness for Primary Activities and Associated Life Cycle Plutonium Finishing Plant Transition Projects.

\begin{tabular}{|c|c|c|c|}
\hline Facilities & $\begin{array}{lc}\text { Operations } & \begin{array}{c}\text { Transition } \\
\text { (Deactivation) }\end{array} \\
\end{array}$ & $\begin{array}{c}\text { Surveillance \& } \\
\text { Maintenance }\end{array}$ & D\&D \\
\hline $232-Z$ & N/A & Bechtel & Bechtel \\
\hline $231-Z$ & 1094 - 2015 Comiplete & - Not Provided - & D \& D Study \\
\hline $234-5 Z$ & 1994 - 2015 Incomplete & - Not Provided - & - D \& D Studs \\
\hline $236-Z$ & 1994 - 2015 Incomplete & Not Provlded & \\
\hline $242-Z$ & 1994-2015 Complete & & \\
\hline $241-Z$ & 1994-2015 Complete & & \\
\hline $291-Z$ & 1994-2015 Complete & & \\
\hline $243-Z$ & 1994-2015 Complete & & \\
\hline $2736-Z$ & 1904 - 2015 Complete & Complete & \\
\hline $2736-Z B$ & 1994- 2015 Complete & Complete & D \& D Stud \\
\hline $2736-Z A$ & 1994-2015 Complete & & \\
\hline $2731-Z$ & 1994 - 2015 Complete & & \\
\hline 2904-ZA & 1994-201s Complete & & \\
\hline $2904-Z B$ & 1994-2015 Complate & N Not: $\mathrm{F}$ & D \& D St \\
\hline
\end{tabular}


Figure 5-5. Forecast Completeness for Primary Activities and Associated Life Cycle PUREX Transition Projects.

\begin{tabular}{|c|c|c|c|}
\hline Primary Activity & $\begin{array}{c}\text { Transition } \\
\text { (Deactivation) }\end{array}$ & $\begin{array}{l}\text { Surveillance \& } \\
\text { Maintenance }\end{array}$ & D\&D \\
\hline $\begin{array}{l}\text { Terminal Clean Out } \\
\text { and Stabilization } \\
\text { of PUREX }\end{array}$ & 1998 Complete & WHC-IP-977 & WHC-IP-977 \\
\hline $\begin{array}{l}\text { Terminal Clean Out } \\
\text { and Stabilization } \\
\text { of } \mathrm{UO}_{3}\end{array}$ & 1995 Complete & WHC-IP-977 & WHC-IP-977 \\
\hline Tunnels & Not Provided & $\longrightarrow$ & WHC-IP-977 \\
\hline
\end{tabular}


Figure 5-6. Forecast Completeness for Primary Activities and Associated Life Cycle B-Plant Transition Projects.

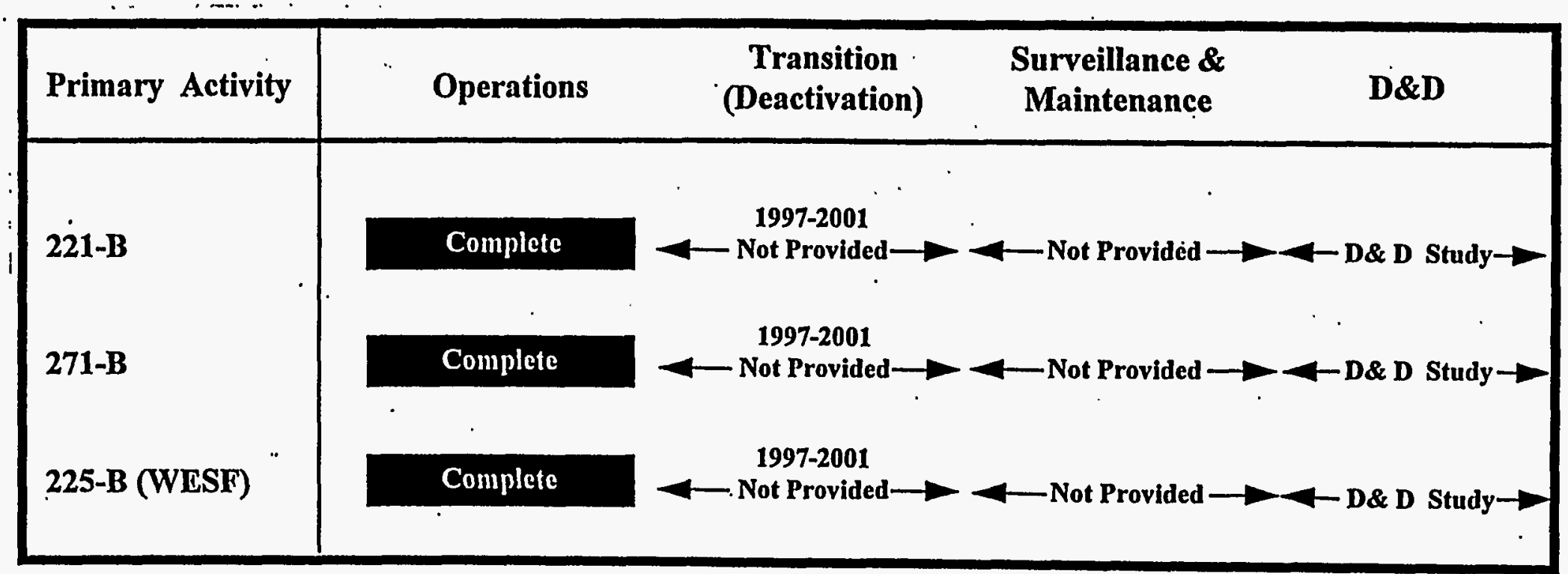


Figure 5-7. Forecast Completeness for Primary Activities and Associated Life Cycle 300 Area Fuel Supply Transition Projects. $\because$

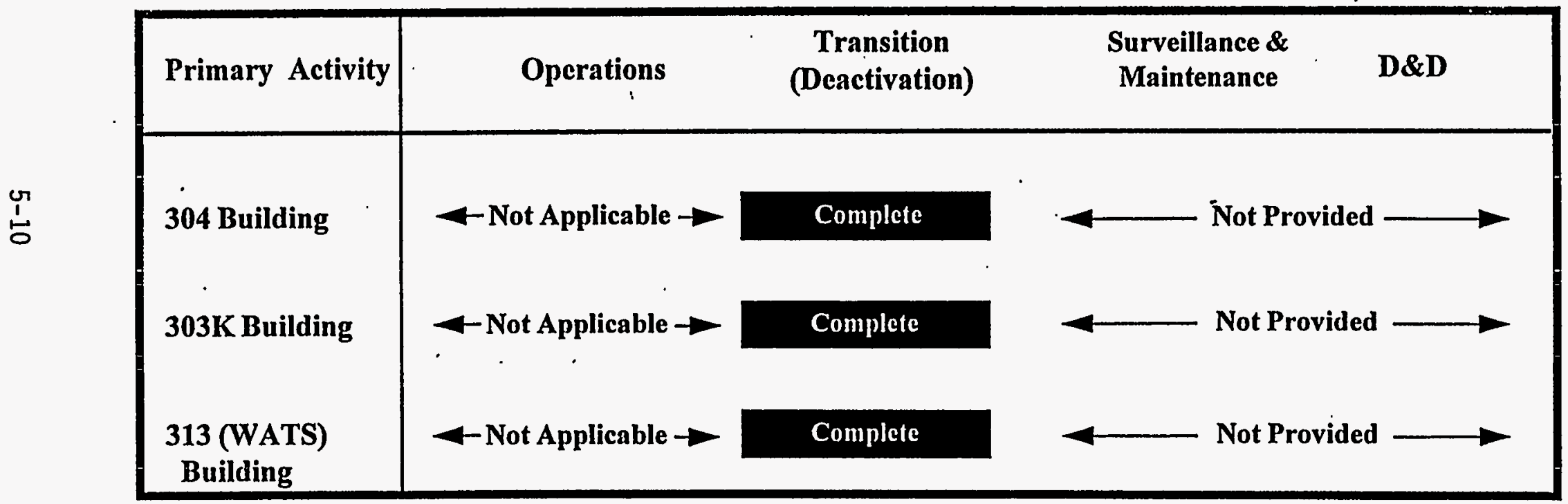


Figure 5-7 displays the 300 Area Fuel Supply's forecast completeness for each facility. The figure shows that the forecasts no longer need to report on operations, because the facilities are in a transitional phase. The forecast is complete in capturing the waste associated with this life-cycle phase; however the surveillance and maintenance and D\&D volumes are not provided in the forecast. The schedule for deactivation is not defined in the forecasts.

In short, waste volumes for the Facility Transitions program are likely to increase significantly. Although PFP could be completely closed down without additional solid waste volumes being generated, this scenario seems unlikely. PFP plutonium stabilization activities, activities associated with PUREX tunnels, B-Plant's deactivation activities, and the 300 Area Fuel Supply's surveillance and maintenance volumes are not currently forecasted. Therefore, it is likely that these activities, especially the deactivation of B-Plant, will increase future solid waste volumes. 
WHC-EP-0865

Intentionally Left Blank 


\subsection{OTHER PROGRAMS}

This section provides waste volumes and programmatic profiles for those programs that generate minimal solid waste volumes. The programs that will be discussed in this section include Liquid Effluent, Pacific Northwest Laboratory (PNL), Analytical Services, Solid Waste, and Spent Nuclear Fuel.

\subsection{BACKGROUND}

Each program in this section has a unique mission. In the course of pursuing their respective missions, they often generate solid waste that will eventualiy be sent to the CHC. The missions are described below:

- Liquid Effluent's mission is to eliminate the use of the soil column for liquid effluent treatment and to manage current and future liquid effluent streams in a safe, responsible, cost-effective and legally compliant manner. This program will generate solid waste during the treatment of 1iquid effluents from TWRS' 242-A Evaporator and from the 300 Area.

- Pacific Northwest Laboratory is a national research Taboratory that conducts research for DOE, other government agencies, and private industry to solve problems of national importance. Solid waste is generated during research and decontamination activities.

- Analytical Services provides analytical field support and process development services to other Site programs, using onsite and offsite anaTytical laboratories. Analytical Services also provides field sampling, field screening, mobile laboratories, and data deliverables.

- Solid Waste's mission is to receive, store, treat, decontaminate, and dispose of solid radioactive and nonradioactive dangerous waste in a safe, cost-effective, and environmentally compliant manner. Secondary solid waste will be generated to achieve this mission.

- Spent Nuclear Fuel's mission is to provide safe storage of SNF; remedy unsafe conditions; design, construct, operate, and maintain interim storage facilities until final disposition of SNF is determined; and stage the SNF for fiñal disposition once further direction is received. The current program mission, as defined in the Multi-Year Program Plan, addresses the SNF stored at the K-Basins only. Solid waste will be generated during the disposition of the spent fuel at K-Basins.

The volumes reported for each of these programs are taken from the 1994 Solid Waste Forecasts. No additional documentation was obtained that provided solid waste volumes for activities not currently forecasted. 


\subsection{OTHER PROGRAMS}

Low-level mixed waste and TRU TRUM waste generation for the next 30 years is estimated to be approximately 5,180 cubic meters for these programs. This waste volume is approximately $2 \%$ of the waste expected at the Site for the next 30 years. However, many activities within these other programs are currently not forecasted, and the associated waste volumes are expected to increase. Figure 6-1 displays the waste volumes for each of these programs. As seen in the figure, $49 \%$ of the waste is forecasted by the Liquid Effluent program, $23 \%$ by PNL, $18 \%$ by Analytical Services, $8 \%$ by Solid Waste, and $2 \%$ by Spent Nuclear FueT.

Following are descriptions of the programs and their primary waste generators, assumptions, and potential waste volume fluctuations.

- Liquid Effluent: The Liquid Effluent program consists of three LLMW and TRU_TRUM waste generators as stated in the forecasts: the 200 Area Effīuent Treatment Facility (ETF), the 300 Area Treatment Effluent Disposal Facility (TEDF), and the 340/307 Buildings. These facilities provide waste volumes only for the operations life-cycle phase. The 200 Area ETF is being constructed to provide effluent treatment and disposal capabilities required to support the continued operation of the 242-A Evaporator and to process the stored evaporator effluents in the Liquid Effluent Retention Facility (LERF). Operations are expected to begin in July 1995 and continue through 2023.

The 300 Area TEDF provides a system for continuous treatment and disposal of 1 iquid effluents from the 300 area that are discharged to the Columbia River. The treated effluent used to be discharged to the

Figure 6-1. Other Programs LLMW and TRU_TRUM Waste Generators.

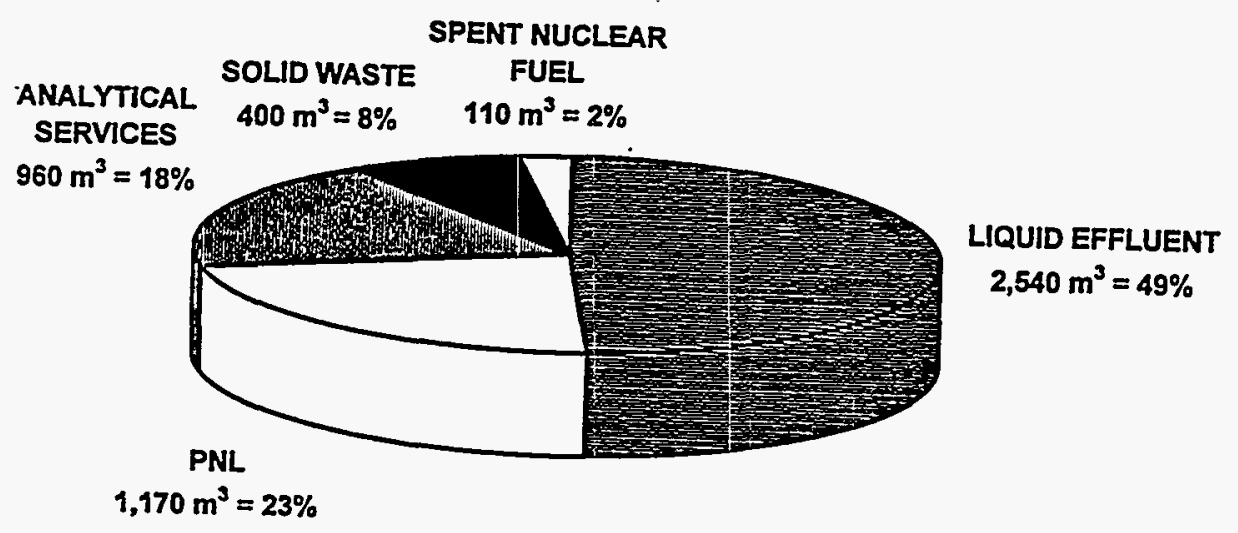


300 Area Process Trenches. TEDF began operations in December 1994 and will continue until 2023. The 340/307 Buildings accumulate and provide interim storage for tank waste analysis effluents generated by the PNL 300 Area Laboratories. These facilities also provide on-line monitoring of the 300 Area Retention Process Sewer to ensure protection of the environment prior to the effluents being sent to the 300 Area TEDF. The forecast for operations of the 340 and 307 facilities does not include upgrades planned for 1997.

Several other facilities are not currentiy included in the forecasts, and it is unknown whether these facilities generate LLMW and TRU TRUM waste. These are the Liquid Effluent Retention Facility (LERF), 200 Area Treatment Effluent Disposal Facility (TEDF), and the 300 Area Process Trenches. LERF is responsible for treating and storing the 242-A Evaporator process condensate until it can be processed by the 200 Area ETF in July 1995. The 200 Area TEDF is intended to provide the collection and disposal system necessary to transport waste streams to a single permitted soil column disposal site. The schedule for this facility is unknown. Lastly, the 300 Area Process Trenches are operated for the disposal of 300 Area effluents; however, they were removed from service in December 1994 with the operation of the TEDF.

- PNL: The majority of these waste streams are associated with research and development activities being accomplished at the Hanford Site. The forecasted volumes were based on decontamination activities and research projects. Operational and transitional waste has been included for this program.

- Analytical Services: The Analytical Services program consists of one LLMW and TRU TRUM waste generator, which is the 222-S Laboratory. The 222-S Laboratory provides analytical services to the Hanford Site. The general types of solid waste that are generated include miscellaneous laboratory equipment such as rubber gloves, paper, and plastic bottles. Some solid waste is generated from construction activities occurring in the laboratory. Most LLMW and TRU TRUM waste is generated from laboratory analysis of Double-Shell and Single-Shell Tank waste samples. It is forecasted that more waste will be generated in FY 1995 due to an increase in tank farm analysis. Operation of the laboratory is the only life-cycle phase forecasted:

One other onsite facility for this program could be a potential LLMW and TRU TRUM waste generator, the Waste Sampling and Characterization Facility (WSCF). Since it began operations in 1994, it has not provideda forecast, so it is unknown whether additional LLMW and TRU TRUM waste volumes should be included. WSCF will provide analytical support to liquid waste treatment plants and anaTyze LLW for environmental monitoring purposes. WSCF will generate primarily lab waste and labpacks. 
Additional waste volumes are not forecasted which are associated with offsite commercial laboratories. Three offsite laboratories are currently providing analytical support to Hanford. These laboratories are storing solid waste at their respective locations until they become official Hanford waste generators.

- Solid Waste: The Solid Waste program has four LLMW and TRU TRUM waste generators within its program: T-Plant, the Mixed-Waste Störage Facility, the Low-Level Burial Grounds, and TRUSAF. T-Plant is a canyon building that is used to decontaminate equipment and sort and package waste from other onsite facilities. D\&D of T-Plant is addressed in Section 4.0. T-Plant forecasts operations and transitional wastes, which include routine activities such as maintenance, decontamination, upgrades, and routine cleanup. The Mixed-Waste Storage Facility currently forecasts only operations that include storage of the LLMW sent to the CWC. The Low-Level Burial Grounds forecasts only operational waste that consists of disposing LLW sent to the CWC by burying the waste in trenches. Lastly, TRUSAF forecasts operational waste that includes only the storage of TRU_TRUM waste sent to the CWC.

- Spent Nuclear Fuel: $K$-Basins is the only waste generator included in the Spent Nuclear Fuel program. Currently, K-Basins provide wet storage for spent fuel. The forecast provides waste volumes only for maintenance of the basins for the next 30 years. The Path Forward document, however, indicates that maintenance activities will be completed in 2001, and several other activities will be initiated that are not currently forecast, including remediation of the K-Basins, interim storage of the fuel prior to stabilization, stabilization of the spent fuel, and 40-year interim storage of the fuel prior to transport to a national repository (Fulton 1994). Not all iife-cycle phases for these disposition activities are forecasted.

\subsection{FORECASTED VOLUMES}

A description of the waste category volumes for 0ther Programs and for each specific waste generator within the programs. is provided in the following sections.

\subsubsection{Forecasted Volumes For Other Programs}

Figure 6-2 displays the overall volumes by waste category for 0ther Programs. Eighty-six percent of the waste is CH_LLMW, 6\% is RH_LLMW, and RH_TRU_TRUM and $\mathrm{CH}_{-}$TRU_TRUM each represent $4 \%$. 
Figure 6-2. Other Programs Total 30-Year Solid Waste Volumes by Waste Category.

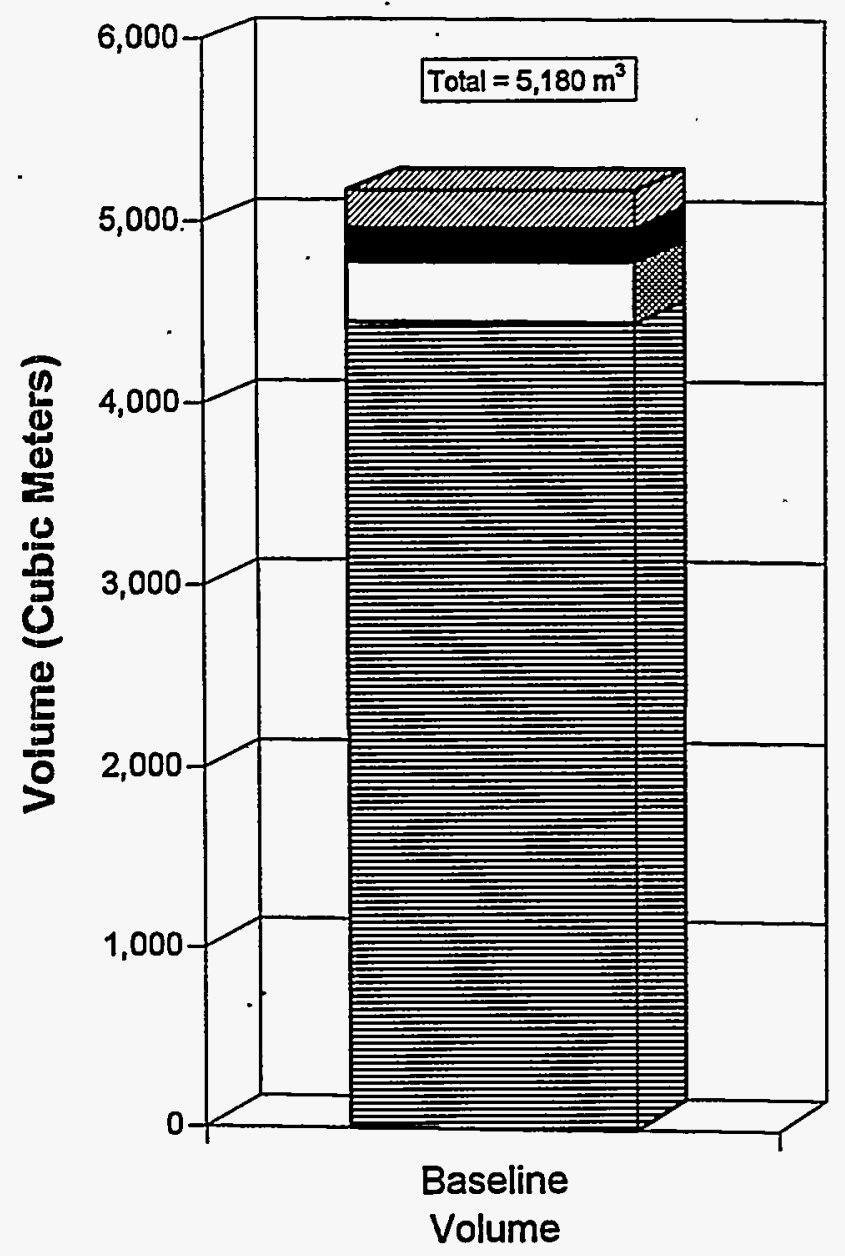

\begin{tabular}{|c|}
\hline ORH_TRU_TRUM \\
$\left(200 \mathrm{~m}^{3}=4 \%\right)$ \\
CH_TRU_TRUM \\
$\left(190 \mathrm{~m}^{3}=4 \%\right)$ \\
DRH_LLMW \\
$\left(330 \mathrm{~m}^{3}=6 \%\right)$ \\
ECH_LLMW \\
$\left(4,450 \mathrm{~m}^{3}=86 \%\right)$
\end{tabular}

Figure 6-3 shows the annual volumes by waste category. As seen in the figure, waste generation is fairly constant at 150 to 200 cubic meters per year. Both CH_LLMW and RH_LLMW remain constant for the 30-year period. CH_TRU_TRUM remains constant from 1996 through 2014, when it is no longer expected to be generated. RH_TRU_TRUM waste generation is constant from 1996 through 2009 with a peak in 1995 . $^{-}$PNL is the driver for this peak.

\subsubsection{Forecasted Volumes by . Program and Waste Generator}

Table 6-1 displays the amount of waste generated for each waste category and waste generator within each program. The majority of CH_LLMW is generated by Liquid Effluent's 200 Area ETF. Other contributors of the $\mathrm{CH}_{-}$LLMW volumes are PNL and Analytical Laboratory's 222-S Laboratory. RH_LLMW is primarily generated by PNL and Solid Waste's T-PTant. RH_TRU TRUM waste volumes are generated mostly by PNL and Spent Nuclear Fuel's K-Basins. And CH_TRU_TRUM waste volumes are primarily generated by PNL. 
WHC-EP-0865

Figure 6-3. Other Programs Annual Solid Waste Volumes by Waste Category.

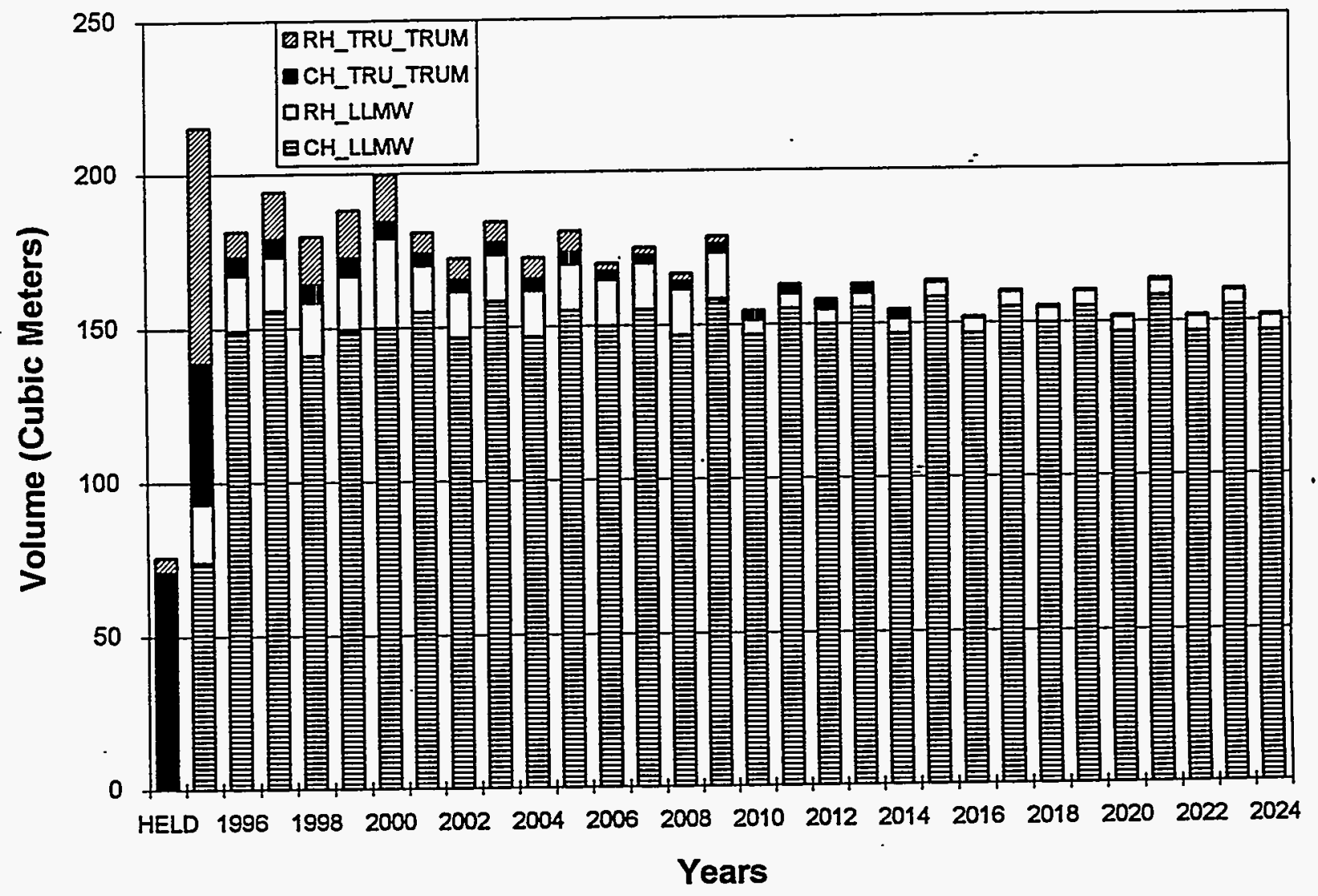

\subsection{DATA OBSERVATIONS}

Figures 6-4, 6-5, and 6-6 display the forecast completeness for Liquid Effluents, Analytical Services, and Spent Nuclear Fuel, respectively. PNL and Solid Waste are completely forecasted since both of these programs expect to be in the operational life-cycle phase for the next 30 years. Additional waste volumes are not expected from these programs unless they undergo mission changes.

Figure 6-4 displays the forecast completeness for a11 facilities within Liquid Effluents. As seen in the figure, the forecasts for LERF and the Process Trenches are not provided, therefore any potential solid waste generated by these two facilities has not been captured. The figure also shows that the forecast is incomplete in capturing the operational phase of the 200 Area ETF and the 300 Area $340 / 307$ Buildings. The ETF has incomplete characterization 
Table 6-1. Other Programs. Waste Generator Volumes. (a)

\begin{tabular}{|c|c|c|c|c|c|c|}
\hline Program & Waste Generator & $\begin{array}{c}\left.\mathrm{CH}_{(\mathrm{m}} \mathrm{m}^{3}\right) \\
\end{array}$ & $\begin{array}{c}\text { RH_L4HH } \\
\left(\mathrm{m}^{3}\right)\end{array}$ & $\begin{array}{c}\text { CH_TRU_TRUM } \\
\left(\mathrm{m}^{3}\right)\end{array}$ & $\begin{array}{c}\text { RH_TRU, TRUM } \\
\frac{\left(\mathrm{m}^{5}\right)}{}\end{array}$ & $\begin{array}{l}\text { Total } \\
\left(\mathrm{m}^{5}\right)\end{array}$ \\
\hline \multirow[t]{4}{*}{ Liquid Effluents } & 200 Area ETF & 2,380 & 0 & 0 & 0 & 2,380 \\
\hline & 300 Area TEDF & 120 & 0 & 0 & 0 & 120 \\
\hline & $340 / 307$ Buildings & 40 & 0 & 0 & 0 & 40 \\
\hline & Total & 2,540 & 0 & 0 & 0 & 2,540 \\
\hline PNL & PNL & 740 & 190 & 150 & 90 & 1,170 \\
\hline $\begin{array}{l}\text { Analytical } \\
\text { Services }\end{array}$ & 222-S Laboratory & 890 & 60 & 0 & 20 & 960 \\
\hline \multirow[t]{5}{*}{ Solid Waste } & $T$-Plant & 210 & 90 & 0 & 30 & 330 \\
\hline & Mixed Waste Storage & 0 & 0 & 40 & 0 & 40 \\
\hline & $\begin{array}{l}\text { Low-Level Burial } \\
\text { Grounds }\end{array}$ & 30 & $0^{\circ}$ & 0 & 0 & 30 \\
\hline & TRUSAF & 3 & 0 & 0 & 0 & 3 \\
\hline & Total & 250 & 90 & 40 & 30 & 400 \\
\hline $\begin{array}{l}\text { Spent Nuclear } \\
\text { Fuel }\end{array}$ & $\mathrm{K}$-Basins & 40 & 0 & 0 & 70 & 110 \\
\hline Total & & 4,450 & 330 & 190 & 200 & 5,180 \\
\hline
\end{tabular}

information, while the $340 / 307$ Buildings' forecasts do not include the planned 1997 upgrades. Lastly, no transitional, surveillance and maintenance, or D\&D wastes are included in the forecasts. Therefore, based on these unforecasted activities and 7 ife-cycle phases, it seems that waste volumes for Liquid Effluents could increase.

Figure 6-5 shows the forecast completeness for the laboratories within Analytical Services. The figure shows that no waste information has been provided for any life-cycle phases of the WSCF. In addition, transitional, surveillance and maintenance and D\&D wastes have not been included for 222-S Laboratory; however, these wastes will not be generated within the next 30 years. Lastly, offsite commercial laboratories have not provided forecasts. Based on these unforecasted life-cycle phases and the lack of waste information for WSCF and the three offsite commercial 1aboratories, it appears that waste volumes could increase for Analytical Services.

Figure 6-6 displays the forecast completeness for Spent Nuclear Fuel's $\mathrm{K}$-Basins. The figure shows that the only activity forecasted is maintenance of the K-Basins. All other activities and life-cycle phases are not forecasted, which indicates a high probability that waste volumes for Spent Nuclear Fuel will increase. 
Figure 6-4. Forecast Completeness for Primary Activities and Associated Life Cycle Liquid Effluents.

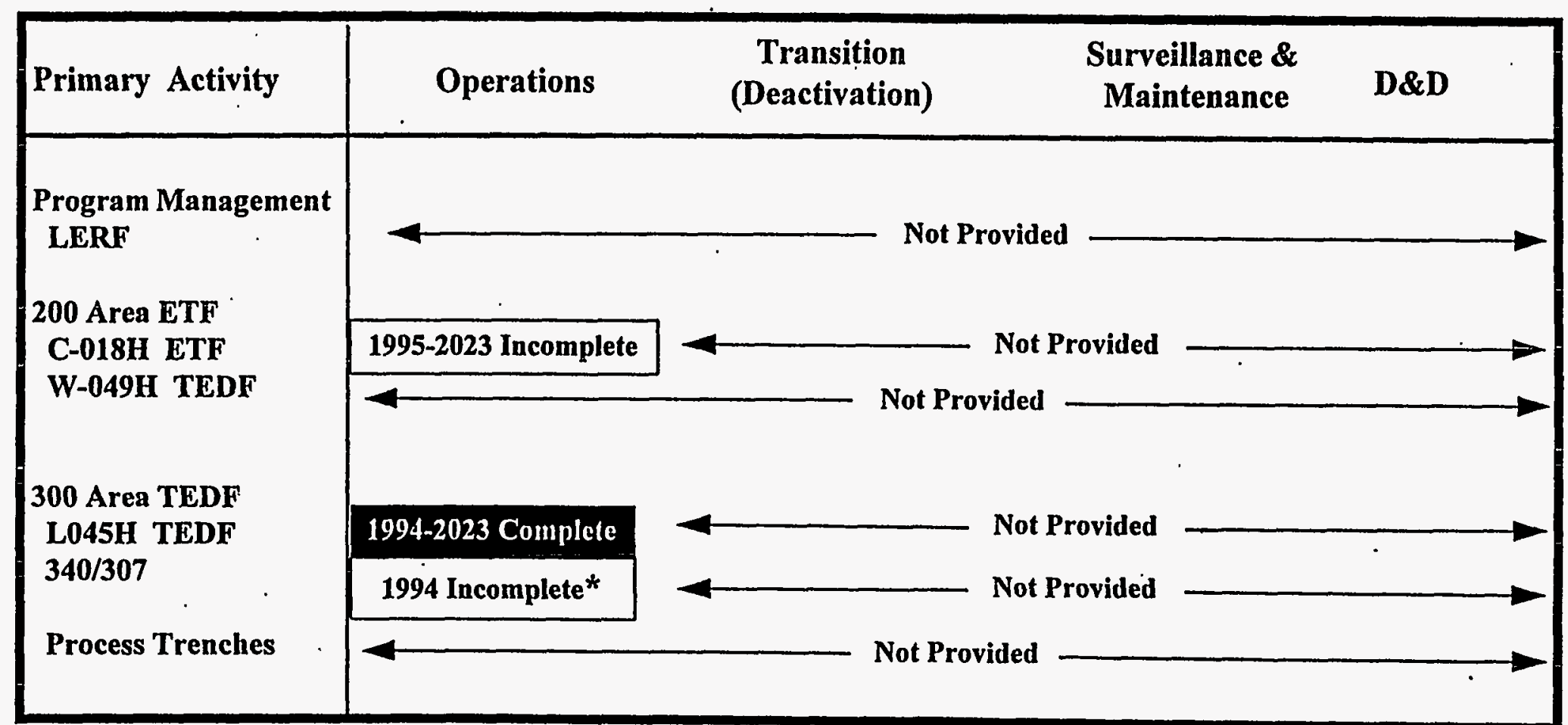

*No 1997 upgrades included 
Figure 6-5. Forecast Completeness for Primary Activities and Associated Life Cycle Analytical Services.

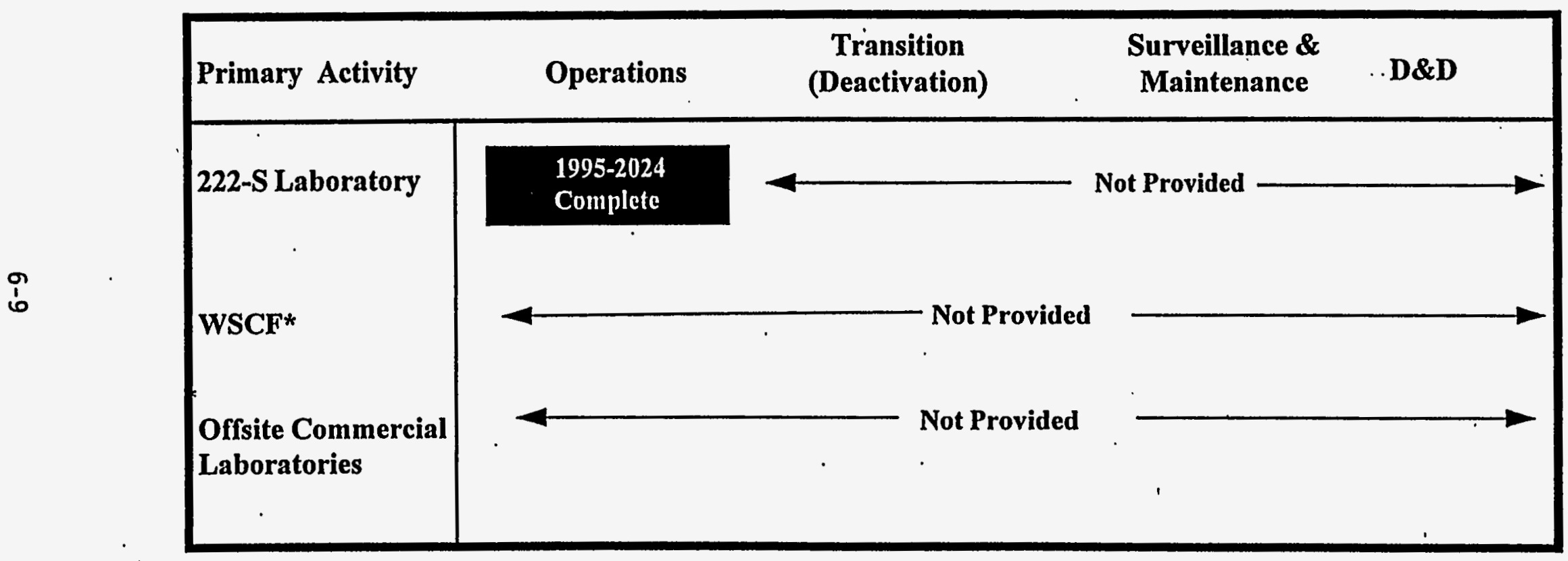

WSCF $=$ Waste Sampling and Characterization Facility 
Figure 6-6. Forecast Completeness for Primary Activates and Associated Life Cycle Spent Nuclear Fuel.

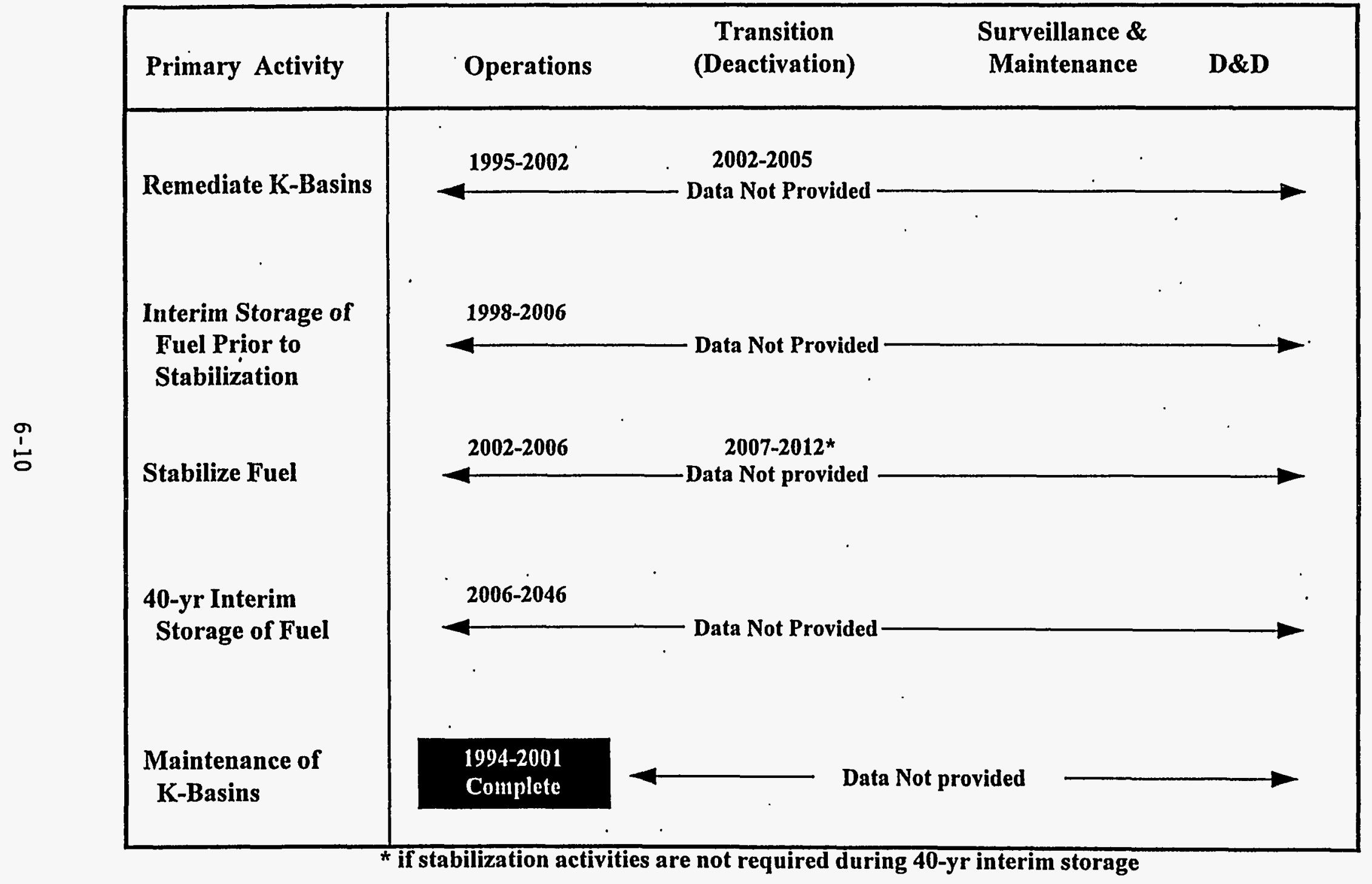




\subsection{NON-PROGRAMMATIC}

Two types of waste generators are described in this section: those waste generators that are currently not within a Hanford program and those waste generators about whom uncertainties arise in determining the program that is responsible for their operations. Waste generator volumes and descriptions of the LLMW and TRU TRUM solid waste expected from these nonprogrammatic sources are discussēd.

\subsection{BACKGROUND}

The volumes reported for the Non-Programmatic waste generators are taken from the 1994 S.olid Waste Forecasts. The Buried Equipment generator did not complete a forecast in 1993 or 1994; therefore, waste volumes from the 1992 forecasts were used for this generator. No additional documentation was used to supplement the forecasts submitted by these waste generators.

\subsection{NON-PROGRAMMATIC}

LLMW and TRU TRUM solid waste generation for the next 30 years is estimated to be approximately 7,420 cubic meters for the Non-Programmatic waste generators. This is approximately $3 \%$ of the total waste expected at Hanford. Figure 7-1 displays the waste volumes for each waste generator in the NonProgrammatic area. Buried Equipment forecasts approximately $99 \%$ of the waste; the remaining $1 \%$ is generated by 100 Area Construction Services, the Geotechnical Engineering Laboratory, the Fuels Development Laboratory, and the Large Sodium Fire Facility.

Figure 7-1. Non-Programmatic LLMW and TRU_TRUM Waste Generators.

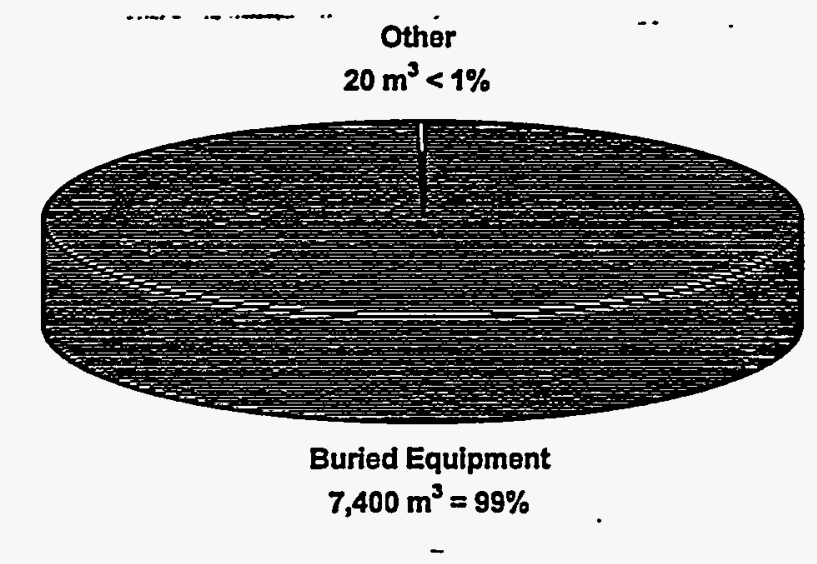


Descriptions of the five waste generators for the Non-Programmatic area are provided below, including the generator's assumptions and potential waste volume fluctuations.

- Buried.Equipment (WHC_BQUIP): This generator disposes of general purpose coded equipment, including vehicles and mobile construction units used in tank farms. A 1994 Solid Waste Forecast was not submitted for this generator because of funding issues; therefore, the 1992 forecast was used. The volumes forecasted are based on past experience and waste that has not been buried in 1992-1994 due to lack of funding. The waste that has not been buried is located with the respective waste generators. Thus the schedule for this generator is uncertain, but the volumes are certain because the waste currently exists. Since maximum and minimum ranges were not collected in 1992, these data are not available for Buried Equipment, and 100\% minimum and maximum ranges were assumed.

- 100 Area Construction Services (KEH 100): Construction Services generates LLMW that consists primarily of soil from past practice sites. Therefore, it is assumed that most of this waste exists. Currently, it is planned to ship all the backlog LLMW in 1995, and no further radioactive waste will be generated. The minimum range for this generator is $8 \%$, which reflects the 1994 Solid Waste Forecast baseline. The maximum range is $100 \%$, which indicates that waste volumes will not increase.

- Geotechnical Engineering Laboratory (WHC 377): Waste from this generator generaliy consists of cleanup wastes, sludge, and sampling waste. Only operational waste has been forecasted for this waste generator. The minimum range for the laboratory is $50 \%$, and the maximum range is $300 \%$, which indicates uncertainty about exact waste volume generation over the next 30 years.

- Fuels Development Laboratory (WHC FUEL DEV): This waste generator forecasts TRU waste generation for the year 1996 . The waste will result from deactivation of the TRIGA Fuel Storage Facility and include metal, absorber control rods, and TRIGA Reactor fuel elements. The waste may not be sent to CWC due to uncertainties with resources and schedules, as indicated by the minimum reported range of $0 \%$. The maximum range is 100\%; therefore, the waste volumes are not expected to increase.

- Large Sodium Fire Facility (WHC SODIUM FAC): LLMW is the only waste forecasted for this waste generätor from 1996 through 1997. The waste is generated from general facility cleanup, where facility equipment is chemically contaminated with sodium carbonate. The forecast assumes that the facility cleanup plan will be approved by the State in CY 1995. The minimum range for the facility is $0 \%$; the maximum range is $300 \%$ due to uncertainties with future waste generation volumes. 


\subsection{FORECASTED VOLUMES}

Descriptions of the waste category volumes for all Non-Programmatic waste generators and for each specific waste generator are provided in the following sections.

\subsubsection{Forecasted Volumes for Non-Programmatic}

Figure 7-2 displays the overall volumes by waste category for NonProgrammatic waste generators. Over 99\% of the waste is CH_LLMW, with less than $1 \%$ forecasted to be $\mathrm{CH}_{-}$TRU_TRUM.

Figure 7-3 shows the annual waste volumes by waste category. As seen in the figure, there are five 350-cubic meter peaks that are driven by Buried Equipment.

Figure 7-2. Non-Progranmatic Total 30-Year Solid Waste Volumes by Waste Category.

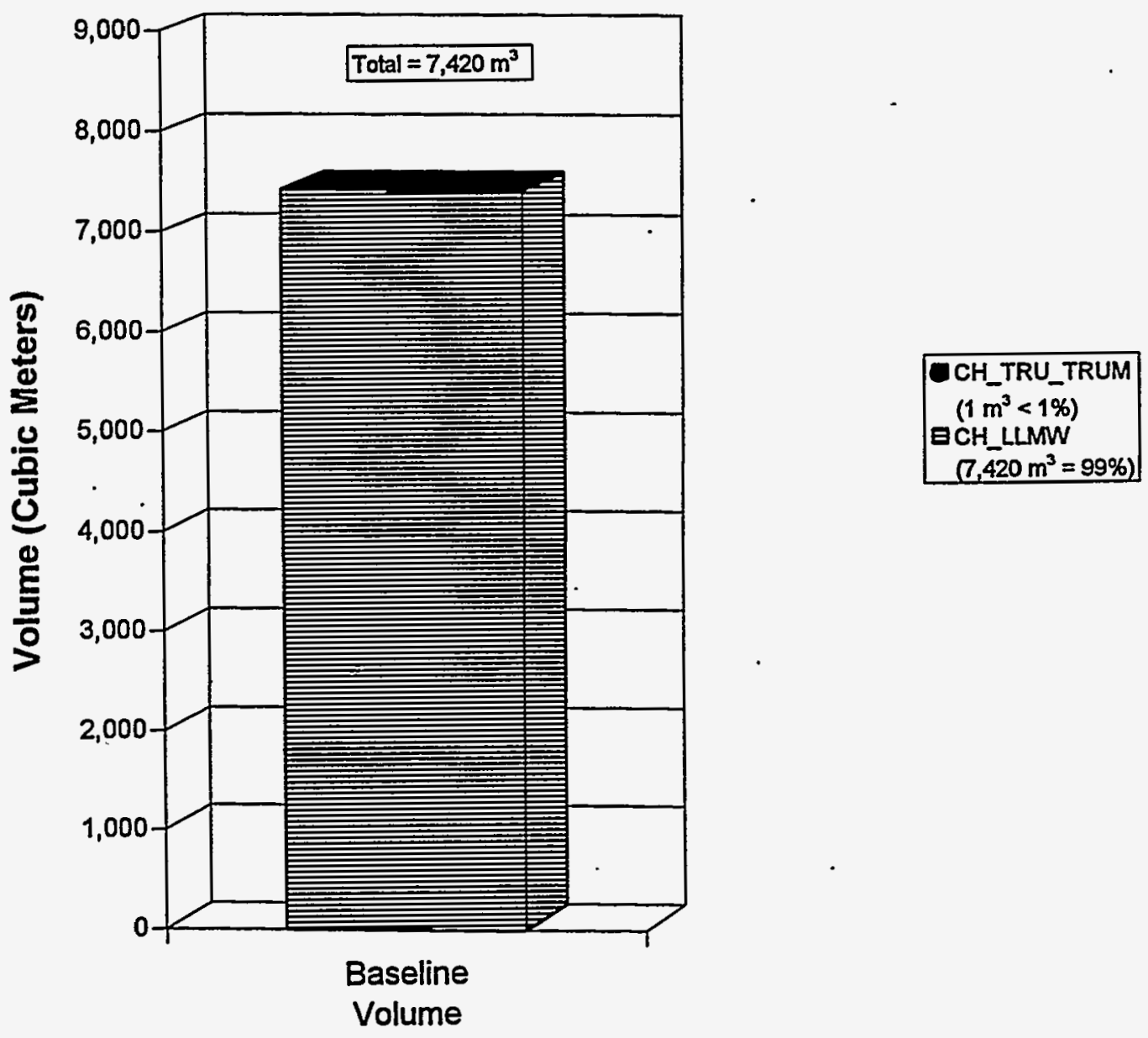


Figure 7-3. Non-Programmatic Annual Solid Waste Volumes by Waste Category.

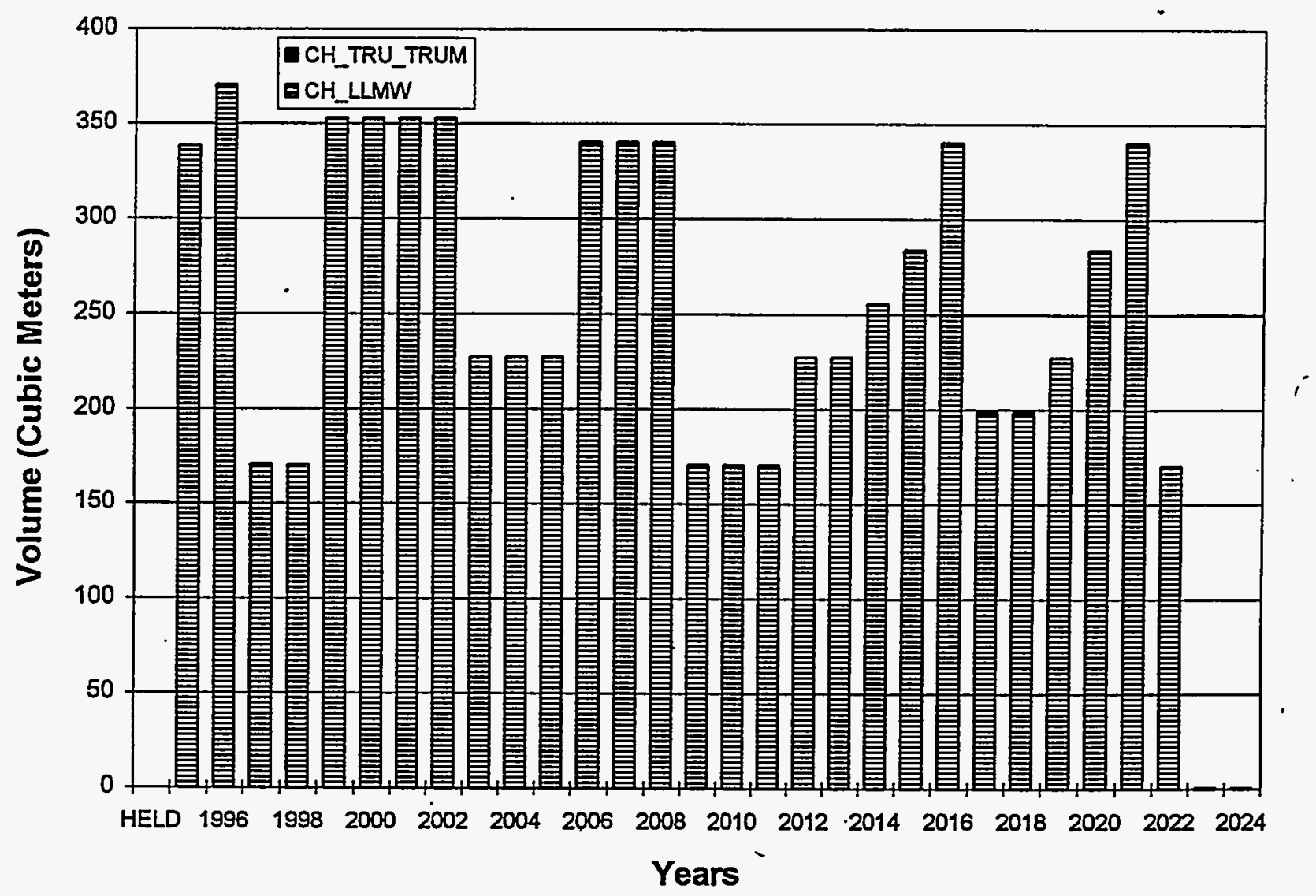

\subsubsection{Forecasted Volumes by Waste Generator}

Table 7-1 displays the amount of waste generated for each waste category and Non-Programmatic waste generator. The majority of the waste is CH_LLMW generated primarily by Buried Equipment. The Fuels Development Laboratory is the only $\mathrm{CH}_{-}$TRU_TRUM waste generator.

\subsection{DATA OBSERVATIONS}

Solid waste volumes for the Non-Programmatic waste generators are unlikely to vary significantly in the future. Buried Equipment, 100 Area Construction Services, the Fuels Development Laboratory, and the Large Sodium Fire Facility all forecast primarily existing waste, so the reported volumes 
Table 7-1. Non-Programmatic Waste Generator Volumes. ${ }^{\text {(a) }}$

\begin{tabular}{||l|c|c|c|c|c|}
\hline \multicolumn{1}{|c|}{ Waste Generator } & $\begin{array}{c}\text { CH LLMW } \\
\left(\mathrm{m}^{3}\right)\end{array}$ & $\begin{array}{c}\text { RH_LLMW } \\
\left(\mathrm{m}^{3}\right)\end{array}$ & $\begin{array}{c}\text { CH_TRU TRUM } \\
\left(\mathrm{m}^{5}\right)\end{array}$ & $\begin{array}{c}\text { RH_TRU TRUM } \\
\left(\mathrm{m}^{5}\right)\end{array}$ & $\begin{array}{c}\text { Total } \\
\left(\mathrm{m}^{3}\right)\end{array}$ \\
\hline \hline Buried Equipment & 7,400 & 0 & 0 & 0 & 7,400 \\
\hline $\begin{array}{l}\text { 100 Area } \\
\text { Construction } \\
\text { Services }\end{array}$ & 10 & 0 & 0 & 0 & 10 \\
\hline $\begin{array}{l}\text { Geotechnical } \\
\text { Engineering } \\
\text { Laboratory }\end{array}$ & 10 & 0 & 0 & 0 & 10 \\
\hline $\begin{array}{l}\text { Fuels Development } \\
\text { Laboratory }\end{array}$ & 0 & 0 & 1 & 0 & 1 \\
\hline $\begin{array}{l}\text { Large Sodium Fire } \\
\text { Facility }\end{array}$ & 1 & 0 & 0 & 0 & 1 \\
\hline \hline Total & 7,420 & 0 & 1 & 0 & 7,420 \\
\hline \hline (a) Sum of individual values may not match totals due to rounding. \\
\hline
\end{tabular}

are fairly certain. The schedule for shipment of this existing waste is not as certain, however, since it is based on mission assumptions. In short, the potential impacts to the solid waste system caused by Non-Programmatic waste generators are minimal. 
WHC-EP-0865

Intentionally Left $B T$ ank 


\subsection{OFFSITE WASTE GENERATORS}

This section provides waste volumes and profiles for offsite waste generators that are certified to ship waste to the Hanford Site. Most offsite waste generators are national Taboratories, universities, or DOE-owned contractor-operated facilities.

\subsection{BACKGROUND}

Offsite missions include activities such as general maintenance and operations, environmental restoration, D\&D, and research and development. The forecasted volumes presented in this section are from the 1994 Solid Waste Forecasts. Additional documentation was not used to supplement these forecasts. It should be noted that the volumes reported for offsite waste generators are considerably greater than what is forecasted for the Proposed Site Treatment Plans (PSTPS).

\subsection{WASTE GENERATORS}

Offsite waste generators forecast that approximately 14,170 cubic meters of waste will be generated over the next 30 years, which is $6 \%$ of the total waste expected at the Hanford Site during this period. Portsmouth Gaseous Diffusion Plant - Energy Systems will generate the largest percentage of offsite waste with 46\% (see Figure 8-1). Paducah Energy Services expects to generate $36 \%$, Argonne National Laboratory - East wi 11 generate $10 \%$, and all other offsite generators will contribute a total of $8 \%$ of the offsite solid waste.

Figure 8-1. Offsite LLMW and TRU_TRUM Waste Generators.

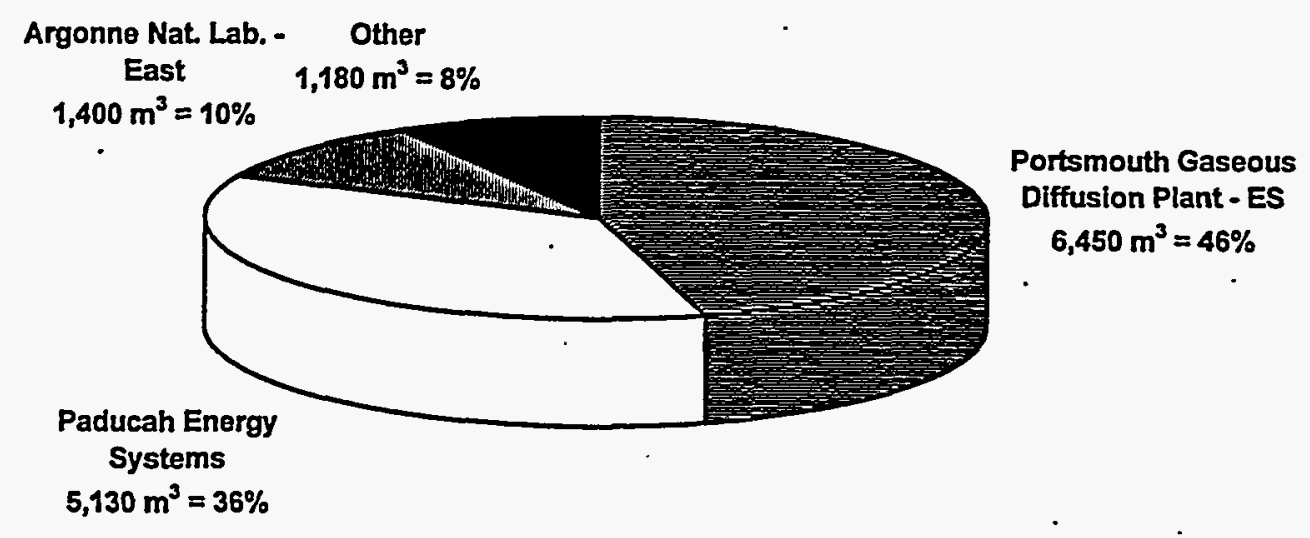


The following text briefly describes each major offsite waste generator's assumptions, missions, and potential waste fluctuations.

- Portsmouth Gaseous Diffusion P1ant - Energy Systems (PORTSMOUTH ES): The energy systems element of Portsmouth is responsible for environmental restoration (ER) waste, including past operational waste and past and future ER waste. D\&D waste is not included in the forecast. The waste primarily consists of soils, sludges, laboratory wastes, filter cakes, and other miscellaneous past operational waste. Portsmouth does not expect the waste volumes that they forecasted to decrease, but they may increase by $20 \%$.

- Paducah Energy Systems (PADUCAH_ES): Forecasted waste volumes for this generator include ER and D\&D projects only; therefore, the waste exists. However, most of the existing waste that is planned for shipment in FY 1995 through FY 1999 has not been certified for acceptance at the CWC. Therefore, the schedule for this waste is uncertain. It has been assumed that all TRU waste will not be sent to the Hanford Site. The LLMW that will be sent to the site has a minimum range of $50 \%$ and a maximum range of $150 \%$.

- Argonne National Laboratory - East (ANLE): Most of the waste volumes (approximately 70\%) associated with Argonne National Laboratory - East will be generated as a result of site remediation cleanup and D\&D activities that will occur from 1995 through 2001 . The remaining waste will result from over 100 waste generators that are working on laboratory bench-scale projects, thus the solid waste generated will be operational waste. Argonne National Laboratory - East indicates that the forecasted baseline volume may not be sent to Hanford at all or that the volume may triple. The reason for the $0 \%$ scenario is possible programmatic changes, whereas the $300 \%$ maximum estimate is due to possible underestimation by Argonne National Laboratory - East.

\subsection{FORECASTED VOLUMES}

A description of the waste category volumes for all offsite waste generators is provided in the following sections.

\subsubsection{Forecasted Volumes for Offsite Haste Generators}

Figure 8-2 shows the overall volumes by waste category for offsite waste generators. Ninety-eight percent of the expected offsite waste is CH_LLMW; CH_TRU_TRUM and RH_TRU_TRUM each make up of $1 \%$ of the total offsite solid waste expected to be shipped to the CWC. No RH_LLMW is expected from offsite waste generators. 
Figure 8-2. Offsite Total 30-Year Solid Waste Volumes by Waste Category.

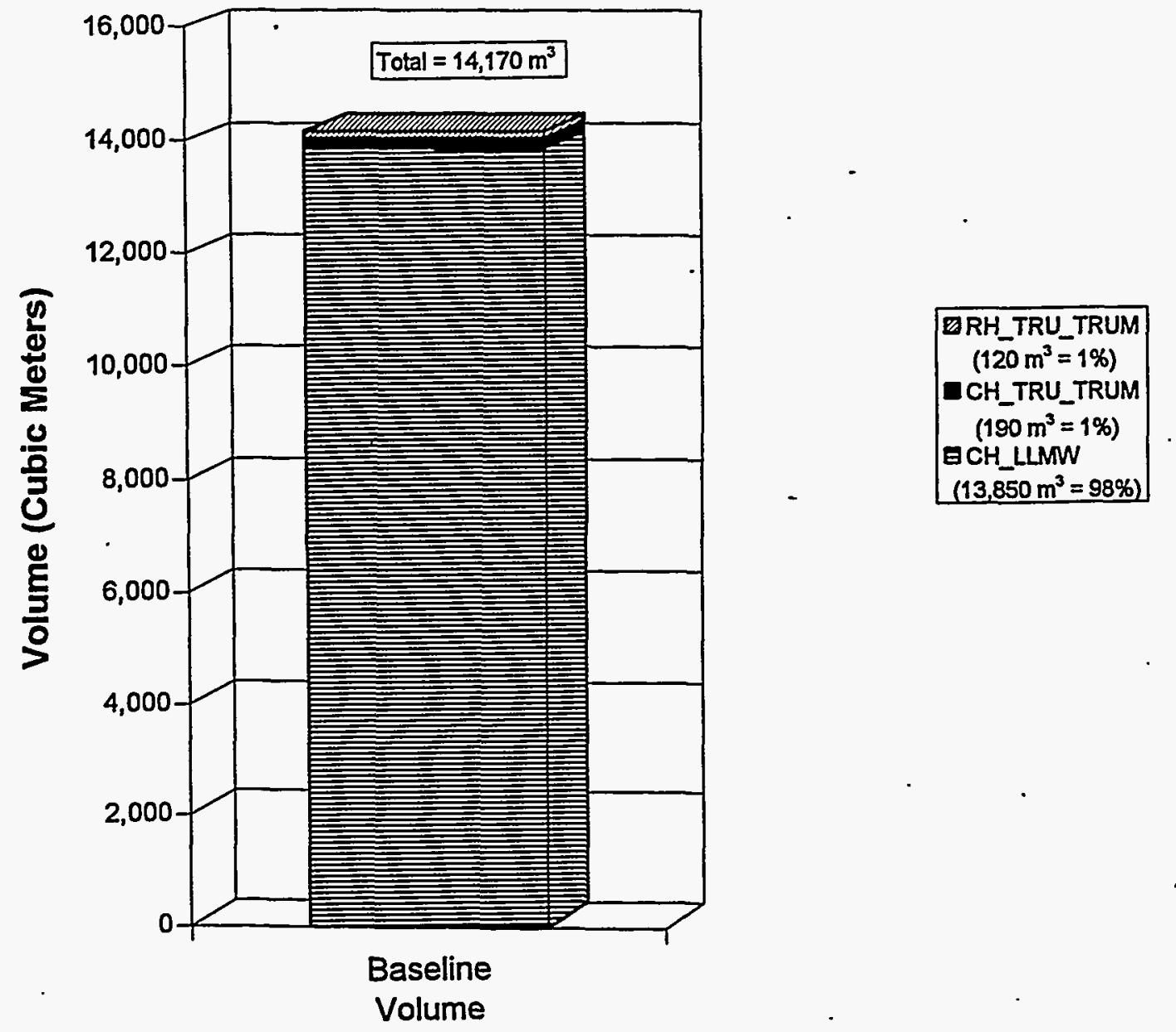

Figure 8-3 displays the annual volumes by waste category. As seen in the figure, 1995 and 1996 waste volumes are approximately 600 cubic meters, which accounts for expected Argonne National Laboratory waste shipments. Waste volumes decrease to approximately 300 cubic meters from 1997 through 1999. In 2000, an increase of about 650 cubic meters is expected. This increase is due to Argonne National Laboratory, which expects to increase shipments from 10 cubic meters to 100 cubic meters in 2000. From 2001 through 2015, waste volumes increase to approximately 500 cubic meters, which reflects Portsmouth Gaseous Diffusion Plant - Energy Systems expected shipping schedule. Volumes level off to approximately 400 cubic meters from 2016 through 2024. 
Figure 8-3. Offsite Annual Solid Waste Volumes by Waste Category.

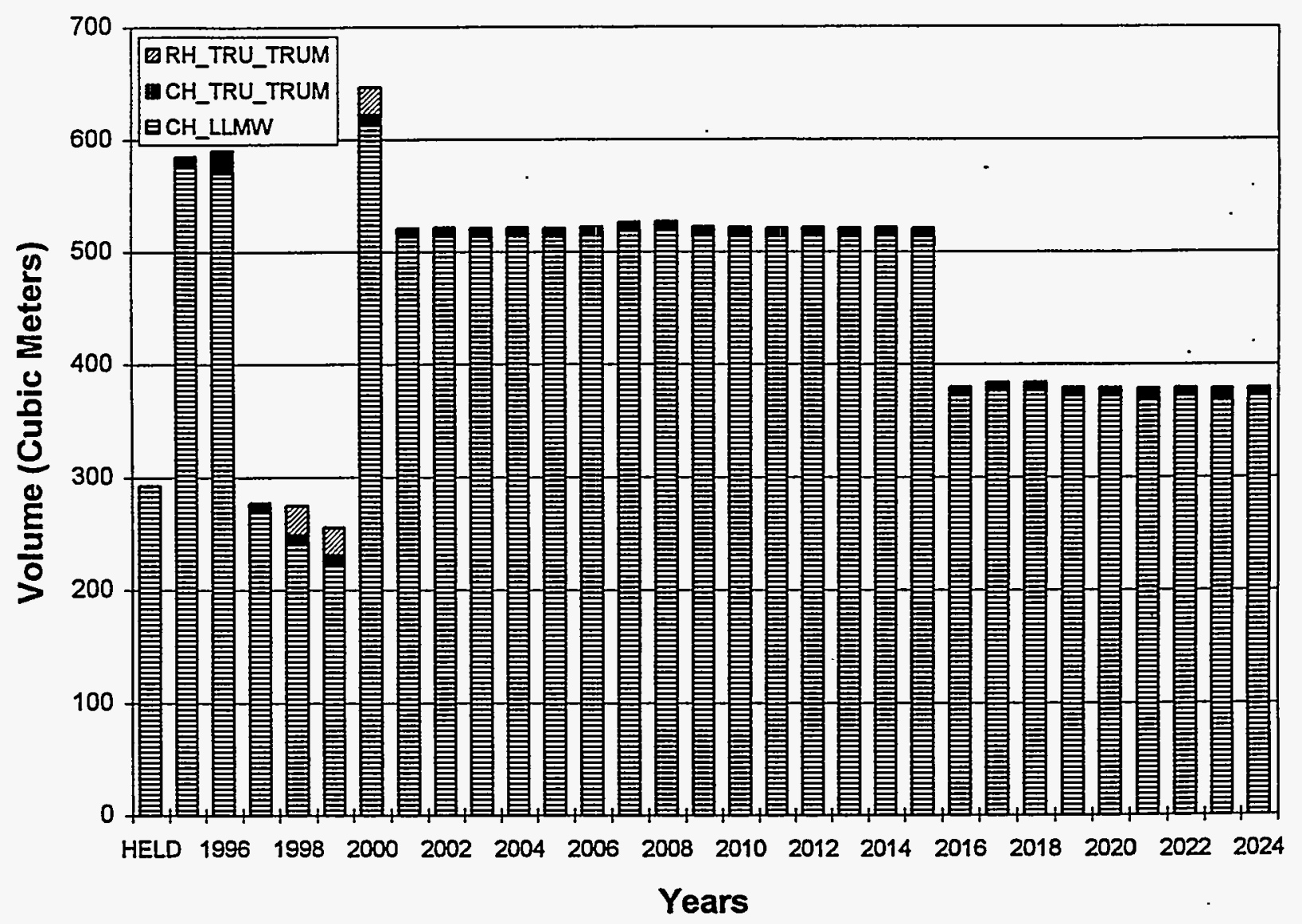

\subsubsection{Forecasted Volumes by Program and Waste Generator}

Table 8-1 displays the amount of waste to be generated for each waste category and offsite waste generator. As seen in the table, all waste generators contribute to the large generation of CH LLMW; however, Portsmouth Gaseous Diffusion PTant - Energy Systems and Paducah Energy Systems are the largest contributors. Argonne National Laboratory - East and Battelle Columbus Laboratory are the only generators of RH.TRU TRUM. Argonne National Laboratory - East is the only generator of $\mathrm{CH}_{-}$TRU_TRUM.

\subsection{DATA OBSERVATIONS}

All offsite generators of LLMW are required by the Federal Facilities Compliance Act to have an approved Site Treatment PIan (STP) by September 30, 1995. As a result of the STPs, several waste streams reported in this document may be directed to another site for treatment, which could significantly 
Table 8.1. Offsite Waste Generator Volumes ${ }^{(a)}$

\begin{tabular}{|c|c|c|c|c|c|}
\hline Haste Generator & $\begin{array}{c}\mathrm{CH} \text { LLMWH } \\
\left.7 \mathrm{~m}^{3}\right)\end{array}$ & $\underset{\left(\mathrm{m}^{3}\right)}{\mathrm{RH}}$ & $\underset{\left.-m^{3}\right)}{C H \_T R U \text { TRUM }}$ & $\begin{array}{c}\text { RH_TRU TRUM } \\
\left(\mathrm{m}^{3}\right)\end{array}$ & $\begin{array}{c}\text { Total } \\
\left(\mathrm{m}^{3}\right)\end{array}$ \\
\hline Portsmouth Energy Systems & 6,450 & 0 & 0 & 0 & 6,450 \\
\hline Paducah Energy Systems & 5,130 & 0 & 0 & 0 & 5,130 \\
\hline $\begin{array}{l}\text { Argonne National } \\
\text { Laboratory - East }\end{array}$ & 1,160 & 0 & 190 & 50 & 1,400 \\
\hline $\begin{array}{l}\text { Lawrence Berkeley } \\
\text { Laboratory }\end{array}$ & 230 & 0 & 0 & 0 & 230 \\
\hline $\begin{array}{l}\text { Portsmouth Utility } \\
\text { Services }\end{array}$ & 210 & 0 & 0 & 0 & 210 \\
\hline $\begin{array}{l}\text { Bettis Atomic Power. } \\
\text { Laboratory }\end{array}$ & 200 & 0 & 0 & 0 & 200 \\
\hline $\begin{array}{l}\text { Knolls Atomic Power } \\
\text { Laboratory }\end{array}$ & 130 & 0 & 0 & 0 & 130 \\
\hline $\begin{array}{l}\text { Battelle Columbus } \\
\text { Laboratory }\end{array}$ & 50 & 0 & 0 & 70 & 120 \\
\hline $\begin{array}{l}\text { FERMI National } \\
\text { Accelerator Laboratory }\end{array}$ & 80 & 0 & 0 & 0 & 80 \\
\hline $\begin{array}{l}\text { Brookhaven National } \\
\text { Laboratory }\end{array}$ & 60 & 0 & 0 & 0 & 60 \\
\hline $\begin{array}{l}\text { Princeton Plasma Physics } \\
\text { Laboratory }\end{array}$ & 50 & 0 & 0 & 0 & 50 \\
\hline General Atomics & 30 & 0 & 0 & 0 & 30 \\
\hline $\begin{array}{l}\text { Paducah - Utility } \\
\text { Services }\end{array}$ & 30 & 0 & 0 & 0 & 30 \\
\hline Rockwell Canoga Park & 20 & $\mathbf{0}$ & 0 & 0 & 20 \\
\hline $\begin{array}{l}\text { University of California } \\
\text { - Davis }\end{array}$ & 10 & 0 & 0 & 0 & 10 \\
\hline $\begin{array}{l}\text { Stanford Linear } \\
\text { Accelerator }\end{array}$ & 10 & 0 & 0 & 0 & 10 \\
\hline University of Utah & 10 & 0 & 0 & 0 & 10 \\
\hline Ames Laboratory & 1 & 0 & 0 & 0 & 1 \\
\hline TOTAL & 13,850 & 0 & 190 & 120 & 14,170 \\
\hline
\end{tabular}

reduce the volume of waste sent to Hanford. Such waste streams include certain wastes from Paducah and Portsmouth. The volumes presented in this report do not reflect the information contained in the STPs, because the forecast requests were completed before the majority of the STPS were completed.

Offsite waste generators seem to have a high degree of certainty based on the minimum and maximum ranges provided. The waste generators indicate 
minimum and maximum ranges that indicate some uncertainty; however, the general range for waste generation is minimal (with the exception of Argonne National Laboratory - East). Some additional waste volumes may be expected if D\&D waste volumes from Portsmouth Gaseous Diffusion Plant - Energy Systems are sent to the CWC. Additional waste may also be sent from Paducah Energy Systems if they decide to send TRU waste to the Hanford Site. In addition, the exact schedule for shipment of the existing waste is uncertain. 


\subsection{REFERENCES}

Bowen, W. W. 1992. B Plant Cleanout and Stabilization Program Plan. WHC-SD-WM-AP-023, Rev.0, Westinghouse Hanford Company, Richland, Washington.

Cabson, M. A. 1993. Hanford Restoration Baseline Approach - Interface Control Document. WHC-SD-ER-EE-002, Rev. 0, Westinghouse Hanford Company, Richland, Washington.

Fulton, J. C. 1994. Hanford Spent Nuclear Fuel Project Recommended Path Forward. WHC-EP-0830, Vol. 1 \& 2, Rev. 0, Westinghouse Hanford Company, Richland, Washington.

Washington State Department of Ecology. 1993. Dangerous Waste Regulations. Washington State Administrative Code, 173-303.

Westinghouse Hanford Company. 1994. Estimation of PUREX Equipment and Materials that are Candidates for Removal and Waste Processing during PUREX Plant Closure. WHC-IP-0977, Rich7and, Washington.

Valero, 0. J., et al. 1994. Solid Waste 30-Year Volume Summary. WHC-EP-0768, Westinghouse Hanford Company, Richland, Washington.

Westinghouse Hanford Company. 1994. Hanford Site Tank Waste Remediation System Technical Strategy, Rev. 0, WHC, Richland, Washington. 
IHC-EP-0865

Intentionally Left Blank 
WHC-EP-0865

\section{APPENDIX A}

\section{ASSUMPTIONS FOR SUPPLEMENTARY SOURCES}


WHC-EP-0865

Intentionally Left Blank 
WHC-EP-0865

APPENDIX A

\section{ASSUMPTIONS FOR SUPPLEMENTARY SOURCE}

This appendix briefly describes the references used to supplement the FY 1994 Solid Waste Forecasts and any assumptions made in using these references.

\section{Fiscal Year (FY) 1993 Solid Waste Forecasts:}

Several generators that completed a FY 1993 forecast did not complete the FY 1994 forecast. These generators include those that were given the option of completing a new forecast (as discussed in Section 1.0) as well as other generators that did not complete forecasts for various reasons. In these cases, the FY 1993 forecast data were included in the FY 1994 data set. Since the FY 1993 forecast covered the years 1994 to 2023, and the FY 1994 forecast covers the years 1995 to 2024, the data for 1994 were not used, and the data for 2023 were duplicated in 2024. The minimum and maximum volumes were assumed to be the same as the baseline.

\section{Draft Report: Waste Stream Evaluation for Long-Term Decontamination Study:}

This special study investigated potential D\&D waste generators that are not on the surplus facilities list. Hundreds of facilities exist on the Hanford Site that will require D\&D. To limit the study, two sources were used to identify 26 facilities with potentially large D\&D volumes: the 1992 solid waste forecast database and the programmatic environmental impact statement database. Waste information was gathered primarily through interviews with operational and waste personnel. Some estimates were developed by briefly touring the building and estimating the amount of equipment or contaminated material. Data include rough estimates of the waste volumes by waste class, physical characteristics, and possible container types. The shipment of this waste was assumed to begin in 2013 and conclude in 2022.

For the purpose of completing the 30-Year Summary Report, the following assumptions and data revisions were made:

- Only the volume and waste category data were used.

- Building demolition and soil excavation activities are not expected to be managed by Solid Waste; therefore, the physical waste category construction material and the waste.generator Secondary Environmental Restoration were not used.

- Volumes for PUREX and the PUREX Tunnels were not used since volumes for these facilities were obtained from another source (WHC-IP-0977). 
WHC-EP-0865

- Volumes for the canyon buildings, B-Plant, T-Plant, and the Plutonium Finishing Plant were used, but a ratio of $80 \%$ contact-handled to $20 \%$ remote-handled was based upon personal conversation with Ken Hladek of WHC.

- Volumes associated with the Hanford Waste Vitrification Plant and the Grout facility were not used because these projects have been cancelled and the projects that are scheduled to replace them will not undergo D\&D until after the 30-year forecast period.

Estimation of PUREX Equipment and Materials that are Candidates for Removal and Waste Processing During PUREX Plant Closure, WHC-IP-0977 (Valero 1994)

This study is a detailed investigation of potential waste from PUREX and the PUREX Tunnels, two facilities not listed on the surplus facilities list that are expected to generate large volumes of D\&D waste. Volumes and waste category information were obtained from this study; however, no distinction was made between contact-handled and remote-handled waste. Therefore, a ratio of $80 \%$ contact-handled to $20 \%$ remote-handled was assumed based upon personal conversation with Ken Hladek.

This document also states that surveillance and maintenance waste will peak at $20 \%$ of the current waste generation rate (pp. 2-6). It was assumed that the current generation rates corresponded to the last year (FY 1998) that volumes were provided in the FY 1994 forecasts for PUREX. The volumes provided in the forecast for 1998 were multiplied by $20 \%$ to obtain the annual generation rate for PUREX surveillance and maintenance waste. A schedule for this waste was not provided. It was assumed that surveillance and maintenance would occur from 1999 through 2011. This assumption is based on completion of transition (deactivation) in 1998 as estimated in the forecast and D\&D beginning in 2012, as assumed by the draft report discussed above.

Hanford Restoration Baseline Approach - Interface Control Document, WHC-SD-ER-EE-002, Rev. 0 (Casbon 1993)

This document describes the responsibilities of the Solid Waste (SWD) and Environmental Restoration (ER) programs in the remediation of Hanford's past practice sites. It also provides waste volume estimates and identifies program responsibilities. The Environmental Restoration Division's responsibilities include planning, field operations, environmental remediation, and site cleanup of unplanned release sites, inactive waste sites, and other areas contaminated with radioactive or chemical hazards. The SWD is responsible for the management of centralized facilities for the receipt, containment, treatment, storage, and disposal of solid waste at hanford. The SWD program will provide storage of LLMW and TRU_TRUM waste; burial of LLW; treatment and disposal of LLW and LLMW; and charācterization, size reduction, certification, packaging and shipment of TRU waste to the Waste Isolation Pilot Plant. 
At this time, TRU TRUM waste will not be handled by ERD; therefore, the SWD Program will receive this waste. Any waste that the SWD program has treatment technologies for may also be sent to this program rather than ERD.

The volumes included in the forecast data were developed from estimates of solid waste generation provided in the Hanford Restoration Baseline Approach. This document states the following assumptions for remedial action wastes:

- $10 \%$ of the LLMW is expected to be sent to SWD.

- All of the TRU, TRUM, and greater-than-class-III (GTC_III) is expected to be sent to SWD. (ATthough WHC-SD-ER-002 did not specify the GTC_III waste as LLW or LLMW, it was assumed to be LLMW for the purposes of this document.)

- All waste is expected to be contact-handled.

The volumes were modified according to these guidelines and included in the Forecast Database under the Past Practice Remediation waste generator. 
WHC-EP-0865

Intentionally Left Blank 
WHC-EP-0865

APPENDIX B

LOW-LEVEL WASTE VOLUMES

$B-i$ 
WHC-EP-0865

Intentionally Left Blank 
WHC-EP-0865

\section{APPENDIX B}

\section{LOW-LEVEL WASTE VOLUME}

In addition to the low-level mixed waste (LLMW) and transuranic and transuranic mixed (TRU TRUM) waste described in this document, a large amount of low-level waste (LLW) will also be sent to the Central Waste Complex (CWC) for processing. Because this waste will require little or no treatment before burial or disposal, it has not been included in previous sections. The LLW volumes are provided here, however, as a source of reference. This information, combined with the information in Sections 2 through 7 of this document, represents the total radioactive and mixed solid waste currently forecasted for treatment, storage, and disposal (TSD) at the CWC.

\section{B.1 BACKGROUND}

A11 of the major program areas identified in this document are expected to generate LLW. Although the volumes of LLW are considerably greater than the LLMW and TRU TRUM waste volumes, they do not represent a great concern because the vast majority of this waste can be sent directly to disposal. Therefore, it is unlikely that the volumes presented in this section will have a significant impact on the solid waste system.

The primary source used for obtaining LLW volumes is the 1994 Solid Waste Forecast. As with LLMW and TRU_TRUM waste, several additional sources more completely represent the LLW volumes expected at the Hanford Site over the next 30 years. These additional sources include the 1993 Solid Waste Forecasts for generators that did not submit a forecast in 1994, a draft report of decontamination and decommissioning (D\&D) waste from facilities not listed as surplus, and a study of D\&D waste from PUREX. These sources are explained in greater detail in Section 4.1 and Appendix A.

\section{B.2 LLW VOLUMES BY PROGRAM AREA}

As shown in Figure B-1, a baseline total of $1,461,580$ cubic meters of LLW is expected at the Hanford Site over the next 30 years. The majority of this waste is from the Environmental Restoration (ER) program, which expects to generate 1,201,750 cubic meters. Other major contributors of LLW include Offsite, TWRS and Other Programs (Analytical Services, Liquid Effluent, PNL, Solid Waste, and Spent Nuclear. Fuel), forecasting 143,670 cubic meters, 53,020 cubic meters, and 47,140 cubic meters, respectively. Facility Transitions is expected to generate less than $1 \%$ of the total LLW volume. Due to the extremely small amount of waste expected from Non-Programmatic generators, this program area cannot be identified in Figure B-1. 
Figure B-1. Total 30-Year Solid Low-Level Waste Volumes by Program Area.

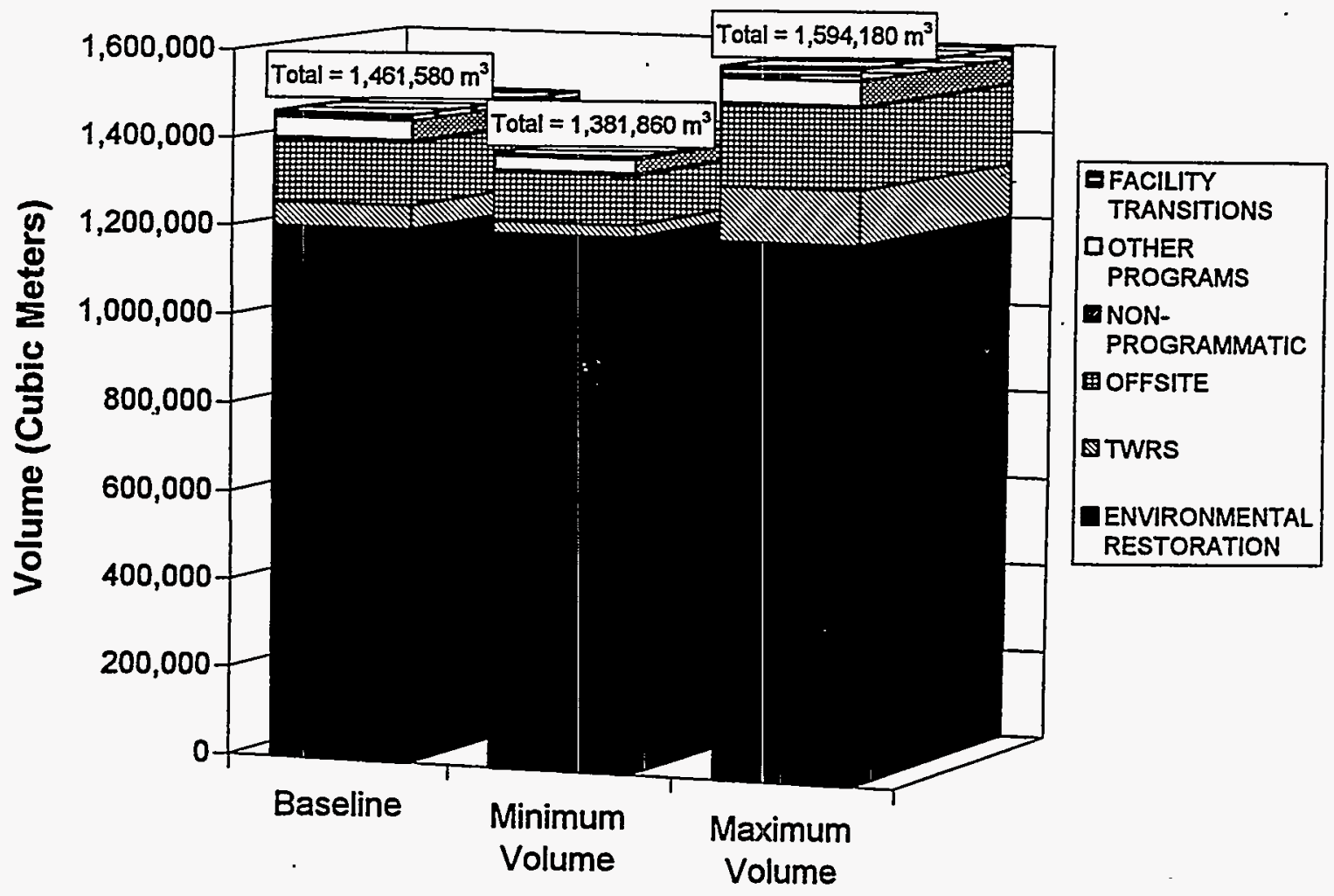

Figure B-1 also shows minimum and maximum estimates of $1,381,860$ cubic meters and 1,594,180 cubic meters. These estimates reflect ranges of variation from the baseline volumes provided by the waste generators. TWRS, Offsite Facilities, Other Programs, and Facility Transitions each contribute to the variations from the baseline volume. As with LLMW and TRU TRUM waste, minimum and maximum estimates were only supplied by generators completing a 1994 forecast. For all other generators, the minimum and maximum volumes were assumed to be the same as the baseline, which accounts for the consistency between the baseline, minimum, and maximum volumes for ER.

Figure B-2 shows the overall baseline, minimum, and maximum annual volumes of LLW. This figure shows a large increase in LLW volumes starting in 2000 and continuing through 2007 where the volumes peak at approximately 220,000 cubic meters. The volumes then decrease rapidly to less than 15,000 cubic meters in 2012. The vast majority of the waste from 2000 to 2012 has been forecasted by Surplus Facilities within the Environmental Restoration program area. Other than the time period from 2000 through 2012, the volumes of LLW are fairly constant, ranging from roughly 5,000 cubic meters to just under 30,000 cubic meters. The overall minimum and maximum volumes vary only slightly from the baseline volumes. This results from the lack of minimum and maximum ranges for the Surplus Facilities generator. 
Figure B-2. Total Annual 30-Year Solid Low-Level Waste Volumes.

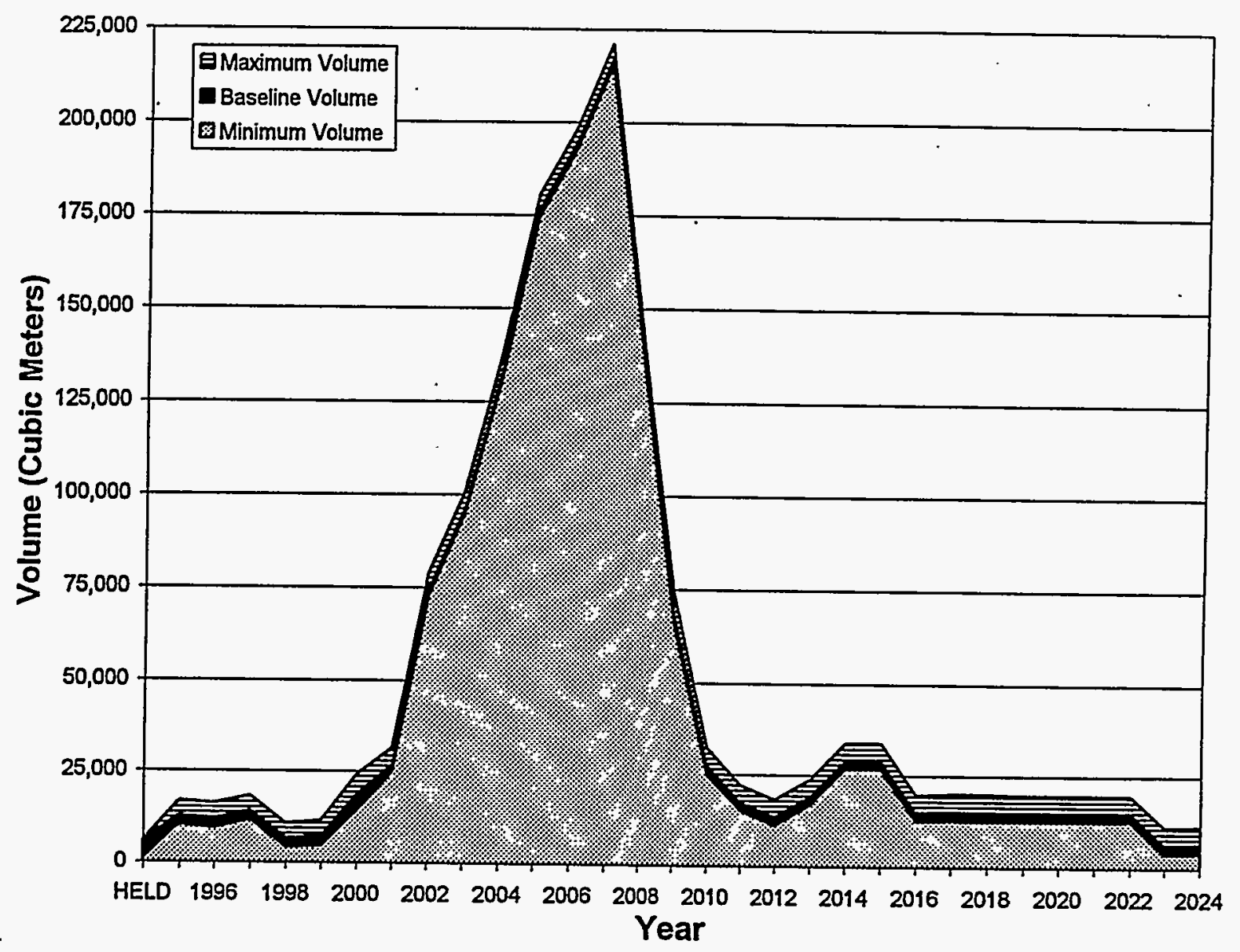

Figures B-3 through B-8 display annual LLW volumes by program area for the baseline, minimum, and maximum volumes. Figure B-3 shows the annual volumes for the TWRS program. This program forecasts a baseline volume of approximateiy 500 cubic meters of waste through 2004. In 2005, the volume increases to just under 1,300 cubic meters, coinciding with the expected startup of the Low-Level Vitrification Project. In 2009, the volumes increase again due to the startup of the High-Level Vitrification Project and remain between 2,500 and 3,000 cubic meters through 2024 . Figure $B-3$ also shows that the minimum and maximum volumes for the TWRS program generally correspond to $50 \%$ and $200 \%$ of the baseline volume, respectively.

Figure B-4 shows the baseline, minimum, and maximum volumes for the - Environmental Restoration program. This figure reflects the trends in Figure B-2 of a large increase in waste volumes from 2000 through 2007 followed by a sharp decrease through 2012. The baseline, minimum, and maximum volumes are essentially the same for this program because the primary generator, Surplus Facilities, did not provide minimum and maximum percent ranges. 
Figure B-3. TWRS Annual 30-Year Solid Low-Level Waste Volumes.

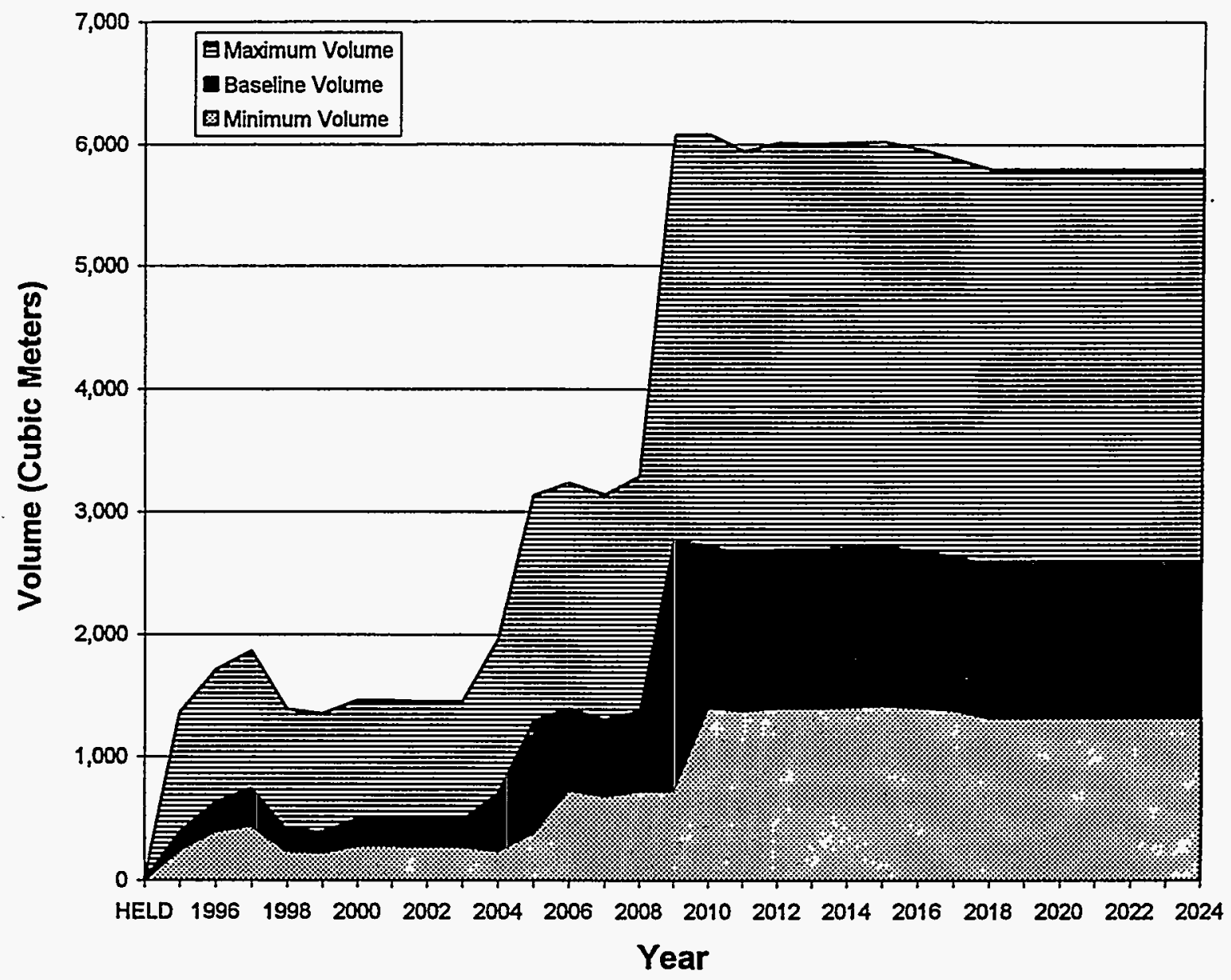

Figure B-5 contains the annual waste volumes for the Facility Transitions program. The figure shows that the baseline volumes decrease from slightly under 1,200 cubic meters in 1995 to approximately 700 cubic meters by 2009. In 2010, the volume decreases to approximately 300 cubic meters due to completion of PFP deactivation activities. The volumes then decrease gradually to roughly 200 cubic meters and continue at this level for the remainder of the forecast. For the majority of the forecast period, the minimum volume expected approximately 100 cubic meters less than the baseline volume. The maximum volume expected is between 400 cubic meters and 800 cubic meters greater than the baseline volume for the first 15 years of the forecast period and approximately 100 cubic meters greater for the last 15 years.

The LLW volumes from the Other Programs area shown in Figure B-6 are greatest in 1995 at just under 2,000 cubic meters. The volumes decrease over the next four years and remain fairly constant at 1,500 cubic meters each year. The minimum and maximum volumes are roughly 400 cubic meters less than and 300 cubic meters greater than the baseline volumes, respectively.

The Non-Programmatic LLW volumes displayed in Figure B-7 show a peak of 220 cubic meters in 1995 . The figure shows approximately 120 cubic meters of 
Figure B-4. Environmental Restoration Annual 30-Year Solid Low-Level Waste Volumes.

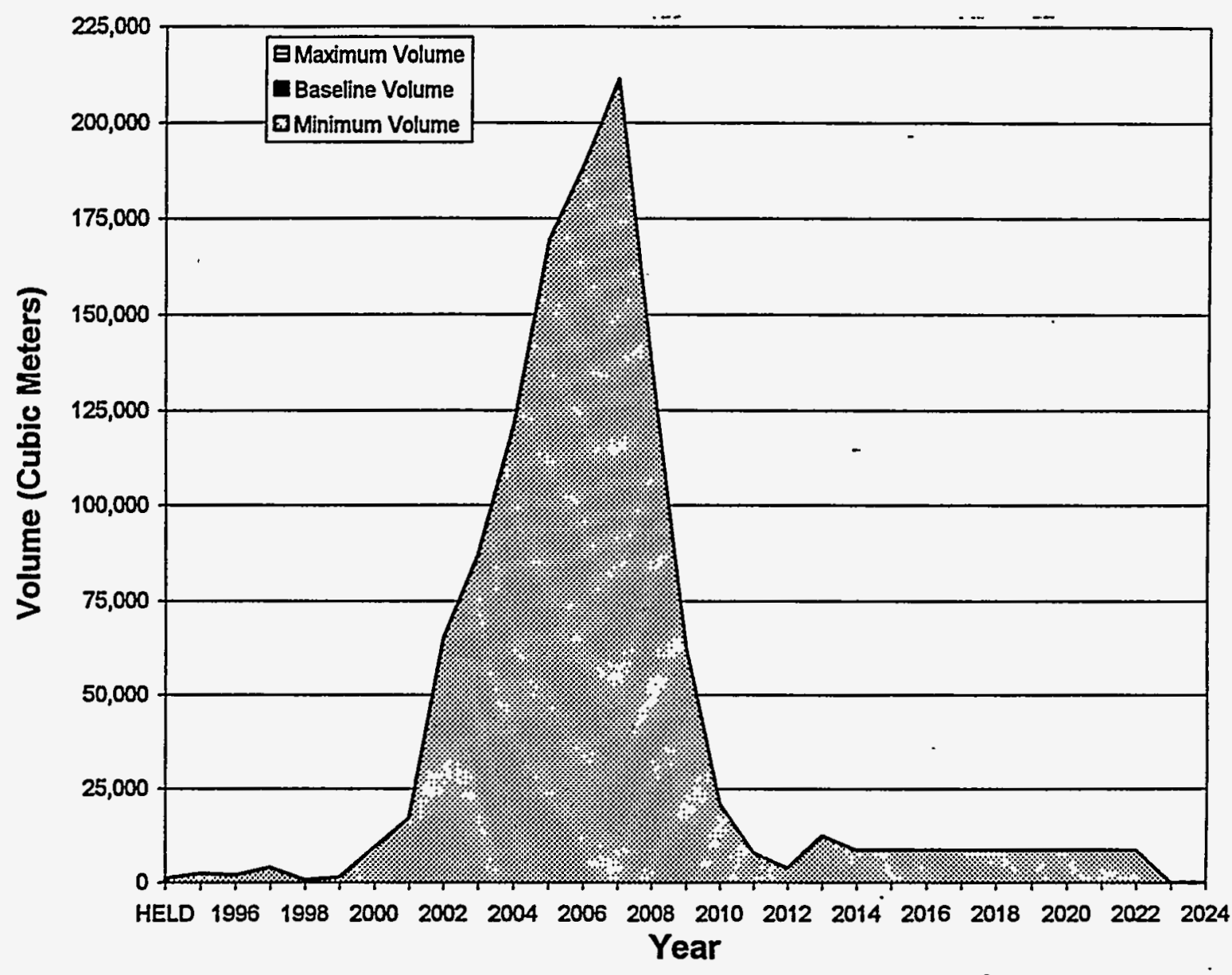

waste each year for the rest of the forecast period. The minimum and maximum volumes are generally within 25 cubic meters of the baseline volumes.

The waste volumes forecasted by offsite waste generators vary greatly over the 30-year forecast period. Figure B-8 shows four large peaks occurring from 1995 to 1997, from 2000 to 2004, from 2011 to 2012, and from 2014 to 2015. The peak from 2014 to 2015 is the largest of these at nearly 16,000 cubic meters. The peak from 1995 to 1997 is well over 6,000 cubic meters and the peak from 2000 to 2004 ranges from over 6,000 cubic meters to over 8,000 cubic meters. The peak from 2011 to 2012 is approximately 5,000 cubic meters. The baseline volumes for the remaining years range between 2,000 cubic meters and 3,000 cubic meters. The minimum and maximum volumes are generally within 500 cubic meters to 1,000 cubic meters of the baseline volumes with the exception of the maximum volume indicated from 1995 to 2004. During this time period, the maximum volume exceeds the baseline volume by as much as 3,300 cubic meters. 
Figure B-5. Facility Transitions Annual 30-Year Solid Low-Level Waste volumes.

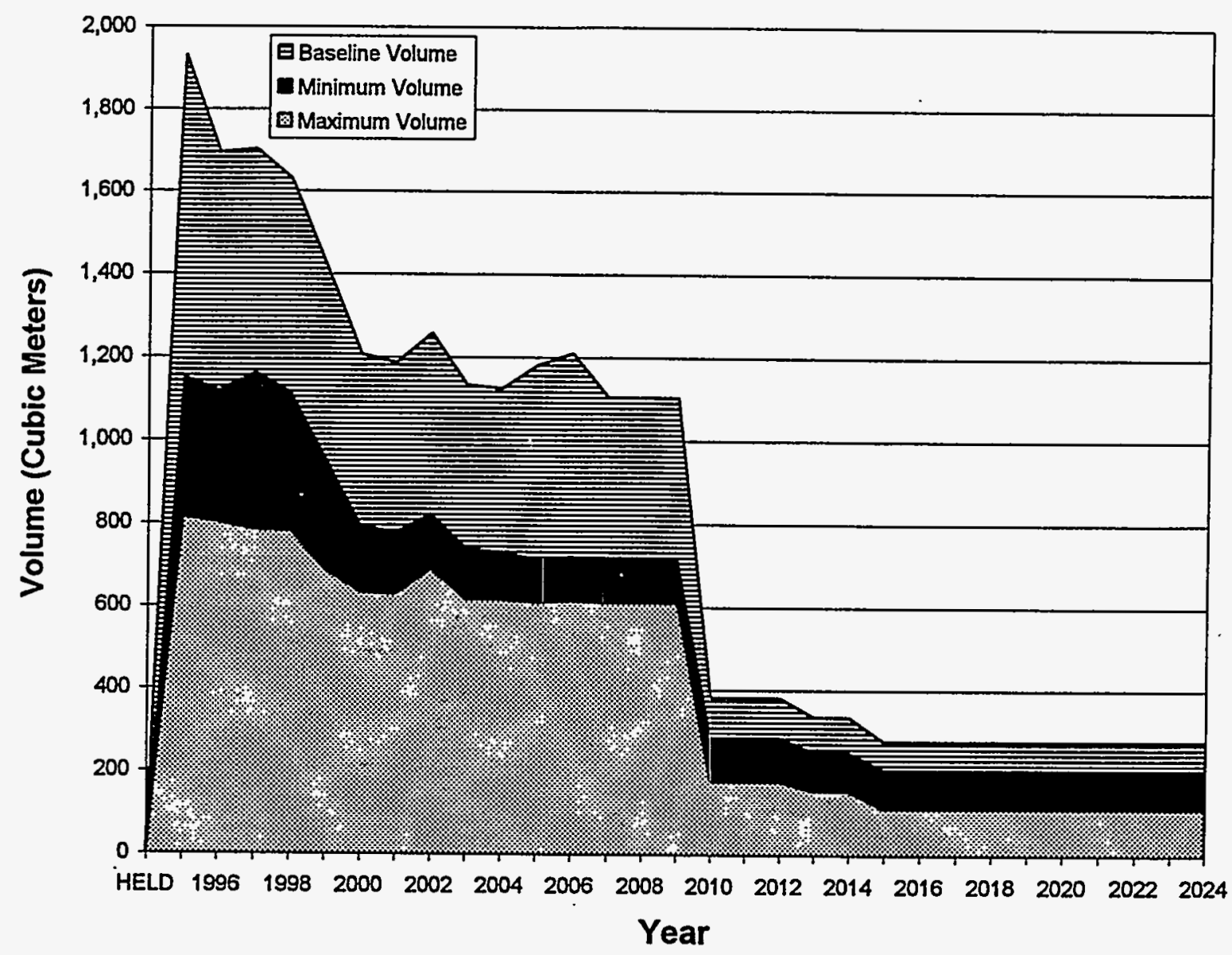

\section{B.3 LLW VOLUMES BY WASTE CATEGORY}

Figure B-9 contains the overall baseline, minimum, and maximum waste volumes by category. The figure shows that the vast majority, 1,403,200 cubic meters, of the LLW is expected to be contact-handled for the baseline, minimum, and maximum volumes. Although the remote-handled $L L W$ is a small percentage of the total LLW volume, it still represents a significant volume, approximately 58,400 cubic meters. This volume may be especially significant because it will be more difficult to transport and dispose of this waste due to remote handling requirements.

Slight variations from the baseline volume for the minimum and maximum volumes of CH_LLW can be seen in Figure B-9. No variations in RH LLW are evident from this figure. Variations do occur, but they are too sma $\overline{7} 7$ to be seen on the figure.

Figures $B-10$ and $B-11$ show the annual baseline, minimum, and maximum volumes, respectively, by waste category. Figure $B-10$ shows a dramatic 
Figure B-6. Other Programs Annual 30-Year Solid Low-Level Waste Volumes.

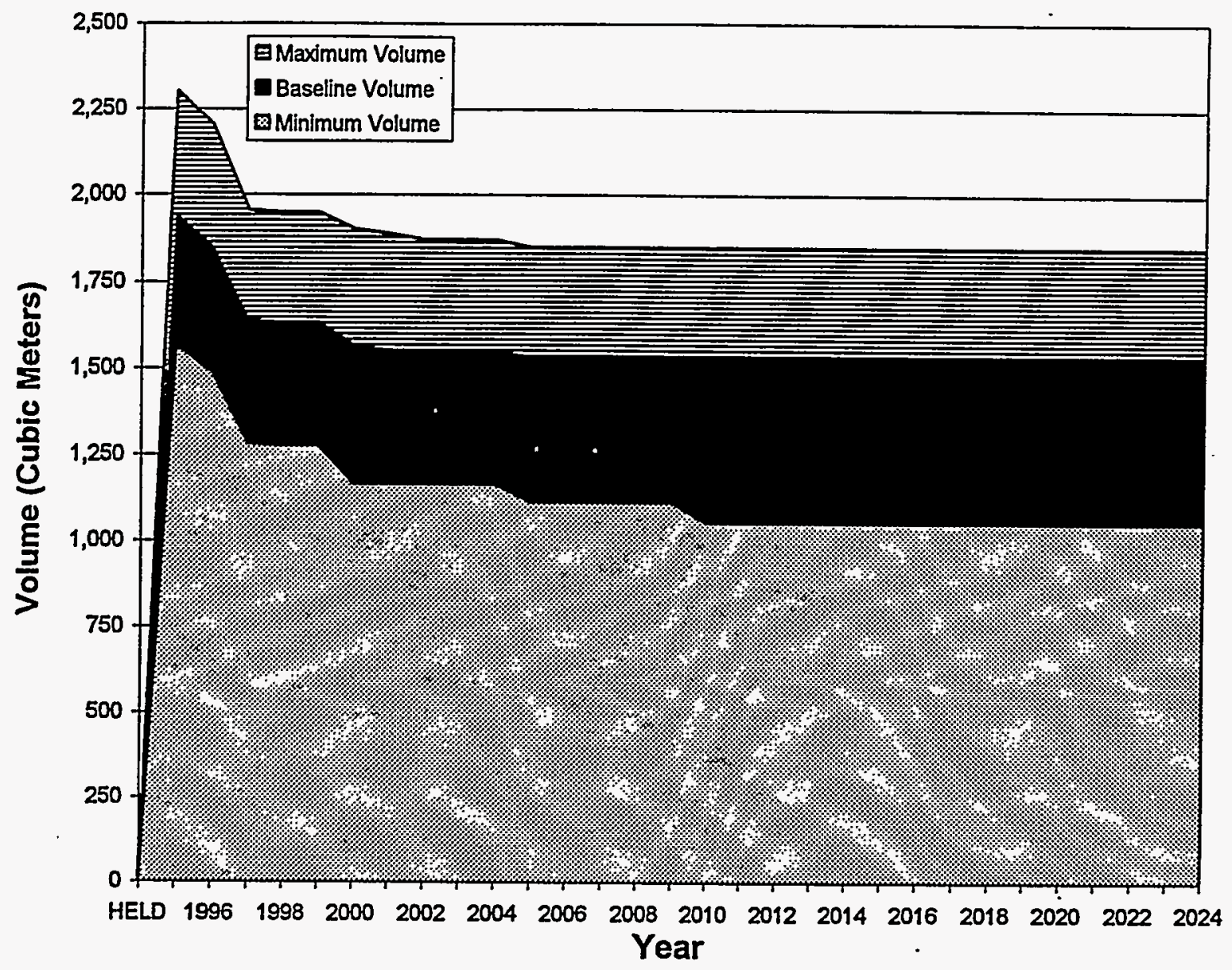

increase of CH_LLW from 2000 through 2007, peaking at approximately 210,000 cubic meters. The volume of $\mathrm{CH}$ LLW then decreases rapidly to less than 30,000 cubic meters in 2010. The minimum and maximum volumes of $\mathrm{CH}$ LLW vary only slightly from the baseline volumes. Both the large peak from $\overline{2} 000$ to 2012 and the slight differences in the minimum and maximum volumes are caused by the Surplus Facilities forecast.

The RH LLW volumes shown in Figure B-11 follow the same general trend of increasing from 2000 to 2007 and then decreasing. The peak volume of RH_LLW, however, is only about 7,500 cubic meters. A fairly significant amount of RH_LLW, approximately 1,400 cubic meters each year, is also expected from 2012 to 2022, primarily from the D\&D of several non-surplus facilities. As with the CH_LLW, the minimum and maximum volumes for RH_LLW do not vary significantly from the baseline due to the lack of ranges for the Environmental Restoration program. 
Figure B-7. Non-Programmatic Annual 30-Year Solid Low-Level Waste Volumes.

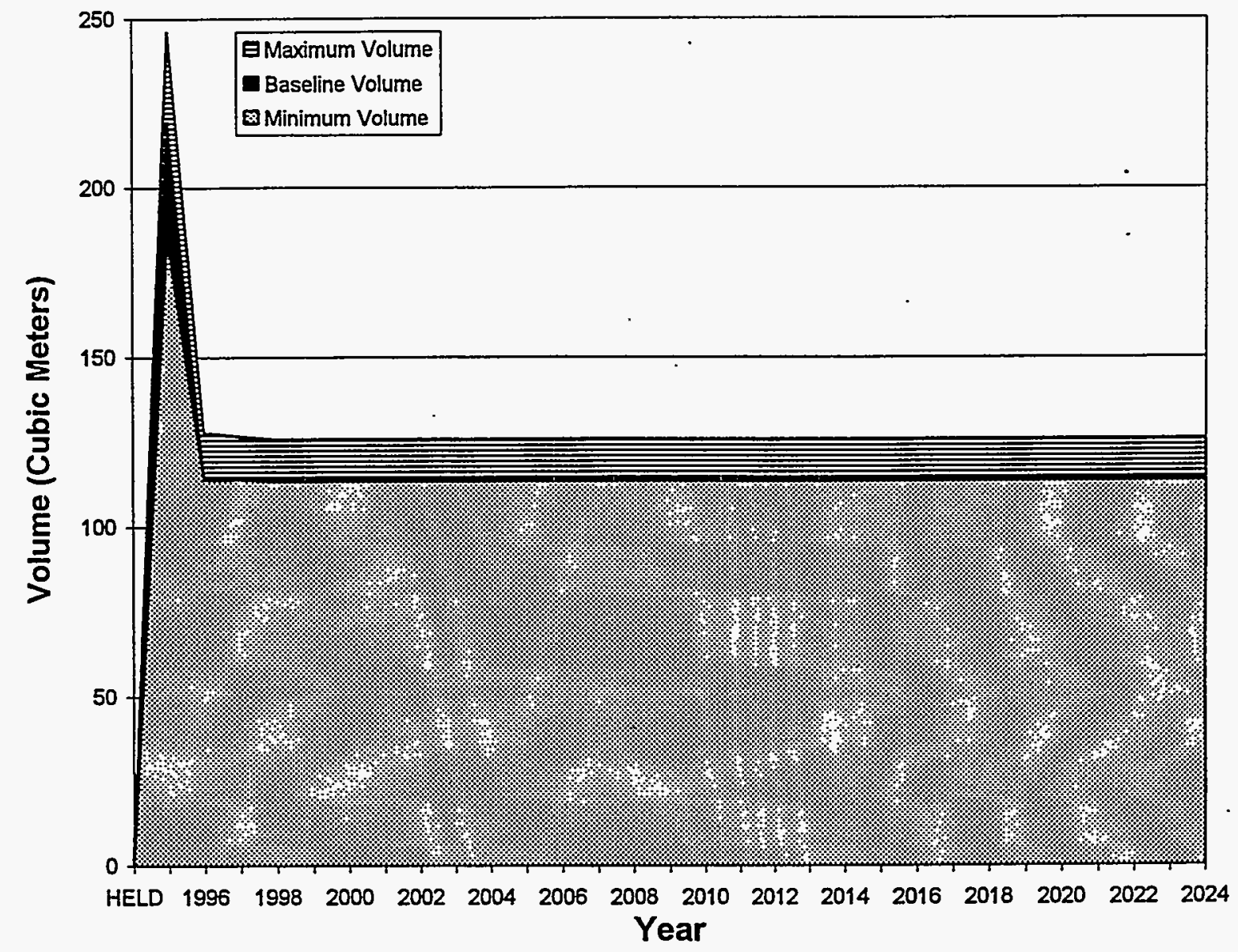

\section{B.4 LLW VOLUMES BY WASTE GENERATOR}

LLW is expected to be generated by 76 waste generators that will send waste to the CWC. TabTe B-1 lists the volumes of CH_LLW and RH_LLW by generator and program area. By far the largest generator of both CH_LLW and RH_LLW is Surplus Facilities. This generator expects to ship over a mīllion cubic meters of CH_LLW and neariy 40,000 cubic meters of RH_LLW. Generators expected to ship at least 10,000 cubic meters of LLW are listed below by program area.

- Environmental Restoration - Surplus Facilities, D\&D of B-Plant, and D\&D of 222-S Analytical Laboratory

- Offsite Facilities - Formerly Utilized Sites Remedial Action Program (FUSRAP), Brookhaven National Laboratory, Lawrence Berkeley Laboratory, and Portsmouth Energy Systems

- TWRS - High-Level Vitrification Project (HLVP) and Low-Level Vitrification Project (LLVP)

- PNL - Pacific Northwest Laboratory 
Figure B-8. Offsite Annual 30-Year Solid Low-Level Waste Volumes.

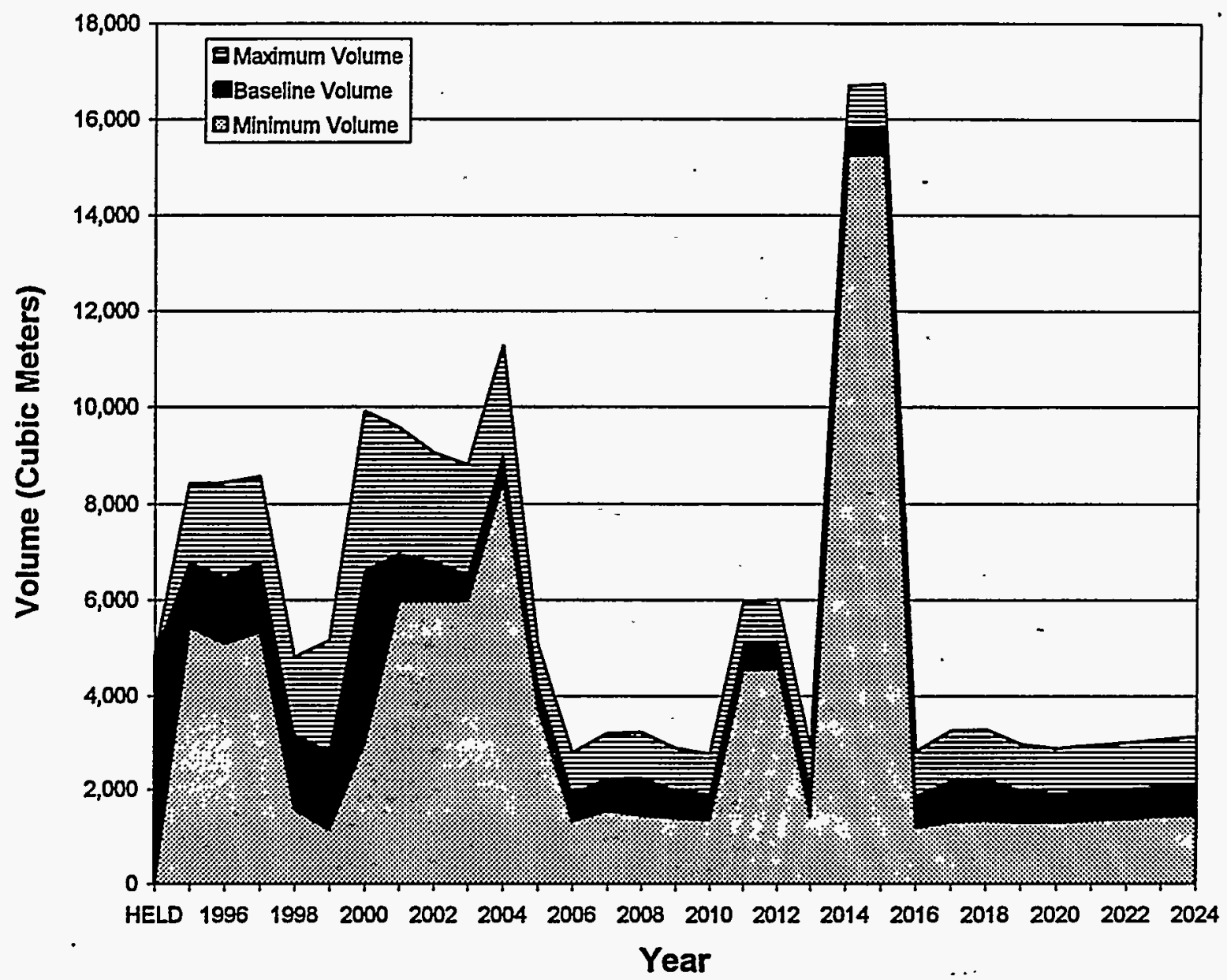

- Liquid Effluent - 300 Area Effluent Treatment (Project L045H)

- Analytical Services - 222-S AnaTytical Laboratory

Facility Transitions, Spent Nuclear Fuel, Non-Programmatic, and Solid Waste do not have any generators that are expected to generate greater than 10,000 cubic meters of LLW.

Most of the primary waste generators of LLW are also primary generators of LLMW and TRU TRUM and have been described in the main document. Those that have not been described are discussed here in order of largest to smallest.

- FUSRAP: FUSRAP is one of several Department of Energy (DOE) programs created to address radiological contamination in excess of guidelines at a number of sites throughout the U.S. Generally, these sites, which became contaminated through uranium and thorium operations, were decontaminated and released for use under the regulations in effect at the time. Since radiological guidelines were not as strict then as today, trace amounts of radioactive 
Figure B-9. Total 30-Year Solid Low-Level Waste Volumes by Waste Category.

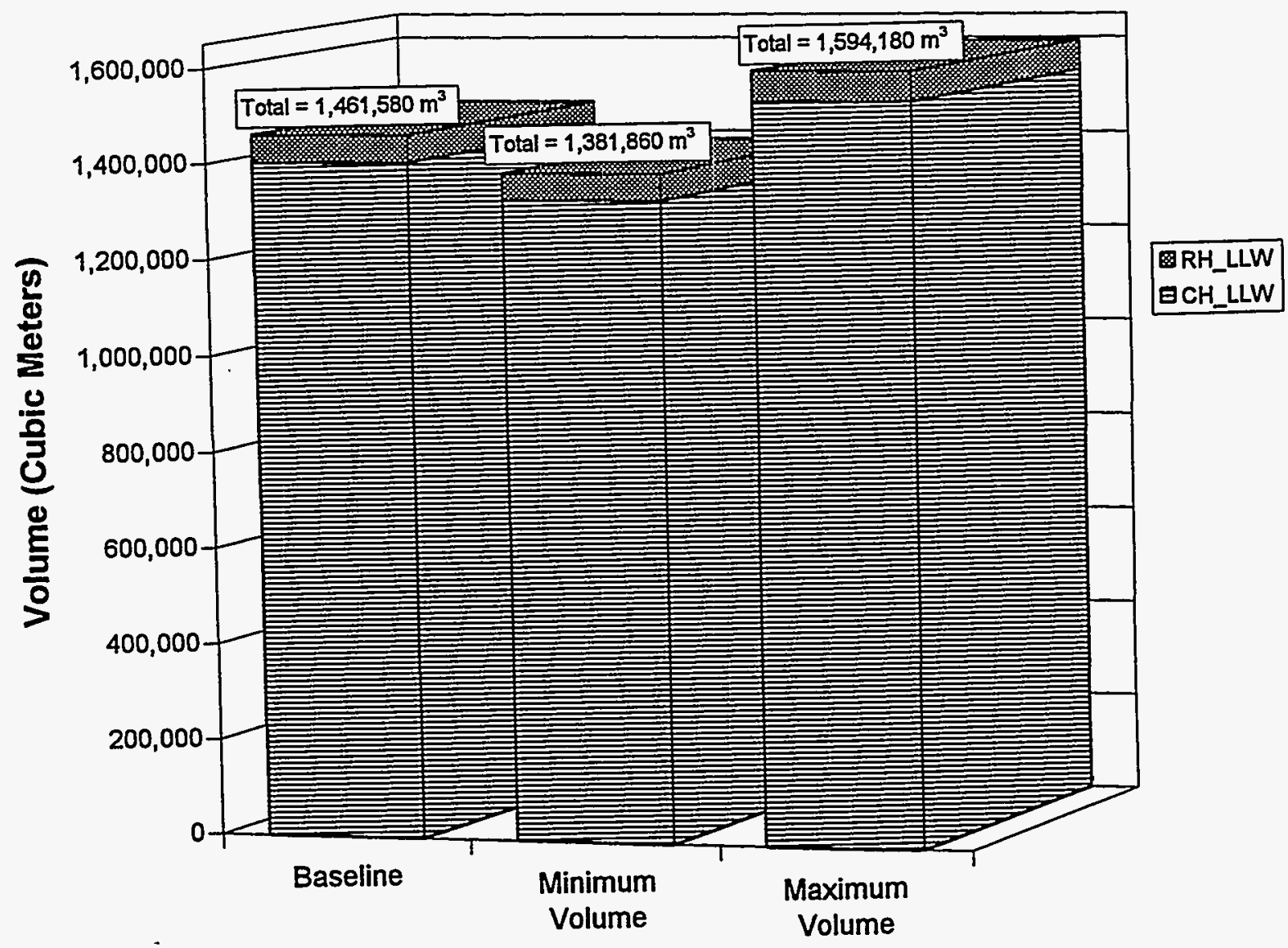

materials remained at some of the sites. Erosion, building demolition, and construction resulted in some of the radioactive residues mixing with largevolumes of soil and rubble, thereby spreading the contamination. If FUSRAP becomes responsible for restoring more sites, their waste volumes could increase. Minimum and maximum ranges were not available for this generator.

- D\&D of B-Plant (TWHC_BPLANT): The volume estimates for B-Plant were obtained by determining the volume of the building from its dimensions. An assumption was made that half of the building volume was contaminated material. In addition, one-third of the Waste Encapsulation and Storage Facility (WESF) was assumed contaminated. Contaminated materials include tanks, jumpers, concentrators, ion exchange columns, filters, ducting, piping, and condensers. Minimum and maximum ranges were not available for this generator.

- Brookhaven National Laboratory (BNL): Brookhaven is a research laboratory that will generate miscellaneous solid laboratory, reactor, and accelerator wastes. Some waste will also be generated 
dnuea lO Ll!ds se yons sə!ł!

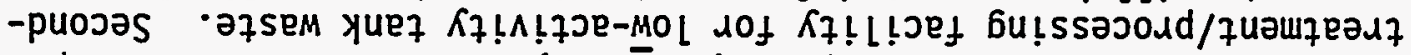

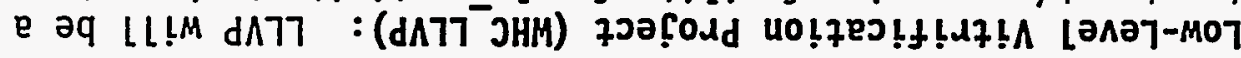

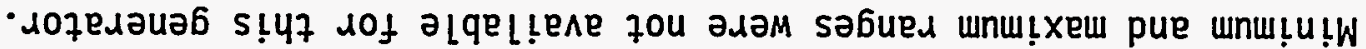

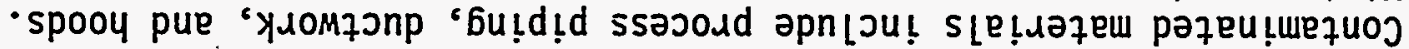

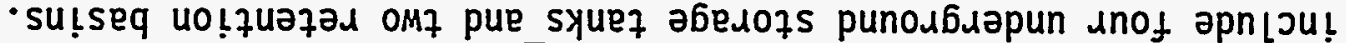

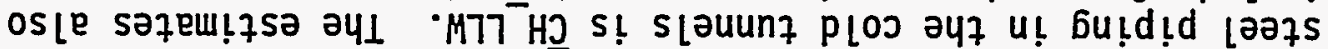

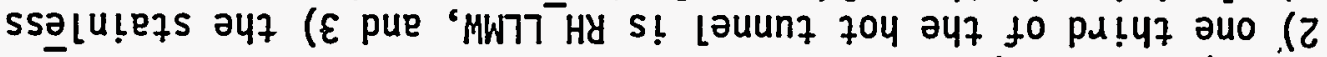

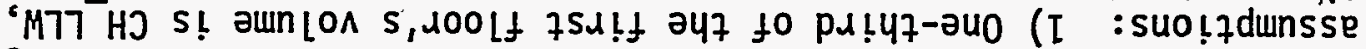

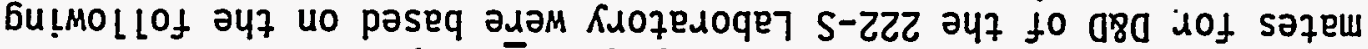

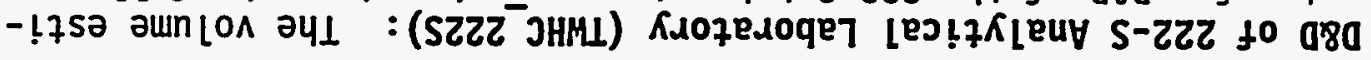

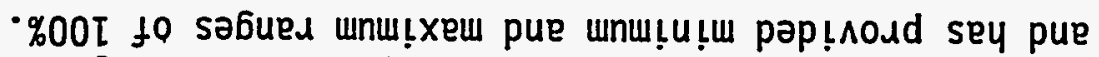

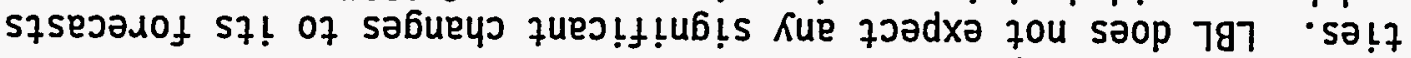

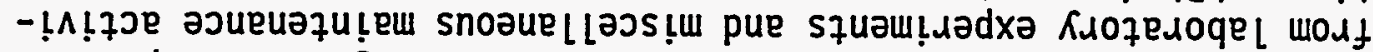

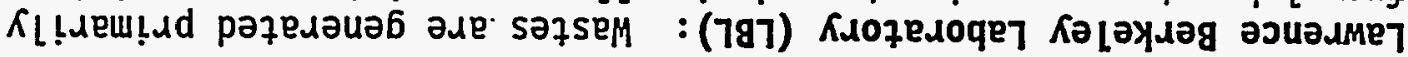

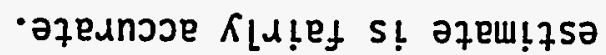

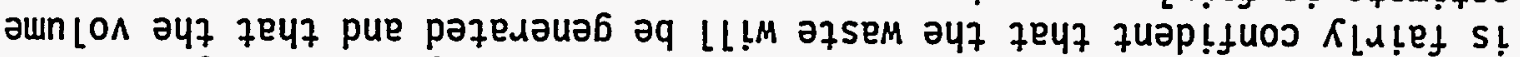

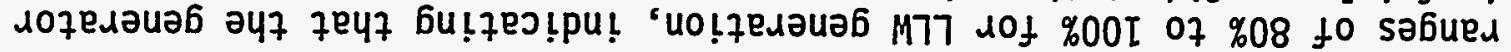

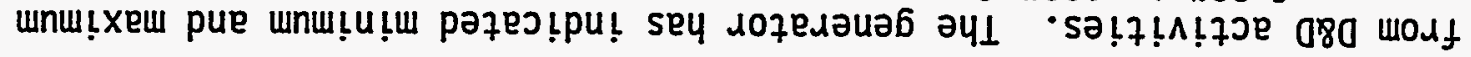

Jeaג

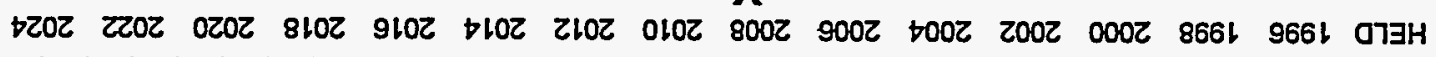

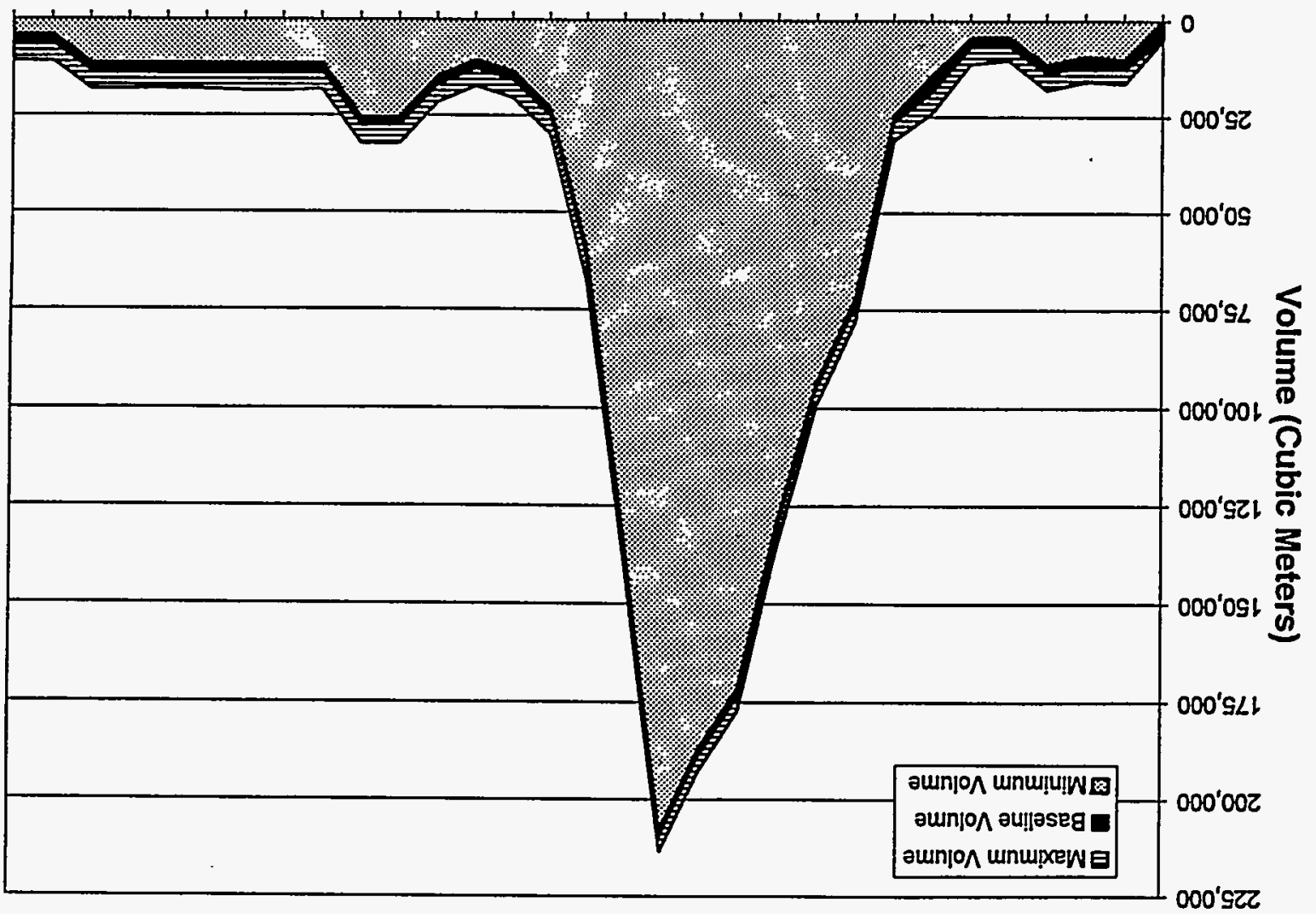

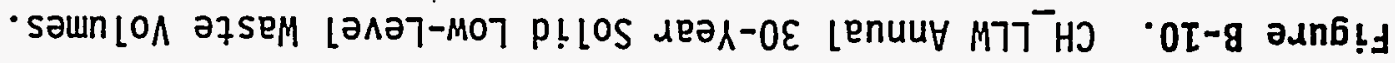


Figure B-11. RH_LLW Annual 30-Year Solid Low-Level Waste Volumes.

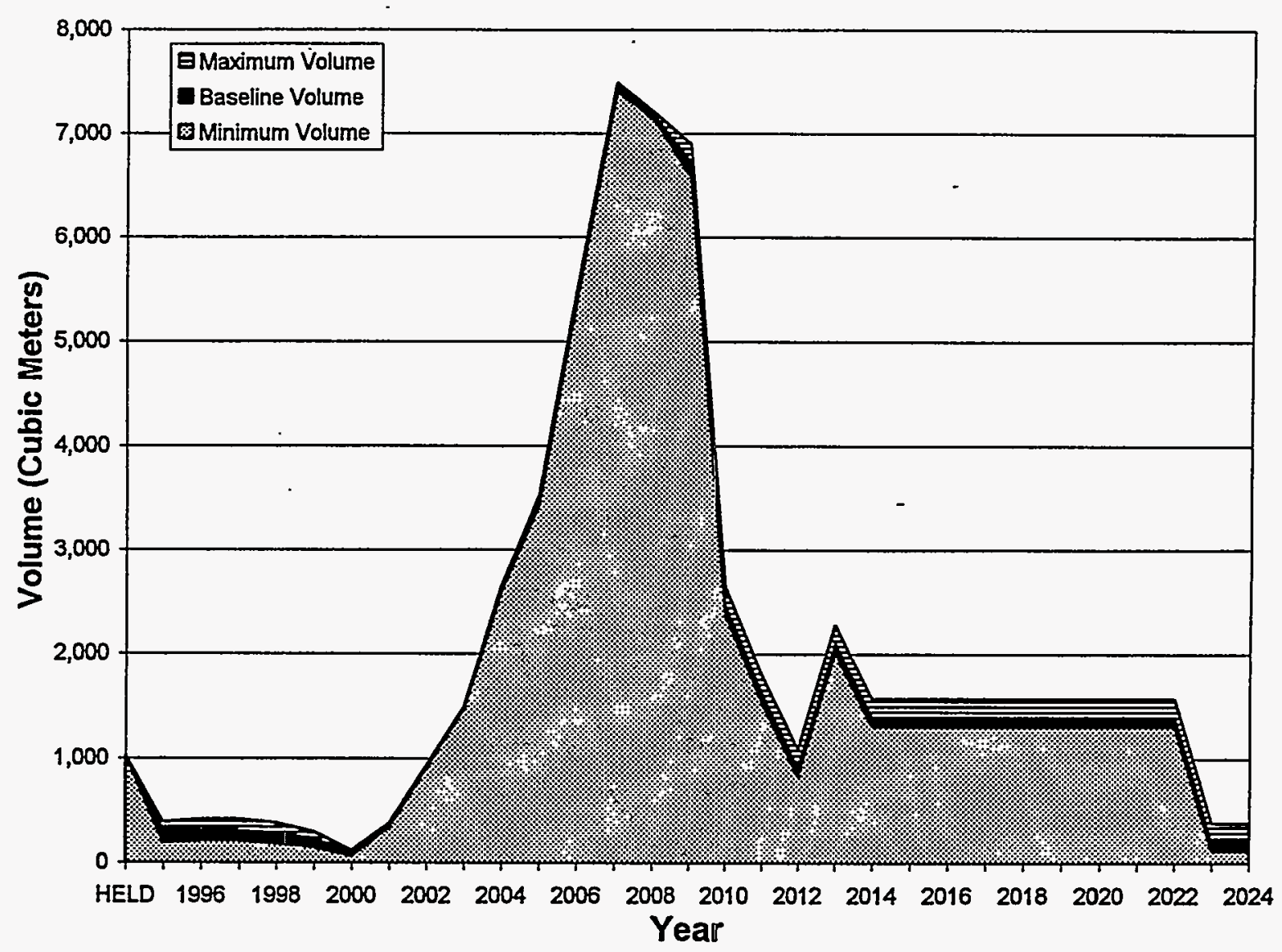

and decontamination, general maintenance and housekeeping, and 1aboratory activities. The waste will generally consist of contaminated failed equipment, metais, paper, plastic, rubber, and other miscellaneous debris. The minimum range for this waste generator is $0 \%$ in the first year of expected operations (2005) and 50\% for the remaining years of operation. The maximum range is $200 \%$ for all years of operation. This indicates that the waste generator is uncertain about waste generation volumes because the facility is not yet operational.

\section{B.5 DATA OBSERVATIONS}

Since the majority of the LLW is expected to be generated by one waste generator, Surplus Facilities, the LLW estimates may vary considerably in the future. The estimates could increase or decrease depending on programmatic uncertainties associated with who has responsibility for treatment, storage, and disposal of D\&D waste. The forecasts will be updated as these issues are resolved and estimates for waste generation are refined. 
Table B-1. Waste Generator Volumes by Program Area.

\begin{tabular}{|c|c|c|c|c|}
\hline Program Area & Waste Generator & $\begin{array}{c}\mathrm{CH} \text { LLW } \\
\left(\mathrm{m}^{3}\right)\end{array}$ & $\begin{array}{c}\mathrm{RH} L L W \\
\left(\mathrm{~m}^{3}\right)\end{array}$ & $\begin{array}{c}\text { Total } \\
\left(\mathrm{m}^{3}\right)\end{array}$ \\
\hline \multirow{17}{*}{$\begin{array}{l}\text { Environmental } \\
\text { Restoration }\end{array}$} & Surplus Facilities & $1,073,020$ & 39,940 & $1,112,960$ \\
\hline & D\&D of B Plant & 34,270 & 8,570 & 42,830 \\
\hline & $\begin{array}{l}\text { D\&D of 222-S Analytical } \\
\text { Laboratory }\end{array}$ & 12,800 & 0 & 12,800 \\
\hline & D\&D of $T$ Plant & 7,140 & 1,790 & 8,930 \\
\hline & D\&D of 100 Area & 8,330 & 0 & 8,330 \\
\hline & D\&D of PUREX & 4,770 & 1,190 & 5,960 \\
\hline & D\&D of PNL 314 Building & 3,180 & 0 & 3,180 \\
\hline & D\&D of PNL 331 Building & 1,910 & 0 & 1,910 \\
\hline & D\&D of PNL 327 Building & 1,150 & 310 & 1,460 \\
\hline & $\begin{array}{l}100 \text { Area Transition } \\
\text { (Bechtel) }\end{array}$ & 1,380 & 60 & 1,440 \\
\hline & PUREX Tunnels & 0 & 1,020 & 1,020 \\
\hline & D\&D of PNL 324 Building & 360 & 30 & 390 \\
\hline & $\begin{array}{l}\text { D\&D of } 340 \text { Waste Neu- } \\
\text { tralization Facility }\end{array}$ & 200 & 0 & 200 \\
\hline & $\begin{array}{l}\text { D\&D of } 308 \text { Fuels Deve1- } \\
\text { opment Laboratory }\end{array}$ & 130 & 0 & 130 \\
\hline & $\begin{array}{l}\text { D\&D of PNL } \\
\text { 242B/BL Building }\end{array}$ & 100 & 0 & 100 \\
\hline & $\begin{array}{l}\text { D\&D of PNL } \\
\text { 306-W Building }\end{array}$ & 50 & 0 & 50 \\
\hline & $\begin{array}{l}\text { Environmental Projects- } \\
\text { RCRA Waste Only }\end{array}$ & 40 & 0 & 40 \\
\hline
\end{tabular}


Table B-1. Waste Generator Volumes by Program Area.

\begin{tabular}{|c|c|c|c|c|}
\hline Program Area & Waste Generator & $\begin{array}{c}\mathrm{CH} L L W \\
\left(\mathrm{~m}^{3}\right)\end{array}$ & $\begin{array}{c}\mathrm{RH} L L W W \\
\left(\mathrm{~m}^{3}\right)\end{array}$ & $\begin{array}{c}\text { Total } \\
\left(\mathrm{m}^{3}\right)\end{array}$ \\
\hline \multirow{4}{*}{$\begin{array}{l}\text { Environmental } \\
\text { Restoration } \\
\text { (cont.) }\end{array}$} & D\&D of PNL 326 Building & 20 & 0 & 20 \\
\hline & $\begin{array}{l}\text { D\&D of } 306 \text { Development/ } \\
\text { Fabrication/Testing } \\
\text { Laboratory }\end{array}$ & 10 & 0 & 10 \\
\hline & $\begin{array}{l}\text { D\&D of PNL } \\
\text { 231-Z Building }\end{array}$ & 4 & 0 & 4 \\
\hline & Total & $1,148,840$ & 52,910 & $1,201,750$ \\
\hline \multirow[t]{10}{*}{ Offsite } & $\begin{array}{l}\text { Formerly Utilized Sites } \\
\text { Remedial Action Program }\end{array}$ & 57,450 & 0 & 57,450 \\
\hline & $\begin{array}{l}\text { Brookhaven National } \\
\text { Laboratory }\end{array}$ & 18,980 & 10 & 18,990 \\
\hline & $\begin{array}{l}\text { Lawrence Berkeley } \\
\text { Laboratory. }\end{array}$ & 15,780 & 0 & 15,780 \\
\hline & $\begin{array}{l}\text { Portsmouth Energy } \\
\text { Systems }\end{array}$ & 15,480 & 0 & 15,480 \\
\hline & $\begin{array}{l}\text { Argonne National } \\
\text { Laboratory-East }\end{array}$ & 9,760 & 40 & 9,790 \\
\hline & $\begin{array}{l}\text { Plasma Physics } \\
\text { Laboratory }\end{array}$ & 8,160 & 0 & 8,160 \\
\hline & Paducah Energy Systems & 6,690 & 0 & 6,690 \\
\hline & $\begin{array}{l}\text { University of } \\
\text { California-Davis }\end{array}$ & 1,840 & 0 & 1,840 \\
\hline & $\begin{array}{l}\text { Portsmouth Utility } \\
\text { Services }\end{array}$ & 1,780 & 0 & 1,780 \\
\hline & $\begin{array}{l}\text { Battelle Columbus } \\
\text { Laboratories }\end{array}$ & 1,500 & 0 & 1,500 \\
\hline
\end{tabular}


Table B-1. Waste Generator Volumes by Program Area.

\begin{tabular}{|c|c|c|c|c|}
\hline Program Area & Waste Generator & $\begin{array}{c}\mathrm{CH} L \mathrm{LLW} \\
\left(\mathrm{m}^{3}\right)\end{array}$ & $\begin{array}{c}\mathrm{RH} L L L W \\
\left(\mathrm{~m}^{3}\right)\end{array}$ & $\begin{array}{c}\text { Total } \\
\left(\mathrm{m}^{3}\right)\end{array}$ \\
\hline \multirow[t]{13}{*}{$\begin{array}{l}\text { Offsite } \\
\text { (cont.) }\end{array}$} & $\begin{array}{l}\text { FERMI National Accel- } \\
\text { erator Laboratory }\end{array}$ & 1,360 & 0 & 1,360 \\
\hline & Rockwell-Canoga Park & 1,270 & 0 & 1,270 \\
\hline & $\begin{array}{l}\text { Stanford Linear Accel- } \\
\text { erator Center }\end{array}$ & 960 & 0 & 960 \\
\hline & $\begin{array}{l}\text { Paducah Utility } \\
\text { Services }\end{array}$ & 930 & 0 & 930 \\
\hline & General Atomics & 710 & 130 & 840 \\
\hline & $\begin{array}{l}\text { Knolls Atomic Power- } \\
\text { Shipyards }\end{array}$ & 300 & 0 & 300 \\
\hline & EG\&G Rocky Flats Plant & 290 & 0 & 290 \\
\hline & $\begin{array}{l}\text { Bettis Atomic Power- } \\
\text { Laboratory }\end{array}$ & 150 & 0 & 150 \\
\hline & $\begin{array}{l}\text { Ames Laboratory-Ames, } \\
\text { Iowa }\end{array}$ & 100 & 0 & 100 \\
\hline & University of Utah & 10 & .0 & 10 \\
\hline & $\begin{array}{l}\text { Bettis Atomic Power- } \\
\text { Shipyards }\end{array}$ & 10 & 0 & 10 \\
\hline & $\begin{array}{l}\text { Bates Accelerator- } \\
\text { Massachusetts }\end{array}$ & 2 & 0 & 2 \\
\hline & Total & 143,490 & 180 & 143,670 \\
\hline \multirow[t]{4}{*}{ TWRS } & $\begin{array}{l}\text { High Level Vitrifica- } \\
\text { tion Project }\end{array}$ & 20,160 & 2,030 & 22,190 \\
\hline & $\begin{array}{l}\text { Low Level Vitrification } \\
\text { Project }\end{array}$ & 11,340 & 440 & 11,780 \\
\hline & $\begin{array}{l}\text { SST/DST Tank Farm } \\
\text { Operations }\end{array}$ & 8,940 & 0 & 8,940 \\
\hline & $\begin{array}{l}\text { Tank Waste Pretreatment } \\
\text { Facility }\end{array}$ & 5,960 & 300 & 6,260 \\
\hline
\end{tabular}


Table B-1. Waste Generator Volumes by Program Area.

\begin{tabular}{|c|c|c|c|c|}
\hline Program Area & Waste Generator & $\begin{array}{c}\mathrm{CH}\left(\mathrm{m}^{3}\right) \\
\left(\mathrm{m}^{3} W\right.\end{array}$ & $\begin{array}{c}\text { RH_LLW } \\
\left(\mathrm{m}^{3}\right)\end{array}$ & $\begin{array}{c}\text { Total } \\
\left(\mathrm{m}^{3}\right)\end{array}$ \\
\hline \multirow[t]{11}{*}{ TWRS (cont.) } & $\begin{array}{l}\text { DST Retrieval Systems } \\
\text { (10 tanks) }\end{array}$ & 1,980 & 0 & 1,980 \\
\hline & $\begin{array}{l}\text { W343 DST Retrieval } \\
\text { (17 Tanks) }\end{array}$ & 670 & 0 & 670 \\
\hline & $\begin{array}{l}\text { SST Retrieval } \\
\text { (149 tanks) }\end{array}$ & 540 & 0 & 540 \\
\hline & $\begin{array}{l}\text { Cross-Site Transfer } \\
\text { System }\end{array}$ & 370 & 0 & 370 \\
\hline & SST Tank 106C Sluicing & 190 & 0 & 190 \\
\hline & $\begin{array}{l}\text { Tank Farm Ventilation } \\
\text { Upgrade }\end{array}$ & 60 & 0 & 60 \\
\hline & $\begin{array}{l}\text { DST 101AZ Retrieval } \\
\text { (1 tank) }\end{array}$ & 20 & 0 & 20 \\
\hline & $\begin{array}{l}\text { SST Tank } 106 C \\
\text { Manipulator }\end{array}$ & 10 & 10 & 10 \\
\hline & $\begin{array}{l}\text { Aging Waste Transfer } \\
\text { Lines }\end{array}$ & 10 & 0 & 10 \\
\hline & $\begin{array}{l}\text { W315 Pilot Scale } \\
\text { Retrieval }\end{array}$ & 1 & 0 & 1 \\
\hline & Total & 50,250 & 2,780 & 53,020 \\
\hline PNL & $\begin{array}{l}\text { Pacific Northwest } \\
\text { Laboratory }\end{array}$ & 18,340 & 1,730 & 20,070 \\
\hline \multirow[t]{4}{*}{$\begin{array}{l}\text { Facility } \\
\text { Transitions }\end{array}$} & $\begin{array}{l}\text { 2345Z Plutonium Finish- } \\
\text { ing Plant }\end{array}$ & 6,170 & 0 & 6,170 \\
\hline & $\begin{array}{l}225 B \text { B-Plant/WESF } \\
\text { Facilities }\end{array}$ & 4,430 & 610 & 5,040 \\
\hline & 202A Purex Operations & 560 & 0 & 560 \\
\hline & $\begin{array}{l}\text { 202A Purex Operations - } \\
\text { Close out }\end{array}$ & 320 & 0 & 320 \\
\hline
\end{tabular}


Table B-1. Waste Generator Volumes by Program Area.

\begin{tabular}{|c|c|c|c|c|}
\hline Program Area & Waste Generator & $\begin{array}{c}\mathrm{CH} L \mathrm{LLW} \\
\left(\mathrm{m}^{3}\right)\end{array}$ & $\begin{array}{c}\text { RH_LLW } \\
\left(\mathrm{m}^{3}\right)\end{array}$ & $\begin{array}{c}\text { Total } \\
\left(\mathrm{m}^{3}\right)\end{array}$ \\
\hline \multirow{3}{*}{$\begin{array}{l}\text { Facility } \\
\text { Transitions } \\
\text { (cont.) }\end{array}$} & $\begin{array}{l}303 \mathrm{~K} \text { Fuels Fabrication } \\
\text { Transition }\end{array}$ & 220 & 0 & 220 \\
\hline & $\begin{array}{l}\text { FFTF/FMEF/MASF } \\
\text { Maintenance }\end{array}$ & 100 & 50 & 150 \\
\hline & Total & 11,800 & 660 & 12,460 \\
\hline \multirow[t]{3}{*}{$\begin{array}{l}\text { Liquid } \\
\text { Effluent }\end{array}$} & $\begin{array}{l}300 \text { Area Effluent } \\
\text { Treatment (Proj- } \\
\text { ect L045H) }\end{array}$ & 11,050 & 0 & 11,050 \\
\hline & $\begin{array}{l}340 \text { Waste Neutraliza- } \\
\text { tion Facility }\end{array}$ & 620 & 0 & 620 \\
\hline & Total & 11,670 & 0 & 11,670 \\
\hline $\begin{array}{l}\text { Analytical } \\
\text { Services }\end{array}$ & $\begin{array}{l}\text { 222-S Analytical } \\
\text { Laboratory }\end{array}$ & 10,220 & 0 & 10,220 \\
\hline $\begin{array}{l}\text { Spent Nuclear } \\
\text { Fuel }\end{array}$ & K-Basin Operations & 4,430 & 50 & 4,470 . \\
\hline \multirow{7}{*}{$\begin{array}{l}\text { Non- } \\
\text { Programmatic }\end{array}$} & $\begin{array}{l}\text { 2724W Laundry/Equipment } \\
\text { Decontamination }\end{array}$ & 3,400 & 0 & 3,400 \\
\hline & $\begin{array}{l}\text { Kaiser Construction } \\
\text { Services-100 Area }\end{array}$ & 80 & 0 & 80 \\
\hline & $\begin{array}{l}377 \text { Geotechnical Eng. } \\
\text { Laboratory }\end{array}$ & 30 & 0 & 30 \\
\hline & $\begin{array}{l}308 \text { Fuels Development } \\
\text { Laboratory }\end{array}$ & 20 & 0 & 20 \\
\hline & $\begin{array}{l}384 \text { Power House, } \\
300 \text { Area }\end{array}$ & 3 & 0 & 3 \\
\hline & $\begin{array}{l}\text { 105DR Large Sodium Fire } \\
\text { Facility }\end{array}$ & 2 & 0 & 2 \\
\hline & Total & 3,530 & 0 & 3,530 \\
\hline Solid Waste & $\begin{array}{l}221 T / 2706 T \text { T-Plañt } \\
\text { Operations }\end{array}$ & 430 & 90 & 510 \\
\hline
\end{tabular}


Table B-1. Waste Generator Volumes by Program Area.

\begin{tabular}{||l|l|r|r|r||}
\hline \hline \multirow{3}{*}{ Program Area } & \multicolumn{1}{|c|}{ Waste Generator } & \multicolumn{1}{c|}{$\begin{array}{c}\text { CH LLW } \\
\left(\mathrm{m}^{3}\right)\end{array}$} & \multicolumn{1}{|r|}{$\begin{array}{c}\text { RH LLW } \\
\left(\mathrm{m}^{3}\right)\end{array}$} & \multicolumn{1}{c|}{$\begin{array}{c}\text { Total } \\
\left(\mathrm{m}^{3}\right)\end{array}$} \\
\hline \hline \multirow{3}{*}{$\begin{array}{l}\text { Solid Waste } \\
\text { cont, })\end{array}$} & $\begin{array}{l}\text { Low Level Burial } \\
\text { Grounds }\end{array}$ & 190 & 0 & 190 \\
\cline { 2 - 5 } & $\begin{array}{l}\text { 224T TRU Storage \& } \\
\text { Assay Facility }\end{array}$ & 10 & 0 & 10 \\
\cline { 2 - 5 } & Total & 630 & 90 & 710 \\
\hline \hline Grand Total & & $1,403,200$ & 58,400 & $1,461,570$ \\
\hline \hline (a) Sums of individual values may not match totals due to rounding. \\
\hline
\end{tabular}


WHC-EP-0865

\section{APPENDIX C}

ANNUAL WASTE GENERATOR, HASTE CLASS VOLUMES 
WHC-EP-0865

Intentionally Left $\mathrm{B}$ lank 
FY-1994 SOLID FASTE EORECAST

KASTE CLASS, HASTE GENERATOR VOLOMES BY YEAR

(in cubic meters)

FasteGenerator \begin{tabular}{lllll} 
Held & 1995 & 1996 & 1997 & 1998 \\
\hline
\end{tabular}

1999

2000

2001

\section{Faste Class: CH_LIF_I}

MMES

vol

$\begin{array}{rr}\text { VOL } & 25.6 \\ \text { MIN } & 0.0 \\ \text { MAX } & 25.6\end{array}$

NN_E

vor

MI\%

$\cdot 0.0$

max'

BAPL_SHIPYDS

Vol

Mis

Max

BAI_CLBS_LAB

$P_{\text {BRGans }}$

B_LEHR_DAV

2656

EEPHI

EUSRAP

GEN_ATOK

voi
HIN
MAX

$\min$

vot.

Mot

max

MOr

max

vol

MIN

$\begin{array}{lll}605.1 & 605.6 & 527.9\end{array}$

$\begin{array}{ll}6.3 & 6.3 \\ 0.1 & 3.1 \\ 9.1 & 9.1\end{array}$

6

$\begin{array}{ll}6.3 & 3.2 \\ 3.1 & 1.6 \\ 9.1 & 1.0\end{array}$

0.01396 .2

$\begin{array}{lll}0.0 & 1.5 & 0.0\end{array}$

0.0
0.0

$\begin{array}{llll}0.0 & 226.6 & 226.6 & 226.6\end{array}$

$0.0 \quad 29.1$

$0.0 \quad 29.1$

0.0

$\begin{array}{llll}0.0 & 111.8 & 297.5 & 312 . \\ 0.0 & 355.0 & 238.0 & 249.0\end{array}$

$\begin{array}{llll}0.0 & 533.7 & 337.0 & 374.7\end{array}$

0.0

0.034.

$\begin{array}{lll}19.0 & 137.1 \quad 216.7\end{array}$

0.0

418.8 137.

$\begin{array}{ll}0.0 & 155 \\ 0.0 & 93.3 \\ 0.0 & 311\end{array}$

$93.3 \quad 0.0 \quad \cdot 0.0$

$\begin{array}{rr}0.0 & 0.0 \\ 54.2 & 54.2\end{array}$

27.211 .3

$11.3 \quad 14.3 \quad 11.3$

$\begin{array}{lll}22.1 & 22.1 & 22.1 \\ 88.6 & 88.6 & -09.6\end{array}$

2.1

vor

HIN

$\max$

Mrs

mIX

$0.0 \quad 0.0$

0.0

0.0 . 0

0.0

0.0

$0.0 \quad 0.0$

0.0

0.0

$\begin{array}{lllll}0.0 & 78.6 & 227.3 & 227.3 & 176.0\end{array}$

$\begin{array}{rrrrr}0.0 & 70.7 & 204.5 & 204.5 & 139.1 \\ 0.0 & 117.9 & 340.9 & 340.9 & 265.2\end{array}$ 
WASTE CLASS, HASTE GENERATOR VOLUMES BY YEAR (in aubla meters)

\begin{tabular}{|c|c|c|c|c|c|c|c|c|c|c|c|c|c|c|c|c|c|}
\hline Was te Gene & & Held & 1995 & 1996 & 1997 & 1998 & 1999 & 2000 & 2001 & 2002 & 2003 & 2004 & 2005 & 2006 & 2007 & 2008 & 2009 \\
\hline \multicolumn{18}{|c|}{ Waste Class: CH_IHF_I } \\
\hline \multirow[t]{3}{*}{ KEH_100 } & Vor & 0.0 & 76.9 & 0.0 & 0.0 & 0.0 & 0.0 & 0.0 & 0.0 & 0.0 & 0.0 & 0.0 & 0.0 & 0.0 & 0.0 & 0.0 & 0.0 \\
\hline & MIN & 0.0 & 53.8 & 0.0 & 0.0 & 0.0 & 0.0 & 0.0 & 0.0 & 0.0 & 0.0 & 0.0 & 0.0 & 0.0 & 0.0 & 0.0 & 0.0 \\
\hline & $\max$ & 0.0 & 76.9 & 0.0 & 0.0 & 0.0 & 0.0 & 0.0 & 0.0 & 0.0 & 0.0 & 0.0 & 0.0 & 0.0 & 0.0 & 0.0 & 0.0 \\
\hline \multirow[t]{3}{*}{ LEL } & vol & 0.0 & 263.6 & 149.3 & 30.8 & 39.5 & 10.2 & 2873.7 & 2873.7 & 2873.7 & 2873.7 & 2873.7 & 10.8 & 10.8 & 10.8 & 40.8 & 10.8 \\
\hline & MIY & 0.0 & 163.6 & 149.3 & 38.0 & 39.5 & 10.2 & 1436.8 & 2073.7 & 2873.7 & 2873.7 & 2873.7 & 10.0 & 10.8 & 10.8 & 10.8 & 10.8 \\
\hline & $\max$ & 0.0 & 163.6 & 149.3 & 38.0 & 39.5 & 40.2 & 2873.7 & 1310.5 & 1310.5 & 1310.5 & 1310.5 & 10.8 & 10.8 & 10.8 & 10.8 & 10.8 \\
\hline \multirow[t]{3}{*}{ MIT_BATES } & Vor & 0.0 & 0.0 & 0.0 & 0.0 & 0.0 & 0.0 & 0.3 & 0.0 & 0.0 & 0.0 & 0.0 & 0.3 & 0.0 & 0.0 & 0.0 & 0.0 \\
\hline & MIN & 0.0 & 0.0 & 0.0 & 0.0 & 0.0 & 0.0 & 0.3 & 0.0 & 0.0 & 0.0 & 0.0 & 0.3 & 0.0 & 0.0 & 0.0 & 0.0 \\
\hline & $\operatorname{MAX}$ & 0.0 & 0.0 & 0.0 & 0.0 & 0.0 & 0.0 & 0.6 & 0.0 & 0.0 & 0.0 & 0.0 & 0.6 & 0.0 & 0.0 & 0.0 & 0.0 \\
\hline \multirow[t]{3}{*}{ PADUCAH_ES } & VoL & 1998.3 & 256.1 & 156.4 & 156.4 & 156.4 & 136.4 & 256.4 & 156.4 & 256.4 & 156.1 & 256.4 & 156.4 & 156.4 & 256.4 & 156.4 & 156.4 \\
\hline & MIM & 0.0 & 78.2 & 78.2 & 78.2 & 78.2 & 78.2 & 70.2 & 70.2 & 78.2 & 78.2 & 78.2 & 78.2 & 78.2 & 78.2 & 78.2 & 78.2 \\
\hline & $\operatorname{MAX}$ & 1998.3 & 234.6 & 234.6 & 231.6 & 234.6 & 234.6 & 234.6 & 234.6 & 234.6 & 234.6 & 234.6 & 234.6 & 234.6 & 234.6 & 234.6 & 234.6 \\
\hline \multirow[t]{3}{*}{ PADUCAH_US } & vol & 926.3 & 0.0 & 0.0 & 0.0 & 0.0 & 0.0 & 0.0 & 0.0 & 0.0 & 0.0 & 0.0 & 0.0 & 0.0 & 0.0 & 0.0 & 0.0 \\
\hline & MIN & 0.0 & 0.0 & 0.0 & 0.0 & 0.0 & 0.0 & 0.0 & 0.0 & 0.0 & 0.0 & 0.0 & 0.0 & 0.0 & 0.0 & 0.0 & 0.0 \\
\hline & $\max$ & 926.3 & 0.0 & 0.0 & 0.0 & 0.0 & 0.0 & 0.0 & 0.0 & 0.0 & 0.0 & 0.0 & 0.0 & 0.0 & 0.0 & 0.0 & 0.0 \\
\hline \multirow[t]{3}{*}{ PNL } & vor. & 0.0 & 566.6 & 566.6 & 566.6 & 566.6 & 566.6 & 566.6 & 566.6 & 566.6 & 566.6 & 566.6 & 566.6 & 566.6 & 566.6 & 366.6 & 566.6 \\
\hline & MIN & 0.0 & 509.9 & 309.9 & 509.9 & 509.9 & 509.9 & 153.2 & 153.2 & 453.2 & 133.2 & 453.2 & 424.9 & 124.9 & 124.9 & 424.9 & 124.9 \\
\hline & $\max$ & 0.0 & 623.2 & 623.2 & 623.2 & 623.2 & 623.2 & 623.2 & 623.2 & 623.2 & 623.2 & 623.2 & 623.2 & 623.2 & 623.2 & 623.2 & 623.2 \\
\hline \multirow[t]{3}{*}{ PORTSHOUTH_ES } & vot & 0.0 & 368.3 & 368.3 & 360.3 & 368.3 & 368.3 & 674.7 & 674.7 & 674.7 & 674.7 & 674.7 & 674.7 & 674.7 & 674.7 & 674.7 & 674.7 \\
\hline & MIN & 0.0 & 368.3 & 368.3 & 368.3 & 368.3 & 368.3 & 674.7 & 674.7 & 674.7 & 674.7 & 674.7 & 674.7 & 674.7 & 674.7 & 674.7 & 674.7 \\
\hline & $\max$ & 0.0 & 141.9 & 141.9 & $\$ 11.9$ & 141.9 & 111.9 & 609.6 & 809.6 & 809.6 & 009.6 & 809.6 & 809.6 & 809.6 & 809.6 & 809.6 & 009.6 \\
\hline \multirow[t]{3}{*}{ PORTSHOUTH_US } & vor & 1775.7 & 0.0 & 0.0 & 0.0 & 0.0 & 0.0 & 0.0 & 0.0 & 0.0 & 0.0 & 0.0 & 0.0 & 0.0 & 0.0 & 0.0 & 0.0 \\
\hline & HIN & 0.0 & 0.0 & 0.0 & 0.0 & 0.0 & 0.0 & 0.0 & 0.0 & 0.0 & 0.0 & 0.0 & 0.0 & 0.0 & 0.0 & 0.0 & 0.0 \\
\hline & $\max$ & 1775.7 & 0.0 & 0.0 & 0.0 & 0.0 & 0.0 & 0.0 & 0.0 & 0.0 & 0.0 & 0.0 & 0.0 & 0.0 & 0.0 & 0.0 & 0.0 \\
\hline \multirow[t]{3}{*}{ PRINCETOH } & vor & 0.0 & 170.0 & 283.3 & 566.6 & 566.6 & 283.3 & 170.0 & 270.0 & 170.0 & 170.0 & 170.0 & 170.0 & 283.3 & 566.6 & 366.6 & 283.3 \\
\hline & HIN & 0.0 & 51.0 & 84.9 & 283.3 & 169.9 & 81.9 & 51.0 & 31.0 & 51.0 & 51.0 & 31.0 & 51.0 & 84.9 & 283.3 & 169.9 & 84.9 \\
\hline & $\sin x$ & 0.0 & 221.0 & 566.6 & 736.5 & 736.5 & 360.2 & 221.0 & 221.0 & $221.0^{\circ}$ & 221.0 & 221.0 & 221.0 & 368.2 & 736.3 & 736.5 & 368.2 \\
\hline \multirow[t]{3}{*}{ RKW_CAMOGA } & vot & 22.1 & 366.7 & 144.5 & 287.0 & 386.7 & 68.0 & 0.0 & 0.0 & 0.0 & 0.0 & 0.0 & 0.0 & 0.0 & 0.0 & 0.0 & 0.0 \\
\hline & MIN & 0.0 & 330.0 & 113.6 & 229.6 & 309.3 & 34.1 & 0.0 & 0.0 & 0.0 & 0.0 & 0.0 & 0.0 & 0.0 & 0.0 & 0.0 & 0.0 \\
\hline & $\max$ & 21.4 & 366.7 & 173.4 & 341.4 & 164.0 & 01.6 & 0.0 & 0.0 & 0.0 & 0.0 & 0.0 & 0.0 & 0.0 & 0.0 & 0.0 & 0.0 \\
\hline
\end{tabular}




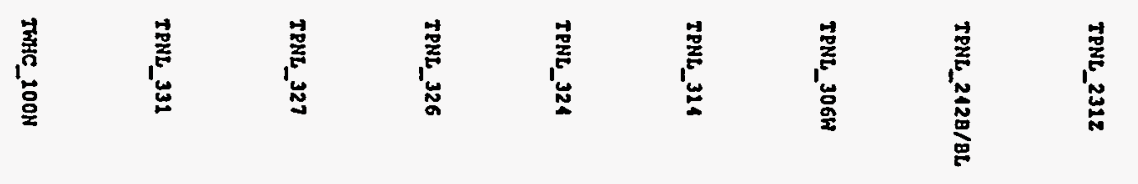

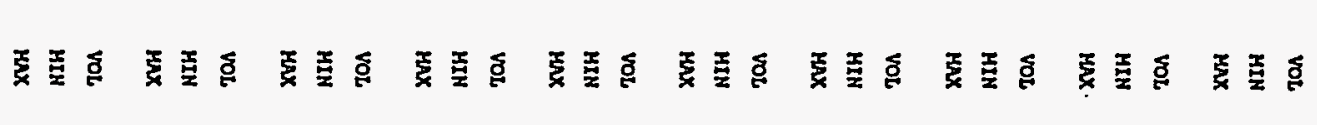

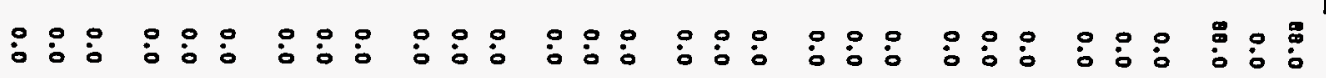

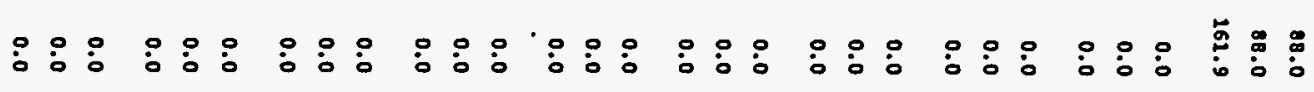

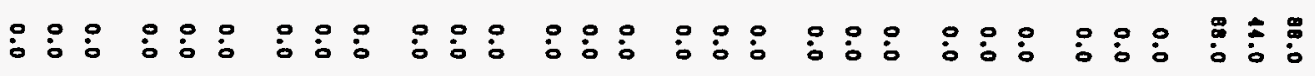

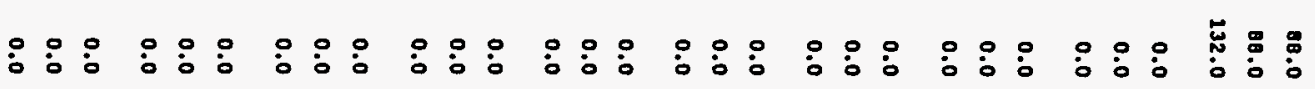

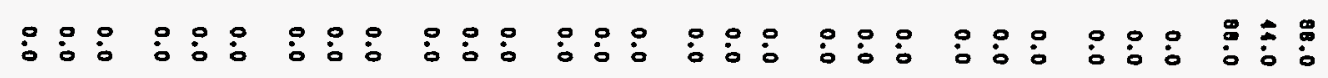

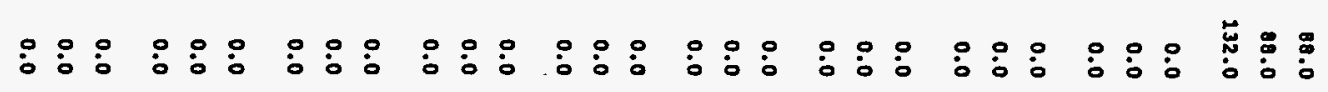

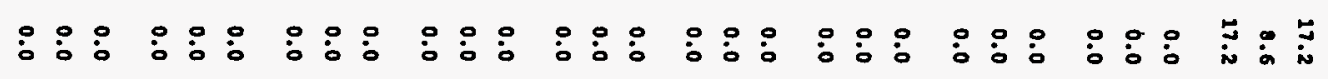

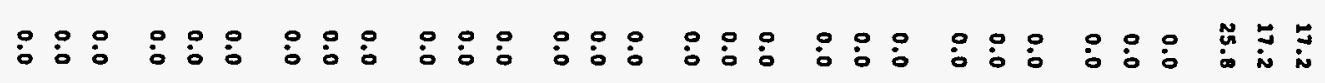

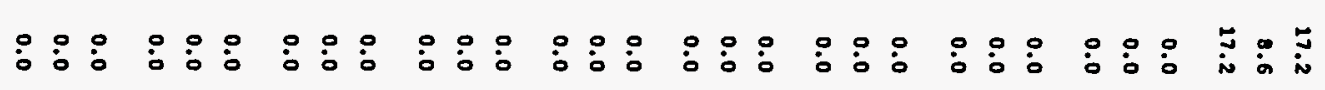

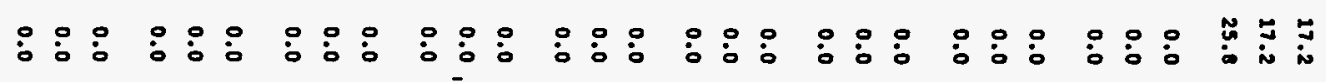

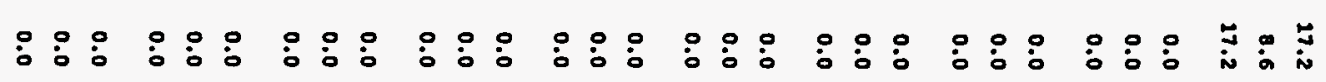

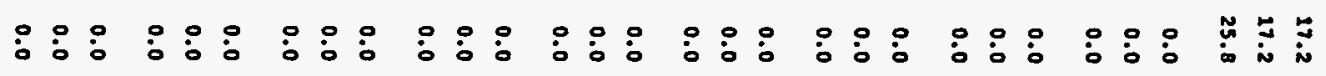

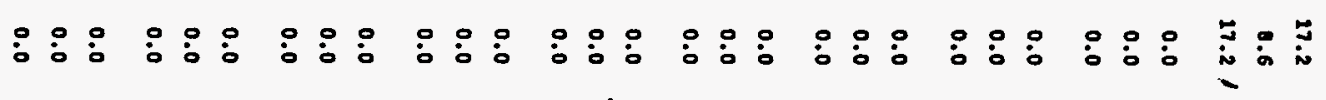

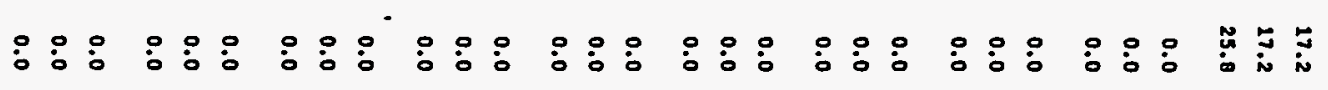

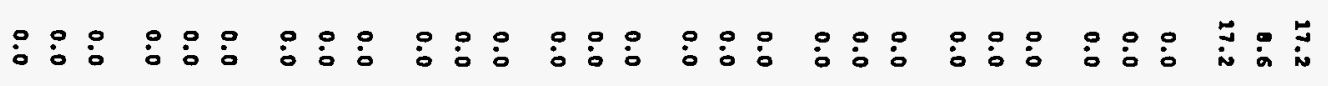

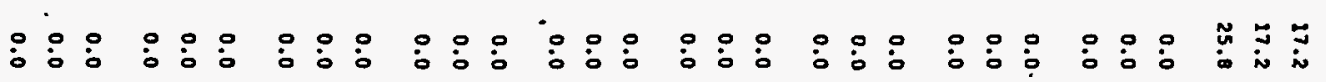




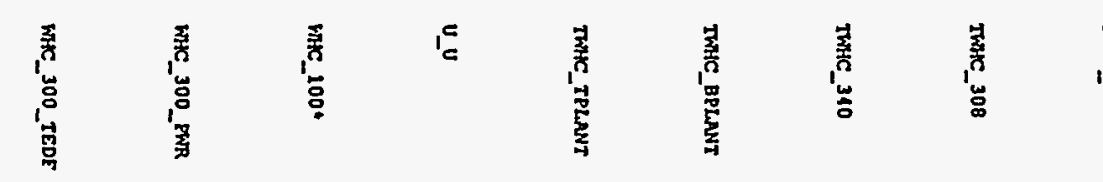

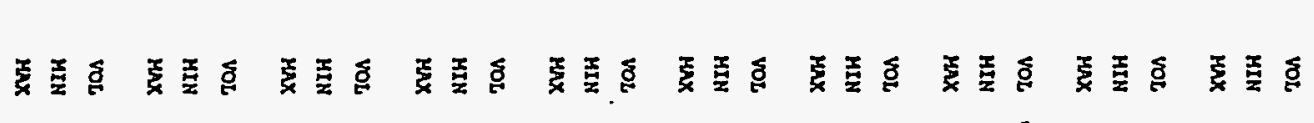

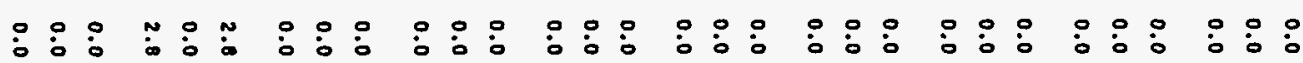

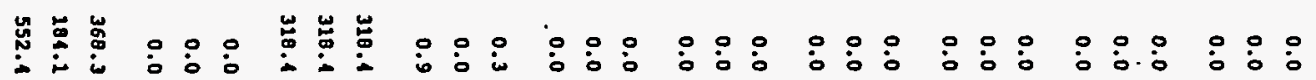

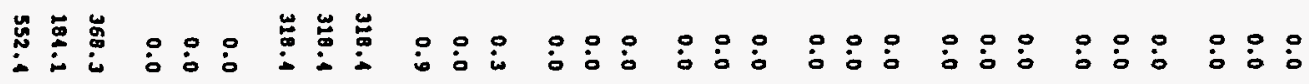

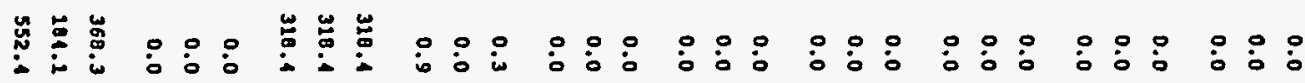

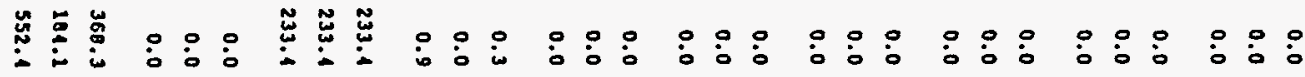

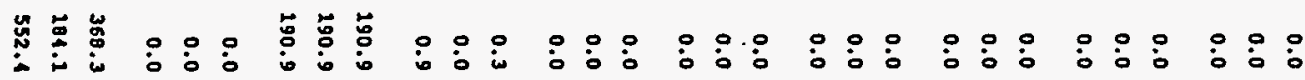

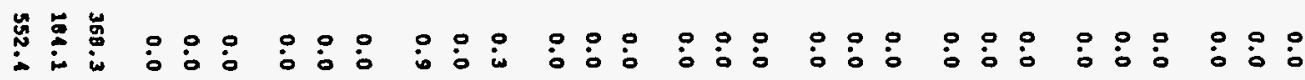

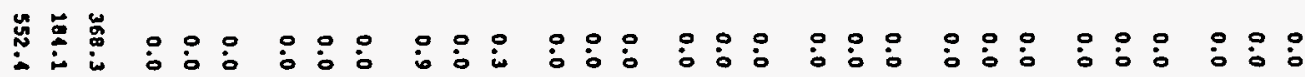

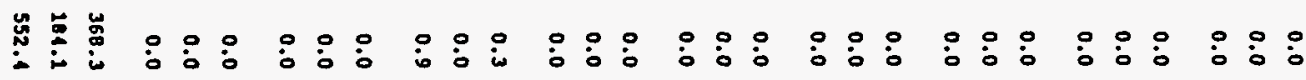

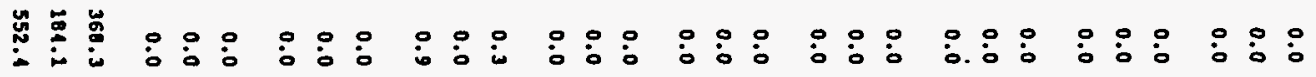

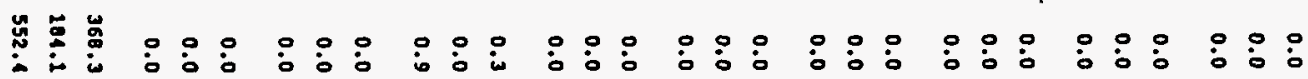

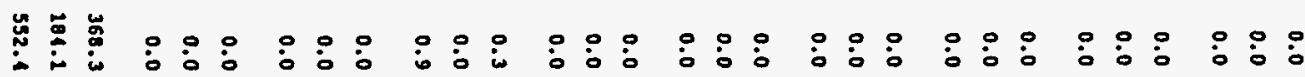

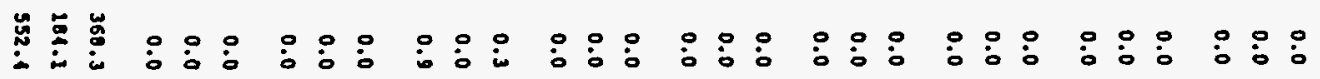

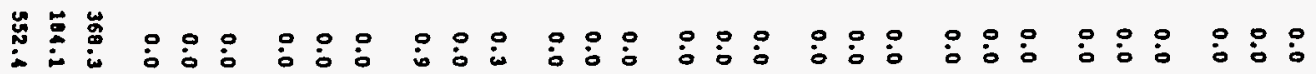

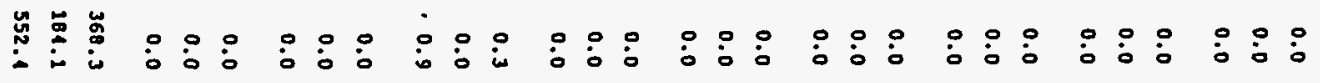

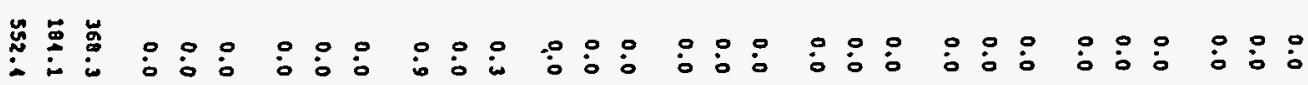




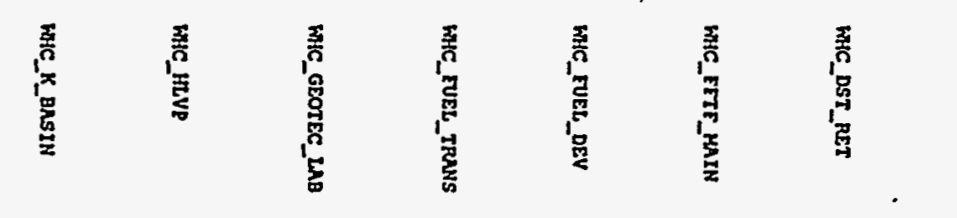

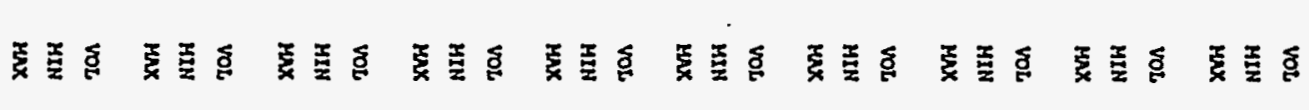

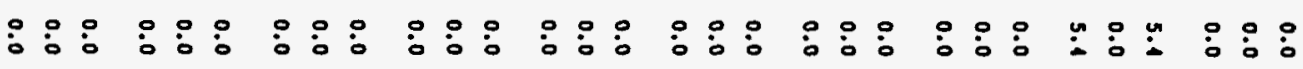

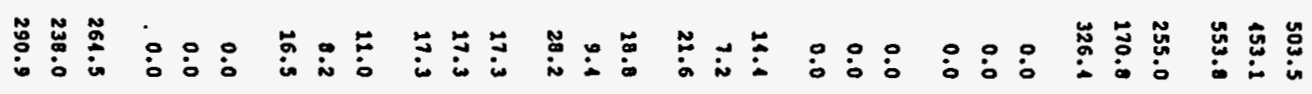

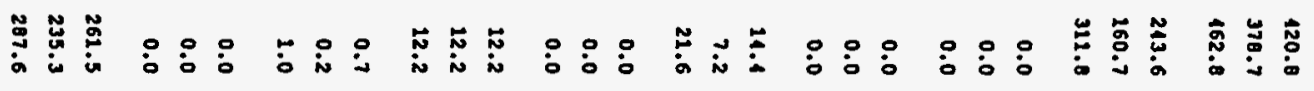

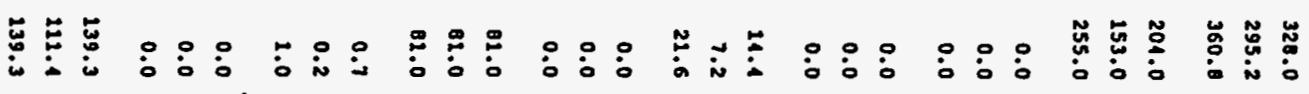

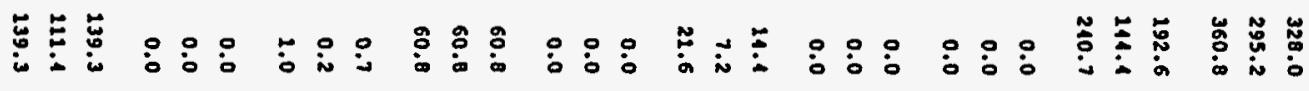

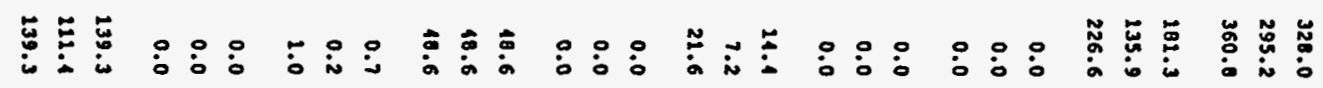

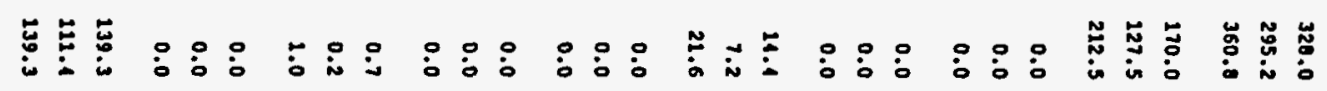

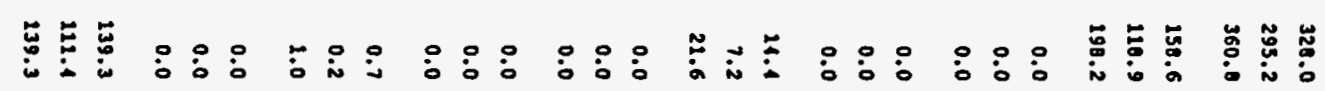

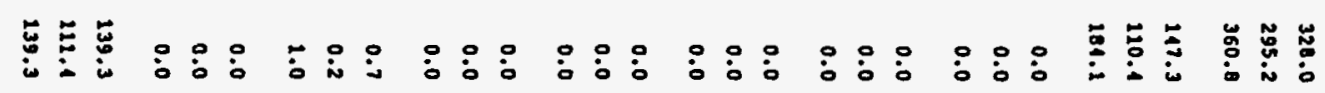

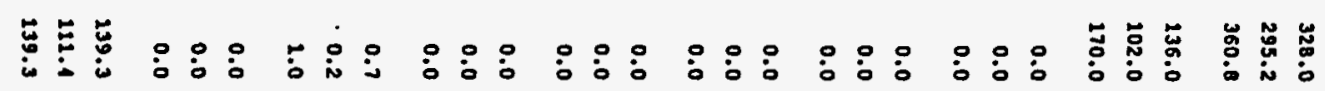

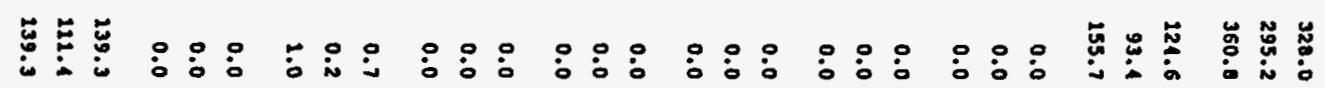

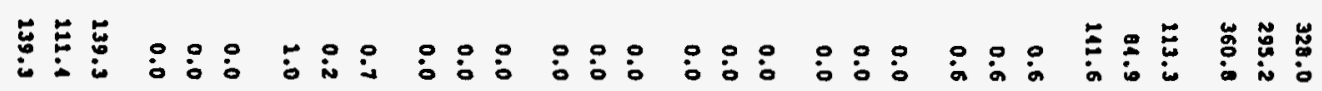

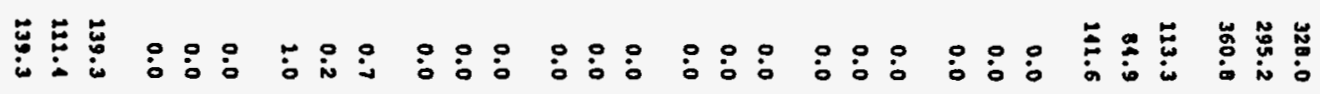

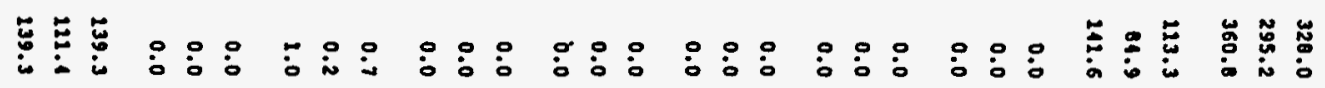

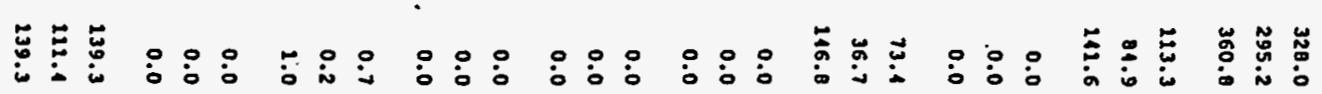

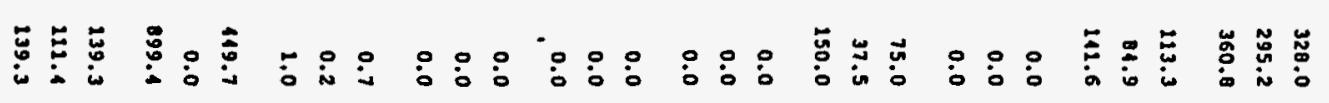

影 
FY-1994 SOLID WASTE FORECAST

WASTE CLASS, WASTE GENERATOR VOLUMES BY YEAR

(In cubio meters)

\begin{tabular}{|c|c|c|c|c|c|c|c|c|c|c|c|c|c|c|c|c|c|}
\hline Fas te Gen & & Held & 1995 & 1996 & 1997 & 1998 & 1999 & 2000 & 2001 & 2002 & 2003 & 2004 & 2005 & 2006 & 2007 & 2008 & 2009 \\
\hline \multicolumn{18}{|c|}{ Waste Class: CH_ILW_I } \\
\hline \multirow[t]{3}{*}{ WHC_LAUNDRY } & vol & 0.0 & $\mathbf{1 1 3 . 3}$ & 113.3 & 123.3 & 113.3 & .213 .3 & 113.3 & 1123.3 & 113.3 & 213.3 & 2123.3 & 213.3 & 113.3 & 213.3 & 113.3 & 113.3 \\
\hline & MIN & 0.0 & 213.3 & 113.3 & 213.3 & 113.3 & 213.3 & 213.3 & 113.3 & 113.3 & 113.3 & 113.3 & 113.3 & 113.3 & 113.3 & 113.3 & 113.3 \\
\hline & $\max$ & 0.0 & 124.6 & 124.6 & 124.6 & 124.6 & 124.6 & 124.6 & 124.6 & 224.6 & 124.6 & 121.6 & 124.6 & 124.6 & 124.6 & 124.6 & 124.6 \\
\hline \multirow[t]{3}{*}{ WHC_LLEG } & vor & 0.0 & 6.3 & 6.3 & 6.3 & 6.3 & 6.3 & 6.3 & 6.3 & 6.3 & 6.3 & 6.3 & 6.3 & 6.3 & 6.3 & 6.3 & 6.3 \\
\hline & HIN & 0.0 & 6.3 & 6.3 & 6.3 & 6.3 & 6.3 & 6.3 & 6.3 & 6.3 & 6.3 & 6.3 & 6.3 & 6.3 & 6.3 & 6.3 & 6.3 \\
\hline & $\max$ & 0.0 & 6.3 & 6.3 & 6.3 & 6.3 & 6.3 & 6.3 & 6.3 & 6.3 & 6.3 & 6.3 & 6.3 & 6.3 & 6.3 & 6.3 & 6.3 \\
\hline \multirow[t]{3}{*}{ Whe_LLVP } & vor & 0.0 & 0.0 & 0.0 & 0.0 & 0.0 & 0.0 & 0.0 & 0.0 & 0.0 & 0.0 & 0.0 & 125.4 & 125.4 & 125.1 & 125.4 & 125.4 \\
\hline & HIM & 0.0 & 0.0 & 0.0 & 0.0 & 0.0 & 0.0 & 0.0 & 0.0 & 0.0 & 0.0 & 0.0 & 0.0 & 212.7 & 212.7 & 212.7 & 212.7 \\
\hline & $\boldsymbol{\mu N X}$ & 0.0 & 0.0 & 0.0 & 0.0 & 0.0 & 0.0 & 0.0 & 0.0 & 0.0 & 0.0 & 0.0 & 850.8 & 950.8 & 850.0 & 950.8 & 850.8 \\
\hline \multirow[t]{3}{*}{ Whic_PFP } & vor & 0.0 & 204.9 & 277.6 & 277.6 & 277.6 & 277.6 & 277.6 & 277.6 & 312.7 & 277.6 & 279.0 & 277.6 & 280.3 & 277.6 & 277.6 & 277.6 \\
\hline & MIN & 0.0 & 0.0 & 0.0 & 0.0 & 0.0 & 0.0 & 0.0 & 0.0 & 0.0 & 0.0 & 0.0 & 0.0 & 0.0 & 0.0 & 0.0 & 0.0 \\
\hline & $\max$ & 0.0 & 854.7 & 832.8 & 832.8 & 832.8 & 832.8 & 832.8 & 832.8 & 930.1 & 832.0 & 837.0 & 032.0 & 840.9 & 832.0 & 032.8 & 032.8 \\
\hline \multirow[t]{3}{*}{ WHE_PRETRT_TW } & vot & 0.0 & 0.0 & 0.0 & 0.0 & 0.0 & 0.0 & 0.0 & 0.0 & 0.0 & 0.0 & 111.0 & 141.0 & 141.0 & 141.8 & 111.8 & 111.8 \\
\hline & MIN & 0.0 & 0.0 & 0.0 & 0.0 & 0.0 & 0.0 & 0.0 & 0.0 & 0.0 & 0.0 & 0.0 & 70.9 & 70.9 & 70.9 & 70.9 & 70.9 \\
\hline & $\max$ & 0.0 & 0.0 & 0.0 & 0.0 & 0.0 & 0.0 & 0.0 & 0.0 & 0.0 & 0.0 & 203.6 & 203.6 & 203.6 & 283.6 & 283.6 & 283.6 \\
\hline \multirow[t]{3}{*}{ RHC_PUREX } & vor & 0.0 & 223.0 & 113.3 & 113.3 & 113.3 & 0.0 & 0.0 & 0.0 & 0.0 & 0.0 & 0.0 & 0.0 & 0.0 & 0.0 & 0.0 & 0.0 \\
\hline & MIN & 0.0 & 111.5 & 56.6 & 56.6 & 56.6 & 0.0 & 0.0 & 0.0 & 0.0 & 0.0 & 0.0 & 0.0 & 0.0 & 0.0 & 0.0 & 0.0 \\
\hline & $\operatorname{mNX}$ & 0.0 & 334.5 & 226.6 & 226.6 & 226.6 & 0.0 & 0.0 & 0.0 & 0.0 & 0.0 & 0.0 & 0.0 & 0.0 & 0.0 & 0.0 & .0 .0 \\
\hline \multirow[t]{3}{*}{ WHC_PUREX_SH } & vor & 0.0 & 0.0 & 0.0 & 0.0 & 0.0 & 22.6 & 22.6 & 22.6 & 22.6 & 22.6 & 22.6 & 22.6 & 22.6 & 22.6 & 22.6 & 22.6 \\
\hline & MIN & 0.0 & 0.0 & 0.0 & 0.0 & 0.0 & 11.3 & 11.3 & 11.3 & 11.3 & 11.3 & 11.3 & 21.3 & 11.3 & 11.3 & 11.3 & 11.3 \\
\hline & $\max$ & 0.0 & 0.0 & 0.0 & 0.0 & 0.0 & 45.3 & 45.3 & 15.3 & 15.3 & 45.3 & 45.3 & 15.3 & 45.3 & 15.3 & 15.3 & 45.3 \\
\hline \multirow[t]{3}{*}{ WHC_SODIUH_EAC } & voL & 0.0 & 0.0 & 1.0 & 0.5 & 0.0 & 0.0 & 0.0 & 0.0 & 0.0 & 0.0 & 0.0 & 0.0 & 0.0 & 0.0 & 0.0 & 0.0 \\
\hline & MIN & 0.0 & 0.0 & 0.3 & 0.5 & 0.0 & 0.0 & 0.0 & 0.0 & 0.0 & 0.0 & 0.0 & 0.0 & 0.0 & 0.0 & 0.0 & 0.0 \\
\hline & $\max$ & 0.0 & 0.0 & 2.0 & 1.0 & 0.0 & 0.0 & 0.0 & 0.0 & 0.0 & 0.0 & 0.0 & 0.0 & 0.0 & 0.0 & 0.0 & 0.0 \\
\hline \multirow[t]{3}{*}{ WHK_SST_RET } & vol & 0.0 & 0.0 & 0.0 & 3.6 & 0.0 & 0.0 & 0.0 & 0.0 & 0.0 & 0.0 & 10.9 & 10.9 & 14.5 & 18.1 & 10.1 & 25.4 \\
\hline & MIH & 0.0 & 0.0 & 0.0 & 3.2 & 0.0 & 0.0 & 0.0 & 0.0 & 0.0 & 0.0 & 9.8 & 9.8 & 13.0 & 16.2 & 16.2 & 22.0 \\
\hline & $\max$ & 0.0 & 0.0 & 0.0 & 3.9 & 0.0 & 0.0 & 0.0 & 0.0 & 0.0 & 0.0 & 11.9 & 11.9 & 15.9 & 19.9 & 19.9 & 21.9 \\
\hline \multirow[t]{3}{*}{ WHC_SURPLS_FAC } & vot & 0.0 & 2039.7 & 1603.4 & 3688.1 & 416.4 & 1246.5 & 8215.3 & 15360.3 & 60617.6 & 60178.5 & 107515.6 & 153767.7 & 165410.8 & 179886.7 & 108411.9 & 38073.7 \\
\hline & MIN & 0.0 & 2039.7 & 1603.1 & 3688.4 & 416.1 & 1246.5 & 8215.3 & 15360.3 & 60617.6 & 80178.5 & 107515.6 & 153767.7 & 165410.8 & 179806.7 & 108411.9 & 38073.7 \\
\hline & $\max$ & 0.0 & 2039.7 & 1603.1 & 3688.4 & 116.4 & 1246.5 & 8215.3 & 13368.3 & 60617.6 & 80178.5 & 107515.6 & 153767.7 & 265410.0 & 179806.7 & 108411.9 & 38073.7 \\
\hline
\end{tabular}




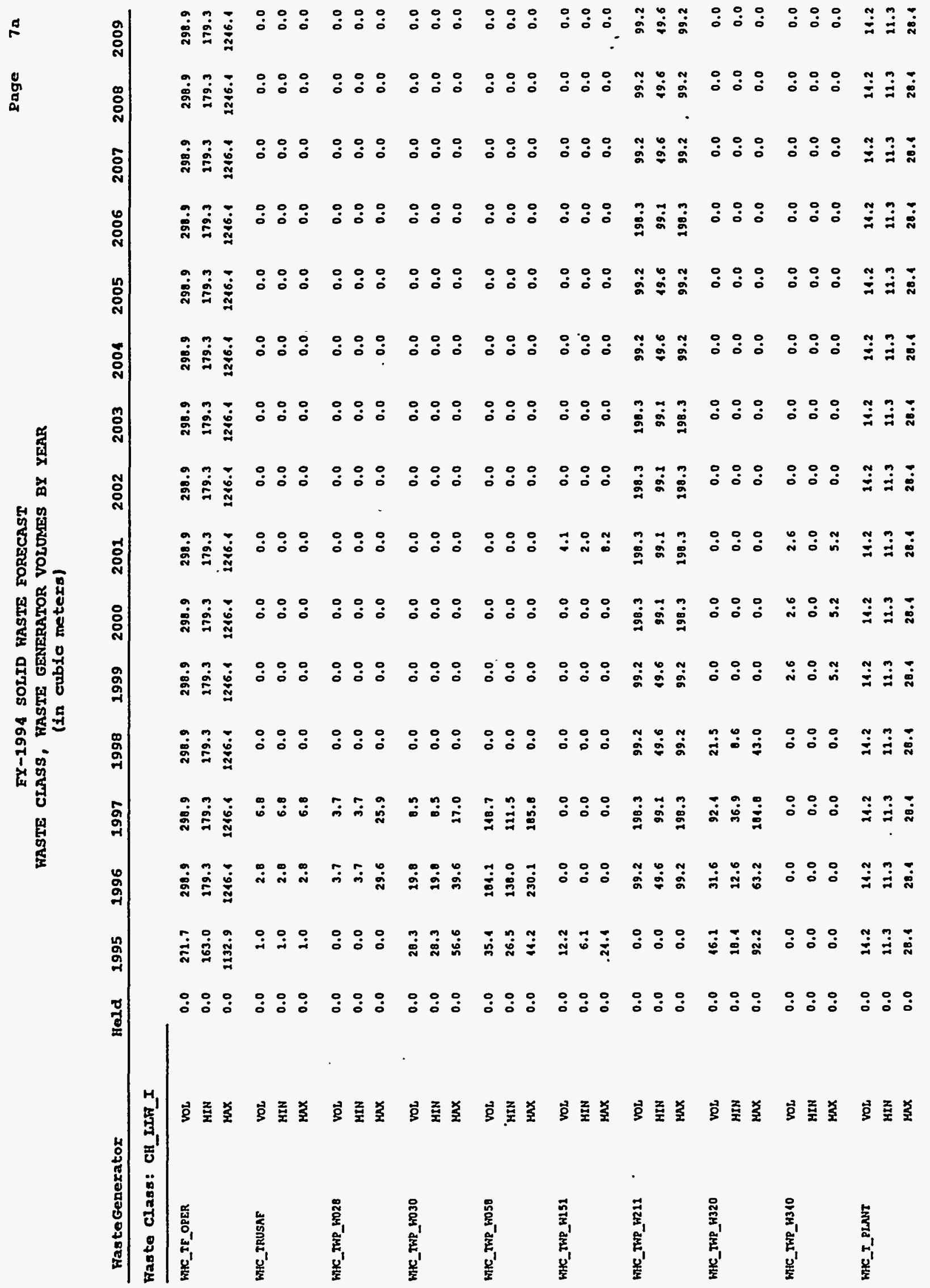


FY-1994 SOLID WASTE FORECAST

WASTE CLASS, HASTE GENERATOR VOLOMES BY YEAR

(in cublo metera)

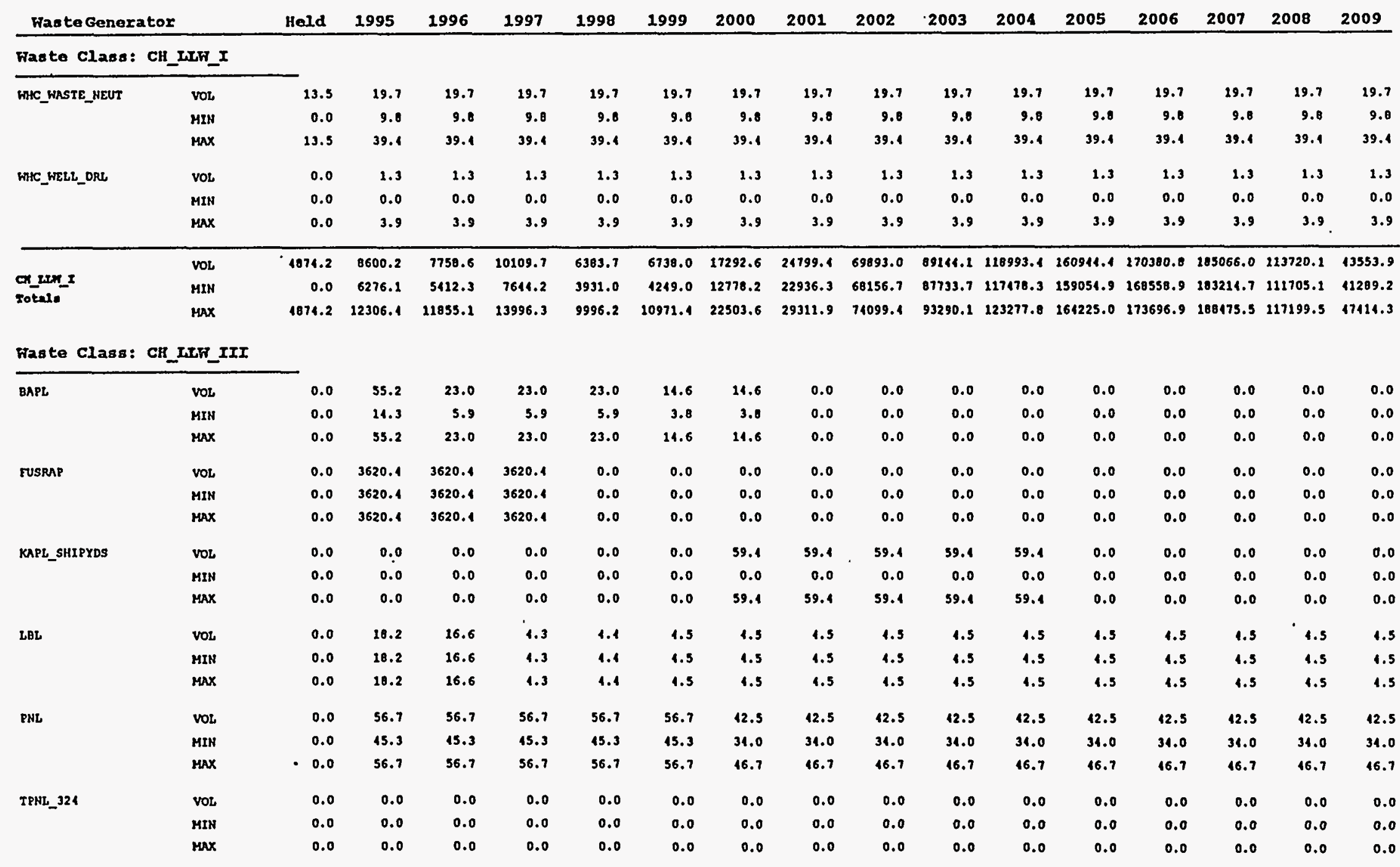




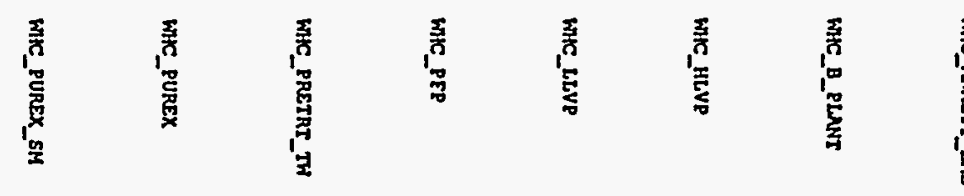

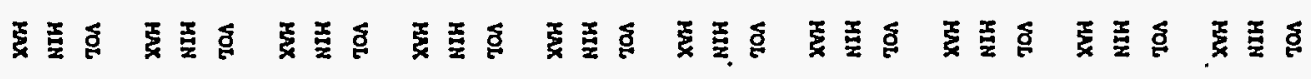

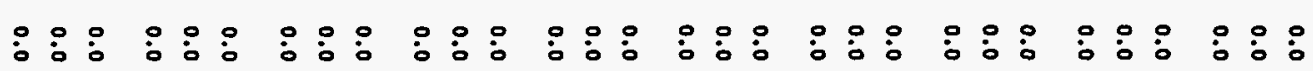

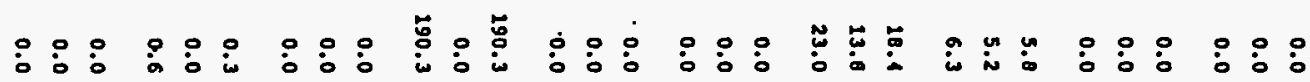

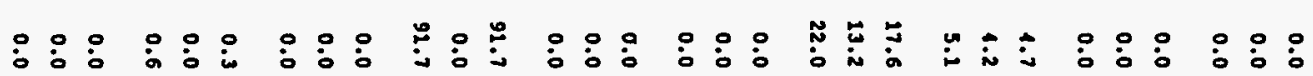

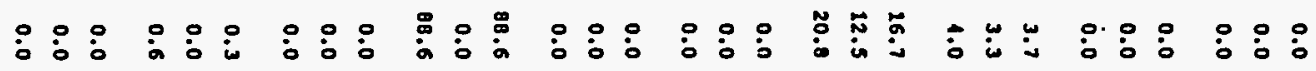

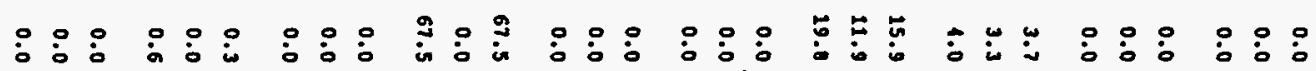

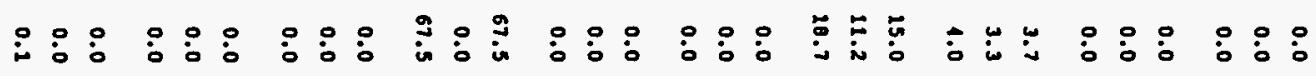

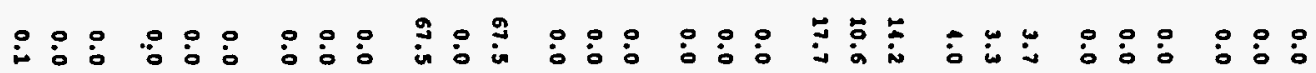

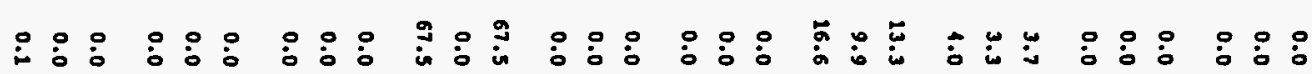

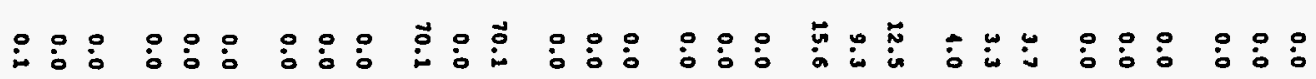

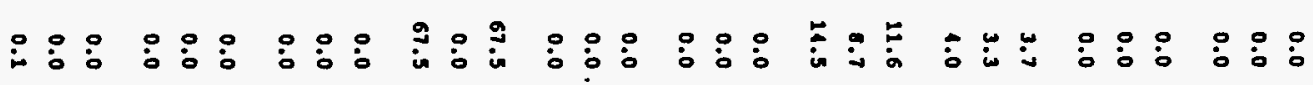

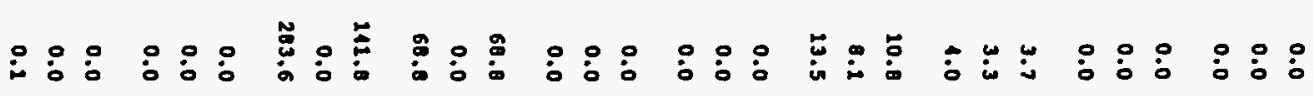

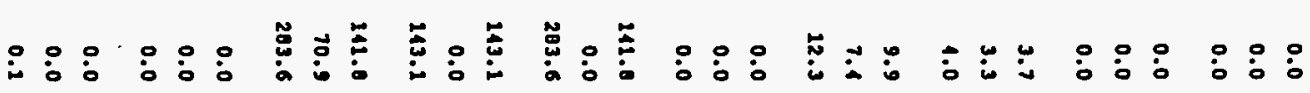

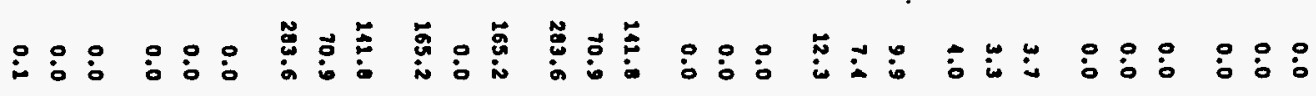

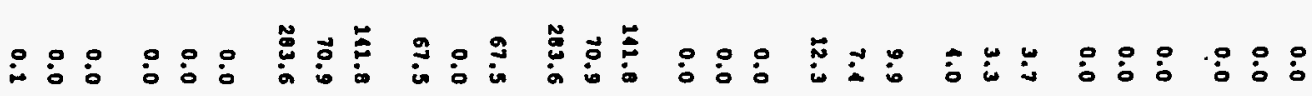

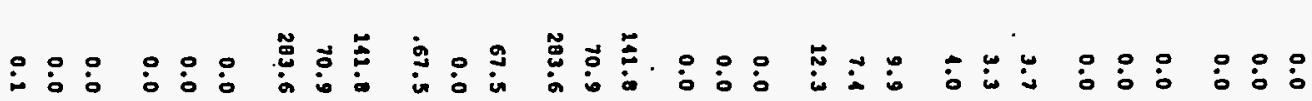

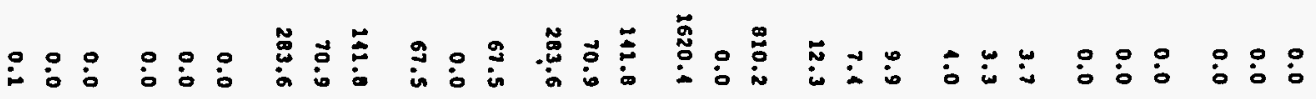




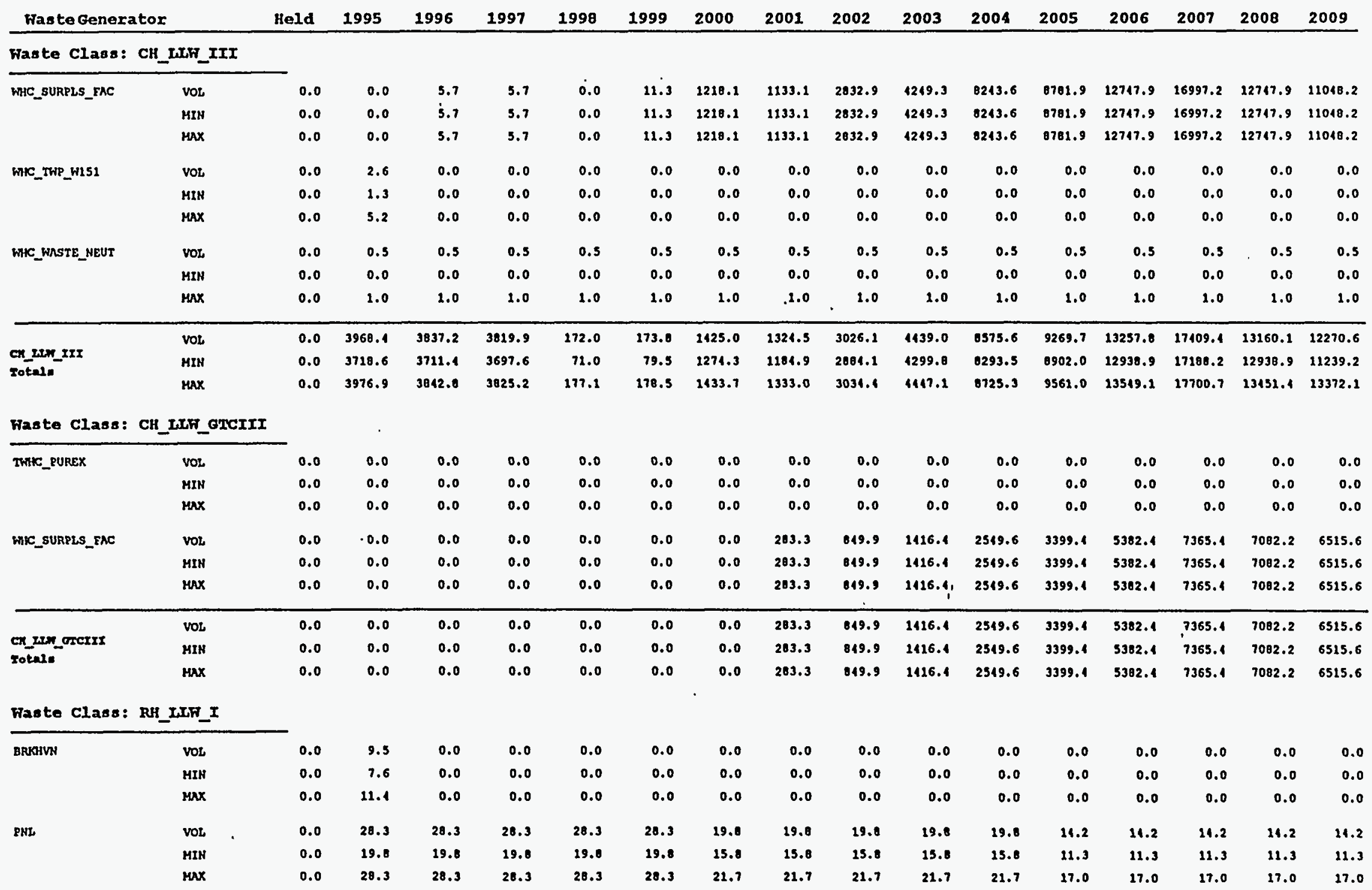




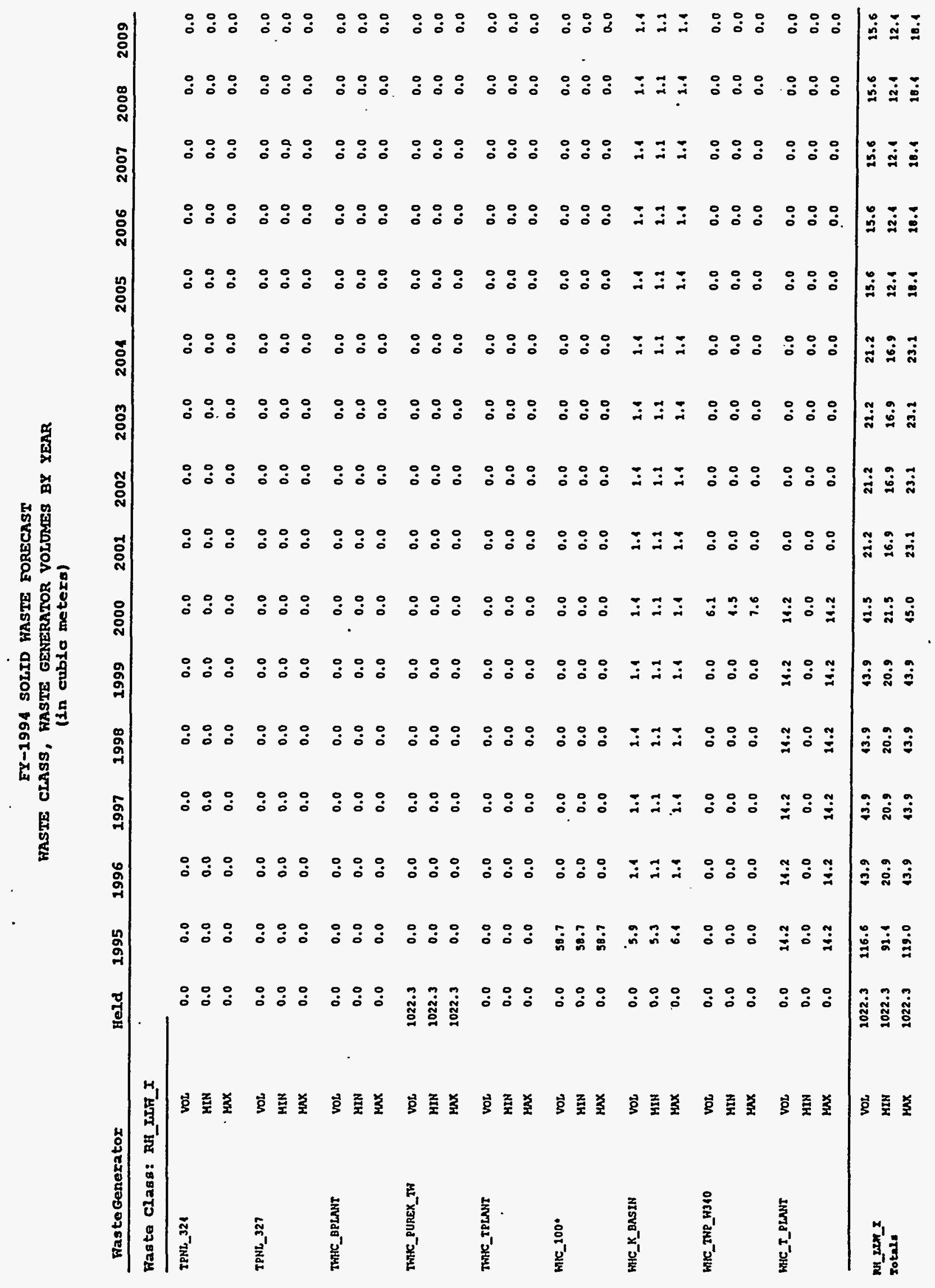




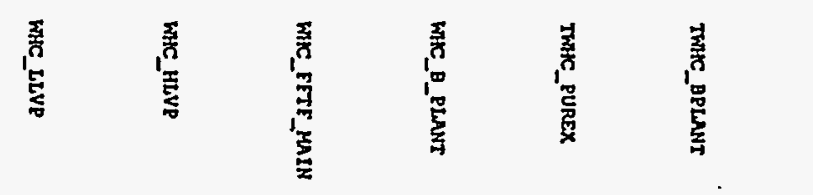

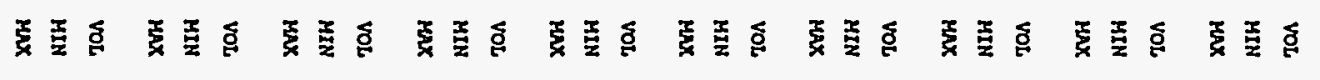

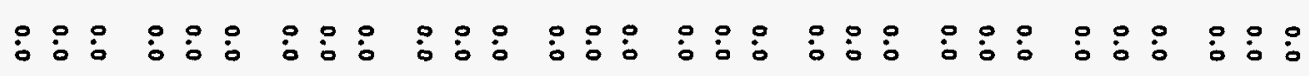

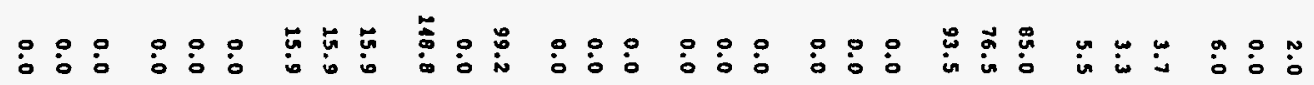

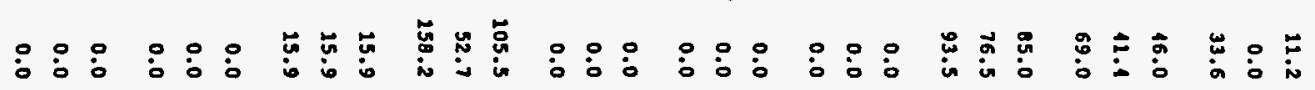

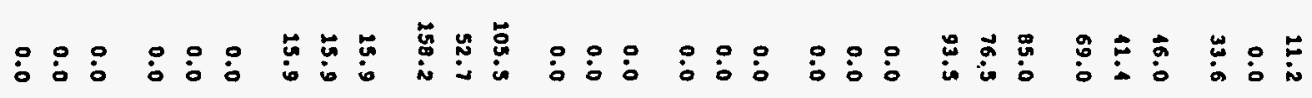

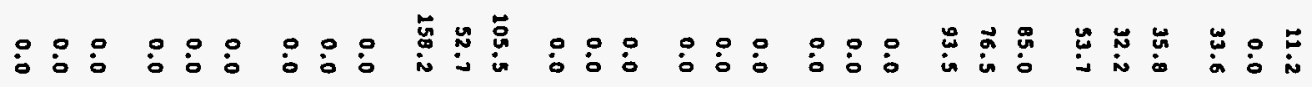

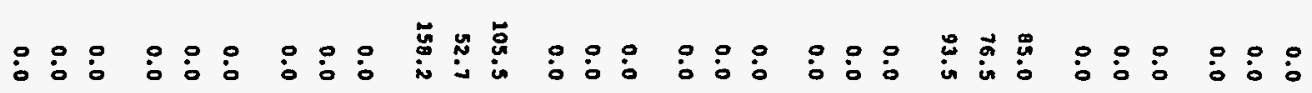

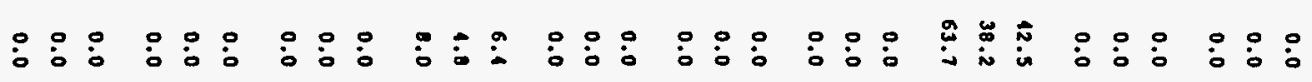

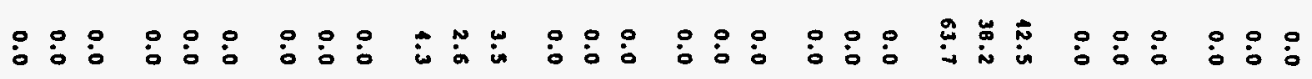

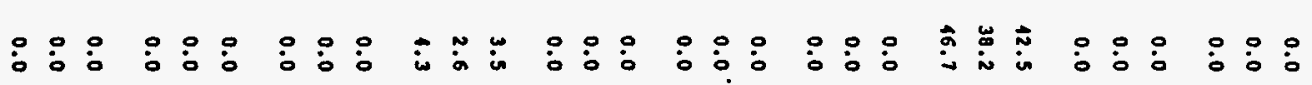

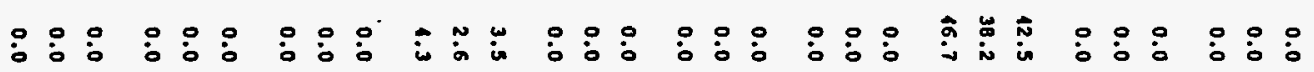

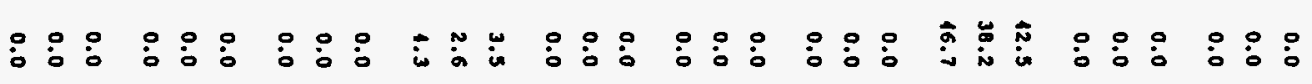

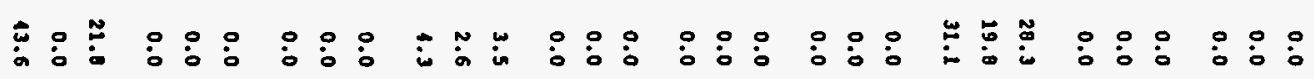
苛

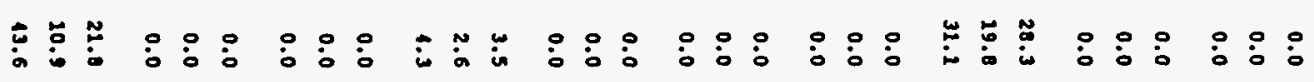

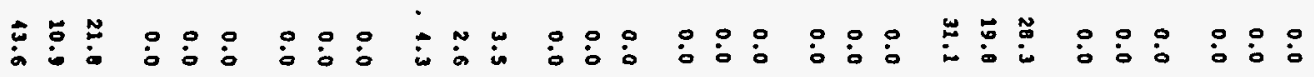

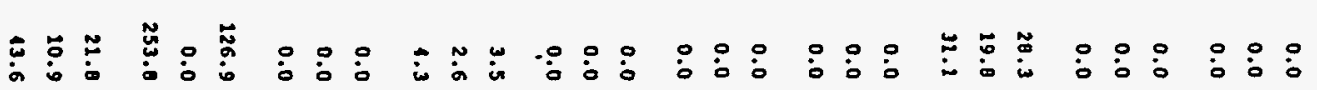


FY-1994 SOLID HASTE FORECAST

HASTE CLASS, HASTE GENERATOR VOLOMES BY YEAR

(In cubic meters)

\begin{tabular}{|c|c|c|c|c|c|c|c|c|c|c|c|c|c|c|c|c|c|}
\hline \multicolumn{2}{|c|}{ Was te Generator } & HeId & 1995 & 1996 & 1997 & 1998 & 1999 & 2000 & 2001 & 2002 & 2003 & 2004 & 2005 & 2006 & 2007 & 2008 & 2009 \\
\hline \multicolumn{18}{|c|}{ Faste Class: RH_ILH_III } \\
\hline \multirow[t]{4}{*}{ WHE_PRETRT_TH } & vot & 0.0 & 0.0 & 0.0 & 0.0 & 0.0 & 0.0 & 0.0 & 0.0 & 0.0 & 0.0 & 14.5 & 14.5 & 14.5 & 14.5 & 14.5 & 14.5 \\
\hline & MIN & 0.0 & 0.0 & 0.0 & 0.0 & 0.0 & 0.0 & 0.0 & 0.0 & 0.0 & 0.0 & 0.0 & 7.2 & 7.2 & 7.2 & 7.2 & 7.2 \\
\hline & $\max$ & 0.0 & $\cdot 0.0$ & 0.0 & 0.0 & 0.0 & 0.0 & 0.0 & 0.0 & 0.0 & 0.0 & 29.0 & 29.0 & 29.0 & 29.0 & 29.0 & 29.0 \\
\hline & vot & 0.0 & 203.0 & 263.6 & 263.6 & 237.5 & 190.5 & 48.9 & 16.0 & .16 .0 & 16.0 & 60.5 & 68.1 & 68.1 & 68.2 & 68.3 & 195.0 \\
\hline \multirow{2}{*}{$\begin{array}{l}\text { ME_LIXIIII } \\
\text { sotale }\end{array}$} & HIN & 0.0 & 95.7 & 186.5 & 186.5 & 161.4 & 129.2 & 43.0 & 40.0 & 10.8 & 10.8 & 10.0 & 29.6 & 10.5 & 10.5 & 40.5 & 10.5 \\
\hline & $\max$ & 0.0 & 269.7 & 370.2 & 370.2 & 339.0 & 251.7 & 71.7 & 60.1 & 51.1 & 31.2 & 00.1 & 108.1 & 108.1 & 108.1 & 108.1 & 361.9 \\
\hline
\end{tabular}

Waste Class: RH_IIF_GICIII

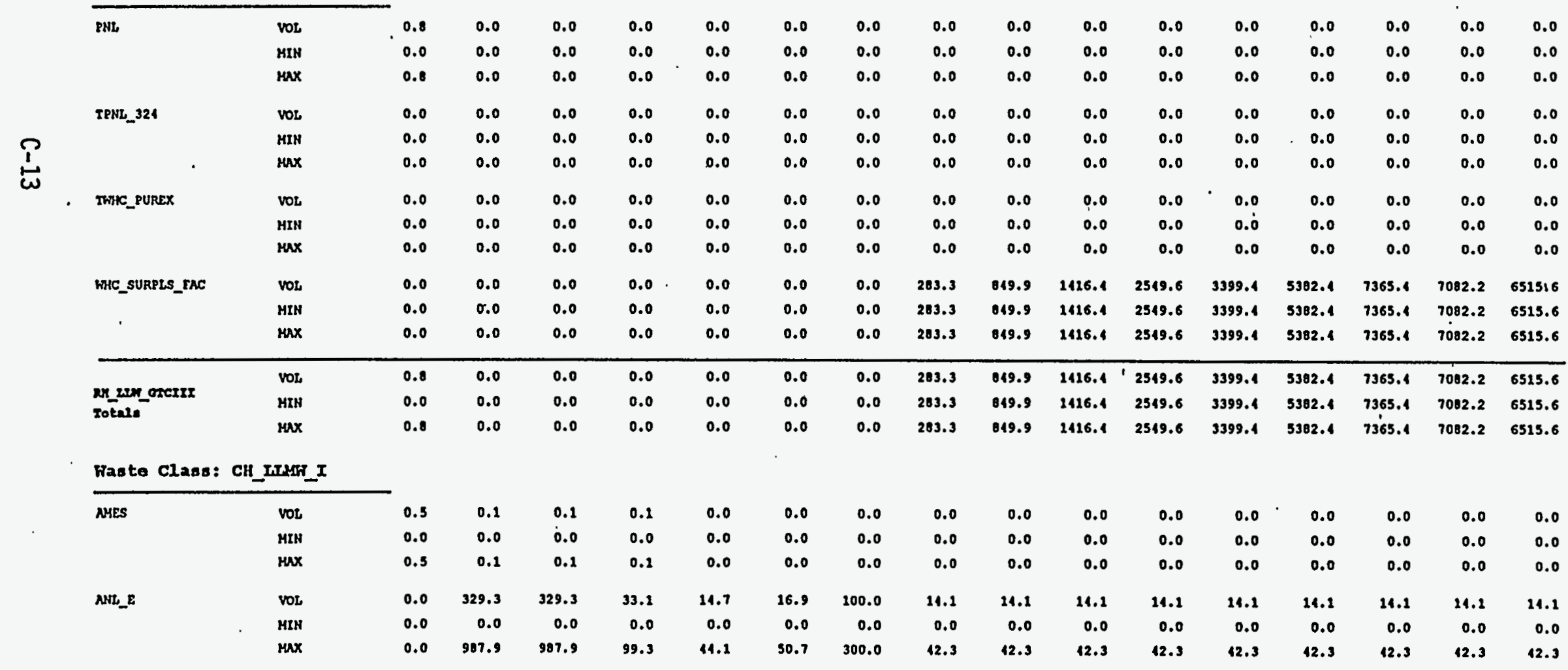




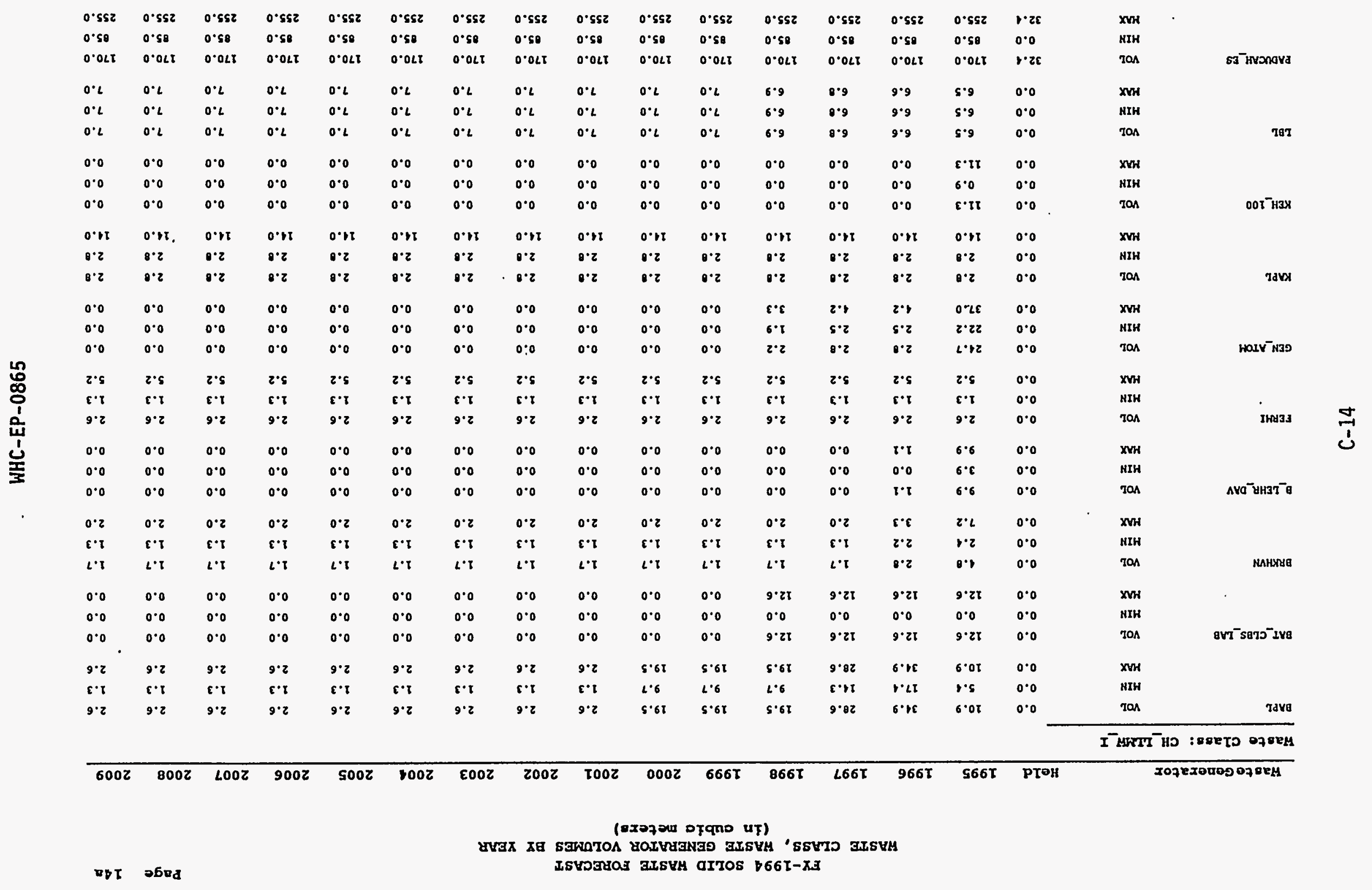


FX-1994 SOLID WASTE FORECAST

WASTE CLASS, FASTE GENERATOR VOLOMES BY YEAR

(In cubic meters)

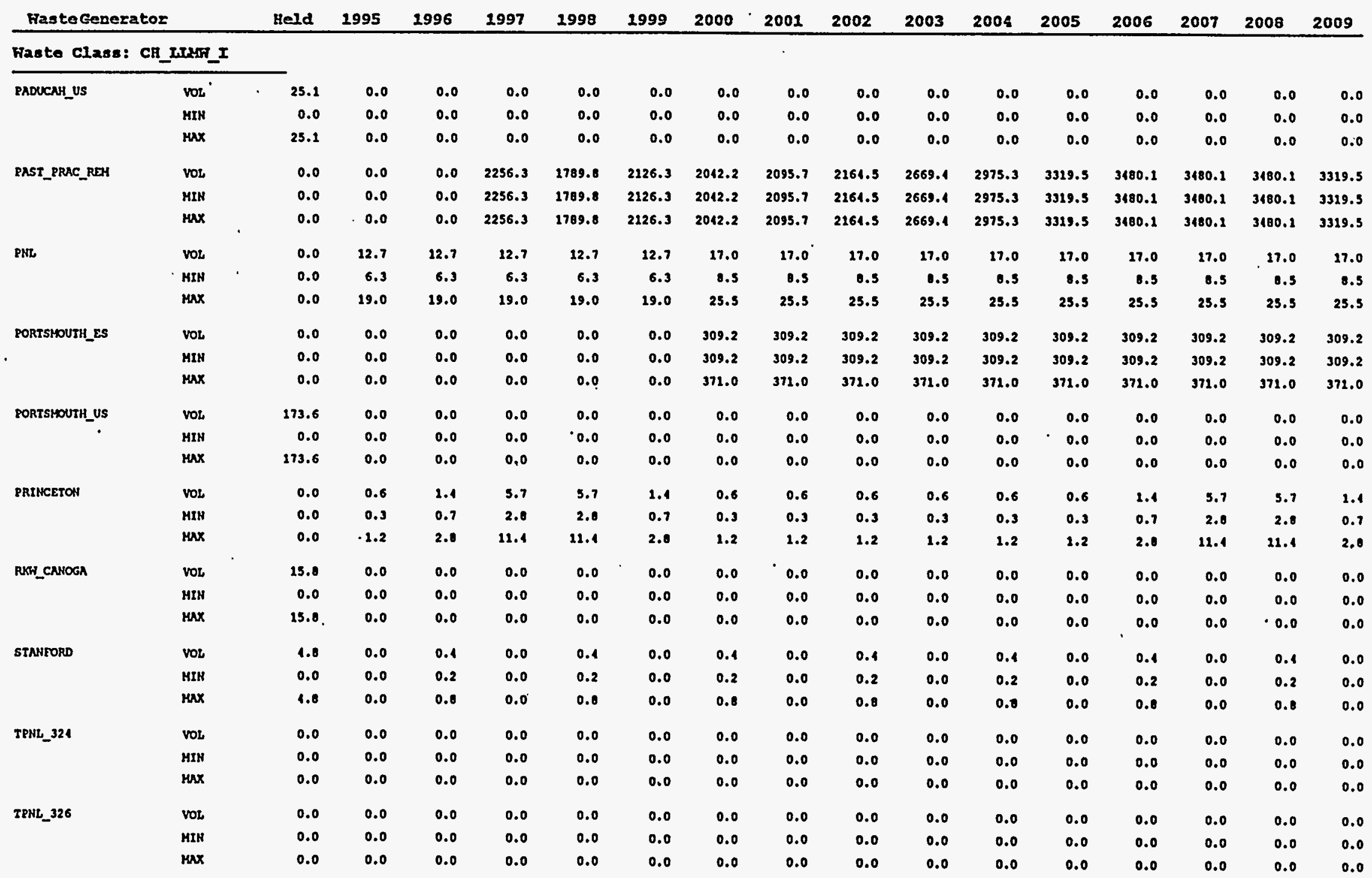




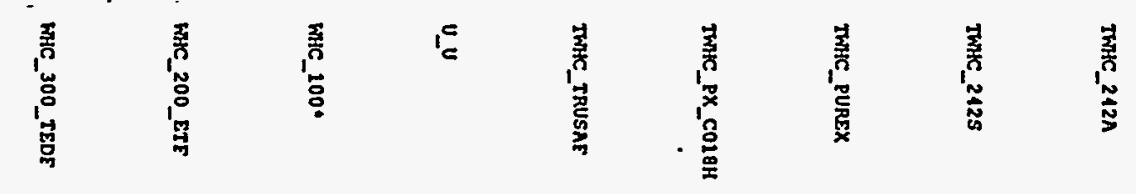

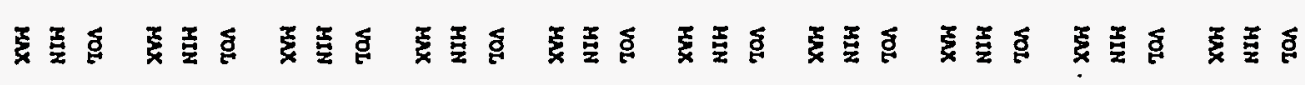

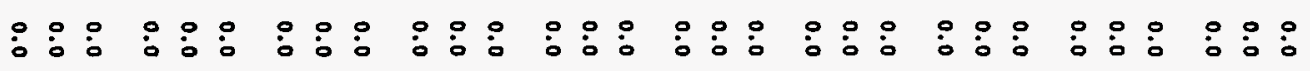

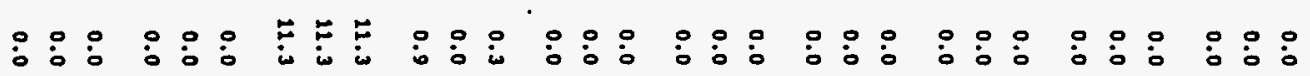

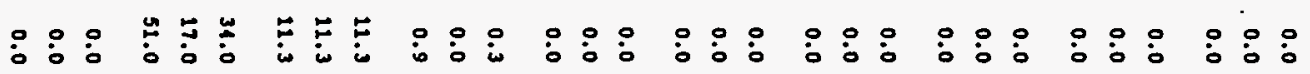

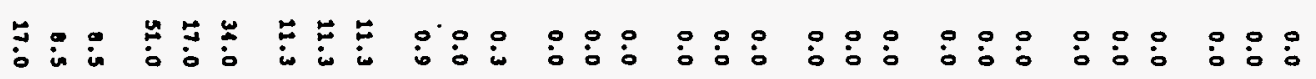

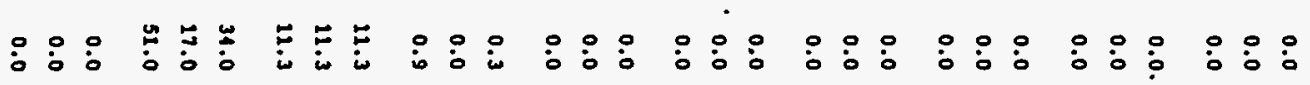

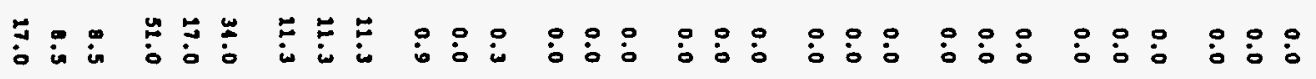

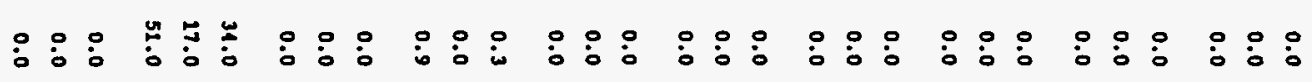

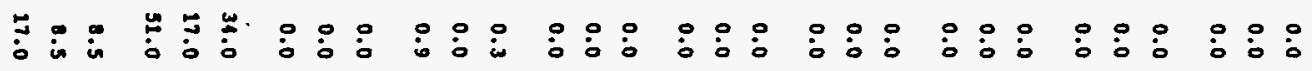

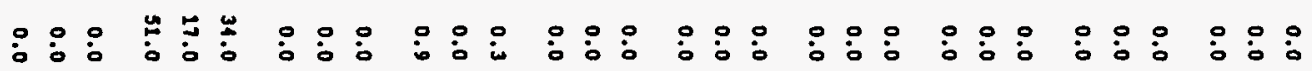

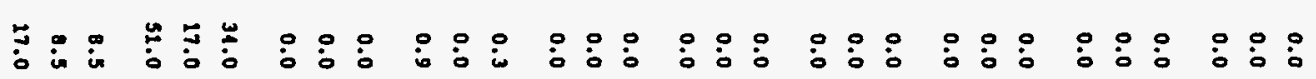

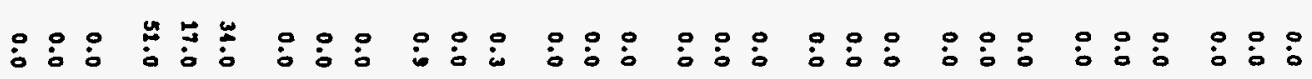

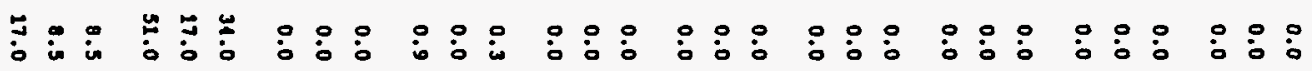

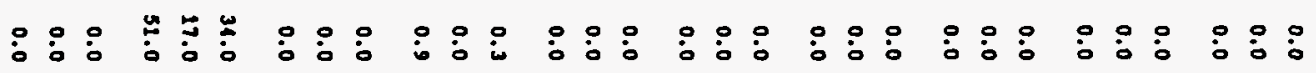

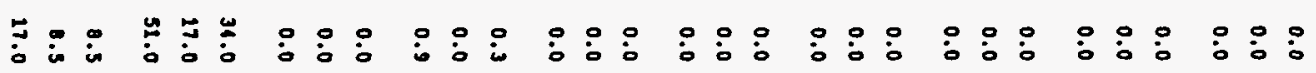

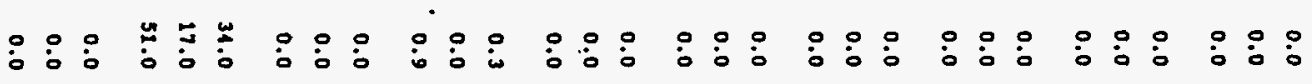

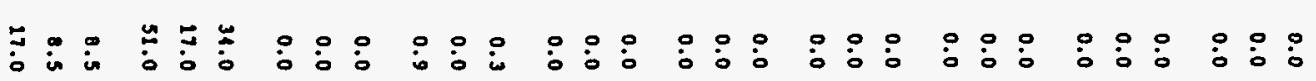


FY-1994 SOLID HASTE FORECAST

HASTE CLASS, FASTE GERTBRATOR VOLOMES BY YEAR

(in oubia metera)

\begin{tabular}{|c|c|c|c|c|c|c|c|c|c|c|c|c|c|c|c|c|c|}
\hline FasteGenex & & Held & 1995 & 1996 & 1997 & 1998 & 1999 & 2000 & 2001 & 2002 & 2003 & 2004 & 2005 & 2006 & 2007 & 2008 & 2009 \\
\hline \multicolumn{18}{|c|}{ Haste Class: CH_ITLen_I } \\
\hline \multirow[t]{3}{*}{ WHC_NNALYT_LAB } & vol & 0.0 & 38.3 & 32.3 & 25.0 & 25.0 & 23.0 & 25.0 & 25.0 & 25.0 & 25.0 & 23.0 & 25.0 & 25.0 & 25.0 & 25.0 & 25.0 \\
\hline & MIM & 0.0 & 34.4 & 29.0 & 22.5 & 22.5 & 22.5 & 22.5 & 22.5 & 22.5 & 22.5 & 22.5 & 22.5 & 22.5 & 22.5 & 22.5 & 22.5 \\
\hline & $\max$ & 0.0 & 12.1 & 35.5 & 27.5 & 27.5 & 27.5 & 27.5 & 27.5 & 27.5 & 27.5 & 27.5 & 27.5 & 27.5 & 27.3 & 27.5 & 27.5 \\
\hline \multirow[t]{2}{*}{ WIX_BQUIP } & vos & 0.0 & 326.3 & 368.3 & 270.0 & 170.0 & 352.7 & 352.7 & 352.7 & 352.7 & 226.6 & 226.6 & 226.6 & 339.9 & 339.9 & 339.9 & 170.0 \\
\hline & MIM & 0.0 & 326.3 & 368.3 & 170.0 & 170.0 & 352.7 & 352.7 & 352.7 & 352.7 & 226.6 & 226.6 & 226.6 & 339.9 & 339.9 & 339.9 & 170.0 \\
\hline . & $\max$ & 0.0 & 326.3 & 368.3 & 170.0 & 170.0 & 352.7 & 332.7 & 332.7 & 352.7 & 226.6 & 226.6 & 226.6 & 339.9 & 339.9 & 339.9 & 170.0 \\
\hline \multirow[t]{3}{*}{ WHC_B_PLNAT } & vot & 0.3 & 2.8 & 2.7 & 2.5 & 2.1 & 2.3 & 2.1 & 2.0 & 2.8 & 1.7 & 1.6 & 1.4 & 1.4 & 1.4 & 1.4 & 1.1 \\
\hline & MIN & 0.0 & 2.1 & 2.0 & 1.0 & 1.0 & 1.7 & 1.3 & 1.5 & 1.3 & 1.2 & 1.2 & 1.0 & 2.0 & 1.0 & 1.0 & 1.0 \\
\hline & $\max$ & 0.3 & 3.5 & $\mathbf{3 . 3}$ & 3.1 & 3.0 & 2.0 & 2.6 & 2.3 & 2.2 & 2.1 & 2.0 & 1.7 & 1.7 & 1.7 & 1.7 & 1.7 \\
\hline \multirow[t]{3}{*}{ WAC_EUEL_TRANS } & vor & 0.0 & 0.8 & 0.0 & 0.3 & 5.2 & 0.0 & 0.0 & 0.0 & 0.0 & 0.0 & 0.0 & 0.0 & 0.0 & 0.0 & 0.0 & 0.0 \\
\hline & HIN & 0.0 & 0.0 & 0.0 & 0.3 & 5.2 & 0.0 . & 0.0 & 0.0 & 0.0 & 0.0 & 0.0 & 0.0 & 0.0 & 0.0 & 0.0 & 0.0 \\
\hline & $\max$ & 0.0 & 0.8 & 0.0 & 8.3 & 3.2 & 0.0 & 0.0 & 0.0 & 0.0 & 0.0 & 0.0 & 0.0 & 0.0 & 0.0 & 0.0 & 0.0 \\
\hline \multirow{3}{*}{ WHC_GEOTEC_LAB } & vor & 0.0 & 0.3 & 0.3 & 0.3 & 0.3 & 0.3 & 0.3 & 0.3 & 0.3 & 0.3 & 0.3 & 0.3 & 0.3 & 0.3 & 0.3 & 0.3 \\
\hline & MIN & 0.0 & 0.1 & 0.1 & 0.1 & 0.1 & 0.1 & 0.1 & 0.1 & 0.1 & 0.2 & 0.1 & 0.1 & 0.1 & 0.1 & 0.1 & 0.1 \\
\hline & $\max$ & 0.0 & 0.9 & 0.9 & 0.9 & 0.9 & 0.9 & 0.9 & 0.9 & 0.9 & 0.9 & 0.9 & 0.9 & 0.9 & 0.9 & 0.9 & 0.9 \\
\hline \multirow[t]{3}{*}{ WHE_HLVP } & vor & 0.0 & 0.0 & 0.0 & 0.0 & 0.0 & 0.0 & 0.0 & 0.0 & 0.0 & 0.0 & 0.0 & 0.0 & 0.0 & 0.0 & 0.0 & 10.2 \\
\hline & MIN & 0.0 & 0.0 & 0.0 & 0.0 & 0.0 & 0.0 & 0.0 & 0.0 & 0.0 & 0.0 & 0.0 & 0.0 & 0.0 & 0.0 & 0.0 & 0.0 \\
\hline & $\max$ & 0.0 & .0 .0 & 0.0 & 0.0 & 0.0 & 0.0 & 0.0 & 0.0 & 0.0 & 0.0 & 0.0 & 0.0 & 0.0 & 0.0 & 0.0 & 36.4 \\
\hline \multirow[t]{3}{*}{ WHC_K_BASIH } & vor & 0.0 & 1.1 & 1.1 & 1.4 & 1.1 & 1.1 & 1.4 & 1.4 & 1.4 & 1.4 & 1.1 & 1.1 & 2.1 & 1.4 & 1.4 & 1.1 \\
\hline & MIM & 0.0 & 1.2 & 1.2 & 1.0 & 1.0 & 1.0 & 1.0 & 1.0 & 1.0 & 2.01 & 1.0 & 1.0 & 2.0 & 1.0 & 1.0 & 1.0 \\
\hline & $\max$ & 0.0 & 1.5 & 1.5 & 1.4 & 1.4 & 2.1 & 1.4 & 1.4 & 1.1 & 1.4 & 1.1 & 1.1 & 1.1 & 2.1 & - 1.4 & 1.4 \\
\hline \multirow[t]{3}{*}{ WHC_LLBG } & vols & 0.0 & 0.0 & 0.0 & 3.1 & 0.0 & 0.0 & 3.1 & 0.0 & 0.0 & 3.1 & 0.0 & 0.0 & 3.4 & 0.0 & 0.0 & 3.4 \\
\hline & HIH & 0.0 & 0.0 & 0.0 & 3.1 & 0.0 & 0.0 & 3.1 & 0.0 & 0.0 & 3.4 & 0.0 & 0.0 & 3.4 & 0.0 & 0.0 & 3.4 \\
\hline & $\max$ & 0.0 & 0.0 & 0.0 & 3.1 & 0.0 & 0.0 & 3.1 & 0.0 & 0.0 & 3.4 & 0.0 & 0.0 & 3.4 & 0.0 & 0.0 & 3.4 \\
\hline \multirow[t]{3}{*}{ WHTC_LLVP } & vol & 0.0 & 0.0 & 0.0 & 0.0 & 0.0 & 0.0 & 0.0 & 0.0 & 0.0 & 0.0 & 0.0 & 2.6 & 1.6 & 1.6 & 1.6 & 1.6 \\
\hline & MIM & 0.0 & 0.0 & 0.0 & 0.0 & 0.0 & 0.0 & 0.0 & 0.0 & 0.0 & 0.0 & 0.0 & 0.0 & 0.0 & 0.0 & 0.8 & 0.8 \\
\hline & $\max$ & 0.0 & 0.0 & 0.0 & 0.0 & 0.0 & 0.0 & 0.0 & 0.0 & 0.0 & 0.0 & 0.0 & 3.2 & 3.2 & 3.2 & 3.2 & 3.2 \\
\hline \multirow[t]{3}{*}{ WHC_PEP } & vol & 0.0 & 126.2 & 126.2 & 126.2 & 126.2 & 126.2 & 126.2 & 126.2 & 126.2 & 126.2 & 126.2 & 126.2 & 126.2 & 126.2 & 126.2 & 126.2 \\
\hline & MIN & 0.0 & 0.0 & 0.0 & 0.0 & 0.0 & 0.0 & 0.0 & 0.0 & 0.0 & 0.0 & 0.0 & 0.0 & 0.0 & 0.0 & 0.0 & 0.0 \\
\hline & $\max$ & 0.0 & 126.2 & 126.2 & 126.2 & 226.2 & 126.2 & 126.2 & 126.2 & 126.2 & 126.2 & 126.2 & 126.2 & 126.2 & 126.2 & 126.2 & 126.2 \\
\hline
\end{tabular}




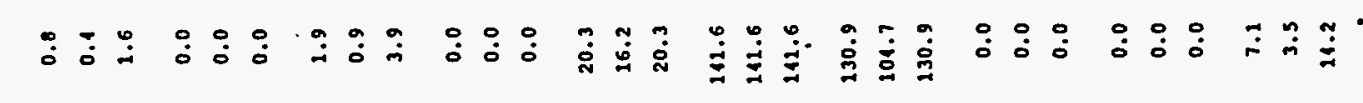

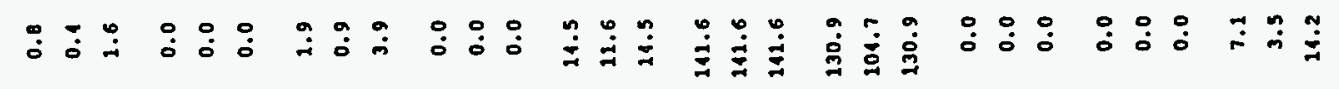

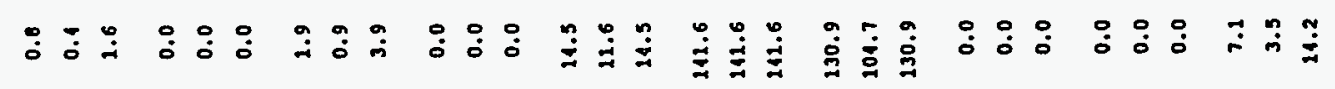

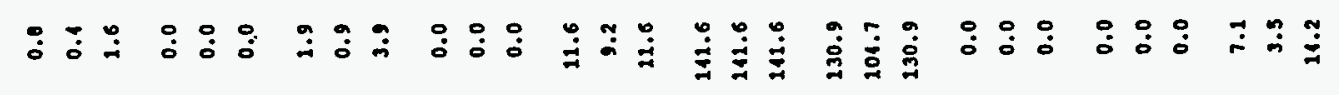

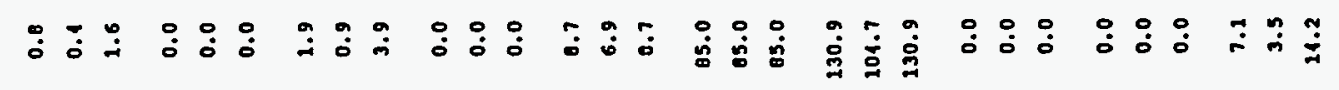

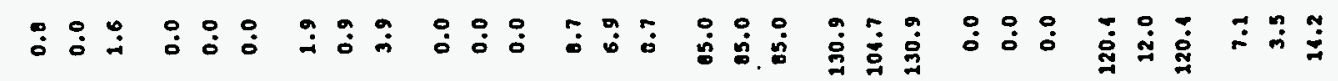

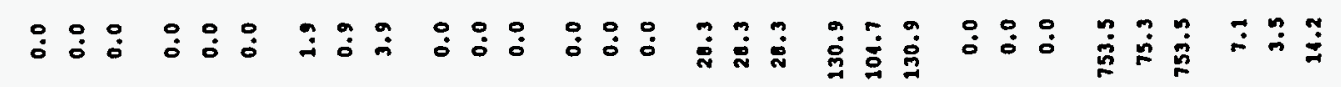

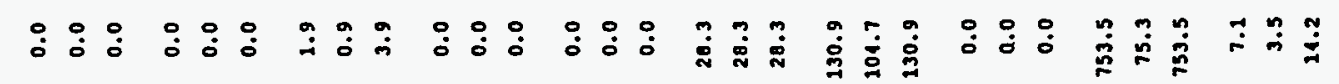

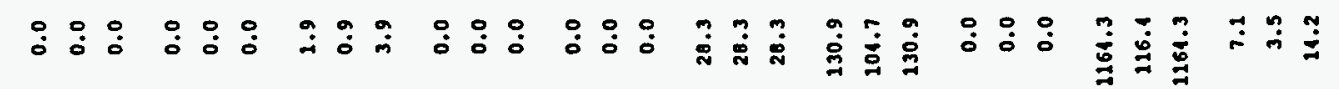

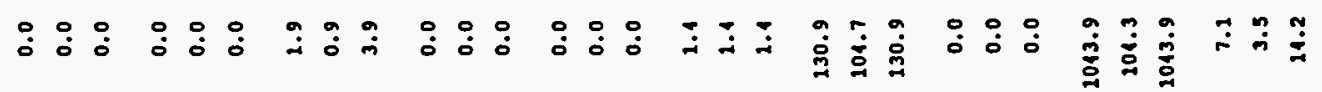

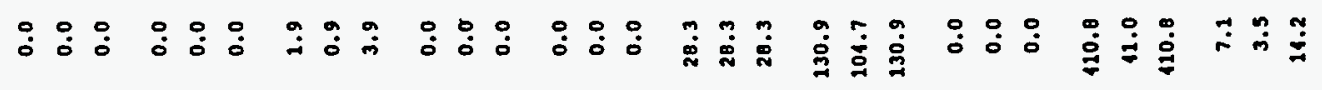
영영

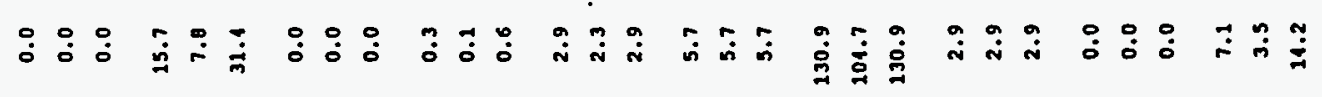

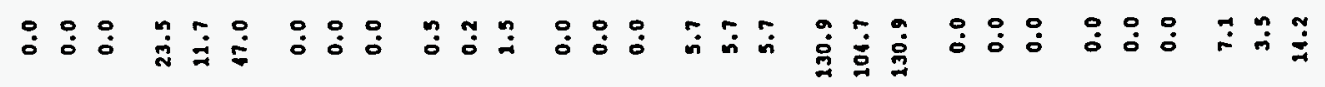

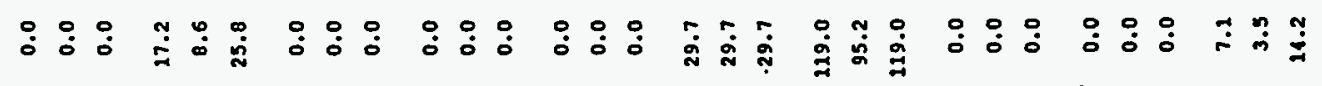

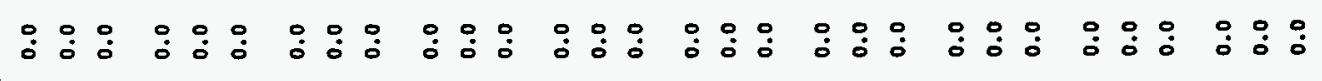

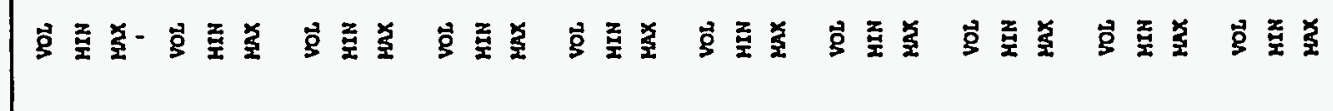




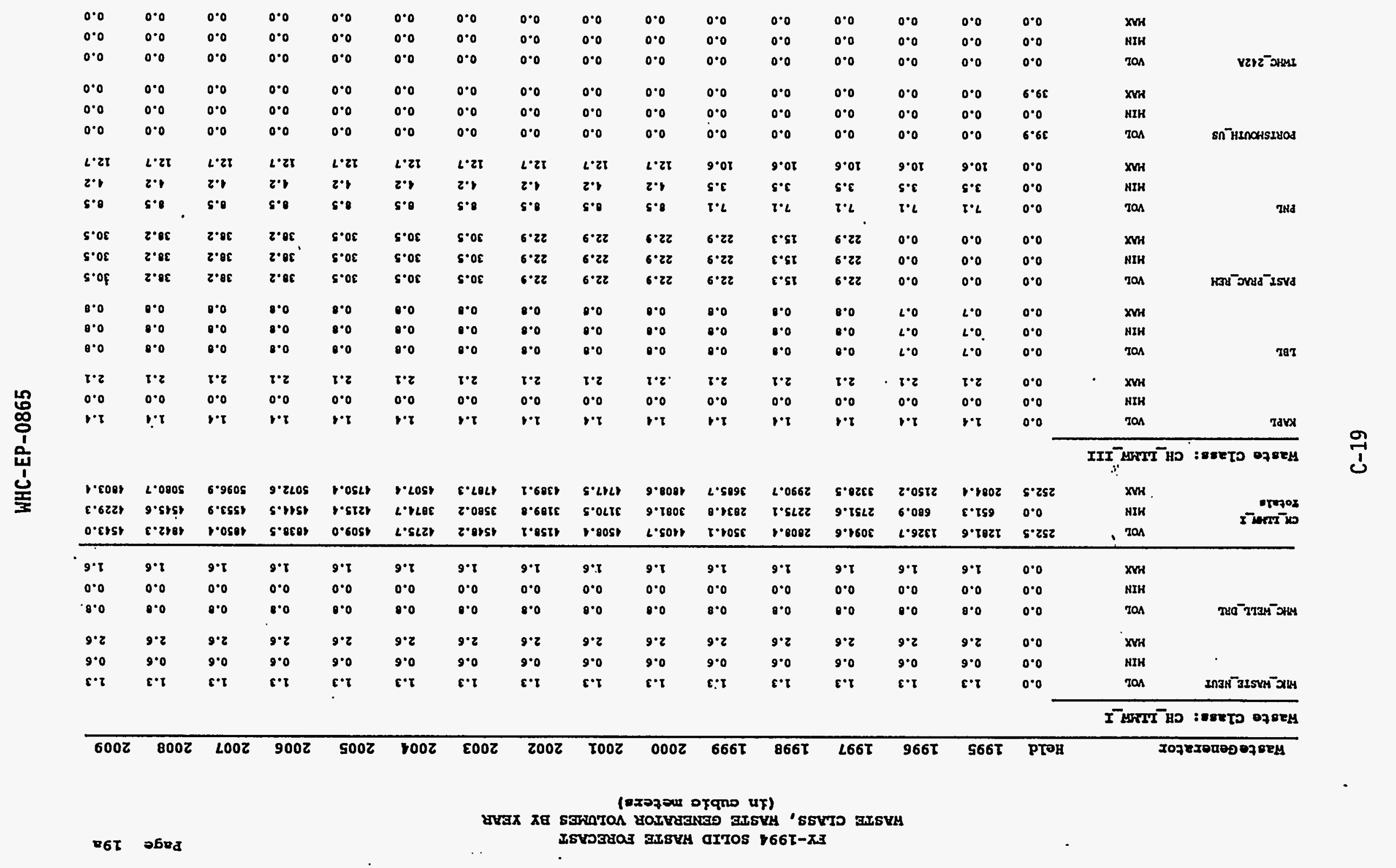




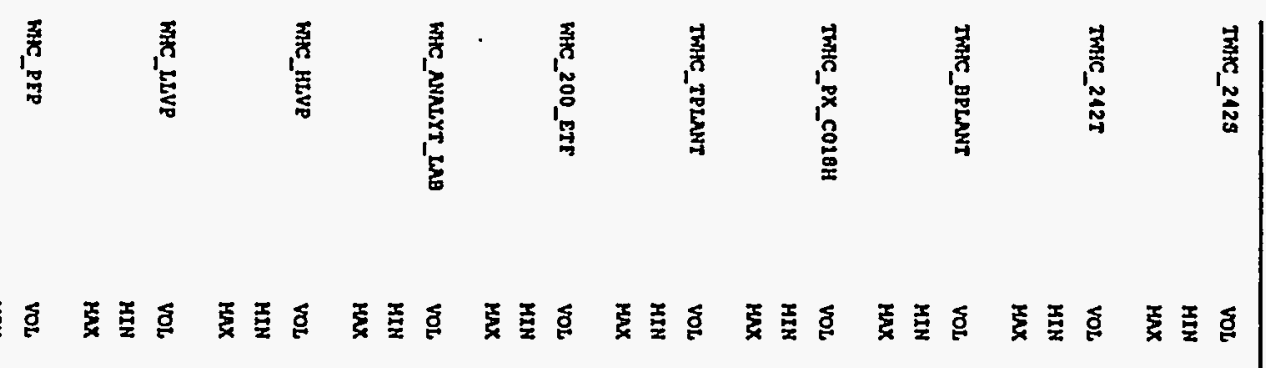

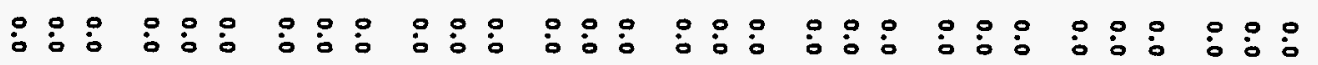

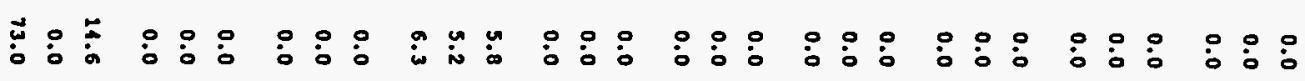

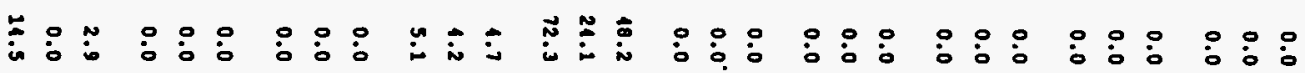

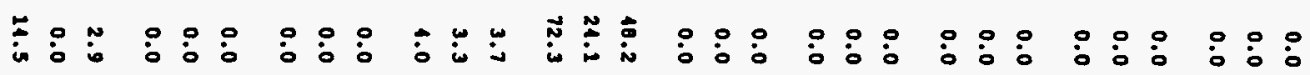

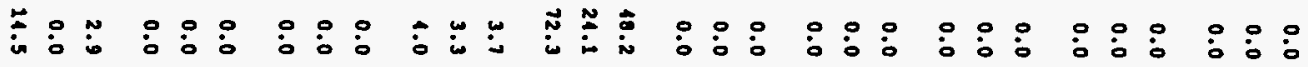

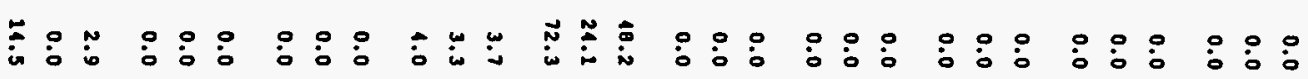

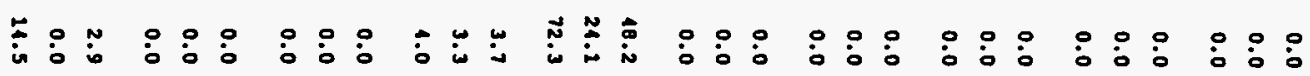

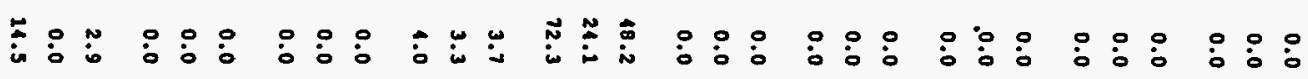

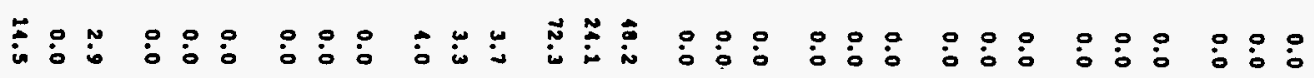

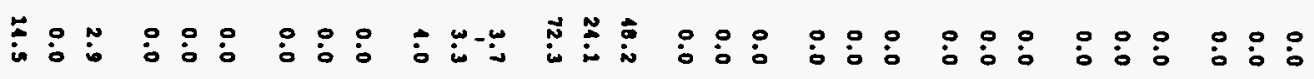

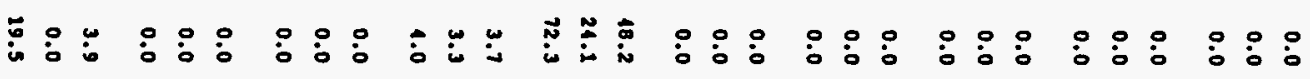

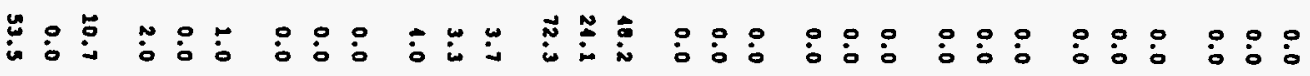

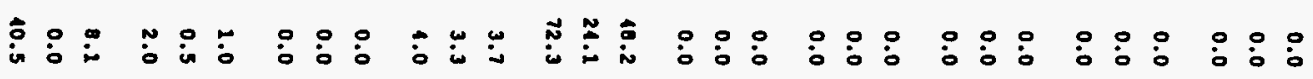

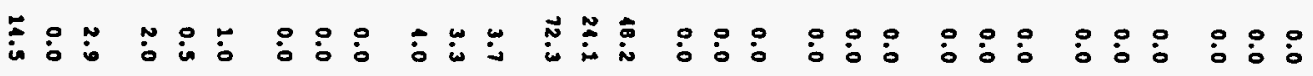

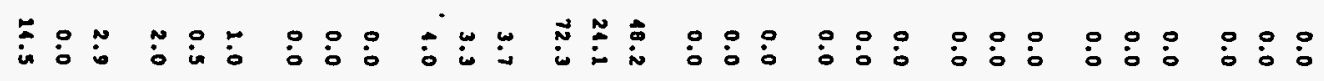

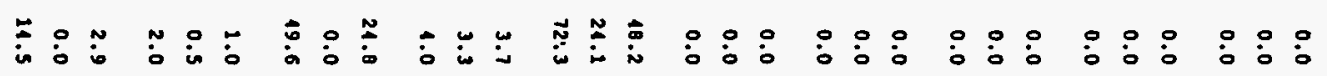


FY-1994 SOLID KASTE FORECAST

FASTE CLASS, HASTE GENERATOR VOLUMIS BY YEAR

Page 21a

(in cubic meters)

\begin{tabular}{|c|c|c|c|c|c|c|c|c|c|c|c|c|c|c|c|c|c|c|}
\hline Fas te Gener & ator & Held & 1995 & 1996 & 1997 & 1998 & 1999 & 2000 & 2001 & 2002 & 2003 & 2004 & 2005 & 2006 & 2007 & 2008 & 2009 & \\
\hline Faste Class: & CH_ITAF_III & & & & & & & & & & & & & & & & & \\
\hline \multirow[t]{3}{*}{ WHC_PRETRT_TH } & vot & 0.0 & 0.0 & 0.0 & 0.0 & 0.0 & 0.0 & 0.0 & 0.0 & 0.0 & 0.0 & 0.5 & 0.5 & 0.5 & 0.5 & 0.5 & 0.5 & \\
\hline & HIM & 0.0 & 0.0 & 0.0 & 0.0 & 0.0 & 0.0 & 0.0 & 0.0 & 0.0 & 0.0 & 0.0 & 0.2 & 0.2 & 0.2 & 0.2 & 0.2 & \\
\hline & $\max$ & 0.0 & 0.0 & 0.0 & 0.0 & 0.0 & 0.0 & 0.0 & 0.0 & 0.0 & 0.0 & 1.0 & 1.0 & 1.0 & 1.0 & 1.0 & 1.0 & \\
\hline \multirow[t]{3}{*}{ RHC_PUREX } & vor & 0.0 & 0.3 & 0.3 & 0.3 & 0.3 & 0.0 & 0.0 & 0.0 & 0.0 & 0.0 & 0.0 & 0.0 & 0.0 & 0.0 & 0.0 & 0.0 & \\
\hline & MIN & 0.0 & 0.0 & 0.0 & 0.0 & 0.0 & 0.0 & 0.0 & 0.0 & 0.0 & 0.0 & 0.0 & 0.0 & 0.0 & 0.0 & 0.0 & 0.0 & \\
\hline & $\max$ & 0.0 & 0.6 & 0.6 & 0.6 & 0.6 & 0.0 & 0.0 & 0.0 & 0.0 & 0.0 & 0.0 & 0.0 & 0.0 & 0.0 & 0.0 & 0.0 & \\
\hline \multirow[t]{3}{*}{ WHC_PUREX_SH } & vor & 0.0 & 0.0 & 0.0 & 0.0 & 0.0 & 0.0 & 0.0 & 0.0 & 0.0 & 0.0 & 0.0 & 0.0 & 0.0 & 0.0 & 0.0 & 0.0 & \\
\hline & HIN & 0.0 & 0.0 & 0.0 & 0.0 & 0.0 & 0.0 & 0.0 & 0.0 & 0.0 & 0.0 & 0.0 & 0.0 & 0.0 & 0.0 & 0.0 & 0.0 & \\
\hline & $\max$ & 0.0 & 0.0 & 0.0 & 0.0 & 0.0 & 0.1 & 0.1 & 0.1 & 0.2 & 0.2 & 0.1 & 0.2 & 0.1 & 0.1 & 0.1 & 0.2 & \\
\hline \multirow[t]{3}{*}{ WHC_SST_LLE } & vol & 0.0 & 16.1 & 223.0 & 398.7 & 369.4 & 194.2 & 544.8 & 643.0 & 713.4 & 297.3 & 2813.2 & 1631.3 & 2934.3 & 2230.0 & 2205.6 & 2633.5 & $\underline{\Sigma}$ \\
\hline & MIN & 0.0 & 39.4 & 109.5 & 508.9 & 323.9 & 120.0 & 163.0 & 546.5 & 631.0 & 677.7 & 2512.9 & 1103.6 & 1614.3 & 1912.5 & 1074.7 & 2230.4 & \\
\hline & $\max$ & 0.0 & 31.0 & 245.3 & 658.5 & 106.3 & 513.6 & 399.2 & 707.3 & 817.7 & 877.0 & 1996.7 & 1816.1 & 2127.9 & 2475.0 & 2426.1 & 2896.0 & \\
\hline \multirow[t]{3}{*}{ HHC_SST_PET } & vor & 0.0 & 0.0 & 0.0 & 1.1 & 0.0 & 0.0 & 0.0 & 0.0 & 0.0 & 0.0 & 13.1 & 13.1 & 27.1 & 21.8 & 21.0 & 30.5 & s \\
\hline & MIN & 0.0 & 0.0 & 0.0 & 3.3 & 0.0 & . 0.0 & 0.0 & 0.0 & 0.0 & 0.0 & 10.1 & 10.1 & 13.9 & 17.4 & 17.4 & 24.1 & 需 \\
\hline & $\max$ & 0.0 & 0.0 & 0.0 & 1.4 & 0.0 & 0.0 & 0.0 & 0.0 & 0.0 & 0.0 & 13.1 & 13.1 & .27 .4 & 21.8 & 21.8 & 30.5 & \\
\hline \multirow[t]{3}{*}{ WHC_SURPLS_FAC } & vor & 0.0 & 0.0 & 0.0 & 0.0 & 0.0 & 5.7 & 0.0 & 20.3 & 28.3 & 20.3 & 85.0 & 85.0 & 141.6 & 141.6 & 111.6 & 111.6 & . \\
\hline & MIN & 0.0 & 0.0 & 0.0 & 0.0 & 0.0 & 3.7 & 0.0 & 28.3 & 28.3 & 28.3 & 03.0 & 85.0 & 111.6 & 142.6 & 141.6 & 112.6 & \\
\hline & $\max$ & 0.0 & 0.0 & 0.0 & 0.0 & 0.0 & 5.7 & 0.0 & 28.3 & 28.3 & 26.3 & 05.0 & 85.0 & 111.6 & 111.6 & 141.6 & 141.6 & \\
\hline \multirow[t]{3}{*}{ WHC_TF_OPER } & vor & 0.0 & 51.0 & 36.1 & 36.1 & 36.1 & 36.1 & 56.1 & 56.1 & 56.1 & 56.1 & 36.1 & 36.1 & 36.1 & 56.1 & 56.1 & 56.1 & \\
\hline & MIN & 0.0 & 10.8 & 11.0 & 11.0 & 11.0 & 14.8 & 14.8 & 14.0 & 11.0 & 14.0 & 11.0 & 11.8 & 14.8 & 11.8 & 11.0 & 11.0 & \\
\hline & $\max$. & 0.0 & 51.0 & 56.1 & 56.1 & 36.2 & 56.1 & 36.1 & 56.1 & 36.1 & 36.1 & 36.1 & 56.2 & ' 36.1 & 56.2 & .36 .1 & 56.1 & \\
\hline \multirow[t]{3}{*}{ WHC_IHP_W32O } & vor & 0.0 & 10.2 & 29.0 & 200.5 & 24.5 & 0.0 & 0.0 & 0.0 & 0.0 & 0.0 & 0.0 & 0.0 & 0.0 & 0.0 & 0.0 & 0.0 & \\
\hline & MIN & 0.0 & 16.0 & 11.6 & 80.2 & 9.0 & 0.0 & 0.0 & 0.0 & 0.0 & 0.0 & 0.0 & 0.0 & 0.0 & 0.0 & 0.0 & 0.0 & \\
\hline & $\max$ & 0.0 & 10.2 & 29.0 & 200.5 & 24.5 & 0.0 & 0.0 & 0.0 & 0.0 & 0.0 & 0.0 & 0.0 & 0.0 & 0.0 & 0.0 & 0.0 & \\
\hline \multirow{3}{*}{ Cotrom_III } & vor & 39.9 & 167.5 & 373.4 & 947.0 & 529.7 & 643.1 & 609.1 & 813.9 & 916.3 & 977.0 & 2067.0 & 1910.9 & 2260.1 & 2374.0 & 2530.4 & 2984.1 & \\
\hline & MIN & 0.0 & 105.7 & 270.6 & 692.2 & 115.7 & 525.3 & 563.3 & 675.1 & 760.5 & 013.9 & 1746.3 & 1607.2 & 1916.2 & 2187.0 & 2150.1 & 2513.1 & \\
\hline & $\max$ & 39.9 & 235.6 & 436.4 & 1047.5 & 607.2 & 732.9 & 704.9 & 921.2 & 2031.7 & 1098.6 & 2291.1 & 2149.0 & 2516.9 & 2842.3 & 2793.5 & 3314.0 & \\
\hline
\end{tabular}




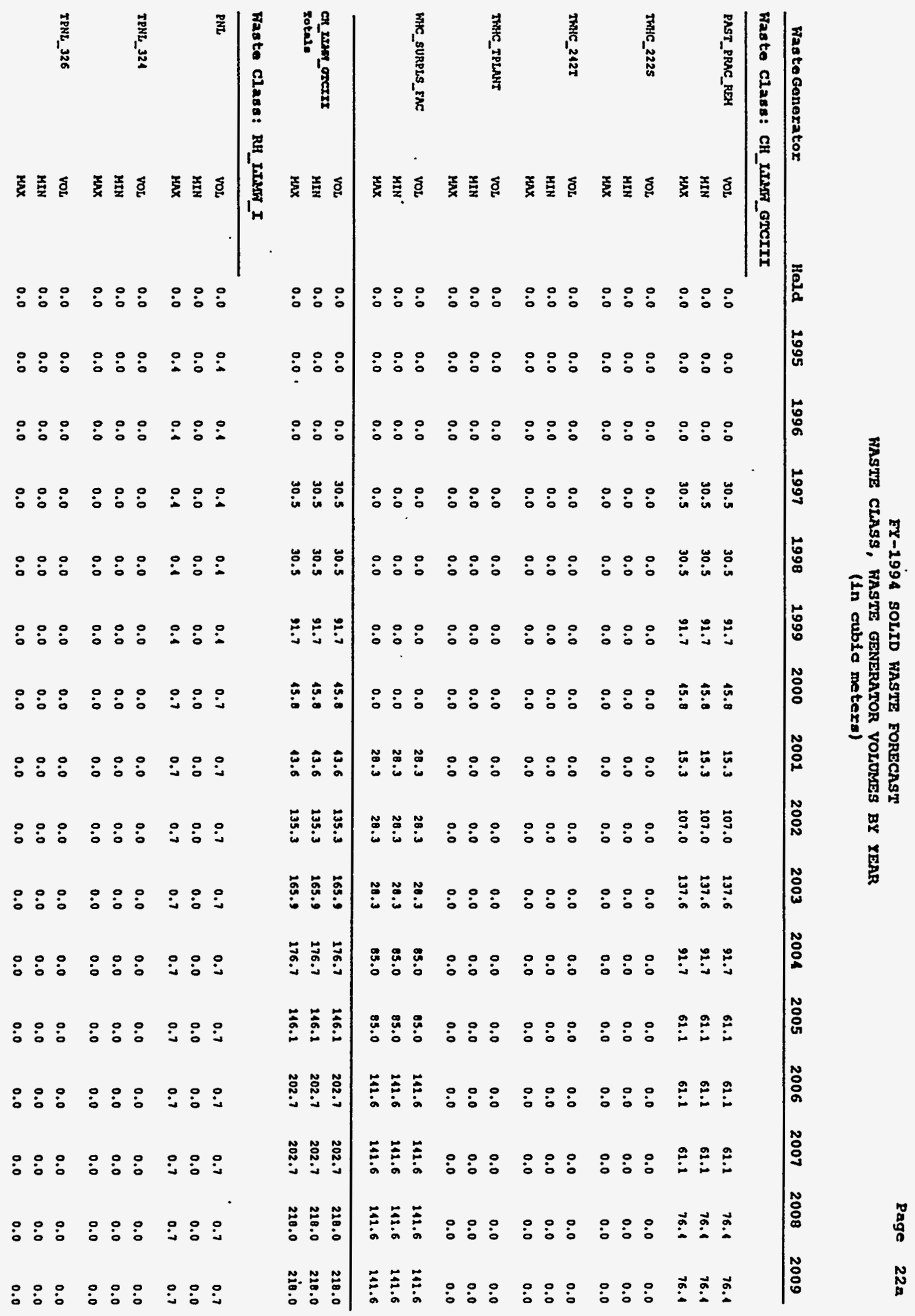




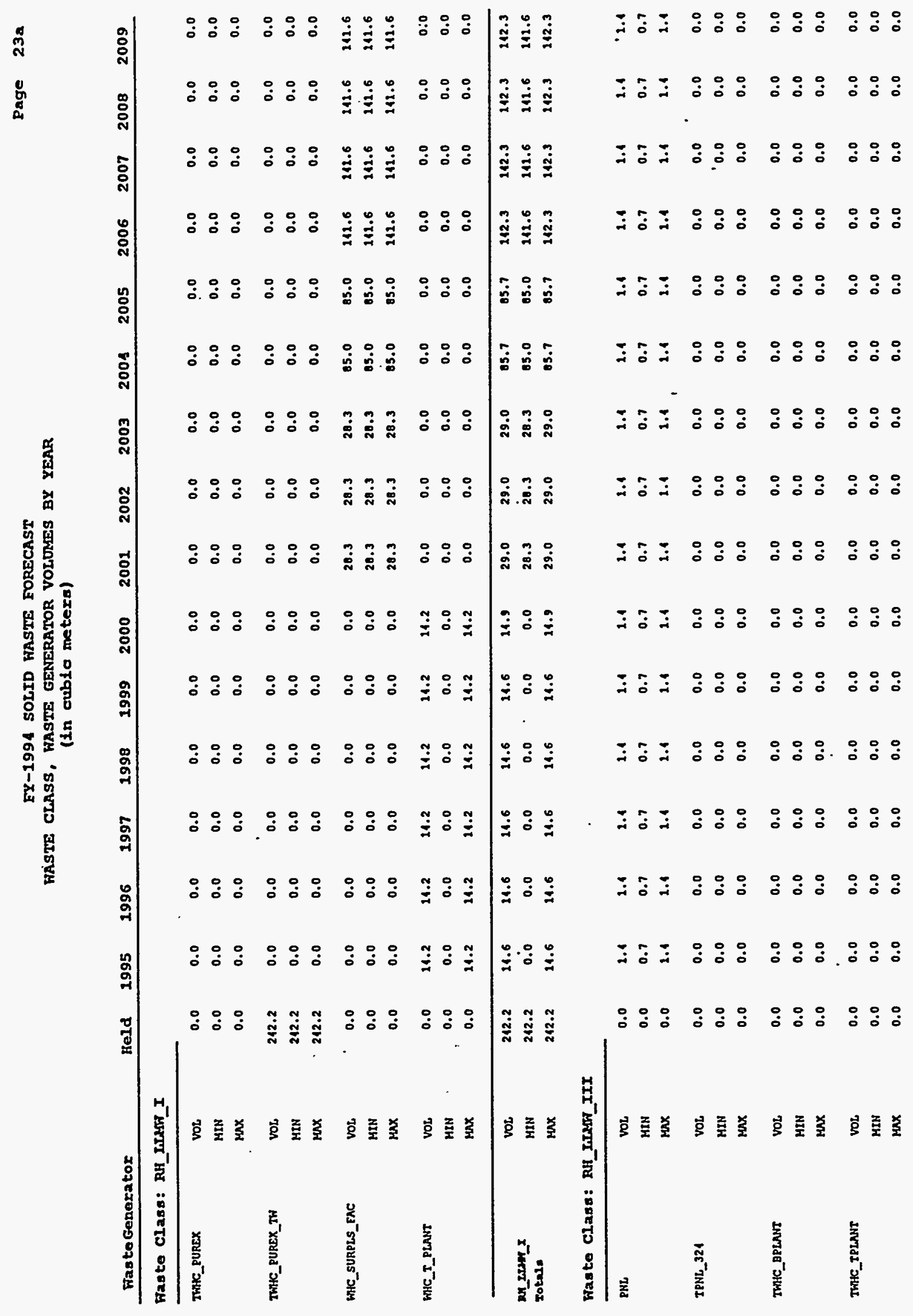


FY-1994 SOLID WASTE DORECAST

WASTE CLASS, WASTE GENERATOR VOLUMES BY YEAR

(In cubio meters)

\begin{tabular}{|c|c|c|c|c|c|c|c|c|c|c|c|c|c|c|c|c|c|c|}
\hline Waste Gener & & He] & Id & 1995 & 1996 & 1997 & 1998 & 1999 & 2000 & 2001 & 2002 & 2003 & 2004 & 2005 & 2006 & 2007 & 2008 & 2009 \\
\hline \multicolumn{19}{|c|}{ Haste C12ss: RH_LreH_III } \\
\hline \multirow[t]{3}{*}{ WHC_ANRLYT_LAB } & voL & & 0.0 & 3.1 & 2.1 & 1.8 & 1.8 & 1.0 & 1.0 & 1.0 & 1.8 & 1.8 & 1.8 & 1.8 & 1.0 & 1.8 & 1.8 & 1.0 \\
\hline & MIN & & 0.0 & 3.0 & 2.1 & 1.6 & 1.6 & 1.6 & 1.6 & 1.6 & 1.6 & 1.6 & 1.6 & 1.6 & 1.6 & 1.6 & 1.6 & 1.6 \\
\hline & $\max$ & & 0.0 & 3.7 & 2.6 & 1.9 & 1.9 & 1.9 & 1.9 & 1.9 & 1.9 & 1.9 & 1.9 & 2.9 & 2.9 & 1.9 & 1.9 & 1.9 \\
\hline \multirow[t]{3}{*}{ WHC_B_PLANT } & vor & & 0.0 & 0.0 & 1.1 & 1.1 & 1.1 & 1.1 & 1.1 & 0.0 & 0.0 & 0.0 & 0.0 & 0.0 & 0.0 & 0.0 & 0.0 & 0.0 \\
\hline & MIN & & 0.0 & 0.0 & 0.0 & 0.3 & 0.5 & 0.5 & 0.5 & 0.0 & 0.0 & 0.0 & 0.0 & 0.0 & 0.0 & 0.0 & 0.0 & 0.0 \\
\hline & $\max$ & & 0.0 & 0.0 & 2.2 & 2.2 & 1.6 & 1.6 & 1.6 & 0.0 & 0.0 & 0.0 & 0.0 & 0.0 & 0.0 & 0.0 & 0.0 & 0.0 \\
\hline \multirow[t]{3}{*}{ WHC_DST_RET } & vot & & 0.0 & 0.0 & 0.0 & 0.0 & 0.0 & 0.0 & 0.0 & 0.0 & 0.0 & 0.0 & 0.0 & 0.0 & 0.0 & 0.0 & 0.0 & 0.0 \\
\hline & MIN & & 0.0 & 0.0 & 0.0 & 0.0 & 0.0 & 0.0 & 0.0 & 0.0 & 0.0 & 0.0 & 0.0 & 0.0 & 0.0 & 0.0 & 0.0 & 0.0 \\
\hline & $\tan x$ & $:$ & 0.0 & 0.0 & 0.0 & 0.0 & 0.0 & 0.0 & 0.0 & 0.0 & 0.0 & 0.0 & 0.0 & 0.0 & 0.0 & 0.0 & 0.0 & 0.0 \\
\hline \multirow[t]{3}{*}{ WITC_SURPLS_EAC } & vot & & 0.0 & 0.0 & 0.0 & 0.0 & 0.0 & 0.0 & 0.0 & 20.3 & 28.3 & 28.3 & 85.0 & 85.0 & 211.6 & 141.6 & 141.6 & 141.6 \\
\hline & MIN & & 0.0 & 0.0 & 0.0 & 0.0 & 0.0 & 0.0 & 0.0 & 28.3 & 28.3 & 26.3 & 85.0 & 85.0 & 141.6 & 111.6 & 111.6 & 111.6 \\
\hline & $\max$ & & 0.0 & 0.0 & 0.0 & 0.0 & 0.0 & 0.0 & 0.0 & 28.3 & 20.3 & 20.3 & 85.0 & 85.0 & 111.6 & 111.6 & 142.6 & 141.6 \\
\hline \multirow[t]{3}{*}{ WHC_TWP_W151 } & vor & & 0.0 & 116.0 & 0.0 & 0.0 & 0.0 & 0.0 & 0.0 & 107.8 & 0.0 & 0.0 & 0.0 & 0.0 & 0.0 & 0.0 & 0.0 & 0.0 \\
\hline & MIN & & 0.0 & 104.4 & 0.0 & 0.0 & 0.0 & 0.0 & 0.0 & 107.0 & 0.0 & 0.0 & 0.0 & 0.0 & 0.0 & 0.0 & 0.0 & 0.0 \\
\hline & $\max$ & & 0.0 & 127.6 & 0.0 & 0.0 & 0.0 & 0.0 & 0.0 & 107.0 & 0.0 & 0.0 & 0.0 & 0.0 & 0.0 & 0.0 & 0.0 & 0.0 \\
\hline \multirow[t]{3}{*}{ WHIC_TWR_W211 } & vor & & 0.0 & 0.0 & 0.0 & 14.2 & 14.2 & 14.2 & 28.3 & 20.3 & 28.3 & 20.3 & 14.2 & 14.2 & 28.3 & 14.2 & 24.2 & 14.2 \\
\hline & MIN & & 0.0 & 0.0 & 0.0 & 7.1 & 7.1 & 7.1 & 14.1 & 14.1 & 14.1 & 14.1 & 7.2 & 7.2 & 14.1 & 7.1 & 7.1 & 7.1 \\
\hline & $\max$ & & 0.0 & 0.0 & 0.0 & 11.2 & 14.2 & 11.2 & 28.3 & 28.3 & 28.3 & 28.3 & 11.2 & 21.2 & 20.3 & 11.2 & 11.2 & .14 .2 \\
\hline \multirow{3}{*}{$\begin{array}{l}\text { Nx_2wne_III } \\
\text { sotale }\end{array}$} & vou & & 0.0 & 120.8 & 4.9 & 10.5 & 18.3 & 10.5 & 32.6 & 167.6 & 39.0 & 39.8 & 102.1 & 102.4 & 173.1 & 259.0 & 139.0 & 159.0 \\
\hline & MIN & & 0.0 & 108.1 & 2.8 & 9.9 & 9.9 & 9.9 & 17.0 & 132.5 & 14.7 & 16.7 & 94.1 & 94.4 & 158.0 & 151.0 & 151.0 & 151.0 \\
\hline & $\max$ & & 0.0 & 232.7 & 6.2 & 29.7 & 29.2 & 29.2 & 33.3 & 267.7 & 39.9 & 39.9 & 102.5 & 102.5 & 173.2 & 159.1 & · 159.1 & 139.1 \\
\hline \multicolumn{19}{|c|}{ Waste Class: RH_LIM_GMCIII } \\
\hline \multirow[t]{3}{*}{ BNL } & vor & & 0.0 & 0.0 & 0.0 & 0.0 & 0.0 & 0.0 & 11.3 & 11.3 & 11.3 & 11.3 & 11.3 & 11.3 & 11.3 & 11.3 & $11: 3^{\circ}$ & 11.3 \\
\hline & HIN & & 0.0 & 0.0 & 0.0 & 0.0 & 0.0 & 0.0 & 0.0 & 0.0 & 0.0 & 0.0 & 0.0 & 0.0 & 0.0 & 0.0 & 0.0 & 0.0 \\
\hline & $\max$ & & 0.0 & 0.0 & 0.0 & 0.0 & 0.0 & 0.0 & 22.6 & 22.6 & 22.6 & 22.6 & 22.6 & 22.6 & 22.6 & 22.6 & 22.6 & 22.6 \\
\hline \multirow[t]{3}{*}{ TWHE_222S } & vor & & 0.0 & 0.0 & 0.0 & 0.0 & 0.0 & 0.0 & 0.0 & 0.0 & 0.0 & 0.0 & 0.0 & 0.0 & 0.0 & 0.0 & 0.0 & 0.0 \\
\hline & MIN & & 0.0 & 0.0 & 0.0 & 0.0 & 0.0 & 0.0 & 0.0 & 0.0 & 0.0 & 0.0 & 0.0 & 0.0 & 0.0 & 0.0 & 0.0 & 0.0 \\
\hline & $\max$ & & 0.0 & 0.0 & 0.0 & 0.0 & 0.0 & 0.0 & 0.0 & 0.0 & 0.0 & 0.0 & 0.0 & 0.0 & 0.0 & 0.0 & 0.0 & 0.0 \\
\hline
\end{tabular}




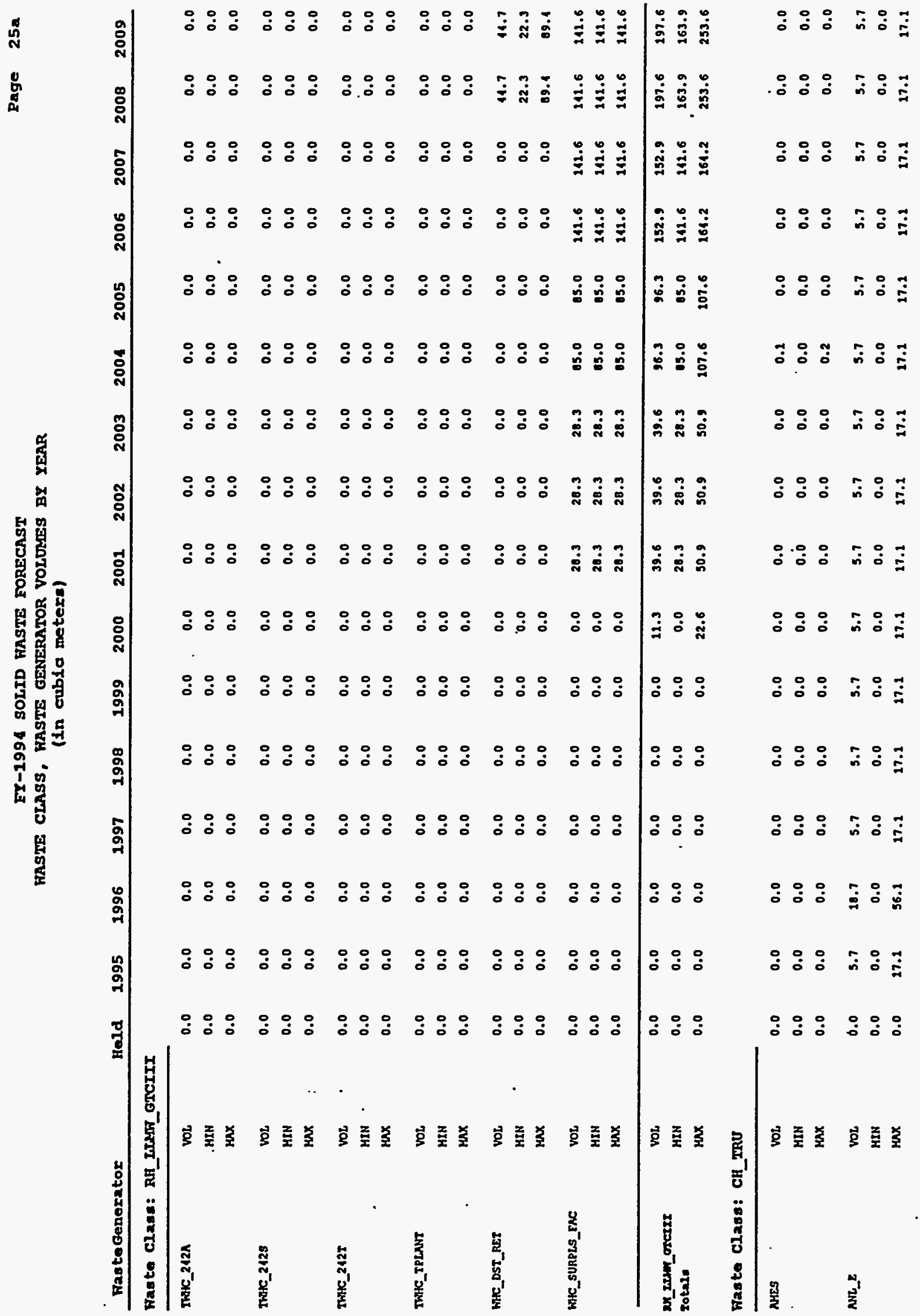




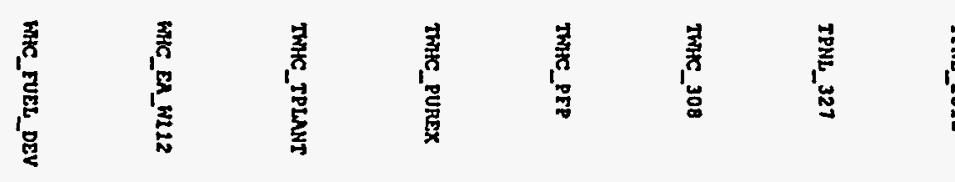

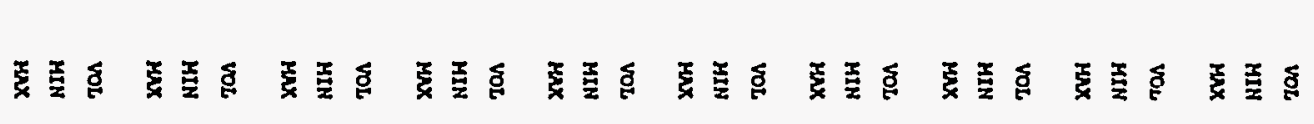

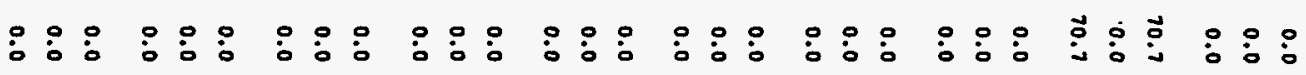

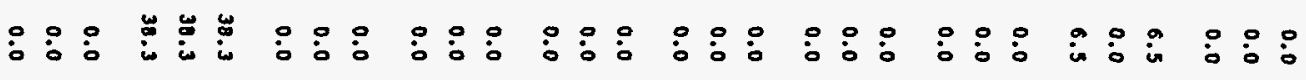

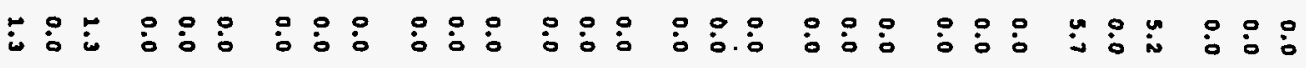

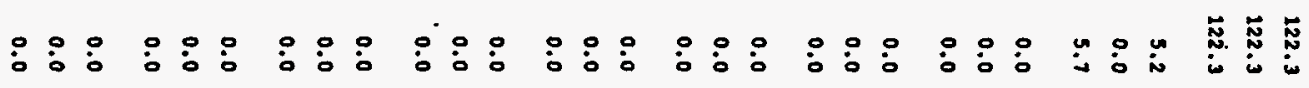

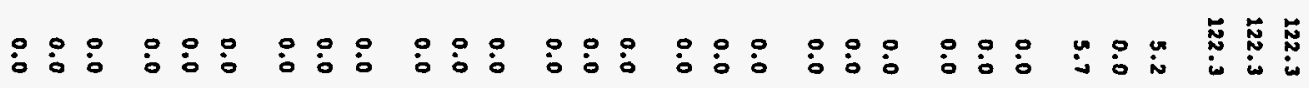

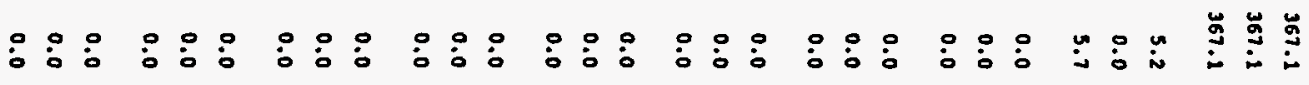

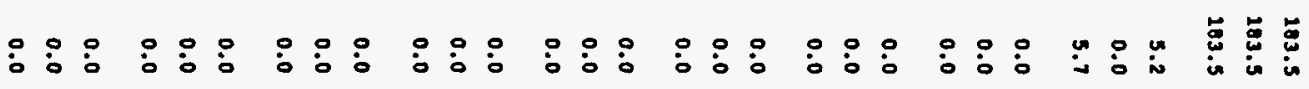

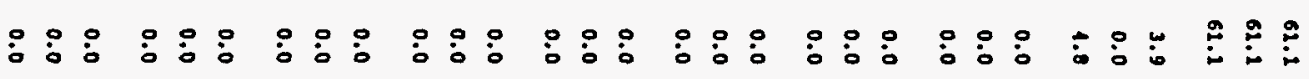

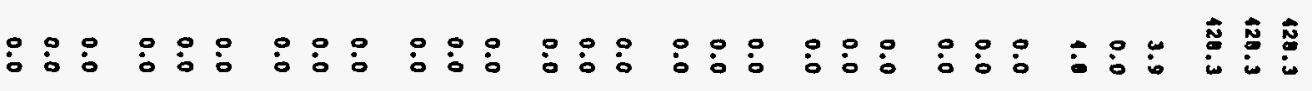

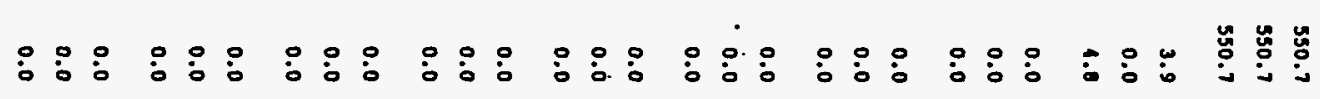

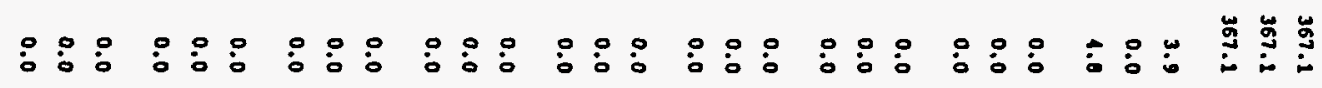

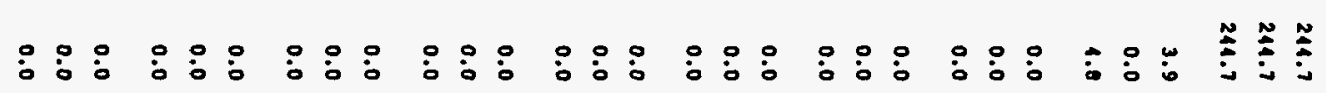

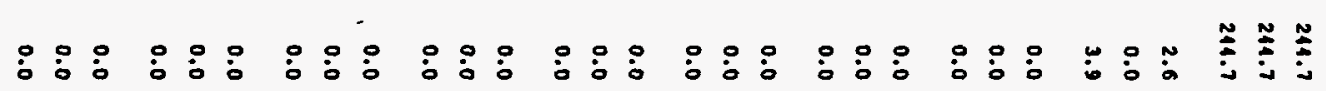

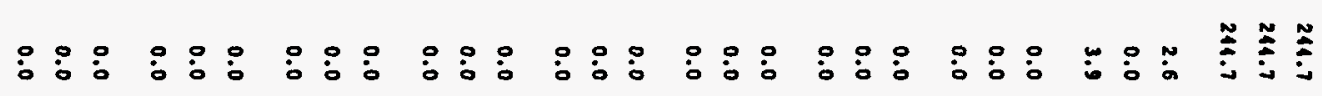

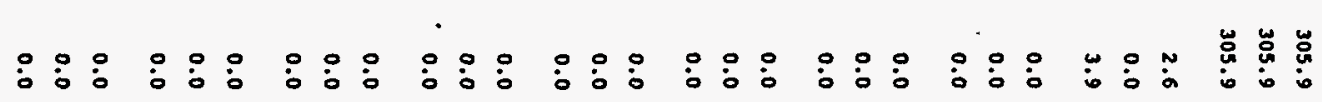

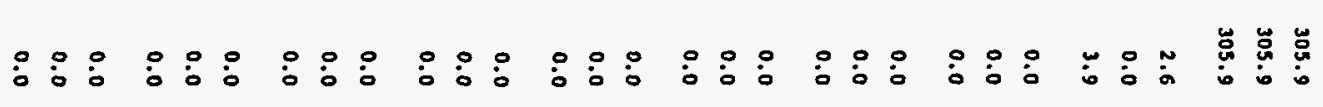

急

两

点氮 


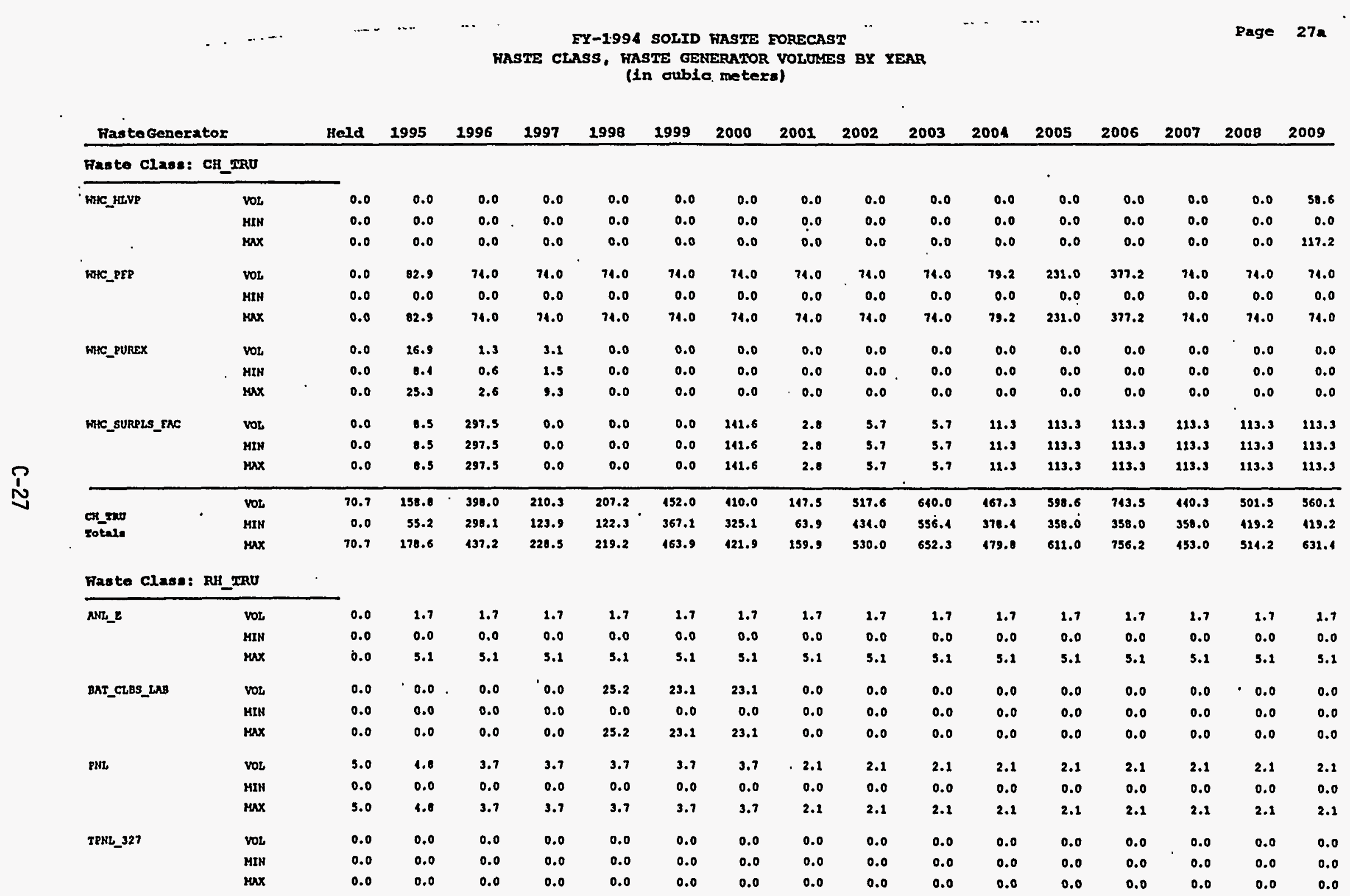


WHC-EP-0865

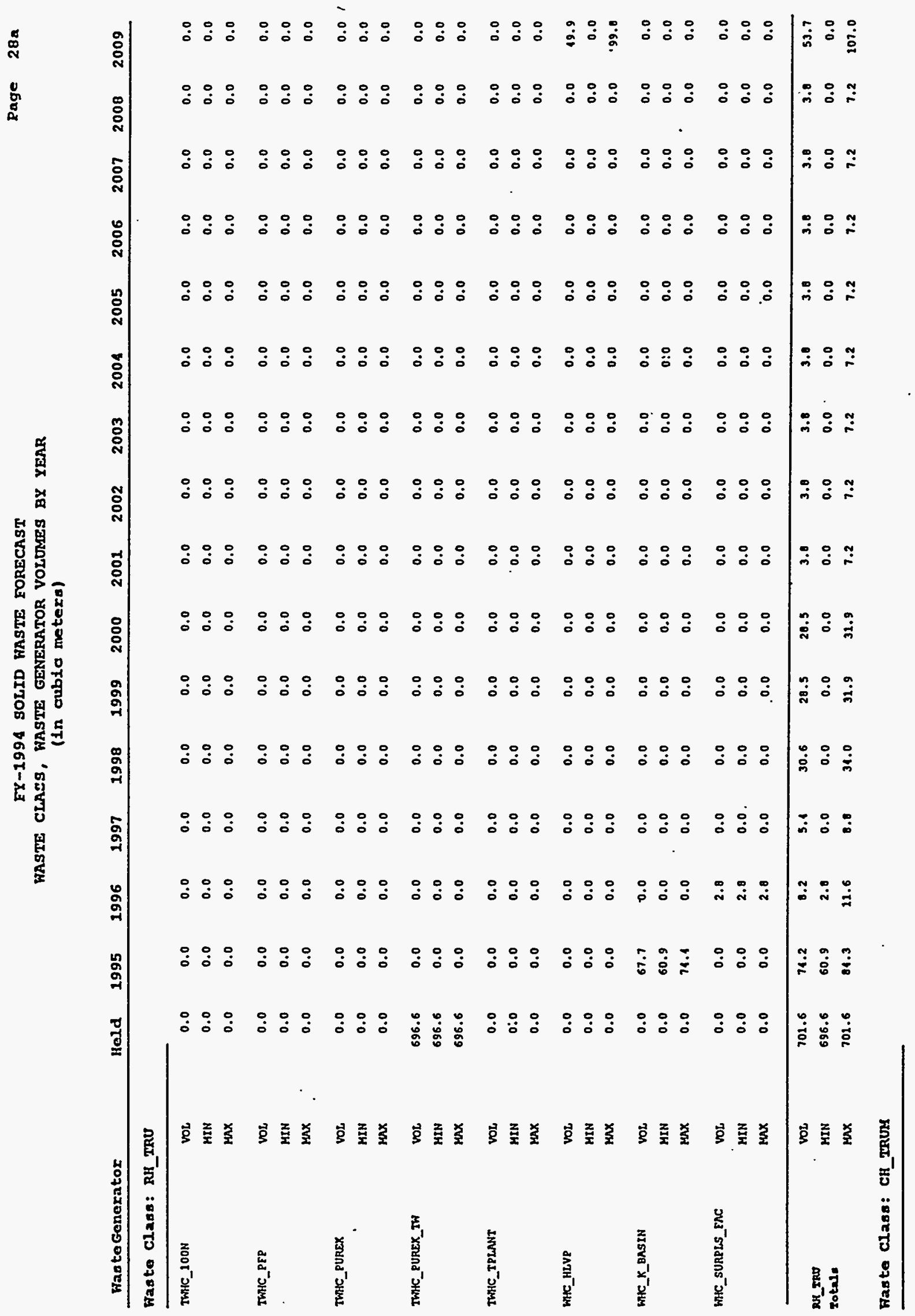




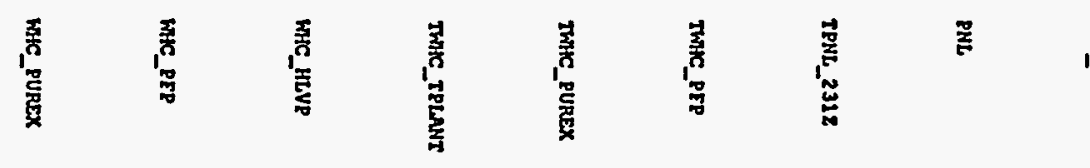

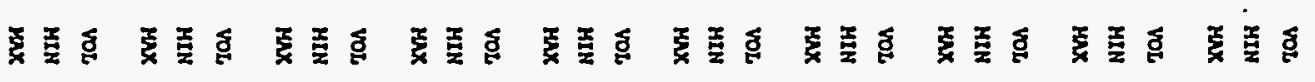

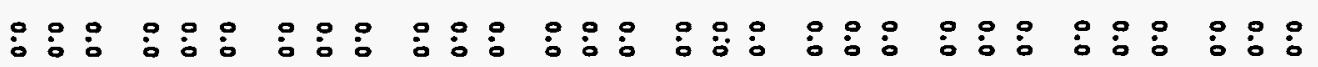

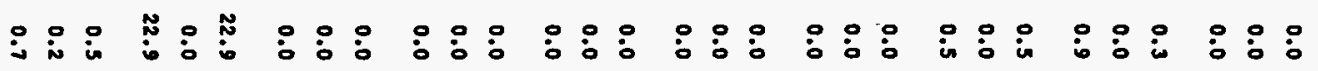

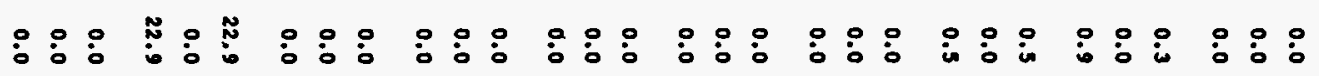

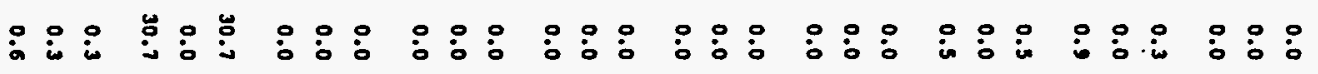

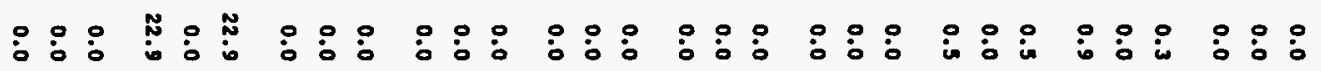

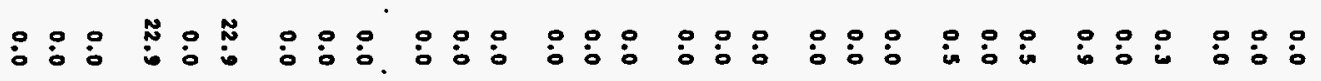

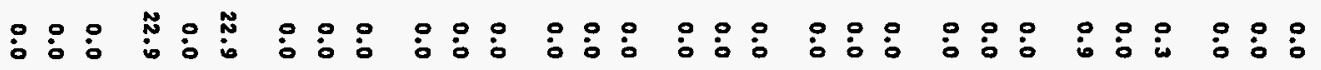

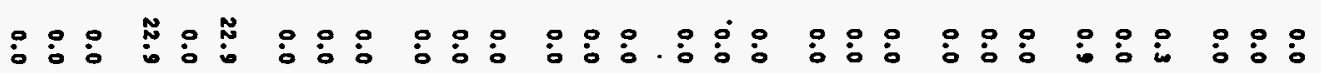

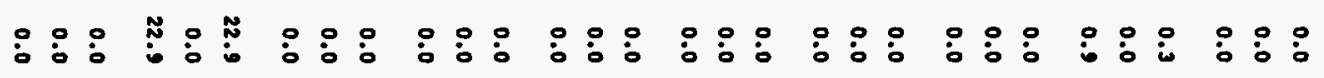

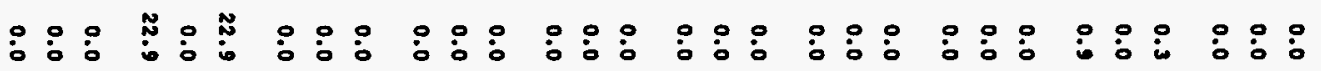

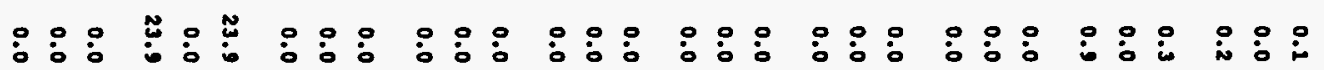

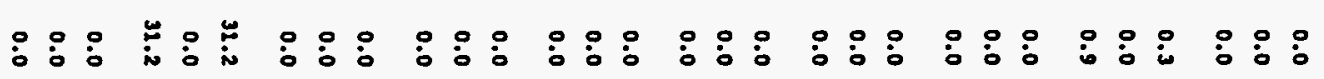

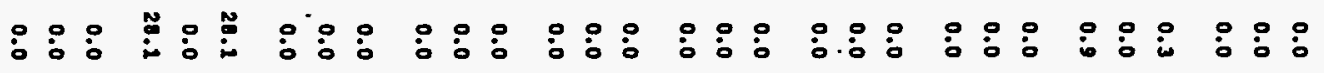

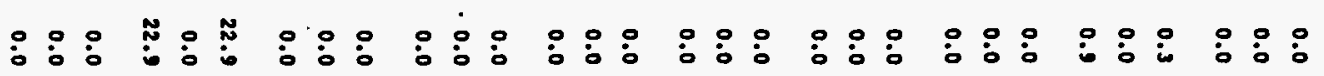

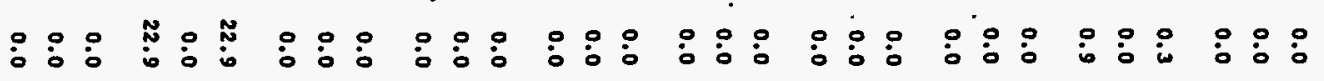

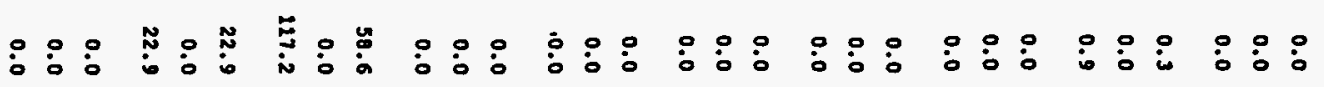




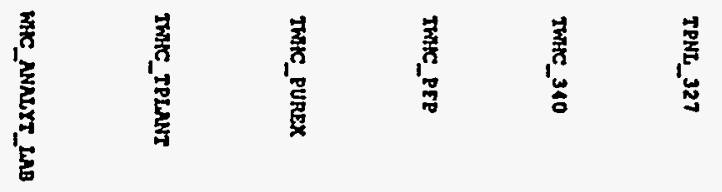

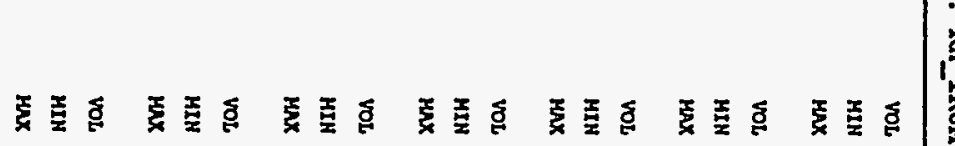

|

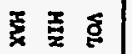

串路

是

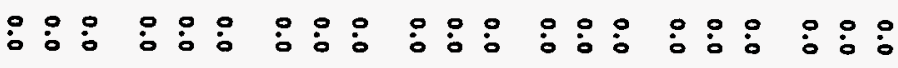

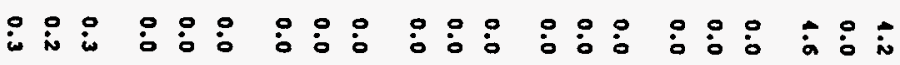

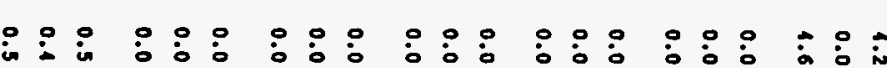

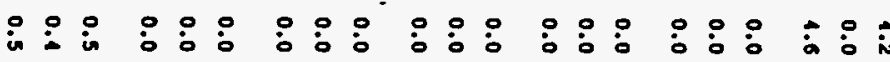

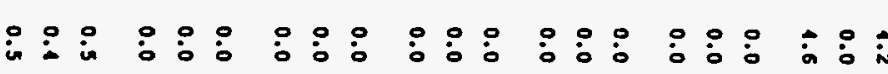

$\mid$

$\therefore: ㅇ: ㅇ$

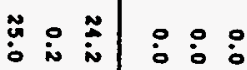

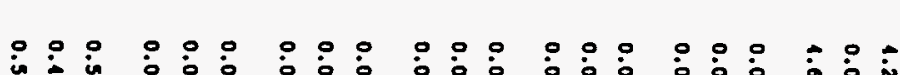

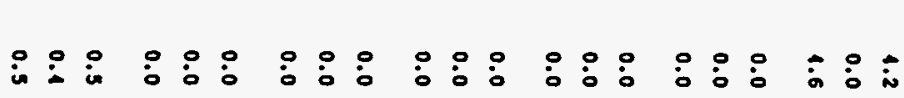

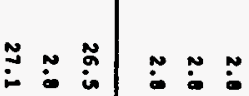

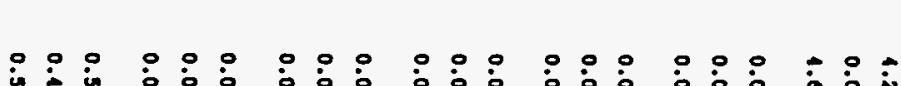

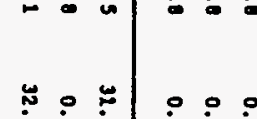

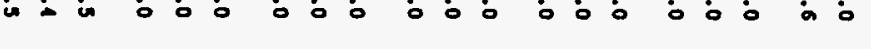

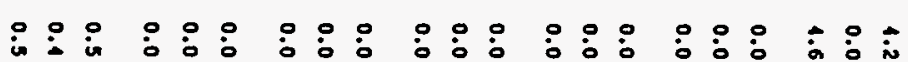

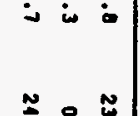

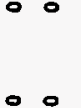

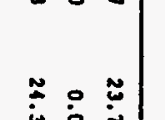

0.0

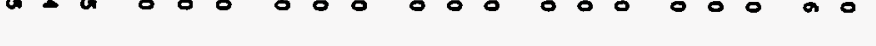

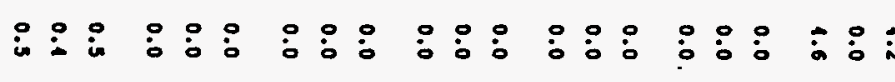

จั

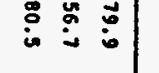

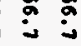

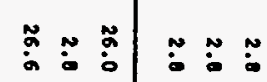

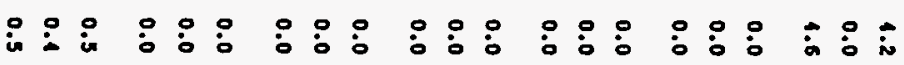

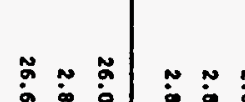

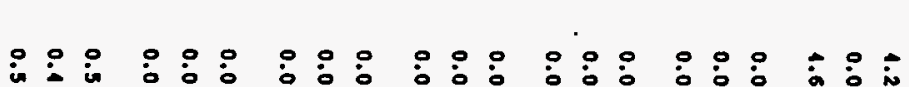

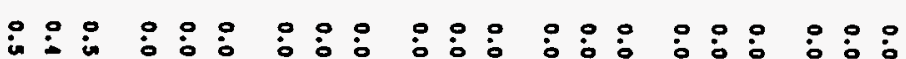

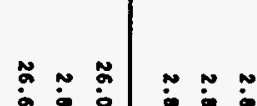

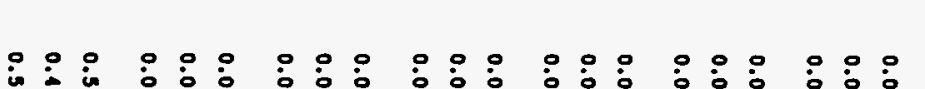

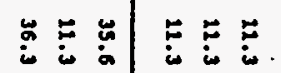

in $\dot{i} \dot{0} \dot{0} \dot{0} \dot{0} \dot{0} \dot{0} \dot{0} \dot{0} \dot{0} \dot{0} \dot{0} \dot{0} \dot{0} \dot{0}:$

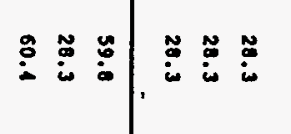

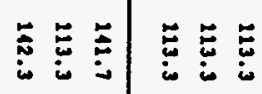

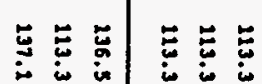

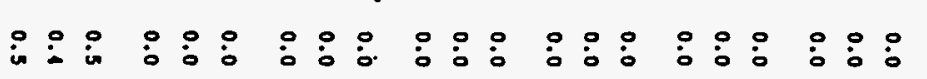

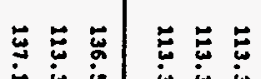

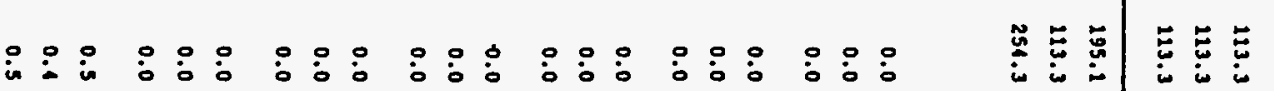




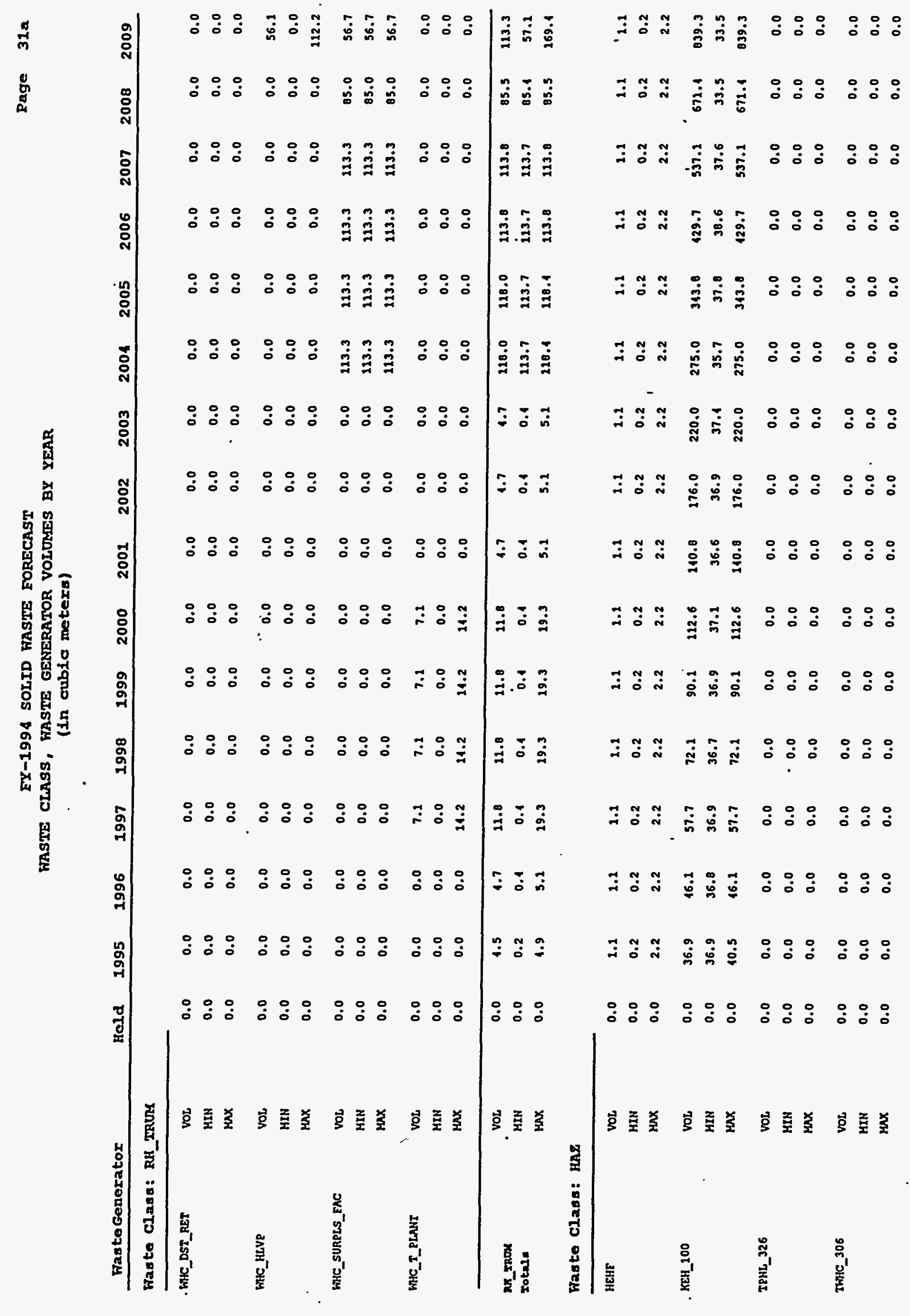




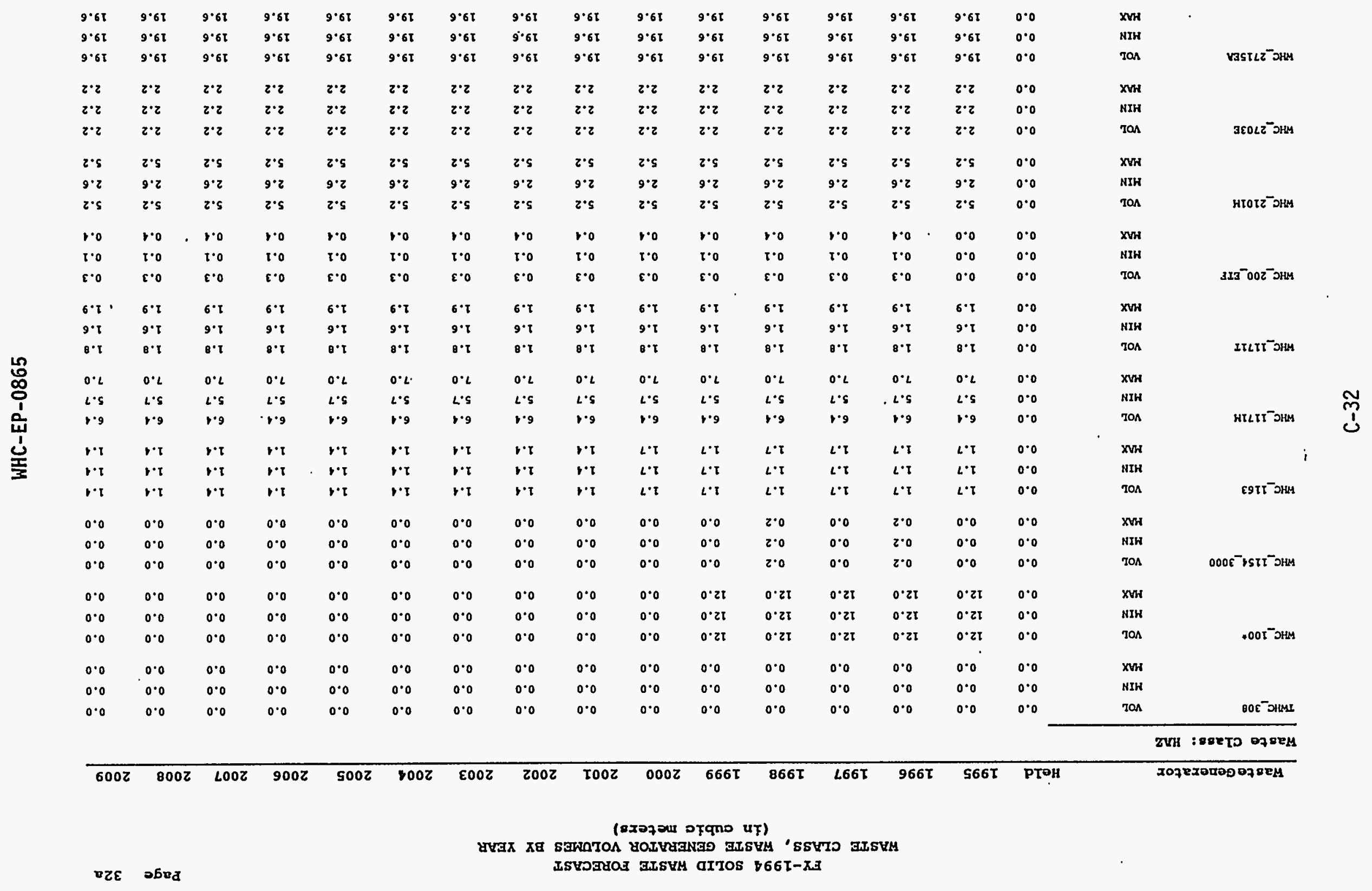


EY-1994 SOLID WASTE FORECAST

WASTE CLASS, WASTE GENERATOR VOLTMES BY YEAR

(in cubic metera)

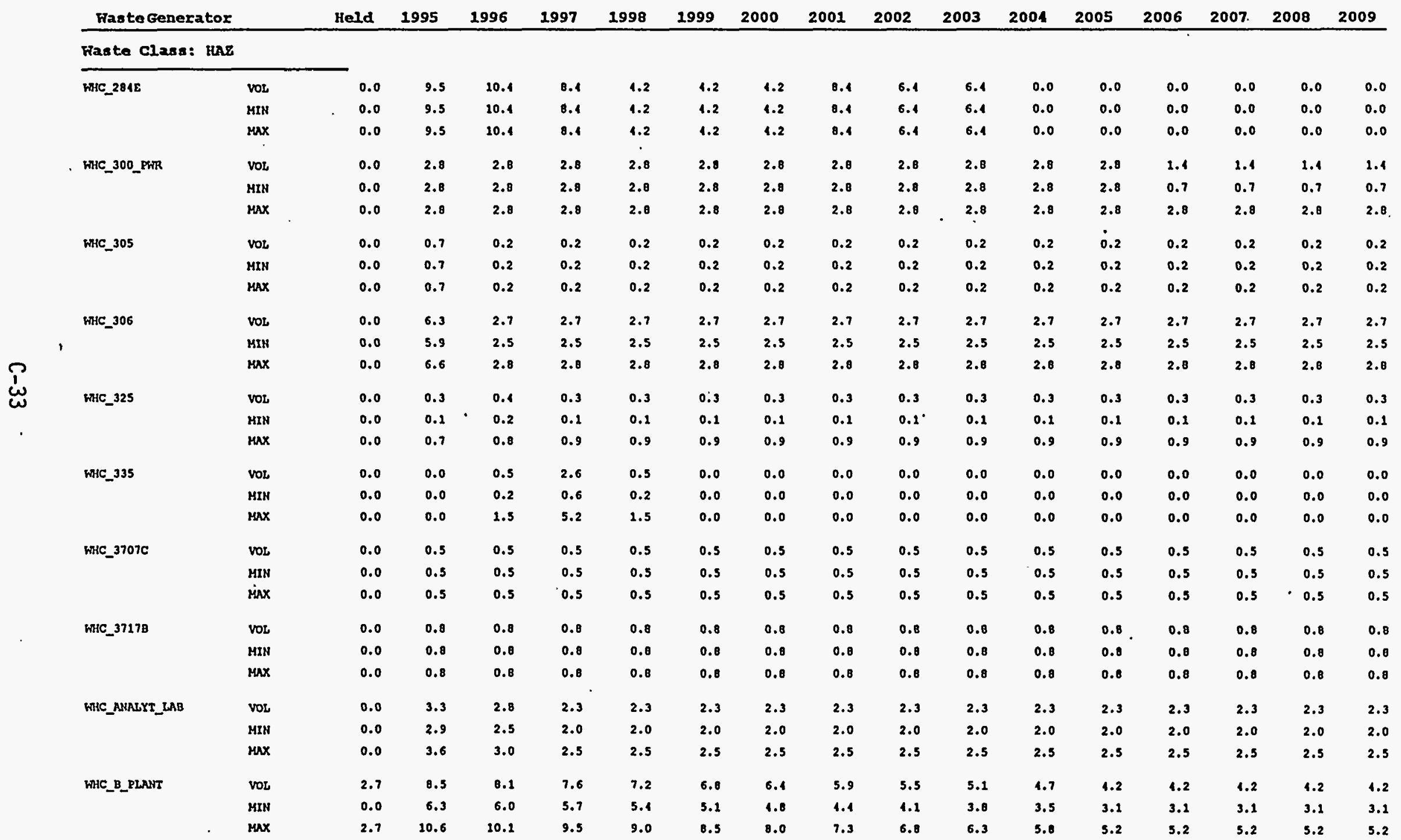


WHC-EP-0865

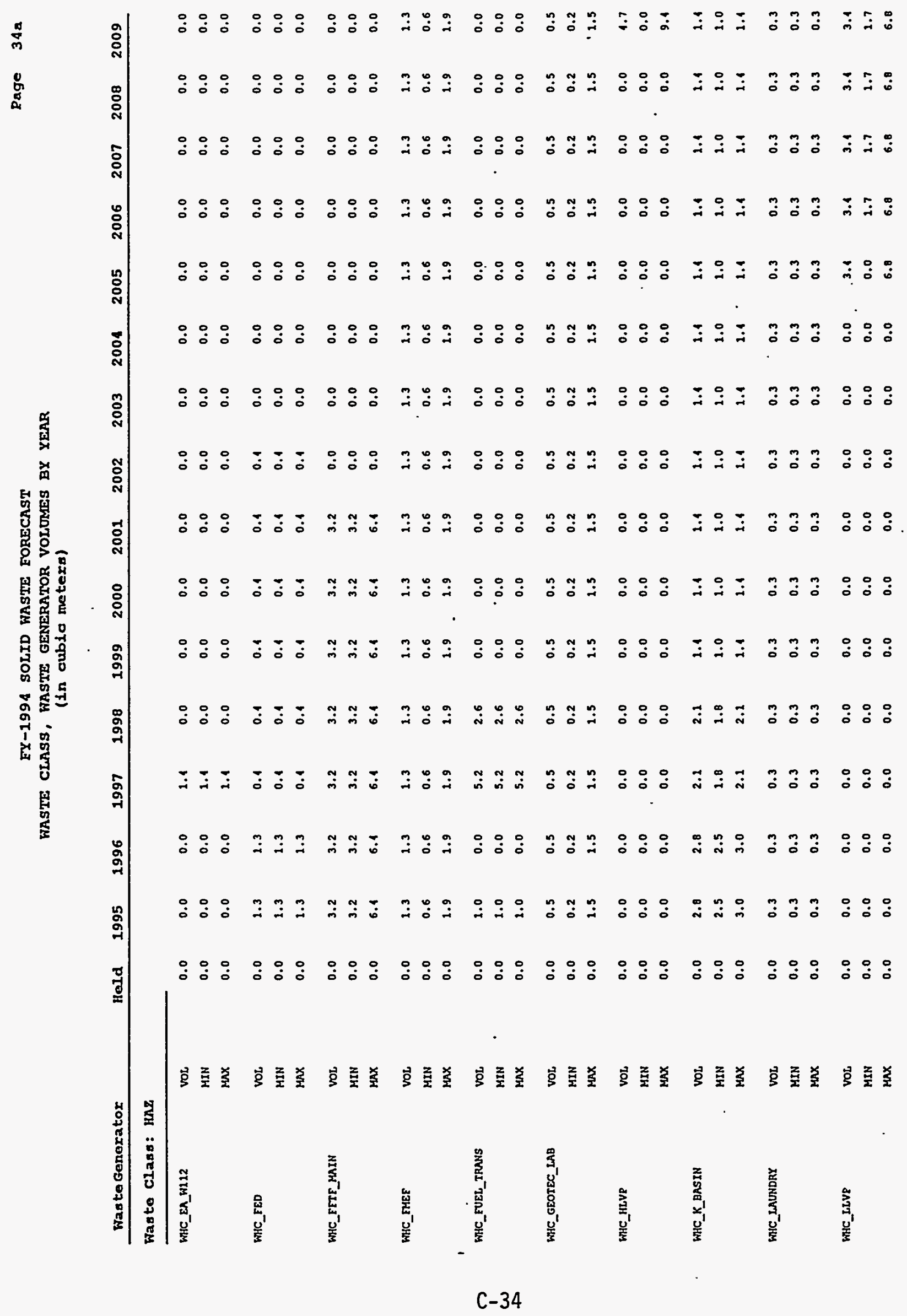


FY-1994 SOLID FASTE FORECAST

WASTE CLASS, FASTE GENERATOR VOLUMES BY YEAR

$$
\text { (in cubia metera) }
$$

\begin{tabular}{|c|c|c|c|c|c|c|c|c|c|c|c|c|c|c|c|c|c|}
\hline Faste Genes & ator & Held & 1995 & 1996 & 1997 & 2998 & 1999 & 2000 & 2001 & 2002 & 2003 & 2004 & 2005 & 2006 & 2007 & 2008 & 2009 \\
\hline Waste Class & HAZ & & & & & & & & & & & & & & & & \\
\hline WHC_PEP & vor & 0.0 & 14.9 & 14.9 & 41.9 & 11.9 & 11.9 & v1.9 & 14.9 & 14.9 & 14.9 & 14.9 & 14.9 & 14.9 & 14.9 & 11.9 & 11.9 \\
\hline$. \quad-5$ & HIN & 0.0 & 0.0 & 0.0 & 0.0 & 0.0 & 0.0 & 0.0 & 0.0 & 0.0 & 0.0 & 0.0 & 0.0 & 0.0 & 0.0 & 0.0 & 0.0 \\
\hline & $\max$ & 0.0 & 14.9 & 11.9 & 11.9 & 11.9 & 11.9 & 14.9 & 14.9 & 14.9 & 14.9 & 14.9 & 41.9 & 14.9 & 14.9 & 11.9 & 14.9 \\
\hline WHC_PRETRT_IH & vor & 0.0 & 0.0 & 0.0 & 0.0 & 0.0 & 0.0 & 0.0 & 0.0 & 0.0 & 0.0 & 1.6 & 1.6 & 1.6 & 1.6 & 1.6 & 1.6 \\
\hline & MrN & 0.0 & 0.0 & 0.0 & 0.0 & 0.0 & 0.0 & 0.0 & 0.0 & 0.0 & 0.0 & 0.0 & 0.8 & 0.8 & 0.8 & 0.8 & 0.8 \\
\hline & $\max$ & 0.0 & 0.0 & 0.0 & 0.0 & 0.0 & 0.0 & 0.0 & 0.0 & 0.0 & 0.0 & 3.2 & 3.2 & 3.2 & 3.2 & 3.2 & 3.2 \\
\hline WHC_PUREX & YoL & 0.0 & 1.2 & 4.8 & 4.2 & 1.2 & 0.0 & 0.0 & 0.0 & 0.0 & 0.0 & 0.0 & 0.0 & 0.0 & 0.0 & 0.0 & 0.0 \\
\hline & HIN & 0.0 & 2.1 & 2.1 & 2.1 & 2.1 & 0.0 & 0.0 & 0.0 & 0.0 & 0.0 & 0.0 & 0.0 & 0.0 & 0.0 & 0.0 & 0.0 \\
\hline & $\max$ & 0.0 & 6.3 & 7.2 & 6.3 & 8.4 & 0.0 & 0.0 & 0.0 & 0.0 & 0.0 & 0.0 & 0.0 & 0.0 & 0.0 & 0.0 & 0.0 \\
\hline WHE_PUREX_SM & vor & 0.0 & 0.0 & 0.0 & 0.0 & 0.0 & 0.8 & 0.8 & 0.8 & 0.8 & 0.8 & 0.8 & 0.8 & 0.8 & 0.8 & 0.8 & 0.8 \\
\hline & MIN & 0.0 & 0.0 & 0.0 & 0.0 & 0.0 & 0.1 & 0.1 & 0.4 & 0.4 & 0.4 & 0.1 & 0.4 & 0.4 & 0.1 & 0.4 & 0.4 \\
\hline , & $\operatorname{MAX}$ & 0.0 & 0.0 & 0.0 & 0.0 & 0.0 & 1.6 & 1.6 & 1.6 & 1.6 & 1.6 & 1.6 & 1.6 & 1.6 & 1.6 & 1.6 & 1.6 \\
\hline WHC_ 5300 & vor & 0.0 & 6.1 & 6.1 & 6.1 & 6.1 & 6.1 & 6.1 & 6.1 & 6.1 & 6.1 & 6.1 & 6.1 & 6.1 & 6.1 & 6.1 & 6.1 \\
\hline & MIN & 0.0 & 6.2 & 6.1 & 6.1 & 6.1 & 6.1 & 6.1 & 6.1 & 6.1 & 6.1 & 6.1 & 6.1 . & 6.2 & 6.1 & 6.1 & 6.1 \\
\hline & $\max$ & 0.0 & 6.1 & 6.1 & 6.1 & 6.1 & 6.1 & 6.1 & 6.1 & 6.1 & 6.1 & 6.1 & 6.1 & 6.1 & 6.1 & 6.1 & 6.1 \\
\hline WHC_SODIUH_FAC & vor & 0.0 & 0.0 & 2.0 & 0.5 & 0.0 & 0.0 & 0.0 & 0.0 & 0.0 & 0.0 & 0.0 & 0.0 & 0.0 & 0.0 & 0.0 & 0.0 \\
\hline & MrM & 0.0 & 0.0 & 0.3 & 0.5 & 0.0 & 0.0 & 0.0 & 0.0 & 0.0 & 0.0 & 0.0 & 0.0 & 0.0 & 0.0 & 0.0 & 0.0 \\
\hline & $\max$ & 0.0 & 0.0 & 2.0 & 1.0 & 0.0 & 0.0 & 0.0 & 0.0 & 0.0 & 0.0 & 0.0 & 0.0 & 0.0 & 0.0 & 0.0 & 0.0 \\
\hline WHC_SURPLS_FAC & vol & 0.0 & 0.5 & 11.3 & 11.3 & 11.3 & 14.2 & 36.8 & 150.1 & 198.3 & 321.6 & 566.6 & 566.6 & 566.6 & 124.9 & 203.3 & 141.6 \\
\hline & MIN & 0.0 & B.5 & 11.3 & 21.3 & 21.3 & 14.2 & 36.8 & 150.2 & 198.3 & 311.6 & 366.6 & 566.6 & 566.6 & 124.9 & 283.3 & 211.6 \\
\hline & $\operatorname{MAX}$ & 0.0 & 8.5 & 11.3 & 11.3 & 11.3 & 14.2 & 36.8 & 150.1 & 198.3 & 311.6 & 566.6 & 566.6 & 566.6 & 124.9 & 283.3 & 211.6 \\
\hline WHE_IF_OPER & vor & 0.0 & 31.3 & 37.8 & 37.8 & 37.8 & 37.0 & 37.8 & 37.8 & 37.0 & 37.8 & 37.8 & 37.8 & 37.0 & 37.0 & 37.8 & 37.8 \\
\hline & MIN & 0.0 & 27.4 & 30.2 & 30.2 & 30.2 & 30.2 & 30.2 & 30.2 & 30.2 & 30.2 & 30.2 & 30.2 & 30.2 & 30.2 & 30.2 & 30.2 \\
\hline & MAX & 0.0 & 34.3 & 37.8 & 37.8 & 37.0 & 37.0 & 37.8 & 37.8 & 37.0 & 37.8 & 37.8 & 37.8 & 37.8 & 37.8 & 37.8 & 37.0 \\
\hline WHC_T_PLANT & vor & 0.0 & 1.4 & 1.4 & 1.4 & 1.4 & 1.4 & 1.4 & 1.1 & 1.1 & 1.4 & 1.4 & 1.4 & 1.4 & 1.4 & 1.4 & 2.4 \\
\hline & MIN & 0.0 & 0.7 & 0.7 & 0.7 & 0.7 & 0.7 & 0.7 & 0.7 & 0.7 & 0.7 & 0.7 & 0.7 & 0.7 & 0.7 & 0.7 & 0.7 \\
\hline & $\max$ & 0.0 & 2.1 & 2.1 & 2.1 & 2.1 & 2.1 & 2.1 & 2.1 & 2.1 & 2.1 & 2.1 & 2.1 & 2.1 & 2.1 & 2.1 & 2.1 \\
\hline WHC_WELL_DRL, & vor & 0.0 & 6.5 & 6.5 & 6.5 & 6.5 & 6.5 & 6.5 & 6.3 & 6.5 & 6.5 & 6.5 & 6.5 & 6.5 & 6.5 & 6.5 & 6.5 \\
\hline & MIN & 0.0 & 0.0 & 0.0 & 0.0 & 0.0 & 0.0 & 0.0 & 0.0 & 0.0 & 0.0 & 0.0 & 0.0 & 0.0 & 0.0 & 0.0 & 0.0 \\
\hline & $\max$ & 0.0 & 13.0 & 13.0 & 13.0 & 13.0 & 13.0 & 13.0 & 13.0 & 13.0 & 13.0 & 13.0 & 13.0 & 13.0 & 13.0 & 13.0 & 13.0 \\
\hline
\end{tabular}


FY-1994 SOLID WASTE FORECAST

WASTE CLASS, FASTE GENERATOR VOLUMES BY YEAR

(In cubic meters)

\begin{tabular}{|c|c|c|c|c|c|c|c|c|c|c|c|c|c|c|c|c|c|}
\hline Fas te Ger & & Held & 1995 & 1996 & 1997 & 1998 & 1999 & 2000 & 2001 & 2002 & 2003 & 2004 & 2005 & 2006 & 2007 & 2008 & 2009 \\
\hline \multirow{3}{*}{$\begin{array}{l}\text { xus } \\
\text { Totar. }\end{array}$} & vor & 2.7 & 233.9 & 249.0 & 263.3 & 266.7 & 279.8 & 312.5 & 457.4 & 335.2 & 691.7 & 996.5 & 1068.2 & 2152.7 & 2128.4 & 2111.1 & 1112.0 \\
\hline & MIN & 0.0 & 166.5 & 169.6 & 170.9 & 161.5 & 158.7 & 169.2 & 285.5 & 328.6 & 441.6 & 680.2 & 690.7 & 691.2 & 510.1 & 102.8 & 261.1 \\
\hline & $\operatorname{mAX}$ & 2.7 & 259.1 & 270.6 & 285.1 & 288.7 & 297.1 & 330.0 & 174.8 & 549.3 & 705.7 & 1012.0 & 1087.0 & 1172.9 & 1138.6 & 1131.3 & 1166.9 \\
\hline \multirow{3}{*}{$\begin{array}{l}\text { Orand } \\
\text { Sotale }\end{array}$} & vol & 7207.0 & 14973.1 & 14309.3 & 18865.1 & 10778.9 & 12214.3 & 24850.8 & 33001.4 & 81111.8 & 103669.9 & 141175.2 & 185795.7 & 204112.5 & 227185.1 & 151056.6 & 79374.8 \\
\hline & MIN & 1961.2 & 11330.3 & 10766.5 & 25329.3 & 7200.3 & 8467.0 & 18376.7 & 29196.9 & 77751.5 & 100587.0 & 138182.9 & 182222.1 & 200696.9 & 223699.3 & 117252.8 & 73880.7 \\
\hline & $\max$ & 7207.0 & 19691.9 & 19471.3 & 23251.6 & 14804.1 & 16826.8 & 30618.3 & 37936.8 & 85722.5 & 108233.3 & 146133.8 & 189937.8 & 208602.0 & 231491.1 & 155161.7 & 85418.5 \\
\hline
\end{tabular}

w 
EX-1994 SOLID HASTE FORECAST

WASTE CLASS, FASTE GENERATOR VOLOMES BY YEAR

(in cuble meters)

Haste Generator

$\begin{array}{llllllllllllllll}2010 & 2011 & 2012 & 2013 & 2014 & 2015 & 2016 & 2017 & 2018 & 2019 & 2020 & 2021 & 2022 & 2023 & 2024 & \text { Total Pct }\end{array}$

Faste Class: CH_LWF_I

MES

NL_E

BAT_CLBS_LAB

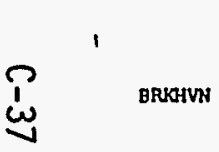

PROHWN

B_LEHR_DAV

EGเG

EEPMI

IVSRAP
20102011

$\begin{array}{cc}\text { voL } & 1.6 \\ \text { MIN } & 0.8 \\ \operatorname{Max} & 2.4\end{array}$

$\begin{array}{ll}1.6 & 2.6 \\ 0.8 & 0.8 \\ 2.4 & 2.4\end{array}$

$1.6 \quad 1.6$

$\begin{array}{ll}0.8 & 0.8 \\ 2.1 & 2.4\end{array}$

1.6
0.0

$1.6 \quad 1.6$

0.8

vor

$\begin{array}{lll}212.5 & 212.5 & 212.5\end{array}$

$212.5 \quad 212.5 \quad 212.5$

$212.5 \quad 212.5$

637.5637 .5

$637.5 \quad 637.5 \quad 637.5$

vol

MIN

HIN

$\max$

vor

MIk

$\max$

Vor

MIN

voL

MIN

$\max$

vor

MIN

yot

MOL

$\operatorname{MIN}$

vos

MIN

$\operatorname{san}$

$\begin{array}{rrrrrrrr}0.0 & 0.0 & 2.8 & 0.0 & 0.0 & 0.0 & 0.0 & 0.0 \\ 0.0 & 0.0 & 0.0 & 0.0 & 0.0 & 0.0 & 0.0 & 0.0 \\ 0.0 & 0.0 & 2.8 & 0.0 & 0.0 & 0.0 & 0.0 & 0.0 \\ 0.0 & 0.0 & 0.0 & 0.0 & 0.0 & 0.0 & 0.0 & 0.0 \\ 0.0 & 0.0 & 0.0 & 0.0 & 0.0 & 0.0 & 0.0 & 0.0 \\ 0.0 & 0.0 & 0.0 & 0.0 & 0.0 & 0.0 & 0.0 & 0.0 \\ 589.0 & 618.4 & 649.3 & 681.0 & 715.9 & 751.7 & 789.2 & 820.7 \\ 171.2 & 191.7 & 519.4 & 545.1 & 572.7 & 601.3 & 631.3 & 662.9 \\ 706.8 & 712.0 & 779.1 & 018.1 & 859.0 & 902.0 & 947.0 & 994.1\end{array}$

$$
0.0 \quad 0.0
$$

$0.0 \quad 0.0$$$
\begin{array}{ll}
0.0 & 0.0 \\
0.0 & 0.0
\end{array}
$$

$\begin{array}{lll}0.0 & 0.0 & 0.0 \\ 0.0 & 0.0 & 0.0\end{array}$

0.0
0.0
0.0

0.0

$$
0.0 \quad 0.0
$$

$0.0 \cdot 0.0$

$0.0 \quad 0.0$

$0.0 \quad 0.0$

0.0
0.0

0.0

0.0
0.0
0.0

41.3

11.3

$\begin{array}{lll}11.3 & 11.3 \quad 14.3\end{array}$

$\begin{array}{ll}.22 .1 & 22.1 \\ 88.6 & 88.6\end{array}$

$0.0 \quad 0.0$

$\begin{array}{llll}22.1 & 22.1 & 22.1 & 22.1\end{array}$

0.0

$$
0.0
$$

$0.0 \quad 0.0$

$\begin{array}{lll}0.0 & 0.0 & 0.0 \\ 0.0 & 0.0 & 0.0\end{array}$

$\begin{array}{lll}0.0 & 0.0 & 0.0\end{array}$

$0.0 \quad 0.0$

$\begin{array}{lll}0.0 & 0.0 & 0.0 \\ 0.0 & 0.0 & 0.0\end{array}$ 


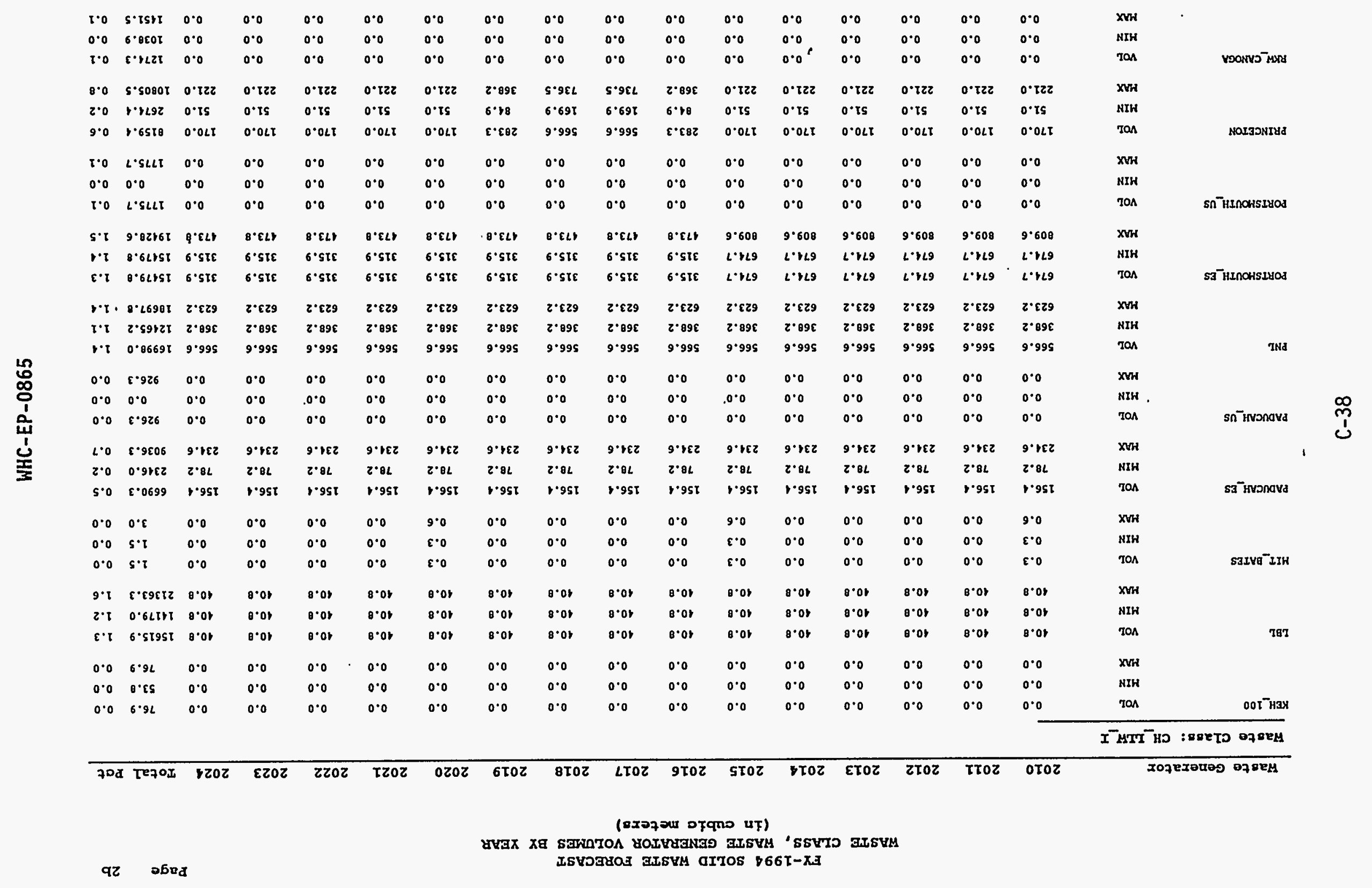


FY-1994 SOLID HASTE FORECAST

WASTE CLASS, HASTE GENERATOR VOLUMES BY YEAR

(in cubic metera)

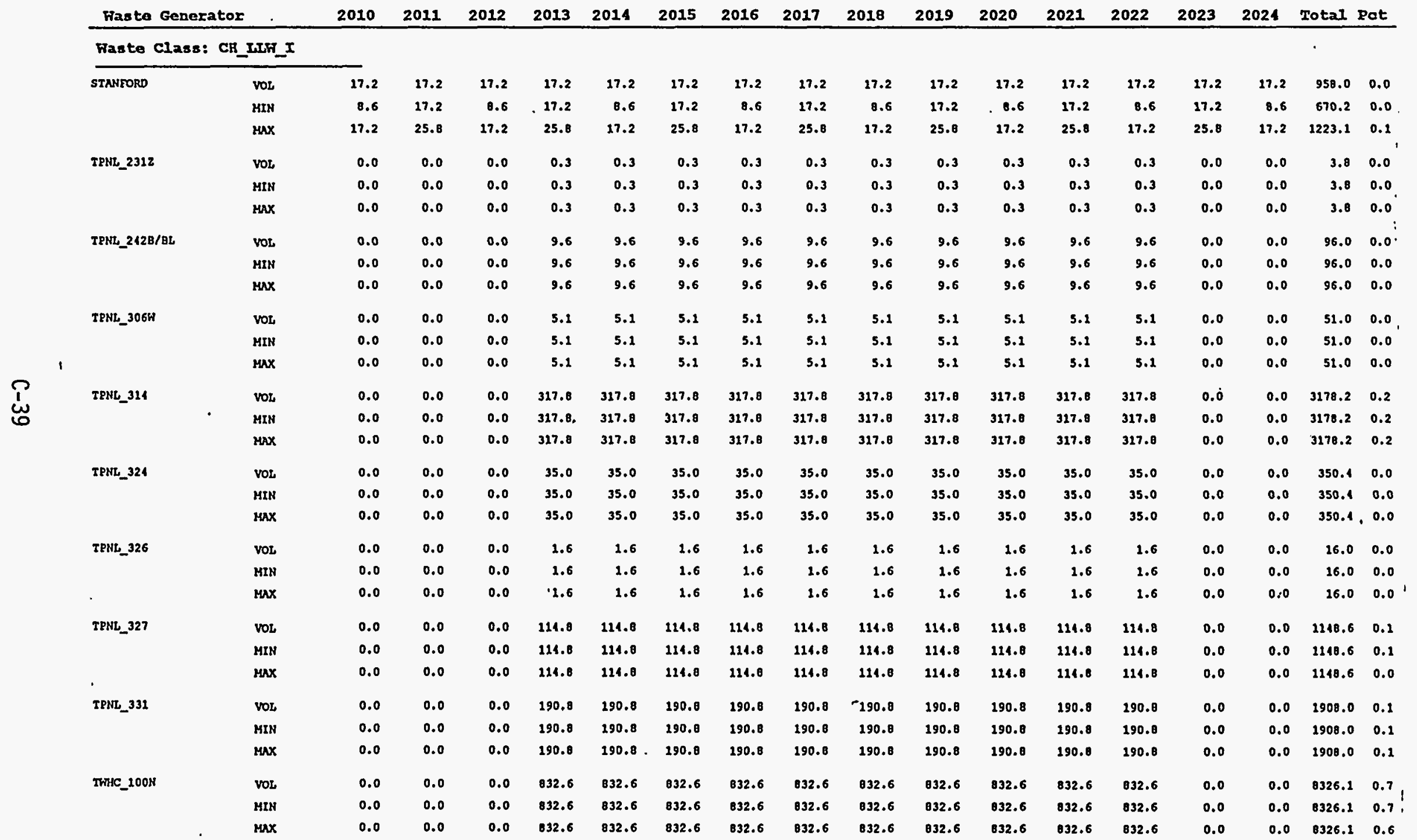


Waste Generator

\begin{tabular}{llllllllllllllll}
2010 & 2011 & 2012 & 2013 & 2014 & 2015 & 2016 & 2017 & 2018 & 2019 & 2020 & 2021 & 2022 & 2023 & 2024 & Total Pot \\
\hline
\end{tabular}

\begin{tabular}{|c|c|c|c|c|c|c|c|c|c|c|c|c|c|c|c|c|c|c|}
\hline \multirow[t]{3}{*}{ TWHC_222S } & vol & $0.0^{\circ}$ & 0.0 & 0.0 & 1280.2 & 1280.2 & 1280.2 & 1280.2 & 1280.2 & 1280.2 & 1280.2 & 1280.2 & 1280.2 & 1280.2 & 0.0 & 0.0 & 12802.7 & 1.0 \\
\hline & MIN & 0.0 & 0.0 & 0.0 & 1200.2 & 1280.2 & 1200.2 & 1200.2 & 1200.2 & 1280.2 & 1280.2 & 1280.2 & 1280.2 & 1280.2 & 0.0 & 0.0 & 12802.7 & 1.1 \\
\hline & $\operatorname{MAX}$ & 0.0 & 0.0 & 0.0 & 1280.2 & 1280.2 & 1280.2 & 1280.2 & 1280.2 & $12 \theta 0.2$ & 1280.2 & 1280.2 & 1280.2 & 1280.2 & 0.0 & 0.0 & 12802.7 & 1.0 \\
\hline \multirow[t]{3}{*}{ TWHC_306 } & you & 0.0 & 0.0 & 0.0 & 0.5 & 0.5 & 0.5 & 0.5 & 0.5 & 0.5 & 0.5 & 0.5 & 0.5 & 0.5 & 0.0 & 0.0 & 5.4 & 0.0 \\
\hline & MIN & 0.0 & 0.0 & 0.0 & 0.5 & 0.5 & 0.5 & 0.5 & 0.5 & 0.5 & 0.5 & 0.5 & 0.5 & 0.5 & 0.0 & 0.0 & 5.4 & 0.0 \\
\hline & $\operatorname{MAX}$ & 0.0 & 0.0 & 0.0 & 0.5 & 0.5 & 0.5 & 0.5 & 0.5 & 0.5 & 0.5 & 0.5 & 0.5 & 0.5 & 0.0 & 0.0 & 5.4 & 0.0 \\
\hline \multirow[t]{3}{*}{ TWHC_30B } & VOL & 0.0 & 0.0 & 0.0 & 12.8 & 12.8 & 12.8 & 12.8 & 12.8 & 12.8 & 12.8 & 12.8 & 12.8 & 12.8 & 0.0 & 0.0 & $12 \theta .7$ & 0.0 \\
\hline & MIN & 0.0 & 0.0 & 0.0 & 12.8 & 12.8 & 12.8 & 12.8 & 12.8 & 12.8 & 12.8 & 12.0 & 12.8 & 12.8 & 0.0 & 0.0 & $12 \theta .7$ & 0.0 \\
\hline & $\operatorname{MAX}$ & 0.0 & 0.0 & 0.0 & 12.8 & 12.8 & 12.8 & 12.8 & 12.8 & 12.8 & 12.8 & 22.0 & 12.8 & 22.8 & 0.0 & 0.0 & 128.7 & 0.0 \\
\hline \multirow[t]{3}{*}{ TWHC_340 } & vor & 0.0 & 0.0 & 0.0 & 19.8 & 19.8 & 19.8 & 19.8 & 29.8 & 19.8 & 19.8 & 29.8 & 19.8 & 19.8 & 0.0 & 0.0 & 198.1 & 0.0 \\
\hline & MIN & 0.0 & 0.0 & 0.0 & 29.0 & 19.8 & 19.8 & 19.8 & 29.8 & 19.8 & 19.8 & 19.8 & 19.8 & 19.8 & 0.0 & 0.0 & 199.1 & 0.0 \\
\hline & $\max$ & 0.0 & 0.0 & 0.0 & 19.8 & 19.8 & 19.8 & 19.8 & 29.8 & 19.8 & 19.8 & 19.8 & 19.8 & 19.8 & 0.0 & 0.0 & 198.1 & 0.0 \\
\hline \multirow[t]{3}{*}{ THHC_BPLANT } & vol & 0.0 & 0.0 & 0.0 & 688.0 & 688.0 & 608.0 & 688.0 & 688.0 & 688.0 & 688.0 & 688.0 & 688.0 & 688.0 & 0.0 & 0.0 & 6880.0 & 0.5 \\
\hline & MIN & 0.0 & 0.0 & 0.0 & 689.0 & 688.0 & 680.0 & 680.0 & 688.0 & 680:0 & 688.0 & 688.0 & 688.0 & 688.0 & 0.0 & 0.0 & $6880: 0$ & 0.6 \\
\hline & $\operatorname{MAX}$ & 0.0 & 0.0 & 0.0 & 688.0 & 688.0 & 688.0 & 688.0 & 688.0 & 688.0 & 688.0 & 688.0 & 680.0 & 688.0 & 0.0 & 0.0 & 6980.0 & 0.5 \\
\hline \multirow[t]{3}{*}{ TWHC_TPLANT } & vor & 0.0 & 0.0 & 0.0 & 724.1 & 714.4 & 714.4 & 714.4 & 724.1 & 714.1 & 714.1 & 714.1 & 714.4 & 714.4 & 0.0 & 0.0 & 7144.2 & 0.6 \\
\hline & MIN & 0.0 & 0.0 & 0.0 & 724.1 & 714.4 & 714.1 & 714.4 & 714.1 & 714.1 & 714.4 & 714.4 & 714.4 & 711.4 & 0.0 & 0.0 & 7144.1 & 0.6 \\
\hline & $\operatorname{MAX}$ & 0.0 & 0.0 & 0.0 & 714.1 & 714.4 & 714.4 & 714.4 & 714.1 & 714.1 & 714.4 & 714.1 & 714.4 & 711.4 & 0.0 & 0.0 & $7141.1^{\prime}$ & 0.5 \\
\hline \multirow[t]{3}{*}{ U_u } & Vol & 0.3 & 0.3 & 0.3 & 0.3 & 0.3 & 0.3 & 0.3 & 0.3 & 0.3 & 0.3 & 0.3 & 0.3 & 0.3 & 0.3 & 0.3 & 9.0 & 0.0 \\
\hline & MIN & 0.0 & 0.0 & 0.0 & 0.0 & 0.0 & 0.0 & 0.0 & 0.0 & 0.0 & 0.0 & 0.0 & 0.0 & 0.0 & 0.0 & 0.0 & 0.0 & 0.0 \\
\hline & $\operatorname{MAX}$ & 0.9 & 0.9 & 0.9 & 0.9 & 0.9 & 0.9 & 0.9 & 0.9 & 0.9 & 0.9 & 0.9 & 0.9 & 0.9 & 0.9 & 0.9 & 27.0 & 0.0 \\
\hline \multirow[t]{3}{*}{ WHC_100" } & VoL & 0.0 & 0.0 & 0.0 & 0.0 & 0.0 & 0.0 & 0.0 & 0.0 & 0.0 & 0.0 & 0.0 & 0.0 & 0.0 & 0.0 & 0.0 & 1379.5 & 0.1 \\
\hline & MIN & 0.0 & 0.0 & 0.0 & 0.0 & 0.0 & 0.0 & 0.0 & 0.0 & 0.0 & 0.0 & 0.0 & 0.0 & 0.0 & 0.0 & 0.0 & 1379.5 & 0.1 \\
\hline & $\max$ & 0.0 & 0.0 & 0.0 & 0.0 & 0.0 & 0.0 & 0.0 & 0.0 & 0.0 & 0.0 & 0.0 & 0.0 & 0.0 & 0.0 & 0.0 & 1379.5 & 0.1 \\
\hline \multirow[t]{3}{*}{ WHC_300_PWR } & vot & 0.0 & 0.0 & 0.0 & 0.0 & 0.0 & 0.0 & 0.0 & 0.0 & 0.0 & 0.0 & 0.0 & 0.0 & 0.0 & 0.0 & 0.0 & 2.8 & 0.0 \\
\hline & MIN & 0.0 & 0.0 & 0.0 & 0.0 & 0.0 & 0.0 & 0.0 & 0.0 & 0.0 & 0.0 & 0.0 & 0.0 & 0.0 & 0.0 & 0.0 & 0.0 & 0.0 \\
\hline & $\operatorname{MAX}$ & 0.0 & 0.0 & 0.0 & 0.0 & 0.0 & 0.0 & 0.0 & 0.0 & 0.0 & 0.0 & 0.0 & 0.0 & 0.0 & 0.0 & 0.0 & 2.8 & 0.0 \\
\hline \multirow[t]{3}{*}{ WHC_300_TEDF } & vor & 368.3 & 368.3 & 368.3 & 368.3 & 368.3 & 360.3 & 368.3 & 368.3 & 368.3 & 368.3 & 368.3 & 368.3 & 368.3 & 368.3 & 368.3 & 11049.0 & 0.9 \\
\hline & MIN & 184.1 & 184.1 & 184.1 & 284.1 . & 184.1 & 184.1 & 284.1 & 284.1 & 184.1 & 184.1 & 104.1 & 104.1 & 186.1 & 184.1 & 184.2 & 5321.5 & 0.5 \\
\hline & $\operatorname{Mx}$ & 552.1 & 552.4 & 552.4 & 552.4 & 552.4 & 552.4 & 552.4 & 552.1 & 552.4 & 352.4 & 552.4 & 552.4 & 552.1 & 552.4 & 352.4 & 16573.5 & 1.2 \\
\hline
\end{tabular}




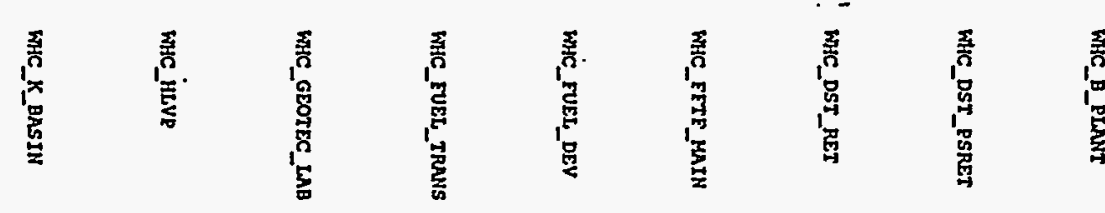

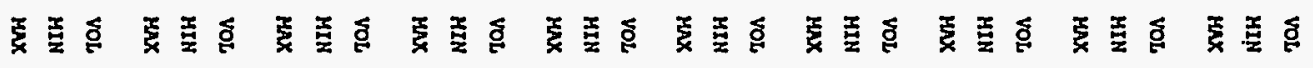

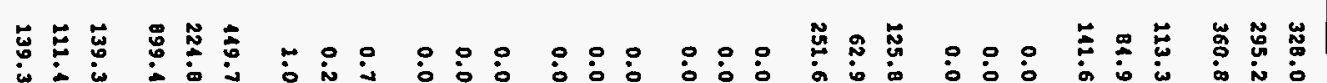

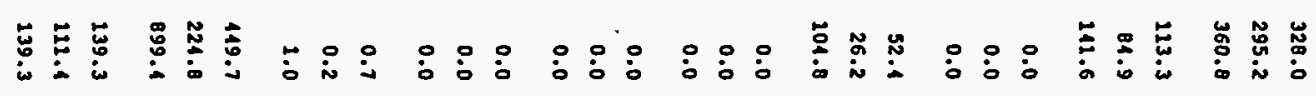

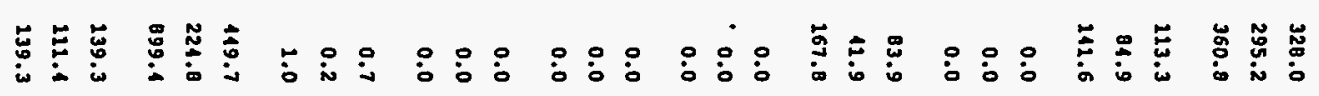

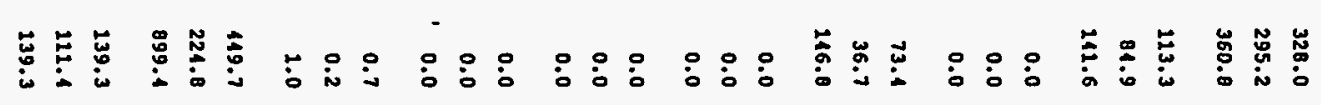

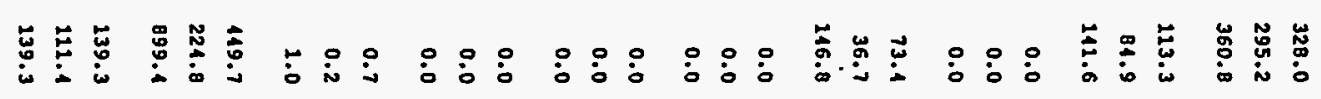

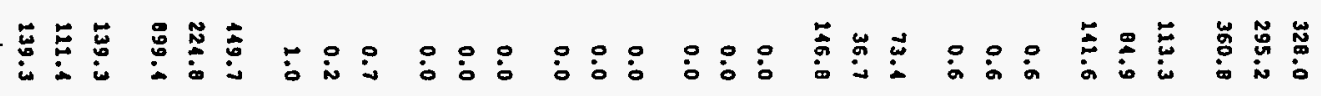

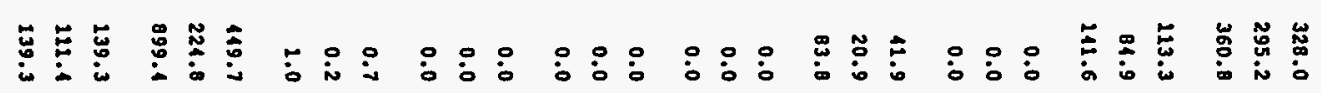

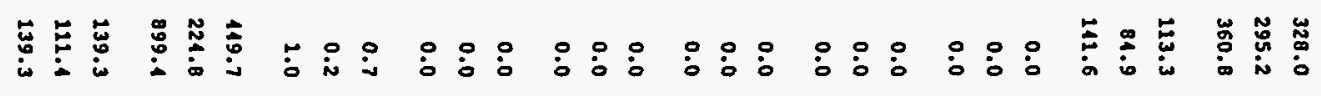

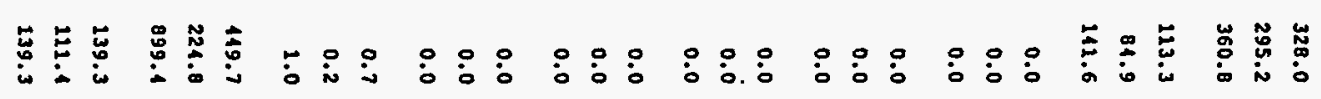

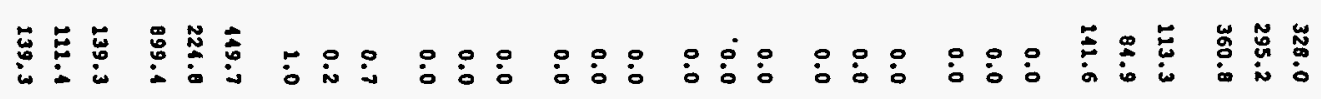

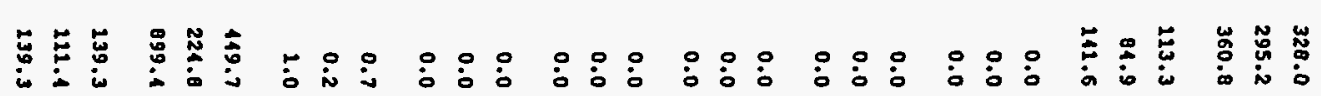

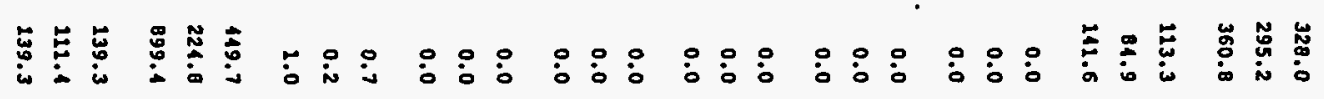

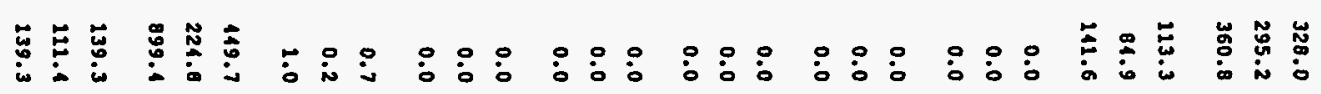

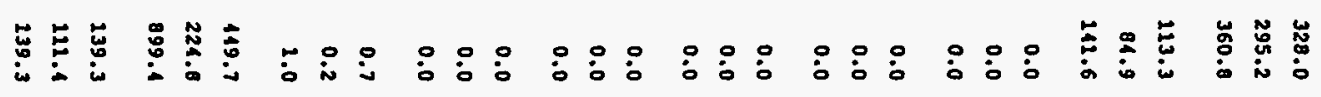

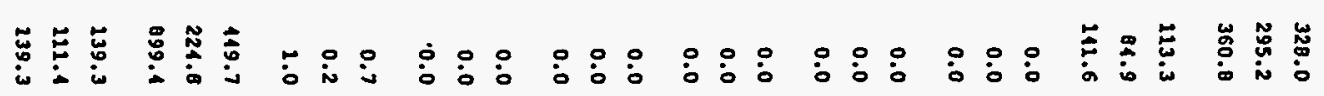

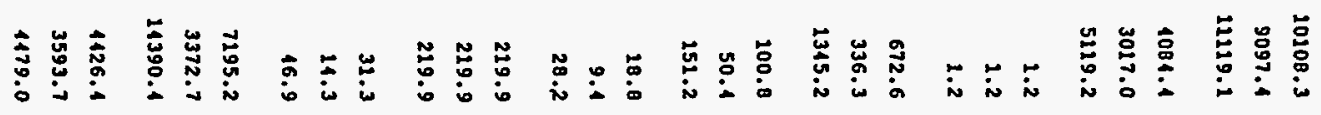

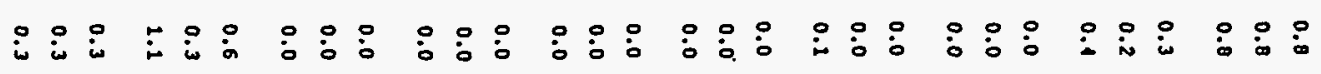




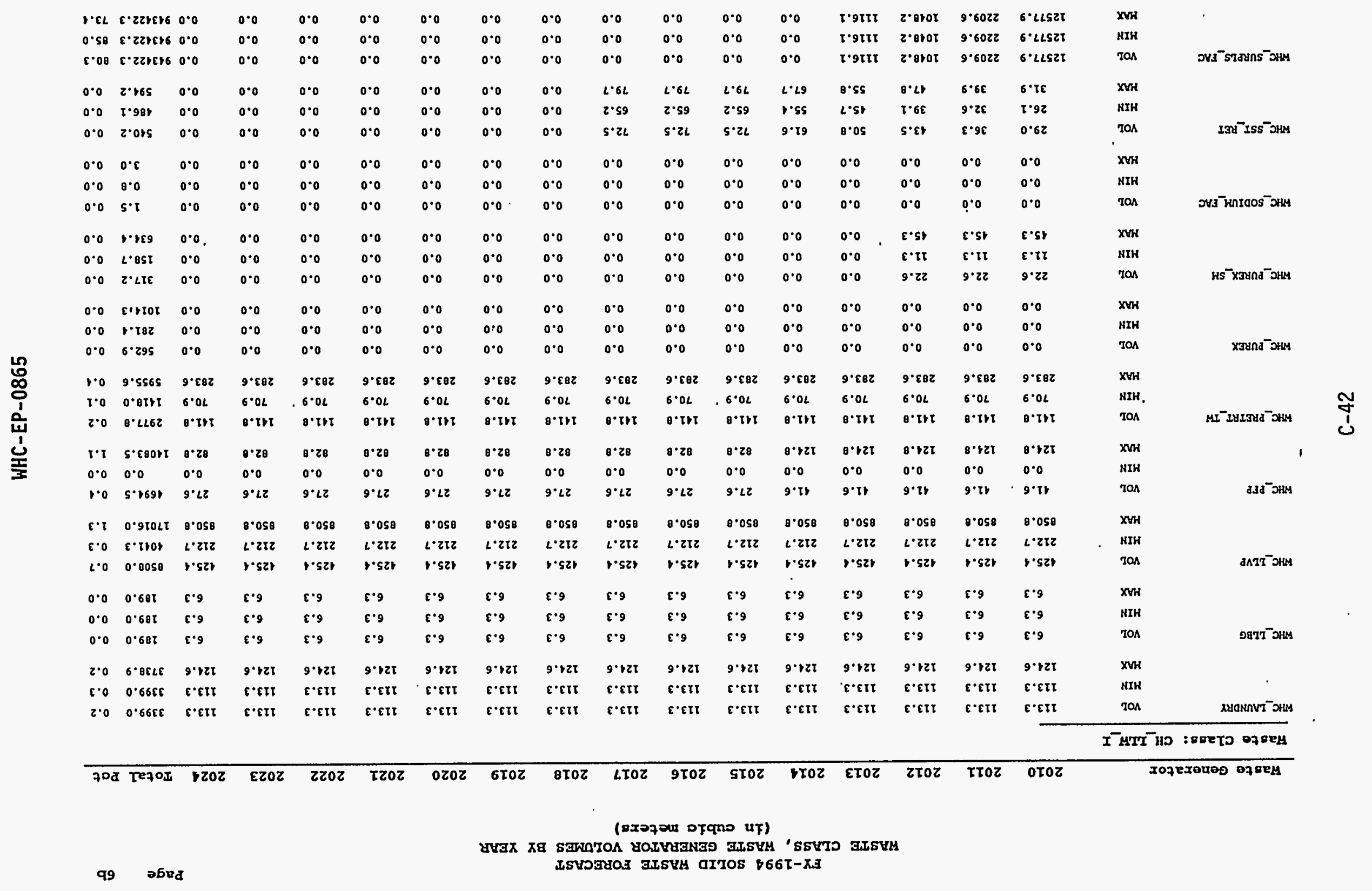




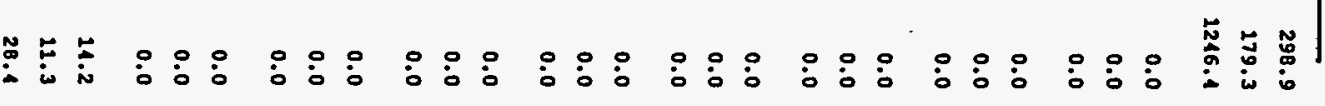

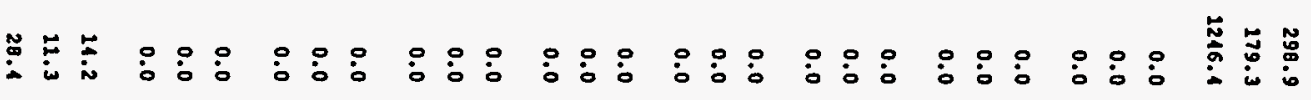

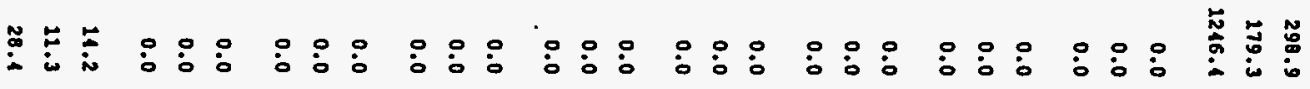

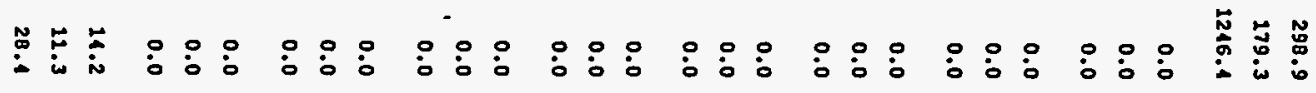

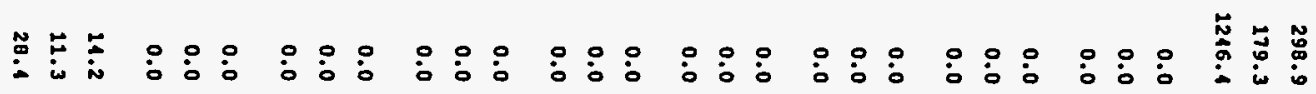

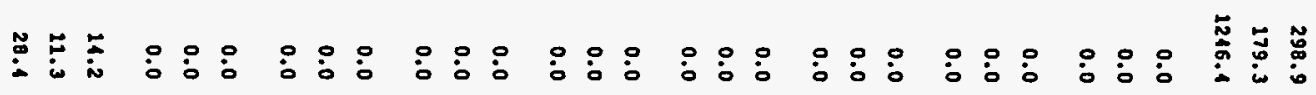

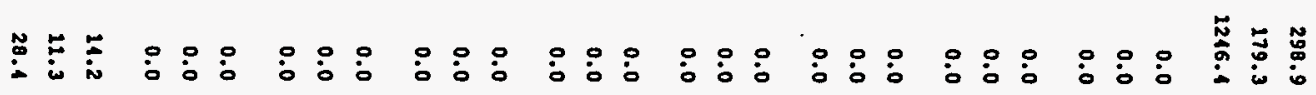

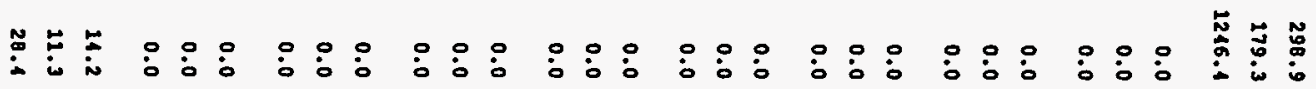

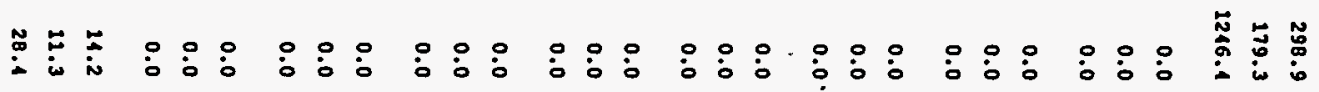

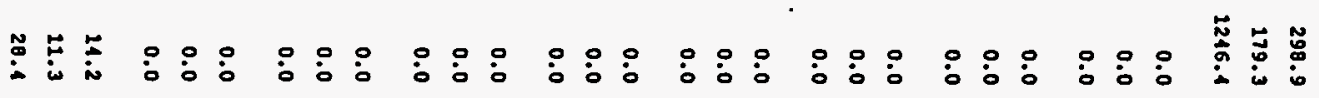

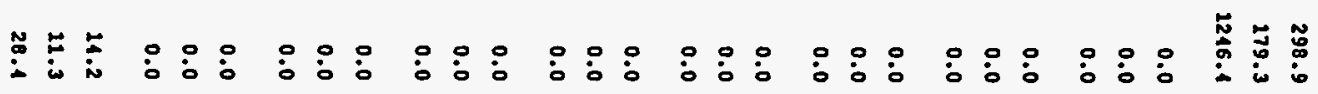

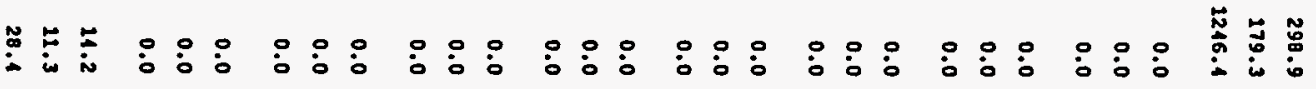
氙芒莣

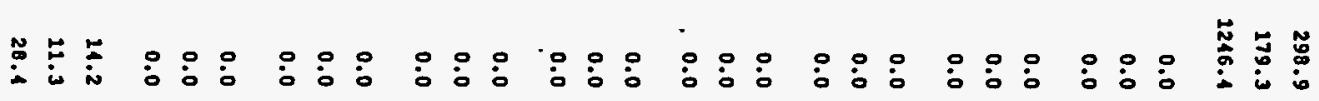

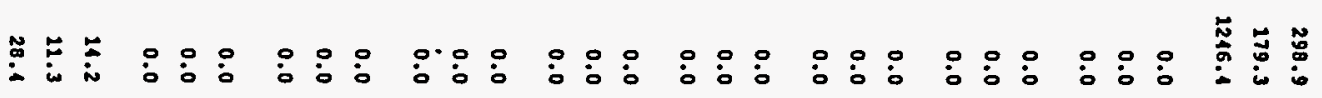

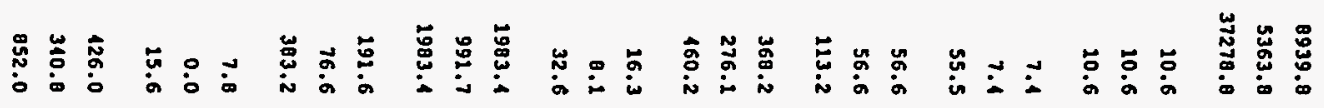

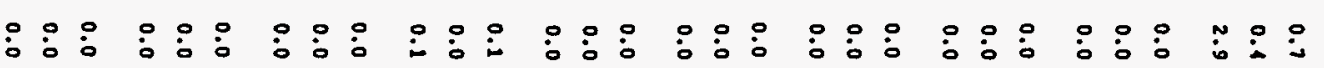


FY-1994 SOLID WASTE EORECAST

WASTE CLASS, WASTE GENERATOR VOLUMES BY YEAR

(in cubic meters)

Waste Generator

$\begin{array}{llllllllllllllll}2010 & 2011 & 2012 & 2013 & 2014 & 2015 & 2016 & 2017 & 2018 & 2019 & 2020 & 2021 & 2022 & 2023 & 2024 & \text { Total pct }\end{array}$ Waste Clasa: CH_LrW_I

\begin{tabular}{|c|c|c|c|c|c|c|c|c|c|c|c|c|c|c|c|c|c|c|}
\hline \multirow[t]{3}{*}{ WHF_WASTE_NEUT } & VoL & 19.7 & 19.7 & 19.7 & 19.7 & 19.7 & 19.7 & 19.7 & 19.7 & 19.7 & 19.7 & 29.7 & 19.7 & 19.7 & 19,7 & 19.7 & 604.5 & 0.0 \\
\hline & MIN & 9.8 & 9.8 & 9.8 & 9.8 & 9.8 & 9.8 & 9.8 & 9.8 & 9.8 & 9.8 & 9.8 & 9.8 & 9.8 & 9.8 & 9.8 & 295.3 & 0.0 \\
\hline & $\max$ & 39.4 & 39.4 & 39.4 & 39.4 & 39.4 & 39.4 & 39.1 & 39.4 & 39.1 & 39.1 & 39.4 & 39.1 & 39.4 & 39.4 & 39.4 & 1195.3 & 0.0 \\
\hline \multirow[t]{3}{*}{ WITC_HELL_DRL } & vol & 1.3 & 1.3 & 2.3 & 2.3 & 1.3 & 1.3 & 1.3 & 1.3 & 1.3 & 1.3 & 1.3 & 1.3 & 1.3 & 1.3 & 1.3 & 39.0 & 0.0 \\
\hline & MIN & 0.0 & 0.0 & 0.0 & 0.0 & 0.0 & 0.0 & 0.0 & 0.0 & 0.0 & 0.0 & 0.0 & 0.0 & 0.0 & 0.0 & 0.0 & 0.0 & 0.0 \\
\hline & $\max$. & 3.9 & 3.9 & 3.9 & 3.9 & 3.9 & 3.9 & 3.9 & 3.9 & 3.9 & 3.9 & 3.9 & 3.9 & 3.9 & 3.9 & 3.9 & 117.0 & 0.0 \\
\hline \multirow{3}{*}{$\begin{array}{l}\text { CR_2LR_I } \\
\text { Totas. }\end{array}$} & vot, & 27690.8 & 1205.5 & 6196.5 & 10492.0 & 9420.8 & 9454.1 & 9214.0 & 9494.9 & 9463.0 & 9223.3 & 9155.9 & 9203.6 & 9254.0 & 5003.2 & $5130^{\circ}$ & 23. & 100.0 \\
\hline & MIN & 15898.8 & 5532.2 & $\$ 109.2$ & 8725.3 & 7637.6 & 7685,6 & 7365.5 & 7469.8 & 7428.6 & 7387.1 & 7381.3 & 7128.0 & 7459.7 & 3287.0 & 3322.81 & .08838 .6 & 100.0 \\
\hline & $\operatorname{MAX}$ & 21092.8 & 20629.1 & 9569.9 & 13848.0 & 12776.1 & 12798.8 & 12582.5 & 12923.0 & 12883.1 & 12575.8 & 12475.2 & 12540.0 & 12592.7 & O411.1 & 8499.11 & 83722.7 & 100.0 \\
\hline
\end{tabular}

Waste Class: CH_IIF_III

\begin{tabular}{|c|c|c|c|c|c|c|c|c|c|c|c|c|c|c|c|c|c|c|}
\hline \multirow[t]{3}{*}{ BAPL } & vor & 0.0 & 0.0 & 0.0 & 0.0 & 0.0 & 0.0 & 0.0 & 0.0 & 0.0 & 0.0 & 0.0 & 0.0 & 0.0 & 0.0 & 0.0 & 153.4 & 0.0 \\
\hline & MIN & 0.0 & 0.0 & 0.0 & 0.0 & 0.0 & 0.0 & 0.0 & 0.0 & 0.0 & 0.0 & 0.0 & 0.0 & 0.0 & 0.0 & 0.0 & 39.8 & 0.0 \\
\hline & $\max$ & 0.0 & .0 .0 & 0.0 & 0.0 & 0.0 & 0.0 & 0.0 & 0.0 & .0 .0 & 0.0 & 0.0 & 0.0 & 0.0 & 0.0 & 0.0 & 153.4 & 0.0 \\
\hline \multirow[t]{3}{*}{ FUSRAP } & vor & 0.0 & 3162.7 & 3162.7 & 0.0 & 13767.7 & 13767.7 & 0.0 & 0.0 & 0.0 & 0.0 & 0.0 & 0.0 & 0.0 & 0.0 & 0.0 & 11722.0 & 23.9 \\
\hline & MIN & 0.0 & 3162.7 & 3162.7 & 0.0 & 13767.7 & 13767.7 & 0.0 & 0.0 & 0.0 & 0.0 & 0.0 & 0.0 & 0.0 & 0.0 & 0.0 & $\$ 1722.0$ & 25.6 \\
\hline & $\max$ & 0.0 & 3162.7 & 3162.7 & 0.0 & 13767.7 & 13767.7 & 0.0 & 0.0 & 0.0 & 0.0 & 0.0 & 0.0 & 0.0 & 0.0 & 0.0 & $\$ 1722.0$ & 21.6 \\
\hline \multirow[t]{3}{*}{ KAPL_SHIPYDS } & vol & 0.0 & 0.0 & 0.0 & 0.0 & 0.0 & 0.0 & 0.0 & 0.0 & 0.0 & 0.0 & 0.0 & 0.0 & 0.0 & 0.0 & 0.0 & 297.0 . & 0.1 \\
\hline & MIN & 0.0 & 0.0 & 0.0 & 0.0 & 0.0 & 0.0 & 0.0 & 0.0 & 0.0 & 0.0 & 0.0 & 0.0 & 0.0 & 0.0 & 0.0 & 0.0 & 0.0 \\
\hline & $\operatorname{MAX}$ & 0.0 & 0.0 & 0.0 & 0.0 & 0.0 & 0.0 & 0.0 & 0.0 & 0.0 & 0.0 & 0.0 & 0.0 & 0.0 & 0.0 & 0.0 & 297.0 & 0.1 \\
\hline \multirow[t]{3}{*}{ LBL } & vor & 1.5 & 4.5 & 1.5 & 4.5 & 4.5 & 4.5 & 4.5 & 4.5 & 4.5 & 1.5 & 4.5 & 1.5 & 4.5 & 4.5 & 1:5 & 160.5 & 0.0 \\
\hline & MIN & 1.5 & 4.5 & 4.5 & 4.5 & 1.5 & 1.5 & 4.5 & 1.5 & 4.5 & 4.5 & 4.5 & 4.5 & 1.5 & 1.5 & 4.5 & 160.5 & 0.0 \\
\hline & $\max$ & 1.5 & 4.5 & 4.5 & 4.5 & 1.5 & 4.5 & 1.5 & 1.5 & 1.5 & 1.5 & 4.5 & 4.5 & 1.5 & 1.5 & 1.5 & 160.5 & 0.0 \\
\hline \multirow[t]{3}{*}{ PNL } & vor & 12.5 & 12.5 & 12.5 & 42.5 & 42.5 & 12.5 & 42.5 & 12.5 & 12.5 & 42.5 & 12.5 & 42.5 & 12.5 & 12.5 & 12.5 & 1346.0 & 0.7 \\
\hline & MIN & 31.8 & 31.8 & 31.8 & 31.0 & 31.8 & 31.8 & 31.8 & 31.8 & 31.8 & 31.8 & 31.8 & 31.8 & 31.8 & 31.8 & 31.8 & 1045.0 & 0.6 \\
\hline & $\max$ & 16.7 & 16.7 & 16.7 & 46.7 & 46.7 & 16.7 & 16.7 & 16.7 & 16.7 & 16.7 & 16.7 & 46.7 & 16.7 & 16.7 & 46.7 & 1452.2 & 0.7 \\
\hline \multirow[t]{3}{*}{ TPNL_324 } & vor & 0.0 & 0.0 & 0.0 & 0.9 & 0.9 & 0.9 & 0.9 & 0.9 & 0.9 & 0.9 & 0.9 & 0.9 & 0.9 & 0.0 & 0.0 & 9.7 & 0.0 \\
\hline & MIN & 0.0 & 0.0 & 0.0 & 0.9 & 0.9 & 0.9 & 0.9 & 0.9 & 0.9 & 0.9 & 0.9 & 0.9 & 0.9 & 0.0 & 0.0 & 9.7 & 0.0 \\
\hline & $\max$ & 0.0 & 0.0 & 0.0 & 0.9 & 0.9 & 0.9 & 0.9 & 0.9 & 0.9 & 0.9 & 0.9 & 0.9 & 0.9 & 0.0 & 0.0 & 9.7 & \\
\hline
\end{tabular}




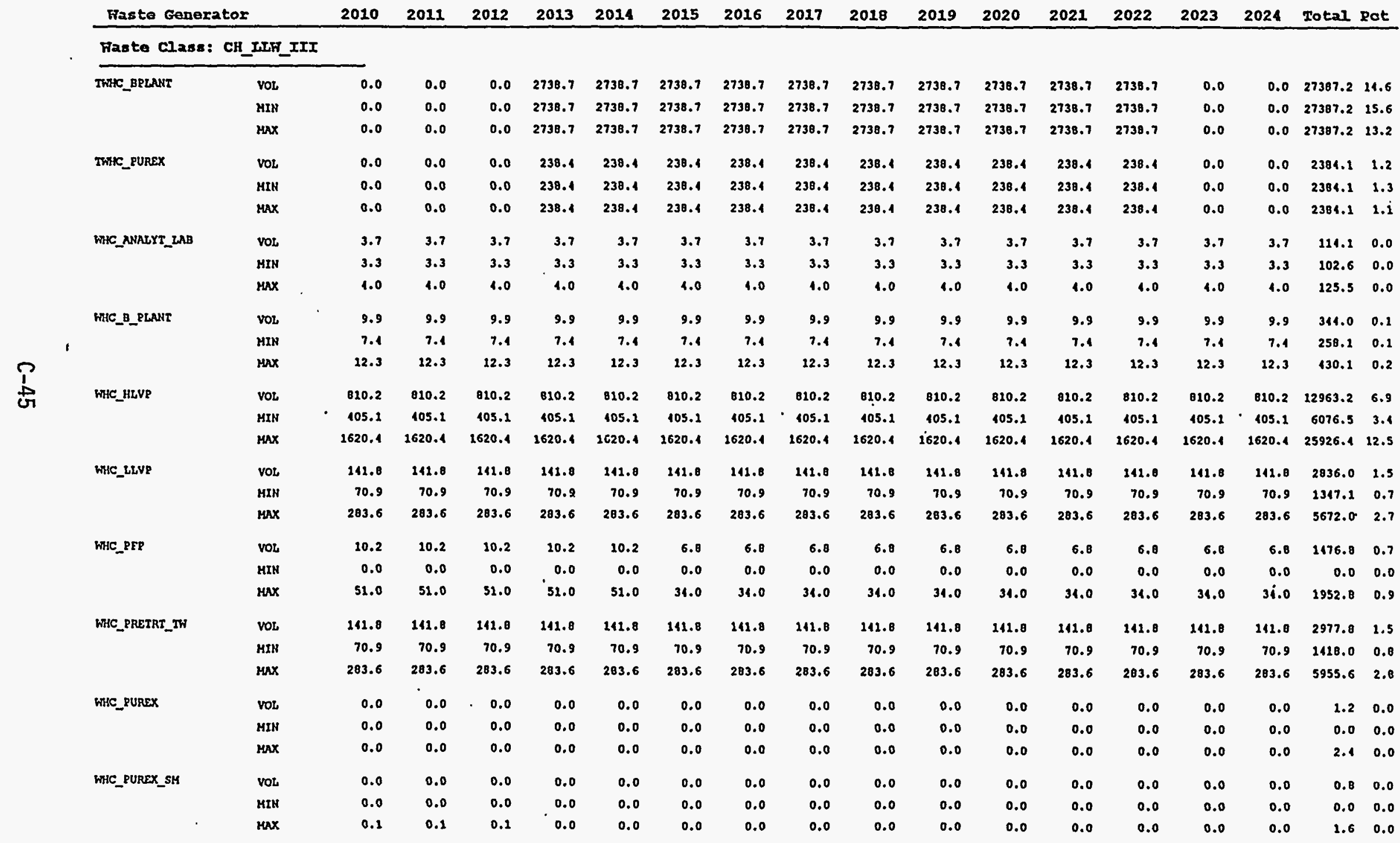




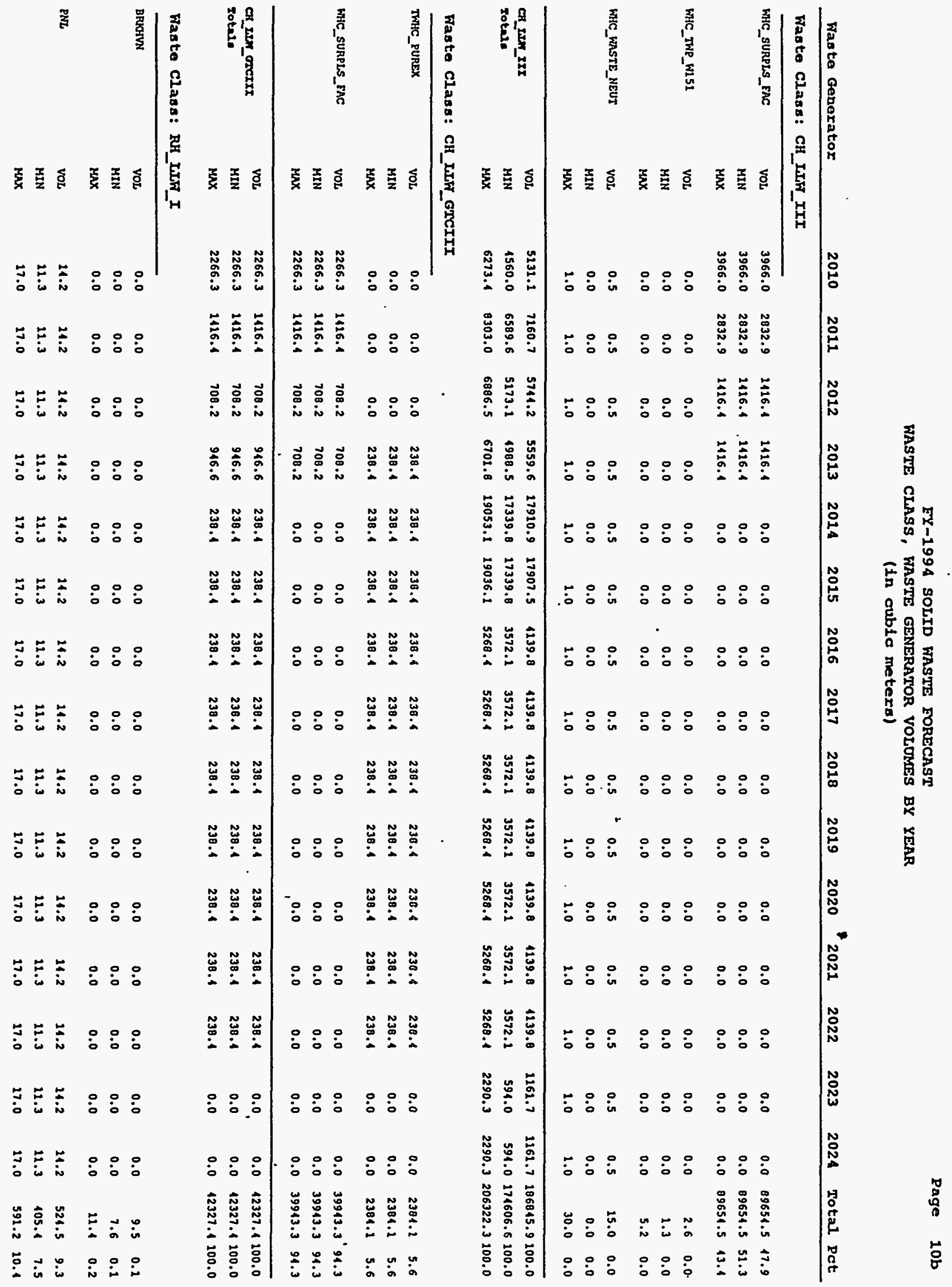


WASTE CLASS, WASTE GENERATOR VOLUMES BY YEAR

(in cubia meters)

Faste Generatox

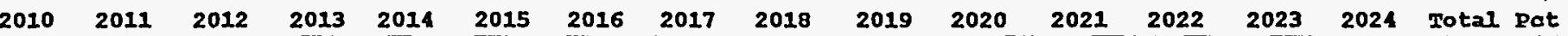

\begin{tabular}{|c|c|c|c|c|c|c|c|c|c|c|c|c|c|c|c|c|c|c|}
\hline \multirow[t]{3}{*}{ IPML_324 } & vor & 0.0 & 0.0 & 0.0 & 1.1 & 1.1 & 1.1 & 1.1 & 1.1 & 1.1 & 1.1 & 1.1 & 1.1 & 1.1 & 0.0 & 0.0 & 21.8 & 0.2 \\
\hline & MIN & 0.0 & 0.0 & 0.0 & 1.1 & 1.1 & 1.1 & 1.1 & 1.1 & 2.1 & 1.1 & 1.1 & 1.1 & 1.1 & 0.0 & 0.0 & 11.8 & 0.2 \\
\hline & $\max$ & 0.0 & 0.0 & 0.0 & 1.1 & 2.1 & 1.1 & 1.1 & 1.1 & 1.1 & 1.1 & 1.1 & 1.1 & 1.1 & 0.0 & 0.0 & 11.0 & 0.2 \\
\hline \multirow[t]{3}{*}{ IPNL_327 } & voL & 0.0 & 0.0 & 0.0 & 31.4 & 31.4 & 31.1 & 31.4 & 31.1 & 31.4 & 31.4 & 31.1 & 31.4 & 31.1 & 0.0 & 0.0 & 314.5 & 5.6 \\
\hline & MIN & 0.0 & 0.0 & 0.0 & 31.4 & 31.4 & 31.4 & 31.4 & 31.1 & 31.4 & 31.1 & 31.4 & 31.4 & 31.4 & 0.0 & 0.0 & 311.5 & 5.8 \\
\hline & MAX & 0.0 & 0.0 & 0.0 & 31.4 & 31.4 & 31.4 & 31.4 & 31.1 & 31.4 & 31.1 & 31.1 & 31.1 & 31.1 & 0.0 & 0.0 & 314.5 & 3.5 \\
\hline \multirow[t]{3}{*}{ TWHC_BPLANT } & vol & 0.0 & 0.0 & 0.0 & 172.0 & 172.0 & 172.0 & 172.0 & 172.0 & 172.0 & 172.0 & 172.0 & 172.0 & 172.0 & 0.0 & 0.0 & 1720.0 & 30.0 \\
\hline & MIH & 0.0 & 0.0 & 0.0 & 272.0 & 172.0 & 172.0 & 172.0 & 272.0 & 172.0 & 172.0 & 172.0 & 272.0 & 272.0 & 0.0 & 0.0 & 1720.0 & 32.0 \\
\hline & $\max$ & 0.0 & 0.0 & 0.0 & 172.0 & 272.0 & 172.0 & 172.0 & 172.0 & 172.0 & 172.0 & 172.0 & 172.0 & 172.0 & 0.0 & 0.0 & 1720.0 & 30.1 \\
\hline \multirow[t]{3}{*}{ TWHC_PUREX_TW } & VoL & 0.0 & 0.0 & 0.0 & 0.0 & 0.0 & 0.0 & 0.0 & 0.0 & 0.0 & 0.0 & 0.0 & 0.0 & 0.0 & 0.0 & 0.0 & 2022.3 & 28.3 \\
\hline & MIN & 0.0 & 0.0 & 0.0 & 0.0 & 0.0 & 0.0 & 0.0 & 0.0 & 0.0 & 0.0 & 0.0 & 0.0 & 0.0 & 0.0 & 0.0 & 1022.3 & 19.0 \\
\hline & $\max$ & 0.0 & 0.0 & 0.0 & 0.0 & 0.0 & 0.0 & 0.0 & 0.0 & 0.0 & 0.0 & 0.0 & 0.0 & 0.0 & 0.0 & 0.0 & 1022.3 & 18.0 \\
\hline \multirow[t]{3}{*}{ TWHC_IPLANI } & VOL & 0.0 & 0.0 & 0.0 & 178.6 & 178.6 & 178.6 & 178.6 & 178.6 & 178.6 & 178.6 & 178.6 & 178.6 & 278.6 & 0.0 & 0.0 & 1786.0 & 31.9 \\
\hline & MIN & 0.0 & 0.0 & 0.0 & 178.6 & 278.6 & 179.6 & 278.6 & 178.6 & 178.6 & 278.6 & 178.6 & 178.6 & 178.6 & 0.0 & 0.0 & 1786.0 & 33.2 \\
\hline & $\max$ & 0.0 & 0.0 & 0.0 & 178.6 & 178.6 & 178.6 & 178.6 & 178.6 & 178.6 & 178.6 & 178.6 & 278.6 & 178.6 & 0.0 & 0.0 & 1786.0 & 31.5 \\
\hline \multirow[t]{3}{*}{ WHC__100" } & VoL & 0.0 & 0.0 & 0.0 & 0.0 & 0.0 & 0.0 & 0.0 & 0.0 & 0.0 & 0.0 & 0.0 & 0.0 & 0.0 & 0.0 & 0.0 & 58.7 & 1.0 \\
\hline & HIN & $0 . \dot{0}$ & 0.0 & 0.0 & 0.0 & 0.0 & 0.0 & 0.0 & 0.0 & 0.0 & 0.0 & 0.0 & 0.0 & 0.0 & 0.0 & 0.0 & 38.7 & 1.0 \\
\hline & $\max$ & 0.0 & 0.0 & 0.0 & 0.0 & 0.0 & 0.0 & 0.0 & 0.0 & 0.0 & 0.0 & 0.0 & 0.0 & 0.0 & 0.0 & 0.0 & 58.7 . & . 1.0 \\
\hline \multirow[t]{3}{*}{ WHC_K_BASIN } & VoL & 1.4 & 1.1 & 1.4 & 1.1 & 1.4 & 1.1 & 1.1 & 1.4 & 1.1 & 2.1 & 1.1 & 1.4 & 1.4 & 1.1 & 2.4 & 16.5 & 0.8 \\
\hline & HIN & 1.1 & 1.1 & 1.1 & 1.1 & 1.1 & 1.1 & 1.1 & 1.1 & 1.1 & 2.1 & 1.2 & 2.1 & 1.1 & 1.1 & 1.1 & 37.7 & 0.7 \\
\hline & $\max$ & 1.4 & 1.4 & 1.4 & 1.4 & 1.4 & 1.1 & 1.1 & 2.1 & 1.4 & 1.4 & 1.1 & 1.1 & 1.1 & 1.4 & $1: 1$ & 17.0 & 0.8 \\
\hline \multirow[t]{3}{*}{ WHIC_TWR_H310 } & vor & 0.0 & 0.0 & 0.0 & 0.0 & 0.0 & 0.0 & 0.0 & 0.0 & 0.0 & 0.0 & 0.0 & 0.0 & 0.0 & 0.0 & 0.0 & 6.1 & 0.1 \\
\hline & MIN & 0.0 & 0.0 & 0.0 & 0.0 & 0.0 & 0.0 & 0.0 & 0.0 & 0.0 & 0.0 & 0.0 & 0.0 & 0.0 & 0.0 & 0.0 & 4.5 & 0.0 \\
\hline & $\max$ & 0.0 & 0.0 & 0.0 & 0.0 & 0.0 & 0.0 & 0.0 & 0.0 & 0.0 & 0.0 & 0.0 & 0.0 & 0.0 & 0.0 & 0.0 & 7.6 & 0.1 \\
\hline \multirow[t]{3}{*}{ WHC_I_PLANT } & vor & 0.0 & 0.0 & 0.0 & 0.0 & 0.0 & 0.0 & 0.0 & 0.0 & 0.0 & 0.0 & 0.0 & 0.0 & 0.0 & 0.0 & 0.0 & 85.2 & 1.5 \\
\hline & MIN & 0.0 & 0.0 & 0.0 & 0.0 & 0.0 & 0.0 & 0.0 & 0.0 & 0.0 & 0.0 & 0.0 & 0.0 & 0.0 & 0.0 & 0.0 & 0.0 & 0.0 \\
\hline & $\max$ & 0.0 & 0.0 & 0.0 & 0.0 & 0.0 & 0.0 & 0.0 & 0.0 & 0.0 & 0.0 & 0.0 & 0.0 & 0.0 & 0.0 & 0.0 & 85.2 & 1.5 \\
\hline \multirow{3}{*}{ 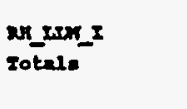 } & vor & 15.6 & 15.6 & 15.6 & 390.8 & 398.0 & 398.8 & 398.8 & 390.8 & 398.0 & 398.8 & 398.8 & 398.8 & 398.8 & 15.6 & 25.6 & 5383.11 & 100.0 \\
\hline & KIN & 12.4 & 12.1 & 22.1 & 395.7 & 395.7 & 395.7 & 395.7 & 395.7 & 395.7 & 395.7 & 395.1 & 395.7 & 395.7 & 12.4 & 12.4 & 5360.8 & 100.0 \\
\hline & $\max$ & 18.4 & 18.4 & 18.4 & 101.6 & 401.6 & 101.6 & 101.6 & 101.6 & 101.6 & 101.6 & 101.6 & 101.6 & 401.6 & 28.1 & 28.4 & 5655.9 & 100.0 \\
\hline
\end{tabular}


FY-1994 - SOLID WASTE FORECAST

WASTE CLASS, HASTE GENERATOR VOLONES BY YEAR

(in cublo meters)

\begin{tabular}{|c|c|c|c|c|c|c|c|c|c|c|c|c|c|c|c|c|c|c|c|}
\hline Waste Gene & & 2010 & 2011 & 2012 & 2013 & 2014 & 2015 & 2016 & 2017 & 2018 & 2019 & 2020 & 2021 & 2022 & 2023 & 2024 & Total 1 & Pet & \\
\hline Waste Clas & H_WW_ & & & & & & & & & & & & & & & & & & \\
\hline \multirow[t]{3}{*}{ ANL_E } & vos & 0.0 & 0.0 & 0.0 & 0.0 & 0.0 & 0.0 & 0.0 & 0.0 & 0.0 & 0.0 & 0.0 & 0.0 & 0.0 & 0.0 & 0.0 & 35.6 & 0.3 & \\
\hline & MIN & 0.0 & 0.0 & 0.0 & 0.0 & 0.0 & 0.0 & 0.0 & 0.0 & 0.0 & 0.0 & 0.0 & 0.0 & 0.0 & 0.0 & 0.0 & 0.0 & 0.0 & \\
\hline & $\max$ & 0.0 & 0.0 & 0.0 & 0.0 & 0.0 & 0.0 & 0.0 & 0.0 & 0.0 & 0.0 & 0.0 & 0.0 & 0.0 & 0.0 & 0.0 & 106.0 & 0.7 & \\
\hline \multirow[t]{3}{*}{ GEN_ATOH } & vos & 0.0 & 0.0 & 0.0 & 0.0 & 0.0 & 0.0 & 0.0 & 0.0 & 0.0 & 0.0 & 0.0 & 0.0 & 0.0 & 0.0 & 0.0 & 131.5 & 1.1 & \\
\hline & MIN & 0.0 & 0.0 & 0.0 & 0.0 & 0.0 & 0.0 & 0.0 & 0.0 & 0.0 & 0.0 & 0.0 & 0.0 & 0.0 & 0.0 & 0.0 & 118.3 & 1.2 & \\
\hline & $\max$ & 0.0 & 0.0 & 0.0 & 0.0 & 0.0 & 0.0 & 0.0 & 0.0 & 0.0 & 0.0 & 0.0 & 0.0 & 0.0 & 0.0 & 0.0 & 197.2 & 1.3 & \\
\hline \multirow[t]{3}{*}{ PNE } & vos & 28.3 & 28.3 & 28.3 & 28.3 & 28.3 & 28.3 & 28.3 & 28.3 & 28.3 & 28.3 & 28.3 & 20.3 & 28.3 & 28.3 & 28.3 & 1203.5 & 10.2 & \\
\hline & MIN & 19.8 & 19.8 & 19.8 & 19.0 & 19.8 & 19.8 & 19.8 & 19.8 & 19.8 & 19.8 & 19.8 & 19.8 & 29.0 & 19.8 & 19.8 & 969.9 & 9.9 & \\
\hline & $\max$ & 31.1 & 31.1 & 31.1 & 31.1 & 31.1 & 31.1 & 31.1 & 31.1 & 31.1 & 31.1 & 31.1 & 31.1 & 31.1 & 31.1 & 31.1 & 1357.8 & 8.9 & \\
\hline \multirow[t]{3}{*}{ TPNL_324 } & vor & 0.0 & 0.0 & 0.0 & 1.3 & 1.3 & 1.3 & 1.3 & 2.3 & 1.3 & 1.3 & 1.3 & 1.3 & 3.3 & 0.0 & 0.0 & 13.8 & 0.1 & \\
\hline & MIN & 0.0 & 0.0 & 0.0 & 1.3 & 1.3 & 1.3 & 1.3 & 1.3 & 1.3 & 1.3 & 1.3 & 1.3 & 1.3 & 0.0 & 0.0 & 13.8 & 0.1 & \\
\hline & $\max$ & 0.0 & 0.0 & 0.0 & 1.3 & 1.3 & 1.3 & 1.3 & 1.3 & 1.3 & 1.3 & 1.3 & 1.3 & 1.3 & 0.0 & 0.0 & 13.8 & 0.0 & \\
\hline \multirow[t]{3}{*}{ TWHC_BPLANT } & vot & 0.0 & 0.0 & 0.0 & 681.6 & 684.6 & 684.6 & 684.6 & 684.6 & 604.6 & 681.6 & 684.6 & 684.6 & 684.6 & 0.0 & 0.0 & 6846.8 & 58.0 & T \\
\hline & MIN & 0.0 & 0.0 & 0.0 . & 684.6 & 684.6 & 684.6 & 684.6 & 604.6 & 684.6 & 694.6 & 684.6 & 684.6 & 684.6 & 0.0 & 0.0 & 6816.0 & 70.4 & \\
\hline & $\operatorname{Max}$ & 0.0 & 0.0 & 0.0 & 684.6 & 681.6 & 684.6 & 684.6 & 684.6 & 684.6 & 684.6 & 684.6 & 684.6 & 684.6 & 0.0 & 0.0 & 6846.8 & .45 .2 & \& \\
\hline \multirow[t]{3}{*}{ TWHC_PUREX } & vor & 0.0 & 0.0 & 0.0 & 23.7 & 13.7 & 13.7 & 13.7 & 13.7 & 13.7 & 13.7 & 13.7 & 13.7 & 13.7 & 0.0 & 0.0 & 137.1 & 1.1 & \\
\hline & MIN & 0.0 & 0.0 & 0.0 & 13.7 & 13.7 & 23.7 & 13.7 & 13.7 & 13.7 & 13.7 & 13.7 & 13.7 & 13.7 & 0.0 & 0.0 & 137.1 & 1.1 & \\
\hline & $\cdot \max$ & 0.0 & 0.0 & 0.0 & 13.7 & 13.7 & 13.7 & 13.7 & 13.7 & 13.7 & 13.7 & 13.7 & 13.7 & 13.7 & 0.0 & 0.0 & $137.1^{\circ}$ & - 0.9 & \\
\hline \multirow[t]{3}{*}{ WHC_B_PLANT } & vor & 3.5 & 3.5 & 3.5 & 3.5 & 3.5 & 3.5 & 3.5 & 3.5 & 3.5 & 3.5 & 3.5 & 3.5 & 3.5 & 3.5 & 3.5 & 611.6 & 5.1 & \\
\hline & MIN & 2.6 & 2.6 & 2.6 & 2.6 & 2.6 & 2.6 & 2.6 & 2.6 & 2.6 & 2.6 & 2.6 & 2.6 & 2.6 & 2.6 & 2.6 & 278.9 & 2.8 & \\
\hline & $\max$ & 4.3 & 4.3 & 1.3 & 4.3 & 4.3 & 4.3 & 4.3 & 1.3 & 4.3 & 1.3 & 4.3 & 4.3 & 1.3 & 1.3 & i.3 & 894.9 & 5.9 & \\
\hline \multirow[t]{3}{*}{ WHC_ETTF_MAIN } & vos & 0.0 & 0.0 & 0.0 & 0.0 & 0.0 & 0.0 & 0.0 & $0.0^{\circ}$ & 0.0 & 0.0 & 0.0 & 0.0 & 0.0 & 0.0 & 0.0 & 17.7 & 0.1 & \\
\hline & MIN & 0.0 & 0.0 & 0.0 & 0.0 & 0.0 & 0.0 & 0.0 & 0.0 & 0.0 & 0.0 & 0.0 & 0.0 & 0.0 & 0.0 & 0.0 & 17.7 & 0.1 & \\
\hline & $\max$ & 0.0 & 0.0 & 0.0 & 0.0 & 0.0 & 0.0 & 0.0 & 0.0 & 0.0 & 0.0 & 0.0 & 0.0 & 0.0 & 0.0 & 0.0 & 17.7 & 0.3 & \\
\hline \multirow[t]{3}{*}{ WHC_HLVP } & Vor & 126.9 & 126.9 & 126.9 & 126.9 & 126.9 & 126.9 & 126.9 & 126.9 & 126.9 & 126.9 & 126.9 & 126.9 & 126.9 & 126.9 & 126.9 & 2030.1 & 17.2 & \\
\hline & MIN & 63.1 & 63.1 & 63.4 & 63.1 & 63.4 & 63.4 & 63.4 & 63.4 & 63.4 & 63.1 & 63.4 & 63.4 & 63.4 & 63.4 & 63.4 & 951.7 & 9.8 & \\
\hline & $\max$ & 253.8 & 253.8 & 253.8 & 253.8 & 253.8 & 253.8 & 253.8 & 253.8 & 253.8 & 253.8 & 253.8 & 253.8 & 253.8 & 253.8 & 253.8 & 4060.0 & 26.8 & \\
\hline \multirow[t]{3}{*}{ WHC_LLVP } & VoL & 21.8 & 21.8 & 21.8 & 21.8 & 21.8 & 21.8 & 21.0 & 21.8 & 21.8 & 21.0 & 21.8 & 21.8 & 21.8 & 21.8 & 21.0 & 436.0 & 3.7 & \\
\hline & MTS & 20.9 & 10.9 & 10.9 & 10.9 & 10.9 & 10.9 & 10.9 & 10.9 & 10.9 & 10.9 & 10.9 & 10.9 & 10.9 & 10.9 & 10.9 & 207.1 & 2.1 & \\
\hline & $\max$ & 13.6 & 13.6 & 43.6 & 13.6 & 43.6 & 13.6 & 43.6 & 43.6 & 13.6 & 43.6 & 13.6 & 43.6 & 13.6 & 13.6 & 13.6 & 872.0 & 5.7 & \\
\hline
\end{tabular}


FY-1994 SOLID WASTE FORECAST

WASTE CLASS, HASTE GENERATOR VOEOMES BY YEAR

(In cubla meters)

Waste Generator

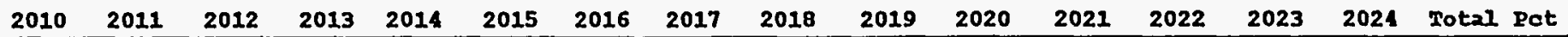

Faste Class: RH_LIF_III

\begin{tabular}{|c|c|c|c|c|c|c|c|c|c|c|c|c|c|c|c|c|c|c|}
\hline \multirow[t]{3}{*}{ WHC_PRETRT_TH } & vor & 14.5 & 14.5 & 14.5 & 14.5 & 24.5 & 14.5 & 14.5 & 14.5 & 14.5 & 14.5 & 14.5 & 14.5 & 14.5 & 14.5 & 14.5 & 304.5 & 2.5 \\
\hline & MIN & 7.2 & 7.2 & 7.2 & 7.2 & 7.2 & 7.2 & 7.2 & 7.2 & 7.2 & 7.2 & 7.2 & 7.2 & 7.2 & 7.2 & 7.2 & 145.0 & 1.4 \\
\hline & $\max$ & 29.0 & 29.0 & 29.0 & 29.0 & 29.0 & 29.0 & 29.0 & 29.0 & 29.0 & 29.0 & 29.0 & 29.0 & 29.0 & 29.0 & 29.0 & 609.0 & 1.0 \\
\hline \multirow{3}{*}{$\begin{array}{l}\text { PH_IIIXIIII } \\
\text { Iotan: }\end{array}$} & vos & 195.0 & 295.0 & 195.0 & 894.7 & 894.7 & 894.7 & 894.7 & 694.7 & 894.7 & 894.7 & 894.7 & 994.7 & 894.7 & 195.0 & 195.0 & 11798.51 & 00.0 \\
\hline & MIN & 104.0 & 104.0 & 104.0 & 803.8 & 803.8 & 803.8 & 603.8 & 803.8 & 803.8 & 803.8 & 803.8 & 803.8 & 803.8 & 104.0 & 204.0 & 9716.4 & 100.0 \\
\hline & $\max$ & 361.9 & 361.9 & 361.9 & 1061.6 & 1061.6 & 1061.6 & 1061.6 & 1061.6 & 1061.6 & 1061.6 & 1051.6 & 2061.6 & 1061.6 & 361.9 & 361.9 & 15141.0 & 100.0 \\
\hline
\end{tabular}

Waste Class: RH_LIF_GTCIII

\begin{tabular}{|c|c|c|c|c|c|c|c|c|c|c|c|c|c|c|c|c|c|c|c|}
\hline \multirow{15}{*}{$\stackrel{?}{b}$} & \multirow[t]{3}{*}{ PNL } & vor & 0.0 & 0.0 & 0.0 & 0.0 & 0.0 & 0.0 & 0.0 & 0.0 & 0.0 & 0.0 & 0.0 & 0.0 & 0.0 & 0.0 & 0.0 & 0.8 & 0.0 \\
\hline & & MIN & 0.0 & 0.0 & 0.0 & 0.0 & 0.0 & 0.0 & 0.0 & 0.0 & 0.0 & 0.0 & 0.0 & 0.0 & 0.0 & 0.0 & 0.0 & 0.0 & 0.0 \\
\hline & & $\max$ & 0.0 & 0.0 & 0.0 & 0.0 & 0.0 & 0.0 & 0.0 & 0.0 & 0.0 & 0.0 & 0.0 & 0.0 & 0.0 & 0.0 & 0.0 & 0.8 & 0.0 \\
\hline & TPNL_324 & vor & 0.0 & 0.0 & 0.0 & 0.4 & 0.4 & 0.1 & 0.4 & 0.1 & 0.4 & 0.1 & 0.1 & 0.4 & 0.1 & 0.0 & 0.0 & 4.0 & 0.0 \\
\hline & . & MIN & 0.0 & 0.0 & 0.0 & 0.4 & 0.4 & 0.1 & 0.4 & 0.1 & 0.1 & 0.1 & 0.1 & 0.4 & 0.1 & 0.0 & 0.0 & 4.0 & 0.0 \\
\hline & . & $\operatorname{MNX}$ & 0.0 & 0.0 & 0.0 & $0 . .4$ & 0.4 & 0.1 & 0.4 & 0.4 & 0.1 & 0.1 & 0.1 & 0.4 & 0.1 & 0.0 & 0.0 & 1.0 & 0.0 \\
\hline & TWHC_PUREX & vor & 0.0 & 0.0 & 0.0 & 105.5 & 105.5 & 105.5 & 105.5 & 205.5 & 105.5 & 105.5 & 105.5 & 105.5 & 105.5 & 0.0 & 0.0 & 1055.0 & 2.5 \\
\hline & & MIN & 0.0 & 0.0 & 0.0 & 105.5 & 105.5 & 205.5 & 105.5 & 105.5 & 105.5 & 205.5 & 105.5 & 105.5 & 105.5 & 0.0 & 0.0 & 1055.0 & 2.5 \\
\hline & & $\max$ & 0.0 & 0.0 & 0.0 & 105.5 & 105.3 & 105.5 & 105.5 & 105.5 & 105.5 & 105.5 & 205.5 & 105.5 & 105.5 & 0.0 & 0.0 & 1055.0 & 2.5 \\
\hline & WHC_SURPLS_EAC & vor & 2266.3 & 1416.4 & 708.2 & 708.2 & 0.0 & 0.0 & 0.0 & 0.0 & 0.0 & 0.0 & 0.0 & 0.0 & 0.0 & 0.0 & 0.0 & $39943.3^{\circ}$ & 97.4 \\
\hline & & MIN & 2266.3 & 1416.4 & 708.2 & 708.2 & 0.0 & 0.0 & 0.0 & 0.0 & 0.0 & 0.0 & 0.0 & 0.0 & 0.0 & 0.0 & 0.0 & 39943.3 & 97.1 \\
\hline & & $\max$ & 2266.3 & 1416.4 & 708.2 & 708.2 & 0.0 & 0.0 & 0.0 & 0.0 & 0.0 & 0.0 & $i^{0.0}$ & 0.0 & 0.0 & 0.0 & 0.0 & 39943.3 & 97.4 \\
\hline & RH Ixw oretrs & vou & 2266.3 & 2416.4 & 708.2 & 814.1 & 105.9 & 205.9 & 205.9 & 205.9 & 105.9 & 205.9 & 105.9 & 105.9 & 105.9 & 0.0 & 0.0 & 41003.1 & 100.0 \\
\hline & Iotale- & MIN & 2266.3 & 1416.4 & 700.2 & 814.1 & 105.9 & 105.9 & 105.9 & 205.9 & 105.9 & 105.9 & 105.9 & 105.9 & 105.9 & $0.0^{\prime}$ & 0.0 & 41002.3 & 100.0 \\
\hline & & $\max$ & 2266.3 & 1416.4 & 708.2 & 814.1 & 105.9 & 105.9 & 105.9 & 105.9 & 105.9 & 205.9 & 105.9 & 103.9 & 105.9 & 0.0 & 0.0 & 11003.1 & 200.0 \\
\hline
\end{tabular}

Waste C12ss: CH_IrMH_I

\begin{tabular}{|c|c|c|c|c|c|c|c|c|c|c|c|c|c|c|c|c|c|c|}
\hline \multirow[t]{3}{*}{ AMES } & vor & 0.0 & 0.0 & 0.0 & 0.0 & 0.0 & 0.0 & 0.0 & 0.0 & 0.0 & 0.0 & 0.0 & 0.0 & 0.0 & 0.0 & 0.0 & 0.8 & 0.0 \\
\hline & MIN & 0.0 & 0.0 & 0.0 & 0.0 & 0.0 & 0.0 & 0.0 & 0.0 & 0.0 & 0.0 & 0.0 & 0.0 & 0.0 & 0.0 & 0.0 & 0.1 & 0.0 \\
\hline & $\operatorname{mAX}$ & 0.0 & 0.0 & 0.0 & 0.0 & 0.0 & 0.0 & 0.0 & 0.0 & 0.0 & 0.0 & 0.0 & 0.0 & 0.0 & 0.0 & 0.0 & 0.9 & 0.0 \\
\hline \multirow[t]{3}{*}{ ANL_E } & VOL & 24.1 & 14.1 & 14.1 & 14.1 & 14.1 & 14.1 & 14.1 & 14.1 & 14.1 & 14.1 & 14.1 & 14.1 & 14.1 & 14.1 & 14.1 & 1161.7 & 1.3 \\
\hline & MIN & 0.0 & 0.0 & 0.0 & 0.0 & 0.0 & 0.0 & 0.0 & 0.0 & 0.0 & 0.0 & 0.0 & 0.0 & 0.0 & 0.0 & 0.0 & 0.0 & 0.0 \\
\hline & $\max$ & 42.3 & 42.3 & 12.3 & 12.3 & 12.3 & 42.3 & 12.3 & 42.3 & 12.3 & 12.3 & 12.3 & 12.3 & 42.3 & 12.3 & 12.3 & 3485.1 & 3.6 \\
\hline
\end{tabular}




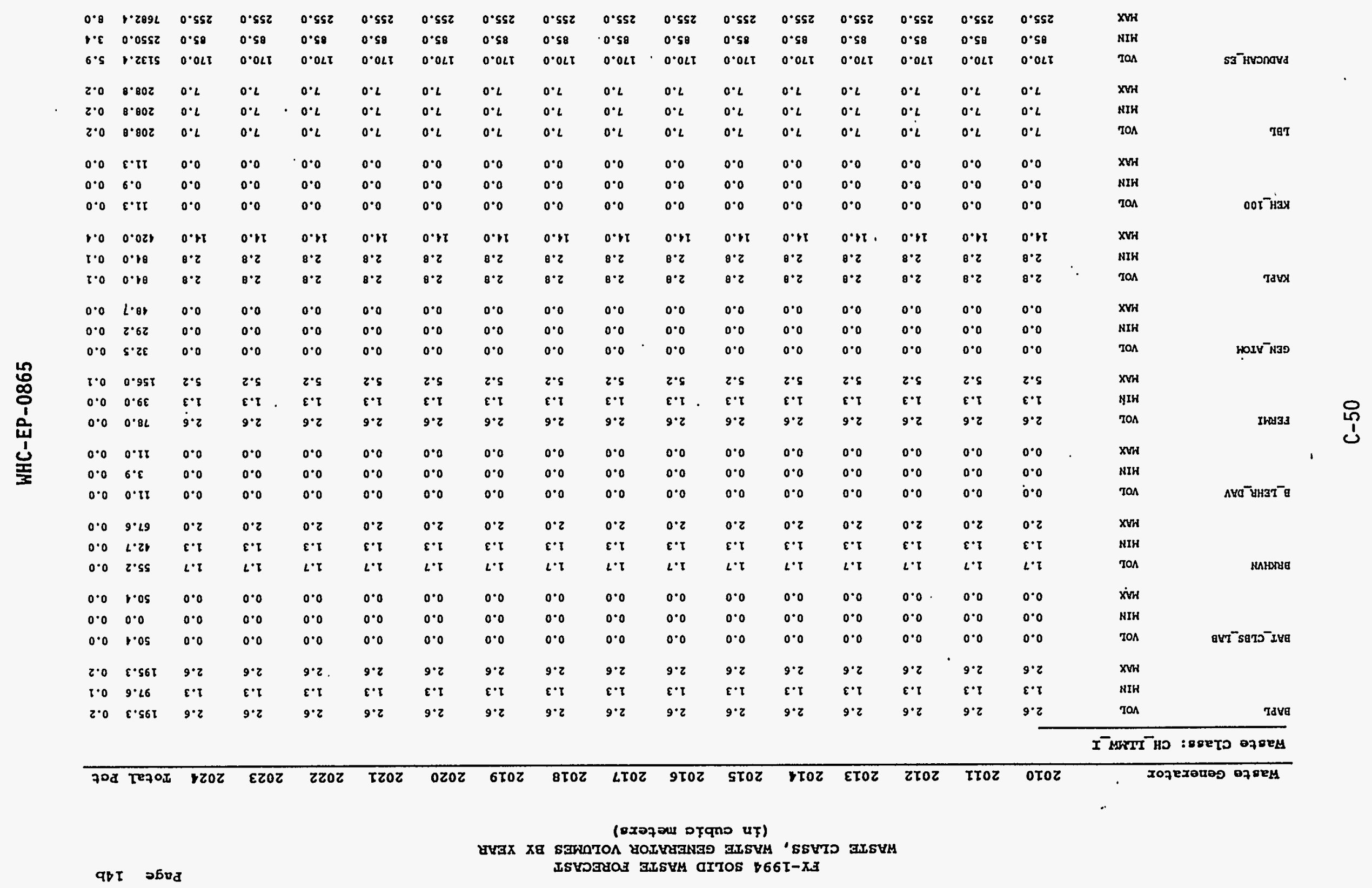




\section{WHC-EP-0865}

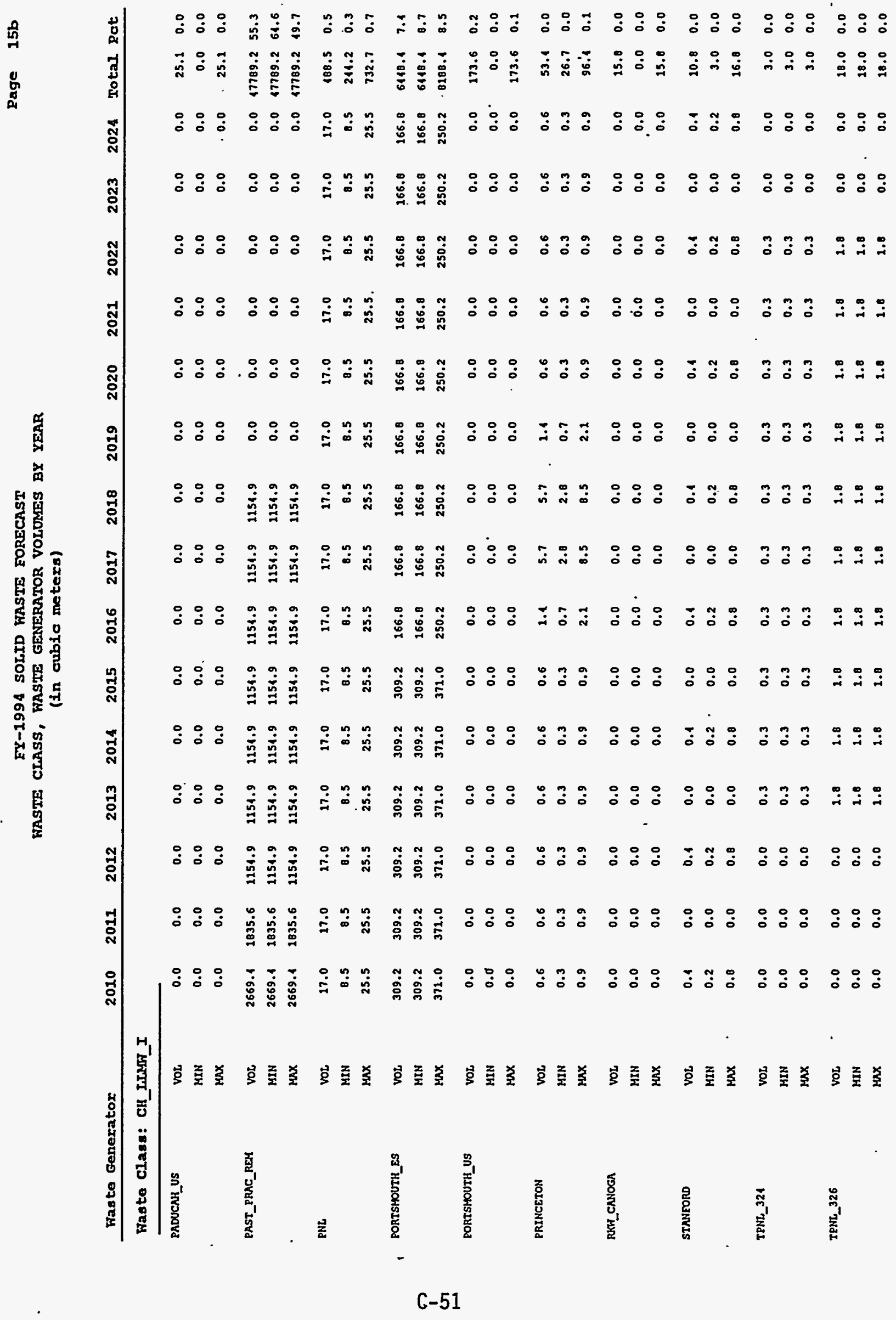


FY-1994 SOLID WASTE FORECAST

WASTE CLASS,' WASTE GENERATOR VOLOMES BY YEAR

(In cublo meters)

\begin{tabular}{|c|c|c|c|c|c|c|c|c|c|c|c|c|c|c|c|c|c|c|}
\hline \multicolumn{2}{|c|}{ Waste Generator } & 2010 & \multirow[t]{2}{*}{2011} & \multirow[t]{2}{*}{2012} & \multirow[t]{2}{*}{2013} & \multirow[t]{2}{*}{2014} & \multirow[t]{2}{*}{2015} & \multirow[t]{2}{*}{2016} & \multirow[t]{2}{*}{2017} & \multirow[t]{2}{*}{2018} & \multirow[t]{2}{*}{2019} & \multirow[t]{2}{*}{2020} & \multirow[t]{2}{*}{2021} & \multirow[t]{2}{*}{2022} & \multirow[t]{2}{*}{2023} & \multirow[t]{2}{*}{2024} & \multirow[t]{2}{*}{ Total } & \multirow[t]{2}{*}{ Pot } \\
\hline Waste Clas & th & & & & & & & & & & & & & & & & & \\
\hline \multirow[t]{3}{*}{ TWHC_222S } & vol & 0.0 & 0.0 & 0.0 & 3.6 & 3.6 & 3.6 & 3.6 & 3.6 & 3.6 & 3.6 & 3.6 & 3.6 & 3.6 & 0.0 & 0.0 & 36.3 & 0.0 \\
\hline & MIN & 0.0 & 0.0 & 0.0 & 3.6 & 3.6 & 3.6 & 3.6 & 3.6 & 3.6 & 3.6 & 3.6 & 3.6 & 3.6 & 0.0 & 0.0 & 36.3 & 0.0 \\
\hline & $\max$ & 0.0 & 0.0 & 0.0 & 3.6 & 3.6 & 3.6 & 3.6 & 3.6 & 3.6 & 3.6 & 3.6 & 3.6 & 3.6 & 0.0 & 0.0 & 36.3 & 0.0 \\
\hline \multirow[t]{3}{*}{ TWHC_212A } & Vor & 0.0 & 0.0 & 0.0 & 20.3 & 20.3 & 20.3 & 20.3 & 20.3 & 20.3 & 20.3 & 20.3 & 20.3 & 20.3 & 0.0 & 0.0 & 203.7 & 0.2 \\
\hline & MIN & 0.0 & 0.0 & 0.0 & 20.3 & 20.3 & 20.3 & 20.3 & 20.3 & 20.3 & 20.3 & 20.3 & 20.3 & 20.3 & 0.0 & 0.0 & 203.7 & 0.2 \\
\hline & $\max$ & 0.0 & 0.0 & 0.0 & 20.3 & 20.3 & 20.3 & 20.3 & 20.3 & 20.3 & 20.3 & 20.3 & 20.3 & 20.3 & 0.0 & 0.0 & 203.7 & 0.2 \\
\hline \multirow[t]{3}{*}{ IWHC_242S } & VoL & 0.0 & 0.0 & 0.0 & 20.3 & 20.3 & 20.3 & 20.3 & 20.3 & 20.3 & 20.3 & 20.3 & 20.3 & 20.3 & 0.0 & 0.0 & 203.7 & 0.2 \\
\hline & MIN & 0.0 & 0.0 & 0.0 & 20.3 & 20.3 & 20.3 & 20.3 & 20.3 & 20.3 & 20.3 & 20.3 & 20.3 & 20.3 & 0.0 & 0.0 & 203.7 & 0.2 \\
\hline & $\max$ & 0.0 & 0.0 & 0.0 & 20.3 & 20.3 & 20.3 & 20.3 & 20.3 & 20.3 & 20.3 & 20.3 & 20.3 & 20.3 & 0.0 & 0.0 & 203.7 & 0.2 \\
\hline \multirow[t]{3}{*}{ TWHC_PUREX } & voL & 0.0 & 0.0 & 0.0 & 23.6 & 23.6 & 23.6 & 23.6 & 23.6 & 23.6 & 23.6 & 23.6 & 23.6 & 23.6 & 0.0 & 0.0 & 236.1 & 0.2 \\
\hline & MIN & 0.0 & 0.0 & 0.0 & 23.6 & 23.6 & 23.6 & 23.6 & 23.6 & 23.6 & 23.6 & 23.6 & 23.6 & 23.6 & 0.0 & 0.0 & 236.4 & 0.3 \\
\hline & $\max$ & 0.0 & 0.0 & 0.0 & 23.6 & 23.6 & 23.6 & 23.6 & 23.6 & 23.6 & 23.6 & 23.6 & 23.6 & 23.6 & 0.0 & 0.0 & 236.1 & 0.2 \\
\hline \multirow[t]{3}{*}{ TWHC_EX_CO18H } & vol & 0.0 & 0.0 & 0.0 & 150.1 & 150.1 & 150.1 & 150.1 & 150.1 & 150.1 & 150.1 & 150.1 & 150.1 & 150.1 & 0.0 & 0.0 & 1501.0 & 1.7 \\
\hline & MIN & 0.0 & 0.0 & 0.0 & 150.1 & 150.1 & 150.1 & 150.1 & 150.1 & 150.1 & $150.1^{\circ}$ & 150.1 & 150.1 & 150.1 & 0.0 & 0.0 & 1501.0 & 2.0 \\
\hline & $\max$ & 0.0 & 0.0 & 0.0 & 150.1 & 250.1 & 150.1 & 150.1 & 150.1 & 150.1 & 150.1 & 150.1 & 150.1 & 150.1 & 0.0 & 0.0 & 1501.0 & 1.5 \\
\hline \multirow[t]{3}{*}{ TWHC_TRUSAF } & vor & 0,0 & 0.0 & 0.0 & 1.3 & 1.3 & 1.3 & 1.3 & 1.3 & 1.3 & 1.3 & 1.3 & 1.3 & 1.3 & 0.0 & 0.0 & 13.4 & 0.0 \\
\hline & MIN & 0.0 & 0.0 & 0.0 & 1.3 & 1.3 & 1.3 & 1.3 & 1.3 & 1.3 & 1,3 & 1.3 & 1.3 & 1.3 & 0.0 & 0.0 & 13.4 & 0.0 \\
\hline & $\operatorname{MAX}$ & 0.0 & 0.0 & 0.0 & 1.3 & 1.3 & 1.3 & 1.3 & 1.3 & 1.3 & 1.3 & 1.3 & 1.3 & 1.3 & 0.0 & 0.0 & 13.4 & 0.0 \\
\hline U_v & VoL & 0,3 & 0.3 & 0.3 & 0.3 & 0.3 & 0.3 & 0.3 & 0.3 & 0.3 & 0.3 & 0.3 & 0.3 & 0.3 & 0.3 & 0.3 & 9.0 & 0.0 \\
\hline & MIN & 0.0 & 0.0 & 0.0 & 0.0 & 0.0 & 0.0 & 0.0 & 0.0 & 0.0 & 0.0 & 0.0 & 0.0 & 0.0 & 0.0 & 0.0 & 0.0 & 0.0 \\
\hline & $\operatorname{MAX}$ & 0.9 & 0.9 & 0.9 & 0.9 & 0.9 & 0.9 & 0.9 & 0.9 & 0.9 & 0.9 & 0.9 & 0.9 & 0.9 & 0.9 & 0.9 & 27.0 & 0.0 \\
\hline WHC_100* & vor & 0.0 & 0.0 & 0.0 & 0.0 & 0.0 & 0.0 & 0.0 & 0.0 & 0.0 & 0.0 & 0.0 & 0.0 & 0.0 & 0.0 & 0.0 & 56.5 & 0.0 \\
\hline & MIN & 0.0 & 0.0 & 0.0 & 0.0 & 0.0 & 0.0 & 0.0 & 0.0 & 0.0 & 0.0 & 0.0 & 0.0 & 0.0 & 0.0 & 0.0 & 56.5 & 0.0 \\
\hline & $\operatorname{MAX}$ & 0.0 & 0.0 & 0.0 & 0.0 & 0.0 & 0.0 & 0.0 & 0.0 & 0.0 & 0.0 & 0.0 & 0.0 & 0.0 & 0.0 & 0.0 & 56.5 & 0.0 \\
\hline KHC_200_ETF & VOL & 34.0 & 34.0 & 34.0 & 31.0 & 34.0 & 34.0 & 34.0 & 34.0 & 34.0 & 34.0 & 34.0 & 34.0 & 34.0 & 34.0 & 31.0 & 986.0 & 1.1 \\
\hline & MIN & 17.0 & 17.0 & 17.0 & 17.0 & 17.0 & 17.0 & 17.0 & 17.0 & 17.0 & 17.0 & 17.0 & 17.0 & 17.0 & 17.0 & 17.0 & 193.0 & 0.6 \\
\hline & $\operatorname{MAX}$ & 51.0 & $\$ 1.0$ & 51.0 & 51.0 & 31.0 & 51.0 & 51.0 & 51.0 & 51.0 & 51.0 & 51.0 & 51.0 & 51.0 & 51.0 & 51.0 & 1479.0 & 1.5 \\
\hline WHE_ 300 TEDE & vol & 0.0 & 8.5 & 0.0 & 8.5 & 0.0 & 8.5 & 0.0 & 8.5 & 0.0 & 8.5 & 0.0 & 8.5 & 0.0 & 8.5 & 0.0 & 119.0 & 0.1 \\
\hline & MIN & 0.0 & 8.5 & 0.0 & 0.5 & 0.0 & 8.5 & 0.0 & 8.5 & 0.0 & 8.5 & 0.0 & 0.5 & 0.0 & 8.5 & 0.0 & 119.0 & 0.1 \\
\hline & $\max$ & 0.0 & 17.0 & 0.0 & 17.0 & 0.0 & 27.0 & 0.0 & 17.0 & 0.0 & 17.0 & 0.0 & 17.0 & 0.0 & 17.0 & 0.0 & 238.0 & 0.2 \\
\hline
\end{tabular}




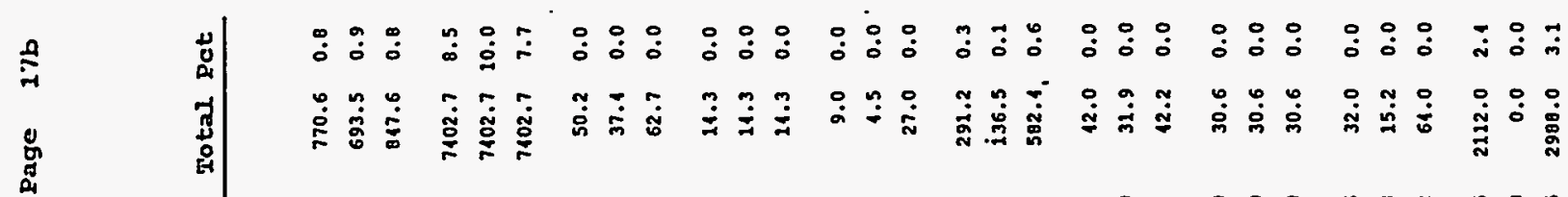

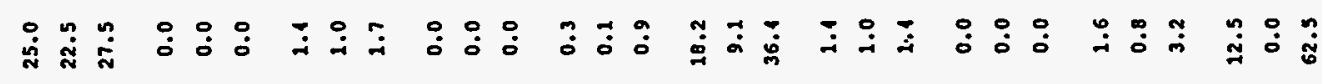

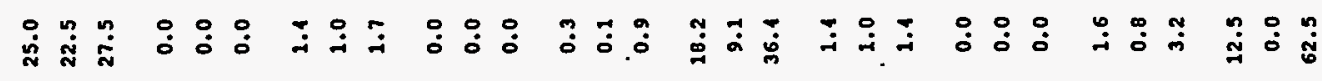

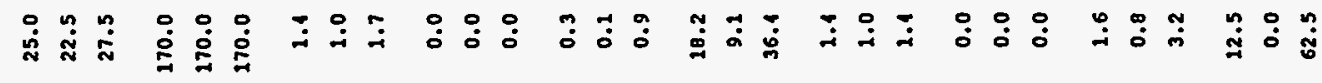

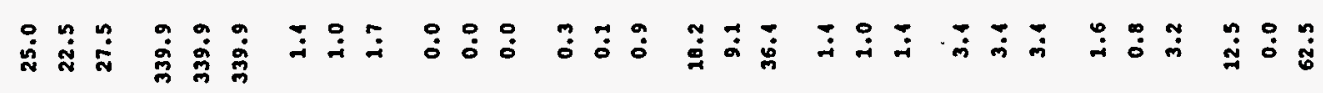

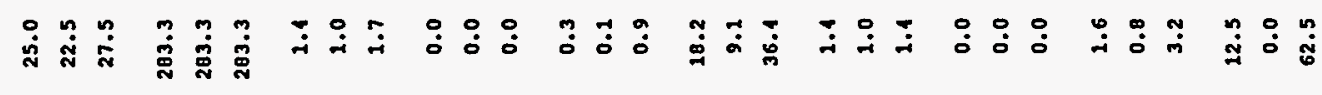

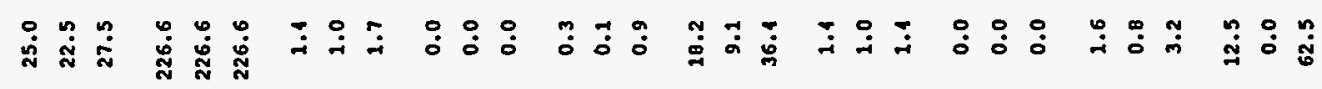

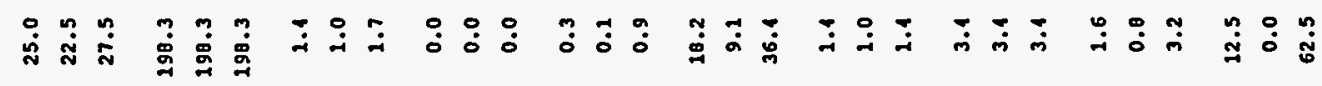

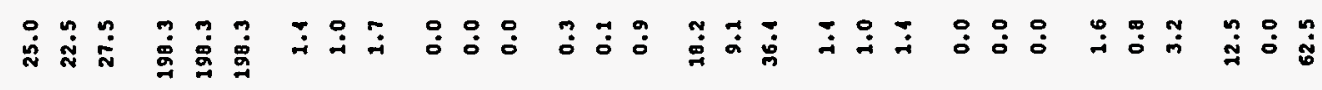

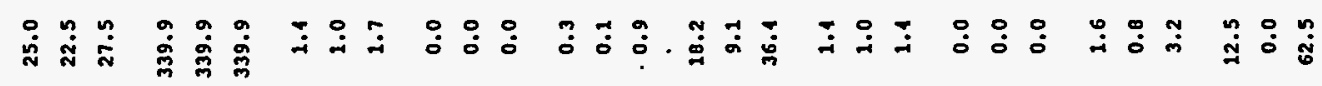

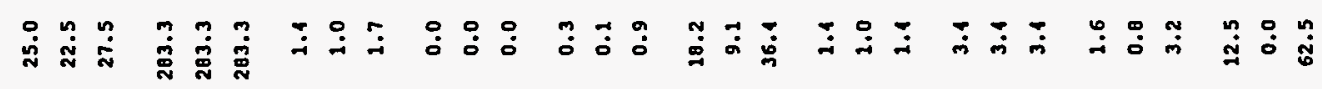

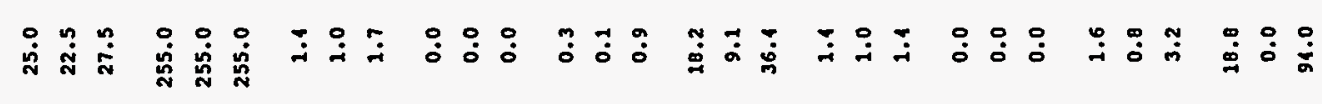

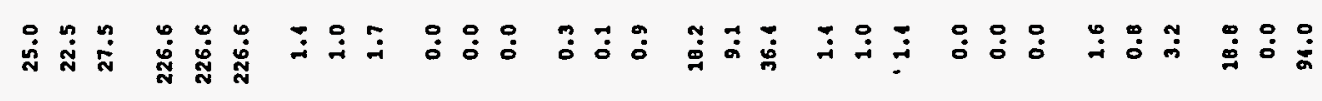

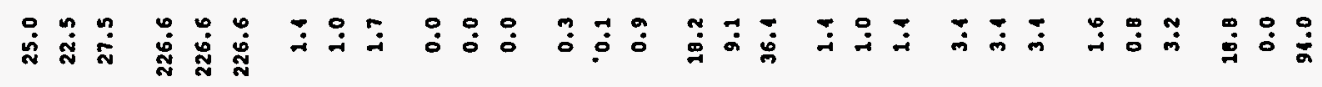

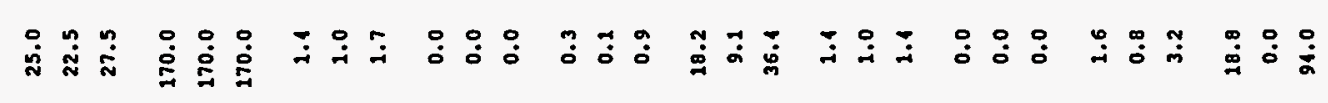

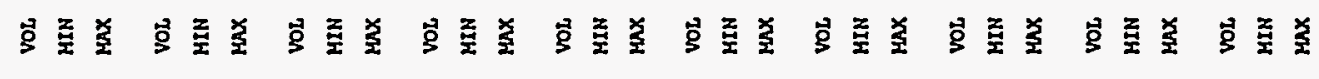

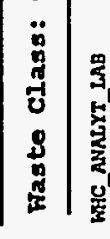
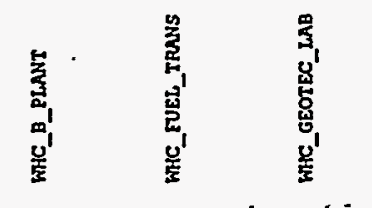

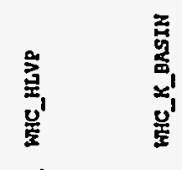

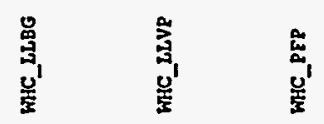


FX-1994 SOLID WASTE FORECAST

Page $18 b$

WASTE CLASS, HASTE GENERATOR VOLOMES BY YEAR

(in cubic meters)

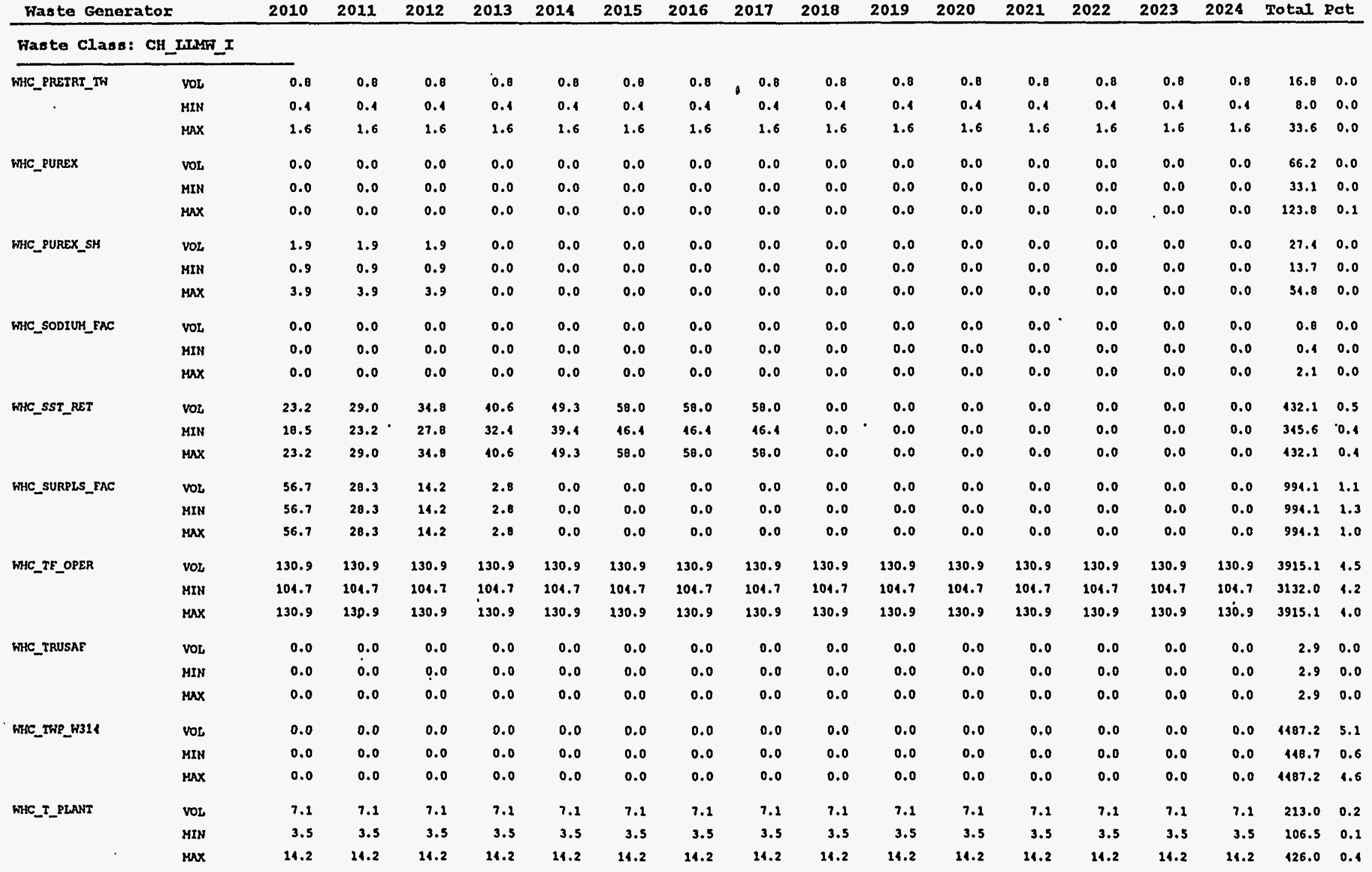


FY-1994 SOLID HASTE FORECAST

HASTE CLASS, WASTE GENERATOR VOLUMIS BY YEAR

(In oubla metera)

\begin{tabular}{|c|c|c|c|c|c|c|c|c|c|c|c|c|c|c|c|c|c|c|}
\hline Faste Gene & & 2010 & 2011 & 2012 & 2013 & 2014 & 2015 & 2016 & 2017 & 2018 & 2019 & 2020 & 2021 & 2022 & 2023 & 2024 & Total & Pet \\
\hline \multicolumn{19}{|c|}{ Faste Class: CH_ITHW_I } \\
\hline \multirow[t]{3}{*}{ WHC_WASTE_NEUT } & voL & 1.3 & 1.3 & 1.3 & 1.3 & 1.3 & 1.3 & 1.3 & 1.3 & 1.3 & 1.3 & 1.3 & 1.3 & 1.3 & 1.3 & 1.3 & 39.0 & 0.0 \\
\hline & MIN & 0.6 & 0.6 & 0.6 & 0.6 & 0.6 & 0.6 & 0.6 & 0.6 & 0.6 & 0.6 & 0.6 & 0.6 & 0.6 & 0.6 & 0.6 & 19.5 & 0.0 \\
\hline & $\max$ & 2.6 & 2.6 & 2.6 & 2.6 & 2.6 & 2.6 & 2.6 & 2.6 & 2.6 & 2.6 & 2.6 & 2.6 & 2.6 & 2.6 & 2.6 & 78.0 & 0.0 \\
\hline \multirow[t]{3}{*}{ WHC_HELL_DRL } & vos & 0.8 & 0.8 & 0.8 & 0.8 & 0.8 & 0.8 & 0.8 & 0.8 & 0.8 & 0.8 & 0.8 & 0.0 & 0.8 & 0.8 & 0.8 & 24.0 & 0.0 \\
\hline & MIN & 0.0 & 0.0 & 0.0 & 0.0 & 0.0 & 0.0 & 0.0 & 0.0 & 0.0 & 0.0 & 0.0 & 0.0 & 0.0 & 0.0 & 0.0 & 0.0 & 0.0 \\
\hline & $\operatorname{sax}$ & 1.6 & 1.6 & 1.6 & 1.6 & 1.6 & 1.6 & 1.6 & 1.6 & 1.6 & 2.6 & 1.6 & 2.6 & 1.6 & 1.6 & 1.6 & 18.0 & 0.0 \\
\hline \multirow{3}{*}{ 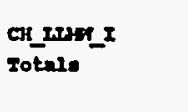 } & vor & 3691.1 & 2012.9 & 2205.8 & 2424.5 & 2430.7 & 2492.9 & 2396.1 & 2267.2 & 2204.5 & 1078.2 & 1126.0 & 1194.1 & 1012.7 & 629.3 & 621.2 & 86385.5 & 100.0 \\
\hline & MIK & 3193.5 & 2614.1 & 2005.9 & 2224.6 & 2240.8 & 2295.8 & 2198.7 & 2067.5 & 2016.2 & 892.3 & 940.3 & 1008.6 & B27.0 & 143.8 & 135.5 & 73923.4 & 100.0 \\
\hline & $\max$ & 1017.5 & 3177.4 & 2532.2 & 2757.0 & 2775.1 & 2800.2 & 2717.6 & 2598.6 & 2527.0 & 1407.5 & 1446.0 & 1523.0 & 1333.5 & 958.1 & 911.9 & 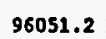 & 00.0 \\
\hline
\end{tabular}

Haste Class: CH_IIMF_III

\begin{tabular}{|c|c|c|c|c|c|c|c|c|c|c|c|c|c|c|c|c|c|c|}
\hline \multirow[t]{3}{*}{ KAPL } & .VOL & 1.1 & 1.1 & 1.1 & 1.1 & 2.1 & 1.1 & 1.1 & 1.4 & 1.4 & 1.4 & 1.4 & 1.4 & 1.4 & 1.1 & 1.1 & 12.0 & 0.0 \\
\hline & MIN & 0.0 & 0.0 & 0.0 & 0.0 & 0.0 & 0.0 & 0.0 & 0.0 & 0.0 & 0.0 & 0.0 & 0.0 & 0.0 & 0.0 & 0.0 & 0.0 & 0.0 \\
\hline & $\max$ & 2.1 & 2.1 & 2.1 & 2.1 & 2.1 & 2.1 & 2.1 & 2.1 & 2.2 & 2.1 & 2.1 & 2.1 & 2.1 & 2.2 & 2.1 & 63.0 & 0.0 \\
\hline \multirow[t]{3}{*}{ LBL } & vor & 0.8 & 0.8 & $0: 8$ & 0.8 & 0.8 & 0.8 & 0.8 & 0.6 & 0.8 & 0.0 & 0.8 & 0.8 & 0.8 & 0.8 & 0.8 & 23.8 & 0.0 \\
\hline & MIN & 0.8 & 0.8 & 0.8 & 0.8 & 0.8 & 0.8 & 0.8 & 0.8 & 0.0 & 0.8 & 0.8 & 0.8 & 0.8 & 0.8 & 0.8 & 23.0 & 0.0 \\
\hline & $\max$ & 0.8 & 0.8 & 0.8 & 0.0 & 0.8 & 0.8 & 0.8 & 0.8 & 0.8 & 0.8 & 0.0 & 0.8 & 0.8 & 0.8 & 0.8 & 23.8 & 0.0 \\
\hline \multirow[t]{3}{*}{ PAST_PRAC_REM } & Vor & 30.5 & 15.3 & 15.3 & 15.3 & 15.3 & 25.3 & 15.3 & 15.3 & 15.3 & 0.0 & 0.0 & 0.0 & 0.0 & 0.0 & 0.0 & $520 . i$ & 0.5 \\
\hline & MIN & 30.5 & 15.3 & 15.3 & 15.3 & 15.3 & 15.3 & 15.3 & 15.3 & 15.3 & 0.0 & 0.0 & 0.0 & 0.0 & 0.0 & 0.0 & 520.1 & 0.6 \\
\hline & $\operatorname{MAX}$ & 30.5 & 15.3 & 25.3 & 25.3 & 15.3 & 15.3 & 15.3 & 15.3 & 15.3 & 0.0 & 0.0 & 0.0 & 0.0 & 0.0 & 0.0 & 520.1 & 0.5 \\
\hline \multirow[t]{3}{*}{ PNL } & vol & 8.5 & 8.5 & 0.5 & 8.5 & 8.5 & 8.5 & 8.5 & 0.5 & 8.5 & 0.5 & 8.5 & B.5 & 0.5 & 8.5 & 8.5 & 240.0 & 0.2 \\
\hline & MIN & 1.2 & 4.2 & 1.2 & 1.2 & 1.2 & 1.2 & 1.2 & 1.2 & 4.2 & 1.2 & 1.2 & 4.2 & 1.2 & 1.2 & 1.2 & 224.0 & 0.1 \\
\hline & $\max$ & 12.7 & 12.7 & 12.7 & 12.7 & 12.7 & 12.7 & 12.7 & 12.7 & 12.7 & 12.7 & 12.7 & 12.7 & 12.7 & 12.7 & 12.7 & 372.0 & 0.3 \\
\hline \multirow[t]{3}{*}{ PORTSHOUTH_US } & Vol & 0.0 & 0.0 & 0.0 & 0.0 & 0.0 & 0.0 & 0.0 & 0.0 & 0.0 & 0.0 & 0.0 & 0.0 & 0.0 & 0.0 & 0.0 & 39.9 & 0.0 \\
\hline & MIN & 0.0 & 0.0 & 0.0 & 0.0 & 0.0 & 0.0 & 0.0 & 0.0 & 0.0 & 0.0 & 0.0 & 0.0 & 0.0 & 0.0 & 0.0 & 0.0 & 0.0 \\
\hline & MAX & 0.0 & 0.0 & 0.0 & 0.0 & 0.0 & 0.0 & 0.0 & 0.0 & 0.0 & 0.0 & 0.0 & 0.0 & 0.0 & 0.0 & 0.0 & 39.9 & 0.0 \\
\hline \multirow[t]{3}{*}{ TWHC_212X } & vol & 0.0 & 0.0 & 0.0 & 12.2 & 12.2 & 12.2 & 12.2 & 12.2 & 12.2 & 12.2 & 12.2 & 12.2 & 12.2 & 0.0 & 0.0 & 122.4 & 0.1 \\
\hline & MIN & 0.0 & 0.0 & 0.0 & 12.2 & 12.2 & 12.2 & 12.2 & 12.2 & 12.2 & 12.2 & 12.2 & 12.2 & 12.2 & 0.0 & 0.0 & 222.4 & 0.1 \\
\hline & $\operatorname{mAX}$ & 0.0 & 0.0 & 0.0 & 12.2 & 12.2 & 12.2 & 12.2 & 22.2 & 12.2 & 12.2 & 12.2 & 12.2 & 12.2 & 0.0 & 0.0 & 122.4 & 0.1 \\
\hline
\end{tabular}


FY-1994 SOLID WASTE FORECAST

WASTE CLASS, WASTE GENERATOR VOLUMES BY YEAR

(in cubia meters)

\begin{tabular}{|c|c|c|c|c|c|c|c|c|c|c|c|c|c|c|c|c|c|c|}
\hline Waste Gene & & 2010 & 2011 & 2012 & 2013 & 2014 & 2015 & 2016 & 2017 & 2018 & 2019 & 2020 & 2021 & 2022 & 2023 & 2024 & Total & Pet \\
\hline \multicolumn{19}{|c|}{ Waste Class: CH_LCHW_III } \\
\hline \multirow[t]{3}{*}{ THHC_212S } & vor & 0.0 & 0.0 & 0.0 & 12.2 & 12.2 & 12.2 & 12.2 & 12.2 & 12.2 & 12.2 & 12.2 & 12.2 & 12.2 & 0.0 & 0.0 & 122.4 & 0.1 \\
\hline & MIN & 0.0 & 0.0 & 0.0 & 12.2 & 12.2 & 12.2 & 12.2 & 12.2 & 12.2 & 12.2 & 12.2 & 12.2 & 12.2 & 0.0 & 0.0 & 122.4 & 0.1 \\
\hline & $\max$ & 0.0 & 0.0 & 0.0 & 12.2 & 12.2 & 12.2 & 12.2 & 12.2 & 12.2 & 12.2 & 12.2 & 12.2 & 12.2 & 0.0 & 0.0 & 122.4 & 0.1 \\
\hline \multirow[t]{3}{*}{ TWHC_242T } & vor & 0.0 & 0.0 & 0.0 & 2.9 & 2.9 & 2.9 & 2.9 & 2.9 & 2.9 & 2.9 & 2.9 & 2.9 & 2.9 & 0.0 & 0.0 & 29.9 & 0.0 \\
\hline & MIN & 0.0 & 0.0 & 0.0 & 2.9 & 2.9 & 2.9 & 2.9 & 2.9 & 2.9 & 2.9 & 2.9 & 2.9 & 2.9 & 0.0 & 0.0 & 29.9 & 0.0 \\
\hline & $\operatorname{MAX}$ & 0.0 & 0.0 & 0.0 & 2.9 & 2.9 & 2.9 & 2.9 & 2.9 & 2.9 & 2.9 & 2.9 & 2.9 & 2.9 & 0.0 & 0.0 & 29.9 & 0.0 \\
\hline \multirow[t]{3}{*}{ TWHC_BPLANT } & VOL, & 0.0 & 0.0 & 0.0 & 27.6 & 27.6 & 27.6 & 27.6 & 27.6 & 27.6 & 27.6 & 27.6 & 27.6 & 27.6 & 0.0 & 0.0 & 276.8 & 0.3 \\
\hline & MIN & 0.0 & 0.0 & 0.0 & 27.6 & 27.6 & 27.6 & 27.6 & 27.6 & 27.6 & 27.6 & 27.6 & .27 .6 & 27.6 & 0.0 & 0.0 & 276.8 & 0.3 \\
\hline & $\max$ & 0.0 & 0.0 & 0.0 & 27.6 & 27.6 & 27.6 & 27.6 & 27.6 & 27.6 & 27.6 & 27.6 & 27.6 & 27.6 & 0.0 & 0.0 & 276.8 & 0.2 \\
\hline \multirow[t]{3}{*}{ TWHC_PX_COLOH. } & vot & 0.0 & 0.0 & 0.0 & 9.8 & 9.8 & 9.8 & 9.8 & 9.8 & 9.8 & 9.8 & 9.8 & 9.8 & 9.8 & 0.0 & 0.0 & 98.0 & 0.1 \\
\hline & HIN & 0.0 & 0.0 & 0.0 & 9.8 & 9.8 & 9.8 & 9.0 & 9.8 & 9,8 & 9.8 & 9.8 & 9.8 & 9.8 & 0.0 & 0.0 & 98.0 & 0.1 \\
\hline & $\max$ & 0.0 & 0.0 & 0.0 & 9.8 & 9.6 & 9.8 & 9.8 & 9.8 & 9.8 & 9.8 & 9.8 & 9.8 & 9.8 & 0.0 & 0.0 & 98.0 & 0.1 \\
\hline \multirow[t]{3}{*}{ TWHAC_TPLANT } & vor & 0.0 & 0.0 & 0.0 & 362.5 & 362.5 & 362.5 & 362.5 & 362.5 & 362.5 & 362.5 & 362.5 & 362.5 & 362.5 & 0.0 & 0.0 & 3625.6 & 3.9 \\
\hline & $\mathrm{MIN}$ & 0.0 & 0.0 & 0.0 & 362.5 & 362.5 & 362.5 & 362.5 & 362.5 & 362.5 & 362.5 & 362.5 & 362.5 & 362.5 & 0.0 & 0.0 & 3625.6 & 4.6 \\
\hline & $\operatorname{MAX}$ & 0.0 & 0.0 & 0.0 & 362.5 & 362.5 & 362.5 & 362.5 & 362.5 & 362.5 & 362.5 . & 362.5 & 362.5 & 362.5 & 0.0 & 0.0 & 3625.6 & 3.5 \\
\hline \multirow[t]{3}{*}{ WHC_200_ETF } & vor & 48.2 & 48.2 & 18.2 & 48.2 & 40.2 & 40.2 & 48.2 & 48.2 & 19.2 & 48.2 & 19.2 & 48.2 & 48.2 & 48.2 & 80.2 & 1397.8 & 1.5 \\
\hline & MIM & 24.1 & 24.1 & 24.1 & 24.1 & 24.1 & 24.2 & 24.1 & 24.1 & 24.1 & 24.1 & 24.1 & 24.1 & 21.1 & 24.1 & 21.1 & 698.9 & 0.9 \\
\hline & $\operatorname{MAX}$ & 72.3 & 72.3 & 72.3 & 72.3 & 72.3 & 72.3 & 72.3 & 72.3 & 72.3 & 72.3 & 72.3 & 72.3 & 72.3 & 72.3 & 72.3 & 2096.7. & 2.0 \\
\hline \multirow[t]{3}{*}{ WHC_ANALYT_LAB } & vor & 3.7 & 3.7 & 3.7 & 3.7 & 3.7 & 3.7 & 3.7 & 3.7 & 3.7 & 3.7 & 3.7 & 3.7 & 3.7 & 3.7 & 3.7 & 114.1 & 0.1 \\
\hline & MIN & 3.3 & 3.3 & 3.3 & 3.3 & 3.3 & 3.3 & 3.3 & 3.3 & 3.3 & 3.3 & 3.3 & 3.3 & 3.3 & 3.3 & 3.3 & 102.6 & 0.1 \\
\hline & $\max$ & 4.0 & 1.0 & 4.0 & 4.0 & 4.0 & 4.0 & 1.0 & 1.0 & 4.0 & 1.0 & 1.0 & 1.0 & 4.0 & 4.0. & 1.0 & 125.5 & 0.1 \\
\hline \multirow[t]{3}{*}{ WHC_HLVP } & Vor & 24.8 & 24.0 & 24.8 & 24.8 & 21.8 & 24.0 & 24.0 & 24.8 & 24.8 & 24.8 & 24.8 & 24.8 & 24.8 & 24.8 & 24.8 & 396.8 & 0.1 \\
\hline & MIN & 12.1 & 12.1 & 12.4 & 12.1 & 12.1 & 12.4 & 12.4 & 12.4 & 12.1 & 12.4 & 12.4 & 12.4 & 12.4 & 12.4 & 12.4 & 186.0 & 0.2 \\
\hline & $\operatorname{MAX}$ & 19.6 & 19.6 & 19.6 & 19.6 & 19.6 & 49.6 & 19.6 & 19.6 & 19.6 & 49.6 & 19.6 & 19.6 & 49.6 & 49.6 & 49.6 & 793.6 & 0.7 \\
\hline \multirow[t]{3}{*}{ WHC_LLVP } & Vor & 1.0 & 1.0 & 1.0 & 1.0 & 1.0 & 1.0 & 1.0 & 1.0 & 1.0 & 1.0 & 1.0 & 1.0 & 1.0 & 1.0 & 2.0 & 20.0 & 0.0 \\
\hline & MIN & 0.5 & 0.5 & 0.5 & 0.5 & 0.5 & 0.5 & 0.5 & 0.5 & 0.5 & 0.5 & 0.5 & 0.5 & 0.5 & 0.5 & 0.5 & 9.5 & 0.0 \\
\hline & $\max$ & 2.0 & 2.0 & 2.0 & 2.0 & 2.0 & 2.0 & 2.0 & 2.0 & 2.0 & 2.0 & 2.0 & 2.0 & 2.0 & 2.0 & 2.0 & 10.0 & 0.0 \\
\hline \multirow[t]{3}{*}{ WHC_PEP } & vor & 0.5 & 0.5 & 0.5 & 0.5 & 0.5 & 0.3 & 0.3 & 0.3 & 0.3 & 0.3 & 0.3 & 0.3 & 0.3 & 0.3 & 0.3 & 74.7 & 0.0 \\
\hline & MIN & 0.0 & 0.0 & 0.0 & 0.0 & 0.0 & 0.0 & 0.0 & 0.0 & 0.0 & 0.0 & 0.0 & 0.0 & 0.0 & 0.0 & 0.0 & 0.0 & 0.0 \\
\hline & $\max$ & 5.0 & 5.0 & 5.0 & 5.0 & 5.0 & 3.0 & 3.0 & 3.0 & 3.0 & 3.0 & 3.0 & 3.0 & 3.0 & 3.0 & 3.0 & 101.0 & o. \\
\hline
\end{tabular}




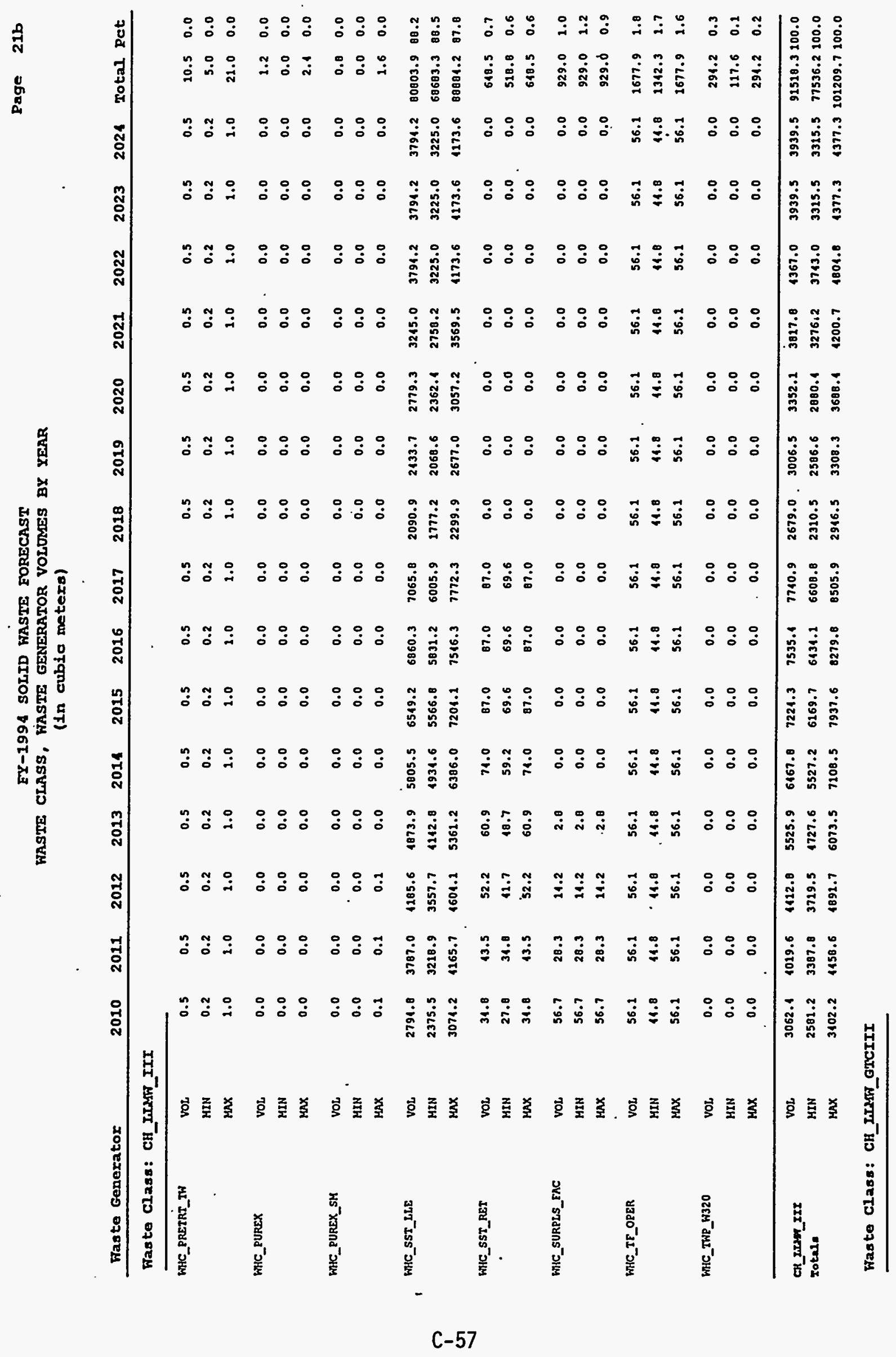




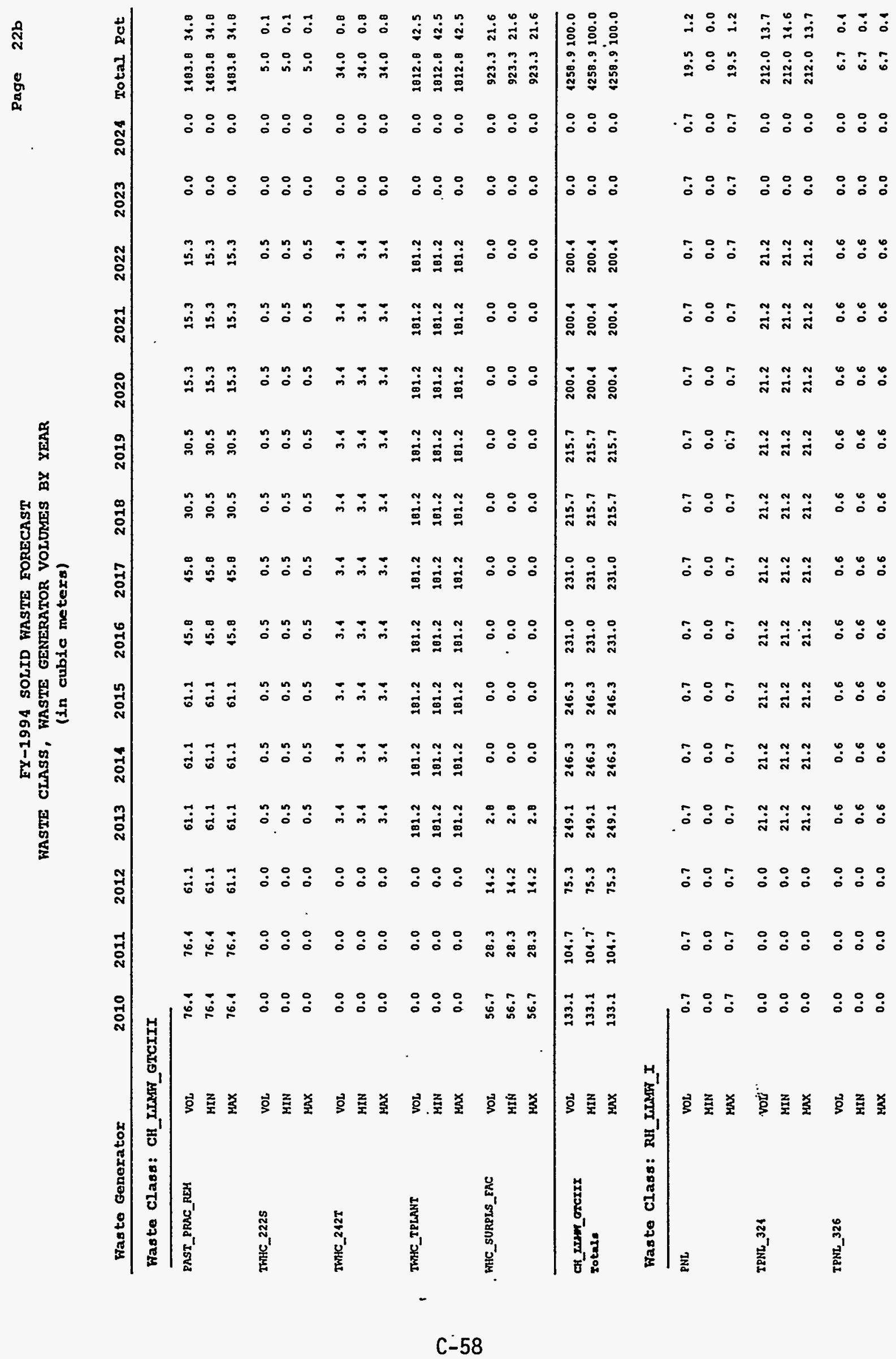




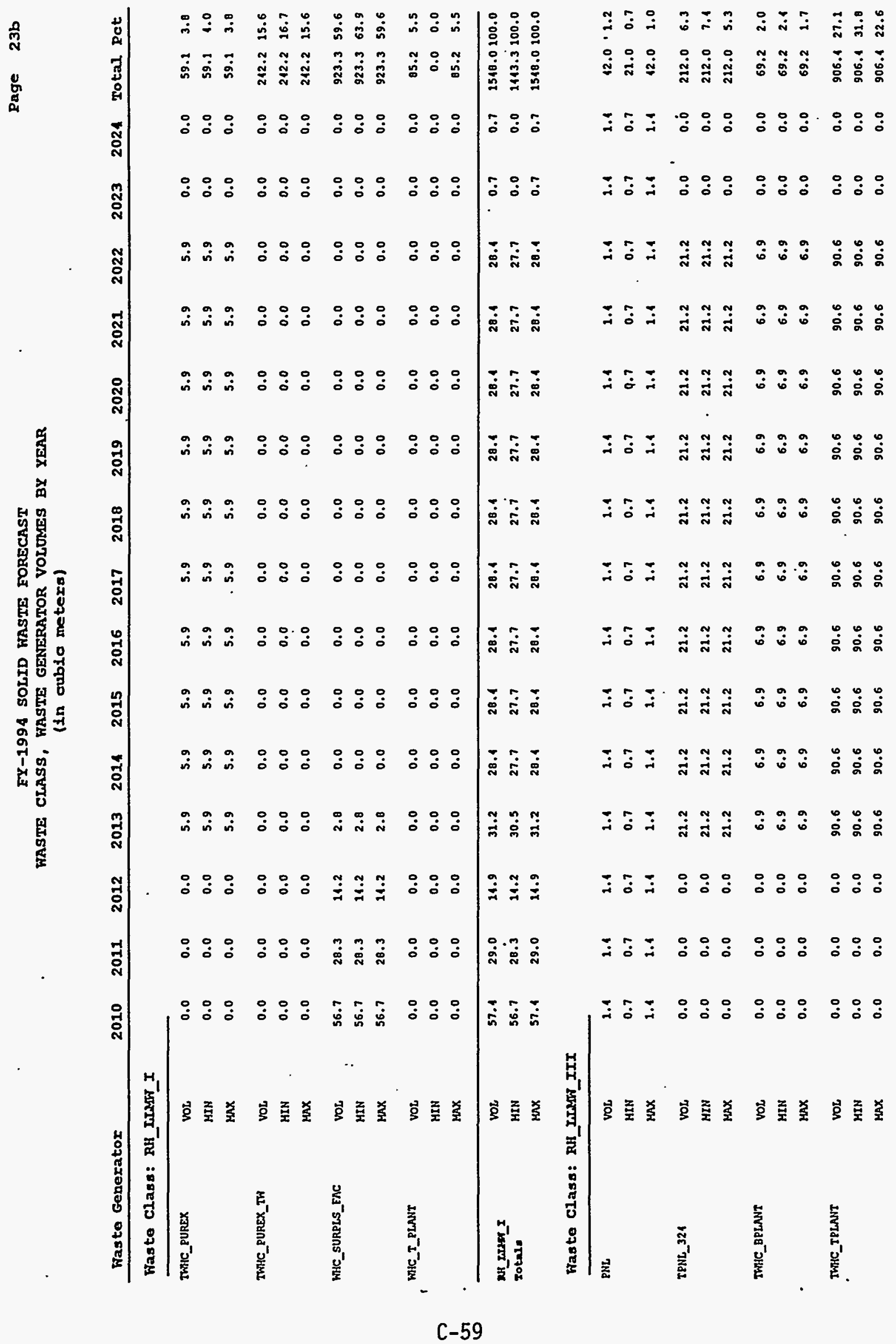


Fi-1994 SOLID HASTE FORECAST

(in cubic metera)

\begin{tabular}{|c|c|c|c|c|c|c|c|c|c|c|c|c|c|c|c|c|c|c|}
\hline Waste Gene & tox & 2010 & 2011 & 2012 & 2013 & 2014 & 2015 & 2016 & 2017 & 2018 & 2019 & 2020 & 2021 & 2022 & 2023 & 2024 & Total & Pat \\
\hline \multicolumn{19}{|c|}{ Fas te Class: RH_IIMH_III } \\
\hline \multirow[t]{3}{*}{ WHC_ANALYT_LAB } & vot & 1.0 & 1.8 & 1.8 & 1.8 & 1.8 & 2.8 & 1.8 & 1.8 & 1.8 & 1.8 & 1.8 & 1.8 & 1.8 & 1.0 & 1.8 & 56.2 & 1.6 \\
\hline & MIN & 1.6 & 2.6 & 1.6 & 1.6 & 1.6 & 1.6 & 2.6 & 1.6 & 1.6 & 1.6 & 1.6 & 1.6 & 1.6 & 1.6 & 1.6 & 50.5 & 1.7 \\
\hline & $\max$ & 1.9 & 1.9 & 1.9 & 1.9 & 1.9 & 1.9 & 1.9 & 1.9 & 1.9 & 1.9 & 1.9 & 1.9 & 1.9 & 1.9 & 1.9 & 61.8 & 1.5 \\
\hline \multirow[t]{3}{*}{ WHTC_B_PLANT } & Vot & 0.0 & 0.0 & 0.0 & 0.0 & 0.0 & 0.0 & 0.0 & 0.0 & 0.0 & 0.0 & 0.0 & 0.0 & 0.0 & 0.0 & 0.0 & 5.5 & 0.1 \\
\hline & MIN & 0.0 & 0.0 & 0.0 & 0.0 & 0.0 & 0.0 & 0.0 & 0.0 & 0.0 & 0.0 & 0.0 & 0.0 & 0.0 & 0.0 & 0.0 & 2.2 & 0.0 \\
\hline & $\max$ & 0.0 & 0.0 & 0.0 & 0.0 & 0.0 & 0.0 & 0.0 & 0.0 & 0.0 & 0.0 & 0.0 & 0.0 & 0.0 & 0.0 & 0.0 & 9.3 & 0.2 \\
\hline \multirow[t]{3}{*}{ WHAC_DST_RET } & vor & 0.0 & 14.7 & 442.4 & 14.7 & 41.7 & 14.7 & 22.4 & 0.0 & 0.0 & 0.0 & 0.0 & 0.0 & 0.0 & 0.0 & 0.0 & 643.6 & 19.2 \\
\hline & MIN & 0.0 & 22.3 & 221.2 & 22.3 & 22.3 & 22.3 & 11.2 & 0.0 & 0.0 & 0.0 & 0.0 & 0.0 & 0.0 & 0.0 & 0.0 & 321.8 & 11.3 \\
\hline & $\max$ & 0.0 & 89.4 & 804.8 & 89.4 & 89.4 & 89.4 & 14.8 & 0.0 & 0.0 & 0.0 & 0.0 & 0.0 & 0.0 & 0.0 & 0.0 & 1287.2 & 32.1 \\
\hline \multirow[t]{3}{*}{ WHC_SURPLS_EAC } & VOL & 56.7 & 28.3 & 14.2 & 2.8 & 0.0 & 0.0 & 0.0 & 0.0 & 0.0 & 0.0 & 0.0 & 0.0 & 0.0 & 0.0 & 0.0 & 923.3 & 27.6 \\
\hline & MIN & 56.7 & 28.3 & 14.2 & 2.8 & 0.0 & 0.0 & 0.0 & 0.0 & 0.0 & 0.0 & 0.0 & 0.0 & 0.0 & 0.0 & 0.0 & 923.3 & 32.4 \\
\hline & $\max$ & 36.7 & 28.3 & 14.2 & 2.8 & 0.0 & 0.0 & 0.0 & 0.0 & 0.0 & 0.0 & 0.0 & 0.0 & 0.0 & 0.0 & 0.0 & 923.3 & 23.0 \\
\hline \multirow[t]{3}{*}{ WHC_TWP_H251 } & vor & 0.0 & 0.0 & 0.0 & 0.0 & 0.0 & 0.0 & 0.0 & 0.0 & 0.0 & 0.0 & 0.0 & 0.0 & 0.0 & 0.0 & 0.0 & 223.8 & 6.7 \\
\hline & $\mathrm{MIN}$ & 0.0 & 0.0 & 0.0 & 0.0 & 0.0 & 0.0 & 0.0 & 0.0 & 0.0 & 0.0 & 0.0 & 0.0 & 0.0 & 0.0 & 0.0 & 212.2 & 7.4 \\
\hline & $\operatorname{MAX}$ & 0.0 & 0.0 & 0.0 & 0.0 & 0.0 & 0.0 & 0.0 & 0.0 & 0.0 & 0.0 & 0.0 & 0.0 & 0.0 & 0.0 & 0.0 & 235.4 & 5.8 \\
\hline \multirow[t]{3}{*}{ WHAC_TWP_W211 } & Vor & 0.0 & 0.0 & 0.0 & 0.0 & 0.0 & 0.0 & 0.0 & 0.0 & 0.0 & 0.0 & 0.0 & 0.0 & 0.0 & 0.0 & 0.0 & 255.1 & 7.6 \\
\hline & MIN & 0.0 & 0.0 & 0.0 & 0.0 & 0.0 & 0.0 & 0.0 & 0.0 & 0.0 & 0.0 & 0.0 & 0.0 & 0.0 & 0.0 & 0.0 & 127.5 & 4.4 \\
\hline & $\max$ & 0.0 & 0.0 & 0.0 & 0.0 & 0.0 & 0.0 & 0.0 & 0.0 & 0.0 & 0.0 & 0.0 & 0.0 & 0.0 & 0.0 & 0.0 & 255.1 & $1 \cdot 6.3$ \\
\hline \multirow{3}{*}{$\begin{array}{l}\text { Nh_rrey_III } \\
\text { sotal. }\end{array}$} & VoL & 59.9 & 76.2 & 459.8 & 169.4 & 166.6 & 166.6 & 144.3 & 121.9 & 121.9 & 121.9 & 121.9 & .121 .9 & 121.9 & 3.2 & 3.2 & 3337.1 & 100.0 \\
\hline & HIN & 59.0 & 52.9 & 237.7 & 146.2 & 143.1 & 143.4 & 132.2 & 121.0 & 121.0 & 121.0 & 121.0 & 121.0 & 121.0 & 2.3 & 2.3 & 2816.2 & 2100.0 \\
\hline & $\max$ & 60.0 & 121.0 & 902.3 & 214.3 & 211.5 & 211.5 & 166.9 & 122.1 & 122.1 & 122.1 & 122.1 & 122.1 & 122.1 & 3.3 & 3.3 & 1001.7 & 100.0 \\
\hline \multicolumn{19}{|c|}{ Waste Class: RH_LHWF_GTCIII } \\
\hline \multirow[t]{3}{*}{ PNL } & Vor & 1.1 & 1.1 & 1.1 & 1.1 & 1.1 & 1.1 & 1.1 & 1.1 & 1.1 & 1.1 & 1.1 & 1.2 & 1.1 & 1.1 & 1.1 & 129.5 & 7.2 \\
\hline & MIN & 0.0 & 0.0 & 0.0 & 0.0 & 0.0 & 0.0 & 0.0 & 0.0 & 0.0 & 0.0 & 0.0 & 0.0 & 0.0 & 0.0 & 0.0 & 0.0 & 0.0 \\
\hline & $\max$ & 1.1 & 1.1 & 1.1 & 1.1 & 1.1 & 2.1 & 1.1 & 1.1 & 1.1 & 1.1 & 1.1 & 1.1 & 1.1 & 1.1 & 1.2 & 212.5 & 312.0 \\
\hline \multirow[t]{3}{*}{ TWHC_222S } & vor & 0.0 & 0.0 & 0.0 & 10.7 & 10.7 & 10.7 & 10.7 & 10.7 & 10.7 & 10.7 & 10.7 & 10.7 & 10.1 & 0.0 & 0.0 & 107.4 & 6.0 \\
\hline & MIN & 0.0 & 0.0 & 0.0 & 10.7 & 10.7 & 10.7 & 10.7 & 10.7 & 10.7 & 10.7 & 10.7 & 10.7 & 10.7 & 0.0 & 0.0 & 207.4 & 6.7 \\
\hline & $\max$ & 0.0 & 0.0 & 0.0 & 20.7 & 10.7 & 10.7 & 10.7 & 10.7 & 20.7 & 10.7 & 10.7 & 10.7 & 10.7 & 0.0 & 0.0 & 107.4 & 5.3 \\
\hline
\end{tabular}


EY-1994 SOLID WASTE FORECAST

HASTE CLASS, WASTE GENERATOR YOLUMES BY YEAR

(in cubio meters)

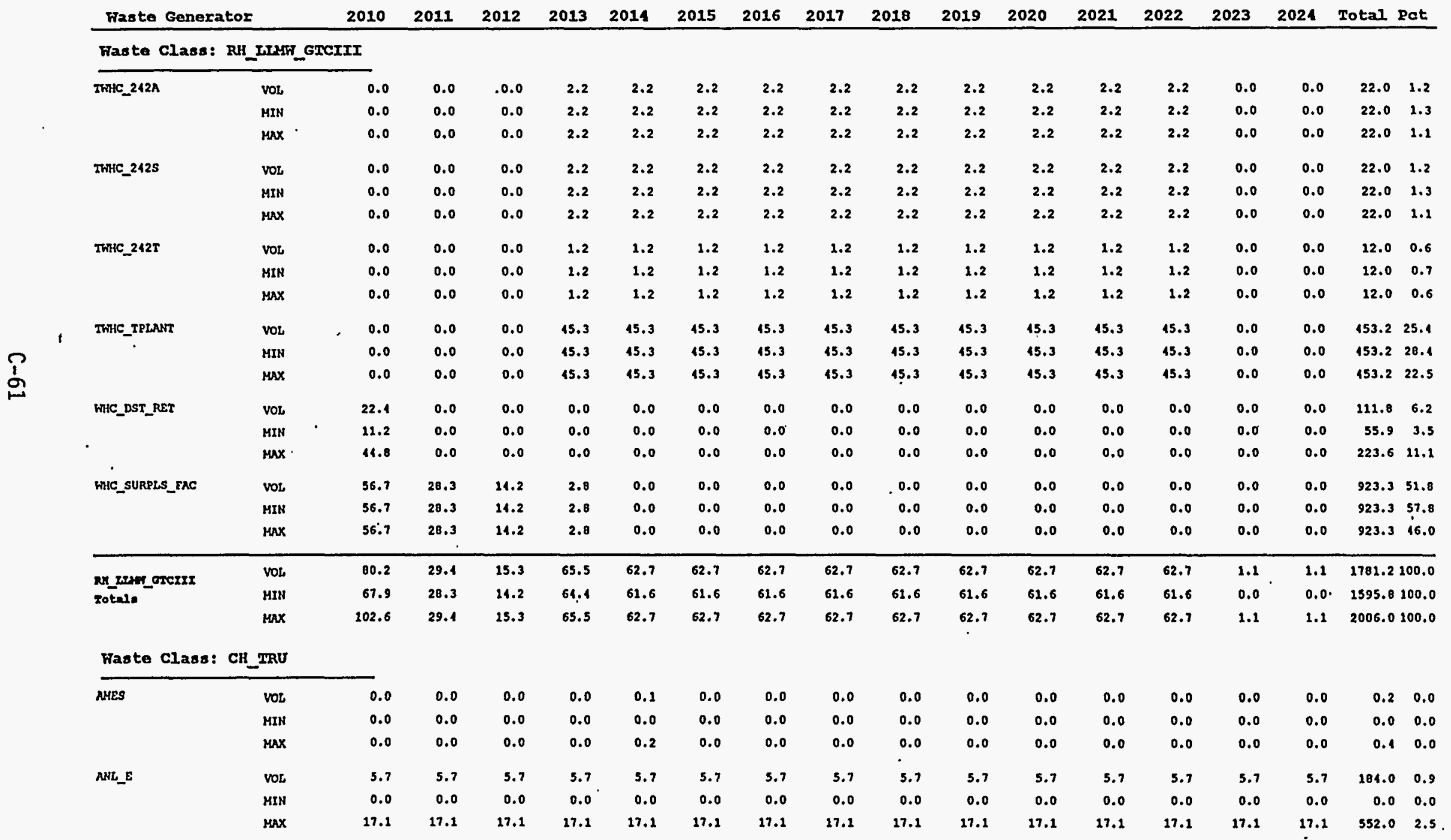


FY-1994 SOLID WASTE EORECAST

WASTE CLASS, HASTE GENERATOR VOLOMES BY YEAR

(in cubla meters)

Wasto Generator

Waste Class: CH_IRU

PAST_PRAC_REY

PNL

กิ

MIN

$\max$

TPNL_2312

vor

MIN

yol

$\overbrace{}^{\text {RPNL_327 }}$

vor

MIN

vor

2010

2011

2012

$2013 \quad 2014$

2015

201

2016

2017

20172018

18

201

2020

$2021 \quad 2022 \quad 2023 \quad 2024$ Total Pat

VOL
MIN
MAX

305.9
305.9
305.9

305.9

214.7

$\begin{array}{llll}244.7 & 244.7 & 244.7 & 183.5\end{array}$

183.5

$22.3 \quad 122.3$

$\begin{array}{lllllll} & 214.7 & 241.7 & 244.7 & 241.7 & 183.5 & 183.5\end{array}$

2.6
0.0

2.6
0.0

$\begin{array}{llll}2.6 & 2.6 & 2.6 & 0.0\end{array}$

$0.0 \quad 0.0$

0.0

$\begin{array}{llll}.1 & 3.1 & 3.1 & 3.1\end{array}$

$0.0 \quad 0.0$

0.0
0.0
0.0

$0.0 \quad 0.0$

$\begin{array}{lll}0.0 & 0.0 & 0.0\end{array}$

61.

$0.0 \quad 3.2 \quad 3.2$

$3.2 \quad 3.2$

3.23 .2

$\begin{array}{lll}0.0 & 0.0 & 0.0\end{array}$

3.2$$
0.0
$$

Twhe_PFP

TWHC_PUREX

TWHC_IPLANT

0.0

0.0

3.2

$3.2 \quad 3.2$

3.2

3.23 .2

3.2

2

3.2

2.6

2.6

$2.6 \quad 2.6$

2.6

$\begin{array}{llll}2.6 & 2.6 & 2.6 & 2.6\end{array}$

$$
\begin{array}{ll}
0.0 & 0.0 \\
0.0 & 0.0 \\
0.0 & 0.0 \\
0.0 & 0.0
\end{array}
$$$$
0.0
$$

vor
$\operatorname{MIN}$
$\operatorname{Max}$$$
0.0
$$$$
0.0 \quad 0.0
$$

vor
MIN

0.0
0.0$$
0.0
$$$$
0.0
$$$$
0.0 \quad 866.3
$$$$
\begin{aligned}
& 0.0 \\
& 0.0 \\
& 0.0
\end{aligned}
$$

$9.0 \quad 9.0 \quad 9.0$

9.0

$2.6 \quad 2.6$

$9.0 \quad 9.0$

9.0

9.0

9.0

9.0

vor

0.0

$\begin{array}{ll}0.0 & 0.0 \\ 0.0 & 0.0\end{array}$

\begin{tabular}{|r|}
\hline 866.3 \\
\hline 59.5 \\
\hline 59.5 \\
\hline
\end{tabular}

MIN

$$
\begin{aligned}
& 0.0 \\
& 0.0
\end{aligned}
$$$$
0.0
$$$$
0.0
$$

$59.5 \quad 59.5$

$$
84.904 .9
$$

$84.9 \quad 84.9 \quad 84.9$

$\begin{array}{lll}84.9 & 84.9 & 84.9 \\ 84.9 & 84.9 & 84.9\end{array}$

WHC_EA_W112

max

MoI,

WHC_rUEL_DEV

$\begin{array}{ll}0.0 & 0.0 \\ 0.0 & 0.0\end{array}$

0.0

0.0
0.0
0.0

$\begin{array}{llll}0.0 & 0.0 & 0.0 & 0.0 \\ 0.0 & 0.0 & 0.0 & 0.0 \\ 0.0 & 0.0 & 0.0 & 0.0\end{array}$

$\begin{array}{llllllll}\operatorname{MAX} & 0.0 & 0.0 & 0.0 & 0.0 & 0.0 & 0.0 & 0.0 \\ \text { VOL } & 0.0 & 0.0 & 0.0 & 0.0 & 0.0 & 0.0 & 0.0 \\ \text { HIN } & 0.0 & 0.0 & 0.0 & 0.0 & 0.0 & 0.0 & 0.0 \\ \operatorname{MAX} & 0.0 & 0.0 & 0.0 & 0.0 & 0.0 & 0.0 & 0.0\end{array}$

9.0
9.0

$9.0 \cdot 9.0$

9.0

$\begin{array}{llll}866.3 & 866.3 & 866.3 & 866.3\end{array}$

\begin{tabular}{|llll}
866.3 & 866.3 & 866.3 & 866.3 \\
866.3 & 866.3 & 866.3 & 866.3 \\
866.3 & 866.3 & 866.3 & 866.3
\end{tabular}

9.0
9.0
9.0

$9.0 \quad 9.0$

$\begin{array}{llllll}866.3 & 866.3 & 0.0 & 0.0 & 8663.1 & 12.6 \\ 866.3 & 866.3 & 0.0 & 0.0 & 8663.1 & 18.5\end{array}$

$\begin{array}{llllll}866.3 & 866.3 & 0.0 & 0.0 & 8663.1 & 39.1\end{array}$

59.5

59.5

$$
59.5
$$

59.5

59.5

59.5

$\begin{array}{ll}59.5 & 59.5 \\ 59.5 & 59.5 \\ 59.5 & 59.5\end{array}$

84.9

$\begin{array}{llllllllll}84.9 & 84.9 & 84.9 & 84.9 & 81.9 & 84.9 & 0.0 & 0.0 & 849.6 & 4.1 \\ 84.9 & 84.9 & 84.9 & 04.9 & 84.9 & 84.9 & 0.0 & 0.0 & 849.6 & 1.7 \\ 84.9 & 84.9 & 84.9 & 84.9 & 84.9 & 84.9 & 0.0 & 0.0 & 849.6 & 3.8\end{array}$

$\begin{array}{lllllllllll}84.9 & 84.9 & 81.9 & 84.9 & 81.9 & 84.9 & 0.0 & 0.0 & 849.6 & 4.1 \\ 84.9 & 84.9 & 84.9 & 84.9 & 84.9 & 84.9 & 0.0 & 0.0 & 849.6 & 1.7 \\ 84.9 & 84.9 & 84.9 & 84.9 & 84.9 & 84.9 & 0.0 & 0.0 & 849.6 & 3.8\end{array}$

59.5

$\begin{array}{llll}0.0 & 0.0 & 595.0 & 2.9\end{array}$

$\begin{array}{llll}0.0 & 0.0 & 595.0 & 3.3 \\ 0.0 & 0.0 & 595.0 & 2.7\end{array}$

$\begin{array}{llll}0.0 & 0: 0 & 595.0 & 2.7\end{array}$

$\begin{array}{rrrrrrrrrr}84.9 & 84.9 & 04.9 & 04.9 & 04.9 & 84.9 & 0.0 & 0.0 & 849.6 & 1.7 \\ 84.9 & 84.9 & 84.9 & 84.9 & 84.9 & 84.9 & 0.0 & 0.0 & 849.6 & 3.8 \\ 0.0 & 0.0 & 0.0 & 0.0 & 0.0 & 0.0 & 0.0 & 0.0 & 38.3 & 0.1 \\ 0.0 & 0.0 & 0.0 & 0.0 & 0.0 & 0.0 & 0.0 & 0.0 & 38.3 & 0.2\end{array}$

$\begin{array}{llllllllll}0.0 & 0.0 & 0.0 & 0.0 & 0.0 & 0.0 & 0.0 & 0.0 & 38.3 & 0.1 \\ 0.0 & 0.0 & 0.0 & 0.0 & 0.0 & 0.0 & 0.0 & 0.0 & 38.3 & 0.2 \\ 0.0 & 0.0 & 0.0 & 0.0 & 0.0 & 0.0 & 0.0 & 0.0 & 38.3 & 0.1\end{array}$

$\begin{array}{llllllllll}0.0 & 0.0 & 0.0 & 0.0 & 0.0 & 0.0 & 0.0 & 0.0 & 1.3 & 0.0 \\ 0.0 & 0.0 & 0.0 & 0.0 & 0.0 & 0.0 & 0.0 & 0.0 & 0.0 & 0.0\end{array}$

$\begin{array}{llllllllll}0.0 & 0.0 & 0.0 & 0.0 & 0.0 & 0.0 & 0.0 & 0.0 & 1.3 & 0.0 \\ 0.0 & 0.0 & 0.0 & 0.0 & 0.0 & 0.0 & 0.0 & 0.0 & 0.0 & 0.0 \\ 0.0 & 0.0 & 0.0 & 0.0 & 0.0 & 0.0 & 0.0 & 0.0 & 1.3 & 0.0\end{array}$


EY-1994 SOLID FASTE FORECAST

Page 27b

HASTE CLASS, WASTE GENERATOR VOLOMES BY YEAR

(in cubic meters)

Waste Generator

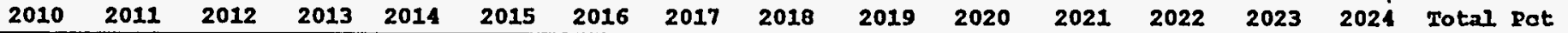
Faste Class: CH_IRU

WiC HLVP

Vol $58.6 \quad 58.6$

$\max$

$\begin{array}{rr}29.3 & 29.3 \\ 117.2 & 117.2\end{array}$

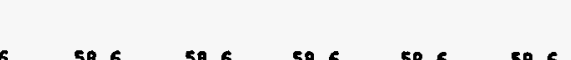

$\begin{array}{rrrrrrr}59.6 & 58.6 & 58.6 & 58.6 & 58.6 & 58.6 & 58.6 \\ 29.3 & 29.3 & 29.3 & 29.3 & 29.3 & 29.3 & 29.3 \\ 117.2 & 117.2 & 117.2 & 117.2 & 117.2 & 117.2 & 117.2\end{array}$

WHC_PEP

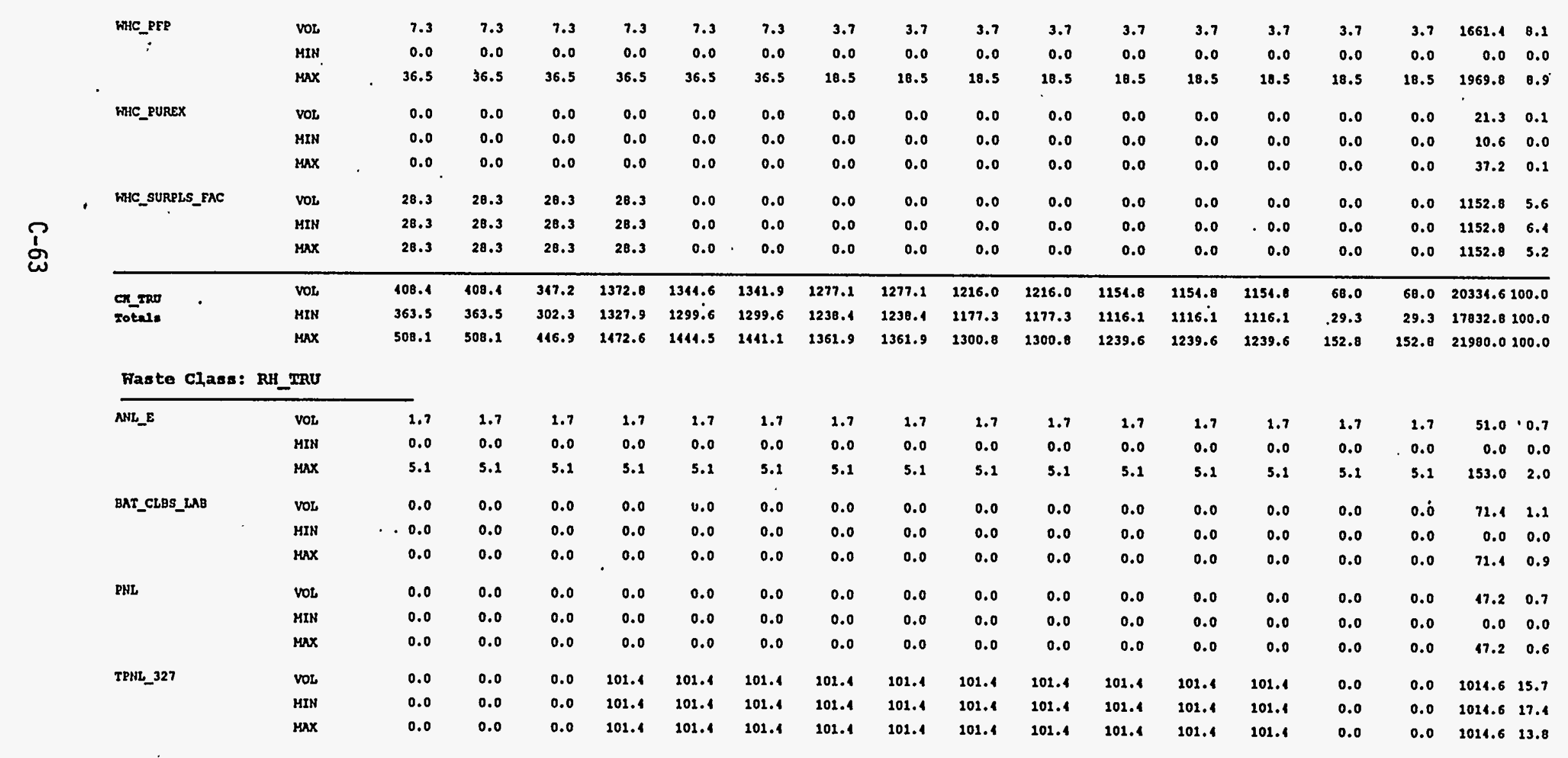

$\begin{array}{lll}117.2 & 217.2\end{array}$

$\begin{array}{rr}29.3 \\ 117.2 & 117.2\end{array}$

9.3

29.3

58.6

\section{6}

58.6

58.6

\section{6}

(a)


EY-1994 SOLID WASTE FORECAST

Page 28b

WASTE CLASS, WASTE GENERATOR VOLOMES BY YEAR

(in cubio metérs)

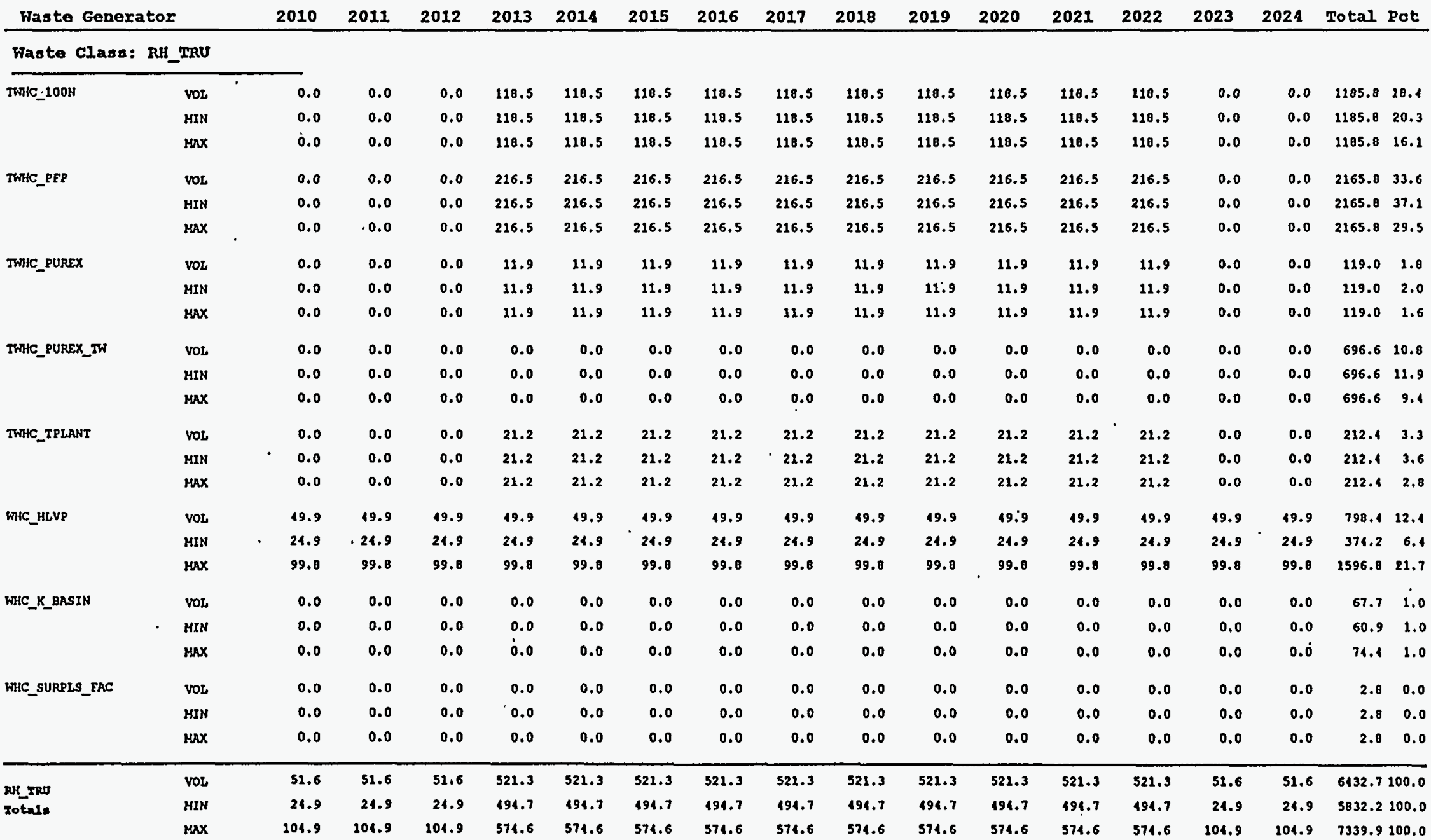

Faste Class: CH_TRUM 


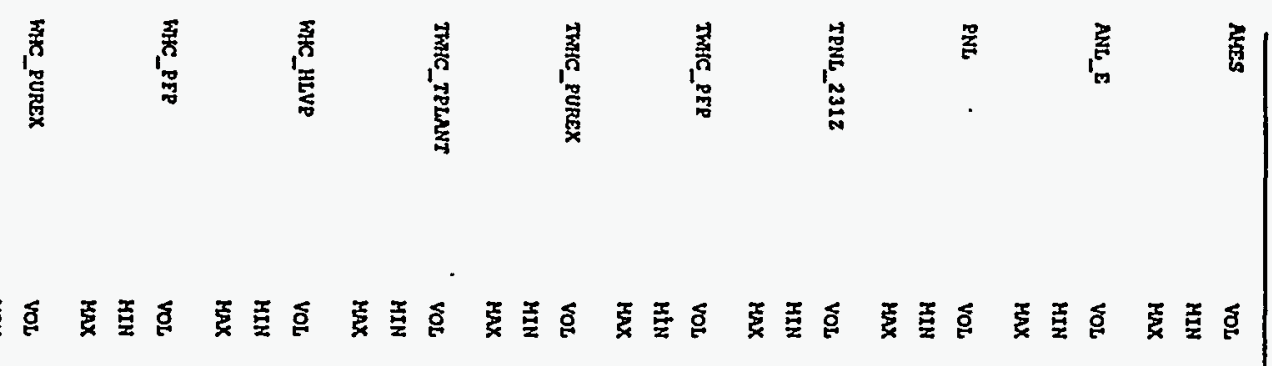

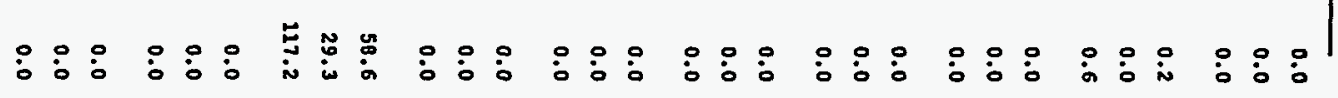

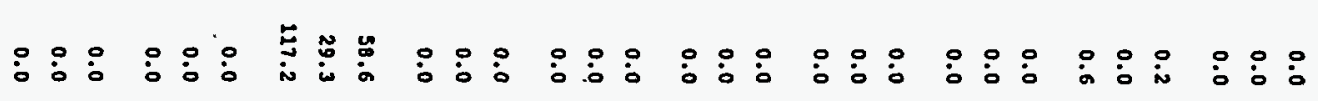

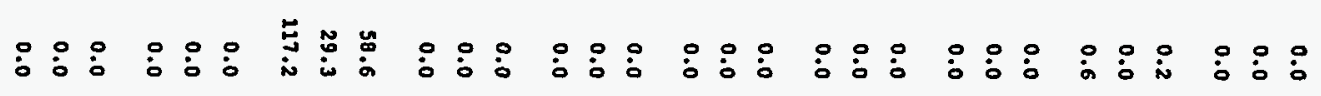

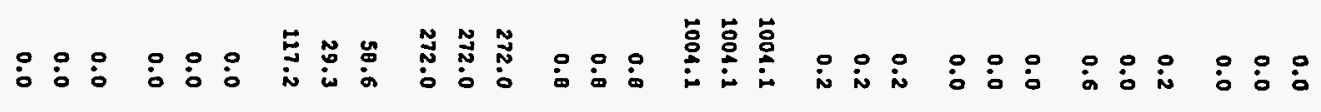

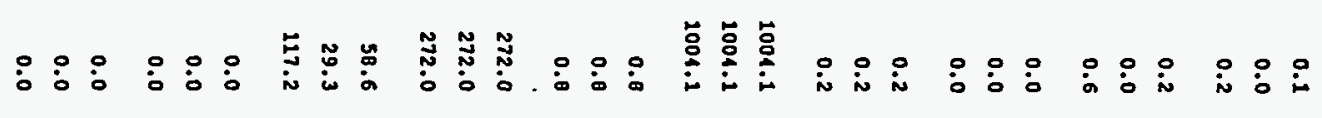

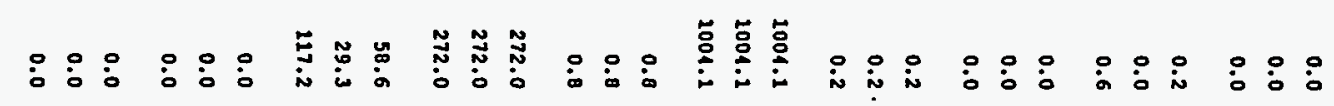

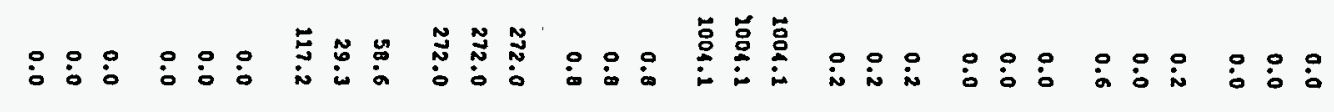

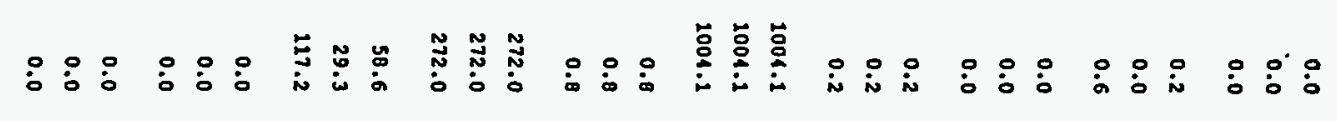

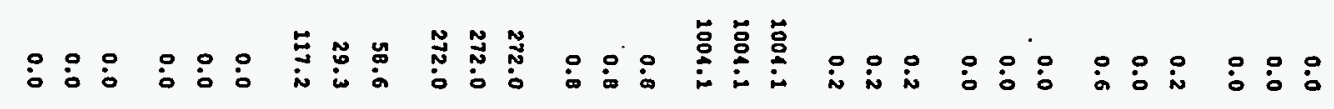

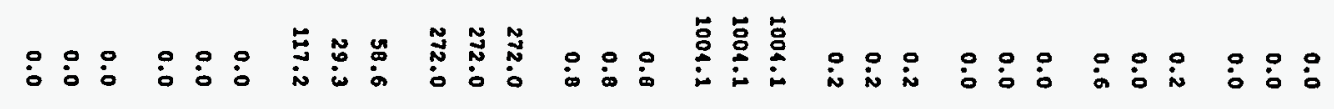

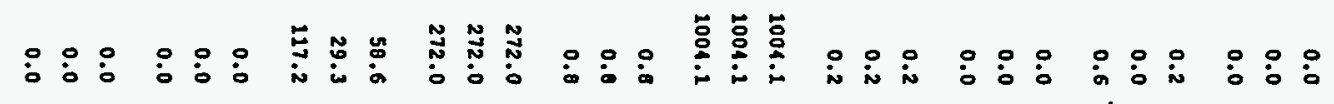

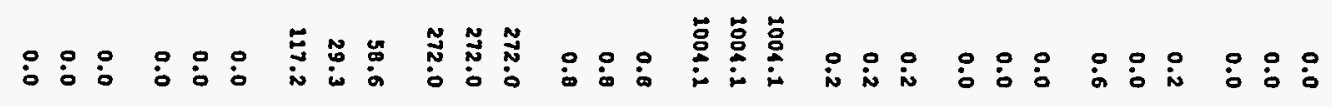

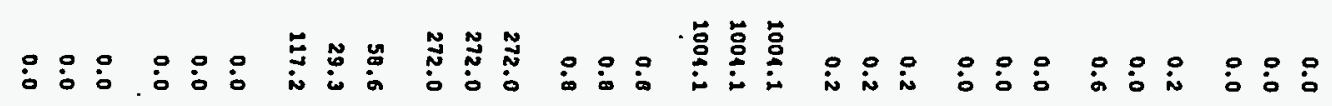

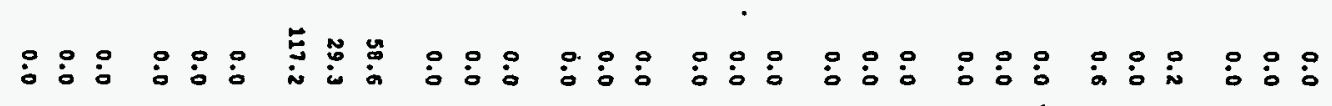

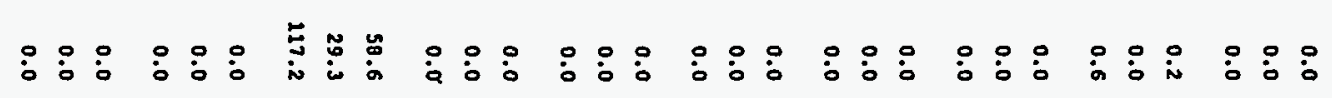

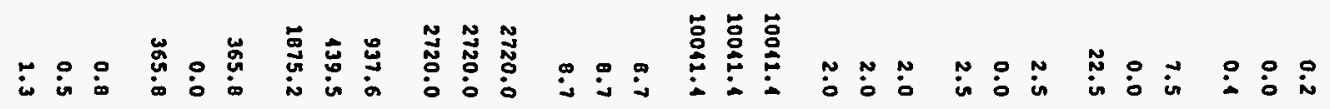

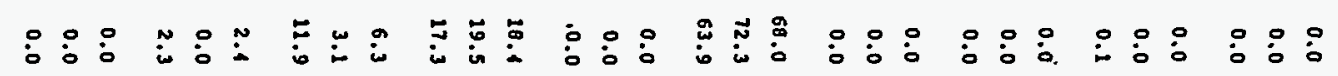


EX-1994 SOLID WASTE FORECAST

WASTE CLASS, WASTE GENERATOR, VOLOMES BY YEAR

(in cublo metexs)

\begin{tabular}{|c|c|c|c|c|c|c|c|c|c|c|c|c|c|c|c|c|c|c|}
\hline Waste Gen & & 2010 & 2011 & 2012 & 2013 & 2014 & 2015 & 2016 & 2017 & 2018 & 2019 & 2020 & 2021 & 2022 & 2023 & 2024 & Total & Pot \\
\hline \multicolumn{19}{|c|}{ Fas te Class: CH_IRURS } \\
\hline \multirow[t]{3}{*}{ WHC_SURPLS_FAC } & Vor & 28.3 & 28.3 & 28.3 & 28.3 & 0.0 & 0.0 & 0.0 & 0.0 & 0.0 & 0.0 & 0.0 & 0.0 & 0.0 & 0.0 & 0.0 & 673.9 & 4.5 \\
\hline & MIN & 28.3 & 28.3 & 28.3 & 28.3 & 0.0 & 0.0 & 0.0 & 0.0 & 0.0 & 0.0 & 0.0 & 0.0 & 0.0 & 0.0 & 0.0 & 673.9 & 4.0 \\
\hline & $\max$ & 20.3 & 28.3 & 28.3 & 28.3 & 0.0 & 0.0 & 0.0 & 0.0 & 0.0 & 0.0 & 0.0 & 0.0 & 0.0 & 0.0 & 0.0 & 673.9 & 4.2 \\
\hline \multirow{3}{*}{$\begin{array}{l}\text { CH_ IROS } \\
\text { rotal. }\end{array}$} & VoL & 87.1 & 07.1 & 87.1 & 1364,3 & 1336.1 & 1336.0 & 1336.0 & 1336.0 & 1336.0 & 1336.0 & 2336.0 & 1336.0 & 1336.0 & 56.8 & 50.8 & 14760.11 & 100.0 \\
\hline & MIN & 57.6 & 57.6 & 57.6 & 1334.8 & 1306.5 & 1306.5 & 1306.5 & 1306.3 & 1306.5 & 1306.5 & 1306.5 & 2306.5 & 1306.5 & 29.3 & 29.3 & 13886.0 & 100.0 \\
\hline & $\max$ & 146.1 & 216.1 & 146.1 & 1423.3 & 1395.2 & 1395.0 & 2395.0 & 1395.0 & 1395.0 & 1395.0 & 1395.0 & 1395.0 & 2395.0 & 217.8 & 117.8 & 15713.7 & 300.0 \\
\hline
\end{tabular}

Waste C1ass: RH_TRUM

\begin{tabular}{|c|c|c|c|c|c|c|c|c|c|c|c|c|c|c|c|c|c|c|}
\hline \multirow[t]{2}{*}{ PNL } & vor & 0.0 & 0.0 & 0.0 & 0.0 & 0.0 & 0.0 & 0.0 & 0.0 & 0.0 & 0.0 & 0.0 & 0.0 & 0.0 & 0.0 & 0.0 & 16.2 & 0.8 \\
\hline & MIN & 0.0 & 0.0 & 0.0 & 0.0 & 0.0 & 0.0 & 0.0 & 0.0 & 0.0 & 0.0 & 0.0 & 0.0 & 0.0 & 0.0 & 0.0 & 0.0 & 0.0 \\
\hline ' & $\max$ & 0.0 & 0.0 & 0.0 & 0.0 & 0.0 & 0.0 & 0.0 & 0.0 & 0.0 & 0.0 & 0.0 & 0.0 & 0.0 & 0.0 & 0.0 & 50.8 & 0.8 \\
\hline \multirow[t]{3}{*}{ TPNL_327 } & VoL & 0.0 & 0.0 & 0.0 & 0.8 & 0.8 & 0.8 & 0.8 & 0.6 & 0.8 & 0.8 & 0.0 & 0.8 & 0.0 & 0.0 & 0.0 & 8.0 & 0.1 \\
\hline & MIN & 0.0 & 0.0 & 0.0 & 0.8 & 0.8 & 0.8 & 0.8 & 0.8 & 0.8 & 0.8 & 0.8 & 0.8 & 0.8 & 0.0 & 0.0 & 8.0 & 0.1 \\
\hline & $\max$ & 0.0 & 0.0 & 0.0 & 0.8 & 0.8 & 0.8 & 0.8 & 0.8 & 0.8 & 0.8 & 0.8 & 0.8 & 0.8 & 0.0 & 0.0 & 8.0 & 0.2 \\
\hline \multirow[t]{3}{*}{ TWHC_340 } & Vot & 0.0 & 0.0 & 0.0 & 2.2 & 2.2 & 2.2 & 2.2 & 2.2 & 2.2 & 2.2 & 2.2 & 2.2 & 2.2 & 0.0 & 0.0 & 22.7 & 0.1 \\
\hline & MIN & 0.0 & 0.0 & 0.0 & 2.2 & 2.2 & 2.2 & 2.2 & 2.2 & 2.2 & 2.2 & 2.2 & 2.2 & 2.2 & 0.0 & 0.0 & 22.7 & 0.5 \\
\hline & $\max$ & 0.0 & 0.0 & 0.0 & 2.2 & 2.2 & 2.2 & 2.2 & 2.2 & 2.2 & 2.2 & 2.2 & 2.2 & 2.2 & 0.0 & 0.0 & 22.7 & 0.3 \\
\hline \multirow[t]{3}{*}{ TWHC_PEP } & vot, & 0.0 & 0.0 & 0.0 & 251.0 & 251.0 & 251.0 & 251.0 & 251.0 & 251.0 & 251.0 & 231.0 & 251.0 & 251.0 & 0.0 & 0.0 & 2510.4 & 48.2 \\
\hline & MIN & 0.0 & 0.0 & 0.0 & 251.0 & 251.0 & 251.0 & 251.0 & 251.0 & 251.0 & 251.0 & 251.0 & 252.0 & 251.0 & 0.0 & 0.0 & 2510.4 & 35.3 \\
\hline & $\operatorname{MAX}$ & 0.0 & 0.0 & 0.0 & 251.0 & 251.0 & 251.0 & 251.0 & 251.0 & 251.0 & 251.0 & 251.0 & 251.0 & 251.0 & 0.0 & 0.0 & 2510.4 & 39.3 \\
\hline \multirow[t]{3}{*}{ TWISC_PUREX } & Vor, & 0.0 & 0.0 & 0.0 & 3.1 & 3.1 & 3.1 & 3.1 & 3.1 & 3.1 & 3.1 & 3.1 & 3.1 & 3.1 & 0.0 & 0.0 & 31.9 & 0.6 \\
\hline & MIN & 0.0 & 0.0 & 0.0 & 3.1 & 3.1 & 3.1 & 3.1 & 3.1 & 3.1 & 3.1 & 3.1 & 3.1 & 3.1 & 0.0 & 0.0 & 31.9 & 0.7 \\
\hline & $\max$ & 0.0 & 0.0 & 0.0 & 3.1 & 3.1 & 3.2 & 3.1 & 3.1 & 3.1 & 3.1 & 3.1 & 3.1 & 3.1 & 0.0 & 0.0 & 31.9 & 0.5 \\
\hline \multirow[t]{3}{*}{ TWRC_TPLANT } & vos & 0.0 & 0.0 & 0.0 & 68.0 & 68.0 & 68.0 & 68.0 & 68.0 & 68.0 & 68.0 & 60.0 & 68.0 & 68.0 & 0.0 & 0.0 & 680.0 & 13.0 \\
\hline & MIN & 0.0 & 0.0 & 0.0 & 68.0 & 68.0 & 68.0 & 60.0 & 68.0 & 68.0 & 68.0 & 68.0 & 68.0 & 68.0 & 0.0 & 0.0 & 680.0 & 15.0 \\
\hline & $\max$ & 0.0 & 0.0 & 0.0 & 68.0 & 68.0 & 68.0 & 68.0 & 68.0 & 68.0 & 68.0 & 68.0 & 68.0 & 68.0 & 0.0 & 0.0 & 680.0 & 10.6 \\
\hline \multirow[t]{3}{*}{ WHC_ANALYT_LAB } & vor & 0.5 & 0.5 & 0.5 & 0.5 & 0.5 & $\cdot \quad 0.5$ & 0.5 & 0.5 & 0.5 & 0.5 & 0.5 & 0.5 & 0.5 & 0.5 & 0.5 & 14.8 & 0.2 \\
\hline & MIN & 0.1 & 0.4 & 0.1 & 0.4 & 0.1 & 0.4 & 0.4 & 0.1 & 0.1 & 0.4 & 0.1 & 0.4 & 0.1 & 0.1 & 0.1 & 13.3 & 0.2 \\
\hline & $\max$ & 0.5 & 0.5 & 0.5 & 0.5 & 0.5 & 0.5 & 0.5 & 0.5 & 0.5 & 0.5 & 0.5 & 0.5 & 0.5 & 0.5 & 0.5 & 16.2 & 0.2 \\
\hline
\end{tabular}




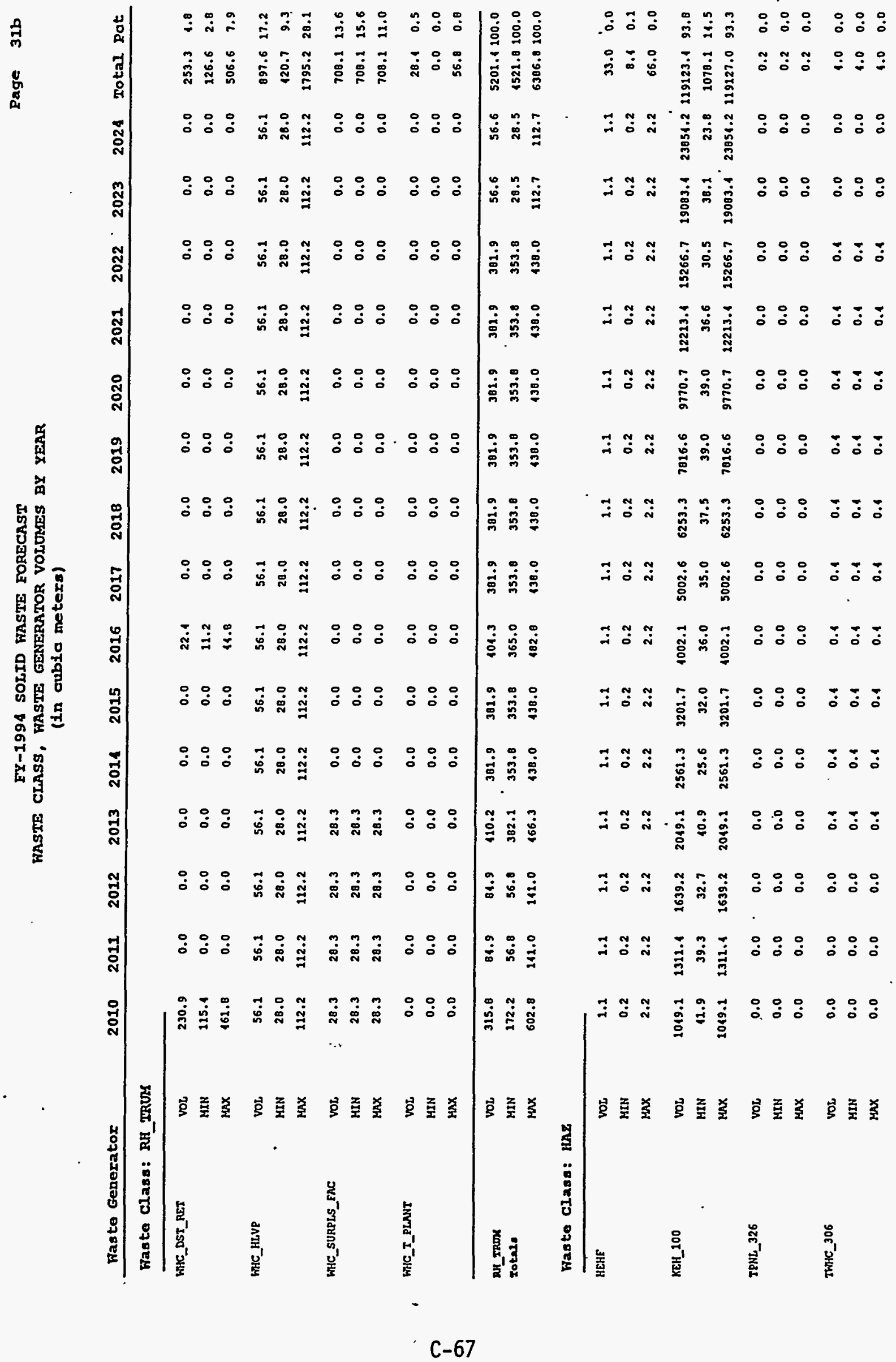


FY-1994 SOLID WASTE FORECAST

WASTE CLASS, KASTE GENERATOR VOLUMES BY YEAR

$$
\text { (in cubic meters) }
$$

Was te Generator

$\begin{array}{lllllllllllllllll}2010 & 2011 & 2012 & 2013 & 2014 & 2015 & 2016 & 2017 & 2018 & 2019 & 2020 & 2021 & 2022 & 2023 & 2024 & \text { Total Pot }\end{array}$

Waste class: HAX

TWR__308

MIN

$\operatorname{MAX}$

WHC_100*

WhC_1163

$?$
1
$\infty$

WHC_1171M

WHC_1171T

VOL
MIN
max

WHC_200_ETE

WHC_21014

MIN

WHC_2703E

MIN

$$
\text { MIN }
$$

WHC_ 2715EA

$016 \quad 201$

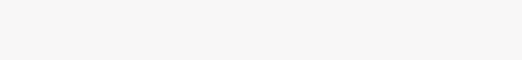

$\begin{array}{ll}0.0 & 0.0 \\ 0.0 & 0.0 \\ 0.0 & 0.0\end{array}$

0.0
0.0

0.0

$\begin{array}{ll}7.0 & 7.0 \\ 7.0 & 7.0\end{array}$

$\begin{array}{ll}7.0 & 7.0 \\ 7.0 & 7.0\end{array}$

7.0
7.0
7.0
0.0
0.0
0.0
0.0
0.0
0.0

7.0

$\begin{array}{lll}0.0 & 0.0 \\ 0.0 & 0.0\end{array}$

$\begin{array}{llll}0.0 & 0.0 & 0.0 & 0.0 \\ 0.0 & 0.0 & 0.0 & 0.0\end{array}$

$\begin{array}{llll}0.0 & 0.0 & 0.0 & 0.0 \\ 0.0 & 0.0 & 0.0 & 0.0\end{array}$

$\begin{array}{llll}0.0 & 0.0 & 0.0 & 0.0\end{array}$

$\begin{array}{llll}0.0 & 0.0 & 0.0 & 0.0 \\ 0.0 & 0.0 & 0.0 & 0.0\end{array}$

0.0

$\begin{array}{lllll}1.1 & 1.4 & 1.4 & 1.4 & 1.4\end{array}$

vot

$\begin{array}{llllll}1.1 & 1.4 & 1.4 & 1.4 & 1.4 & 1.4\end{array}$

$$
1.4
$$$$
1.4
$$$$
\begin{array}{lllll}
6.4 & 6.4 & 6.4 & 6.4 & 6.4
\end{array}
$$$$
\begin{array}{llllll}
6.1 & 6.1 & 6.4 & 6.1 & 6.4 & 6.1 \\
5.7 & 5.7 & 5.7 & 5.7 & 5.7 & 5.7
\end{array}
$$$$
6.4
$$$$
5.7
$$$$
7.0
$$

1.0

$0.0 \quad 0.0$$$
0.0 \quad 0.0
$$$$
0.0 \quad 0.0
$$$$
0.0
$$$$
0.0
$$$$
0.0
$$$$
1.4
$$$$
1.1
$$$$
1.1 \quad 1.4
$$$$
6.1
$$$$
\begin{aligned}
& 6.1 \\
& 5.7
\end{aligned}
$$$$
1.4
$$$$
6.4
$$$$
5.7
$$$$
6.4
$$$$
\begin{aligned}
& 6.4 \\
& 5.7
\end{aligned}
$$$$
5.7
$$$$
1.8
$$

$1.8 \quad 1.8 \quad 1.0$

$1.0 \quad 1.8 \quad 1.8$

$\begin{array}{lll}1.6 & 1.6 & 1.6 \\ 1.9 & 1.9 & 1.9\end{array}$

1.8

1.9

1.9

1.8

$\begin{array}{ll}1.6 & 1.6 \\ 1.9 & 1.9\end{array}$

vos 0.3

30.3

30

$$
\begin{array}{lll}
0.3 & 0.3 & 0.3
\end{array}
$$

0.3

$$
\begin{array}{lll}
0.3 & 0.3 & 0.3 \\
0.1 & 0.1 & 0.1
\end{array}
$$$$
0.4
$$$$
0.1
$$$$
0.1
$$$$
10.1
$$

$\begin{array}{lll}5.2 & 5.2 & 5.2 \\ 2.6 & 2.6 & 2.6\end{array}$

$\begin{array}{llll}5.2 & 5.2 & 5.2\end{array}$

5.2

2.6
5.2

5.2

$5.2 \quad 5.2$

$2.6 \quad 2.6$

$\begin{array}{lll}5.2 & 5.2 & 5.2\end{array}$

$2.2 \quad 2.2$

$\begin{array}{ll}2.2 & 2.2 \\ 2.2 & 2.2\end{array}$

$\begin{array}{ll}2.2 & 2.2\end{array}$

$\begin{array}{ll}2.2 & 2.2 \\ 2.2 & 2.2\end{array}$

$\begin{array}{lllllll}19.6 & 19.6 & 29.6 & 19.6 & 19.6 & 19.6\end{array}$

$\begin{array}{llll}19.6 & 19.6 & 19.6 & 19.6 \\ 19.6 & 19.6 & 19.6 & 19.6\end{array}$ $\operatorname{mIN}$

$\begin{array}{lllllll} & 19.6 & 19.6 & 19.6 & 19.6 & 19.6 & 19.6 \\ & 19.6 & 19.6 & 19.6 & 19.6 & 19.6 & 19.6\end{array}$

19.6

$\begin{array}{llll}19.6 & 19.6 & 19.6 & 19.6 \\ 19.6 & 19.6 & 19.6 & 19.6\end{array}$

$\begin{array}{lllllll}19.6 & 19.6 & 19.6 & 19.6 & 19.6 & 588.0 & 0.4 \\ 19.6 & 19.6 & 19.6 & 19.6 & 19.6 & 588.0 & 7.9 \\ 19.6 & 19.6 & 19.6 & 19.6 & 19.6 & 588.0 & 0.4\end{array}$ 


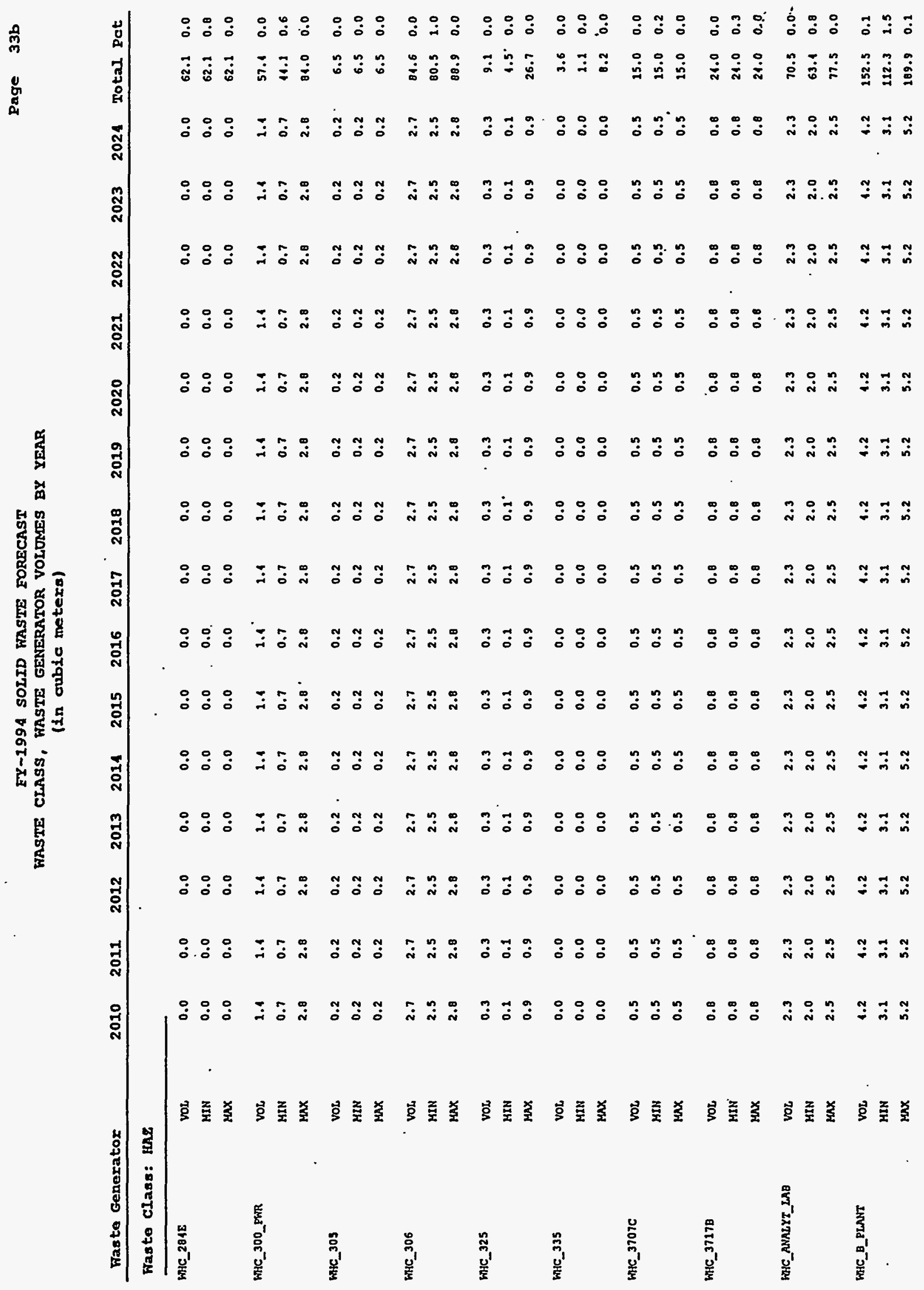


FX-1994 SOLID WASTE FORECAST

Page 34b

WASTE CLASS, WASTE GENERATOR VOLUMES BY YEAR

(in aubla meters)

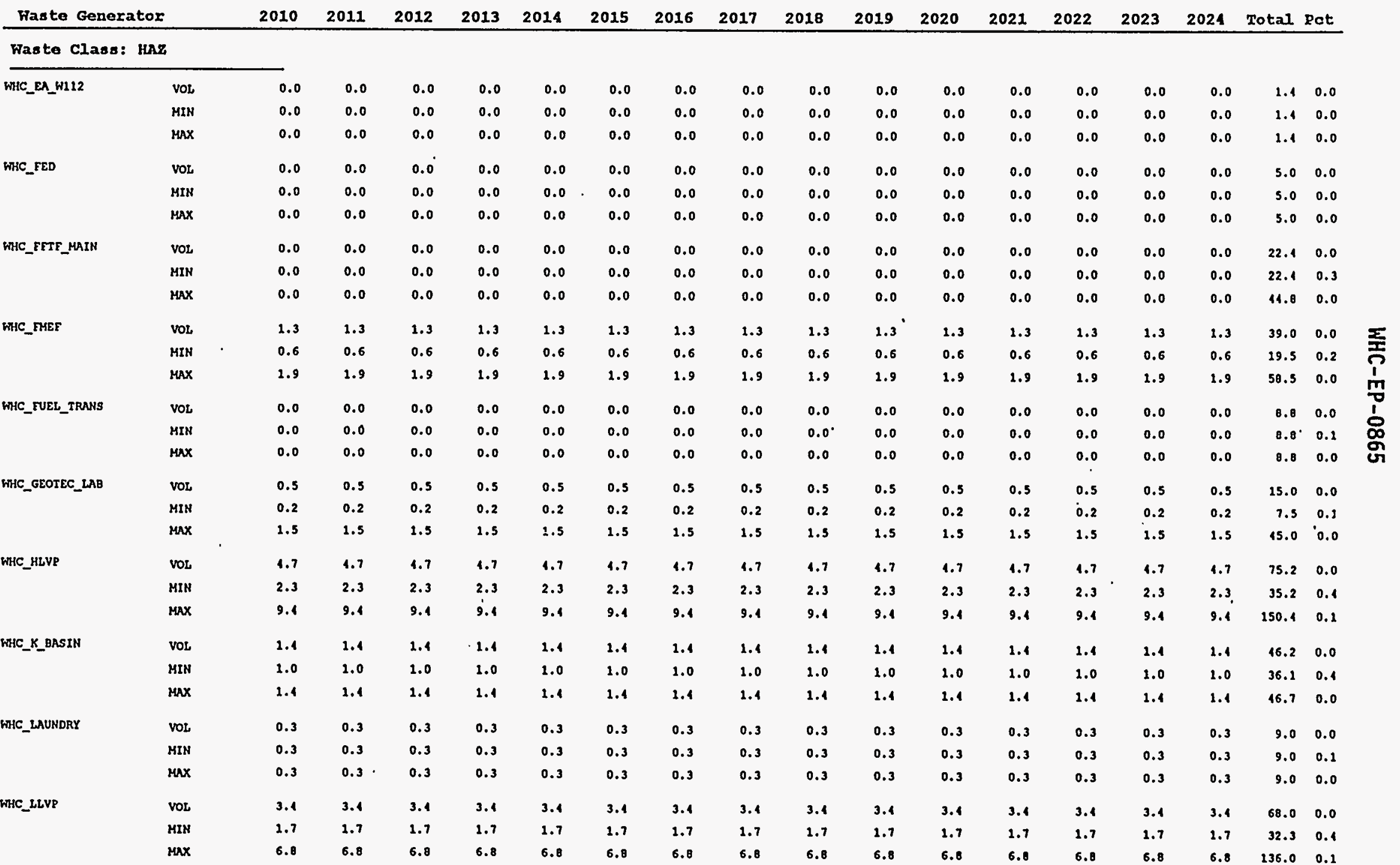




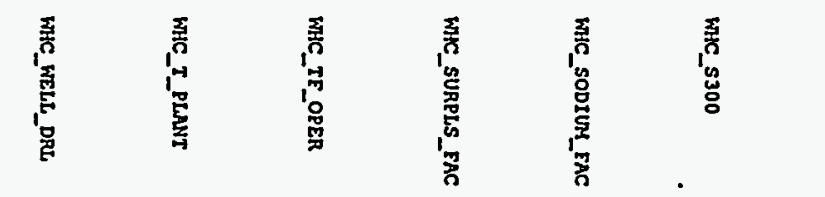

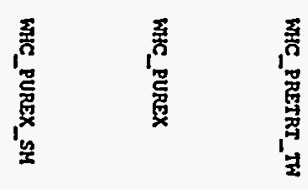

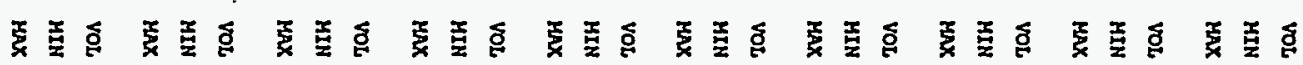

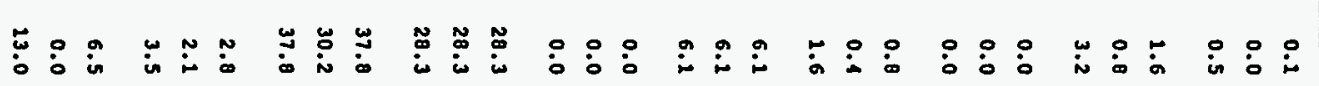

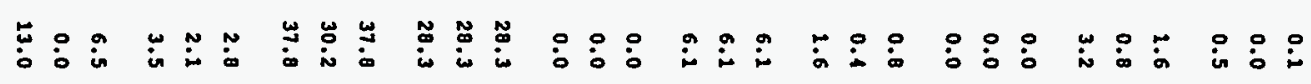

苛

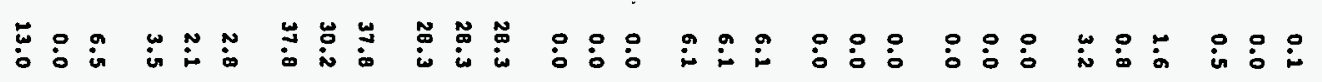

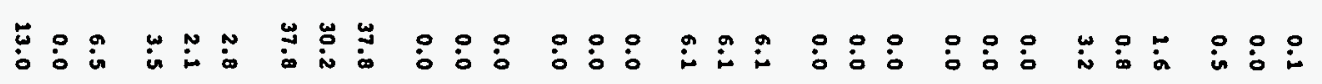

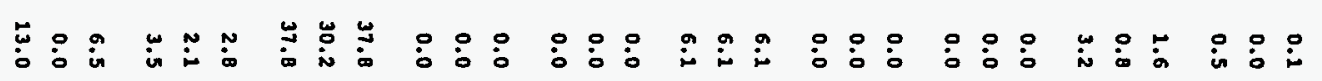

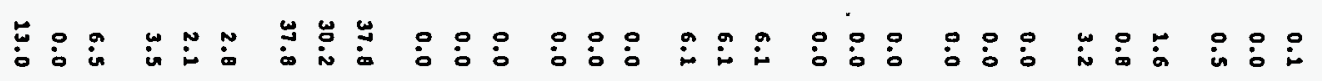

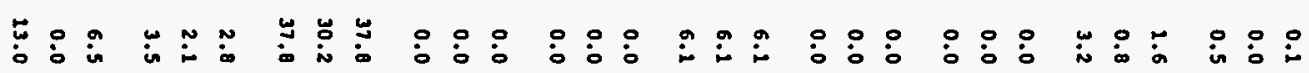

苛

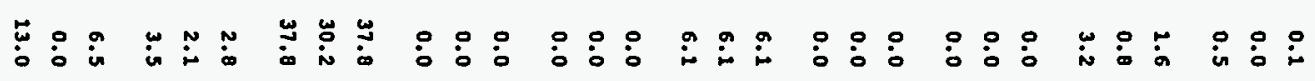

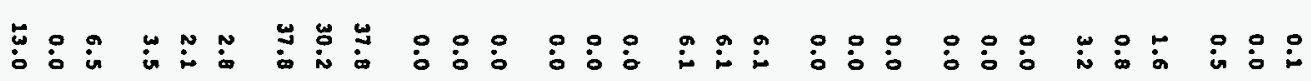

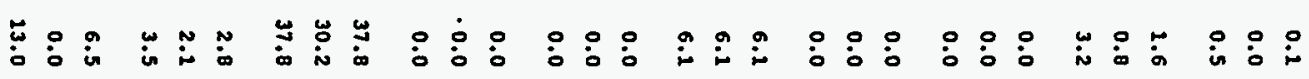

苛

苛

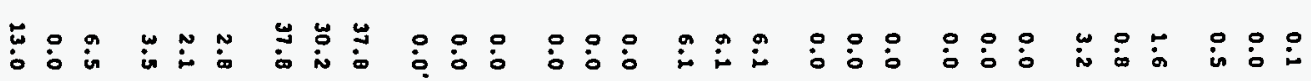

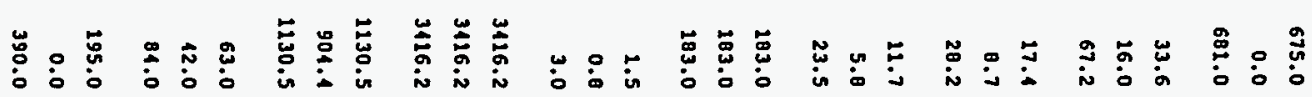

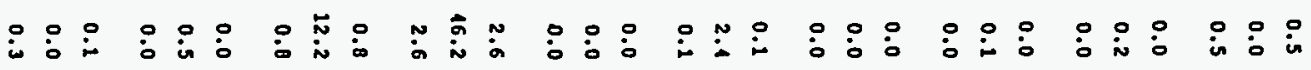


FY-1994 SOLID WASTE FORECAST

Page $36 b$

WASTE CLASS, WASTE GENERATOR VOLUMES BY YEAR

(in cubla meters)

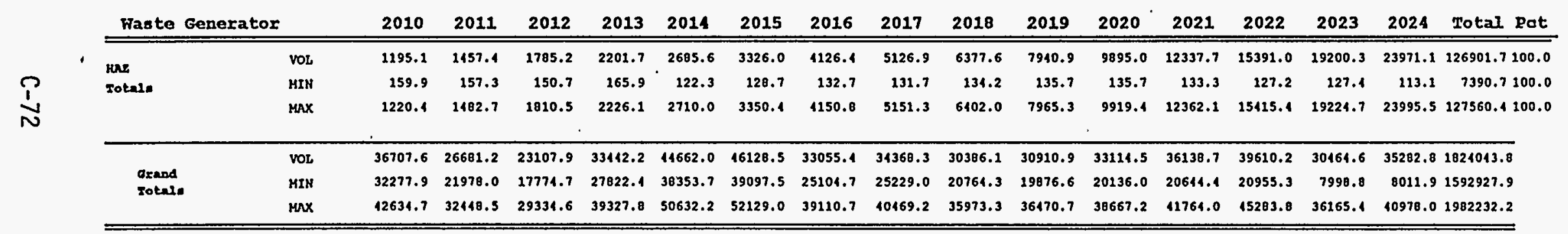


WHC-EP-0865

APPENDIX D

ANNUAL WASTE CLASS, WASTE GENERATOR VOLUMES

D- $\mathbf{i}$ 
WHC-EP-0865

Intentionally Left Blank 


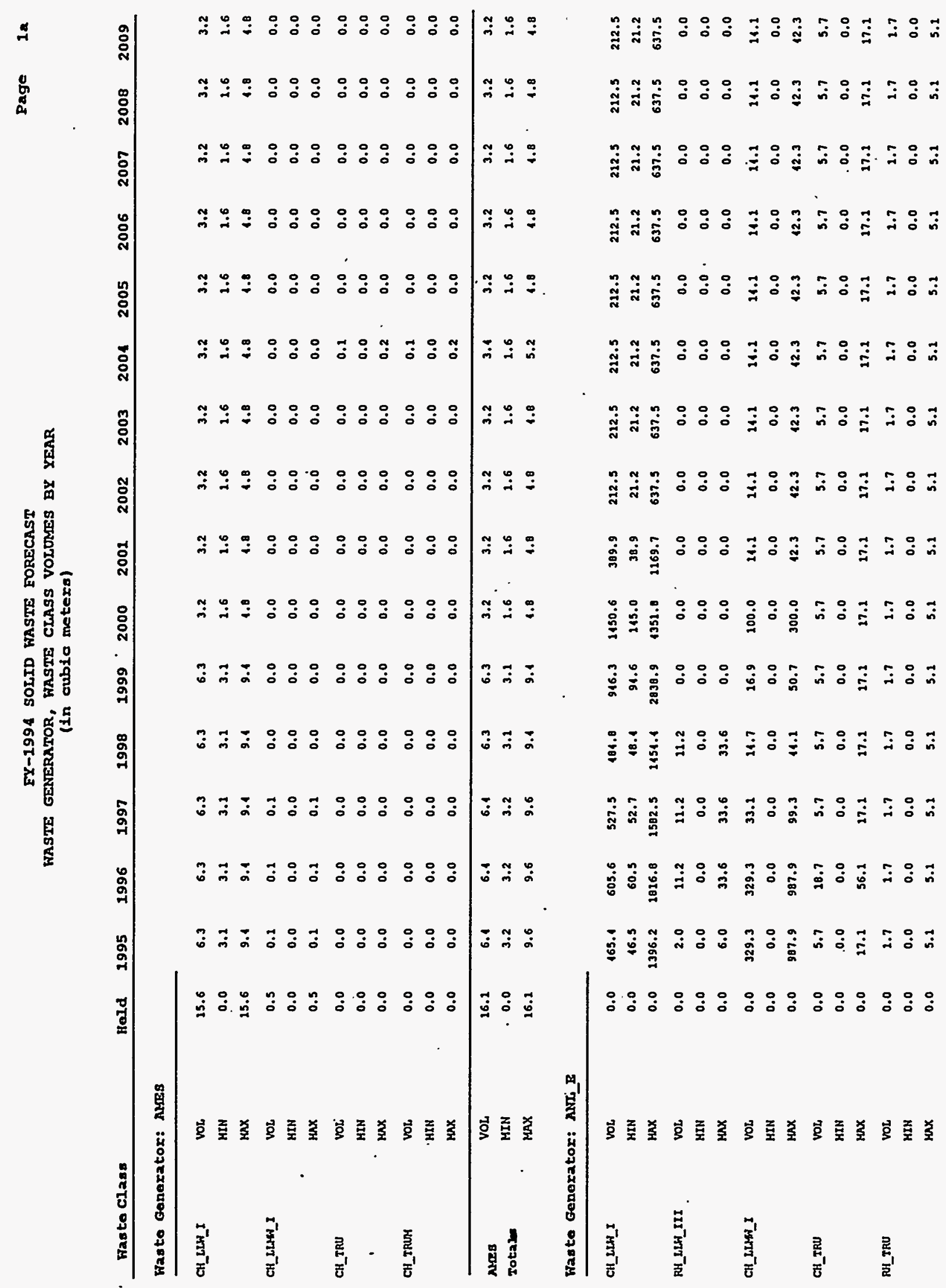


FY-1994 SOLID WASTE EORECA.ST

WASTE GENERATOR, HASTE CLASS VOLUMES BY YEAR

(in cubic metera)

\begin{tabular}{|c|c|c|c|c|c|c|c|c|c|c|c|c|c|c|c|c|c|}
\hline \multicolumn{2}{|c|}{ Was to Class } & Held & 1995 & 1996 & 1997 & 1998 & 1999 & 2000 & 2001 & 2002 & 2003 & 2004 & 2005 & 2006 & 2007 & 2008 & 2009 \\
\hline \multicolumn{18}{|c|}{ Fas to Generator: ANL_B } \\
\hline \multirow[t]{2}{*}{ CH_IRUM } & vot & 0.0 & 0.3 & 0.3 & 0.3 & 0.3 & 0.3 & 0.3 & 0.3 & 0.3 & 0.3 & 0.3 & 0.3 & 0.3 & 0.3 & 0.3 & 0.3 \\
\hline & $\max$ & 0.0 & 0.9 & 0.9 & 0.9 & 0.9 & 0.9 & 0.9 & 0.9 & 0.9 & 0.9 & 0.9 & 0.9 & 0.9 & 0.9 & 0.9 & 0.9 \\
\hline \multirow{2}{*}{$\begin{array}{l}\text { ANL_X } \\
\text { Total= }\end{array}$} & VoL & 0.0 & 001.4 & 966.8 & 379.5 & 518.4 & 970.9 & 1558.3 & 111.7 & 234.3 & 234.3 & 234.3 & 234.3 & 234.3 & 234.3 & 234.3 & 234.3 \\
\hline & $\operatorname{MIN}$ & 0.0 & 16.5 & 60.5 & 52.7 & 48.1 & 94.6 & 145.0 & 38.9 & 21.2 & 21.2 & 21.2 & 21.2 & 21.2 & 21.2 & 21.2 & 21.2 \\
\hline
\end{tabular}

Wasto Genorator: BAPL

\begin{tabular}{|c|c|c|c|c|c|c|c|c|c|c|c|c|c|c|c|c|c|}
\hline \multirow[t]{3}{*}{ CH_LLH_III } & VOL & 0.0 & 55.2 & 23.0 & 23.0 & 23.0 & 14.6 & 14.6 & 0.0 & 0.0 & 0.0 & 0.0 & 0.0 & 0.0 & 0.0 & 0.0 & 0.0 \\
\hline & MIN & 0.0 & 24.3 & 5.9 & 5.9 & 5.9 & 3.6 & 3.8 & 0.0 & 0.0 & 0.0 & 0.0 & 0.0 & 0.0 & 0.0 & 0.0 & 0.0 \\
\hline & $\max$ & 0.0 & 35.2 & 23.0 & 23.0 & 23.0 & 14.6 & 14.6 & 0.0 & 0.0 & 0.0 & 0.0 & 0.0 & 0.0 & 0.0 & 0.0 & 0.0 \\
\hline \multirow[t]{3}{*}{ CH_LLHA_I } & vot & 0.0 & 10.9 & 34.9 & 28.6 & 19.5 & 19.5 & 19.5 & 2.6 & 2.6 & 2.6 & 2.6 & 2.6 & 2.6 & 2.6 & 2.6 & 2.6 \\
\hline & MIN & 0.0 & 5.4 & 17.4 & 14.3 & 9.7 & 9.7 & 9.7 & 1.3 & 1.3 & 1.3 & 1.3 & 1.3 & 1.3 & 1.3 & 1.3 & 1.3 \\
\hline & $\max$ & 0.0 & 10.9 & 34.9 & 28.6 & 29.5 & 19.5 & 19.5 & 2.6 & 2.6 & 2.6 & 2.6 & 2.6 & 2.6 & 2.6 & 2.6 & 2.6 \\
\hline \multirow{2}{*}{$\begin{array}{l}\text { BAPI } \\
\text { sotzl. }\end{array}$} & VOL & 0.0 & 66.1 & 57.9 & 31.6 & 12.5 & 34.1 & 31.1 & 2.6 & 2.6 & 2.6 & 2.6 & 2.6 & 2.6 & 2.6 & 2.6 & 2.6 \\
\hline & MIN & 0.0 & 19.8 & 23.4 & 20.2 & 15.7 & 13.5 & 13.5 & 1.3 & 1.3 & 1.3 & 1.3 & 1.3 & 1.3 & 1.3 & 1.3 & 1.3 \\
\hline
\end{tabular}

Fas te Gonerator: BAPL_SHIPXDs

\begin{tabular}{|c|c|c|c|c|c|c|c|c|c|c|c|c|c|c|c|c|c|}
\hline \multirow[t]{3}{*}{ CH_LLH_I } & VOL & 0.0 & 1.5 & 0.0 & 0.0 & 0.0 & 0.0 & 0.0 & 0.0 & 0.0 & 0.0 & 0.0 & 0.0 & 0.0 & 0.0 & 0.0 & 0.0 \\
\hline & MIN & 0.0 & 0.0 & 0.0 & 0.0 & 0.0 & 0.0 & 0.0 & 0.0 & 0.0 & 0.0 & 0.0 & 0.0 & 0.0 & 0.0 & 0.0 & 0.0 \\
\hline & $\max$ & 0.0 & 4.5 & 0.0 & 0.0 & 0.0 & 0.0 & 0.0 & 0.0 & 0.0 & 0.0 & 0.0 & 0.0 & 0.0 & 0.0 & 0.0 & 0.0 \\
\hline \multirow{3}{*}{$\begin{array}{l}\text { BAPL_BHIPYDB } \\
\text { Tatal. }\end{array}$} & Vor & 0.0 & 1.5 & 0.0 & 0.0 & 0.0 & 0.0 & 0.0 & 0.0 & 0.0 & 0.0 & 0.0 & 0.0 & 0.0 & 0.0 & 0.0 & 0.0 \\
\hline & MIN & 0.0 & 0.0 & 0.0 & 0.0 & 0.0 & 0.0 & 0.0 & 0.0 & 0.0 & 0.0 & 0.0 & 0.0 & 0.0 & 0.0 & 0.0 & 0.0 \\
\hline & MAX & 0.0 & 4.5 & 0.0 & 0.0 & 0.0 & 0.0 & 0.0 & 0.0 & 0.0 & 0.0 & 0.0 & 0.0 & 0.0 & 0.0 & 0.0 & 0.0 \\
\hline
\end{tabular}

Waste Generator: BAI_CLBS_LAB

\begin{tabular}{|c|c|c|c|c|c|c|c|c|c|c|c|c|c|c|c|c|c|}
\hline \multirow{3}{*}{ CH_LLH_I } & vor & 0.0 & 226.6 & 226.6 & 226.6 & 203.3 & 283.3 & 255.0 & 0.0 & 0.0 & 0.0 & 0.0 & 0.0 & 0.0 & 0.0 & 0.0 & 0.0 \\
\hline & MIN & 0.0 & 29.4 & 29.1 & 29.4 & 28.3 & 28.3 & 28.0 & 0.0 & 0.0 & 0.0 & 0.0 & 0.0 & 0.0 & 0.0 & 0.0 & 0.0 \\
\hline & $\max$ & 0.0 & 226.6 & 226.6 & 226.6 & 283.3 & 283.3 & 255.0 & 0.0 & 0.0 & 0.0 & 0.0 & 0.0 & 0.0 & 0.0 & 0.0 & 0.0 \\
\hline CH_LIHA_I & VOL & 0.0 & 12.6 & 12.6 & 12.6 & 12.6 & 0.0 & 0.0 & 0.0 & 0.0 & 0.0 & 0.0 & 0.0 & 0.0 & 0.0 & 0.0 & 0.0 \\
\hline
\end{tabular}


FY-1994 SOLID WASTE FORECAST

HASTE GENERATOR, HASTE CLASS VOLUMES BY YEAR

(in cuble meters)

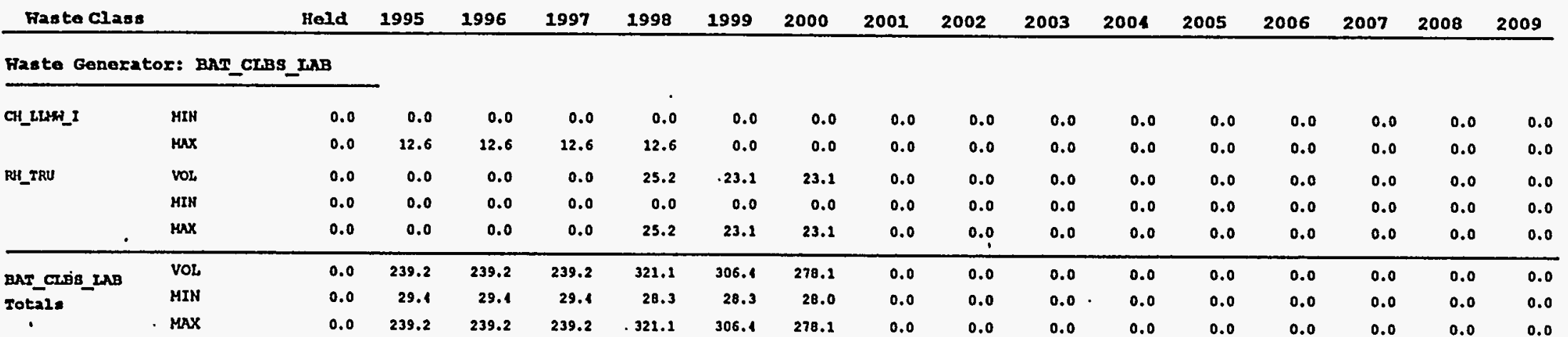

Waste Generator: BRurus

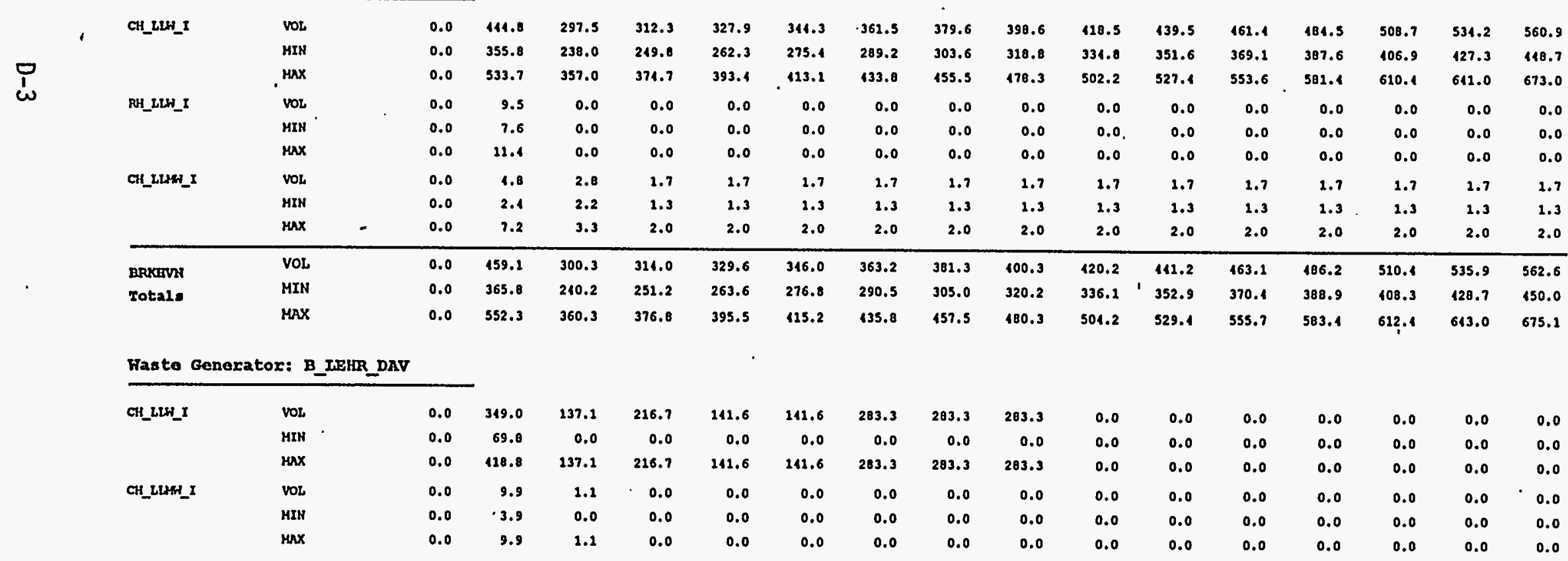


FY-1994 SOLID WASTE FORECA ST

WASTE GENERATOR, WASTT CLASS VOLUMES BY YEAR (in oublo metera)

\begin{tabular}{|c|c|c|c|c|c|c|c|c|c|c|c|c|c|c|c|c|c|}
\hline Waste Clas: & & Held & 1995 & 1996 & 1997 & 1998 & 1999 & 2000 & 2001 & 2002 & 2003 & 2004 & 2005 & 2006 & 2007 & 2008 & 2009 \\
\hline \multirow{3}{*}{$\begin{array}{l}\text { B_LRER_DAV } \\
\text { Totale }\end{array}$} & VOL & 0.0 & 350.9 & 138.2 & 216.7 & 141.6 & 141.6 & 283.3 & 203.3 & 203.3 & 0.0 & 0.0 & 0.0 & 0.0 & 0.0 & 0.0 & 0.0 \\
\hline & MIN & 0.0 & 73.7 & 0.0 & 0.0 & 0.0 & 0.0 & 0.0 & 0.0 & 0.0 & 0.0 & 0.0 & 0.0 & 0.0 & 0.0 & 0.0 & 0.0 \\
\hline & MAX & 0.0 & 128.7 & 238.2 & 226.7 & 141.6 & 141.6 & 283.3 & 283.3 & 283.3 & 0.0 & 0.0 & 0.0 & 0.0 & 0.0 & 0.0 & 0.0 \\
\hline
\end{tabular}

Fasto Generator: EGLG

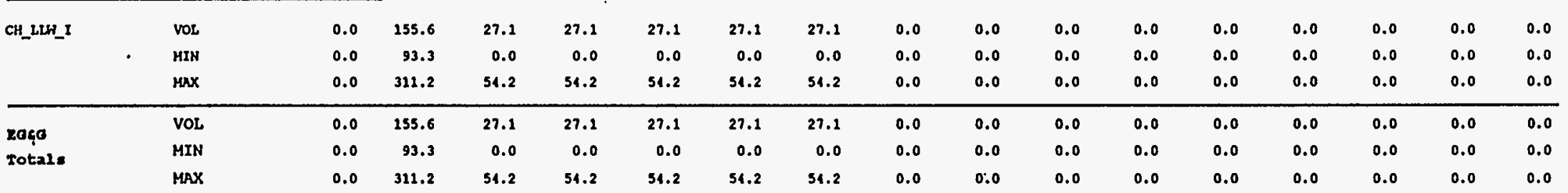

Waste Generator: FERM

\begin{tabular}{|c|c|c|c|c|c|c|c|c|c|c|c|c|c|c|c|c|c|}
\hline \multirow[t]{3}{*}{ CH_LLA_I } & VOL & 27.2 & 44.3 & 14.3 & 14.3 & 11.3 & 11.3 & 11.3 & 11.3 & 11.3 & 14.3 & 44.3 & 41.3 & 11.3 & 11.3 & 11.3 & 11.3 \\
\hline & MIN & $0.0^{\circ}$ & 22.1 & 22.1 & 22.1 & 22.1 & 22.1 & 22.1 & $22: 1$ & 22.1 & 22.1 & 22.1 & 22.1 & 22.1 & 22.1 & 22.1 & 22.1 \\
\hline & $\operatorname{MAX}$ & 27.2 & 88.6 & 88.6 & 88.6 & 88.6 & 88.6 & 08.6 & 88.6 & 88.6 & 08.6 & 08.6 & 80.6 & 89.6 & 88.6 & 88.6 & 88.6 \\
\hline \multirow[t]{2}{*}{ CH_LLW_I } & VoL & 0.0 & 2.6 & 2.6 & 2.6 & 2.6 & 2.6 & 2.6 & 2.6 & 2.6 & 2.6 & 2.6 & 2.6 & 2.6 & 2.6 & 2.6 & 2.6 \\
\hline & MAX & 0.0 & 5.2 & 5.2 & 5.2 & 5.2 & 5.2 & 3.2 & 3.2 & 5.2 & 5.2 & 3.2 & 5.2 & 5.2 & 3.2 & 5.2 & 5.2 \\
\hline \multirow{2}{*}{$\begin{array}{l}\text { FeRMY } \\
\text { Totale }\end{array}$} & VOL & 27.2 & 16.9 & 16.9 & 46.9 & 16.9 & 16.9 & 16.9 & 16.9 & 16.9 & 16.9 & 16.9 & 16.9 & 16.9 & 16.9 & 16.9 & 16.9 \\
\hline & MIN & 0.0 & 23.4 & 23.1 & 23.4 & 23.4 & 23.4 & 23.4 & 23.4 & 23.1 & 23.4 & 23.1 & 23.4 & 23.4 & 23.4 & 23.4 & 23.4 \\
\hline
\end{tabular}

Wasto Generator: FUSRAP

\begin{tabular}{|c|c|c|c|c|c|c|c|c|c|c|c|c|c|c|c|c|c|}
\hline \multirow[t]{3}{*}{ CH_LLH_I } & vor & 0.0 & 0.0 & 0.0 & 0.0 & 0.0 & 0.0 & 217.6 & 1903.7 & 1903.7 & 1903.7 & 4351.3 & 2447.6 & 0.0 & 0.0 & 0.0 & 0.0 \\
\hline & MIN & 0.0 & 0.0 & 0.0 & 0.0 & 0.0 & 0.0 & 217.6 & 1903.7 & 1903.7 & 1903.7 & 4351.3 & 2447.6 & 0.0 & 0.0 & 0.0 & 0.0 \\
\hline & $\max$ & 0.0 & 0.0 & 0.0 & 0.0 & 0.0 & 0.0 & 217.6 & 2903.7 & 1903.7 & 2903.7 & 1351.3 & 2147.6 & 0.0 & 0.0 & 0.0 & 0.0 \\
\hline \multirow[t]{3}{*}{ CH_LLN_III } & Vol. & 0.0 & 3620.1 & 3620.1 & 3620.4 & 0.0 & 0.0 & 0.0 & 0.0 & 0.0 & 0.0 & 0.0 & 0.0 & 0.0 & 0.0 & 0.0 & 0.0 \\
\hline & HIH & 0.0 & 3620.4 & 3620.1 & 3620.4 & 0.0 & 0.0 & 0.0 & 0.0 & 0.0 & 0.0 & 0.0 & 0.0 & 0.0 & 0.0 & 0.0 & 0.0 \\
\hline & $\max$ & 0.0 & 3620.4 & 3620.4 & 3620.4 & 0.0 & 0.0 & 0.0 & 0.0 & 0.0 & 0.0 & 0.0 & 0.0 & 0.0 & 0.0 & 0.0 & 0.0 \\
\hline
\end{tabular}




\begin{tabular}{|c|c|c|c|c|c|c|c|c|c|c|c|c|c|c|c|c|c|}
\hline I.2 & $r \cdot z$ & $\tau \cdot z$ & $t \cdot 2$ & $x \cdot z$ & $i \cdot 2$ & $x \cdot z$ & $t \cdot z$ & $: \cdot z$ & $\tau \cdot z$ & $\tau \cdot z$ & $t \cdot z$ & I'Z & $t \cdot 2$ & $\tau \cdot z$ & 0.0 & $x w$ & \\
\hline 0.0 & 0.0 & 0.0 & 0.0 & 0.0 & 0.0 & 0.0 & 0.0 & 0.0 & 0.0 & 0.0 & 0.0 & 0.0 & 0.0 & 0.0 & 0.0 & MIH & \\
\hline $1 \cdot \tau$ & $1 \cdot \tau$ & $\mapsto \tau$ & $\because r$ & $\cdot \cdot t$ & $\bullet \tau$ & $\cdot \tau$ & 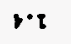 & $\cdot 1 \cdot \tau$ & $r \cdot t$ & $\cdot \cdot \tau$ & $\cdot r$ & $P \cdot \tau$ & $r \cdot r$ & $2 \cdot \tau$ & 0.0 & TON & 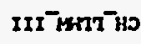 \\
\hline 0.12 & $0 . b t$ & 0.48 & 0.11 & $0 \cdot n$ & 0.15 & $0 . n$ & $0 . b \mathrm{t}$ & $0 .+1$ & $0.1 \pi$ & $0 \cdot r t$ & $0.4 \pi$ & $0 . r t$ & 0.01 & $0 . r t$ & 0.0 & $\mathbf{X N H}$ & \\
\hline 0.2 & $8 \cdot 2$ & $0 \cdot 2$ & $\theta \cdot z$. & $\theta \cdot \tau$ & 8.2 & $\theta \cdot z$ & $8 \cdot 2$ & $0 \cdot z$ & 0.2 & $g \cdot z$ & $8 \cdot 2$ & $\theta \cdot z$ & $8 \cdot z$ & $\theta \cdot z$ & 0.0 & MIH & \\
\hline $8 \cdot z$ & $\theta \cdot 2$ & $\theta \cdot z$ & $8 \cdot 2$ & $9 \cdot z$ & 8.2 & $\theta \cdot z$ & $8 \cdot z$ & $B \cdot z$ & $\theta \cdot \tau$ & $\theta \cdot z$ & $\theta \cdot \tau$ & 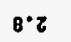 & $0 \cdot 2$ & $\theta \cdot z$ & 0.0 & zon & І \\
\hline
\end{tabular}

Td4X :

\begin{tabular}{|c|c|c|c|c|c|c|c|c|c|c|c|c|c|c|c|c|c|}
\hline $2 \cdot z$ & $z \cdot z$ & $\tau \cdot z$ & $z \cdot z$ & $z \cdot z$ & $z \cdot z$ & $2 \cdot 2$ & $z \cdot z$ & $z \cdot z$ & $2 \cdot z$ & $z \cdot z$ & $z^{\circ} \cdot \tau$ & $\tau \cdot \tau$ & $\tau \cdot z$ & $2 \cdot z$ & 0.0 & $x \forall W$ & \\
\hline $2 \cdot 0$ & $2 \cdot 0$ & $2 \cdot 0$ & $2 \cdot 0$ & $z \cdot 0$ & $2 \cdot 0$ & $2 \cdot 0$ & $z \cdot 0$ & $2 \cdot 0$ & $2 \cdot 0$ & $z \cdot 0$ & $2 \cdot 0$ & $z \cdot 0$ & $2 \cdot 0$ & $2 \cdot 0$ & 0.0 & NIW & - TEFOI \\
\hline 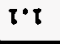 & $\tau \cdot \tau$ & $t \cdot r$ & I.T & T't & $\tau \cdot \tau$ & $\tau \cdot \tau$ & $\tau \cdot \tau$ & $\tau \cdot \tau$ & $x \cdot t$ & $\tau \cdot \tau$ & $t \cdot \tau$ & $\tau \cdot \tau$ & $\tau \cdot \tau$ & $\tau \cdot \tau$ & 0.0 & TOA & AHYB \\
\hline$i \cdot z$ & $z \cdot z$ & $2 \cdot 2$ & $z \cdot z$ & $z \cdot \tau$ & $z \cdot z$ & $2 \cdot 2$ & $2 \cdot 2$ & $i^{\prime} z$ & $z \cdot z$ & $z \cdot z$ & $z \cdot z$ & $z \cdot \tau$ & $2 \cdot 2$ & $z \cdot z$ & 0.0 & $x \mathbf{W H}$ & \\
\hline $2 \cdot 0$ & $2 \cdot 0$ & $2 \cdot 0$ & $2 \cdot 0$ & $2 \cdot 0$ & 2.0 & 200 & 2.0 & $2 \cdot 0$ & $z \cdot 0$ & 200 & $2 \cdot 0$ & $z \cdot 0$ & $2 \cdot 0$ & 200 & 0.0 & NIH & \\
\hline$\tau$ & $\tau \cdot \boldsymbol{\tau}$ & $\tau \cdot \tau$ & $\boldsymbol{t} \cdot \tau$ & $\tau \cdot \tau$ & $\boldsymbol{I} \cdot \boldsymbol{\tau}$ & $t \cdot t$ & $\tau \cdot \tau$ & $\tau \cdot \tau$ & $\tau \cdot \tau$ & $\tau \cdot \tau$ & $t \cdot t$ & $\tau \cdot \tau$ & $\tau \cdot \tau$ & $\tau \cdot \tau$ & 0.0 & IOA & \\
\hline
\end{tabular}

1
0
0
0
1
0
1
1
1.
至

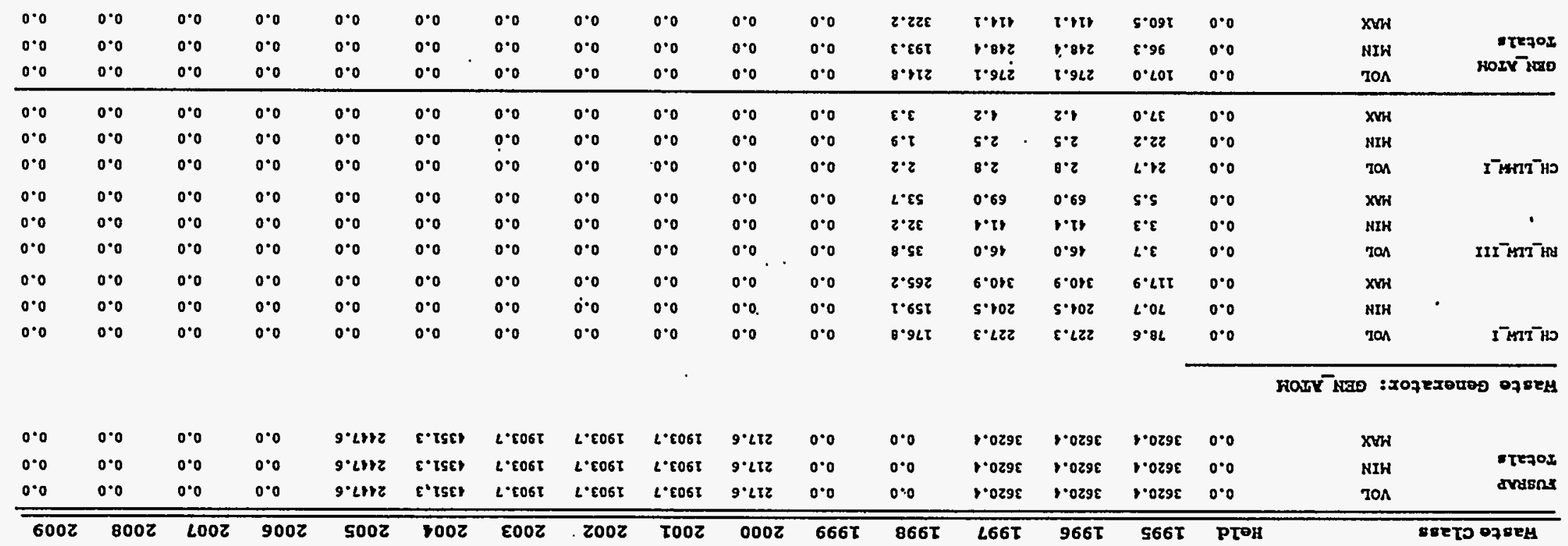

es s5ea

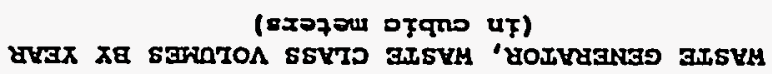

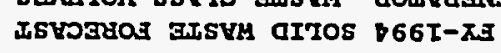


FY-1994 SOLID HASTE FORECAST

WASTE GENERATOR, WASTE CLASS VOLUMES BY YEAR

(in cubio metera)

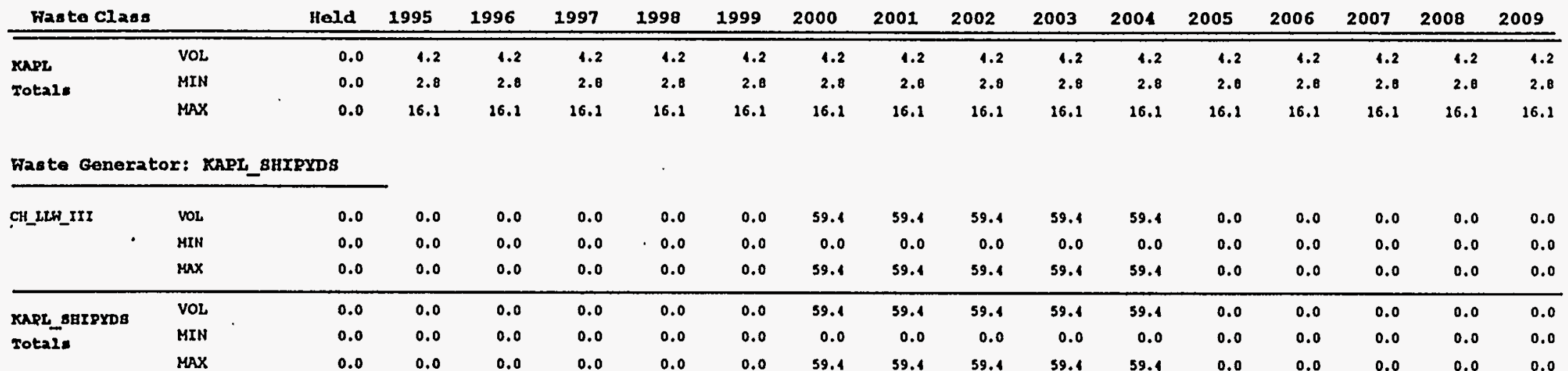

Waste Generator: KEH_100

\begin{tabular}{|c|c|c|c|c|c|c|c|c|c|c|c|c|c|c|c|c|c|c|}
\hline \multirow[t]{3}{*}{ CH_LLH_I } & vos & & 0.0 & 76.9 & 0.0 & 0.0 & 0.0 & 0.0 & 0.0 & 0.0 & 0.0 & 0.0 & 0.0 & 0.0 & 0.0 & 0.0 & 0.0 & 0.0 \\
\hline & MIN $\cdot$ & & 0.0 & 53.8 & 0.0 & 0.0 & 0.0 & 0.0 & 0.0 & 0.0 & 0.0 & 0.0 & 0.0 & 0.0 & 0.0 & 0.0 & 0.0 & 0.0 \\
\hline & $\max$ & & 0.0 & .. 76.9 & 0.0 & 0.0 & 0.0 & 0.0 & 0.0 & 0.0 & 0.0 & 0.0 & 0.0 & 0.0 & 0.0 & 0.0 & 0.0 & 0.0 \\
\hline \multirow[t]{3}{*}{ CH_LLWA_I } & VoL & & 0.0 & 11.3 & 0.0 & 0.0 & 0.0 & 0.0 & 0.0 & 0.0 & 0.0 & 0.0 & 0.0 & 0.0 & 0.0 & 0.0 & 0.0 & 0.0 \\
\hline & HIN & & 0.0 & 0.9 & 0.0 & 0.0 & 0.0 & 0.0 & 0.0 & 0.0 & 0.0 & 0.0 & 0.0 & 0.0 & 0.0 & 0.0 & 0.0 & 0.0 \\
\hline & $\operatorname{sax}$ & & 0.0 & 11.3 & 0.0 & 0.0 & 0.0 & 0.0 & 0.0 & 0.0 & 0.0 & 0.0 & 0.0 & 0.0 & 0.0 & 0.0 & 0.0 & 0.0 \\
\hline \multirow[t]{3}{*}{ Hne } & VoL & & 0.0 & 36.9 & 46.2 & 37.7 & 72.1 & 90.1 & 112.6 & 140.8 & 176.0 & 220.0 & 275.0 & 343.8 & 429.7 & 537.1 & 671.4 & 839.3 \\
\hline & MIN & & 0.0 & 36.9 & 36.0 & 36.9 & 36.7 & 36.9 & 37.1 & 36.6 & 36.9 & 37.4 & 35.7 & 37.8 & 38.6 & 37.6 & 33.5 & 33.5 \\
\hline & $\max$ & - & 0.0 & 10.5 & 16.1 & 57.7 & 72.1 & 90.1 & 112.6 & 110.8 & 176.0 & 220.0 & 275.0 & 343.8 & 129.7 & 537.1 & 671.4 & 839.3 \\
\hline \multirow{3}{*}{$\begin{array}{l}\text { KR日_100 } \\
\text { Total. }\end{array}$} & VOL & & 0.0 & 125.1 & 16.1 & 57.7 & 72.1 & 90.1 & 212.6 & 160.8 & 176.0 & 220.0 & 275.0 & 343.0 & 429.7 & 537.1 & 671.4 & 839.3 \\
\hline & MIN & & 0.0 & 91.6 & 36.0 & 36.9 & 36.7 & 36.9 & 37.1 & 36.6 & 36.9 & 37.1 & 35.7 & 37.8 & 30.6 & 37.6 & 33.5 & 33.5 \\
\hline & $\operatorname{MAX}$ & & 0.0 & 128.7 & 16.1 & 57.7 & 72.1 & 90.1 & 112.6 & 140.8 & 176.0 & 220.0 & 275.0 & 343.8 & 129.7 & 537.1 & 671.1 & 839.3 \\
\hline
\end{tabular}

Waste Generator: IBL

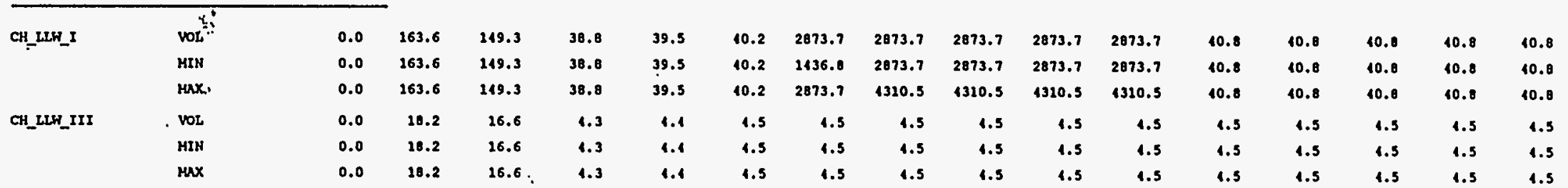


FX-1994 SOLID WASTE FORECAST

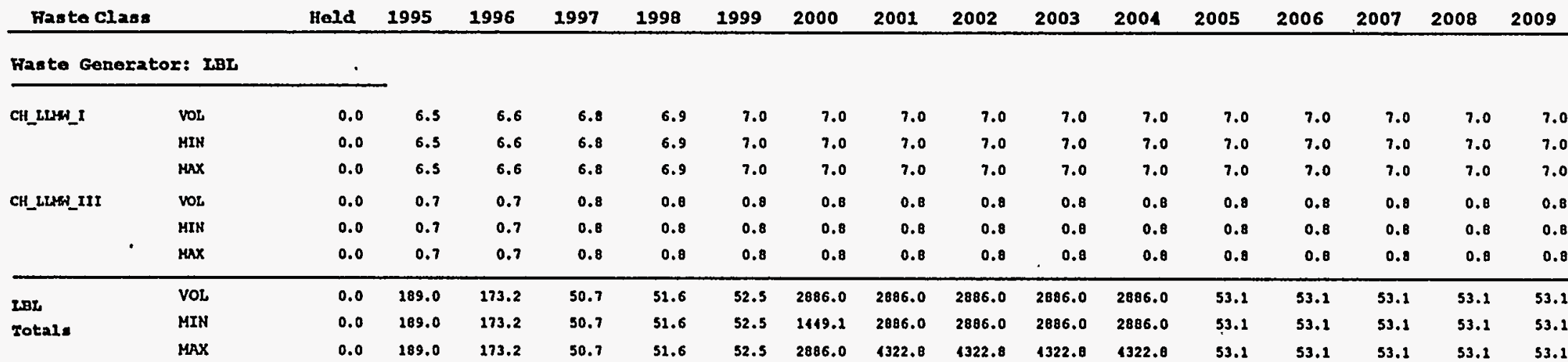

Faste Generator: MIT_BATzS

\begin{tabular}{|c|c|c|c|c|c|c|c|c|c|c|c|c|c|c|c|c|c|}
\hline \multirow[t]{3}{*}{ CH_LLH_I } & vor & 0.0 & 0.0 & 0.0 & 0.0 & 0.0 & 0.0 & 0.3 & 0.0 & 0.0 & 0.0 & 0.0 & 0.3 & 0.0 & 0.0 & 0.0 & 0.0 \\
\hline & MIH & 0.0 & 0.0 & 0.0 & 0.0 & 0.0 & 0.0 & 0.3 & 0.0 & 0.0 & 0.0 & 0.0 & 0.3 & 0.0 & 0.0 & 0.0 & 0.0 \\
\hline & $\max$ & 0.0 & 0.0 & 0.0 & $0.0^{\circ}$ & 0.0 & 0.0 & 0.6 & 0.0 & 0.0 & 0.0 & 0.0 & 0.6 & 0.0 & 0.0 & 0.0 & 0.0 \\
\hline \multirow{3}{*}{$\begin{array}{l}\text { MIT_BATzs } \\
\text { zotal. }\end{array}$} & VOL & 0.0 & 0.0 & 0.0 & 0.0 & 0.0 & 0.0 & 0.3 & 0.0 & 0.0 & 0.0 & 0.0 & 0.3 & 0.0 & 0.0 & 0.0 & 0.0 \\
\hline & MIN & 0.0 & 0.0 & 0.0 & 0.0 & 0.0 & 0.0 & 0.3 & 0.0 & 0.0 & 0.0 & 0.0 & 0.3 & 0.0 & 0.0 & 0.0 & 0.0 \\
\hline & $\operatorname{MAX}$ & 0.0 & 0.0 & 0.0 & 0.0 & 0.0 & 0.0 & 0.6 & 0.0 & 0.0 & 0.0 & 0.0 & 0.6 & 0.0 & 0.0 & 0.0 & 0.0 \\
\hline
\end{tabular}

Has to Genoratox: PADUCAH_ES

\begin{tabular}{|c|c|c|c|c|c|c|c|c|c|c|c|c|c|c|c|c|c|}
\hline \multirow[t]{3}{*}{ CH_LLH_I } & VoL & 1998.3 & 256.4 & 156.4 & 156.4 & 156.4 & 156.1 & 156.1 & 256.4 & 156.4 & 136.4 & 156.4 & 156.1 & 156.4 & 156.1 & 156.4 & 156.1 \\
\hline & HIN & 0.0 & 78.2 & 78.2 & 78.2 & 78.2 & 78.2 & 78.2 & 70.2 & 78.2 & 78.2 & 78.2 & 78.2 & 78.2 & 78.2 & 78.2 & 78.2 \\
\hline & $\max$ & 1998.3 & 231.6 & 234.6 & 234.6 & 234.6 & 234.6 & 234.6 & 234.6 & 234.6 & 234.6 & 234.6 & 234.6 & 234.6 & 234.6 & 234.6 & 234.6 \\
\hline \multirow[t]{3}{*}{ CH_LLWW_I } & vor & 32.4 & 170.0 & 170.0 & 170.0 & 170.0 & 170.0 & 170.0 & 170.0 & 270.0 & 170.0 & 170.0 & 170.0 & 170.0 & 270.0 & 170.0 & 170.0 \\
\hline & HIN & 0.0 & 85.0 & 05.0 & 05.0 & 85.0 & 85.0 & 85.0 & 85.0 & 85.0 & 85.0 & 85.0 & 85.0 & B5.0 & 85.0 & 85.0 & 85.0 \\
\hline & $\max$ & 32.4 & 255.0 & 235.0 & 255.0 & 255.0 & 255.0 & 255.0 & 255.0 & 255.0 & 255.0 & 255.0 & 235.0 & 255.0 & 255.0 & 255.0 & 255.0 \\
\hline \multirow{3}{*}{$\begin{array}{l}\text { PADUCAR_z8 } \\
\text { Total: }\end{array}$} & VOL & 2030.7 & 326.1 & 326.4 & 326.4 & 326.4 & 326.1 & 326.4 & 326.4 & 326.4 & 326.4 & 326.1 & 326.1 & 326.1 & 326.1 & 326.1 & 326.4 \\
\hline & MIN & 0.0 & 163.2 & 163.2 & 163.2 & 163.2 & 163.2 & 163.2 & 163.2 & 163.2 & 263.2 & 163.2 & 163.2 & 163.2 & 163.2 & 163.2 & 163.2 \\
\hline & $\operatorname{MAX}$ & 2030.7 & 489.6 & 189.6 & 189.6 & 489.6 & 189.6 & 189.6 & 189.6 & 489.6 & 189.6 & 189.6 & 489.6 & 189.6 & 489.6 & 189.6 & 489.6 \\
\hline
\end{tabular}




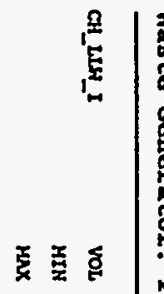

$\therefore:$

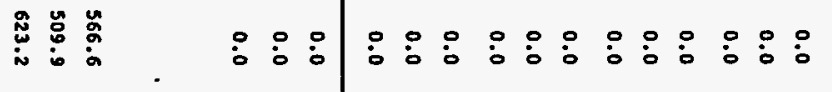

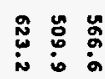

$\therefore: 0$

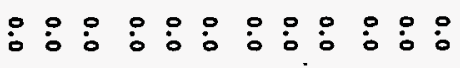

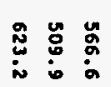

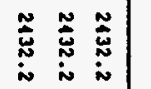

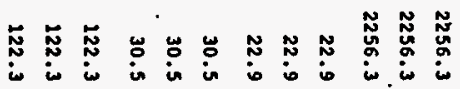

芯䒺

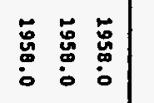

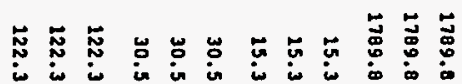

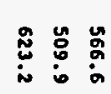

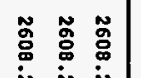

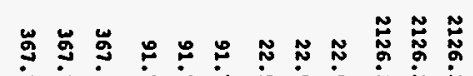

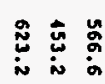

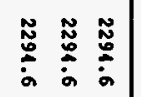

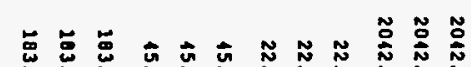

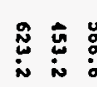

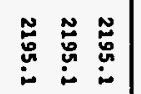

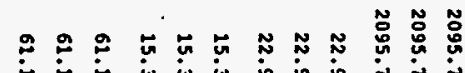

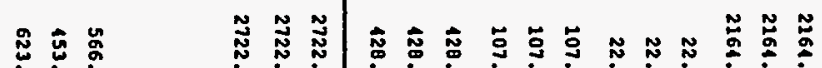

$\stackrel{2}{*}$

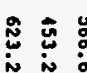

总芯总

这

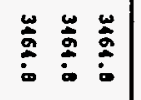

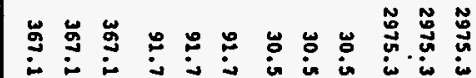

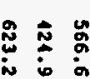

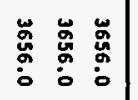

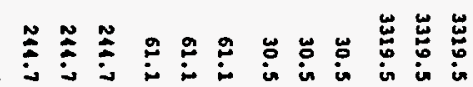

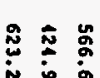

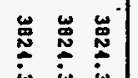

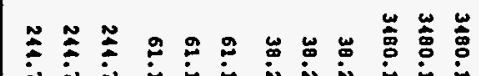

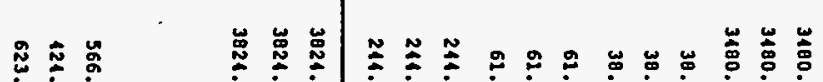

¿.:

密

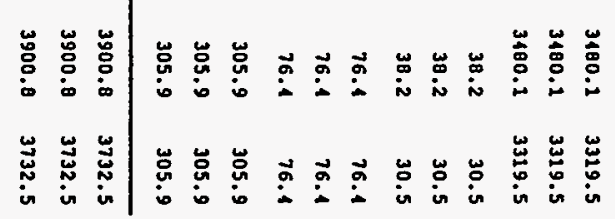

:

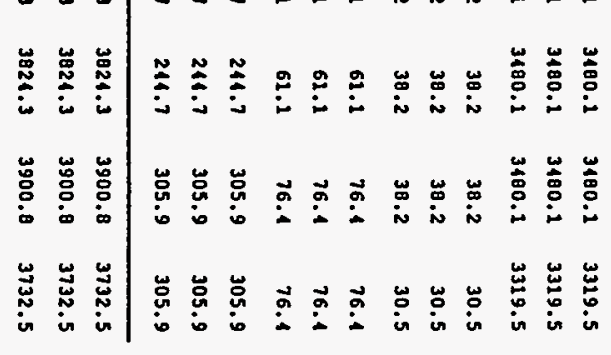

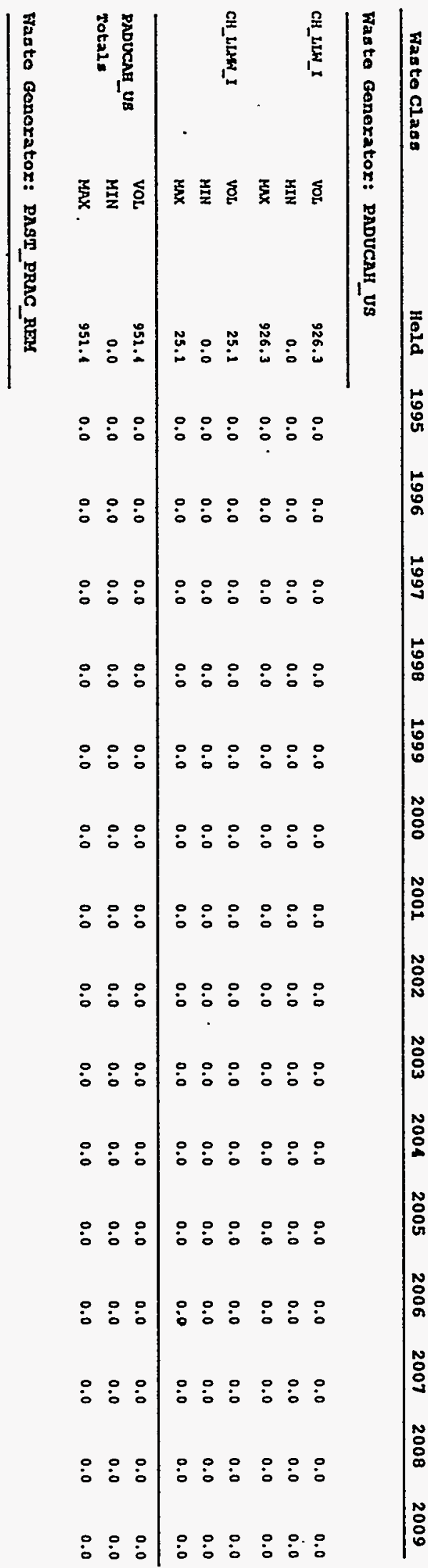

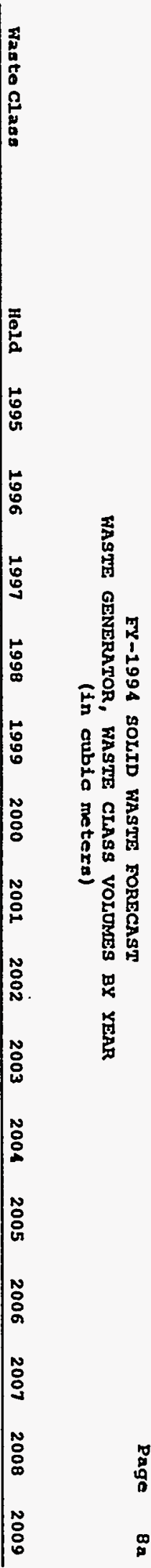


FX-1994 SOLID FASTE FORECAST

WASTE GENERATOR, HASTE CLASS VOLUMES BY YEAR (in cubia meters)

Haste class

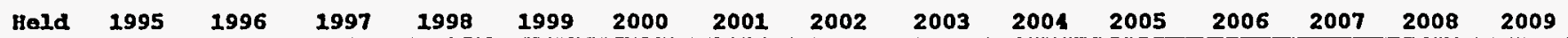

Faste Generator: PNT

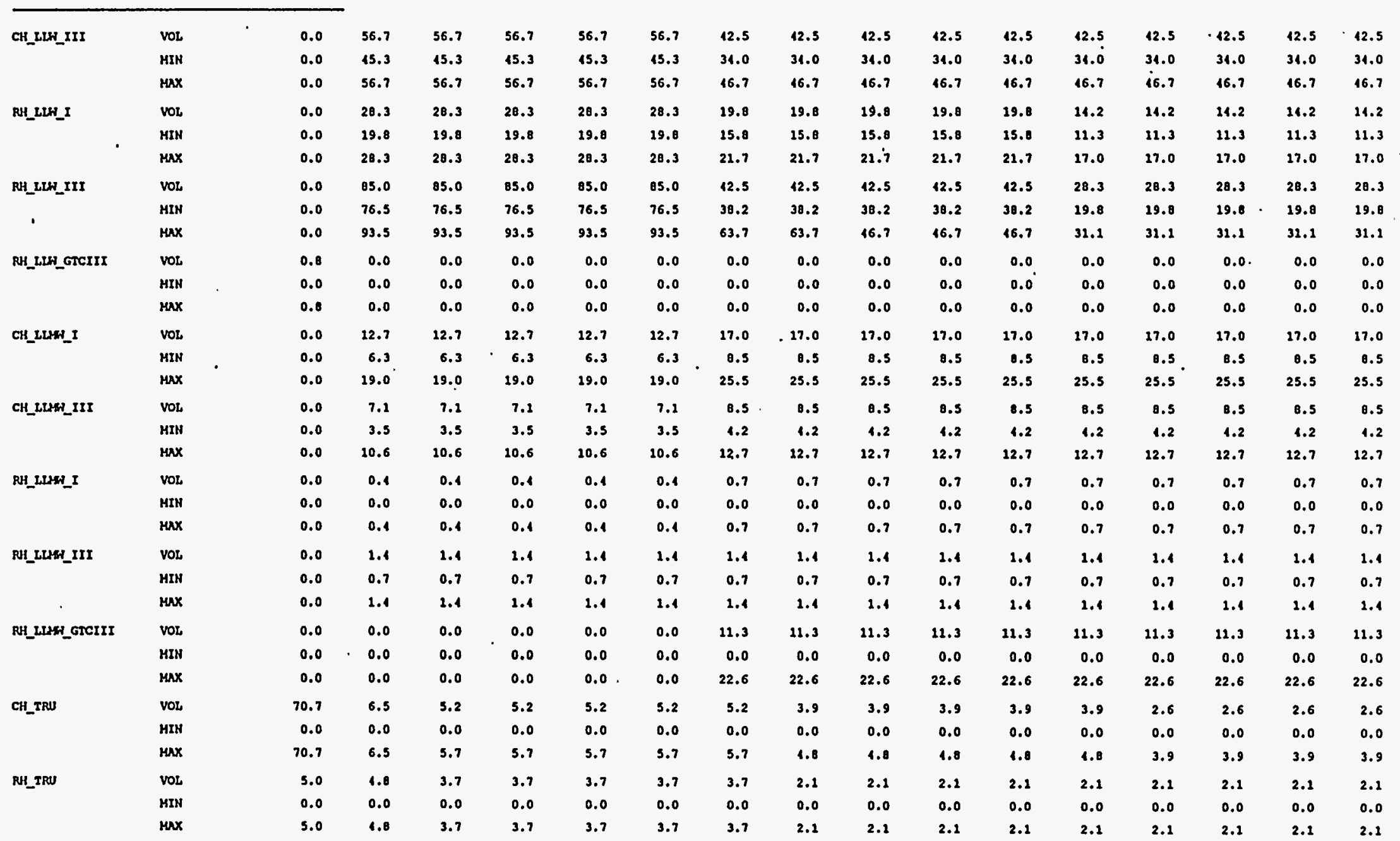


FY-1994 SOLID HASTE FORECAST

WASTE GENERATOR, HASTE CLASS VOLUMES BY YEAR

(in cubia meter:)

\begin{tabular}{|c|c|c|c|c|c|c|c|c|c|c|c|c|c|c|c|c|c|c|}
\hline \multicolumn{3}{|c|}{ Waste Class } & Held & 1995 & 1996 & 1997 & 1998 & 1999 & 2000 & 2001 & 2002 & 2003 & 2004 & 2005 & 2006 & 2007 & 2008 & 2009 \\
\hline \multicolumn{19}{|c|}{ Faste Genorator: PNI } \\
\hline \multirow{2}{*}{ CH_TRUM } & & voL & 0.0 & 0.5 & 0.5 & 0.5 & 0.5 & 0.5 & 0.0 & 0.0 & 0.0 & 0.0 & 0.0 & 0.0 & 0.0 & 0.0 & 0.0 & 0.0 \\
\hline & & $\max$ & 0.0 & 0.5 & 0.5 & 0.5 & 0.5 & 0.5 & 0.0 & 0.0 & 0.0 & 0.0 & 0.0 & 0.0 & 0.0 & 0.0 & 0.0 & 0.0 \\
\hline \multirow[t]{3}{*}{ RH_TRUM } & & vot & 0.0 & 1.2 & 1.2 & 4.2 & 1.2 & 1.2 & 4.2 & 4.2 & 4.2 & 1.2 & 1.2 & 4.2 & 0.0 & 0.0 & 0.0 & 0.0 \\
\hline & & HIN & 0.0 & 0.0 & 0.0 & 0.0 & 0.0 & 0.0 & 0.0 & 0.0 & 0.0 & 0.0 & 0.0 & 0.0 & 0.0 & 0.0 & 0.0 & 0.0 \\
\hline & & $\max$ & 0.0 & 4.6 & 1.6 & 1.6 & 1.6 & 4.6 & 1.6 & 4.6 & 4.6 & 1.6 & 1.6 & 1.6 & 0.0 & 0.0 & 0.0 & 0.0 \\
\hline \multirow{3}{*}{$\begin{array}{l}\text { PNR } \\
\text { Total. }\end{array}$} & & VoL & 76.5 & 711.2 & 271.8 & 771.8 & 771.8 & 711.8 & 723.1 & 720.5 & 720.5 & 720.5 & 720.5 & 100.7 & 695.2 & 695.2 & 695.2 & 695.2 \\
\hline & & MIN & 0.0 & 662.2 & 662.2 & 662.2 & 662.2 & 662.2 & 554.8 & 354.8 & 554.8 & 554.8 & 554.0 & 503.5 & 503.5 & 503.5 & 503.5 & 503.5 \\
\hline & & $\operatorname{MAX}$ & 76.5 & 849.6 & 847.8 & 847.8 & 047.8 & 847.8 & 832.5 & 830.0 & 813.0 & 823.0 & 823.0 & 792.7 & 707.1 & 787.1 & 787.1 & 787.1 \\
\hline
\end{tabular}

Waste Generator: PORTSMOUTh_Es

1
0
0

\begin{tabular}{|c|c|c|c|c|c|c|c|c|c|c|c|c|c|c|c|c|c|}
\hline \multirow{3}{*}{ CH_ILW__I } & vor & 0.0 & 360.3 & 368.3 & 368.3 & 368.3 & 368.3 & 674.7 & 674.7 & 674.7 & 674.7 & 674.7 & 674.7 & 674.7 & 674.7 & 674.7 & 674.7 \\
\hline & MIN & 0.0 & 368.3 & 368.3 & 368.3 & 360.3 & 360.3 & 674.7 & 674.7 & 674.7 & 674.7 & 674.7 & 674.7 & 674.7 & 674.7 & 674.7 & 674.7 \\
\hline & $\operatorname{HaX}$ & 0.0 & 412.9 & 112.9 & 441.9 & 141.9 & 111.9 & 609.6 & 809.6 & 009.6 & 809.6 & 809.6 & 809.6 & 809.6 & 809.6 & 809.6 & 809.6 \\
\hline \multirow[t]{3}{*}{ CH_LLHF_I } & Vol & 0.0 & 0.0 & 0.0 & 0.0 & 0.0 & 0.0 & 309.2 & 309.2 & 309.2 & 309.2 & 309.2 & 309.2 & 309.2 & 309.2 & 309.2 & 309.2 \\
\hline & HIN & 0.0 & 0.0 & 0.0 & 0.0 & 0.0 & 0.0 & 309.2 & 309.2 & 309.2 & 309.2 & 309.2 & 309.2 & 309.2 & 309.2 & 309.2 & 309.2 \\
\hline & $\operatorname{Max}$. & 0.0 & 0.0 & 0.0 & 0.0 & 0.0 & 0.0 & 371.0 & 371.0 & 371.0 & 371.0 & 371.0 & 371.0 & 371.0 & 371.0 & 371.0 & 371.0 \\
\hline \multirow{3}{*}{$\begin{array}{l}\text { PORTBMOUTa_Es } \\
\text { Tot21. }\end{array}$} & VOL & 0.0 & 368.3 & 360.3 & 368.3 & 368.3 & 368.3 & 983.9 & 983.9 & 983.9 & 983.9 & 983.9 & 983.9 & 983.9 & 983.9 & 983.9 & 903.9 \\
\hline & MIN & 0.0 & 360.3 & 368.3 & 368.3 & 368.3 & 368.3 & 983.9 & 983.9 & 983.9 & 983.8 & 983.9 & 983.9 & 983.9 & 983.9 & 983.9 & 983.9 \\
\hline & $\operatorname{MAX}$ & 0.0 & 111.9 & 111.9 & 111.9 & 111.9 & 111.9 & 1180.6 & 1180.6 & 1180.6 & 1180.6 & 1180.6 & 1180.6 & 1180.6 & 1180.6 & 1180.6 & 1180.6 \\
\hline
\end{tabular}

Waste Generator: PORTsMoUrH_Us

\begin{tabular}{|c|c|c|c|c|c|c|c|c|c|c|c|c|c|c|c|c|c|}
\hline \multirow[t]{3}{*}{ CH_LLH_I } & vot & 2775.7 & 0.0 & 0.0 & 0.0 & 0.0 & 0.0 & 0.0 & 0.0 & 0.0 & 0.0 & 0.0 & 0.0 & 0.0 & 0.0 & 0.0 & 0.0 \\
\hline & MIN & 0.0 & 0.0 & 0.0 & 0.0 & 0.0 & 0.0 & 0.0 & 0.0 & 0.0 & 0.0 & 0.0 & 0.0 & 0.0 & 0.0 & 0.0 & 0.0 \\
\hline & $\max$ & 1775.7 & 0.0 & 0.0 & 0.0 & 0.0 & 0.0 & 0.0 & 0.0 & 0.0 & 0.0 & 0.0 & 0.0 & 0.0 & 0.0 & 0.0 & 0.0 \\
\hline \multirow[t]{2}{*}{ CH_LLSW_I } & vor & 173.6 & 0.0 & 0.0 & 0.0 & 0.0 & 0.0 & 0.0 & 0.0 & 0.0 & 0.0 & 0.0 & 0.0 & 0.0 & 0.0 & 0.0 & 0.0 \\
\hline & $\operatorname{Max}$ & 173.6 & 0.0 & 0.0 & 0.0 & 0.0 & 0.0 & 0.0 & 0.0 & 0.0 & 0.0 & 0.0 & 0.0 & 0.0 & 0.0 & 0.0 & 0.0 \\
\hline \multirow[t]{3}{*}{ CH_LLW_III } & vot & 39.9 & 0.0 & 0.0 & 0.0 & 0.0 & 0.0 & 0.0 & 0.0 & 0.0 & 0.0 & 0.0 & 0.0 & 0.0 & 0.0 & 0.0 & 0.0 \\
\hline & MIN & 0.0 & 0.0 & 0.0 & 0.0 & 0.0 & 0.0 & 0.0 & 0.0 & 0.0 & 0.0 & 0.0 & 0.0 & 0.0 & 0.0 & 0.0 & 0.0 \\
\hline & $\max$ & 39.9 & 0.0 & 0.0 & 0.0 & 0.0 & 0.0 & 0.0 & 0.0 & 0.0 & 0.0 & 0.0 & 0.0 & 0.0 & 0.0 & 0.0 & 0.0 \\
\hline
\end{tabular}


EY-1994 SOLID KASTE FORECAST

WASTE GENERATOR, WASTE CLASS VOLOMES BY YEAR

(In cubla meters)

\begin{tabular}{|c|c|c|c|c|c|c|c|c|c|c|c|c|c|c|c|c|c|}
\hline Was te Clasa & & Held & 1995 & 1996 & 1997 & 1998 & 1999 & 2000 & 2001 & 2002 & 2003 & 2004 & 2005 & 2006 & 2007 & 2008 & 2009 \\
\hline \multirow{3}{*}{$\begin{array}{l}\text { PORTBHOUTR_US } \\
\text { Totals }\end{array}$} & VOL & 1989.2 & 0.0 & 0.0 & 0.0 & 0.0 & 0.0 & 0.0 & 0.0 & 0.0 & 0.0 & 0.0 & 0.0 & 0.0 & 0.0 & 0.0 & 0.0 \\
\hline & MIN & 0.0 & 0.0 & 0.0 & 0.0 & 0.0 & 0.0 & 0.0 & 0.0 & 0.0 & 0.0 & 0.0 & 0.0 & 0.0 & 0.0 & 0.0 & 0.0 \\
\hline & $\max$ & 1989.2 & 0.0 & 0.0 & 0.0 & 0.0 & 0.0 & 0.0 & 0.0 & 0.0 & 0,0 & 0.0 & 0.0 & 0.0 & 0.0 & 0.0 & 0.0 \\
\hline
\end{tabular}

Was to Generator: PRINCEroN

\begin{tabular}{|c|c|c|c|c|c|c|c|c|c|c|c|c|c|c|c|c|c|}
\hline \multirow[t]{2}{*}{ CH_LLH_I } & $\begin{array}{l}\text { VOI } \\
\text { MIN }\end{array}$ & $\begin{array}{l}0.0 \\
0.0\end{array}$ & $\begin{array}{r}170.0 \\
51.0\end{array}$ & $\begin{array}{r}203.3 \\
81.9\end{array}$ & $\begin{array}{l}566.6 \\
283.3\end{array}$ & $\begin{array}{l}566.6 \\
169.9\end{array}$ & $\begin{array}{r}283.3 \\
84.9\end{array}$ & $\begin{array}{r}270.0 \\
51.0\end{array}$ & $\begin{array}{r}170.0 \\
51.0\end{array}$ & $\begin{array}{r}170.0 \\
51.0\end{array}$ & $\begin{array}{r}170.0 \\
51.0\end{array}$ & $\begin{array}{r}170.0 \\
51.0\end{array}$ & $\begin{array}{r}170.0 \\
51.0\end{array}$ & $\begin{array}{r}283.3 \\
84.9\end{array}$ & $\begin{array}{l}566.6 \\
283.3\end{array}$ & $\begin{array}{l}566.6 \\
169.9\end{array}$ & $\begin{array}{r}283.3 \\
04.9\end{array}$ \\
\hline & $\max$ & 0.0 & 221.0 & 566.6 & 736.5 & 736.5 & 368.2 & 221.0 & 221.0 & 221.0 & 221.0 & 221.0 & 221.0 & 360.2 & 736.5 & 736.5 & 360.2 \\
\hline \multirow[t]{3}{*}{ CH_LLAH_I } & VoL & 0.0 & 0.6 & 1.4 & 5.7 & 5.7 & 1.1 & 0.6 & 0.6 & 0.6 & 0.6 & 0.6 & 0.6 & 1.1 & 5.7 & 5.7 & 1.4 \\
\hline & MIN & 0.0 & 0.3 & 0.7 & 2.8 & 2.8 & 0.7 & 0.3 & 0.3 & 0.3 & 0.3 & 0.3 & 0.3 & 0.7 & 2.8 & 2.8 & 0.7 \\
\hline & $\max$ & 0.0 & 1.2 & 2.8 & 11.1 & 21.4 & 2.8 & 2.2 & 1.2 & 1.2 & 1.2 & 1.2 & 1.2 & 2.0 & 11.1 & 11.4 & 2.8 \\
\hline \multirow{3}{*}{$\begin{array}{l}\text { PRUNCETON } \\
\text { Total. }\end{array}$} & VoL & 0.0 & 270.6 & 284.7 & 372.3 & 372.3 & 284.7 & 170.6 & 270.6 & 170.6 & 170.6 & 270.6 & 170.6 & 284.7 & 572.3 & 572.3 & 284.7 \\
\hline & MIN & 0.0 & 31.3 & 85.6 & 286.1 & 172.8 & 85.6 & 31.3 & 31.3 & 31.3 & 51.3 & 51.3 & 51.3 & 85.6 & 286.1 & 172.8 & 85.6 \\
\hline & $\operatorname{MAX}$ & 0.0 & 222.2 & 369.4 & 747.9 & 717.9 & 371.0 & 222.2 & 222.2 & 222.2 & 222.2 & 222.2 & 222.2 & 371.0 & 717.9 & 717.9 & 371.0 \\
\hline
\end{tabular}

Faste Ġenerator: RKW_CNroaA

\begin{tabular}{|c|c|c|c|c|c|c|c|c|c|c|c|c|c|c|c|c|c|}
\hline \multirow[t]{3}{*}{ CH_LLH_I } & vor & 21.4 & 366.7 & 144.5 & 287.0 & 386.7 & 68.0 & 0.0 & 0.0 & 0.0 & 0.0 & 0.0 & 0.0 & 0.0 & 0.0 & 0.0 & 0.0 \\
\hline & MIM & 0.0 & 330.0 & 115.6 & 229.6 & 309.3 & 54.4 & 0.0 & 0.0 & 0.0 & 0.0 & 0.0 & 0.0 & 0.0 & 0.0 & 0.0 & 0.0 \\
\hline & $\max$ & 21.4 & 366.7 & 173.1 & 364.1 & 164.0 & 82.6 & 0.0 & 0.0 & 0.0 & 0.0 & 0.0 & 0.0 & 0.0 & 0.0 & 0.0 & 0.0 \\
\hline \multirow[t]{3}{*}{ CH_LLW_I } & vol & 15.8 & 0.0 & 0.0 & 0.0 & 0.0 & 0.0 & 0.0 & 0.0 & 0.0 & 0.0 & 0.0 & 0.0 & 0.0 & 0.0 & 0.0 & 0.0 \\
\hline & HIN & 0.0 & 0.0 & 0.0 & 0.0 & 0.0 & 0.0 & 0.0 & 0.0 & 0.0 & 0.0 & 0.0 & 0.0 & 0.0 & 0.0 & 0.0 & 0.0 \\
\hline & $\max$ & 15.8 & 0.0 & 0.0 & 0.0 & 0.0 & 0.0 & 0.0 & 0.0 & 0.0 & 0.0 & 0.0 & 0.0 & 0,0 & 0.0 & 0.0 & 0.0 \\
\hline \multirow{3}{*}{$\begin{array}{l}\text { RuKH_CANOaA } \\
\text { Total: }\end{array}$} & vos & 37.2 & 366.7 & 214.5 & 287.0 & 386.7 & 68.0 & 0.0 & 0.0 & 0.0 & 0.0 & 0.0 & 0.0 & 0.0 & 0.0 & 0.0 & 0.0 \\
\hline & MIN & 0.0 & 330.0 & 213.6 & 229.6 & 309.3 & 54.4 & 0.0 & 0.0 & 0.0 & 0.0 & 0.0 & 0.0 & 0.0 & 0.0 & 0.0 & 0.0 \\
\hline & $\operatorname{MAX}$ & 37.2 & 366.7 & 173.4 & 344.4 & 164.0 & 01.6 & 0.0 & 0.0 & 0.0 & 0.0 & 0.0 & 0.0 & 0.0 & 0.0 & 0.0 & 0.0 \\
\hline
\end{tabular}

Waste Generator: 8TANEORD

\begin{tabular}{|c|c|c|c|c|c|c|c|c|c|c|c|c|c|c|c|c|c|}
\hline \multirow[t]{3}{*}{ CH_LLW_I } & vor & 80.0 & 88.0 & 80.0 & 80.0 & 80.0 & 88.0 & 17.2 & 17.2 & 17.2 & 17.2 & 17.2 & 17.2 & 17.2 & 17.2 & 17.2 & 17.2 \\
\hline & HIN & 0.0 & 80.0 & 11.0 & 88.0 & 41.0 & 80.0 & 8.6 & 17.2 & 8.6 & 17.2 & 8.6 & 17.2 & 0.6 & 17.2 & 0.6 & 37.2 \\
\hline & $\max$ & 88.0 & 161.9 & 88.0 & 132.0 & 88.0 & 132.0 & 17.2 & 25.0 & 17.2 & 25.8 & 17.2 & 25.0 & 37.2 & 25.8 & 17.2 & 25.8 \\
\hline \multirow[t]{3}{*}{ CH_LLHA_II } & vor & 1.8 & 0.0 & 0.1 & 0.0 & 0.1 & 0.0 & 0.1 & 0.0 & 0.1 & 0.0 & 0.1 & 0.0 & 0.1 & 0.0 & 0.1 & 0.0 \\
\hline & MIN & 0.0 & 0.0 & 0.2 & 0.0 & 0.2 & 0.0 & 0.2 & 0.0 & 0.2 & 0.0 & 0.2 & 0.0 & 0.2 & 0.0 & 0.2 & 0.0 \\
\hline & $\max$ & 1.8 & 0.0 & 0.8 & 0.0 & 0.0 & 0.0 & 0.0 & 0.0 & 0.8 & 0.0 & 0.8 & 0.0 & 0.8 & 0.0 & 0.0 & 0.0 \\
\hline
\end{tabular}


FY-1994 SOLID WASTE FORECAST

Page 12a

WASTE GENERATOR, WASTE CLASS VOLOMES BY YEAR

(in cubio metera)

\begin{tabular}{|c|c|c|c|c|c|c|c|c|c|c|c|c|c|c|c|c|c|}
\hline Wasto & & Hold & 1995 & 1996 & 1997 & 1998 & 1999 & 2000 & 2001 & 2002 & 2003 & 2001 & 2005 & 2006 & 2007 & 2008 & 2009 \\
\hline \multirow{3}{*}{$\begin{array}{l}\text { ATAMPORD } \\
\text { TotzL. }\end{array}$} & VoL & 92.8 & 80.0 & 80.4 & 88.0 & 80.4 & 80.0 & 17.6 & 17.2 & 17.6 & 27.2 & 27.6 & 17.2 & 17.6 & 17.2 & 17.6 & 17.2 \\
\hline & MIN & 0.0 & 80.0 & 11.2 & 88.0 & 14.2 & 88.0 & 8.8 & 17.2 & 8.8 & 17.2 & 8.8 & 17.2 & 0.8 & 17.2 & 8.8 & 17.2 \\
\hline & $\operatorname{MAX}$ & 92.8 & 261.9 & 80.8 & 232.0 & 88.8 & 132.0 & 28.0 & 25.8 & 18.0 & 25.8 & 28.0 & 25.8 & 18.0 & 25.8 & 18.0 & 25.8 \\
\hline
\end{tabular}

Fasto Generator: TPNL_2312

\begin{tabular}{|c|c|c|c|c|c|c|c|c|c|c|c|c|c|c|c|c|c|c|}
\hline \multirow{19}{*}{$\vec{v}$} & \multirow[t]{2}{*}{ CH_LLA_I } & vor & 0.0 & 0.0 & 0.0 & 0.0 & 0.0 & 0.0 & 0.0 & 0.0 & 0.0 & 0.0 & 0.0 & 0.0 & 0.0 & 0.0 & 0.0 & 0.0 \\
\hline & & MIM & 0.0 & 0.0 & 0.0 & 0.0 & 0.0 & 0.0 & 0.0 & 0.0 & 0.0 & 0.0 & 0.0 & 0.0 & 0.0 & 0.0 & 0.0 & 0.0 \\
\hline & $\cdot$ & HAX & 0.0 & 0.0 & 0.0 & 0.0 & 0.0 & 0.0 & 0.0 & 0.0 & 0.0 & 0.0 & 0.0 & 0.0 & 0.0 & 0.0 & 0.0 & 0.0 \\
\hline & \multirow{3}{*}{$\mathrm{CH}_{-}^{\mathrm{TRU}}$} & VoL & 0.0 & 0.0 & 0.0 & 0.0 & 0.0 & 0.0 & 0.0 & 0.0 & 0.0 & 0.0 & 0.0 & 0.0 & 0.0 & 0.0 & 0.0 & 0.0 \\
\hline & & MIN & 0.0 & 0.0 & 0.0 & 0.0 & 0.0 & 0.0 & 0.0 & 0.0 & 0.0 & 0.0 & 0.0 & 0.0 & 0.0 & 0.0 & 0.0 & 0.0 \\
\hline & & $\max$ & 0.0 & 0.0 & 0.0 & 0.0 & 0.0 & 0.0 & 0.0 & 0.0 & 0.0 & 0.0 & 0.0 & 0.0 & 0.0 & 0.0 & 0.0 & 0.0 \\
\hline & \multirow[t]{3}{*}{$\mathrm{CH}_{-} \mathrm{TRUM}$} & VoL & 0.0 & 0.0 & 0.0 & 0.0 & 0.0 & 0.0 & 0.0 & 0.0 & 0.0 & 0.0 & 0.0 & 0.0 & 0.0 & 0.0 & 0.0 & 0.0 \\
\hline & & HIN & 0.0 & 0.0 & 0.0 & 0.0 & 0.0 & 0.0 & 0.0 & 0.0 & 0.0 & 0.0 & 0.0 & 0.0 & 0.0 & 0.0 & 0.0 & 0.0 \\
\hline & & $\max$ & 0.0 & 0.0 & 0.0 & 0.0 & 0.0 & 0.0 & 0.0 & 0.0 & 0.0 & 0.0 & 0.0 & 0.0 & 0.0 & 0.0 & 0.0 & 0.0 \\
\hline & \multirow{3}{*}{$\begin{array}{l}\text { TPNI_.2312 } \\
\text { Total. }\end{array}$} & VoL & 0.0 & 0.0 & 0.0 & 0.0 & 0.0 & 0.0 & 0.0 & 0.0 & 0.0 & 0.0 & 0.0 & 0.0 & 0.0 & 0.0 & 0.0 & 0.0 \\
\hline & & MIN & 0.0 & 0.0 & 0.0 & 0.0 & 0.0 & 0.0 & 0.0 & 0.0 & 0.0 & 0.0 & 0.0 & 0.0 & 0.0 & 0.0 & 0.0 & 0.0 \\
\hline & & $\operatorname{MAX}$ & 0.0 & 0.0 & 0.0 & 0.0 & 0.0 & 0.0 & 0.0 & 0.0 & 0.0 & 0.0 & 0.0 & 0.0 & 0.0 & 0.0 & 0.0 & 0.0 \\
\hline & \multicolumn{3}{|c|}{ Hasto Generator: IFNI_242B/BL } & & & & & & & & & & & & & & & \\
\hline & \multirow[t]{3}{*}{ CH_LLW_I } & Vor & 0.0 & 0.0 & 0.0 & 0.0 & 0.0 & 0.0 & 0.0 & 0.0 & 0.0 & 0.0 & 0.0 & 0.0 & 0.0 & 0.0 & 0.0 & 0.0 \\
\hline & & MIN & 0.0 & 0.0 & 0.0 & 0.0 & 0.0 & 0.0 & 0.0 & 0.0 & 0.0 & 0.0 & 0.0 & 0.0 & 0.0 & 0.0 & 0.0 & 0.0 \\
\hline & & $\max$ & 0.0 & 0.0 & 0.0 & 0.0 & 0.0 & 0.0 & 0.0 & 0.0 & 0.0 & 0.0 & 0.0 & 0.0 & 0.0 & 0.0 & 0.0 & 0.0 \\
\hline & \multirow{3}{*}{$\begin{array}{l}\operatorname{TPA} \sqrt{2} 2428 / B \mathrm{~B} \\
\text { Total: }\end{array}$} & voL & 0.0 & 0.0 & 0.0 & 0.0 & 0.0 & 0.0 & 0.0 & 0.0 & 0.0 & 0.0 & 0.0 & 0.0 & 0.0 & 0.0 & 0.0 & 0.0 \\
\hline & & MIN & 0.0 & 0.0 & 0.0 & 0.0 & 0.0 & 0.0 & 0.0 & 0.0 & 0.0 & 0.0 & 0.0 & 0.0 & 0.0 & 0.0 & 0.0 & 0.0 \\
\hline & & $\operatorname{MAX}$ & 0.0 & 0.0 & 0.0 & 0.0 & 0.0 & 0.0 & 0.0 & 0.0 & 0.0 & 0.0 & 0.0 & 0.0 & 0.0 & 0.0 & 0.0 & 0.6 \\
\hline
\end{tabular}

Haste Generator: TRN]_30ศ

\begin{tabular}{llllllllllllllllll}
\hline CH_LLEI & VOL & 0.0 & 0.0 & $0: 0$ & 0.0 & 0.0 & 0.0 & 0.0 & 0.0 & 0.0 & 0.0 & 0.0 & 0.0 & 0.0 & 0.0 & 0.0 & 0.0 \\
& $\operatorname{HIN}$ & 0.0 & 0.0 & 0.0 & 0.0 & 0.0 & 0.0 & 0.0 & 0.0 & 0.0 & 0.0 & 0.0 & 0.0 & 0.0 & 0.0 & 0.0 & 0.0 \\
& $\max$ & 0.0 & 0.0 & 0.0 & 0.0 & 0.0 & 0.0 & 0.0 & 0.0 & 0.0 & 0.0 & 0.0 & 0.0 & 0.0 & 0.0 & 0.0 & 0.0
\end{tabular}




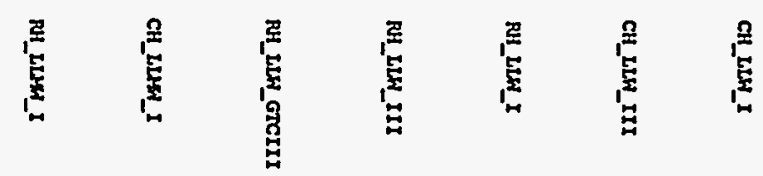

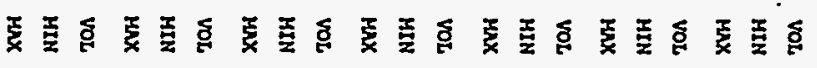

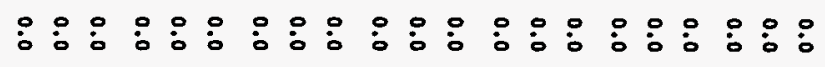

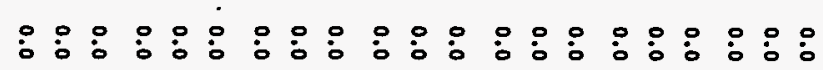

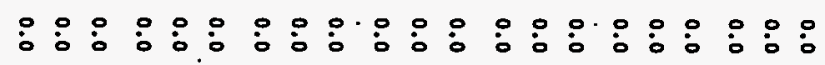

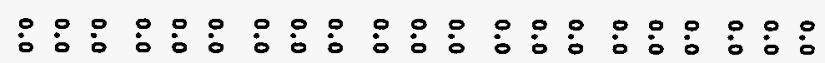

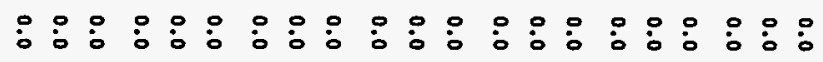

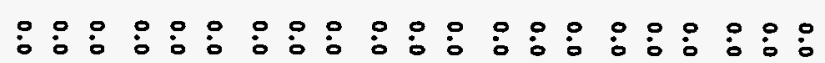

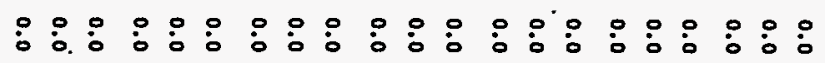

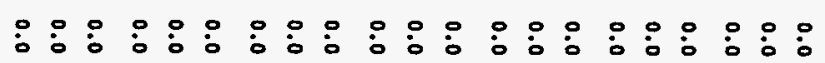

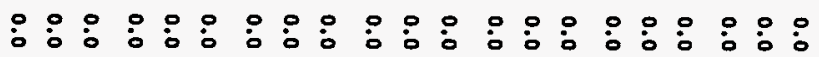

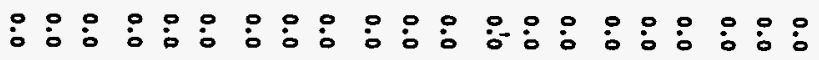

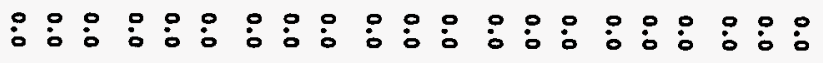

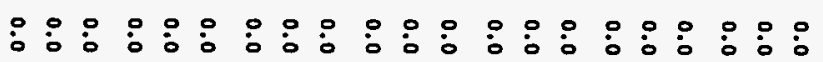

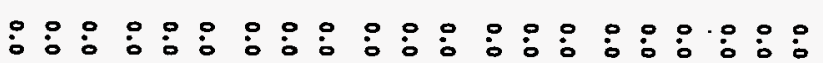

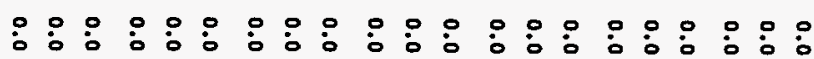

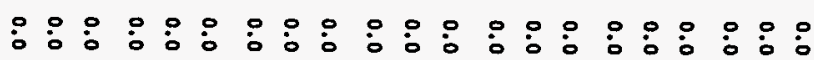

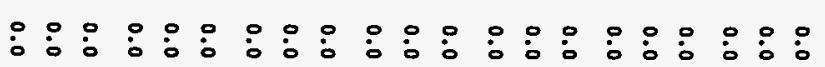

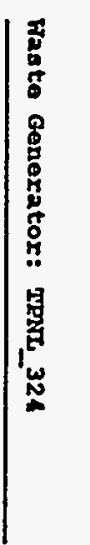

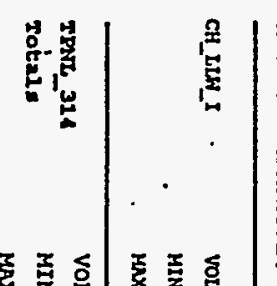

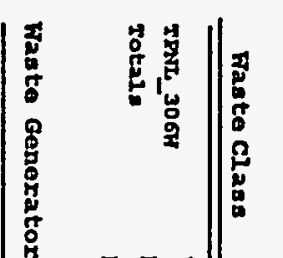

$\therefore$$$
:
$$

$\therefore:: 0:: 0$

$$
\therefore
$$

$\therefore: ㅇ ㅇ ㅇ$

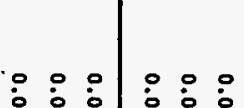

10

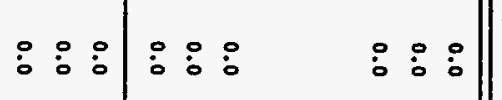

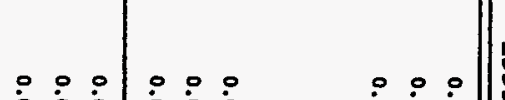

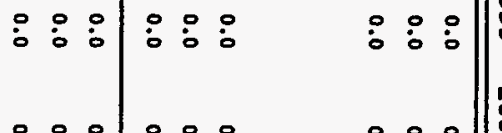

$\therefore: \div: \div$

$\dot{\circ}: \stackrel{\circ}{\circ}: \stackrel{\circ}{\circ}$

ํํㅇํำ

$\therefore \circ: \circ:$

$\therefore \circ: \circ:$

$\therefore: \square$

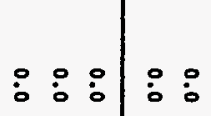

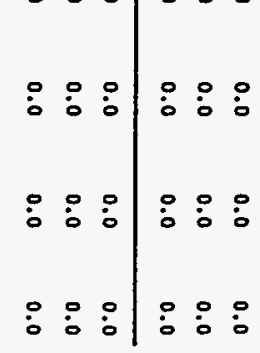




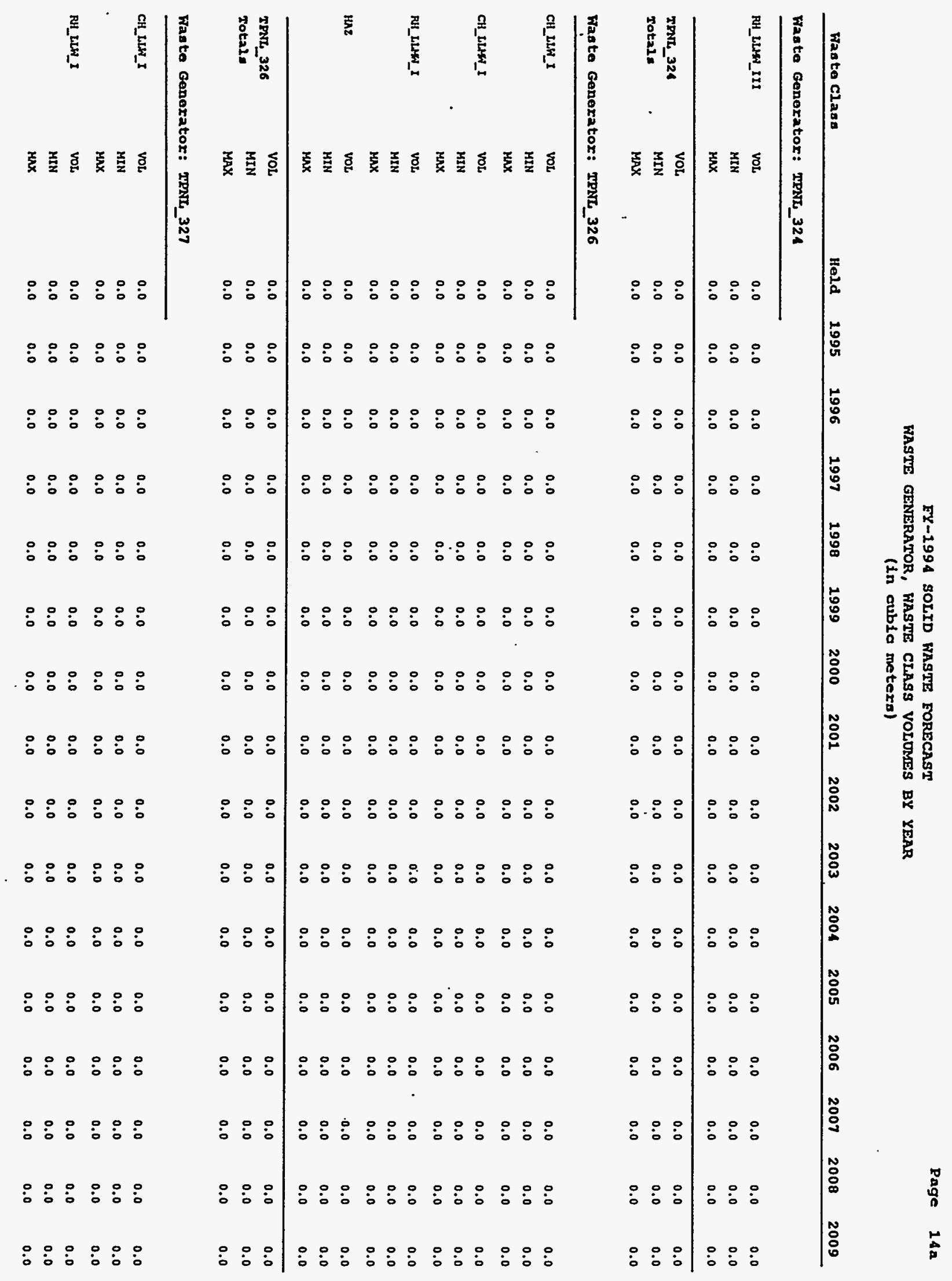




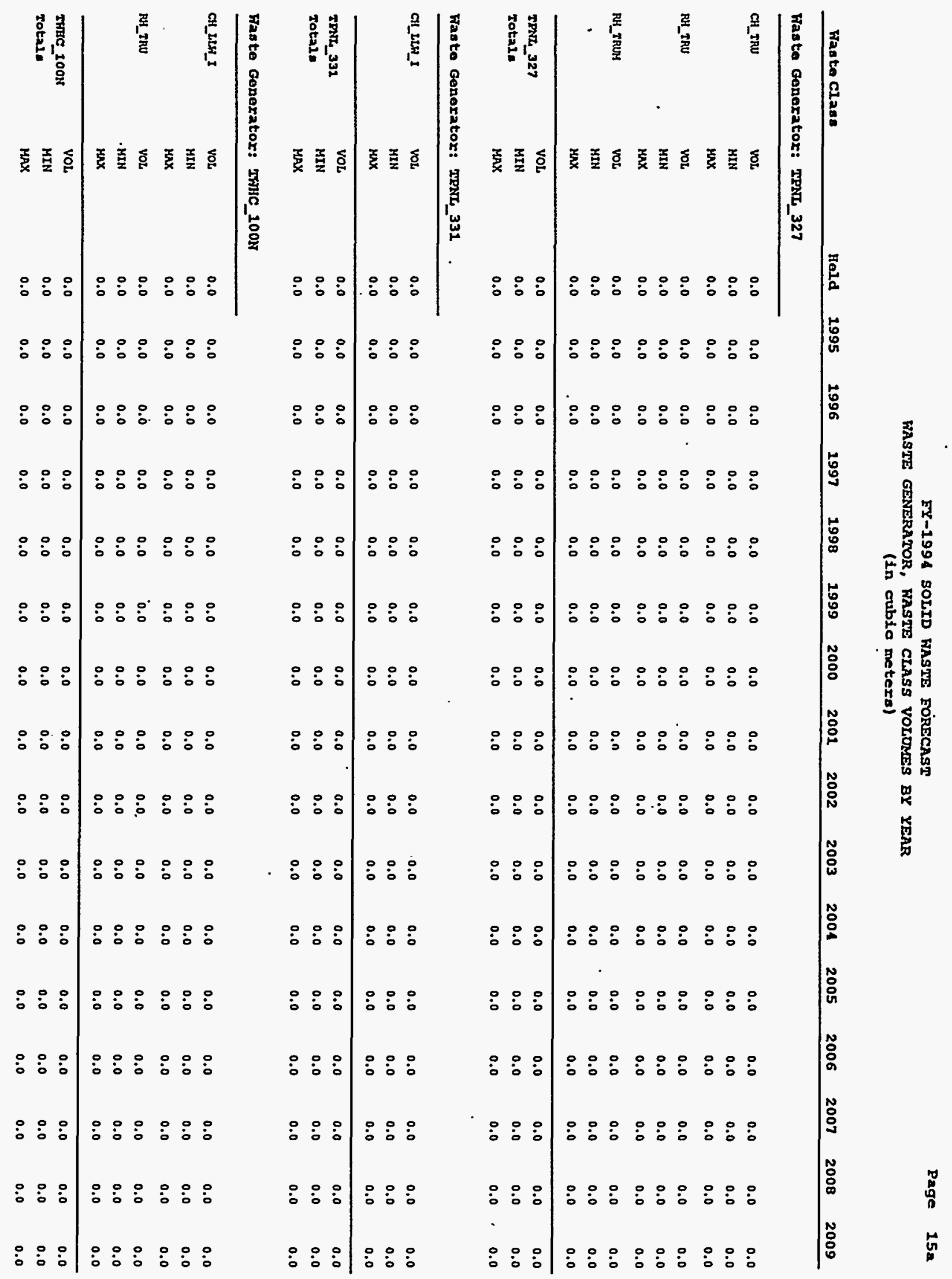




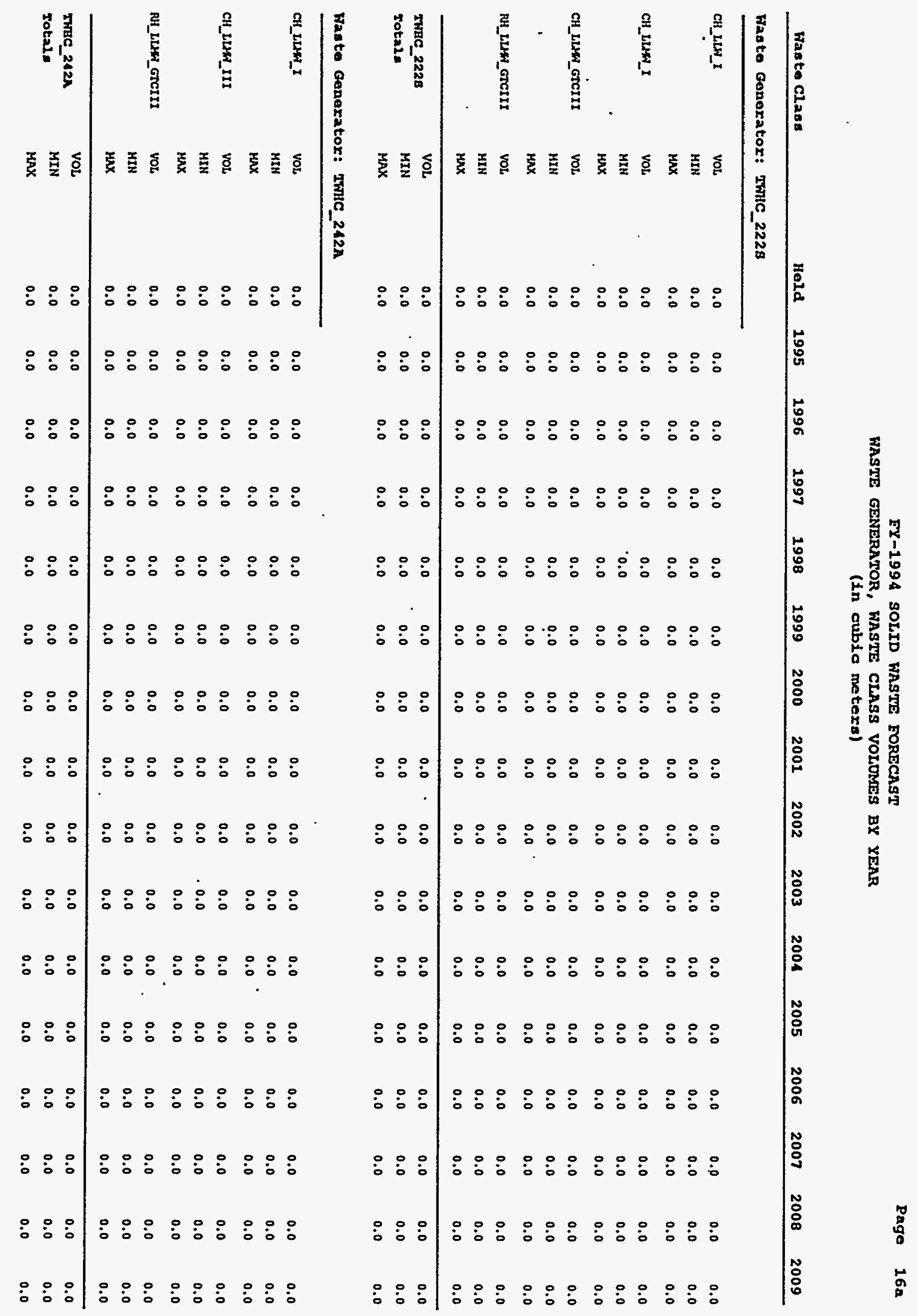


HHC-EP-0865

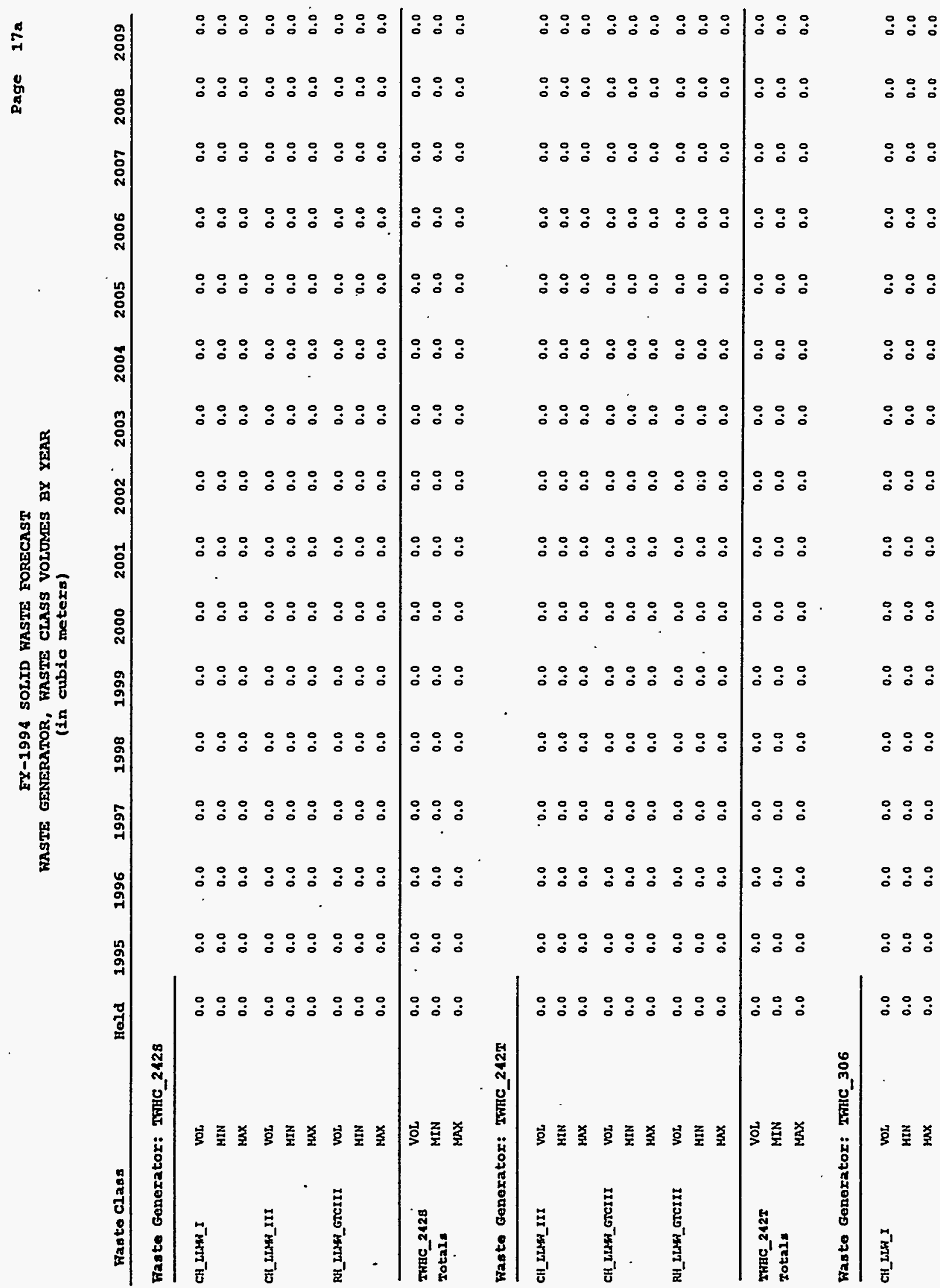




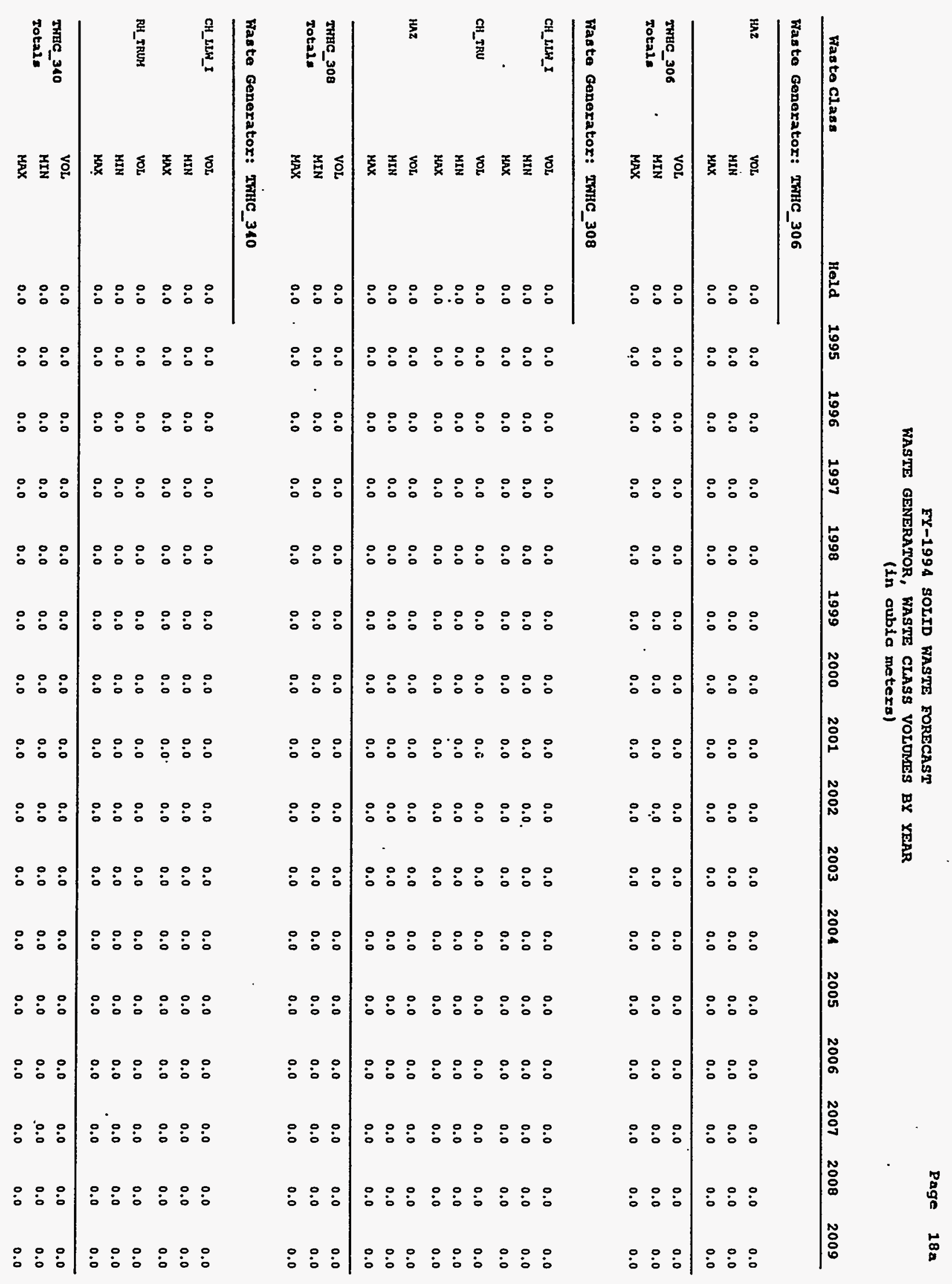


高

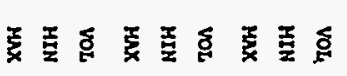

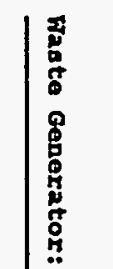

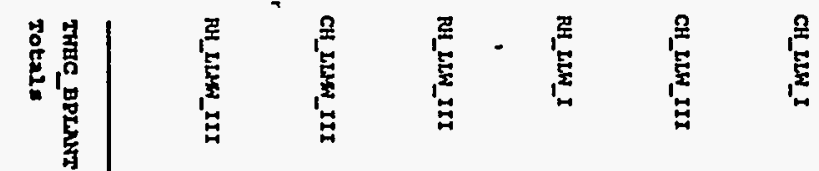

$\therefore:::: ㅇ$

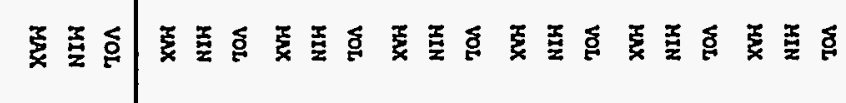

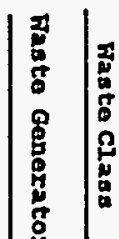

$\therefore: ㅇ:: ㅇ: ㅇ$

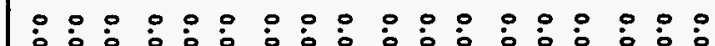

$\therefore \circ: 0.00000000000 .00$

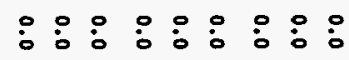

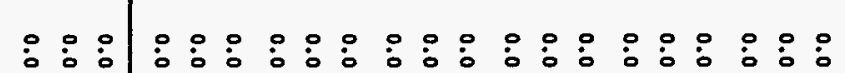

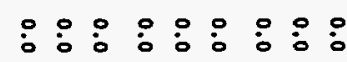

\section{$\because \because$}

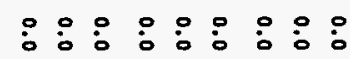

\section{0}

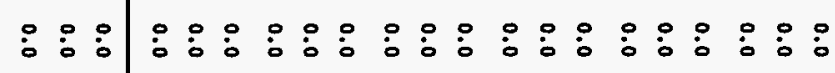

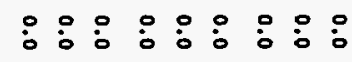

$\therefore: \div$

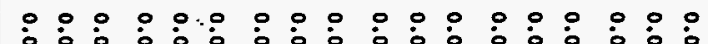

$\therefore: ㅇ ㅠ: ㅇ: ㅇ$

$\therefore: \div$

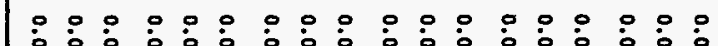

$\because \because \div \div \div: ㅇ$

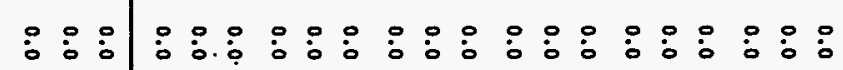

$\because: ㅇ: ㅇ: ㅇ: 0$

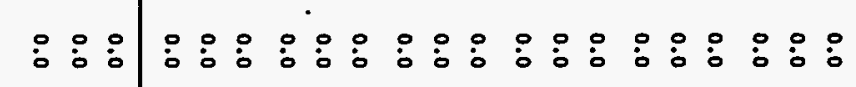

$\stackrel{\circ}{\circ}: \stackrel{\circ}{0}: \stackrel{\circ}{0}: \stackrel{0}{0}:$

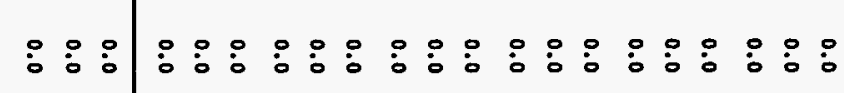

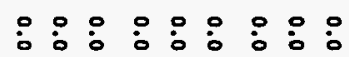

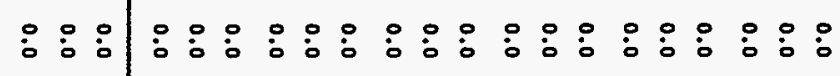

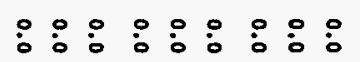

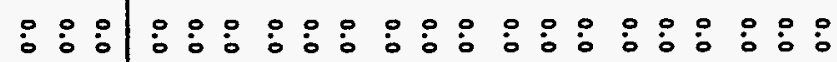

$\stackrel{0}{0}: \stackrel{\circ}{0}: \circ:: 0$

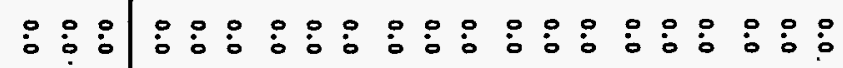

$\dot{0}: \stackrel{\circ}{0}: \stackrel{\circ}{0}: \dot{0}$

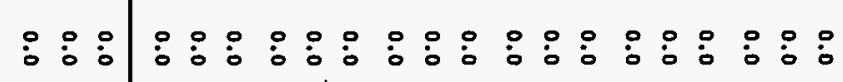

$: ㅇ:: ㅇ::$

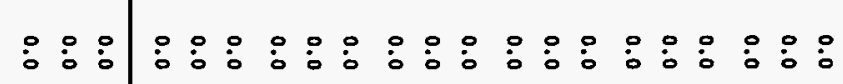

$\because: \therefore:: \circ: \circ:$

$\dot{0}: \dot{0} \mid \dot{0}: \dot{0}: \dot{0}: \dot{0}: \dot{0}: \dot{0}: \dot{0}: \dot{0}:$

蛋 
WHC-EP-0865

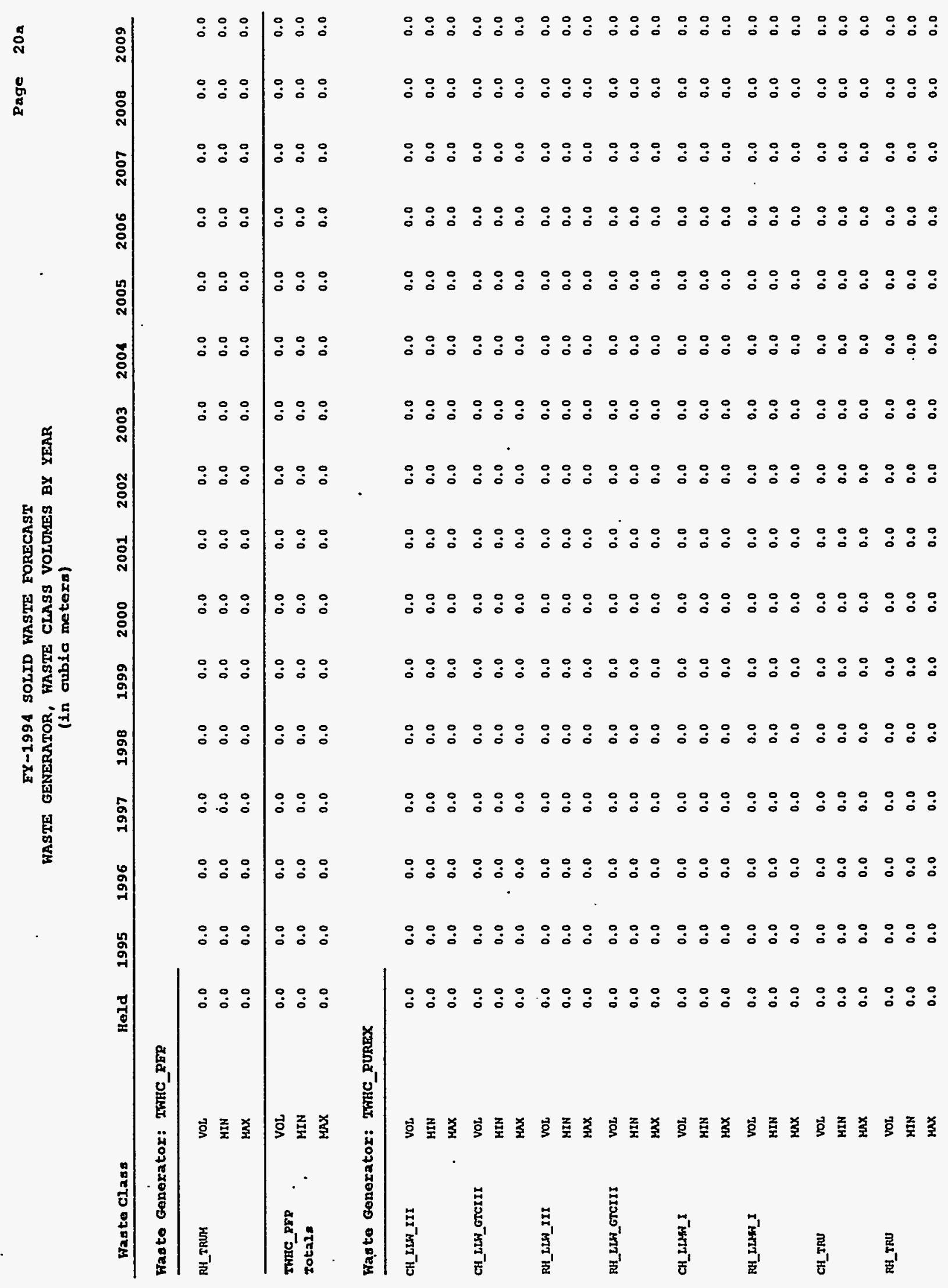


WHC-EP-0865

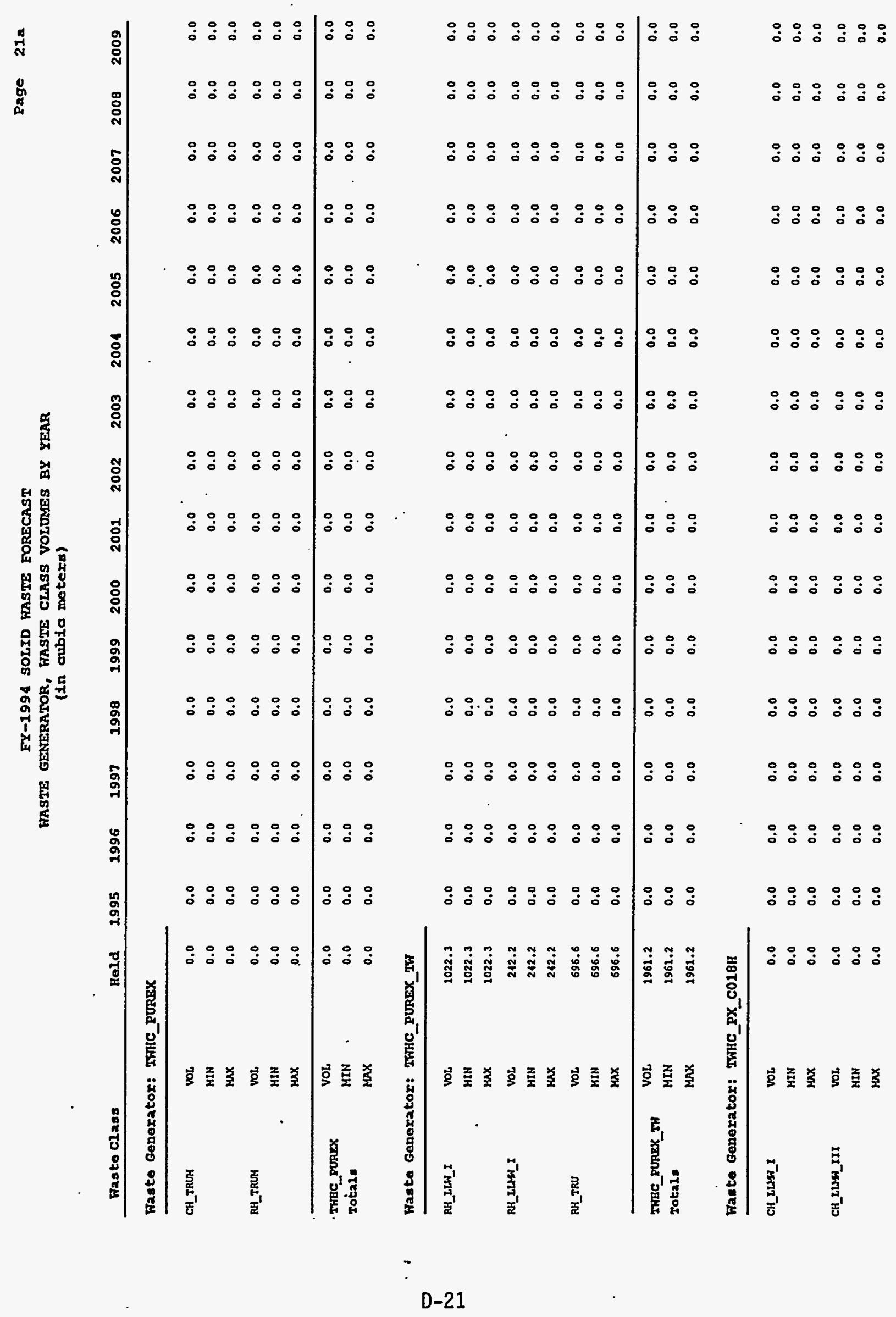




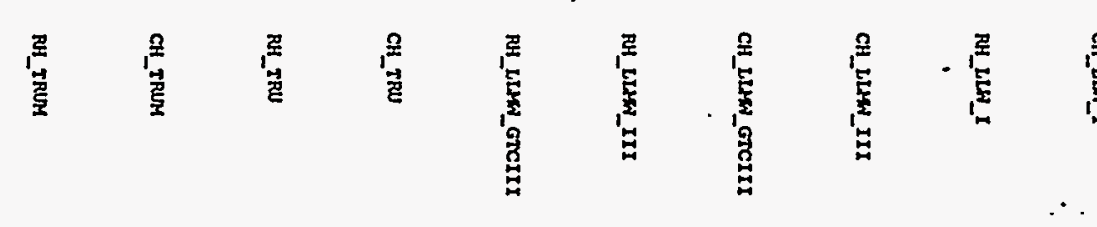

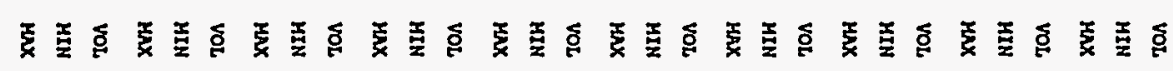

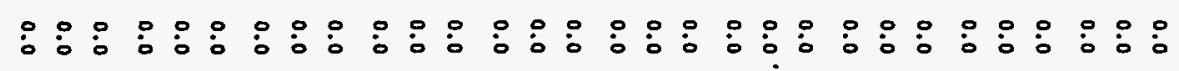

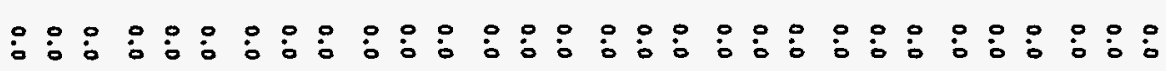

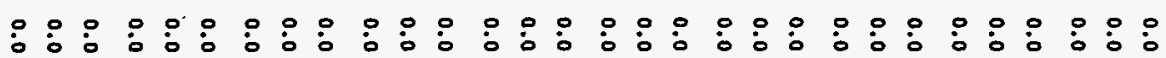

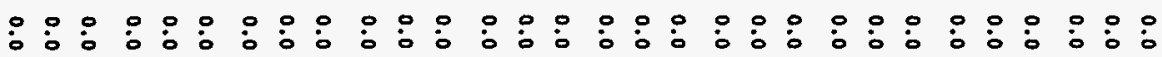

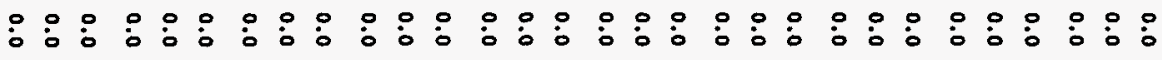

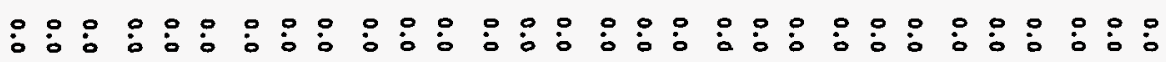

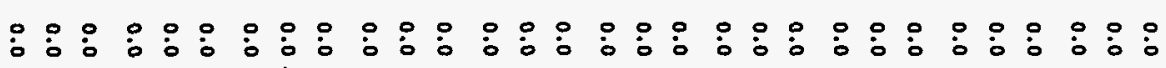

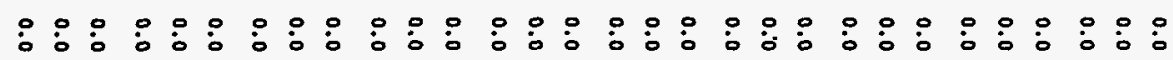

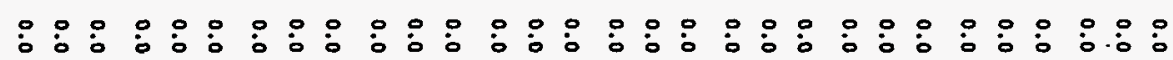

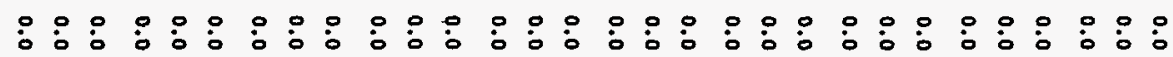

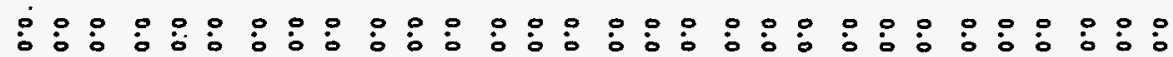

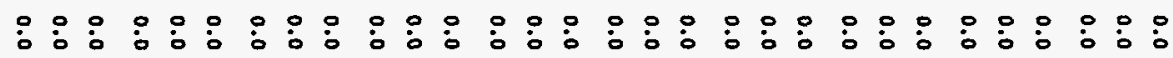

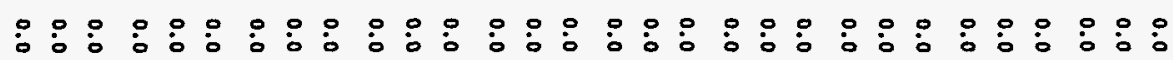

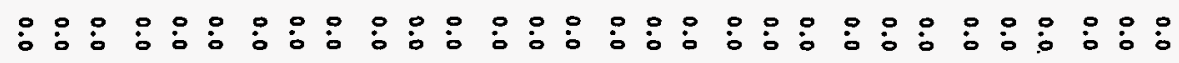

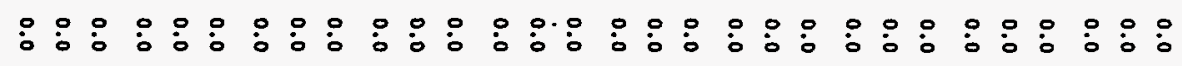

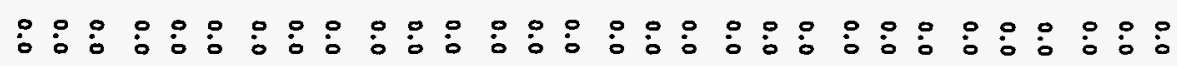

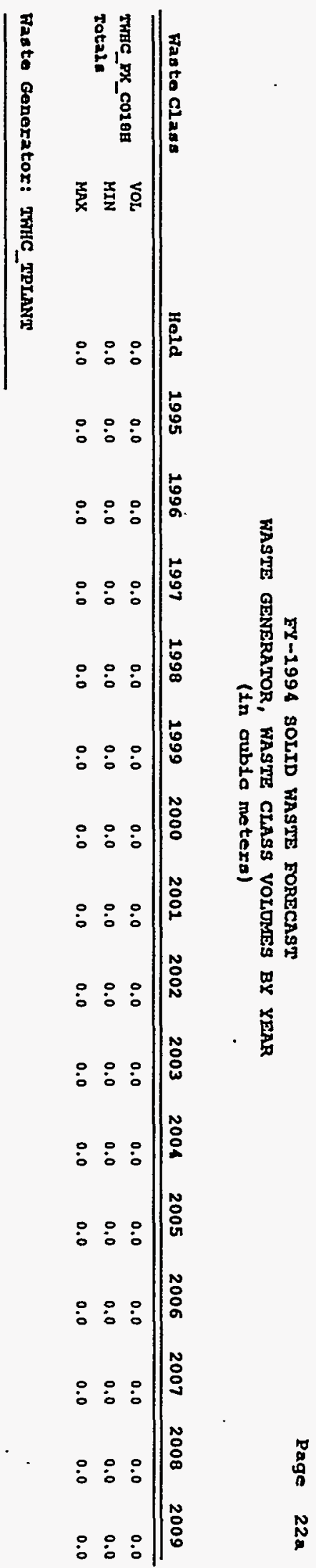




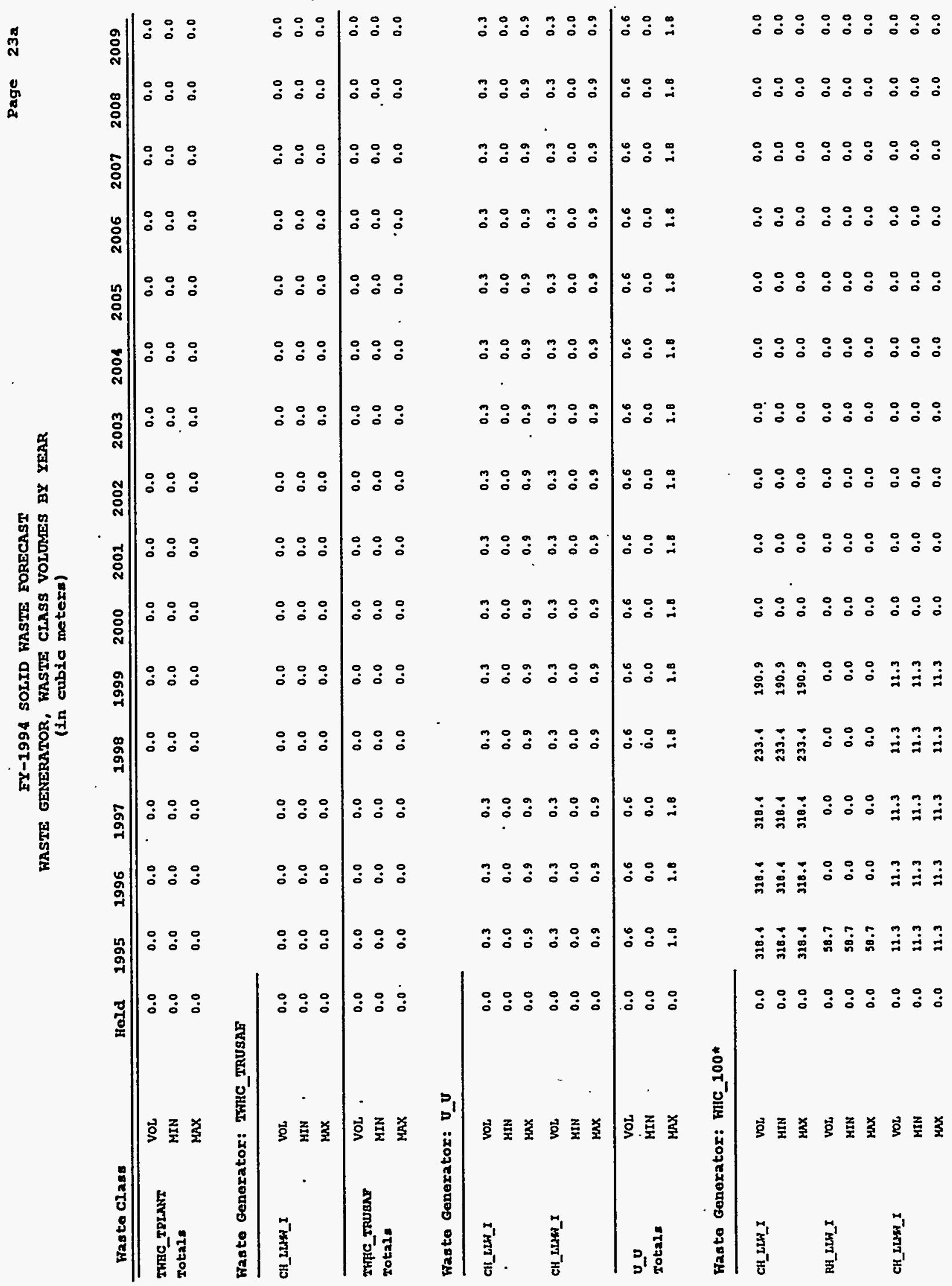


WHC-EP-0865

\begin{tabular}{|c|c|c|c|c|c|c|c|c|c|c|c|c|c|}
\hline$\stackrel{5}{\sim}$ & :े & & $\stackrel{0}{0}: \stackrel{0}{0}:$ & $\mid \begin{array}{lll}0 & 0 \\
0 & 0 & 0\end{array}$ & & $\stackrel{0}{\circ}: \stackrel{0}{0}$ & $\stackrel{0}{\circ} \stackrel{\circ}{0}:$ & & $\dot{I} \dot{I}$ & $\mid \bar{i} \dot{i}$ & & $\dot{\vec{b}} \dot{\dot{n}} \stackrel{0}{\dot{r}}$ & $\vec{i} \dot{i}: \stackrel{0}{i}$ \\
\hline & : & & $\stackrel{0}{0} \stackrel{0}{0}:$ & $\stackrel{\circ}{\circ} \stackrel{\circ}{\circ} \stackrel{0}{0}$ & & $\stackrel{0}{0}: \stackrel{0}{0}$ & $\begin{array}{lll}0 & 0 \\
0 & 0 & 0 \\
0 & 0\end{array}$ & & 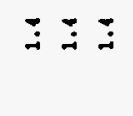 & $\dot{I} \dot{I}$ & & 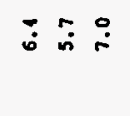 & $\overrightarrow{\dot{0}} \dot{\mathrm{i}}$ \\
\hline & : & & $\stackrel{9}{\stackrel{0}{0}} \stackrel{0}{0}:$ & $\stackrel{0}{\stackrel{0}{0}} \stackrel{0}{0} \stackrel{0}{0}$ & & $\stackrel{0}{0}: \stackrel{0}{0}:$ & $\stackrel{0}{0} \stackrel{0}{\circ} \stackrel{0}{0}$ & & $\bar{I} \dot{I}$ & $\dot{I} \dot{I}$ & & $\overrightarrow{\dot{b}} \dot{\mathrm{s}} \stackrel{0}{\mathrm{O}}$ & $\overline{0}$ is : \\
\hline & : & & $\stackrel{0}{\stackrel{0}{0}} \stackrel{0}{0} \stackrel{0}{\dot{0}}$ & $\stackrel{0}{\stackrel{0}{0}} \stackrel{0}{0}:$ & & $\begin{array}{lll}0 & 0 & 0 \\
0 & 0 & 0\end{array}$ & $\mid \begin{array}{lll}0 & 0 & 0 \\
0 & \vdots & 0 \\
0\end{array}$ & & $\bar{i} \dot{I}$ & $\dot{I} \dot{I}$ & & $\dot{\dot{b}} \dot{\dot{m}} \stackrel{0}{\dot{\gamma}}$ & $\overrightarrow{0}$ in \\
\hline & : & & $\stackrel{0}{\circ} \stackrel{0}{\dot{0}} \stackrel{0}{\dot{0}}$ & $\stackrel{0}{0}: \stackrel{0}{0}$ & . & $\begin{array}{lll}\stackrel{0}{0} & : & 0 \\
0 & 0 & 0\end{array}$ & $\mid \begin{array}{lll}0 & 0 & 0 \\
0 & \vdots & 0\end{array}$ & & $\dot{I} \dot{I}$ & $\dot{I} \dot{I}$ & & $\dot{\dot{b}} \dot{i} \stackrel{0}{\dot{0}}$ & $\dot{0}: \stackrel{\circ}{i}$ \\
\hline & :े & & $\stackrel{0}{0}: \stackrel{0}{0}:$ & 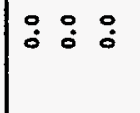 & & $\begin{array}{lll}0 & 0 & 0 \\
0 & \vdots & 0\end{array}$ & $\mid \begin{array}{lll}0 & 0 \\
0 & \vdots & 0 \\
0\end{array}$ & & $\dot{I} \dot{I}$ & $\dot{I} \dot{I} \dot{I}$ & & $\dot{\dot{b}} \dot{n} \stackrel{0}{\circ}$ & 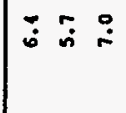 \\
\hline 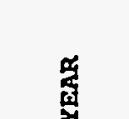 & : & & $\stackrel{0}{0}: \stackrel{0}{0}:$ & $\stackrel{0}{0}: \stackrel{0}{0}:$ & & $\begin{array}{lll}0 & 0 & 0 \\
0 & 0 & \vdots \\
0\end{array}$ & 过 & & $\dot{i}$ & $\dot{i} \dot{i}$ & & $\dot{i}$ i & $\ddot{\dot{0}} \dot{\mathrm{n}}$ \\
\hline 崮 & 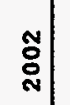 & & $\stackrel{0}{\circ}: \stackrel{0}{0}:$ & 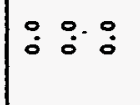 & & $\stackrel{0}{0} \stackrel{0}{0} \stackrel{0}{0}$ & $\stackrel{0}{0} \stackrel{0}{0} \stackrel{0}{0}$ & & $\dot{I} \dot{I}$ & $\dot{I} \dot{i}$ & & $\dot{i} \dot{0} \stackrel{0}{\dot{0}}$ & : \\
\hline 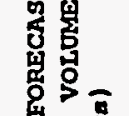 & :ే & & $\stackrel{0}{:} \stackrel{0}{0}$ & {$\left[\begin{array}{lll}0 & 0 \\
0 & 0 & 0 \\
0\end{array}\right.$} & & $\begin{array}{lll}0 & 0 & 0 \\
0 & \stackrel{0}{0} & 0\end{array}$ & $\mid \begin{array}{lll}0 & 0 & 0 \\
0 & 0 & 0 \\
0\end{array}$ & & $\bar{i} \dot{i}$ & $\dot{I} \dot{I}$ & & $\overrightarrow{\dot{b}} \dot{i} \stackrel{0}{\dot{0}}$ & $\dot{b} \dot{0}:$ \\
\hline 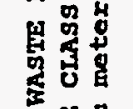 & : & & $\stackrel{0}{:}: 0$ & $\stackrel{0}{0}: \stackrel{0}{0}$ & & $\stackrel{0}{0}: \stackrel{0}{0}:$ & $\stackrel{0}{0}: \stackrel{0}{0}$ & & $\Xi \Xi \Xi$ & $=\Xi$ & & $\bar{i} \dot{i} \stackrel{\circ}{\dot{S}}$ & $\overrightarrow{0} \tilde{i} \stackrel{\circ}{r}$ \\
\hline 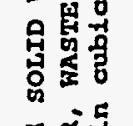 & ลे & & 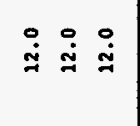 & 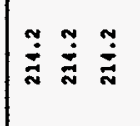 & & $\begin{array}{lll}0 & 0 & 0 \\
0 & \stackrel{0}{0} & 0\end{array}$ & $\stackrel{0}{0} \stackrel{0}{0}$ & & $\Xi \Xi$ & 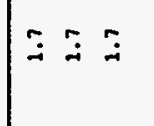 & & $\dot{\dot{0}} \dot{i} \stackrel{0}{\circ}$ & $\dot{\dot{b}} \dot{i} \stackrel{\circ}{\dot{\gamma}}$ \\
\hline 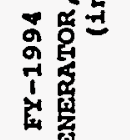 & : & & 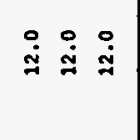 & 客灾 & & 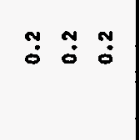 & 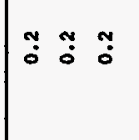 & & $\Xi \Xi$ & 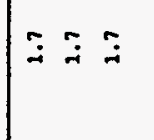 & & $\ddot{0}$ is & $\ddot{0} \dot{\circ} \stackrel{\circ}{\circ}$ \\
\hline 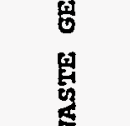 & बे & & 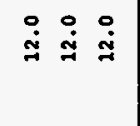 & $\dot{=}$ & & $\stackrel{0}{\circ}: \stackrel{0}{\dot{0}}:$ & 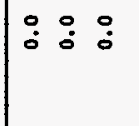 & & 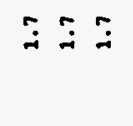 & $\Xi \mp$ & & 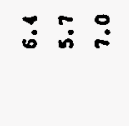 & $\dot{0} \dot{0} \stackrel{\circ}{\circ}$ \\
\hline & $\stackrel{\circ}{2}$ & & 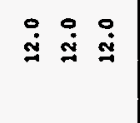 & 兽焉雍 & & 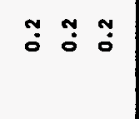 & : : : : : & & 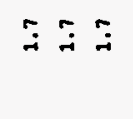 & تテ & & 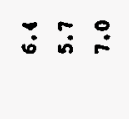 & 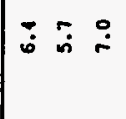 \\
\hline & $\begin{array}{l}2 \\
\stackrel{2}{7}\end{array}$ & & 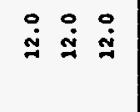 & $\dot{\dot{g}} \dot{\dot{g}} \dot{\dot{g}}$ & & $\stackrel{0}{0} \stackrel{0}{0}:$ & 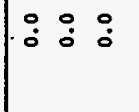 & & ت & $\exists \Im$ & & 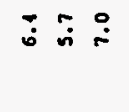 & 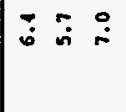 \\
\hline & $\begin{array}{l}0 \\
: \\
\vdots \\
8 \\
8 \\
8 \\
8\end{array}$ & 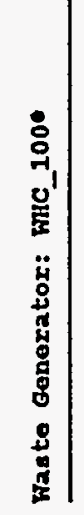 & 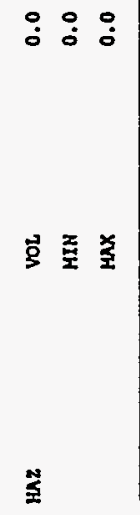 & 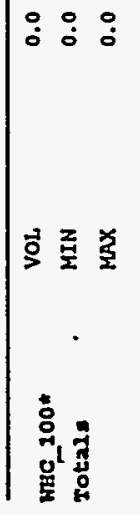 & 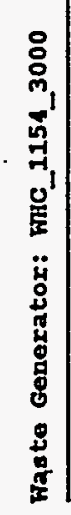 & 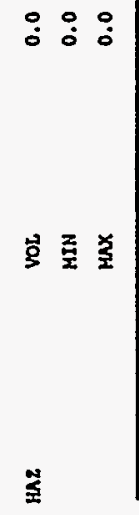 & 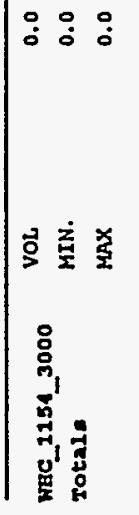 & 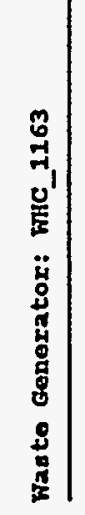 & 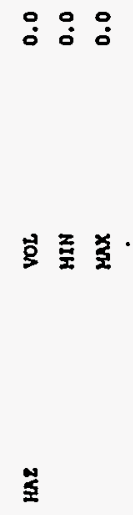 & 要希 & 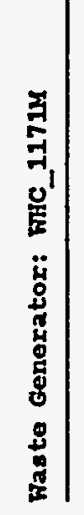 & g至 & 호를 줄 \\
\hline
\end{tabular}


EX-1994 SOLID WASTE FORECAST

HASTE GENERATOR, WASTE CLASS VOLUMES BY YEAP

(In cubio meters)

Faste Class

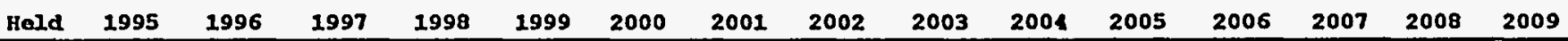

Faste Generator: FHC $1171 \mathrm{~T}$

\begin{tabular}{|c|c|c|c|c|c|c|c|c|c|c|c|c|c|c|c|c|c|}
\hline \multirow[t]{3}{*}{$\operatorname{HAz}$} & vor & 0.0 & 1.8 & 2.8 & 1.8 & 1.8 & 1.8 & 1.0 & 1.8 & 1.8 & 1.8 & 1.8 & 1.8 & 1.8 & 1.8 & 1.8 & 1.8 \\
\hline & MIN & 0.0 & 1.6 & 1.6 & 1.6 & 1.6 & 1.6 & 1.6 & 1.6 & 1.6 & 1.6 & 1.6 & 1.6 & 1.6 & 1.6 & 1.6 & 1.6 \\
\hline & $\operatorname{mnx}$ & 0.0 & 1.9 & 1.9 & 1.9 & 1.9 & 1.9 & 1.9 & 1.9 & 1.9 & 1.9 & 1.9 & 1.9 & 1.9 & 1.9 & 1.9 & 1.9 \\
\hline \multirow{2}{*}{$\begin{array}{l}\text { KBC_1171T } \\
\text { Jotal: }\end{array}$} & VOL & 0.0 & 1.8 & 1.0 & 1.8 & 1.0 & 2.0 & 1.0 & 1.8 & 1.8 & 1.0 & 1.8 & 1.0 & 1.0 & 1.8 & 1.8 & 1.8 \\
\hline & $\operatorname{MAX}$ & 0.0 & 1.9 & 1.9 & 1.9 & 1.9 & 1.9 & 1.9 & 1.9 & 1.' & 1.9 & '1.9 & 1.9 & 1.9 & 1.9 & 1.9 & 1.9 \\
\hline
\end{tabular}

Haste Generator: WilC_200_EIF

\begin{tabular}{|c|c|c|c|c|c|c|c|c|c|c|c|c|c|c|c|c|c|}
\hline \multirow[t]{3}{*}{ CH_LHAN_I } & Vor & 0.0 & 0.0 & 34.0 & 34.0 & 34.0 & 34.0 & 34.0 & 34.0 & 34.0 & 34.0 & 34.0 & 34.0 & 34.0 & 31.0 & 34.0 & 34.0 \\
\hline & MIN & 0.0 & 0.0 & 17.0 & 27.0 & 17.0 & 17.0 & 17.0 & 27.0 & 27.0 & 27.0 & 27.0 & 17.0 & 17.0 & 17.0 & 17.0 & 17.0 \\
\hline & $\max$ & 0.0 & 0.0 & 51.0 & 51.0 & 51.0 & 51.0 & 31,0 & 51.0 & 51.0 & 51.0 & 51.0 & 51.0 & 51.0 & 51.0 & 51.0 & 51.0 \\
\hline \multirow[t]{2}{*}{ CH_LLAN_III } & vor & 0.0 & 0.0 & 49.2 & 18.2 & 18.2 & 48.2 & 10.2 & 18.2 & 18.2 & 18.2 & 40.2 & 40.2 & 18.2 & 48.2 & 18.2 & 18.2 \\
\hline & $\max$ & 0.0 & 0.0 & 72.3 & 72.3 & 72.3 & 72.3 & 72.3 & 72.3 & 72.3 & 72.3 & 72.3 & 72.3 & 72.3 & 72.3 & 72.3 & 72.3 \\
\hline \multirow[t]{3}{*}{ HAz } & voL & 0.0 & 0.0 & 0.3 & 0.3 & 0.3 & 0.3 & 0.3 & 0.3 & 0.3 & 0.3 & 0.3 & 0.3 & 0.3 & 0.3 & 0.3 & 0.3 \\
\hline & MIN & 0.0 & 0.0 & 0.1 & 0.1 & 0.1 & 0.1 & 0.1 & 0.1 & 0.1 & 0.1 & 0.1 & 0.1 & 0.1 & 0.1 & 0.1 & 0.1 \\
\hline & $\max$ & 0.0 & 0.0 & 0.1 & 0.4 & 0.1 & 0.1 & 0.4 & 0.1 & 0.1 & 0.4 & 0.1 & 0.1 & 0.1 & 0.1 & 0.1 & 0.4 \\
\hline \multirow{3}{*}{$\begin{array}{l}\text { भRG_200_XTF } \\
\text { Tota21. }\end{array}$} & Vor & 0.0 & 0.0 & B2.5 & 82.5 & 82.5 & 82.5 & 82.5 & 82.5 & 82.5 & 82.5 & 02.5 & 82.5 & 82.5 & 02.5 & 82.5 & 82.5 \\
\hline & MIN & 0.0 & 0.0 & 11.2 & 41.2 & 11.2 & 41.2 & 11.2 & 11.2 & 11.2 & 11.2 & 11.2 & 11.2 & 11.2 & 11.2 & 11.2 & 11.2 \\
\hline & $\operatorname{MAX}$ & 0.0 & 0.0 & 123.7 & 123.7 & 123.7 & 123.7 & 123.7 & 123.7 & 223.7 & 123.7 & 123.7 & 123.7 & 123.7 & 123.7 & 123.7 & 123.7 \\
\hline
\end{tabular}

Was te Generatox: HHC 2101M

\begin{tabular}{|c|c|c|c|c|c|c|c|c|c|c|c|c|c|c|c|c|c|}
\hline \multirow[t]{3}{*}{ HARZ } & VoL & 0.0 & 5.2 & 5.2 & 3.2 & 5.2 & 5.2 & 5.2 & 5.2 & 5.2 & 5.2 & 5.2 & 5.2 & 5.2 & 5.2 & 5.2 & 5.2 \\
\hline & MIN & 0.0 & 2.6 & 2.6 & 2.6 & 2.6 & 2.6 & 2.6 & 2.6 & 2.6 & 2.6 & 2.6 & 2.6 & 2.6 & 2.6 & 2.6 & 2.6 \\
\hline & $\max$ & 0.0 & 5,2 & 5.2 & 3.2 & 5.2 & 3.2 & 3.2 & 5.2 & 5.2 & 5.2 & 5.2 & 5.2 & 5.2 & 3.2 & 5.2 & 5.2 \\
\hline \multirow{3}{*}{$\begin{array}{l}\text { Kac_2101K } \\
\text { Total: }\end{array}$} & VOL & 0.0 & 5.2 & 5.2 & 5.2 & 5.2 & 5.2 & 5.2 & 5.2 & 5.2 & 3.2 & 5.2 & 5.2 & 5.2 & 3.2 & 5.2 & 5.2 \\
\hline & MIN & 0.0 & 2.6 & 2.6 & 2.6 & 2.6 & 2.6 & 2.6 & 2.6 & 2.6 & 2.6 & 2.6 & 2.6 & 2.6 & 2.6 & 2.6 & 2.6 \\
\hline & $\operatorname{MAX}$ & 0.0 & 5.2 & 5.2 & 5.2 & 5.2 & 3.2 & 5.2 & 5.2 & 5.2 & 3.2 & 3.2 & 5.2 & 5.2 & 5.2 & 5.2 & 5.2 \\
\hline
\end{tabular}


FY-1994 SOLID WASTE EORECAST

HASTE GENERATOR, HASTE CLASS VOLUMES BY YEAR

(in cubic meters)

\begin{tabular}{|c|c|c|c|c|c|c|c|c|c|c|c|c|c|c|c|c|c|}
\hline Waste $\mathrm{C}$ & & Held & 1995 & 1996 & 1997 & 1998 & 1999 & 2000 & 2001 & 2002 & 2003 & 2004 & 2005 & 2006 & 2007 & 2008 & 2009 \\
\hline Waste Ger & $r: F$ & & & & & & & & & & & & & & & & \\
\hline \multirow[t]{2}{*}{$\operatorname{HAz}$} & VoL & 0.0 & 2.2 & 2.2 & 2.2 & 2.2 & 2.2 & 2.2 & 2.2 & 2.2 & 2.2 & 2.2 & 2.2 & 2.2 & 2.2 & 2.2 & 2.2 \\
\hline & $\max$ & 0.0 & 2.2 & 2.2 & 2.2 & 2.2 & 2.2 & 2.2 & 2.2 & 2.2 & 2.2 & 2.2 & 2.2 & 2.2 & 2.2 & 2.2 & 2.2 \\
\hline \multirow{2}{*}{$\begin{array}{l}\text { WaC_27038 } \\
\text { Total: }\end{array}$} & VoL & 0.0 & 2.2 & 2.2 & 2.2 & 2.2 & 2.2 & 2.2 & 2.2 & 2.2 & 2.2 & 2.2 & 2.2 & 2.2 & 2.2 & 2.2 & 2.2 \\
\hline & MIN & 0.0 & 2.2 & 2.2 & 2.2 & 2.2 & 2.2 & 2.2 & 2.2 & 2.2 & 2.2 & 2.2 & 2.2 & 2.2 & 2.2 & 2.2 & 2.2 \\
\hline
\end{tabular}

Waste Generator: WHC_2715EA

\begin{tabular}{|c|c|c|c|c|c|c|c|c|c|c|c|c|c|c|c|c|c|c|}
\hline \multirow{6}{*}{ مَ' } & \multirow[t]{3}{*}{ HAZ } & VOL & 0.0 & 19.6 & 19.6 & 19.6 & 19.6 & 19.6 & 19.6 & 19.6 & 19.6 & 19.6 & 19.6 & 19.6 & 19.6 & 19.6 & 19.6 & 19.6 \\
\hline & & MIN & 0.0 & 19.6 & 19.6 & 19.6 & 19.6 & 19.6 & 19.6 & 19.6 & 19.6 & 19.6 & 19.6 & 19.6 & 19.6 & 19.6 & 19.6 & 19.6 \\
\hline & & $\max$ & 0.0 & 19.6 & 19.6 & 19.6 & 19.6 & 19.6 & 19.6 & 19.6 & 19.6 & 19.6 & 19.6 & 19.6 & 19.6 & 19.6 & 19.6 & 19.6 \\
\hline & \multirow{3}{*}{$\begin{array}{l}\text { NBC_2715RA } \\
\text { Total. }\end{array}$} & VOL & 0.0 & 19.6 & 19.6 & 19.6 & 19.6 & 19.6 & 19.6 & 19.6 & 19.6 & 19.6 & 19.6 & 19.6 & 19.6 & 19.6 & 19.6 & 19.6 \\
\hline & & MIN & 0.0 & 19.6 & 19.6 & 19.6 & 19.6 & 19.6 & 19.6 & 19.6 & 19.6 & 19.6 & 19.6 & 19.6 & 19.6 & 19.6 & 19.6 & 19.6 \\
\hline & & $\operatorname{MAX}$ & 0.0 & 19.6 & 29.6 & 19.6 & 19.6 & 19.6 & 19.6 & 19.6 & 19.6 & 19.6 & 19.6 & 29.6 & 19.6 & 19.6 & 19.6 & 29.6 \\
\hline
\end{tabular}

Waste Genorator: WHC_2BAE

\begin{tabular}{|c|c|c|c|c|c|c|c|c|c|c|c|c|c|c|c|c|c|}
\hline \multirow[t]{3}{*}{ HAZ } & VoL & 0.0 & 9.5 & 10.1 & 0.1 & 6.2 & 1.2 & 4.2 & 0.4 & 6.4 & 6.1 & 0.0 & 0.0 & 0.0 & 0.0 & 0.0 & 0.0 \\
\hline & MIN & 0.0 & 9.5 & 10.4 & 0.1 & 4.2 & 1.2 & 1.2 & 0.1 & 6.4 & 6.1 & 0.0 & 0.0 & 0.0 & 0.0 & 0.0 & 0.0 \\
\hline & $\operatorname{Max}$ & 0.0 & 9.5 & 10.4 & 0.1 & 4.2 & 4.2 & 1.2 & 0.1 & 6.4 & 6.1 & 0.0 & 0.0 & 0.0 & 0.0 & 0.0 & 0.0 \\
\hline \multirow{2}{*}{$\begin{array}{l}\text { FBC_2812 } \\
\text { Totals }\end{array}$} & VOL & 0.0 & 9.5 & 10.1 & 0.1 & 4.2 & 4.2 & 4.2 & 0.1 & 6.1 & 6.1 & 0.0 & 0.0 & 0.0 & 0.0 & 0.0 & 0.0 \\
\hline & $\operatorname{MAX}$ & 0.0 & 9.5 & 10.4 & 8.1 & 4.2 & 1.2 & 4.2 & 8.1 & 6.4 & 6.1 & 0.0 & 0.0 & 0.0 & 0.0 & 0.0 & 0.0 \\
\hline
\end{tabular}

Waste Generator: WHC_300_PWR

\begin{tabular}{|c|c|c|c|c|c|c|c|c|c|c|c|c|c|c|c|c|c|}
\hline \multirow[t]{3}{*}{$\mathrm{CH}_{-} \mathrm{LLW}_{-} \mathrm{I}$} & Vor & 2.0 & 0.0 & 0.0 & 0.0 & 0.0 & 0.0 & 0.0 & 0.0 & 0.0 & 0.0 & 0.0 & 0.0 & 0.0 & 0.0 & 0.0 & 0.0 \\
\hline & MIN & 0.0 & 0.0 & 0.0 & 0.0 & 0.0 & 0.0 & 0.0 & 0.0 & 0.0 & 0.0 & 0.0 & 0.0 & 0.0 & 0.0 & 0.0 & 0.0 \\
\hline & $\boldsymbol{\mu} \boldsymbol{\mu} X$ & 2.8 & 0.0 & 0.0 & 0.0 & 0.0 & 0.0 & 0.0 & 0.0 & 0.0 & 0.0 & 0.0 & 0.0 & 0.0 & 0.0 & 0.0 & 0.0 \\
\hline \multirow[t]{3}{*}{ HAZ } & VOL & 0.0 & 2.0 & 2.0 & 2.0 & 2.8 & 2.8 & 2.0 & 2.8 & 2.8 & 2.8 & 2.8 & 2.0 & 1.1 & 1.1 & 1.1 & 1.1 \\
\hline & MIN & 0.0 & 2.8 & 2.8 & 2.8 & 2.8 & 2.8 & 2.8 & 2.8 & 2.8 & 2.8 & 2.8 & 2.0 & 0.7 & 0.7 & 0.7 & 0.7 \\
\hline & $\operatorname{mix}$ & 0.0 & 2.8 & 2.8 & 2.8 & 2.8 & 2.8 & 2.8 & 2.8 & 2.8 & 2.8 & 2.8 & 2.8 & 2.0 & 2.8 & 2.8 & 2.0 \\
\hline
\end{tabular}


FY-1994 SOLID WASTE FORECAST

WASTE GENERATOR, WASTE CLASS VOLUMES BY YEAR

(in cubic meters)

\begin{tabular}{llcccccccccccccccc} 
Haste Class & & Held & 1995 & 1996 & 1997 & 1998 & 1999 & 2000 & 2001 & 2002 & 2003 & 2004 & 2005 & 2006 & 2007 & 2008 & 2009 \\
\hline \hline \multirow{2}{*}{ HRC_300_PHR } & VOL & 2.0 & 2.8 & 2.8 & 2.8 & 2.8 & 2.8 & 2.8 & 2.8 & 2.8 & 2.8 & 2.8 & 2.8 & 1.1 & 1.1 & 1.1 & 1.1 \\
Tota1: & MIN & 0.0 & 2.8 & 2.8 & 2.8 & 2.8 & 2.8 & 2.8 & 2.8 & 2.8 & 2.8 & 2.8 & 2.8 & 0.7 & 0.7 & 0.7 & 0.7 \\
& MAX & 2.8 & 2.8 & 2.8 & 2.8 & 2.8 & 2.8 & 2.8 & 2.8 & 2.8 & 2.8 & 2.8 & 2.8 & 2.8 & 2.8 & 2.8 & 2.8
\end{tabular}

Wasto Genorator: HHC_300_TEDE

\begin{tabular}{|c|c|c|c|c|c|c|c|c|c|c|c|c|c|c|c|c|c|}
\hline \multirow[t]{2}{*}{ CH_LLW_I } & $\begin{array}{l}\text { Vor } \\
\text { MIN }\end{array}$ & $\begin{array}{l}0.0 \\
0.0\end{array}$ & $\begin{array}{l}368.3 \\
284.1\end{array}$ & $\begin{array}{l}368.3 \\
184.1\end{array}$ & $\begin{array}{l}369.3 \\
104.1\end{array}$ & $\begin{array}{l}368.3 \\
184.1\end{array}$ & $\begin{array}{l}368.3 \\
104.1\end{array}$ & $\begin{array}{l}368.3 \\
284.1\end{array}$ & $\begin{array}{l}368.3 \\
184.1\end{array}$ & $\begin{array}{l}368.3 \\
184 . .1\end{array}$ & $\begin{array}{l}368.3 \\
184.1\end{array}$ & $\begin{array}{l}368.3 \\
184.1\end{array}$ & $\begin{array}{l}368.3 \\
181.1\end{array}$ & $\begin{array}{l}368.3 \\
184.1\end{array}$ & $\begin{array}{l}368.3 \\
184.1\end{array}$ & $\begin{array}{l}368.3 \\
104.1\end{array}$ & $\begin{array}{l}368.3 \\
184.1\end{array}$ \\
\hline & $\max$ & 0.0 & 552.4 & 532.1 & 552.4 & 552.1 & 552.4 & 552.4 & 552.4 & 552.1 & 552.4 & 552.4 & 552.4 & 552.4 & 552.4 & 552.4 & 552.4 \\
\hline \multirow[t]{3}{*}{ CH_LLWW_I } & vor & 0.0 & 0.0 & 0.0 & 8.5 & 0.0 & 8.5 & 0.0 & 8.5 & 0.0 & 0.5 & 0.0 & 8.5 & 0.0 & 8.5 & 0.0 & 8.5 \\
\hline & MIN & 0.0 & 0.0 & 0.0 & B.5 & 0.0 & 8.5 & 0.0 & 8.5 & 0.0 & 8.5 & 0.0 & 8.5 & 0.0 & 8.5 & 0.0 & 8.5 \\
\hline & $\max$ & 0.0 & 0.0 & 0.0 & 27.0 & 0.0 & 17.0 & 0.0 & 17.0 & 0.0 & 17.0 & 0.0 & 27.0 & 0.0 & 17.0 & 0.0 & 17.0 \\
\hline \multirow{2}{*}{$\begin{array}{l}\text { HEC_300_TEDF } \\
\text { Totale }\end{array}$} & VOL & 0.0 & 368.3 & 360.3 & 376.8 & 368.3 & 376.8 & 360.3 & 376.8 & 360.3 & 376.8 & 368.3 & 376.8 & 368.3 & 376.8 & 368.3 & 376.8 \\
\hline & $\begin{array}{l}\text { MIN } \\
\text { MAX }\end{array}$ & $\begin{array}{l}0.0 \\
0.0\end{array}$ & $\begin{array}{l}104.1 \\
552.4\end{array}$ & $\begin{array}{l}184.1 \\
552.1\end{array}$ & $\begin{array}{l}192.6 \\
569.4\end{array}$ & $\begin{array}{l}284.1 \\
552.1\end{array}$ & $\begin{array}{l}192.6 \\
369.4\end{array}$ & $\begin{array}{l}184.1 \\
552.4\end{array}$ & $\begin{array}{l}192.6 \\
569.4\end{array}$ & $\begin{array}{l}184.1 \\
552.4\end{array}$ & $\begin{array}{l}192.6 \\
569.4\end{array}$ & $\begin{array}{l}184.1 \\
532.4\end{array}$ & $\begin{array}{l}192.6 \\
569.4\end{array}$ & $\begin{array}{l}181.1 \\
552.4\end{array}$ & $\begin{array}{l}192.6 \\
569.4\end{array}$ & $\begin{array}{l}184.1 \\
552.4\end{array}$ & $\begin{array}{l}192.6 \\
569.4\end{array}$ \\
\hline
\end{tabular}

Wante Generator: WHC_305

\begin{tabular}{|c|c|c|c|c|c|c|c|c|c|c|c|c|c|c|c|c|c|}
\hline \multirow[t]{3}{*}{ HAz } & VOL & 0.0 & 0.7 & 0.2 & 0.2 & 0.2 & 0.2 & 0.2 & 0.2 & 0.2 & 0.2 & 0.2 & 0.2 & 0.2 & 0.2 & 0.2 & 0.2 \\
\hline & MIN & 0.0 & 0.7 & 0.2 & 0.2 & 0.2 & 0.2 & 0.2 & 0.2 & 0.2 & 0.2 & 0.2 & 0.2 & 0.2 & 0.2 & 0.2 & 0.2 \\
\hline & $\max$ & 0.0 & 0.7 & 0.2 & 0.2 & 0.2 & 0.2 & 0.2 & 0.2 & 0.2 & 0.2 & 0.2 & 0.2 & 0.2 & 0.2 & 0.2 & 0.2 \\
\hline \multirow{3}{*}{$\begin{array}{l}\text { FRC_305 } \\
\text { Tota1. }\end{array}$} & VOL & 0.0 & 0.7 & 0.2 & 0.2 & 0.2 & 0.2 & 0.2 & 0.2 & 0.2 & 0.2 & 0.2 & 0.2 & 0.2 & 0.2 & 0.2 & 0.2 \\
\hline & MIN & 0.0 & 0.7 & 0.2 & 0.2 & 0.2 & 0.2 & 0.2 & 0.2 & 0.2 & 0.2 & 0.2 & 0.2 & 0.2 & 0.2 & 0.2 & 0.2 \\
\hline & $\operatorname{MAX}$ & 0.0 & 0.7 & 0.2 & 0.2 & 0.2 & 0.2 & 0.2 & 0.2 & 0.2 & 0.2 & 0.2 & 0.2 & 0.2 & 0.2 & 0.2 & 0.2 \\
\hline
\end{tabular}

Waste Generator: WHC 306

\begin{tabular}{|c|c|c|c|c|c|c|c|c|c|c|c|c|c|c|c|c|c|}
\hline \multirow[t]{3}{*}{ HA2 } & Vor & 0.0 & 6.3 & 2.7 & 2.7 & 2.7 & 2.7 & 2.7 & 2.7 & 2.7 & 2.7 & 2.7 & 2.7 & 2.7 & 2.7 & 2.7 & 2.7 \\
\hline & MIN & 0.0 & 5.9 & 2.5 & 2.5 & 2.5 & 2.5 & 2.5 & 2.5 & 2.5 & 2.5 & 2.5 & $2.5^{\circ}$ & 2.5 & 2.5 & 2.5 & 2.5 \\
\hline & $\operatorname{HAX}$ & 0.0 & 6.6 & 2.8 & 2.8 & 2.0 & 2.8 & 2.8 & 2.8 & 2.0 & 2.0 & 2.0 & 2.0 & 2.8 & 2.8 & 2.8 & 2.8 \\
\hline \multirow{2}{*}{$\begin{array}{l}\text { HBC_306 } \\
\text { Iotal. }\end{array}$} & VOL & 0.0 & 6.3 & 2.7 & 2.7 & 2.7 & 2.7 & 2.7 & 2.7 & 2.7 & 2.7 & 2.7 & 2.7 & 2.7 & 2.7 & 2.7 & 2.7 \\
\hline & MIN & 0.0 & 5.9 & 2.5 & 2.5 & 2.5 & 2.5 & 2.5 & 2.5 & 2.5 & 2.5 & 2.5 & 2.5 & 2.5 & 2.5 & 2.5 & 2.5 \\
\hline
\end{tabular}




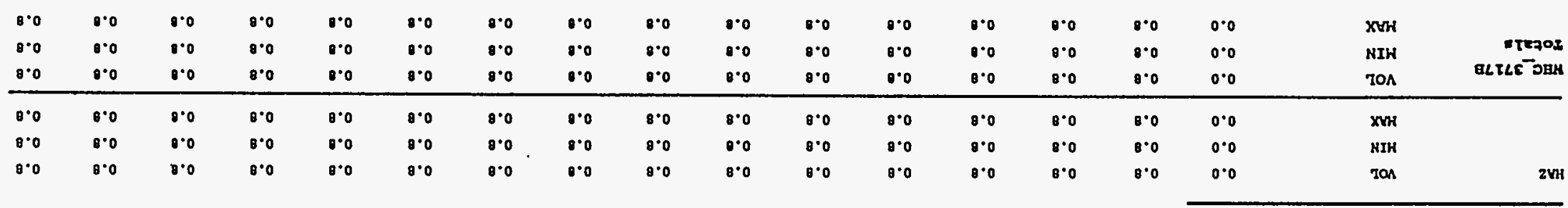

\begin{tabular}{|c|c|c|c|c|c|c|c|c|c|c|c|c|c|c|c|c|c|}
\hline 5.0 & s.o & $s \cdot 0$ & s.o & s.o & $s \cdot 0$ & $s \cdot 0$ & s.0 & $s \cdot 0$ & $s \cdot 0$ & $s \cdot 0$ & s.o & $5 \cdot 0$ & $s \cdot 0$ & $5 \cdot 0$ & 0.0 & $X Y W$ & \\
\hline s.0 & s.o & s:0 & s.o & s.0 & s.o & s.o & s.0 & s.o & s.0 & s.o & s.o & $s \cdot 0$ & s.o & $s \cdot 0$ & 0.0 & NIH & $=$ TE70I \\
\hline s.o & s.o & s.0. & s.o & s.0 & s.o & s.o & $s \cdot 0$ & $5 \cdot 0$ & s.o & $s \cdot 0$ & s.o & s.o & s.o & 5.0 & 0.0 & TOA & OLOLE РHН \\
\hline s:0 & s.o & s.o & s.o & s:o & s.o & , s.0 & $s \cdot 0$ & 5.0 & s.o & s.o & s.o & s.o & s.0 & $s=0$ & 0.0 & $x \mathbf{x w}$ & \\
\hline s.0 & s.o & s.o & s.o & $5: 0$ & s.0 & s.o & $5 \cdot 0$ & s.0 & s.o & s.o & s.0 & s.o & s.o & $s \cdot 0$ & 0.0 & Jon & $2 \mathbf{V}$ \\
\hline
\end{tabular}

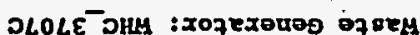

\begin{tabular}{|c|c|c|c|c|c|c|c|c|c|c|c|c|c|}
\hline 0.0 & 0.0 & 0.0 & 0.0 & 0.0 & 0.0 & 0.0 & 0.0 & 0.0 & 0.0 & 0.0 & S.I & $z \cdot \delta$ & S.T \\
\hline 0.0 & 0.0 & 0.0 & 0.0 & 0.0 & 0.0 & 0.0 & 0.0 & 0.0 & 0.0 & 0.0 & $z \cdot 0$ & $9 \cdot 0$ & $2 \cdot 0$ \\
\hline 0.0 & 0.0 & 0.0 & 0.0 & 0.0 & 0.0 & 0.0 & 0.0 & 0.0 & 0.0 & 0.0 & s.o & $9 \cdot z$ & s.0 \\
\hline 0.0 & 0.0 & 0.0 & 0.0 & 0.0 & 0.0 & 0.0 & 0.0 & 0.0 & 0.0 & 0.0 & $S \cdot I$ & $2 \cdot 5$ & $s \cdot t$ \\
\hline 0.0 & 0.0 & 0.0 & 0.0 & 0.0 & 0.0 & 0.0 & 0.0 & 0.0 & 0.0 & 0.0 & $2 \cdot 0$ & 9.0 & $z \cdot 0$ \\
\hline 0.0 & 0.0 & 0.0 & 0.0 & 0.0 & 0.0 & 0.0 & 0.0 & 0.0 & 0.0 & 0.0 & S.0 & $9 \cdot z$ & s.o \\
\hline
\end{tabular}

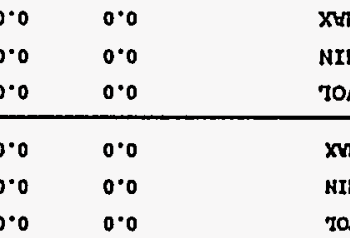

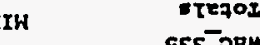

$\mathbf{x w}$

Ton

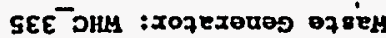

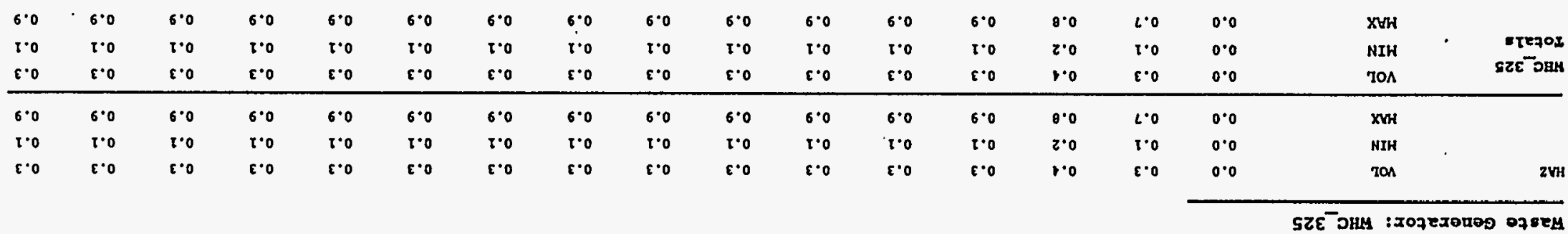

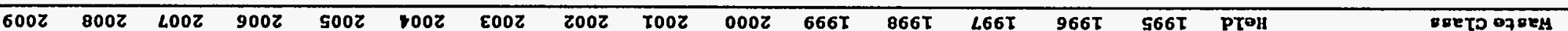

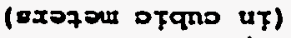

e8z o5va

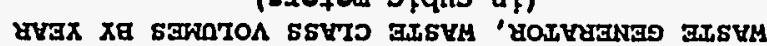

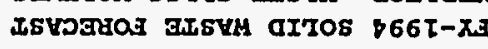


HASTE GENERATOR, HASTE CLASS VOLUMES BY YEAR (in cubio meters)

Haste Class

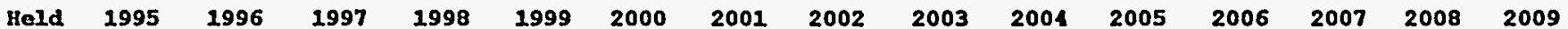
Waste Generator: WHC_AKHIYY__LAB

\begin{tabular}{|c|c|c|c|c|c|c|c|c|c|c|c|c|c|c|c|c|c|}
\hline \multirow[t]{3}{*}{ CH_LLH_I } & vor & 0.0 & 503.5 & 120.8 & 328.0 & 328.0 & 328.0 & 328.0 & 328.0 & 328.0 & 328.0 & 328.0 & 328.0 & 328.0 & 328.0 & 328.0 & 328.0 \\
\hline & MIN & 0.0 & 453.1 & 378.7 & 295.2 & 295.2 & 295.2 & 295.2 & 295.2 & 295.2 & 295.2 & 295.2 & 295.2 & 295.2 & 295.2 & 295.2 & 295.2 \\
\hline & $\max$ & 0.0 & 553.0 & 162.8 & 360.8 & 360.8 & 360.8 & 360.8 & 360.8 & 360.8 & 360.8 & 360.8 & 360.8 & 360.8 & 360.8 & 360.8 & 360.8 \\
\hline \multirow[t]{3}{*}{ CH_LLW_III } & vor & 0.0 & 5.8 & 1.7 & 3.7 & 3.7 & 3.7 & 3.7 & 3.7 & 3.7 & 3.7 & 3.7 & 3.7 & 3.7 & 3.7 & 3.7 & 3.7 \\
\hline & MIN & 0.0 & 5.2 & 1.2 & 3.3 & 3.3 & 3.3 & 3.3 & 3.3 & 3.3 & 3.3 & 3.3 & 3.3 & 3.3 & 3.3 & 3.3 & 3.3 \\
\hline & $\operatorname{HAx}$ & 0.0 & 6.3 & 5.1 & 4.0 & 1.0 & 4.0 & 1.0 & 1.0 & 4.0 & 1.0 & 1.0 & 1.0 & 1.0 & 4.0 & 1.0 & 1.0 \\
\hline \multirow[t]{3}{*}{ CH_LLSA_I } & vor & 0.0 & 30.3 & 32.3 & 25.0 & 25.0 & 25.0 & 25.0 & 25.0 & 25.0 & 25.0 & 25.0 & 25.0 & 25.0 & 25.0 & 25.0 & 25.0 \\
\hline & HIN & 0.0 & 34.4 & 29.0 & 22.5 & 22.5 & 22.5 & 22.5 & 22.5 & 22.3 & 22.5 & 22.5 & 22.5 & 22.5 & 22.5 & 22.5 & 22.5 \\
\hline & $\operatorname{MAX}$ & 0.0 & 12.1 & 35.5 & 27.5 & 27.5 & 27.5 & 27.5 & 27.5 & 27.5 & 27.5 & 27.5 & 27.5 & 27.5 & 27.5 & 27.5 & 27.5 \\
\hline \multirow[t]{3}{*}{ CH_LLH_III } & Vor & 0.0 & 5.8 & 4.7 & 3.7 & 3.7 & 3.7 & 3.7 & .3 .7 & 3.7 & 3.7 & 3.7. & 3.7 & 3.7 & 3.7 & 3.7 & 3.7 \\
\hline & MIN & 0.0 & 5.2 & 4.2 & 3.3 & 3.3 & 3.3 & 3.3 & 3.3 & 3.3 & 3.3 & 3.3 & 3.3 & 3.3 & 3.3 & 3.3 & 3.3 \\
\hline & $\max$ & 0.0 & 6.3 & 5.1 & 1.0 & 1.0 & 1.0 & 1.0 & 1.0 & 1.0 & 1.0 & 1.0 & 1.0 & 1.0 & 4.0 & 1.0 & 1.0 \\
\hline \multirow[t]{3}{*}{ RH_LLWF_III } & vor & 0.0 & 3.1 & 2.1 & 1.8 & 1.8 & 1.8 & 1.8 & 1.8 & 1.8 & 1.8 & 1.8 & 1.8 & 1.8 & 1.8 & 1.8 & 1.0 \\
\hline & MIN & 0.0 & 3.0 & 2.1 & 1.6 & 1.6 & 1.6 & 1.6 & 1.6 & 1.6 & 1.6 & 1.6 & 1.6 & 1.6 & 2.6 & 1.6 & 1.6 \\
\hline & $\max$ & 0.0 & 3,7 & 2.6 & 1.9 & 1.9 & 1.9 & 1.9 & 1.9 & 1.9 & 1.9 & 1.9 & 1.9 & 1.9 & 1.9 & 1.9 & 1.9 \\
\hline \multirow[t]{3}{*}{ RH_TRUYM } & vor & 0.0 & 0.3 & 0.5 & 0.5 & 0.5 & 0.5 & 0.5 & 0.5 & 0.5 & 0.5 & $0.5^{\circ}$ & 0.5 & 0.5 & 0.5 & 0.5 & 0.5 \\
\hline & MIN & 0.0 & 0.2 & 0.1 & 0.4 & 0.1 & 0.1 & 0.1 & 0.1 & 0.4 & 0.4 & 0.4 & 0.1 & 0.1 & 0.1 & 0.1 & 0.4 \\
\hline & $\max$ & 0.0 & 0.3 & 0.5 & $\cdot 0.5$ & 0.5 & 0.5 & 0.5 & 0.5 & 0.5 & 0.5 & 0.5 & 0.5 & 0.5 & 0.5 & 0.5 & 0.5 \\
\hline \multirow[t]{3}{*}{ HAZ } & vot & 0.0 & 3.3 & 2.8 & 2.3 & 2.3 & 2.3 & 2.3 . & 2.3 & 2.3 & 2.3 & 2.3 & 2.3 & 2.3 & 2.3 & 2.3 & 2.3 \\
\hline & MIN & 0.0 & 2.9 & 2.5 & 2.0 & 2.0 & 2.0 & 2.0 & 2.0 & 2.0 & 2.0 & 2.0 & 2.0 & 2.0 & 2.0 & 2.0 & 2.0 \\
\hline & $\max$ & 0.0 & 3.6 & 3.0 & 2.5 & 2.3 & 2.5 & 2.5 & 2.5 & 2.5 & 2.5 & 2.5 & 2.5 & 2.5 & 2.5 & 2.5 & 2.5 \\
\hline \multirow{3}{*}{$\begin{array}{l}\text { WHC_ANASYY_LNAB } \\
\text { Totals }\end{array}$} & voL & 0.0 & 560.4 & 168.2 & 365.0 & 365.0 & 365.0 & 365.0 & 365.0 & 365.0 & 365.0 & 365.0 & 365.0 & 365.0 & 365.0 & 365.0 & 365.0 \\
\hline & MIN & 0.0 & 504.3 & 121.3 & 328.5 & 328.5 & 328.5 & 328.5 & 328.5 & 328.5 & 328.5 & 328.5 & 328.5 & 320.5 & 328.5 & 329.5 & 328.5 \\
\hline & $\operatorname{MAX}$ & 0.0 & 626.4 & 515.0 & 101.5 & 101.5 & 101.5 & 101.5 & 101.5 & 101.5 & 101.5 & 101.5 & 101.5 & 101.5 & 101.5 & 101.5 & 101.5 \\
\hline
\end{tabular}

Waste Generator: FHC_BQUIP

\begin{tabular}{|c|c|c|c|c|c|c|c|c|c|c|c|c|c|c|c|c|c|}
\hline \multirow[t]{3}{*}{ CH_LUH_I } & vor & 0.0 & 326.3 & 368.3 & 170.0 & 170.0 & 352.7 & 352.7 & 352.7 & 352.7 & 226.6 & 226.6 & 226.6 & 339.9 & 339.9 & 339.9 & 170.0 \\
\hline & MIN & 0.0 & 326.3 & 368.3 & 170.0 & 170.0 & 352.7 & 352.7 & 352.7 & 352.7 & 226.6 & 226.6 & 226.6 & 339.9 & 339.9 & 339.9 & 170.0 \\
\hline & $\operatorname{man} x$ & 0.0 & 326.3 & 368.3 & 270.0 & 170.0 & 352.7 & 352.7 & 352.7 & 352.7 & 226.6 & 226.6 & 226.6 & 339.9 & 339.9 & 339.9 & 170.0 \\
\hline \multirow{3}{*}{$\begin{array}{l}\text { HRC_BQUTP } \\
\text { Totall }\end{array}$} & VOL & 0.0 & 326.3 & 368.3 & 170.0 & 270.0 & 352.7 & 352.7 & 352.7 & 352.7 & 226.6 & 226.6 & 226.6 & 339.9 & 339.9 & 339.9 & 170.0 \\
\hline & MIN & 0.0 & 326.3 & 360.3 & 170.0 & 170.0 & 352.7 & 352.7 & 352.7 & 352.7 & 226.6 & 226.6 & 226.6 & 339.9 & 339.9 & 339.9 & 170.0 \\
\hline & MAX & 0.0 & 326.3 & 368.3 & 170.0 & 170.0 & 352.7 & 352.7 & 352.7 & 352.7 & 226.6 & 226.6 & 226.6 & 339.9 & . 339.9 & 339.9 & 170.0 \\
\hline
\end{tabular}




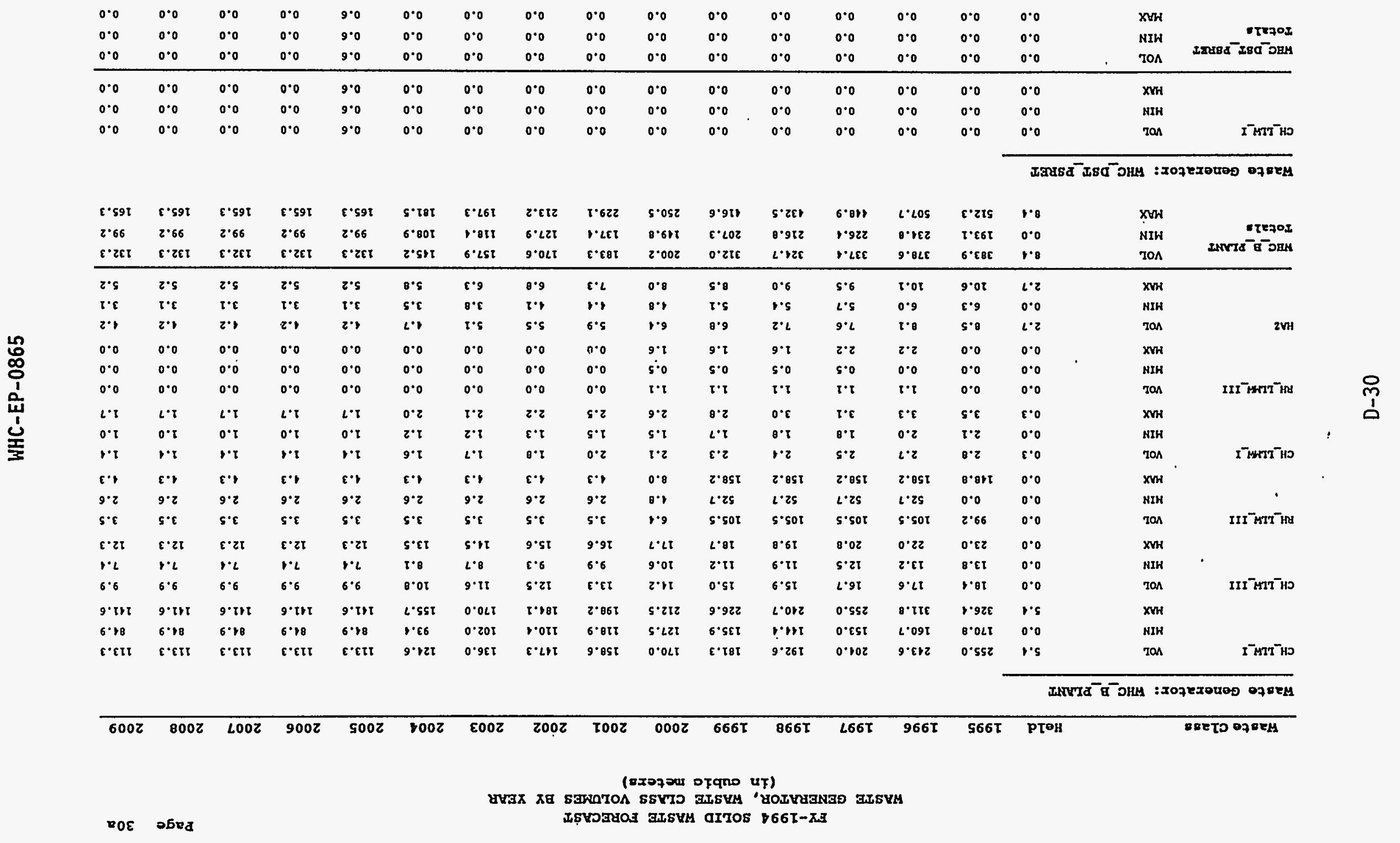


FY-1994 SOLID WASTE FORECAST

WASTE GENERATOR, HASTE CIASS VOLOMES BY YEAR

(in cubic meters)

Fasto Class

$1996 \quad 1997$

1998

\begin{tabular}{lllllll}
2003 & 2004 & 2005 & 2006 & 2007 & 2008 & 2009 \\
\hline
\end{tabular}

\begin{tabular}{|c|c|c|c|c|c|c|c|c|c|c|c|c|c|c|c|c|c|}
\hline \multirow[t]{3}{*}{ CH_LLW_I } & vot & 0.0 & 0.0 & 0.0 & 0.0 & 0.0 & 0.0 & 0.0 & 0.0 & 0.0 & 0.0 & 0.0 & 0.0 & 0.0 & 0.0 & 73.1 & 75.0 \\
\hline & MIN & 0.0 & 0.0 & 0.0 & 0.0 & 0.0 & 0.0 & 0.0 & 0.0 & 0.0 & 0.0 & 0.0 & 0.0 & 0.0 & 0.0 & 36.7 & 37.5 \\
\hline & $\max$ & 0.0 & 0.0 & 0.0 & 0.0 & 0.0 & 0.0 & 0.0 & 0.0 & 0.0 & 0.0 & 0.0 & 0.0 & 0.0 & 0.0 & 146.8 & 150.0 \\
\hline \multirow[t]{3}{*}{ PH_LLH__III } & vor & 0.0 & 0.0 & 0.0 & 0.0 & 0.0 & 0.0 & 0.0 & 0.0 & 0.0 & 0.0 & 0.0 & 0.0 & 0.0 & 0.0 & 0.0 & 0.0 \\
\hline & MIN & 0.0 & 0.0 & 0.0 & 0.0 & 0.0 & 0.0 & 0.0 & 0.0 & 0.0 & 0.0 & 0.0 & 0.0 & 0.0 & 0.0 & 0.0 & 0.0 \\
\hline & $\max$ & 0.0 & 0.0 & 0.0 & 0.0 & 0.0 & 0.0 & 0.0 & 0.0 & 0.0 & 0.0 & 0.0 & 0.0 & 0.0 & 0.0 & 0.0 & 0.0 \\
\hline \multirow[t]{3}{*}{ RH_LLAF_GTCIII } & vor & 0.0 & 0.0 & 0.0 & 0.0 & 0.0 & 0.0 & 0.0 & 0.0 & 0.0 & 0.0 & 0.0 & 0.0 & 0.0 & 0.0 & 14.7 & 11.7 \\
\hline & MIN & 0.0 & 0.0 & 0.0 & 0.0 & 0.0 & 0.0 & 0.0 & 0.0 & 0.0 & 0.0 & 0.0 & 0.0 & 0.0 & 0.0 & 22.3 & 22.3 \\
\hline & $\max$ & 0.0 & 0.0 & 0.0 & 0.0 & 0.0 & 0.0 & 0.0 & 0.0 & 0.0 & 0.0 & 0.0 & 0.0 & 0.0 & 0.0 & 89.1 & 89.1 \\
\hline \multirow[t]{3}{*}{ RH_IRUMA } & vor & 0.0 & 0.0 & 0.0 & 0.0 & 0.0 & 0.0 & 0.0 & 0.0 & 0.0 & 0.0 & 0.0 & $0.0^{\circ}$ & 0.0 & 0.0 & 0.0 & 0.0 \\
\hline & MIN & 0.0 & 0.0 & 0.0 & 0.0 & 0.0 & 0.0 & 0.0 & 0.0 & 0.0 & 0.0 & 0.0 & 0.0 & 0.0 & 0.0 & 0.0 & 0.0 \\
\hline & $\operatorname{mix}$ & 0.0 & 0,0 & 0.0 & 0.0 & 0.0 & 0.0 & 0.0 & 0.0 & 0.0 & 0.0 & 0.0 & 0.0 & 0.0 & 0.0 & 0.0 & 0.0 \\
\hline \multirow{3}{*}{$\begin{array}{l}\text { FRC_DST_RBT } \\
\text { Total: }\end{array}$} & VOL & 0.0 & 0.0 & 0.0 & 0.0 & 0.0 & 0.0 & 0.0 & 0.0 & 0.0 & 0.0 & 0.0 & 0.0 & 0.0 & 0.0 & 118.1 & 119.7 \\
\hline & MIN & 0.0 & 0.0 & .0 .0 & 0.0 & 0.0 & 0.0 & 0.0 & 0.0 & 0.0 & 0.0 & 0.0 & 0.0 & 0.0 & 0.0 & 59.0 & 59.8 \\
\hline & $\operatorname{MAX}$ & 0.0 & 0.0 & 0.0 & 0.0 & 0.0 & 0.0 & 0.0 & 0.0 & 0.0 & 0.0 & 0.0 & 0.0 & 0.0 & 0.0 & 236.2 & 239.4 \\
\hline
\end{tabular}

Was to Generator: WHC_En_W112

\begin{tabular}{|c|c|c|c|c|c|c|c|c|c|c|c|c|c|c|c|c|c|}
\hline \multirow[t]{3}{*}{ CH_tRU } & Vor & 0.0 & 30.3 & 0.0 & 0.0 & 0.0 & 0.0 & 0.0 & 0.0 & 0.0 & 0.0 & 0.0 & 0.0 & 0.0 & 0.0 & 0.0 & 0.0 \\
\hline & HIN & 0.0 & 30.3 & 0.0 & 0.0 & 0.0 & 0.0 & 0.0 & 0.0 & 0.0 & 0.0 & 0.0 & 0.0 & 0.0 & 0.0 & 0.0 & 0.0 \\
\hline & $\operatorname{mAx}$ & 0.0 & 38.3 & 0.0 & 0.0 & 0.0 & 0.0 & 0.0 & 0.0 & 0.0 & 0.0 & 0.0 & 0.0 & 0.0 & 0.0 & 0.0 & 0.0 \\
\hline \multirow[t]{3}{*}{ HAZ } & vor & 0.0 & 0.0 & 0.0 & 1.1 & 0.0 & 0.0 & 0.0 & 0.0 & 0.0 & 0.0 & 0.0 & 0.0 & 0.0 & 0.0 & 0.0 & 0.0 \\
\hline & MIN & 0.0 & 0.0 & 0.0 & 1.1 & 0.0 & 0.0 & 0.0 & 0.0 & 0.0 & 0.0 & 0.0 & 0.0 & 0.0 & 0.0 & 0.0 & 0.0 \\
\hline & $\max$ & 0.0 & 0.0 & 0.0 & 1.4 & 0.0 & 0.0 & 0.0 & 0.0 & 0.0 & 0.0 & 0.0 & 0.0 & 0.0 & 0.0 & 0.0 & 0.0 \\
\hline \multirow{3}{*}{$\begin{array}{l}\text { HBC_2X_H112 } \\
\text { Tot:21: }\end{array}$} & VOL & 0.0 & 38.3 & 0.0 & 1.4 & 0.0 & 0.0 & 0.0 & $0.0^{\circ}$ & 0.0 & 0.0 & 0.0 & 0.0 & 0.0 & 0.0 & 0.0 & 0.0 \\
\hline & MIN & 0.0 & 38.3 & 0.0 & 1.4 & 0.0 & 0.0 & 0.0 & 0.0 & 0.0 & 0.0 & 0.0 & 0.0 & 0.0 & 0.0 & 0.0 & 0.0 \\
\hline & $\operatorname{MAX}$ & 0.0 & 38.3 & 0.0 & 1.4 & 0.0 & 0.0 & 0.0 & 0.0 & 0.0 & 0.0 & 0.0 & 0.0 & 0.0 & 0.0 & 0.0 & 0.0 \\
\hline
\end{tabular}

Wasto Genoratox: WHC_EED

\begin{tabular}{|c|c|c|c|c|c|c|c|c|c|c|c|c|c|c|c|c|c|}
\hline \multirow[t]{3}{*}{ Han } & Vor & 0.0 & 1.3 & 2.3 & 0.4 & 0.1 & 0.4 & 0.1 & 0.1 & 0.1 & 0.0 & 0.0 & 0.0 & 0.0 & 0.0 & 0.0 & 0.0 \\
\hline & MIN & 0.0 & 1.3 & 1.3 & 0.1 & 0.1 & 0.4 & 0.4 & 0.1 & 0.1 & 0.0 & 0.0 & 0.0 & 0.0 & 0.0 & 0.0 & 0.0 \\
\hline & $\operatorname{sax}$ & 0.0 & 1.3 & 1.3 & 0.1 & 0.1 & 0.4 & 0.4 & 0.1 & 0.1 & 0.0 & 0.0 & 0.0 & 0.0 & 0.0 & 0.0 & 0.0 \\
\hline
\end{tabular}


FY-1994 SOLID WASTE FORECAST

WASTE GENERATOR, WASTE CLASg VOLUMES BY YEAR

(In cublo meters)

\begin{tabular}{|c|c|c|c|c|c|c|c|c|c|c|c|c|c|c|c|c|c|}
\hline Waste & & Held & 1995 & 1996 & 1997 & 1998 & 1999 & 2000 & 2001 & 2002 & 2003 & 2004 & 2005 & 2006 & 2007 & 2008 & 2009 \\
\hline \multirow{3}{*}{$\begin{array}{l}\text { Hac_Fro } \\
\text { Total. }\end{array}$} & VoL & 0.0 & 1.3 & 1.3 & 0.4 & 0.1 & 0.1 & 0.4 & 0.4 & 0.4 & 0.0 & 0.0 & 0.0 & 0.0 & 0.0 & 0.0 & 0.0 \\
\hline & MIN & 0.0 & 1.3 & 1.3 & 0.1 & 0.1 & 0.1 & 0.1 & 0.4 & 0.1 & 0.0 & 0.0 & 0.0 & 0.0 & 0.0 & 0.0 & 0.0 \\
\hline & $\max$ & 0.0 & 1.3 & 1.3 & 0.1 & 0.4 & 0.1 & 0.1 & 0.1 & 0.1 & 0.0 & 0.0 & 0.0 & 0.0 & 0.0 & 0.0 & 0.0 \\
\hline
\end{tabular}

Waste Generator: WHC_FETE_ManK

\begin{tabular}{|c|c|c|c|c|c|c|c|c|c|c|c|c|c|c|c|c|}
\hline \multirow[t]{3}{*}{ CH_LLH_I } & vos & 0.0 & 14.1 & 14.4 & 14.4 & 14.4 & 34.1 & 14.1 & 31.4 & 0.0 & 0.0 & 0.0 & 0.0 & 0.0 & 0.0 & 0.0 \\
\hline & MIN & 0.0 & 7.2 & 7.2 & 7.2 & 7.2 & 7.2 & 7.2 & 7.2 & 0.0 & 0.0 & 0.0 & 0.0 & 0.0 & 0.0 & 0.0 \\
\hline & $\max$ & 0.0 & 21.6 & 21.6 & 21.6 & 21.6 & 21.6 & 21.6 & 21.6 & 0.0 & 0.0 & 0.0 & 0.0 & 0.0 & 0.0 & 0.0 \\
\hline \multirow[t]{2}{*}{ PH_LLW_III } & vot & 0.0 & 25.9 & 15.9 & 15.9 & 0.0 & 0.0 & 0.0 & 0.0 & 0.0 & 0.0 & 0.0 & 0.0 & 0.0 & 0.0 & 0.0 \\
\hline & $\max$ & 0.0 & 25.9 & 15.9 & 15.9 & 0.0 & 0.0 & 0.0 & 0.0 & 0.0 & 0.0 & 0.0 & 0.0 & 0.0 & 0.0 & 0.0 \\
\hline \multirow[t]{3}{*}{ HAZ } & vor & 0.0 & 3.2 & 3.2 & 3.2 & 3.2 & 3.2 & 3.2 & 3.2 & 0.0 & 0.0 & 0.0 & 0.0 & 0.0 & 0.0 & 0.0 \\
\hline & HIH & 0.0 & 3.2 & 3.2 & 3.2 & 3.2 & 3.2 & 3.2 & 3.2 & 0.0 & 0.0 & 0.0 & 0.0 & 0.0 & 0.0 & 0.0 \\
\hline & $\operatorname{mnx}$ & 0.0 & 6.1 & 6.4 & 6.1 & 6.1 & 6.1 & 6.1 & 6.1 & 0.0 & 0.0 & 0.0 & 0.0 & 0.0 & 0.0 & 0.0 \\
\hline \multirow{3}{*}{$\begin{array}{l}\text { WHC_mTr_maxN } \\
\text { rotaly }\end{array}$} & VOL & 0.0 & 33.5 & 33.5 & 33.5 & 17.6 & 17.6 & 27.6 & 17.6 & 0.0 & 0.0 & 0.0 & 0.0 & 0.0 & 0.0 & 0.0 \\
\hline & MIN & 0.0 & 26.3 & 26.3 & 26.3 & $10 . i$ & 10.1 & 10.1 & 10.4 & 0.0 & 0.0 & 0.0 & 0.0 & 0.0 & 0.0 & 0.0 \\
\hline & $\operatorname{MAX}$ & 0.0 & 43.9 & 43.9 & 13.9 & 28.0 & 28.0 & 28.0 & 28.0 & 0.0 & 0.0 & 0.0 & 0.0 & 0.0 & 0.0 & 0.0 \\
\hline
\end{tabular}

Waste Generatox: FHC_FMEE

\begin{tabular}{|c|c|c|c|c|c|c|c|c|c|c|c|c|c|c|c|c|c|}
\hline \multirow[t]{3}{*}{ HAZ } & YOL & 0.0 & 1.3 & 1.3 & 1.3 & 1.3 & 1.3 & 1.3 & 1.3 & 1.3 & 1.3 & 1.3 & 1.3 & 1.3 & 1.3 & 1.3 & 1.3 \\
\hline & MIN & 0.0 & 0.6 & 0.6 & 0.6 & 0.6 & 0.6 & 0.6 & 0.6 & 0.6 & 0.6 & 0.6 & 0.6 & 0.6 & 0.6 & 0.6 & 0.6 \\
\hline & $\operatorname{HAX}$ & 0.0 & 1.9 & 1.9 & 1.9 & 1.9 & 1.9 & 1.9 & 1.9 & 1.9 & 1.9 & 1.9 & 1.9 & 1.9 & 1.9 & 1.9 & 1.9 \\
\hline \multirow{3}{*}{$\begin{array}{l}\text { WRC_FRBP } \\
\text { Totals }\end{array}$} & VoL & 0.0 & 1.3 & 1.3 & 1.3 & 1.3 & 1.3 & 1.3 & 1.3 & 1.3 & 1.3 & 1.3 & 1.3 & 1.3 & 1.3 & 1.3 & 1.3 \\
\hline & MIN & 0.0 & 0.6 & 0.6 & 0.6 & 0.6 & 0.6 & 0.6 & 0.6 & 0.6 & 0.6 & 0.6 & 0.6 & 0.6 & 0.6 & 0.6 & 0.6 \\
\hline & $\operatorname{MAX}$ & 0.0 & 3.9 & 1.9 & 1.9 & 1.9 & 1.9 & 1.9 & 1.9 & 1.9 & 1.9 & 1.9 & 1.9 & 1.9 & 1.9 & 1.9 & 1.9 \\
\hline
\end{tabular}

Waste Generator: HHC_EUEr_DEV

\begin{tabular}{|c|c|c|c|c|c|c|c|c|c|c|c|c|c|c|c|c|}
\hline \multirow{3}{*}{$\mathrm{CH}_{-} \mathrm{LLH} \mathrm{H}_{-} \mathrm{I}$} & Vor & 0.0 & 18.0 & 0.0 & 0.0 & 0.0 & 0.0 & 0.0 & 0.0 & 0.0 & 0.0 & 0.0 & 0.0 & 0.0 & 0.0 & 0.0 \\
\hline & MIH & 0.0 & 9.4 & 0.0 & 0.0 & 0.0 & 0.0 & 0.0 & 0.0 & 0.0 & 0.0 & 0.0 & 0.0 & 0.0 & 0.0 & 0.0 \\
\hline & $\max$ & 0.0 & 28.2 & 0.0 & 0.0 & 0.0 & 0.0 & 0.0 & 0.0 & 0.0 & 0.0 & 0.0 & 0.0 & 0.0 & 0.0 & 0.0 \\
\hline \multirow[t]{2}{*}{$\mathrm{CH}_{-} \mathrm{IRU}$} & Vol & 0.0 & 0.0 & 1.3 & 0.0 & 0.0 & 0.0 & 0.0 & 0.0 & 0.0 & 0.0 & 0.0 & 0.0 . & 0.0 & 0.0 & 0.0 \\
\hline & $\max$ & 0.0 & 0.0 & 1.3 & 0.0 & 0.0 & 0.0 & 0.0 & 0.0 & 0.0 & 0.0 & 0.0 & 0.0 & 0.0 & 0.0 & 0.0 \\
\hline
\end{tabular}




\begin{tabular}{|c|c|c|c|c|c|c|c|c|c|c|c|c|c|c|c|c|c|}
\hline $\begin{array}{l}\text { lo } \\
\qquad \cdot \varepsilon \\
9 \cdot 0 \\
s \cdot \tau\end{array}$ & $\begin{array}{l}. \cdot \varepsilon \\
9.0 \\
5 \cdot t\end{array}$ & $\begin{array}{l}r \cdot \varepsilon \\
9 \cdot 0 \\
5 \cdot 1\end{array}$ & $\begin{array}{l}1 \% \varepsilon \\
9.0 \\
9.6\end{array}$ & $\begin{array}{l}1 \cdot \varepsilon \\
9.0 \\
2 . t\end{array}$ & $\begin{array}{l}P \cdot c \\
9 \cdot 0 \\
s \cdot t\end{array}$ & $\begin{array}{l}1.6 \\
9.0 \\
2.1\end{array}$ & $\begin{array}{l}P \cdot \varepsilon \\
9 \cdot 0 \\
S \cdot \tau\end{array}$ & $\begin{array}{l}1 \cdot c \\
9.0 \\
2 \cdot 1\end{array}$ & $\begin{array}{l}\because \cdot \varepsilon \\
9 \cdot 0 \\
s \cdot t\end{array}$ & $\begin{array}{l}. \cdot \varepsilon \\
9.0 \\
2.1\end{array}$ & $\begin{array}{l}\because \cdot \varepsilon \\
9 \cdot 0 \\
s \cdot \tau\end{array}$ & $\begin{array}{l}. \cdot \varepsilon \\
9 \cdot 0 \\
s \cdot t\end{array}$ & $\begin{array}{l}. \cdot \varepsilon \\
9 \cdot 0 \\
S \cdot \tau\end{array}$ & $\begin{array}{l}0.05 \\
0 \cdot 8 \\
8 \cdot 4 t\end{array}$ & $\begin{array}{l}0.0 \\
0.0 \\
0.0\end{array}$ & $\begin{array}{l}X W W \\
\text { NIW } \\
\text { TON }\end{array}$ & 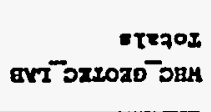 \\
\hline$S \cdot \tau$ & $s \cdot t$ & $S^{\circ} \tau$ & $s \cdot \tau$ & $5 \cdot t$ & $s \cdot \tau$ & $s \cdot t$ & $s \cdot \tau$ & $c \cdot t$ & s.t & s.t & $S \cdot T$ & $5 \cdot \tau$ & $s \cdot \tau$ & S.I & 0.0 & 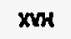 & \\
\hline$z \cdot 0$ & $z \cdot 0$ & $z \cdot 0$ & $2 \cdot 0$ & $2 \cdot 0$ & $2 \cdot 0$ & $\tau \cdot 0$ & $z \cdot 0$ & $2 \cdot 0$ & $2 \cdot 0$ & $2 \cdot 0$ & $2 \cdot 0$ & $2 \cdot 0$ & $2 \cdot 0$ & $2 \cdot 0$ & 0.0 & NIK & \\
\hline$s \cdot 0$ & $s \cdot 0$ & $s \cdot o$ & s.o & $5 \cdot 0$ & s.o & $s \cdot 0$ & s.o & s.o & $5 \cdot 0$ & s.0 & $s \cdot 0$ & s.o & $s \cdot 0$ & $s \cdot 0$ & 0.0 & $\operatorname{Tan}$ & $2 \mathrm{VH}$ \\
\hline 6.0 & 6.0 & $6^{\circ} 0$ & 6.0 & 6.0 & 6.0 & 6.0 & 6.0 & 6.0 & 6.0 & 6.0 & 6.0 & 6.0 & 6.0 & 6.0 & 0.0 & $\mathbf{X V H}$ & \\
\hline ז00 & $\tau=0$ & 700 & $1 \cdot 0$ & $\tau \cdot 0$ & I.0 & I:0 & r.0 & 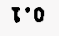 & $\tau \cdot 0$ & $\tau: 0$ & $\tau 0$ & $\tau \cdot 0$ & r.0 & $r \cdot 0$ & 0.0 & MIH & \\
\hline$\varepsilon \cdot 0$ & $c \cdot 0$ & $c^{\circ} 0$ & $\varepsilon \cdot 0$ & $c \cdot 0$ & $\varepsilon \cdot 0$ & 6.0 & $\varepsilon \cdot 0$ & E.0 & $\varepsilon \cdot 0$ & $c \cdot 0$ & $E \cdot 0$ & $\varepsilon \cdot 0$ & $\varepsilon^{\circ} 0$ & $c \cdot 0$ & 0.0 & TOS & $I^{-} \min T^{-}$HD \\
\hline $0 . \tau$ & $0 . \tau$ & $0 . \tau$ & $0 . \tau$ & $0 \cdot \tau$ & $0 . \tau$ & 0.1 & $0 . \tau$ & 0.1 & $0 \cdot \mathfrak{r}$ & $0 . t$ & $0 \cdot \tau$ & 0.1 & 0.7 & S.9t & 0.0 & XWH & \\
\hline $2 \cdot 0$ & $2 \cdot 0$ & $2 \cdot 0$ & $2 \cdot 0$ & $2 \cdot 0$ & $z \cdot 0$ & $z \cdot 0$ & $2 \cdot 0$ & $2 \cdot 0$ & $2 \cdot 0$ & $2 \cdot 0$ & $\tau \cdot 0$ & $z \cdot 0$ & $2 \cdot 0$ & $2 \cdot 8$ & 0.0 & NIH & \\
\hline L.O & $L \cdot 0$ & $\iota \cdot 0$ & $\iota \cdot 0$ & L.O & $L \cdot 0$ & L.O & $2 \cdot 0$ & $l \cdot 0$ & $\iota \cdot 0$ & $L \cdot 0$ & $L \cdot O$ & $L \cdot O$ & $1 \cdot 0$ & $0 . \tau \tau$ & 0.0 & 201 & $I^{-}$МTT \\
\hline
\end{tabular}

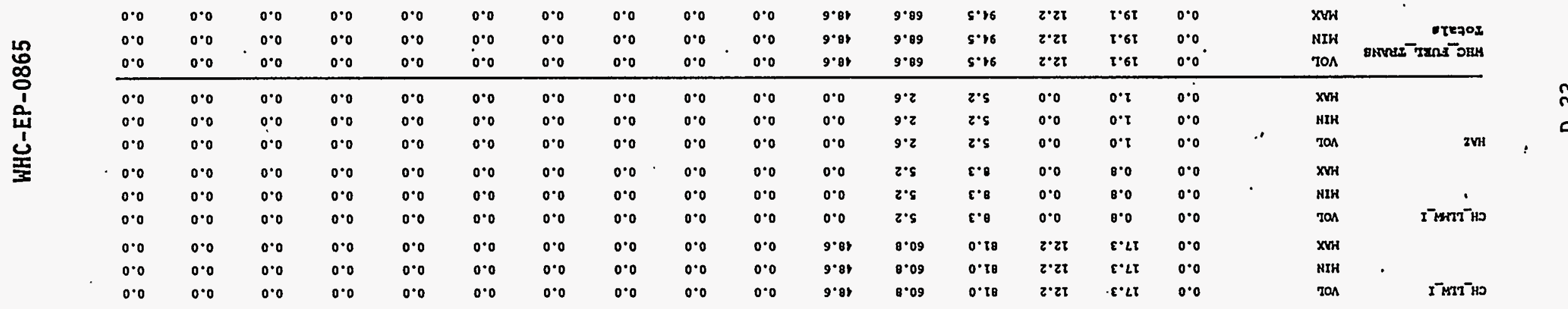

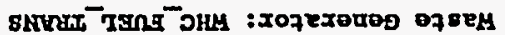

\begin{tabular}{|c|c|c|c|c|c|c|c|c|c|c|c|c|c|c|c|c|c|}
\hline 0.0 & 0.0 & 0.0 & 0.0 & 0.0 & 0.0 & 0.0 & 0.0 & $0: 0$ & 0.0 & 0.0 & 0.0 & 0.0 & $\boldsymbol{\epsilon} \cdot \boldsymbol{\tau}$ & $z \cdot \theta z$ & 0.0 & $x W$ & \\
\hline 0.0 & 0.0 & 0.0 & 0.0 & 0.0 & 0.0 & 0.0 & 0.0 & 0.0 & 0.0 & 0.0 & 0.0 & 0.0 & 0.0 & 1.6 & 0.0 & NIK & 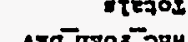 \\
\hline 0.0 & 0.0 & 0.0 & 0.0 & 0.0 & 0.0 & 0.0 & 0.0 & 0.0 & 0.0 & 0.0 & 0.0 & 0.0 & $\varepsilon \cdot \tau$ & $0.8 \mathrm{~B}$ & 0.0 & TON & \\
\hline
\end{tabular}

(25070ux orqno ur)

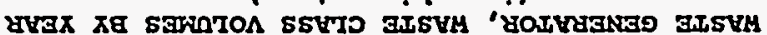

УEE गБษd ISYDTROT IISYM AITOS B66I-XI 


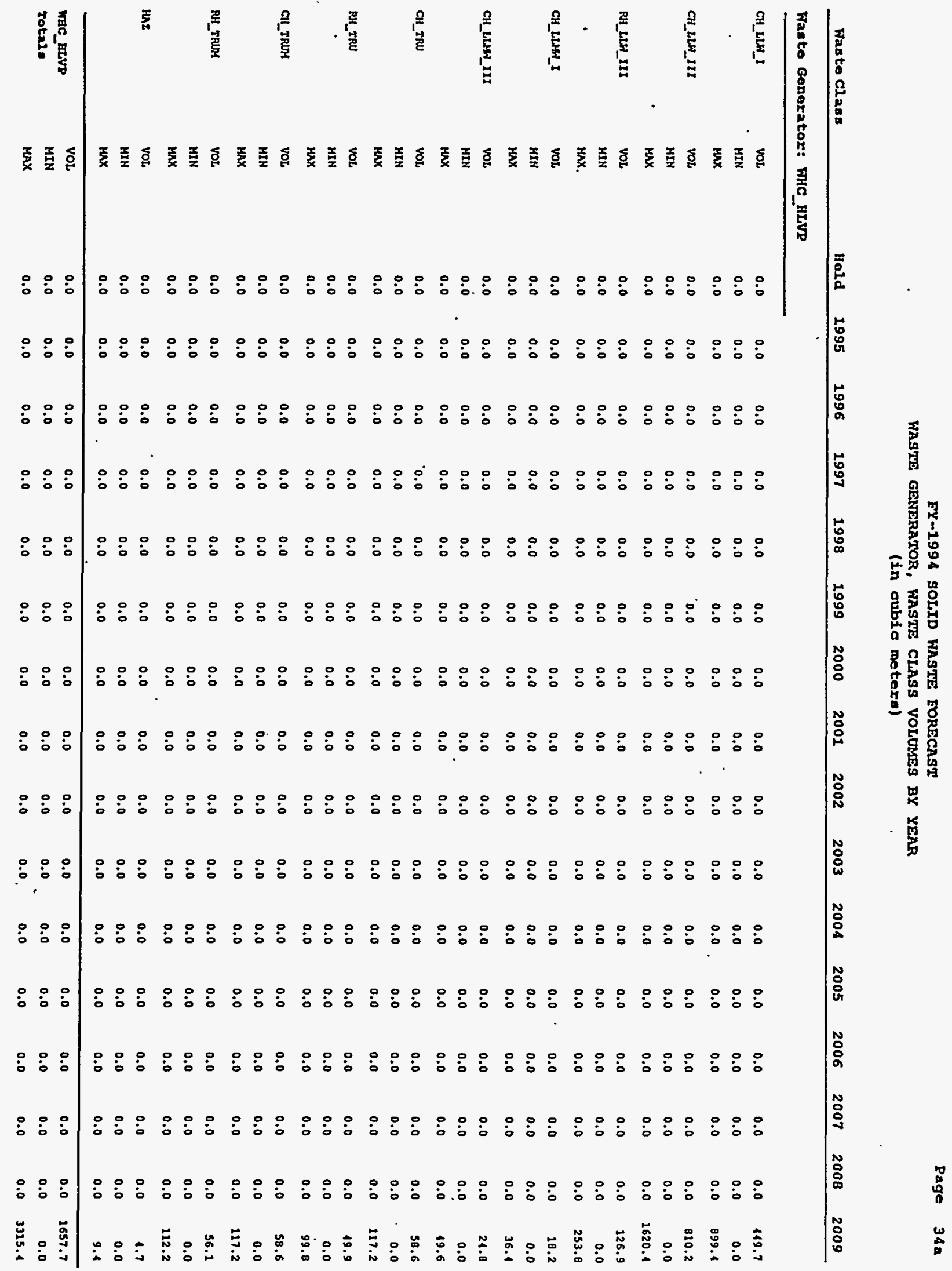


FY-1994 SOLID FASTE FORECAST

WASTE GENERATOR, WASTE CLASS VOROMES BY YEAR

(in cublo metera)

\begin{tabular}{|c|c|c|c|c|c|c|c|c|c|c|c|c|c|c|c|c|c|}
\hline Waste Class & & Held & 1995 & 1996 & 1997 & 1998 & 1999 & 2000 & 2001 & 2002 & 2003 & 2004 & 2005 & 2006 & 2007 & 2008 & 2009 \\
\hline \multicolumn{18}{|c|}{ Wasto Generator: FHC_K_BAsIN } \\
\hline \multirow[t]{3}{*}{$\mathrm{CH}_{-} \mathrm{LLH} \mathrm{H}_{-} \mathrm{I}$} & vor & 0.0 & 264.5 & 261.5 & 139.3 & 139.3 & 239.3 & 139.3 & 139.3 & 139.3 & 139.3 & 139.3 & 239.3 & 139.3 & 139.3 & 239.3 & 139.3 \\
\hline & MIN & 0.0 & $238: 0$ & 235.3 & 111.4 & 111.4 & 111.1 & 111.1 & 111.1 & 111.1 & 111.4 & 121.1 & 211.4 & 111.4 & 111.4 & 211.4 & 211.4 \\
\hline & $\max$ & 0.0 & 290.9 & 287.6 & 139.3 & 139.3 & 139.3 & 139.3 & 139.3 & 139.3 & 239.3 & 139.3 & 239.3 & 139.3 & 139.3 & 139.3 & 139.3 \\
\hline \multirow[t]{3}{*}{ PH_LLH_I } & vor & 0.0 & 5.9 & 1.1 & 1.1 & 1.4 & 1.4 & 1.1 & 1.4 & 1.4 & 1.4 & 1.4 & 1.1 & 1.4 & 1.1 & 1.1 & 1.1 \\
\hline & MIN & 0.0 & 5.3 & 1.1 & 1.1 & 1.1 & 1.1 & 1.1 & 1.1 & 1.1 & 1.1 & 1.1 & 1.1 & 1.1 & 1.1 & 1.1 & 1.1 \\
\hline & $\max$ & 0.0 & 6.1 & 1.1 & 1.1 & 1.4 & 2.4 & 1.1 & 1.4 & 2.4 & 1.1 & 2.1 & 1.4 & 1.4 & 1.1 & 1.1 & 1.1 \\
\hline \multirow[t]{3}{*}{ CH_LLWN_I } & vot, & 0.0 & 1.1 & 1.1 & 1.4 & 1.4 & 1.4 & 1.1 & 1.4 & 1.4 & 1.4 & 1.1 & 1.1 & 1.4 & 2.4 & 1.1 & 1.1 \\
\hline & MIN & 0.0 & 1.2 & 1.2 & 1.0 & 1.0 & 1.0 & 1.0 & 1.0 & 1.0 & 2.0 & 1.0 & 1.0 & 1.0 & 1.0 & 1.0 & 1.0 \\
\hline & $\max$ & 0.0 & 1.5 & 1.5 & 1.4 & 1.1 & 1.1 & 1.4 & 1.4 & 1.1 & 1.4 & 1.1 & 1.4 & 1.1 & 1.1 & 1.4 & 1.1 \\
\hline \multirow[t]{3}{*}{$\mathrm{RH}_{-} \mathrm{TRU}$} & var & 0.0 & 67.7 & 0.0 & 0.0 & 0.0 & 0.0 & 0.0 & 0.0 & 0.0 & 0.0 & 0.0 & 0.0 & 0.0 & 0.0 & 0.0 & 0.0 \\
\hline & MIM & 0.0 & 60.9 & 0.0 & 0.0 & 0.0 & 0.0 & 0.0 & 0.0 & 0.0 & 0.0 & 0.0 & 0.0 & 0.0 & 0.0 & 0.0 & 0.0 \\
\hline & $\max$ & 0.0 & 71.4 & 0.0 & 0.0 & 0.0 & 0.0 & 0.0 & 0.0 & 0.0 & 0.0 & 0.0 & 0.0 & 0.0 & 0.0 & 0.0 & 0.0 \\
\hline \multirow[t]{3}{*}{$\operatorname{Hnz}$} & voL & 0.0 & 2.8 & 2.8 & 2.1 & 2.1 & 1.4 & 1.1 & 1.1 & 1.4 & 1.4 & 1.4 & 2.4 & 1.1 & 1.4 & 1.1 & 1.1 \\
\hline & MIN & 0.0 & 2.5 & 2.5 & 1.8 & 1.8 & 1.0 & 1.0 & 1.0 & 1.0 & 1.0 & 1.0 & 1.0 & 1.0 & 1.0 & 1.0 & 2.0 \\
\hline & $\max$ & 0.0 & 3.0 & 3.0 & 2.1 & 2.1 & 1.4 & 1.4 & 1.1 & 1.4 & 1.4 & 1.4 & 2.4 & 1.4 & 1.4 & 1.4 & 1.4 \\
\hline \multirow{3}{*}{$\begin{array}{l}\text { HaC_X_angry } \\
\text { Totals }\end{array}$} & VOL & 0.0 & 312.3 & 267.1 & 111.2 & 144.2 & 243.5 & 143.5 & 243.5 & 213.5 & 143.5 & 143.5 & 243.5 & 143.5 & 143.5 & 143.5 & 243.5 \\
\hline & MIN & 0.0 & 308.0 & 240.2 & 215.5 & 115.3 & 214.6 & 114.6 & 114.6 & 114.6 & 114.6 & 114.6 & 121.6 & 114.6 & 114.6 & 114.6 & 114.6 \\
\hline & $\max$ & 0.0 & 376.5 & 293.6 & 111.2 & 114.2 & 243.5 & 243.5 & 143.5 & 113.5 & 243.5 & 143.5 & 143.5 & 214.5 & 143.5 & 243.5 & 143.5 \\
\hline \multicolumn{18}{|c|}{ Waste Genorator: WHC_IMUNDRX } \\
\hline \multirow[t]{3}{*}{ CH_LL_I } & vor & 0.0 & 113.3 & 113.3 & 113.3 & 113.3 & 113.3 & 213.3 & 113.3 & 113.3 & 113.3 & 113.3 & 113.3 & 113.3 & 113.3 & 113.3 & 113.3 \\
\hline & MtN & 0.0 & 113.3 & 113.3 & 113.3 & 113.3 & 113.3 & 113.3 & 113.3 & 213.3 & 213.3 & 113.3 & 113.3 & 113.3 & 113.3 & 213.3 & 213.3 \\
\hline & max & 0.0 & 124.6 & 124.6 & 124.6 & 124.6 & 224.6 & 124.6 & 124.6 & 124.6 & 124.6 & 124.6 & 124.6 & 124.6 & 224.6 & 124.6 & 221.6 \\
\hline \multirow[t]{3}{*}{ HAZ } & vol & 0.0 & 0.3 & 0.3 & 0.3 & 0.3 & 0.3 & 0.3 & 0.3 & 0.3 & 0.3 & 0.3 & 0.3 & 0.3 & 0.3 & 0.3 & 0.3 \\
\hline & MIN & 0.0 & 0.3 & 0.3 & 0.3 & 0.3 & 0.3 & 0.3 & 0.3 & 0.3 & 0.3 & 0.3 & 0.3 & 0.3 & 0.3 & 0.3 & 0.3 \\
\hline & $\operatorname{MAX}$ & 0.0 & 0.3 & 0.3 & 0.3 & 0.3 & 0.3 & 0.3 & 0.3 & 0.3 & 0.3 & 0.3 & 0.3 & 0.3 & 0.3 & 0.3 & 0.3 \\
\hline \multirow{3}{*}{$\begin{array}{l}\text { HHC_LuUNDRY } \\
\text { Iotal: }\end{array}$} & VOL & 0.0 & 213.6 & 113.6 & 113.6 & 113.6 & 113.6 & 113.6 & 113.6 & 113.6 & 113.6 & 213.6 & 113.6 & 123.6 & 113.6 & 113.6 & 113.6 \\
\hline & MIN & 0.0 & 223.6 & .113 .6 & 113.6 & 113.6 & 113.6 & 113.6 & 113.6 & 113.6 & 113.6 & 213.6 & 113.6 & 113.6 & 113.6 & 113.6 & 133.6 \\
\hline & $\operatorname{MAX}$ & 0.0 & 124.9 & 124.9 & 121.9 & 124.9 & 121.9 & 124.9 & 121.9 & 124.9 & 124.9 & 124.9 & 124.9 & 124.9 & 124.9 & 124.9 & 121.9 \\
\hline
\end{tabular}


FY-1994 SOLID WASTE TORECAST

WASTE GENERATOR, WASTE CLASS VOLUMES BY YEAR

(in cubia meters)

Waste class

\begin{tabular}{llllllllllllllll} 
Held & 1995 & 1996 & 1997 & 1998 & 1999 & 2000 & 2001 & 2002 & 2003 & 2004 & 2005 & 2006 & 2007 & 2008 & 2009 \\
\hline
\end{tabular}

Faste Generator: HHC_ruBg

\begin{tabular}{|c|c|c|c|c|c|c|c|c|c|c|c|c|c|c|c|c|c|}
\hline \multirow[t]{3}{*}{ CH_LLW_I } & VOL & 0.0 & 6.3 & 6.3 & 6.3 & 6.3 & 6.3 & 6.3 & 6.3 & 6.3 & 6.3 & 6.3 & 6.3 & 6.3 & 6.3 & 6.3 & 6.3 \\
\hline & MIN & 0.0 & 6.3 & 6.3 & 6.3 & 6.3 & 6.3 & 6.3 & .6 .3 & 6.3 & 6.3 & 6.3 & 6.3 & 6.3 & 6.3 & 6.3 & 6.3 \\
\hline & $\operatorname{MAX}$ & 0.0 & 6.3 & 6.3 & 6.3 & 6.3 & 6.3 & 6.3 & 6.3 & 6.3 & 6.3 & 6.3 & 6.3 & 6.3 & 6.3 & 6.3 & 6.3 \\
\hline \multirow[t]{3}{*}{ CH_LLAS_I } & VOL & 0.0 & 0.0 & 0.0 & 3.1 & 0.0 & 0.0 & 3.4 & 0.0 & 0.0 & 3.4 & 0.0 & 0.0 & 3.1 & 0.0 & 0.0 & 3.1 \\
\hline & HIN & 0.0 & 0.0 & 0.0 & 3.1 & 0.0 & 0.0 & 3.1 & 0.0 & 0.0 & 3.4 & 0.0 & 0.0 & 3.4 & 0.0 & 0.0 & 3.4 \\
\hline & $\operatorname{HAX}$ & 0.0 & 0.0 & 0.0 & 3.4 & 0.0 & 0.0 & 3.1 & 0.0 & 0.0 & 3.4 & 0.0 & 0.0 & 3.4 & 0.0 & 0.0 & 3.4 \\
\hline \multirow{2}{*}{$\begin{array}{l}\text { KRE_LIREO } \\
\text { Totale }\end{array}$} & VOL & 0.0 & 6.3 & 6.3 & 9.7 & 6.3 & 6.3 & 9.7 & 6.3 & 6.3 & 9.7 & 6.3 & 6.3 & 9.7 & 6.3 & 6.3 & 9.7 \\
\hline & MIN & 0.0 & 6.3 & 6.3 & 9.7 & 6.3 & 6.3 & 9.7 & 6.3 & 6.3 & 9.7 & 6.3 & 6.3 & 9.7 & 6.3 & 6.3 & 9.7 \\
\hline
\end{tabular}

Fasto Genorator: KHC_InVP

\begin{tabular}{|c|c|c|c|c|c|c|c|c|c|c|c|c|c|c|c|c|c|}
\hline \multirow[t]{3}{*}{ CH_LLH_I } & voL & 0.0 & 0.0 & 0.0 & 0.0 & 0.0 & 0.0 & 0.0 & 0.0 & 0.0 & 0.0 & 0.0 & 425.4 & 125.1 & 125.4 & 125.1 & $\$ 25.4$ \\
\hline & MIN & 0.0 & 0.0 & 0.0 & 0.0 & 0.0 & 0.0 & 0.0 & 0.0 & 0.0 & 0.0 & 0.0 & 0.0 & 212.7 & 212.7 & 212.7 & 212.7 \\
\hline & $\operatorname{MAX}$ & 0.0 & 0.0 & 0.0 & 0.0 & 0.0 & 0.0 & 0.0 & 0.0 & 0.0 & 0.0 & 0.0 & 850.8 & 850.8 & 850.8 & B50.8 & 850.8 \\
\hline \multirow[t]{3}{*}{ CH_LLL_IIII } & vor & 0.0 & 0.0 & 0.0 & 0.0 & 0.0 & 0.0 & 0.0 & 0.0 & 0.0 & 0.0 & 0.0 & 211.0 & 111.8 & 141.8 & 161.8 & 111.8 \\
\hline & MIN & 0.0 & 0.0 & 0.0 & 0.0 & 0.0 & 0.0 & 0.0 & 0.0 & 0.0 & 0.0 & 0.0 & 0.0 & 70.9 & 70.9 & 70.9 & 70.9 \\
\hline & $\operatorname{MAX}$ & 0.0 & 0.0 & 0.0 & 0.0 & 0.0 & 0.0 & 0.0 & 0.0 & 0.0 & 0.0 & 0.0 & 203.6 & 283.6 & 283.6 & 283.6 & 283.6 \\
\hline \multirow[t]{3}{*}{ RH_LLWA_III } & vol & 0.0 & 0.0 & 0.0 & 0.0 & 0.0 & 0.0 & 0.0 & 0.0 & 0.0 & 0.0 & 0.0 & 21.0 & 21.8 & 21.8 & 21.6 & 21.8 \\
\hline & MIN & 0.0 & 0.0 & 0.0 & 0.0 & 0.0 & 0.0 & 0.0 & 0.0 & 0.0 & 0.0 & 0.0 & 0.0 & 10.9 & 10.9 & 20.9 & 10.9 \\
\hline & $\max$ & 0.0 & 0.0 & 0.0 & 0.0 & 0.0 & 0.0 & 0.0 & 0.0 & 0.0 & 0.0 & 0.0 & 13.6 & 13.6 & 43.6 & 43.6 & $\$ 3.6$ \\
\hline \multirow[t]{3}{*}{ CH_LUWE_I } & Vol. & 0.0 & 0.0 & 0.0 & 0.0 & 0.0 & 0.0 & 0.0 & 0.0 & 0.0 & 0.0 & 0.0 & 1.6 & 1.6 & 1.6 & 1.6 & 1.6 \\
\hline & MIN & 0.0 & 0.0 & 0.0 & 0.0 & 0.0 & 0.0 & 0.0 & 0.0 & 0.0 & 0.0 & 0.0 & 0.0 & 0.8 & 0.8 & 0.8 & 0.8 \\
\hline & $\operatorname{MAX}$ & 0.0 & 0.0 & 0.0 & 0.0 & 0.0 & 0.0 & 0.0 & 0.0 & 0.0 & 0.0 & 0.0 & 3.2 & 3.2 & 3.2 & 3.2 & 3.2 \\
\hline \multirow[t]{3}{*}{ CH_LUW_IXI } & Vol & 0.0 & 0.0 & 0.0 & 0.0 & 0.0 & 0.0 & 0.0 & 0.0 & 0.0 & 0.0 & 0.0 & 1.0 & 1.0 & 1.0 & 1.0 & 1.0 \\
\hline & MIN & 0.0 & 0.0 & 0.0 & 0.0 & 0.0 & 0.0 & 0.0 & 0.0 & 0.0 & 0.0 & 0.0 & 0.0 & 0.5 & 0.5 & 0.5 & 0.5 \\
\hline & $\max$ & 0.0 & 0.0 & 0.0 & 0.0 & 0.0 & 0.0 & 0.0 & 0.0 & 0.0 & 0.0 & 0.0 & 2.0 & 2.0 & 2.0 & 2.0 & 2.0 \\
\hline \multirow[t]{3}{*}{ HAZ } & VOL & 0.0 & 0.0 & 0.0 & 0.0 & 0.0 & 0.0 & 0.0 & 0.0 & 0.0 & 0.0 & 0.0 & 3.1 & 3.1 & 3.1 & 3.1 & 3.1 \\
\hline & MIN & 0.0 & 0.0 & 0.0 & 0.0 & 0.0 & 0.0 & 0.0 & 0.0 & 0.0 & 0.0 & 0.0 & 0.0 & 1.7 & 1.7 & 1.7 & 1.7 \\
\hline & $\max$ & 0.0 & 0.0 & 0.0 & 0.0 & 0.0 & 0.0 & 0.0 & 0.0 & 0.0 & 0.0 & 0.0 & 6.0 & 6.8 & 6.8 & 6.8 & 6.8 \\
\hline \multirow{3}{*}{$\begin{array}{l}\text { NBC_LrVP } \\
\text { Total. }\end{array}$} & VOL & 0.0 & 0.0 & 0.0 & 0.0 & 0.0 & 0.0 & 0.0 & 0.0 & 0.0 & 0.0 & 0.0 & 595.0 & 595.0 & 595.0 & 595.0 & 595.0 \\
\hline & MIN & 0.0 & 0.0 & 0.0 & 0.0 & 0.0 & 0.0 & 0.0 & 0.0 & 0.0 & 0.0 & 0.0 & 0.0 & 297.5 & 297.5 & 297.5 & 297.5 \\
\hline & $\max$ & 0.0 & 0.0 & 0.0 & 0.0 & 0.0 & 0.0 & 0.0 & 0.0 & 0.0 & 0.0 & 0.0 & 1190.0 & 1190.0 & 1190.0 & 1190.0 & 1290.0 \\
\hline
\end{tabular}




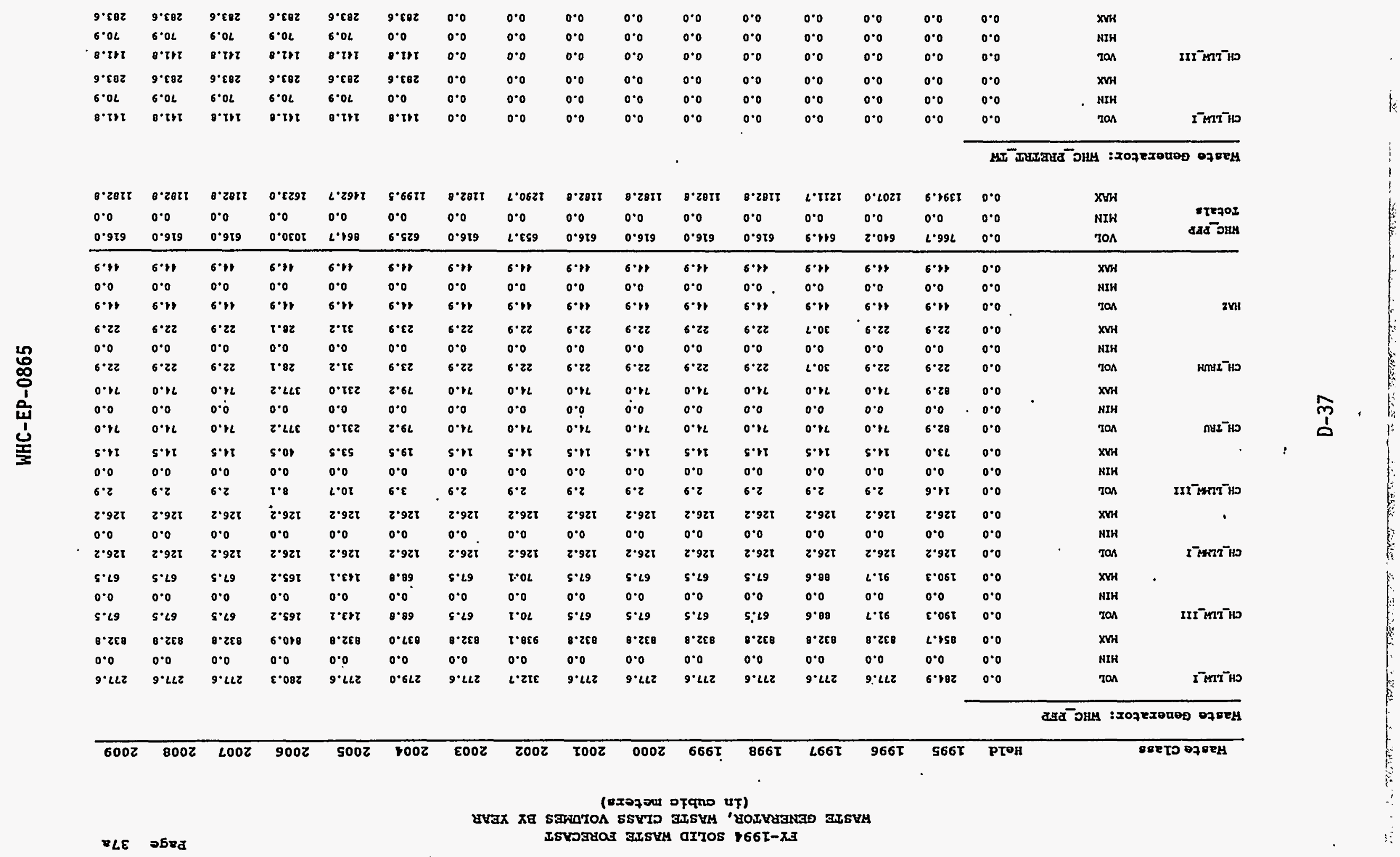




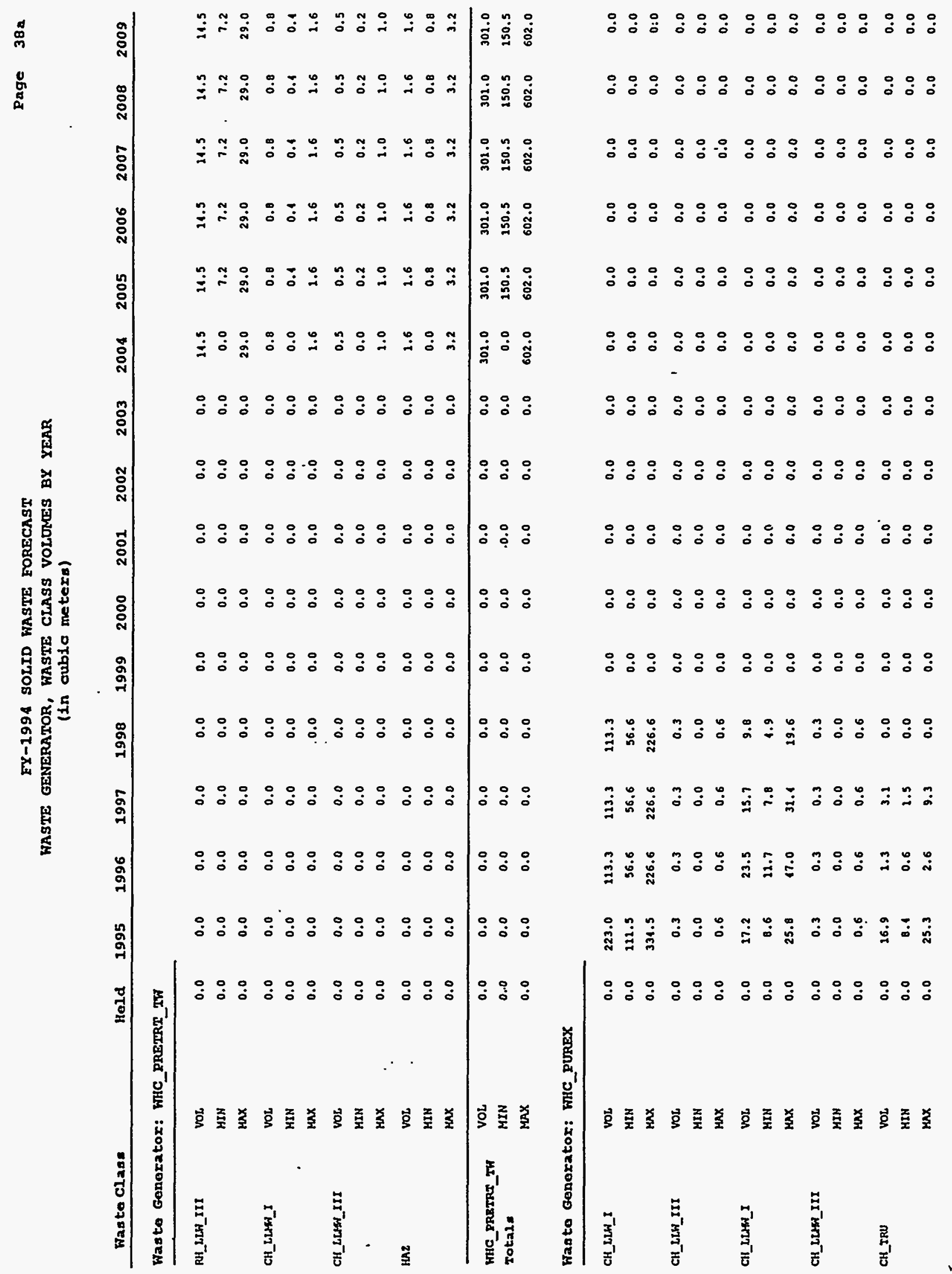


FY-1994 SOLID HASTE FORECAST

WASTE GENERATOR, HASTE CLASS VOLOMES BY YEAR

(in cubic meters)

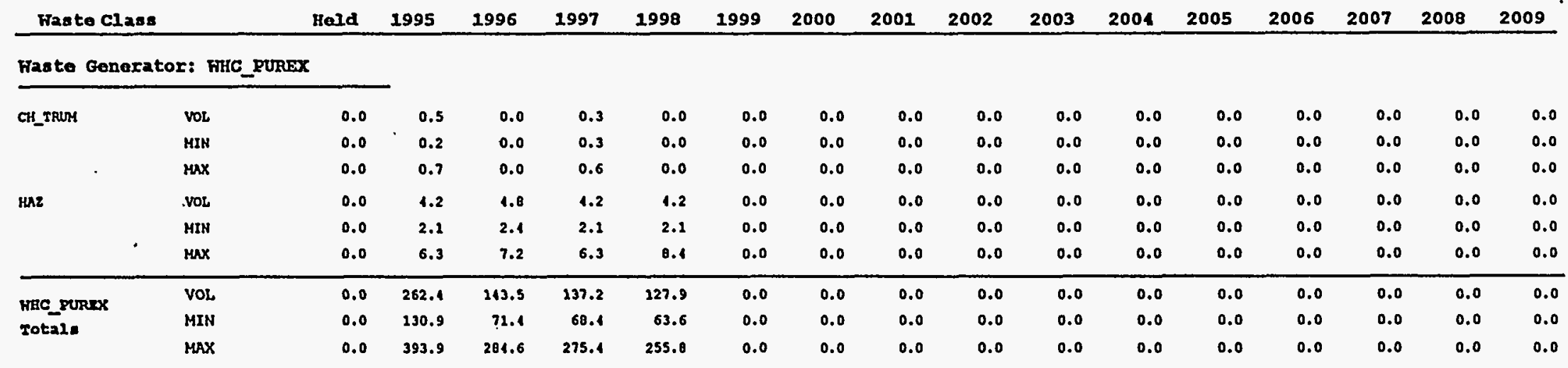

\begin{tabular}{|c|c|c|c|c|c|c|c|c|c|c|c|c|c|c|c|c|c|c|}
\hline \multirow{2}{*}{$\hat{u}$} & \multicolumn{3}{|c|}{ Fasto Generator: WHC_PUREX_SM } & \multirow[b]{2}{*}{0.0} & \multirow[b]{2}{*}{0.0} & \multirow[b]{2}{*}{0.0} & \multirow[b]{2}{*}{0.0} & \multirow[b]{2}{*}{22.6} & \multirow[b]{2}{*}{22.6} & \multirow[b]{2}{*}{22.6} & \multirow[b]{2}{*}{22.6} & \multirow[b]{2}{*}{22.6} & \multirow[b]{2}{*}{22.6} & \multirow[b]{2}{*}{22.6} & \multirow[b]{2}{*}{22.6} & \multirow[b]{2}{*}{22.6} & \multirow[b]{2}{*}{22.6} & \multirow[b]{2}{*}{22.6} \\
\hline & CH_LLW_I & vor & 0.0 & & & & & & & & & & & & & & & \\
\hline & $-3-$ & MIM & 0.0 & 0.0 & 0.0 & 0.0 & 0.0 & 21.3 & 11.3 & 11.3 & 11.3 & 11.3 & 11.3 & 11.3 & 11.3 & 11.3 & 11.3 & 11.3 \\
\hline & • & $\max$ & $0.0^{\circ}$ & 0.0 & 0.0 & 0.0 & 0.0 & 45.3 & 45.3 & 15.3 & 45.3 & $15.3^{\circ}$ & 45.3 & 45.3 & 15.3 & 45.3 & 15.3 & 45.3 \\
\hline & CH_LLW_III & vor & 0.0 & 0.0 & 0.0 & 0.0 & 0.0 & 0.0 & 0.0 & 0.0 & 0.0 & 0.0 & 0.0 & 0.0 & 0.0 & 0.0 & 0.0 & 0.0 \\
\hline & & MIM & 0.0 & 0.0 & 0.0 & 0.0 & 0.0 & 0.0 & 0.0 & 0.0 & 0.0 & 0.0 & 0.0 & 0.0 & 0.0 & 0.0 & 0.0 & 0.0 \\
\hline • & & $\max$ & 0.0 & 0.0 & 0.0 & 0.0 & 0.0 & 0.1 & 0.1 & 0.1 & 0.1 & 0.1 & 0.1 & 0.1 & 0.1 & 0.1 . & 0.1 & 0.1 \\
\hline & CH_LLWW_I & vor & 0.0 & 0.0 & 0.0 & 0.0 & 0.0 & 1.9 & 1.9 & 1.9 & 1.9 & 1.9 & 1.9 & 1.9 & 1.9 & 1.9 & 1.9 & 1.9 \\
\hline & & MIN & 0.0 & 0.0 & 0.0 & 0.0 & 0.0 & 0.9 & 0.9 & 0.9 & 0.9 & 0.9 & 0.9 & 0.9 & 0.9 & 0.9 & 0.9 & 0.9 \\
\hline • & & $\max$ & 0.0 & 0.0 & 0.0 & 0.0 & 0.0 & 3.9 & 3.9 & 3.9 & 3.9 & 3.9 & 3.9 & 3.9 & 3.9 & 3.9 & 3.9 & 3.9 \\
\hline & CH_LLWA_III & vor & 0.0 & 0.0 & 0.0 & 0.0 & 0.0 & 0.0 & 0.0 & 0.0 & $0 . \dot{0}$ & 0.0 & 0.0 & 0.0 & 0.0 & 0.0 & 0.0 & 0.0 \\
\hline & & MIN & 0.0 & 0.0 & 0.0 & 0.0 & 0.0 & 0.0 & 0.0 & 0.0 & 0.0 & 0.0 & 0.0 & 0.0 & 0.0 & 0.0 & 0.0 & 0.0 \\
\hline & & $\max$ & 0.0 & 0.0 & 0.0 & 0.0 & 0.0 & 0.1 & 0.1 & 0.1 & 0.1 & 0.1 & 0.1 & 0.1 & 0.2 & 0.1 & 0.1 & 0.1 \\
\hline & HA2 & vot & 0.0 & 0.0 & 0.0 & 0.0 & 0.0 & 0.8 & 0.0 & 0.8 & 0.8 & 0.8 & 0.8 & 0.8 & 0.8 & 0.0 & 0.8 & 0.8 \\
\hline & & МIH & 0.0 & 0.0 & 0.0 & 0.0 & 0.0 & 0.1 & 0.1 & 0.1 & 0.1 & 0.1 & 0.1 & 0.1 & 0.4 & 0.1 & 0.1 & 0.1 \\
\hline & & $\max$ & 0.0 & 0.0 & 0.0 & 0.0 & 0.0 & 1.6 & 1.6 & 1.6 & 1.6 & 2.6 & 1.6 & 1.6 & 1.6 & 1.6 & 1.6 & 1.6 \\
\hline & & VoL & 0.0 & 0.0 & 0.0 & 0.0 & 0.0 & 25.5 & 25.5 & 23.5 & 25.5 & 25.5 & 25.5 & 25.5 & 25.5 & 25.5 & 25.5 & 25.5 \\
\hline & sotal. & MIN & 0.0 . & 0.0 & 0.0 & 0.0 & 0.0 & 12.7 & 12.7 & 12.7 & 12.7 & 12.7 & 12.7 & 12.7 & 12.7 & 12.7 & 12.7 & 12.7 \\
\hline & & $\operatorname{MAX}$ & 0.0 & 0.0 & 0.0 & 0.0 & 0.0 & 31.1 & 51.1 & 51.1 & 31.1 & 51.1 & 51.1 & 51.1 & 51.1 & 51.1 & 51.1 & 51.1 \\
\hline
\end{tabular}




\begin{tabular}{|c|c|c|c|c|c|c|c|c|c|c|c|c|c|c|c|c|c|}
\hline $\begin{array}{l}\theta \cdot 968 z \\
\text { P. } 9 \varepsilon z z \\
\text { S.Ecgz }\end{array}$ & $\begin{array}{l}r \cdot 9 z r z \\
\text { L·LLI } \\
9 \cdot 50 z z\end{array}$ & $\begin{array}{l}0.5 L 12 \\
\text { s.z1st } \\
0.05 z z\end{array}$ & 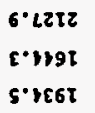 & $\begin{array}{l}\text { P.9tat } \\
9 \cdot \operatorname{cort} \\
\varepsilon \cdot \tau \text { 'tsgt }\end{array}$ & $\begin{array}{l}1.966 t \\
6.285 t \\
2.5 t \theta t\end{array}$ & $\begin{array}{l}0 \cdot \angle L \theta \\
L \cdot L L Q \\
\varepsilon \cdot L 6 L\end{array}$ & 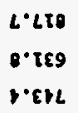 & $\begin{array}{l}\varepsilon \cdot L 0 L \\
S \cdot 915 \\
0 \cdot c y 9\end{array}$ & $\begin{array}{l}2.665 \\
0.898 \\
8.315\end{array}$ & $\begin{array}{l}9.815 \\
0.026 \\
2 \cdot 158\end{array}$ & $\begin{array}{l}\varepsilon \cdot 901 \\
6 \cdot \varepsilon(\varepsilon \\
.69 \varepsilon\end{array}$ & $\begin{array}{l}5.959 \\
6.805 \\
2 \cdot 865\end{array}$ & $\begin{array}{l}\varepsilon \cdot s ı z^{\circ} \\
\text { s.68t } \\
0 . \varepsilon z z\end{array}$ & $\begin{array}{l}0.15 \\
1.6 \varepsilon \\
1.98\end{array}$ & $\begin{array}{l}0.0 \\
0.0 \\
0.0\end{array}$ & $\begin{array}{l}\text { XXW } \\
\text { NIK } \\
\text { TON }\end{array}$ & 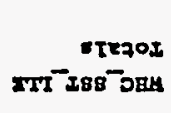 \\
\hline $8.968 z$ & $l \cdot 9 z r z$ & 0. SLIZ & G. LzाZ & P.grar & $L \cdot 966 \mathrm{~T}$ & $0 . \angle 2 \theta$ & L'LT8 & $\varepsilon \cdot \angle 0 L$ & $2 \cdot 665$ & 9.ers & $\varepsilon \cdot 90\}$ & $5 \cdot 859$ & E.spz & 0.0 & 0.0 & $\mathbf{x w} \mathbf{w}$ & \\
\hline S.regr & $9 \cdot 50 z 2$ & 0.0522 & $s \cdot \operatorname{re6t}$ & $\varepsilon \cdot\{59 \tau$ & Z'stgt & $\varepsilon \cdot L 6 L$ & PEमL & $0 \cdot 0$ & Q.urs & $2 \cdot 161$ & $1.69 \varepsilon$ & $L \cdot 865$ & $0 . \varepsilon z z$ & .91 & 0.0 & Tan & 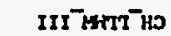 \\
\hline
\end{tabular}

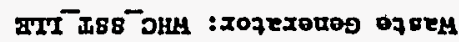

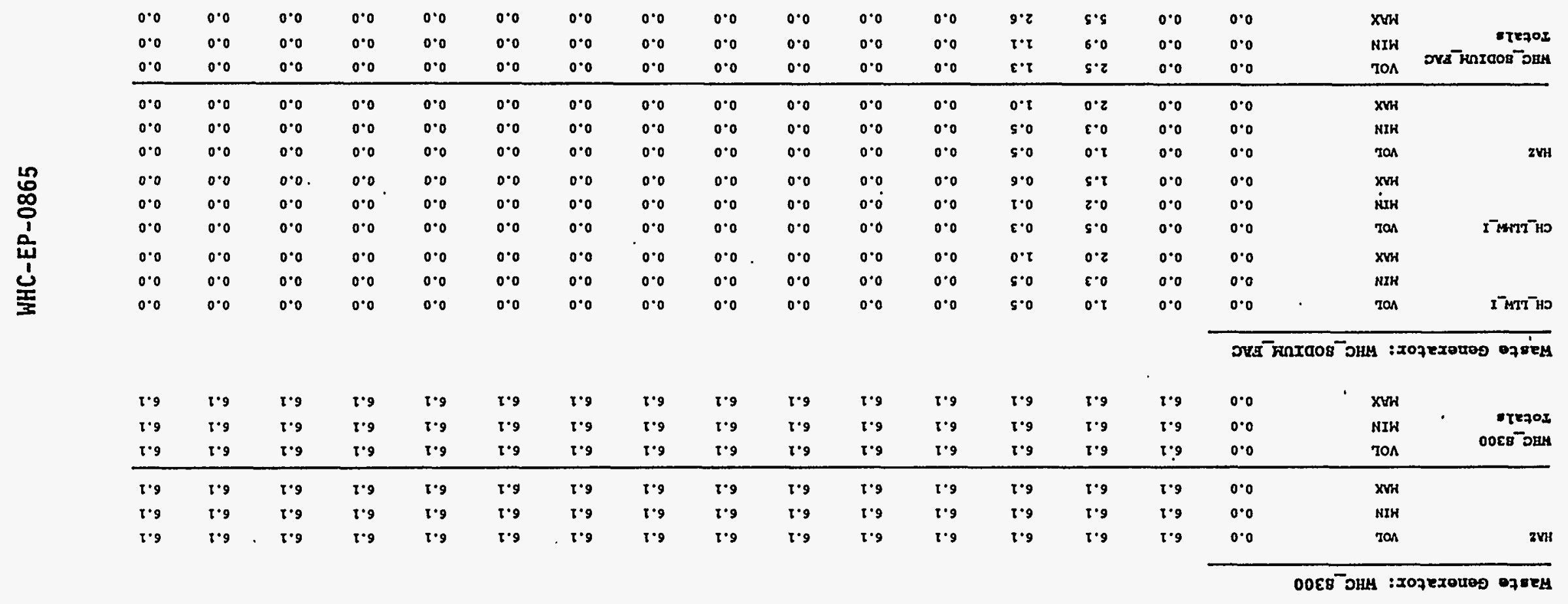

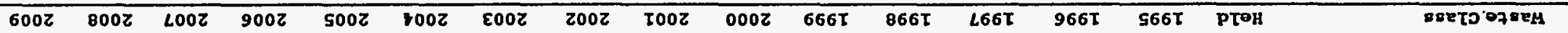

(8xo7var. DTqRo UT)

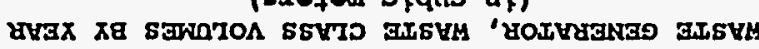

rob כerd

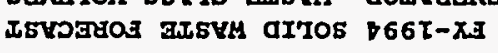


FY-1994 SOLID WASTE FORECAST

WASTE GENERATOR, WASTE CLASS VOLOMES BY YEAR

(in cubic meters)

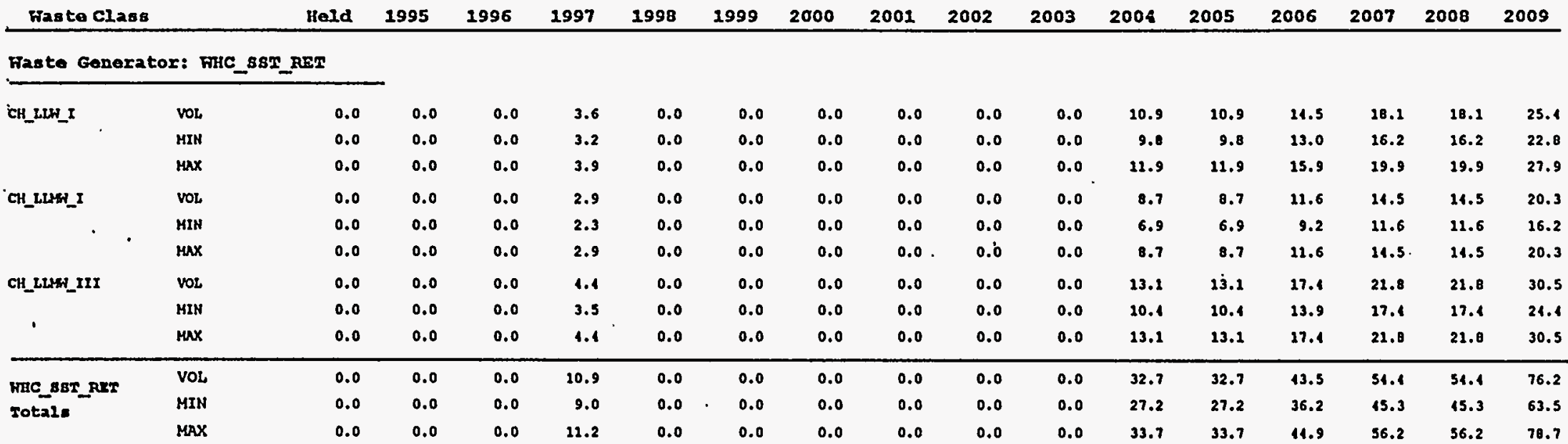

Waste Generator: FHC_SURPLS_EAC

\begin{tabular}{|c|c|c|c|c|c|c|c|c|c|c|c|c|c|c|c|c|c|}
\hline \multirow[t]{3}{*}{ CH_LLW_I } & vor & 0.0 & 2039.7 & 1603.1 & 3688.4 & 126.4 & 1246.5 & 8215.3 & 15368.3 & 60617.6 & 80178.5 & 107515.6 & 153767.7 & 165110.8 & 179886.7 & 100111.9 & 38073.7 \\
\hline & MIN & 0.0 & 2039.7 & 1603.4 & 3688.1 & 416.4 & 1216.5 & 8215.3 & 15368.3 & 60617.6 & 00170.5 & 107515.6 & 153767.7 & 165110.8 & 279886.7 & 200111.9 & 38073.7 \\
\hline & $\max$ & 0.0 & 2039.7 & 1603.4 & 3680.4 & 126.4 & 1246.5 & 8215.3 & 13368.3 & 60617.6 & 80178.5 & 207515.6 & 153767.7 & 265110.8 & 179886.7 & 108111.9 & 38073.7 \\
\hline \multirow[t]{3}{*}{ CH_ILH_III } & vor & 0.0 & 0.0 & 5.7 & 3.7 & 0.0 & 11.3 & 1218.1 & 1133.1 & 2832.9 & 4249.3 & 8243.6 & 8781.9 & 12747.9 & 16997.2 & 12747.9 & 11048.2 \\
\hline & MTN & 0.0 & 0.0 & 5.7 & 5.7 & 0.0 & 11.3 & 1218.1 & 1133.1 & 2032.9 & 1249.3 & 8243.6 & 0781.9 & 12747.9 & 26997.2 & 12747.9 & 11048.2 \\
\hline & $\max$ & 0.0 & 0.0 & 5.7 & 5.7 & 0.0 & 11.3 & 1218.1 & 2133.1 & 2832.9 & 1249.3 & 8243.6 & 8781.9 & 12747.9 & 16997.2 & 12747.9 & 11048.2 \\
\hline \multirow[t]{3}{*}{ CH_LLW_GTCXII } & vor & 0.0 & 0.0 & 0.0 & 0.0 & 0.0 & 0.0 & 0.0 & 283.3 & 849.9 & 2416.4 & 2549.6 & 3399.4 & 5382.4 & 7365.1 & 7082.2 & 6515.6 \\
\hline & HIN & 0.0 & 0.0 & 0.0 & 0.0 & 0.0 & 0.0 & 0.0 & 283.3 & 819.9 & 1416.4 & 2549.6 & 3399.4 & 5382.4 & 7365.1 . & - 7082.2 & 6515.6 \\
\hline & $\max$ & 0.0 & 0.0 & 0.0 & 0.0 & 0.0 & 0.0 & 0.0 & 283.3 & 819.9 & 1416.1 & 2549.6 & 3399.4 & 5382.1 & 7365.1 & 7002.2 & 6515.6 \\
\hline \multirow[t]{3}{*}{ RH_ILH_GTCIYY } & vor & 0.0 & 0.0 & 0.0 & 0.0 & 0.0 & 0.0 & 0.0 & 283.3 & 849.9 & 1416.4 & 2549.6 & 3399.4 & 5382.4 & 7365.1 & 7082.2 & 6515.6 \\
\hline & MIN & 0.0 & 0.0 & 0.0 & 0.0 & 0.0 & 0.0 & 0.0 & 283.3 & 849.9 & 2116.4 & 2519.6 & 3399.4 & 5382.1 & 7365.1 & 7082.2 & 6515.6 \\
\hline & $\operatorname{man} x$ & 0.0 & 0.0 & 0.0 & 0.0 & 0.0 & 0.0 & 0.0 & 283.3 & 849.9 & 1116.4 & 2549.6 & 3399.4 & 3382.1 & 7365.1 & 7082.2 & 6515.6 \\
\hline \multirow[t]{3}{*}{ CH_LLWH_I } & vor & 0.0 & 29.7 & 5.7 & 3.7 & 0.0 & 28.3 & 1.4 & 20.3 & 28.3 & 28.3 & 85.0 & 85.0 & 141.6 & 141.6 & 111.6 & 141.6 \\
\hline & HIH & 0.0 & 29.7 & 3.7 & 5.7 & 0.0 & 20.3 & 1.4 & 28.3 & 28.3 & 28.3 & e5.0 & 85.0 & 241.6 & 141.6 & 111.6 & 141.6 \\
\hline & $\operatorname{mnx}$ & 0.0 & 29.7 & 5.7 & 5.7 & 0.0 & 28.3 & 1.4 & 28.3 & 28.3 & 28.3 & 05.0 & 05.0 & 141.6 & 311.6 & 141.6 & 141.6 \\
\hline \multirow[t]{3}{*}{ CH_LLHH_III } & VoL & 0.0 & 0.0 & 0.0 & 0.0 & 0.0 & 5.7 & 0.0 & 28.3 & 28.3 & 20.3 & 85.0 & 85.0 & 241.6 & 141.6 & 211.6 & 111.6 \\
\hline & MIN & 0.0 & 0.0 & 0.0 & 0.0 & 0.0 & 5.7 & 0.0 & 28.3 & 28.3 & 28.3 & 05.0 & 15.0 & 111.6 & 112.6 & 111.6 & 111.6 \\
\hline & $\max$ & 0.0 & 0.0 & 0.0 & 0.0 & 0.0 & 5.7 & 0.0 & 28.3 & 28.3 & 28.3 & 05.0 & 85.0 & 141.6 & 111.6 & 211.6 & 141.6 \\
\hline
\end{tabular}


FY-1994 SOLID HASTE FORECAST

HASTE GENERATOR, HASTE CLASS VOLOMES BY YEAR

(In cubio meters)

\begin{tabular}{|c|c|c|c|c|c|c|c|c|c|c|c|c|c|c|c|c|c|}
\hline Wasto Class & & Held & 1995 & 1996 & 1997 & $1998^{\circ}$ & 1999 & 2000 & 2001 & 2002 & 2003 & 2004 & 2005 & 2006 & 2007 & 2008 & 2009 \\
\hline \multicolumn{3}{|c|}{ Waste Gonerator: WHC_SURPLS_EAC } & & & & & & & & & & & & & & & \\
\hline \multirow[t]{3}{*}{ CH_LLHE_GTCIII } & vos & 0.0 & 0.0 & 0.0 & 0.0 & 0.0 & 0.0 & 0.0 & 28.3 & 28.3 & 28.3 & 85.0 & 85.0 & 141.6 & 111.6 & 141.6 & 111.6 \\
\hline & MIN & 0.0 & 0.0 & 0.0 & 0.0 & 0.0 & 0.0 & 0.0 & 28.3 & 20.3 & 28.3 & 85.0 & 85.0 & 211.6 & 141.6 & 141.6 & 111.6 \\
\hline & $\max$ & 0.0 & 0.0 & 0.0 & 0.0 & 0.0 & 0.0 & 0.0 & 28.3 & 28.3 & 28.3 & 85.0 & 85.0 & 141.6 & 141.6 & 141.6 & 111.6 \\
\hline \multirow[t]{3}{*}{ PH_LLHE_I } & voL & 0.0 & 0.0 & 0.0 & 0.0 & 0.0 & 0.0 & 0.0 & 28.3 & 28.3 & 28.3 & B5.0 & 85.0 & 111.6 & 141.6 & 141.6 & 141.6 \\
\hline & MIN & 0.0 & 0.0 & 0.0 & 0.0 & 0.0 & 0.0 & 0.0 & 28.3 & 20.3 & 28.3 & 85.0 & 85.0 & 111.6 & 141.6 & 141.6 & 111.6 \\
\hline & $\max$ & 0.0 & 0.0 & 0.0 & 0.0 & 0.0 & 0.0 & 0.0 & 28.3 & 20.3 & 28.3 & 85.0 & 85.0 & 141.6 & 111.6 & 141.6 & 111.6 \\
\hline \multirow[t]{3}{*}{ RH_LLW__III } & voL & 0.0 & 0.0 & 0.0 & 0.0 & 0.0 & 0.0 & 0.0 & 28.3 & 20.3 & 28.3 & 85.0 & 85.0 & 111.6 & 111.6 & 111.6 & 111.6 \\
\hline & HIN & 0.0 & 0.0 & 0.0 & 0.0 & 0.0 & 0.0 & 0.0 & 28.3 & 20.3 & 28.3 & 85.0 & 85.0 & 141.6 & 141.6 & 111.6 & 111.6 \\
\hline & $\max$ & 0.0 & 0.0 & 0.0 & 0.0 & 0.0 & 0.0 & 0.0 & 28.3 & 28.3 & 28.3 & 85.0 & 85.0 & 141.6 & 211.6 & 141.6 & 111.6 \\
\hline \multirow[t]{3}{*}{ RH_LLHW_GTCIII } & vos & 0.0 & 0.0 & 0.0 & 0.0 & 0.0 & 0.0 & 0.0 & 28.3 & 28.3 & 28.3 & 85.0 & 85.0 & 141.6 & 311.6 & 141.6 & 111.6 \\
\hline & HIH & 0.0 & 0.0 & 0.0 & 0.0 & 0.0 & 0.0 & 0.0 & 20.3 & 28.3 & 28.3 & 85.0 & 85.0 & 211.6 & 141.6 & 111.6 & 111.6 \\
\hline & $\max$ & 0.0 & 0.0 & 0.0 & 0.0 & 0.0 & 0.0 & 0.0 & 28.3 & 28.3 & 28.3 & 95.0 & 95.0 & 141.6 & 141.6 & 141.6 & 111.6 \\
\hline \multirow[t]{3}{*}{ CH_IRU } & vor & 0.0 & 8.5 & 297.5 & 0.0 & 0.0 & 0.0 & 141.6 & 2.8 & 5.7 & 5.7 & 11.3 & 113.3 & 123.3 & 213.3 & 113.3 & 213.3 \\
\hline & MIN & 0.0 & 8.5 & 297.5 & 0.0 & 0.0 & 0.0 & 141.6 & 2.8 & 5.7 & 5.7 & 21.3 & 113.3 & 113.3 & 113.3 . & 113.3 & 113.3 \\
\hline & $\operatorname{MAX}$ & 0.0 & 8.5 & 297.5 & 0.0 & 0.0 & 0.0 & 141.6 & 2.8 & 5.7 & 5.7 & 11.3 & 113.3 & 113.3 & $113.3^{\circ}$ & 113.3 & 113.3 \\
\hline \multirow[t]{3}{*}{ RH_TRU } & vor & 0.0 & 0.0 & 2.8 & 0.0 & 0.0 & 0.0 & 0.0 & 0.0 & 0.0 & 0.0 & 0.0 & 0.0 & 0.0 & 0.0 & 0.0 & 0.0 \\
\hline & MIM & 0.0 & 0.0 & 2.8 & 0.0 & 0.0 & 0.0 & 0.0 & 0.0 & 0.0 & 0.0 & 0.0 & 0.0 & 0.0 & 0.0 & 0.0 & 0.0 \\
\hline & $\max$ & 0.0 & 0.0 & 2.8 & 0.0 & 0.0 & 0.0 & 0.0 & 0.0 & 0.0 & 0.0 & 0.0 & 0.0 & 0.0 & 0.0 & 0.0 & 0.0 \\
\hline \multirow[t]{3}{*}{ CH_TRUM } & vot. & 0.0 & 0.0 & 2.8 & 0.0 & 0.0 & 0.0 & 56.7 & 2.8 & 2.0 & 2.8 & 11.3 & 28.3 & 123.3 & 213.3 & 113.3 & 113.3 \\
\hline & MIN & 0.0 & 0.0 & 2.8 & 0.0 & 0.0 & 0.0 & 56.7 & 2.8 & 2.8 & 2.8 & 11.3 & 28.3 & 113.3 & 213.3 & 113.3 & 113.3 \\
\hline & MAX & 0.0 & 0.0 & 2.0 & 0.0 & 0.0 & 0.0 & 56.7 & 2.8 & 2.8 & 2.8 & 11.3 & 20.3 & 113.3 & 113.3 & 113.3 & 113.3 \\
\hline \multirow[t]{3}{*}{ RH_TRUY } & vol & 0.0 & 0.0 & 0.0 & 0.0 & 0.0 & 0.0 & 0.0 & 0.0 & 0.0 & 0.0 & 213.3 & 123.3 & 113.3 & 113.3 & 85.0 & 56.7 \\
\hline & MIN & 0.0 & 0.0 & 0.0 & 0.0 & 0.0 & 0.0 & 0.0 & 0.0 & 0.0 & 0.0 & 113.3 & 113.3 & 113.3 & 113.3 & 85.0 & 56.7 \\
\hline & $\max$ & 0.0 & 0.0 & 0.0 & 0.0 & 0.0 & 0.0 & 0.0 & 0.0 & 0.0 & 0.0 & 213.3 & 113.3 & 213.3 & 213.3 & 85.0 & 36.7 \\
\hline \multirow[t]{3}{*}{ HA2 } & vol & 0.0 & 0.5 & 11.3 & 11.3 & 11.3 & 14.2 & 36.0 & 150.1 & 198.3 & 311.6 & 566.6 & 566.6 & 566.6 & 124.9 & 283.3 & 112.6 \\
\hline & MIM & 0.0 & 8.5 & 11.3 & 21.3 & 11.3 & 14.2 & 36.8 & 150.1 & 198.3 & 311.6 & 566.6 & 566.6 & 566.6 & 124.9 & 283.3 & 141.6 \\
\hline & $\max$ & 0.0 & 8.5 & 11.3 & 21.3 & 11.3 & 14.2 & 36.8 & 150.1 & 198.3 & 312.6 & 566.6 & 566.6 & 566.6 & 124.9 & 203.3 & 141.6 \\
\hline \multirow{3}{*}{$\begin{array}{l}\text { NHC_BURPLB_EAC } \\
\text { Tota1: }\end{array}$} & VoL & 0.0 & 2086.4 & 1929.2 & 3711.1 & 127.7 & 1306.0 & 9669.9 & 17393.5 & 65526.9 & 87750.5 & 122070.9 & 170679.9 & 190679.6 & 213229.1 & 136798.7 & 63427.6 \\
\hline & MIN & 0.0 & 2086.4 & 1929.2 & 3711.1 & 127.7 & 2306.0 & 9669.9 & 17393.5 & 65526.9 & 87750.5 & 122070.9 & 170679.9 & 190679.6 & 213229.1 & 136798.7 & 63427.6 \\
\hline & $\max$ & 0.0 & 2086.1 & 1929.2 & 3711.1 & 127.7 & 3306.0 & 9669.9 & 27393.5 & 65526.9 & 87750.5 & 122070.9 & 170679.9 & 190679.6 & 213229.1 & 136798.7 & 63127.6 \\
\hline
\end{tabular}


Waisto Class

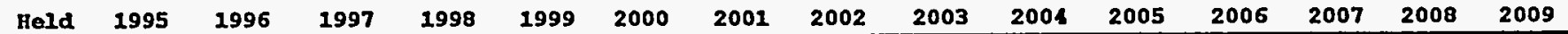

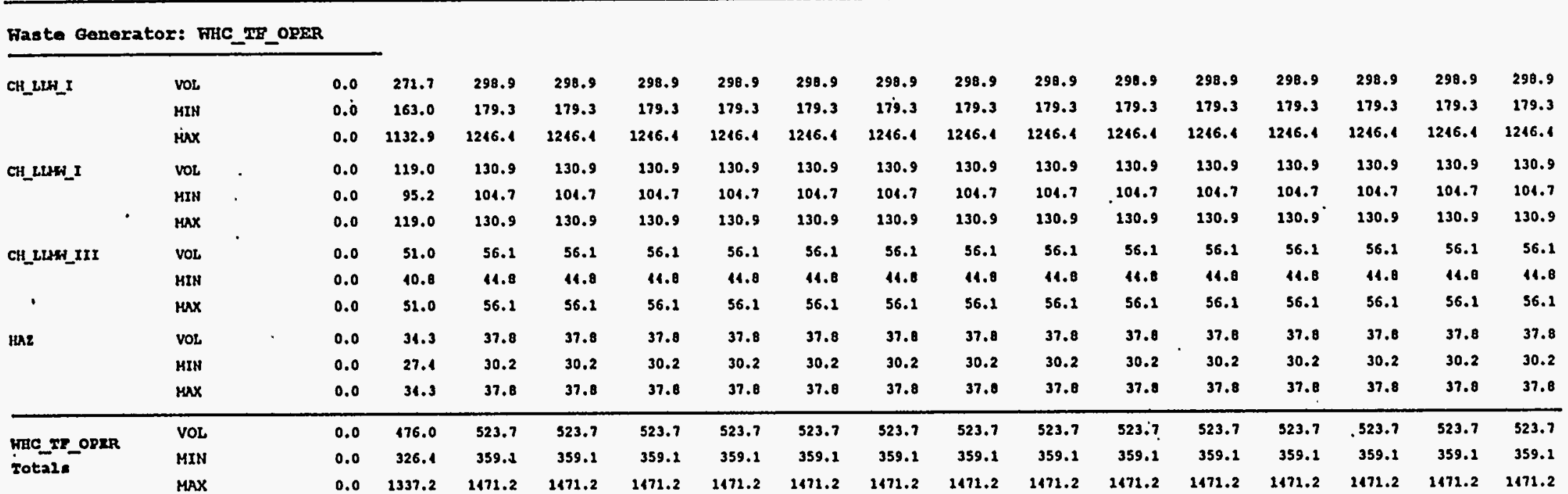

Wasto Ganorator: WIIC_IRUSAF

\begin{tabular}{|c|c|c|c|c|c|c|c|c|c|c|c|c|c|c|c|c|c|}
\hline \multirow[t]{3}{*}{$\mathrm{CH}_{-} \mathrm{LIN} \mathrm{I}$} & vos & 0.0 & 1.0 & 2.8 & 6.8 & 0.0 & 0.0 & 0.0 & 0.0 & 0.0 & 0.0 & 0.0 & 0.0 & 0.0 & 0.0 & 0.0 & 0.0 \\
\hline & MIM & $0.0^{\circ}$ & 1.0 & 2.8 & 6.8 & 0.6 & 0.0 & 0.0 & 0.0 & 0.0 & 0.0 & 0.0 & 0.0 & 0.0 & 0.0 & 0.0 & 0.0 \\
\hline & $\max$ & 0.0 & 1.0 & 2.8 & 6.8 & 0.0 & 0.0 & 0.0 & 0.0 & 0.0 & 0.0 & 0.0 & 0.0 & 0.0 & 0.0 & 0.0 & 0.0 \\
\hline \multirow[t]{2}{*}{ CH_LLW_I } & Vot & 0.0 & 0.0 & 0.0 & 2.9 & 0.0 & 0.0 & 0.0 & 0.0 & 0.0 & 0.0 & 0.0 & 0.0 & 0.0 & 0.0 & 0.0 & 0.0 \\
\hline & $\max$ & 0.0 & 0.0 & 0.0 & 2.9 & 0.0 & 0.0 & 0.0 & 0.0 & 0.0 & 0.0 & 0.0 & 0.0 & 0.0 & 0.0 & 0.0 & 0.0 \\
\hline \multirow{2}{*}{$\begin{array}{l}\text { HBC_LRUAAR } \\
\text { Totale }\end{array}$} & VoL & 0.0 & 1.0 & 2.0 & 9.7 & 0.0 & 0.0 & 0.0 & 0.0 & 0.0 & 0.0 & 0.0 & 0.0 & 0.0 & 0.0 & 0.0 & 0.0 \\
\hline & MIN & 0.0 & 1.0 & 2.8 & 9.7 & 0.0 & 0.0 & 0.0 & 0.0 & 0.0 & 0.0 & 0.0 & 0.0 & 0.0 & 0.0 & 0.0 & 0.0 \\
\hline
\end{tabular}

Waste Generator: HIC_IFP_W028

\begin{tabular}{|c|c|c|c|c|c|c|c|c|c|c|c|c|c|c|c|c|c|}
\hline CH_LLH_I & vol & 0.0 & 0.0 & 3.7 & 3.7 & 0.0 & 0.0 & 0.0 & 0.0 & 0.0 & 0.0 & 0.0 & 0.0 & 0.0 & 0.0 & 0.0 & 0.0 \\
\hline & MIN & 0.0 & 0.0 & 3.7 & 3.7 & 0.0 & 0.0 & 0.0 & 0.0 & 0.0 & 0.0 & 0.0 & 0.0 & 0.0 & 0.0 & 0.0 & 0.0 \\
\hline & $\max$ & 0.0 & 0.0 & 29.6 & 25.9 & 0.0 & 0.0 & 0.0 & 0.0 & 0.0 & 0.0 & 0.0 & 0.0 & 0.0 & 0.0 & 0.0 & 0.0 \\
\hline
\end{tabular}


WHC-EP-0865

\begin{tabular}{|c|c|c|c|c|c|c|c|c|c|c|}
\hline$\$$ & : & $\mid \begin{array}{lll}0 & 0 & 0 \\
0 & 0 & 0 \\
0\end{array}$ & & $\stackrel{0}{\dot{0}}: \stackrel{0}{0}$ & $\mid \begin{array}{lll}0 & 0 & 0 \\
0 & 0 & 0\end{array}$ & & $\stackrel{0}{0}: \stackrel{0}{0}$ & $\mid \begin{array}{lll}0 & 0 & 0 \\
0 & 0 & 0\end{array}$ & & 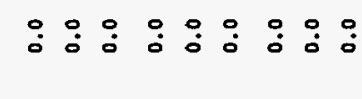 \\
\hline & : & $\stackrel{0}{0}: \vdots$ & & $\stackrel{0}{0}: \stackrel{0}{0}$ & $\stackrel{0}{:}: \stackrel{0}{0}$ & & $\stackrel{0}{\circ} \stackrel{0}{0}:$ & $\stackrel{0}{0}: \stackrel{0}{0}$ & & 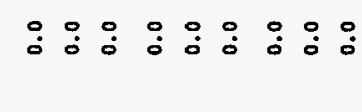 \\
\hline & s: & $\stackrel{0}{0}: 0$ & & $\stackrel{0}{0}: \stackrel{0}{0}$ & $0: \stackrel{0}{0}$ & & $\stackrel{0}{0} \stackrel{0}{0}: 0$ & $\stackrel{0}{0}:$ & & $\stackrel{0}{0}: 000$ \\
\hline & $\stackrel{\circ}{\circ}$ & $\dot{0}: \stackrel{0}{0}$ & & $\stackrel{0}{:}: \stackrel{0}{0}$ & $\ddot{0} \stackrel{0}{0} \stackrel{0}{0}$ & & $\stackrel{0}{0}: \stackrel{0}{0}$ & $\stackrel{9}{\dot{0}} \stackrel{0}{0}:$ & & 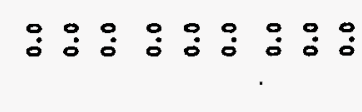 \\
\hline & 怘 & $\stackrel{0}{0}: \stackrel{0}{0}$ & & $\stackrel{0}{0}: \stackrel{0}{0}$ & $\stackrel{0}{0}: \stackrel{0}{0}$ & & $\stackrel{0}{0} \dot{0}: 00$ & $\stackrel{0}{0} \stackrel{0}{0}:$ & & $\stackrel{0}{0}: 000$ \\
\hline & : & $\stackrel{0}{0} \stackrel{0}{0} \stackrel{0}{0}$ & & $\stackrel{0}{0}: \stackrel{0}{0}$ & $\stackrel{0}{0}: \stackrel{0}{0}:$ & & $\stackrel{\circ}{0} \stackrel{0}{0} \stackrel{0}{0}$ & $\stackrel{0}{0}: \stackrel{0}{0}:$ & & 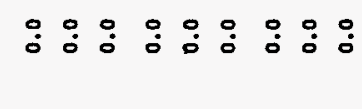 \\
\hline 路 & co & $\begin{array}{lll}0 & 0 & 0 \\
0 & 0 & 0\end{array}$ & & $\stackrel{0}{0}: \stackrel{0}{0}$ & $\stackrel{0}{0}: \stackrel{0}{0}$ & & $\stackrel{0}{0}: \stackrel{0}{0}$ & $\stackrel{0}{\dot{0}} \stackrel{0}{0} \stackrel{0}{0}$ & & 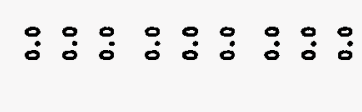 \\
\hline 풀 & ઠิ & $\stackrel{0}{0}: \stackrel{0}{0}$ & & $\stackrel{0}{0}: \stackrel{0}{0}$ & $\stackrel{0}{0}: \stackrel{0}{0}$ & & $\stackrel{0}{0}: \stackrel{0}{0}$ & $\stackrel{0}{0} \stackrel{0}{0}: 0$ & & 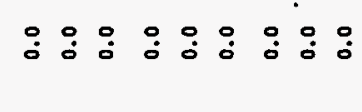 \\
\hline 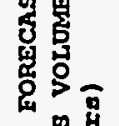 & :ั. & $0: 00$ & & $\stackrel{0}{\circ} \stackrel{0}{0} \stackrel{0}{0}$ & 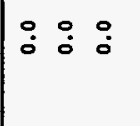 & & 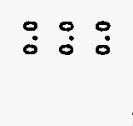 & $\stackrel{0}{0}: \stackrel{0}{0}$ & & 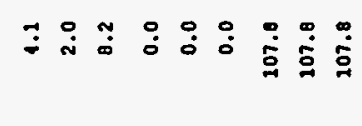 \\
\hline 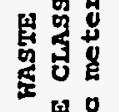 & : & $\stackrel{0}{0}: \stackrel{0}{0}:$ & & $\stackrel{9}{9}: \stackrel{0}{0}$ & $\begin{array}{lll}0 & 0 & 0 \\
0 & \vdots & \vdots \\
0\end{array}$ & & $\stackrel{0}{0}: \stackrel{0}{0}: \stackrel{0}{0}$ & $\mid \begin{array}{lll}0 & 0 & 0 \\
0 & 0 & 0\end{array}$ & & 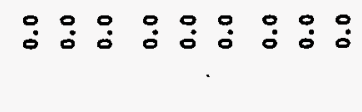 \\
\hline 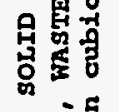 & aे & $\stackrel{0}{0}: 00$ & & $\stackrel{0}{0}: \stackrel{0}{0}$ & $\begin{array}{rll}0 & 0 \\
0 & : & 0 \\
0 & :\end{array}$ & & $\stackrel{0}{0} \stackrel{0}{0}: \stackrel{0}{0}$ & $\stackrel{0}{0}: \stackrel{0}{0}:$ & & $\stackrel{0}{0} \stackrel{0}{0} \stackrel{0}{0}: \stackrel{0}{0} \stackrel{0}{0} \stackrel{0}{0} \stackrel{0}{0} \stackrel{0}{0} \dot{0}$ \\
\hline 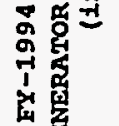 & 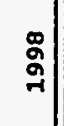 & $\stackrel{0}{0}: \stackrel{0}{0}$ & & $\stackrel{0}{0}: \stackrel{0}{0}$ & $\stackrel{0}{0}: \stackrel{0}{0}:$ & & $\stackrel{0}{0}: \stackrel{0}{0}$ & $\stackrel{0}{0}: \stackrel{0}{0}$ & & 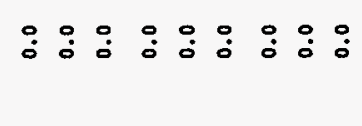 \\
\hline 帠 & ầ & $\dot{i} \dot{i} \dot{\phi}$ & & 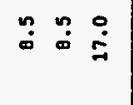 & 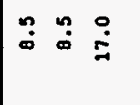 & & 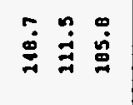 & 兽号离 & & 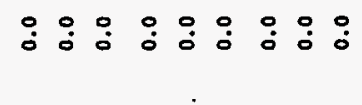 \\
\hline & : & 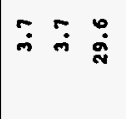 & & 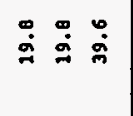 & 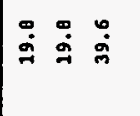 & & 芒咅芦 & 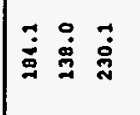 & & $\stackrel{0}{0} \stackrel{0}{0} \stackrel{0}{0} \stackrel{0}{0} \stackrel{0}{0} \stackrel{0}{0} \stackrel{0}{0} \stackrel{0}{0} \stackrel{0}{0}$ \\
\hline & คั & $\stackrel{0}{0} \stackrel{0}{0} \stackrel{0}{0}$ & & 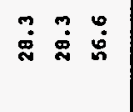 & 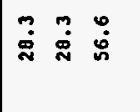 & & 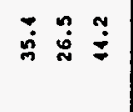 & 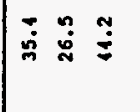 & & 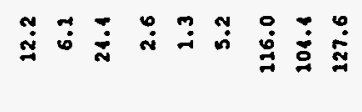 \\
\hline & 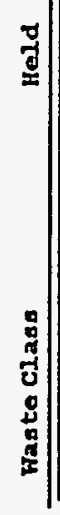 & 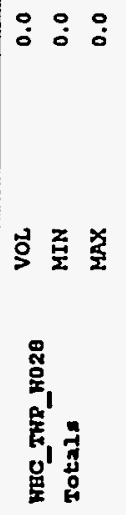 & 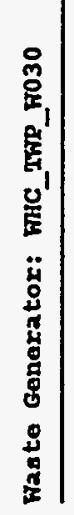 & 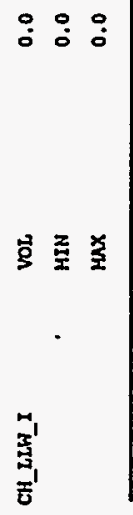 & 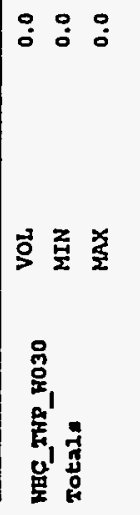 & 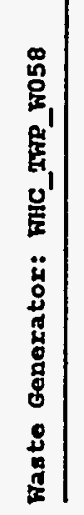 & 岁要旁 & 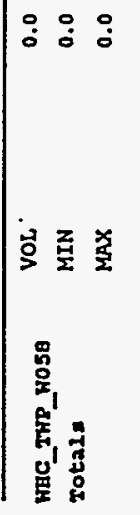 & 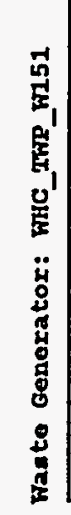 & 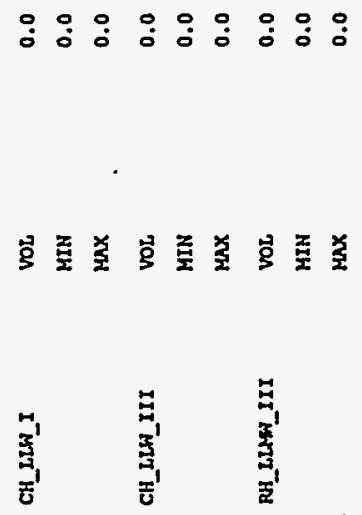 \\
\hline
\end{tabular}


FY-1994 SOLID WASTE FORECAST

WASTE GENERATOR, HASTE CLASS VOLUMES BY YEAR

(in cubic meters)

\begin{tabular}{|c|c|c|c|c|c|c|c|c|c|c|c|c|c|c|c|c|c|}
\hline Was te Class & & Held & 1995 & 1996 & 1997 & 1998 & 1999 & 2000 & 2001 & 2002 & 2003 & 2004 & 2005 & 2006 & 2007 & 2008 & 2009 \\
\hline \multirow{3}{*}{$\begin{array}{l}\text { HHC_FHP_H151 } \\
\text { Total: }\end{array}$} & vor & 0,0 & 130,8 & 0.0 & 0.0 & 0.0 & 0.0 & 0.0 & 111.9 & 0.0 & 0.0 & 0.0 & 0.0 & 0.0 & 0.0 & 0.0 & 0.0 \\
\hline & MIN & 0.0 & 111.8 & 0.0 & 0.0 & 0.0 & 0.0 & 0.0 & 109.8 & 0.0 & 0.0 & 0.0 & 0.0 & 0.0 & 0.0 & 0.0 & 0.0 \\
\hline & $\operatorname{Max}$ & 0.0 & 157.2 & 0.0 & 0.0 & 0.0 & 0.0 & 0.0 & 116.0 & 0.0 & 0.0 & 0.0 & 0.0 & 0.0 & 0.0 & 0.0 & 0.0 \\
\hline
\end{tabular}

\section{Was to Generator: FHC_IFP_W211}

\begin{tabular}{|c|c|c|c|c|c|c|c|c|c|c|c|c|c|c|c|c|c|c|c|}
\hline & \multirow[t]{3}{*}{ CH_LLW_I } & vor & & 0.0 & 0.0 & 99.2 & 198.3 & 99.2 & 99.2 & 198.3 & 198.3 & 198.3 & 198.3 & 99.2 & 99.2 & 198.3 & 99.2 & 99.2 & 99.2 \\
\hline & & MIN & & 0.0 & 0.0 & 19.6 & 99.1 & 19.6 & 49.6 & 99.1 & 99.1 & 99.1 & 99.1 & 19.6 & 19.6 & 99.1 & 49.6 & 19.6 & 19.6 \\
\hline & & $\boldsymbol{m a x}$ & & 0.0 & 0.0 & 99.2 & $\cdot 298.3$ & 99.2 & 99.2 & 298.3 & 298.3 & 298.3 & 198.3 & 99.2 & 99.2 & 198.3 & 99.2 & 99.2 & 99.2 \\
\hline & \multirow{3}{*}{ RH_LLW_III } & vor & - & 0.0 & 0.0 & 0.0. & 14.2 & 14.2 & 14.2 & 28.3 & 28.3 & 28.3 & 20.3 & 14.2 & 14.2 & 28.3 & 14.2 & 14.2 & 14.2 \\
\hline & & MIN & & 0.0 & 0.0 & 0.0 & 7.1 & 2.1 & 7.1 & 14.1 & 14.1 & 14.1 & 24.1 & 7.1 & 7.1 & 14.2 & 7.1 & 7.1 & 7.1 \\
\hline & & $\max$ & & 0.0 & 0.0 & 0.0 & 14.2 & 14.2 & 14.2 & 28.3 & 28.3 & 28.3 & 28.3 & 14.2 & 34.2 & 28.3 & 14.2 & 24.2 & 14.2 \\
\hline$i$ & \multirow{3}{*}{$\begin{array}{l}\text { KBC_FHP_H211 } \\
\text { TOt21. }\end{array}$} & Vor. & & 0.0 & 0.0 & 99.2 & 212.5 & 113.4 & 113.4 & 226.6 & 226.6 & 226.6 & 226.6 & 113.4 & 213.4 & 226.6 & 113.4 & 113.4 & 113.4 \\
\hline & & MIN & & 0.0 & 0.0 & 19.6 & 105.2 & 56.7 & 56.7 & 213.3 & 113.3 & 113.3 & 113.3 & 56.7 & 56.7 & 113.3 & 56.7 & 56.7 & 56.7 \\
\hline 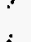 & & $\operatorname{MAX}$ & & 0.0 & 0.0 & 99.2 & 212.5 & 113.4 & 113.1 & 226.6 & 226.6 & 226.6 & 226.6 & 113.4 & 213.4 & 226.6 & 213.4 & 213.4 & 113.4 \\
\hline
\end{tabular}

Wasto Generator: WHC_TWP_F314

\begin{tabular}{|c|c|c|c|c|c|c|c|c|c|c|c|c|c|c|c|c|c|}
\hline \multirow[t]{3}{*}{ CH_LLHE_I } & vor & 0.0 & 0.0 & 0.0 & 0.0 & 210.8 & 420.8 & 1043.9 & 1164.3 & 753.5 & 753.5 & 120.4 & 0.0 & 0.0 & 0.0 & 0.0 & 0.0 \\
\hline & MIN & 0.0 & 0.0 & 0.0 & 0.0 & 24.0 & 11.0 & 101.3 & 116.1 & 75.3 & 75.3 & 12.0 & 0.0 & 0.0 & 0.0 & 0.0 & 0.0 \\
\hline & $\max$ & 0.0 & 0.0 & 0.0 & 0.0 & 240.8 & 110.8 & 1043.9 & 1164.3 & 753.5 & 753.5 & 120.4 & 0.0 & 0.0 & 0.0 & 0.0 & 0.0 \\
\hline \multirow{3}{*}{$\begin{array}{l}\text { Níc_2WP_H314 } \\
\text { Tota1. }\end{array}$} & VOL & 0.0 & 0.0 & 0.0 & 0.0 & 240.8 & $\$ 10.0$ & 1043.9 & 1264.3 & 753.5 & 753.5 & 120.4 & 0.0 & 0.0 & 0.0 & 0.0 & 0.0 \\
\hline & MIN & 0.0 & 0.0 & 0.0 & 0.0 & 24.0 & 11.0 & 101.3 & 116.4 & 75.3 & 75.3 & 12.0 & 0.0 & 0.0 & 0.0 & 0.0 & 0.0 \\
\hline & $\operatorname{MAX}$ & 0.0 & 0.0 & 0.0 & 0.0 & $240 . \theta$ & 110.8 & 1043.9 & 1164.3 & 753.5 & 753.5 & $220.1^{\circ}$ & 0.0 & 0.0 & 0.0 & 0.0 & 0.1 \\
\hline
\end{tabular}

Wasto Generatox: WHC_IFP_F320

\begin{tabular}{|c|c|c|c|c|c|c|c|c|c|c|c|c|c|c|c|c|c|}
\hline \multirow[t]{3}{*}{ CH_LLW_I } & Vor & 0.0 & 16.1 & 31.6 & 92.1 & 21.5 & 0.0 & 0.0 & 0.0 & 0.0 & 0.0 & 0.0 & 0.0 & 0.0 & 0.0 & 0.0 & 0.0 \\
\hline & MIN & 0.0 & 18.4 & 12.6 & 36.9 & 0.6 & 0.0 & 0.0 & 0.0 & 0.0 & 0.0 & 0.0 & 0.0 & 0.0 & 0.0 & 0.0 & 0.0 \\
\hline & $\max$ & 0.0 & 92.2 & 63.2 & 184.8 & 13.0 & 0.0 & 0.0 & 0.0 & 0.0 & 0.0 & 0.0 & 0.0 & 0.0 & 0.0 & 0.0 & 0.0 \\
\hline \multirow[t]{3}{*}{ CH_LLWH_III } & vor & 0.0 & 10.2 & 29.0 & 200.5 & 24.5 & 0.0 & 0.0 & 0.0 & 0.0 & 0.0 & 0.0 & 0.0 & 0.0 & 0.0 & 0.0 & 0.0 \\
\hline & HIM & 0.0 & 16.0 & 11.6 & 80.2 & 9.8 & 0.0 & 0.0 & 0.0 & 0.0 & 0.0 & 0.0 & 0.0 & 0.0 & 0.0 & 0.0 & 0.0 \\
\hline & $\max$ & 0.0 & 10.2 & 29.0 & 200.5 & 24.5 & 0.0 & 0.0 & 0.0 & 0.0 & 0.0 & 0.0 & 0.0 & 0.0 & 0.0 & 0.0 & 0.0 \\
\hline
\end{tabular}


WHC-EP-0865

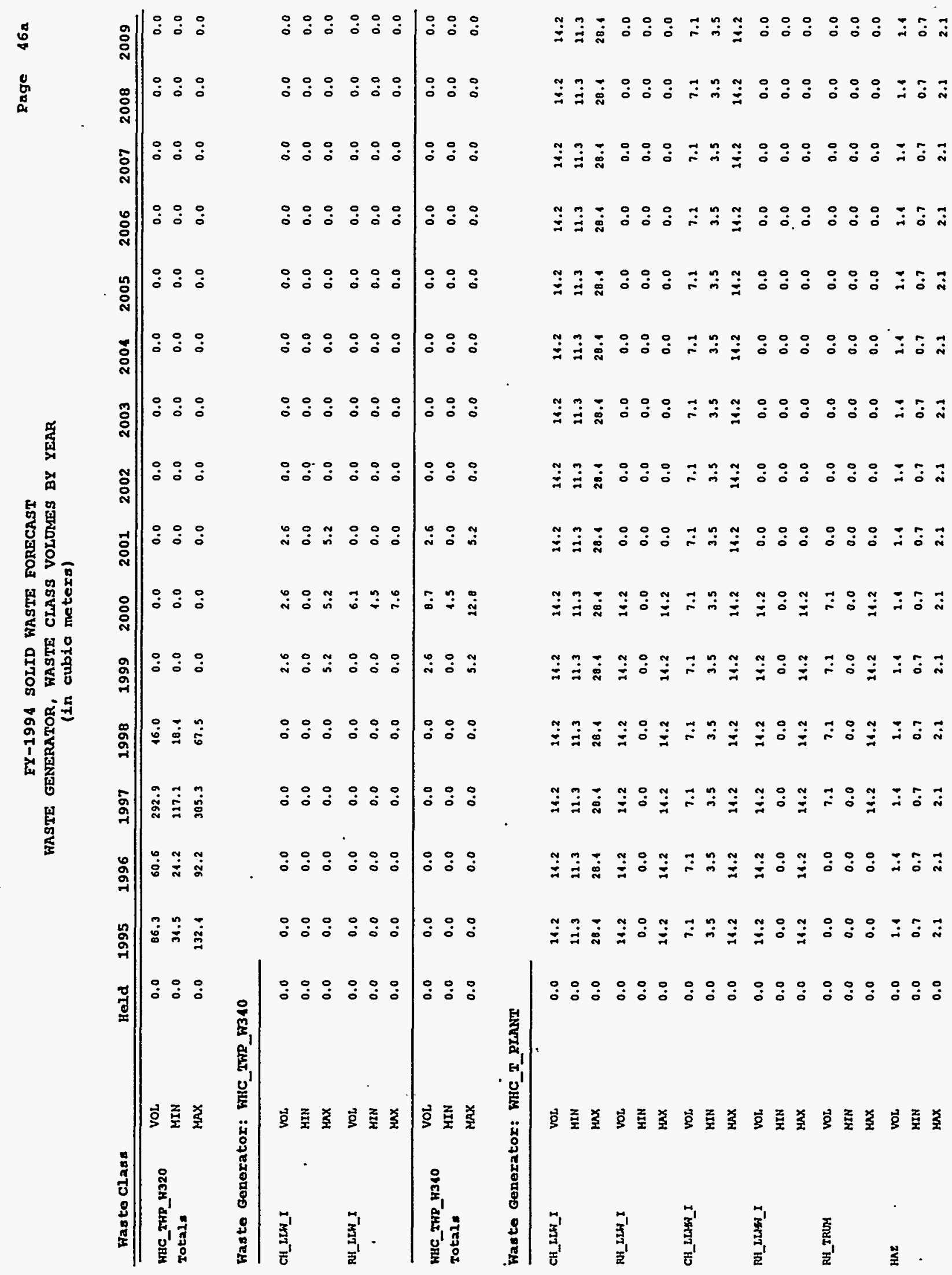




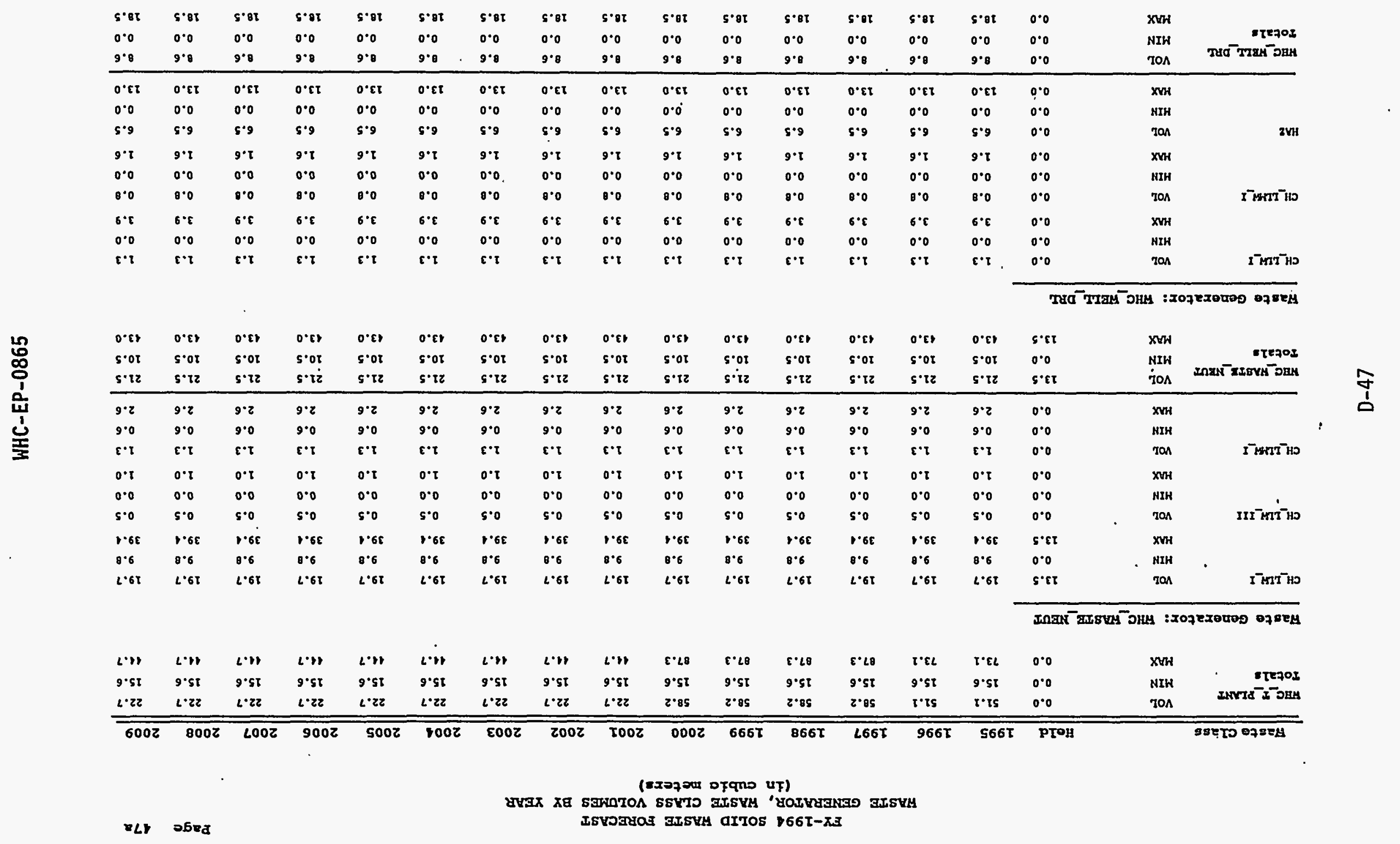


(in cubio meters)

\begin{tabular}{|c|c|c|c|c|c|c|c|c|c|c|c|c|c|c|c|c|c|}
\hline Fas te & & Held & 1995 & 1996 & 1997 & 1998 & 1999 & 2000 & 2001 & 2002 & 2003 & 2004 & 2005 & 2006 & 2007 & 2008 & 2009 \\
\hline \multirow{3}{*}{$\begin{array}{l}\text { Grand } \\
\text { Totals }\end{array}$} & VoL & 7207.0 & 14973.1 & 14309.3 & 20865.1 & 10778.9 & 12214.3 & 24850.8 & 33001.4 & 81211.8 & 203669.9 & 111275.2 & 285795.7 & 204112.5 & 227185.1 & 151056.6 & 79371.8 \\
\hline & MIN & 1961.2 & 21330.3 & 10766.5 & 15329.3 & 7200.3 & 8167.0 & 10376.7 & 29196.9 & 77751.5 & 100587.0 & 230182.9 & 202222.1 & 200696.9 & 223699.3 & 147252.8 & 73880.7 \\
\hline & $\max$ & 7207.0 & 29691.9 & 29471.3 & 23251.6 & 14804.4 & 16826.8 & 30648.3 & 37936.8 & 85722.5 & 300233.3 & 166133.8 & 209937.8 & 208602.0 & 231491.1 & 155464.7 & 85428.5 \\
\hline
\end{tabular}


FY-1994 SOLID HASTE FORECAST

WASTE GENERATOR, WASTE CLASS VOLONES BY YEAR

(in cubio meters)

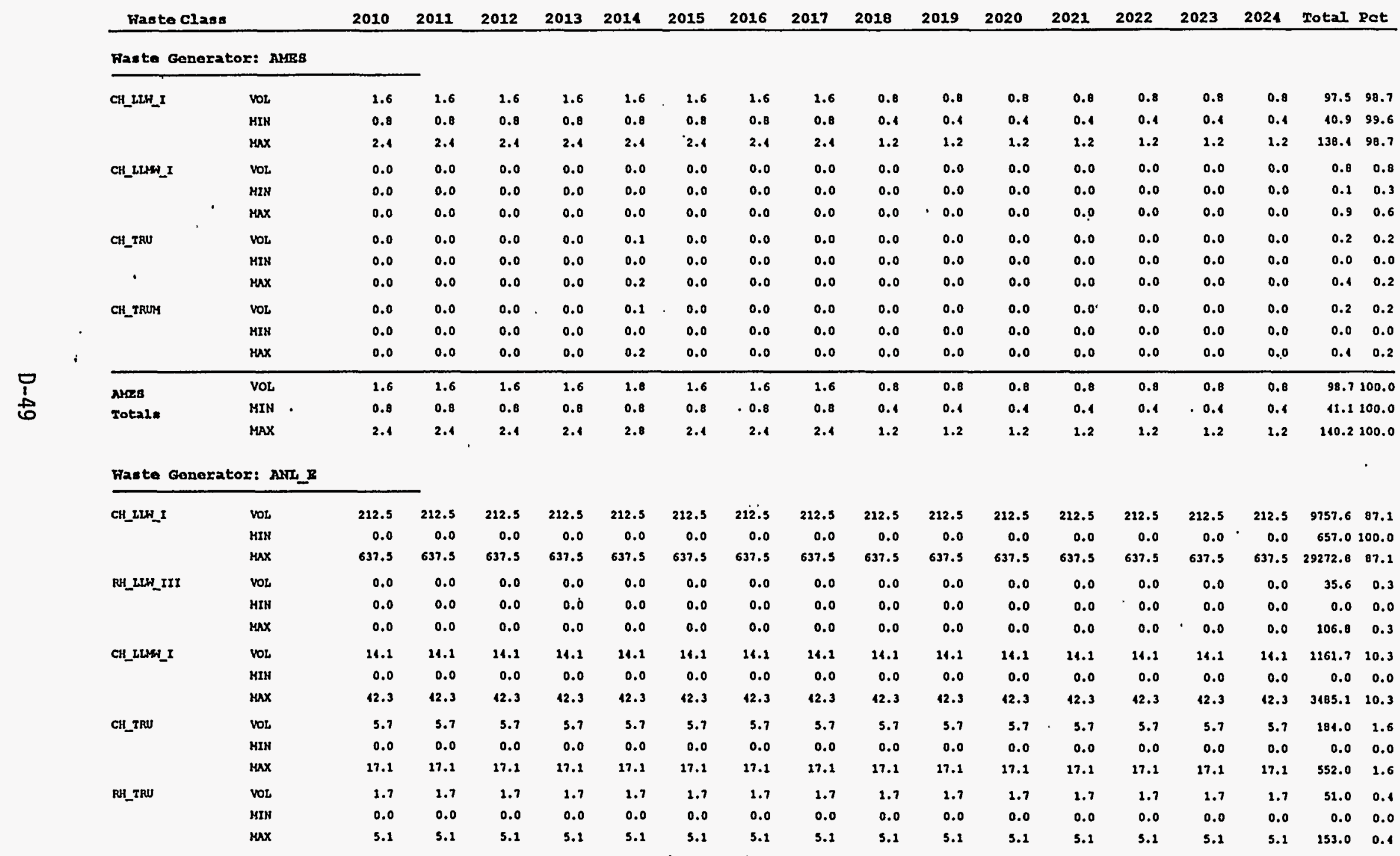


FY-1994 SOLID WASTE FORECAST

HASTE GENERATOR, HASTE CLASS VOLOMES BY YEAR

(in cubic meters)

Faste Class

$\begin{array}{llllllllllllllll}2010 & 2011 & 2012 & 2013 & 2014 & 2015 & 2016 & 2017 & 2018 & 2019 & 2020 & 2021 & 2022 & 2023 & 2021 & \text { Total Pct }\end{array}$

\section{Wasto Generator: ANL_E}

\begin{tabular}{|c|c|c|c|c|c|c|c|c|c|c|c|c|c|c|c|c|c|c|}
\hline \multirow[t]{3}{*}{ CH_tPUM } & vor & 0.2 & 0.2 & 0.2 & 0.2 & 0.2 & 0.2 & 0.2 & 0.2 & 0.2 & 0.2 & 0.2 & 0.2 & 0.2 & 0.2 & 0.2 & 7.5 & 0.0 \\
\hline & MIN & 0.0 & 0.0 & 0.0 & 0.0 & 0.0 & 0.0 & 0.0 & 0.0 & 0.0 & 0.0 & 0.0 & 0.0 & 0.0 & 0.0 & 0.0 & 0.0 & 0.0 \\
\hline & $\operatorname{mAx}$ & 0.6 & 0.6 & 0.6 & 0.6 & 0.6 & 0.6 & 0.6 & 0.6 & 0.6 & 0.6 & 0.6 & 0.6 & 0.6 & 0.6 & 0.6 & 22.5 & 0.0 \\
\hline \multirow{3}{*}{$\begin{array}{l}\text { NNS_X } \\
\text { Total. }\end{array}$} & Vor & 234.2 & 234.2 & 234.2 & 234.2 & 234.2 & 234.2 & 234.2 & 234.2 & 234.2 & 234.2 & 231.2 & 234.2 & 234.2 & 234.2 & 234.2 & 11197.4 & 100.0 \\
\hline & MIN & 0.0 & 0.0 & 0.0 & 0.0 & 0.0 & 0.0 & 0.0 & 0.0 & 0.0 & 0.0 & 0.0 & 0.0 & 0.0 & 0.0 & 0.0 & 657.0 & 100.0 \\
\hline & $\operatorname{MAX}$ & 702.6 & 702.6 & 702.6 & 702.6 & 702.6 & 702.6 & 702.6 & 702.6 & 702.6 & 702.6 & 702.6 & 702.6 & 702.6 & 702.6 & 702.6 & 33592.2 & 200.0 \\
\hline
\end{tabular}

Was te Generator: BAPI

\begin{tabular}{|c|c|c|c|c|c|c|c|c|c|c|c|c|c|c|c|c|c|c|}
\hline \multirow[t]{3}{*}{ CH_LIW_III } & vor & 0.0 & 0.0 & 0.0 & 0.0 & 0.0 & 0.0 & 0.0 & 0.0 & 0.0 & 0.0 & 0.0 & 0.0 & 0.0 & 0.0 & 0.0 & 153.4 & 13.9 \\
\hline & HIH & 0.0 & 0.0 & 0.0 & 0.0 & 0.0 & 0.0 & 0.0 & 0.0 & 0.0 & 0.0 & 0.0 & 0.0 & 0.0 & 0.0 & 0.0 & 39.8 & 29.0 \\
\hline & $\max$ & 0.0 & 0.0 & 0.0 & 0.0 & 0.0 & 0.0 & 0.0 & 0.0 & 0.0 & 0.0 & 0.0 & 0.0 & 0.0 & 0.0 & 0.0 & 253.1 & 13.9 \\
\hline \multirow[t]{2}{*}{ CH_LLH" I } & VoL & 2.6 & 2.6 & 2.6 & 2.6 & 2.6 & 2.6 & 2.6 & 2.6 & 2.6 & 2.6 & 2.6 & 2.6 & 2.6 & 2.6 & 2.6 & 295.3 & 56.0 \\
\hline & $\max$ & 2.6 & 2.6 & 2.6 & 2.6 & 2.6 & 2.6 & 2.6 & 2.6 & 2.6 & 2.6 & 2.6 & 2.6 & 2.6 & 2.6 & 2.6 & 295.3 & 56.0 \\
\hline \multirow{3}{*}{$\begin{array}{l}\text { gAPL } \\
\text { Tota1. }\end{array}$} & vot & 2.6 & 2.6 & 2.6 & 2.6 & 2.6 & 2.6 & 2.6 & 2.6 & 2.6 & 2.6 & 2.6 & 2.6 & 2.6 & 2.6 & 2.6 & 348.7 & 200.0 \\
\hline & MIN & 1.3 & 1.3 & 1.3 & 1.3 & 1.3 & 1.3 & 1.3 & 1.3 & 1.3 & 1.3 & 1.3 & 1.3 & 1.3 & 1.3 & 1.3 & 237.5 & 100.0 \\
\hline & $\operatorname{MAX}$ & $2.6^{\circ}$ & 2.6 & 2.6 & 2.6 & 2.6 & 2.6 & 2.6 . & 2.6 & 2.6 & 2.6 & 2.6 & 2.6 & 2.6 & 2.6 & 2.6 & 340.7 & 200.0 \\
\hline
\end{tabular}

Waste Generator: BAPL_BHIPXDs

\begin{tabular}{|c|c|c|c|c|c|c|c|c|c|c|c|c|c|c|c|c|c|}
\hline \multirow[t]{3}{*}{ CH_LLW_I } & vor & 0.0 & 0.0 & 2.8 & 0.0 & 0.0 & 0.0 & 0.0 & 0.0 & 0.0 & 0.0 & 0.0 & 0.0 & 0.0 & 0.0 & 0.0 & 1.3100 .0 \\
\hline & MIN & 0.0 & 0.0 & 0.0 & 0.0 & 0,0 & 0.0 & 0.0 & 0.0 & 0.0 & 0.0 & 0.0 & 0.0 & 0.0 & 0.0 & 0.0 & $0.0 \quad 0.0$ \\
\hline & $\max$ & 0.0 & 0.0 & 2.8 & 0.0 & 0.0 & 0.0 & 0.0 & 0.0 & 0.0 & 0.0 & 0.0 & 0.0 & 0.0 & 0.0 & 0.0 & 7.3100 .0 \\
\hline \multirow{3}{*}{$\begin{array}{l}\text { gAPR_BRIPYDB } \\
\text { Tota1: }\end{array}$} & VOL & 0.0 & 0.0 & 2.8 & 0.0 & 0.0 & 0.0 & 0.0 & 0.0 & 0.0 & 0.0 & 0.0 & 0.0 & 0.0 & 0.0 & 0.0 & 7.3100 .0 \\
\hline & MIN & 0.0 & 0.0 & 0.0 & 0.0 & 0.0 & 0.0 & 0.0 & 0.0 & 0.0 & 0.0 & 0.0 & 0.0 & 0.0 & 0.0 & 0.0 & 0.0100 .0 \\
\hline & MAS & 0.0 & 0.0 & 2.8 & 0.0 & 0.0 & 0.0 & 0.0 & 0.0 & 0.0 & 0.0 & 0.0 & 0.0 & 0.0 & 0.0 & 0.0 & 7.3100 .0 \\
\hline
\end{tabular}

Wasto Generator: BAT_CrBs_LAB

\begin{tabular}{|c|c|c|c|c|c|c|c|c|c|c|c|c|c|c|c|c|c|}
\hline \multirow[t]{3}{*}{ CH_LLW_I } & Vor & 0.0 & 0.0 & 0.0 & 0.0 & 0.0 & 0.0 & 0.0 & 0.0 & 0.0 & 0.0 & 0.0 & 0.0 & 0.0 & 0.0 & 0.0 & 1501.192 .5 \\
\hline & MIN & 0.0 & 0.0 & 0.0 & 0.0 & 0.0 & 0.0 & 0.0 & 0.0 & 0.0 & 0.0 & 0.0 & 0.0 & 0.0 & 0.0 & 0.0 & 173.0100 .0 \\
\hline & $\max$ & 0.0 & 0.0 & 0.0 & 0.0 & 0.0 & 0.0 & 0.0 & 0.0 & 0.0 & 0.0 & 0.0 & 0.0 & 0.0 & 0.0 & 0.0 & $1501.1 \quad 92.5$ \\
\hline
\end{tabular}




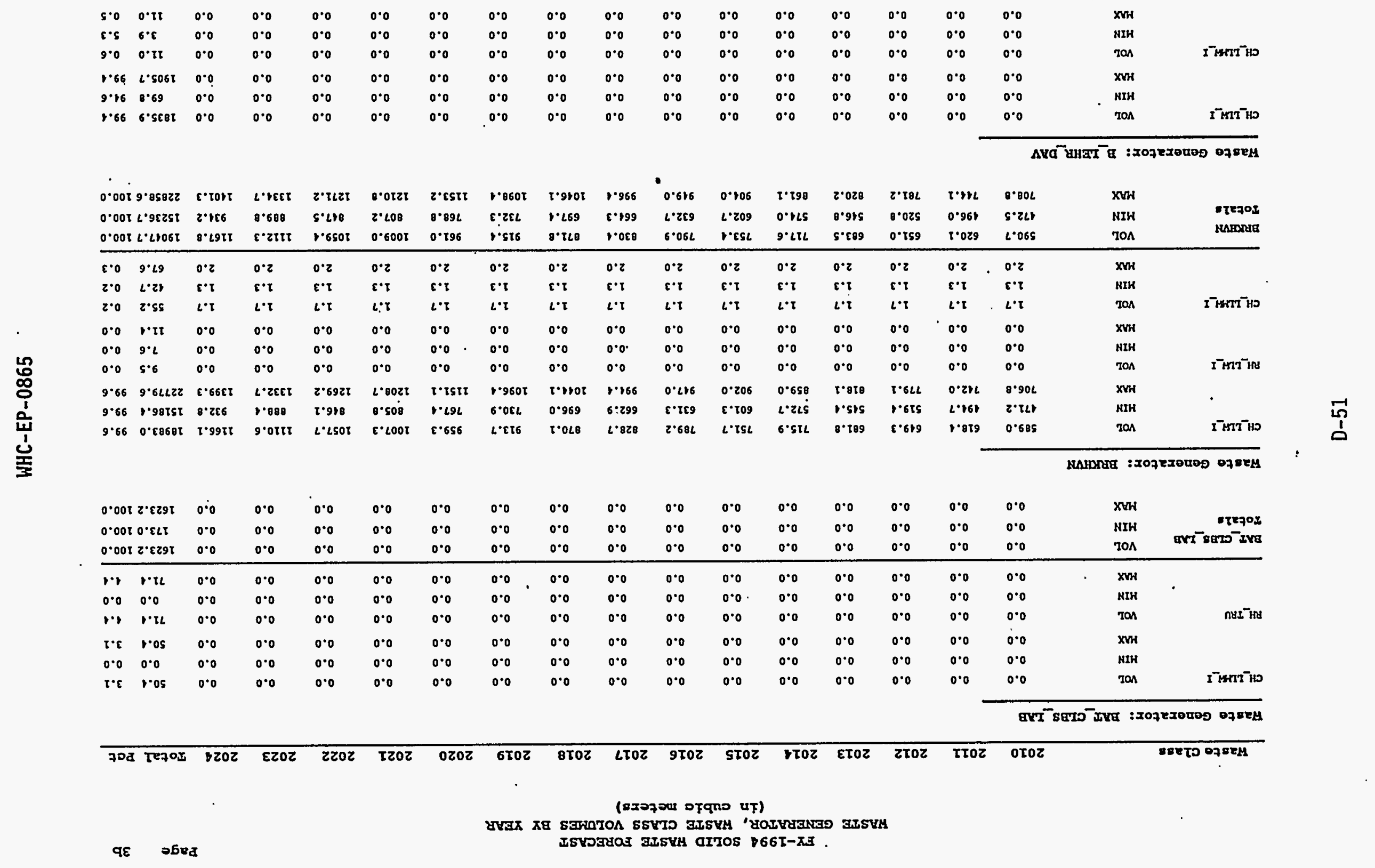




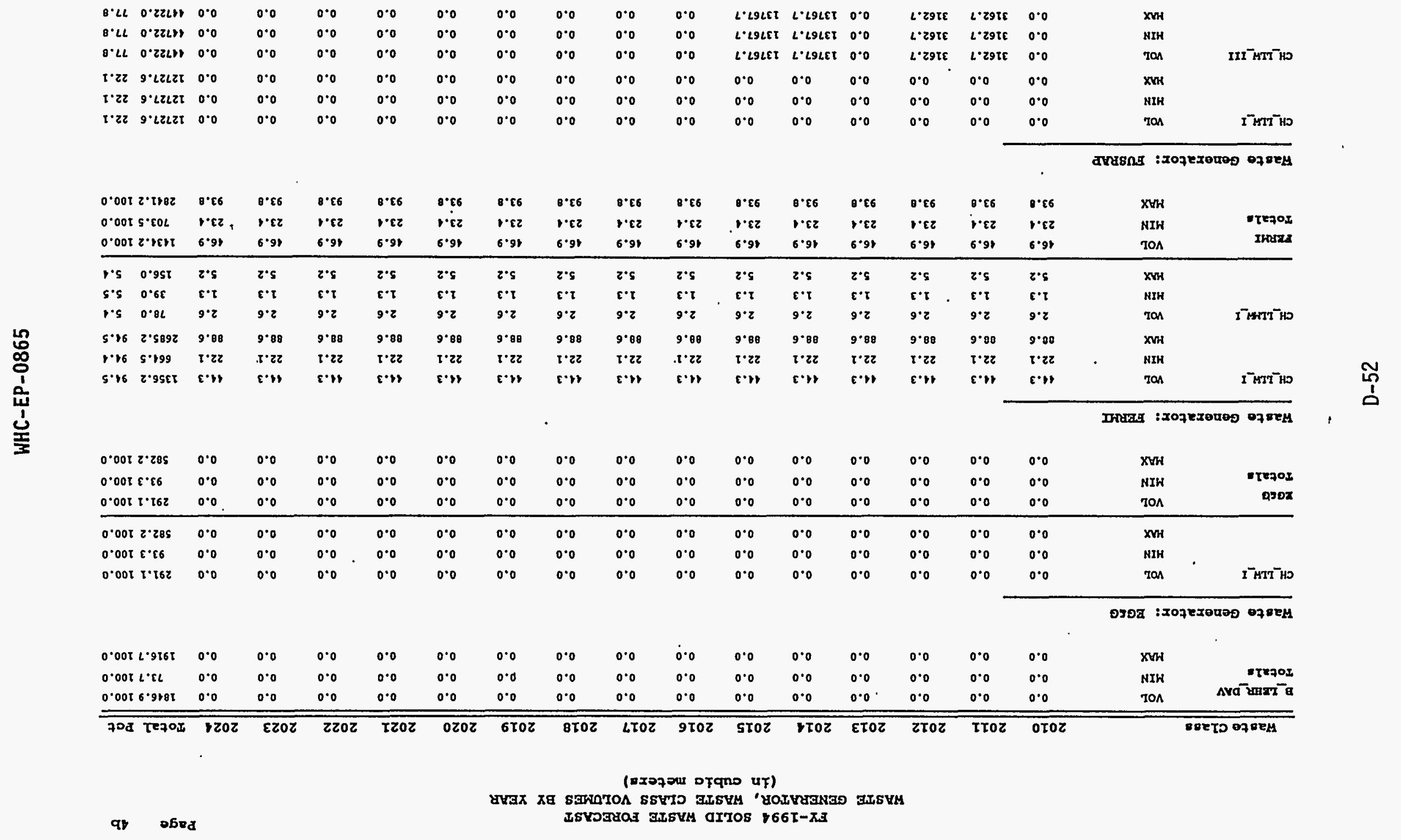




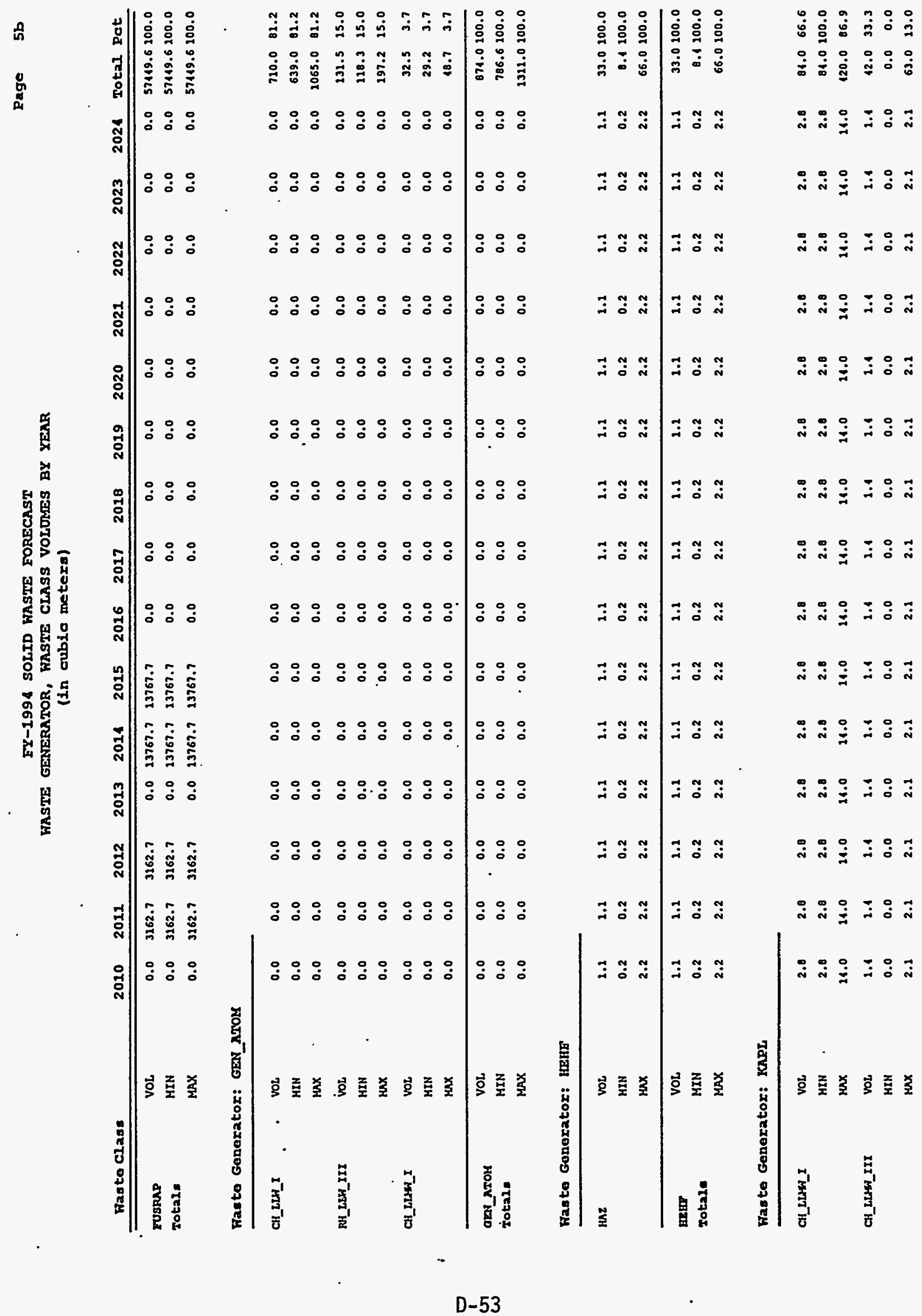


WASTE GENERATOR, WASTE CLASS VOLUMES BY YEAR (in cubic meters)

\begin{tabular}{|c|c|c|c|c|c|c|c|c|c|c|c|c|c|c|c|c|c|}
\hline Waste Class & & 2010 & 2011 & 2012 & 2013 & 2014 & 2015 & 2016 & 2017 & 2018 & 2019 & 2020 & 2021 & 2022 & 2023 & 2024 & Total Pct \\
\hline \multirow{3}{*}{$\begin{array}{l}\text { KAPL. } \\
\text { Total: }\end{array}$} & VOL & 4.2 & 4.2 & 4.2 & 4.2 & 4.2 & 4.2 & 4.2 & 1.2 & 1.2 & 4.2 & 1.2 & 1.2 & 1.2 & 4.2 & 1.2 & 126.0100 .0 \\
\hline & MIN & 2.8 & 2.8 & 2.8 & 2.0 & 2.8 & 2.8 & 2.8 & 2.8 & 2.8 & 2.8 & 2.8 & 2.8 & 2.8 & 2.8 & 2.8 & 84.0100 .0 \\
\hline & $\operatorname{MAX}$ & 16.1 & 26.1 & 16.1 & 16.1 & 16.1 & 26.1 & 16.1 & 26.1 & 16.1 & 26.1 & 16.1 & 16.1 & 16.1 & 16.1 & 16.1 & 183.0100 .0 \\
\hline
\end{tabular}

Waste Generator: KAPX_sHrPYDS

\begin{tabular}{|c|c|c|c|c|c|c|c|c|c|c|c|c|c|c|c|c|c|}
\hline \multirow[t]{3}{*}{ CH_LLW_III } & vor & 0.0 & 0.0 & 0.0 & 0.0 & 0.0 & 0.0 & 0.0 & 0.0 & 0.0 & 0.0 & 0.0 & 0.0 & 0.0 & 0.0 & 0.0 & $297.0 \quad 100.0$ \\
\hline & MIN & 0.0 & 0.0 & 0.0 & 0.0 & 0.0 & 0.0 & 0.0 & 0.0 & 0.0 & 0.0 & 0.0 & 0.0 & 0.0 & 0.0 & 0.0 & $0.0 \quad 0.0$ \\
\hline & $\max$ & 0.0 & 0.0 & 0.0 & 0.0 & 0.0 & 0.0 & 0.0 & 0.0 & 0.0 & 0.0 & 0.0 & 0.0 & 0.0 & 0.0 & 0.0 & 297.0100 .0 \\
\hline \multirow{3}{*}{$\begin{array}{l}\text { xAPL_8asPXDB } \\
\text { rotai: }\end{array}$} & VOL & 0.0 & 0.0 & 0.0 & 0.0 & 0.0 & 0.0 & 0.0 & 0.0 & 0.0 & 0.0 & 0.0 & 0.0 & 0.0 & 0.0 & 0.0 & 297.0200 .0 \\
\hline & MIN & 0.0 & 0.0 & 0.0 & 0.0 & 0.0 & 0.0 & 0.0 & 0.0 & 0.0 & 0.0 & 0.0 & 0.0 & 0.0 & 0.0 & 0.0 & 0.0100 .0 \\
\hline & $\max$ & 0.0 & 0.0 & 0.0 & 0.0 & 0.0 & 0.0 & 0.0 & 0.0 & 0.0 & 0.0 & 0.0 & 0.0 & 0.0 & 0.0 & 0.0 & 297.0100 .0 \\
\hline
\end{tabular}

\section{Waste Generator: KEH_100}

\begin{tabular}{|c|c|c|c|c|c|c|c|c|c|c|c|c|c|c|c|c|c|c|}
\hline \multirow[t]{3}{*}{$\mathrm{CH}_{-} \mathrm{LLW} \mathrm{IL}_{-}$} & VoL & 0.0 & 0.0 & 0.0 & 0.0 & 0.0 & 0.0 & 0.0 & 0.0 & 0.0 & 0.0 & 0.0 & 0.0 & 0.0 & 0.0 & 0.0 & 76.9 & 0.0 \\
\hline & MIN & 0.0 & 0.0 & 0.0 & 0.0 & 0.0 & 0.0 & 0.0 & 0.0 & 0.0 & 0.0 & 0.0 & 0.0 & 0.0 & 0.0 & 0.0 & 53.8 & 4.7 \\
\hline & $\max$ & 0.0 & 0.0 & 0.0 & 0.0 & 0.0 & 0.0 & 0.0 & 0.0 & 0.0 & 0.0 & 0.0 & 0.0 & 0.0 & 0.0 & 0.0 & 76.9 & 0.0 \\
\hline \multirow[t]{3}{*}{ CH_LLMW_I } & VoL & 0.0 & 0.0 & 0.0 & 0.0 & 0.0 & 0.0 & 0.0 & 0.0 & 0.0 & 0.0 & 0.0 & 0.0 & 0,0 & 0.0 & 0.0 & 11.3 & 0.0 \\
\hline & HIN & 0.0 & 0.0 & 0.0 & 0.0 & 0.0 & 0.0 & 0.0 & 0.0 & 0.0 & 0.0 & 0.0 & 0.0 & 0.0 & 0.0 & 0.0 & 0.9 & 0.0 \\
\hline & $\max$ & 0.0 & 0.0 & 0.0 & 0.0 & 0.0 & 0.0 & 0.0 & 0.0 & 0.0 & 0.0 & 0.0 & 0.0 & 0.0 & 0.0 & 0.0 & 11.3 & 0.0 \\
\hline \multirow[t]{3}{*}{ HAZ } & vor & 1049.1 & 1311.4 & 1639.2 & 2019.1 & 2561.3 & 3201.7 & 1002.1 & 5002.6 & 6253.3 & 7816.6 & 9770.7 & 12213.4 & 15265.7 & 29083.1 & 23854.2 & 119123.4 & 99.9 \\
\hline & MIN & 11.9 & 39.3 & 32.7 & 10.9 & 25.6 & 32.0 & 36.0 & 35.0 & 37.5 & 39.0 & 39.0 & 36.6 & 30.5 & 38.1 & 23.8 & 2078.1 & 95.1 \\
\hline & $\max$ & 1019.1 & 1311.1 & 1639.2 & 2019.1 & 2561.3 & 3201.7 & 4002.1 & 5002.6 & 6253.3 & 7816.6 & 9770.7 & 12213.4 & 15266.7 & 19003.4 & 23854.2 & 119127.0 & 99.9 \\
\hline \multirow{3}{*}{$\begin{array}{l}\text { X8H_100 } \\
\text { Total. }\end{array}$} & VOL & 1049.1 & 1311.4 & 1639.2 & 2049.1 & 2561.3 & 3201.7 & 1002.1 & 5002.6 & 6253.3 & 7826.6 & 9770.7 & 12213.4 & 15266.7 & 19083.4 & 23854.2 & 119211.6 & 100.0 \\
\hline & MIN & 11.9 & 39.3 & 32.7 & 10.9 & 25.6 & 32.0 & 36.0 & 35.0 & 37.5 & 39.0 & 39.0 & 36.6 & 30.5 & 38.1 & 23.0 & 1132.8 & 100.0 \\
\hline & $\operatorname{MAX}$ & 1049.1 & 1311.4 & 1639.2 & 2049.1 & 2561.3 & 3202.7 & 1002.1 & 5002.6 & 6253.3 & 7816.6 & 9270.7 & 12213.4 & 15266.7 & 19083.4 & 23854.2 & 119215.2 & 100.0 \\
\hline
\end{tabular}

Waste Gederator: I.BL

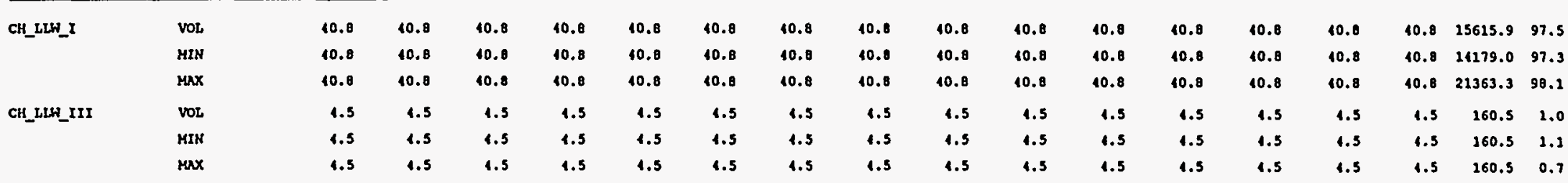


FY-1994 SOLID KASTE FORECAST

WASTE GENERATOR, WASTE CLASS YOLUMES BY YEAR

(in cubic meters)

Fas to Class

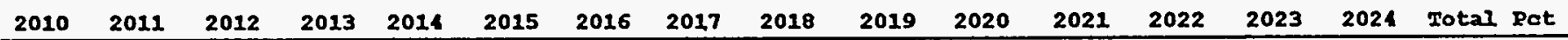

\section{Wasto Generator: L.8L}

\begin{tabular}{|c|c|c|c|c|c|c|c|c|c|c|c|c|c|c|c|c|c|c|}
\hline \multirow{3}{*}{ CH_LLWA_I } & vot & 7.0 & 7.0 & 7.0 & 7.0 & 7.0 & 7.0 & 7.0 & 7.0 & 7.0 & 7.0 & 7.0 & 7.0 & 7.0 & 7.0 & 7.0 & 208.0 & 1.3 \\
\hline & HIN & 7.0 & 7.0 & 7.0 & 7.0 & 7.0 & 7.0 & 7.0 & 7.0 & 7.0 & 7.0 & 7.0 & 7.0 & 7.0 & 7.0 & 7.0 & 208.8 & 1.4 \\
\hline & $\max$ & 7.0 & 1.0 & 7.0 & 7.0 & 7.0 & 7.0 & 7.0 & 7.0 & 7.0 & 7.0 & 7.0 & 7.0 & 7.0 & 7.0 & 7.0 & 200.8 & 0.9 \\
\hline \multirow[t]{2}{*}{ CH_LLW_III } & vor & 0.8 & 0.8 & 0.8 & 0.8 & 0.8 & 0.8 & 0.8 & 0.8 & 0.8 & 0.8 & 0.8 & 0.8 & 0.8 & 0.0 & 0.8 & 23.8 & 0.1 \\
\hline & $\max$ & 0.8 & 0.8 & 0.8 & 0.8 & 0.8 & 0.8 & 0.8 & 0.8 & 0.8 & 0.8 & 0.8 & 0.8 & 0.8 & 0.8 & 0.8 & 23.8 & 0.1 \\
\hline \multirow{2}{*}{$\begin{array}{l}\text { LoI, } \\
\text { Total. }\end{array}$} & VoL & 53.1 & 53.1 & 53.1 & 33.1 & 53.1 & 53.1 & 53.1 & 53.1 & 53.1 & 53.1 & 33.1 & 53.1 & 53.1 & 53.1 & 53.1 & 16009.0 & 100.0 \\
\hline & MIN & 33.1 & 53.1 & 53.1 & 33.1 & 53.2 & 53.1 & 53.1 & 53.1 & 53.1 & 53.1 & 53.1 & 53.1 & 53.1 & 53.1 & 33.1 & 14572.1 & 100.0 \\
\hline
\end{tabular}

Waste Gonerator: HIT_BATES

\begin{tabular}{|c|c|c|c|c|c|c|c|c|c|c|c|c|c|c|c|c|c|}
\hline \multirow[t]{3}{*}{$\mathrm{CH}_{-} \mathrm{LLH} \mathrm{H}_{-} \mathrm{I}$} & VoL & 0.3 & 0.0 & 0.0 & 0.0 & 0.0 & 0.3 & 0.0 & 0.0 & 0.0 & 0.0 & 0.3 & 0.0 & 0.0 & 0.0 & 0.0 & 1.5100 .0 \\
\hline & MIN & 0.3 & 0.0 & 0.0 & 0.0 & 0.0 & 0,3 & 0.0 & 0.0 & 0.0 & 0.0 & 0.3 & 0.0 & 0.0 & 0.0 & 0.0 & 1.5100 .0 \\
\hline & $\operatorname{MNX}$ & 0.6 & 0.0 & 0.0 & 0.0 & 0.0 & 0.6 & 0.0 & 0.0 & 0.0 & 0.0 & 0.6 & 0.0 & 0.0 & 0.0 & 0.0 & 3.0100 .0 \\
\hline \multirow{3}{*}{$\begin{array}{l}\text { MIT_parrs } \\
\text { Totals }\end{array}$} & VOL & 0.3 & 0.0 & 0.0 & 0.0 & 0.0 & 0.3 & 0.0 & 0.0 & 0.0 & 0.0 & 0.3 & 0.0 & 0.0 & 0.0 & 0.0 & 1.5100 .0 \\
\hline & MIN & 0.3 & 0.0 & 0.0 & 0.0 & 0.0 & 0.3 & 0.0 & 0.0 & 0.0 & 0.0 & 0.3 & 0.0 & 0.0 & 0.0 & 0.0 & 1.5100 .0 \\
\hline & $\operatorname{MAX}$ & 0.6 & 0.0 & 0.0 & 0.0 & 0.0 & 0.6 & 0.0 & 0.0 & 0.0 & 0.0 & 0.6 & 0.0 & 0.0 & 0.0 & 0.0 & 3.0100 .0 \\
\hline
\end{tabular}

Haste Gonerator: PADUCAH_Es

\begin{tabular}{|c|c|c|c|c|c|c|c|c|c|c|c|c|c|c|c|c|c|c|}
\hline \multirow[t]{3}{*}{ CH_LLH_I } & VOL & 156.4 & 156.1 & 156.4 & 156.4 & 156.4 & 156.4 & 156.4 & 156.4 & 156.4 & 156.1 & 156.1 & 156.4 & 136.4 & 256.4 & 156.4 & 6690.3 & 56.5 \\
\hline & MIN & 78.2 & 78.2 & 78.2 & 79.2 & 78.2 & 78.2 & 78.2 & 78.2 & 70.2 & 78.2 & 78.2 & 78.2 & 78.2 & 78.2 & 78.2 & 2346.0 & 17.9 \\
\hline & $\max$ & 234.6 & 234.6 & 234.6 & 234.6 & 234.6 & 234.6 & 231.6 & 234.6 & 234.6 & 234.6 & 234.6 & 234.6 & 234.6 & 234.6 & 234.6 & 9036.3 & 54.0 \\
\hline \multirow[t]{3}{*}{ CH_LLME_I } & Vor & 170.0 & 170.0 & 170.0 & 170.0 & 170.0 & 170.0 & 170.0 & 170.0 & 270.0 & 170.0 & 170.0 & 170.0 & 170.0 & 170.0 & 270.0 & 5132.4 & 13.4. \\
\hline & MIN & 85.0 & 85.0 & 05.0 & 85.0 & 85.0 & 05.0 & 85.0 & 85.0 & 85.0 & 85.0 & 85.0 & 85.0 & 85.0 & 85.0 & 85.0 & 2550.0 & 52.0 \\
\hline & $\max$ & 255.0 & 255.0 & 255.0 & 255.0 & 255.0 & 255.0 & 255.0 & 255.0 & 255.0 & 235.0 & 255.0 & 255.0 & 255.0 & 255.0 & 255.0 & 7692.1 & 15.9 \\
\hline \multirow{3}{*}{$\begin{array}{l}\text { PADUCAR_Y8 } \\
\text { Total. }\end{array}$} & VOL & 326.4 & 326.4 & 326.4 & 326.4 & 326.4 & 326.1 & 326.4 & 326.4 & 326.4 & 326.1 & 326.1 & 326.4 & 326.4 & 326.4 & 326.4 & 11022.7 & 100.0 \\
\hline & MIN & 163.2 & 163.2 & 163.2 & 263.2 & 163.2 & 163.2 & 163.2 & 163.2 & 163.2 & 163.2 & 163.2 & 163.2 & 163.2 & 163.2 & 163.2 & 1896.0 & 100.0 \\
\hline & $\max$ & 409.6 & 409.6 & 489.6 & 109.6 & 489.6 & 489.6 & 199.6 & 489.6 & 189.6 & 489.6 & 489.6 & 189.6 & 189.6 & 189.6 & 489.6 & 16718.7 & 100.0 \\
\hline
\end{tabular}


FY-1994 SOLID WASTE FORECAST

WASTE GENERATOR, WASTE CLASS VOLOMES BY YEAR

(in cubic meters)

Haste Class 2010

\begin{tabular}{|c|c|c|c|c|c|c|c|c|c|c|c|c|c|c|c|c|c|c|}
\hline \multirow[t]{3}{*}{$\mathrm{CH}_{-} \mathrm{LLH}_{-} \mathrm{I}$} & vol & 0.0 & 0.0 & 0.0 & 0.0 & 0.0 & 0.0 & 0.0 & 0.0 & 0.0 & 0.0 & 0.0 & 0.0 & 0.0 & 0.0 & 0.0 & 926.3 & 97.3 \\
\hline & MIN & 0.0 & 0.0 & 0.0 & 0.0 & 0.0 & 0.0 & 0.0 & 0.0 & 0.0 & 0.0 & 0.0 & 0.0 & 0.0 & 0.0 & 0.0 & 0.0 & 0.0 \\
\hline & $\max$ & 0.0 & 0.0 & 0.0 & 0.0 & 0.0 & 0.0 & 0.0 & 0.0 & 0.0 & 0.0 & 0.0 & 0.0 & 0.0 & 0.0 & 0.0 & 926.3 & 97.3 \\
\hline \multirow[t]{3}{*}{ CH_LLWN_I } & VoL & 0.0 & 0.0 & 0.0 & 0.0 & 0.0 & 0.0 & 0.0 & 0.0 & 0.0 & 0.0 & 0.0 & 0.0 & 0.0 & 0.0 & 0.0 & 25.1 & 2.6 \\
\hline & MIN & 0.0 & 0.0 & 0.0 & 0.0 & 0.0 & 0.0 & 0.0 & 0.0 & 0.0 & 0.0 & 0.0 & 0.0 & 0.0 & 0.0 & 0.0 & 0.0 & 0.0 \\
\hline & $\max$ & 0.0 & 0.0 & 0.0 & 0.0 & 0.0 & 0.0 & 0.0 & 0.0 & 0.0 & 0.0 & 0.0 & 0.0 & 0.0 & 0.0 & 0.0 & 25.1 & 2.6 \\
\hline \multirow{3}{*}{$\begin{array}{l}\text { PADUCAB_UB } \\
\text { Total: }\end{array}$} & VOL & 0.0 & 0.0 & 0.0 & 0.0 & 0.0 & 0.0 & 0.0 & 0.0 & 0.0 & 0.0 & 0.0 & 0.0 & 0.0 & 0.0 & 0.0 & \multicolumn{2}{|c|}{951.4100 .0} \\
\hline & MIN & 0.0 & 0.0 & 0.0 & 0.0 & 0.0 & 0.0 & 0.0 & 0.0 & 0.0 & 0.0 & 0.0 & 0.0 & 0.0 & 0.0 & 0.0 & \multicolumn{2}{|c|}{0.0100 .0} \\
\hline & $\operatorname{MAX}$ & 0.0 & 0.0 & 0.0 & 0.0 & 0.0 & 0.0 & 0.0 & 0.0 & 0.0 & 0.0 & 0.0 & 0.0 & 0.0 & 0.0 & 0.0 & \multicolumn{2}{|c|}{951.1100 .0} \\
\hline
\end{tabular}

Was te Generator: PAST_PRAC_REM

\begin{tabular}{|c|c|c|c|c|c|c|c|c|c|c|c|c|c|c|c|c|c|c|}
\hline \multirow[t]{3}{*}{ CH_LLFE_I } & vol & 2669.4 & 1835.6 & 1154.9 & 1154.9 & 1154.9 & 1151.9 & 1151.9 & 1254.9 & 1154.9 & 0.0 & 0.0 & 0.0 & 0.0 & 0.0 & 0.0 & 17789.2 & 85.7 \\
\hline & HIN & 2669.4 & 2035.6 & 1154.9 & 1154.9 & 1154.9 & 2151.9 & 1151.9 & 1151.9 & 1154.9 & 0.0 & 0.0 & 0.0 & 0.0 & 0.0 & 0.0 & 47789.2 & 85.7 \\
\hline & $\max$ & 2669.4 & 2835.6 & 1154.9 & 1154.9 & 1151.9 & 1154.9 & 1154.9 & 1151.9 & 1154.9 & 0.0 & 0.0 & 0.0 & 0.0 & 0.0 & 0.0 & 47789.2 & 85.7 \\
\hline \multirow{3}{*}{ CH_LLME_III } & vol & 30.5 & 15.3 & 15.3 & 15.3 & 15.3 & 15.3 & 15.3 & 15.3 & 15.3 & 0.0 & 0.0 & 0.0 & 0.0 & 0.0 & 0.0 & 520.1 & 0.9 \\
\hline & MIN & 30.5 & 15.3 & 15.3 & 15.3 & 15.3 & 15.3 & 15.3 & 15.3 & 15.3 & 0.0 & 0.0 & 0.0 & 0.0 & 0.0 & 0.0 & 320.1 & 0.9 \\
\hline & $\operatorname{sen} x$ & 30.5 & 15.3 & 15.3 & 15.3 & 15.3 & 15.3 & 13.3 & 13.3 & 25.3 & 0.0 & 0.0 & 0.0 & 0.0 & 0.0 & 0.0 & 520.1 & 0.9 \\
\hline \multirow[t]{3}{*}{ CH_LLW_GTCIII } & vor & 76.4 & 76.4 & 61.1 & 61.1 & 61.1 & 61.1 & 15.8 & 45.8 & 30.5 & 30.5 & 15.3 & 15.3 & 15.3 & 0.0 & 0.0 & 1103.8 & 2.6 \\
\hline & MIN & 76.1 & 76.1 & 62.1 & 61.2 & 61.1 & 61.1 & 15.0 & 15.8 & 30.5 & 30.5 & 15.3 & 15.3 & 15.3 & 0.0 & 0.0 & 1483.8 & 2.6 \\
\hline & $\max$ & 76.1 & 76.1 & 61.1 & 61.1 & 61.1 & 61.1 & 15.8 & 15.8 & 30.5 & 30.3 & 15.3 & 13.3 & 25.3 & 0.0 & 0.0 & 1483.8 & 2.6 \\
\hline \multirow[t]{3}{*}{ CH_IRU } & VoL & 305.9 & 305.9 & 241.7 & 241.7 & 214.7 & 214.7 & 283.5 & 103.5 & 122.3 & 122.3 & $61 ! 1$ & 61.1 & 61.1 & 0.0 & 0.0 & 3935.4 & 10.6 \\
\hline & MIN & 305.9 & 305.9 & 214.7 & 244.7 & 214.7 & 211.7 & 183.5 & 183.5 & 222.3 & 122.3 & 61.1 & 61.1 & 61.1 & 0.0 . & 0.0 & 3935.1 & 10.6 \\
\hline & $\operatorname{MAX}$ & 305.9 & 305.9 & 214.7 & 244.7 & 211.7 & 214.7 & 193.5 & 183.3 & 122.3 & 122.3 & 61.1 & 61.1 & 61.1 & 0.0 & 0.0 & 5935.1 & 10.6 \\
\hline \multirow{3}{*}{$\begin{array}{l}\text { PAOT_PRAC_REM } \\
\text { Total: }\end{array}$} & VOL & 3082.1 & 2233.4 & 1476.2 & 1476.2 & 2476.2 & 1476.2 & 1399.7 & 1399.7 & 1323.2 & 152.9 & 76.4 & 76.4 & 76.4 & 0.0 & 0.0 & 55728.6 & 100.0 \\
\hline & MIN & 3082.4 & 2233.4 & 2476.2 & 1476.2 & 1476.2 & 1476.2 & 1399.7 & 1399.7 & 1323.2 & 152.9 & 76.1 & 76.4 & 76.1 & 0.0 & 0.0 & 55728.6 & 100.0 \\
\hline & MAX & 3082.1 & 2233.4 & 1476.2 & 1476.2 & 1176.2 & 1476.2 & 1399.7 & 1399.7 & 1323.2 & 152.9 & 76.1 & 76.4 & 76.4 & 0.0 & 0.0 & 55728.6 & 100.0 \\
\hline \multicolumn{19}{|c|}{ Waste Generator: PRI } \\
\hline \multirow[t]{3}{*}{$\mathrm{CH}_{-} \mathrm{LLH}_{-} \mathrm{I}$} & VOL & 566.6 & 566.6 & 366.6 & 566.6 & 566.6 & 566.6 & 566.6 & 566.6 & 566.6 & 566.6 & 566.6 & 566.6 & 566.6 & 566.6 & 366.6 & 16998.0 & 80.0 \\
\hline & MIN & 368.2 & 368.2 & 368.2 & 368.2 & 368.2 & 368.2 & 368.2 & 368.2 & 368.2 & 363.2 & 368.2 & 368.2 & 360.2 & 368.2 & 368.2 & 12465.2 & 81.6 \\
\hline & $\max$ & 623.2 & 623.2 & 623.2 & 623.2 & 623.2 & 623.2 & 623.2 & 623.2 & 623.2 & 623.2 & 623.2 & 623.2 & 623.2 & 623.2 & 623.2 & 18697.8 & 78.6 \\
\hline
\end{tabular}


WASTE GENERATOR, HASTE CLASS VOLUMES BY YEAR

(in cubic meters)

Faste Class

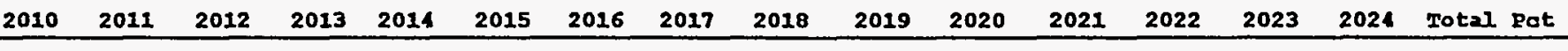

Fas to Generator: Frr

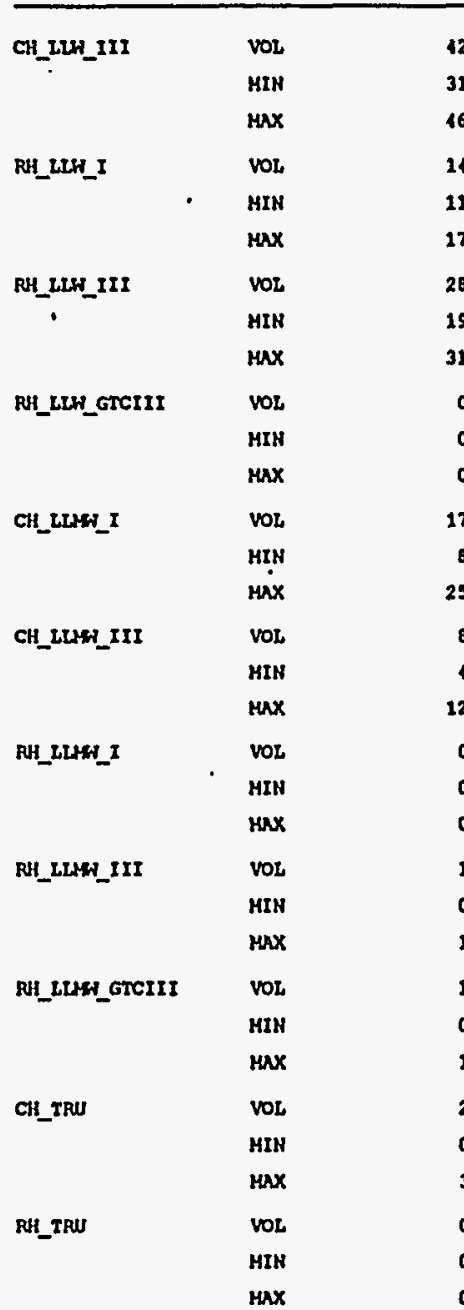

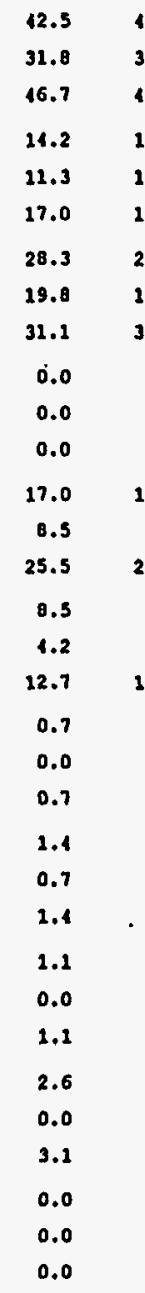

\begin{tabular}{|c|c|c|c|}
\hline 12.5 & 12.5 & 12.5 & 12.5 \\
\hline 31.8 & $31 . \dot{8}$ & 31.8 & 31.8 \\
\hline 46.7 & 16.7 & 16.7 & 16.7 \\
\hline 14.2 & 14.2 & 14.2 & 14.2 \\
\hline 11.3 & 11.3 & 11.3 & 11.3 \\
\hline 17.0 & 17.0 & 17.0 & 17.0 \\
\hline 28.3 & 28.3 & 28.3 & 20.3 \\
\hline 19.8 & 19.8 & 19.8 & 19.8 \\
\hline 31.1 & 31.1 & 31.1 & 31.1 \\
\hline 0.0 & 0.0 & 0.0 & 0.0 \\
\hline 0.0 & 0.0 & 0.0 & 0.0 \\
\hline 0.0 & 0.0 & 0.0 & 0.0 \\
\hline 17.0 & 17.0 & 17.0 & 17.0 \\
\hline 8.5 & 8.5 & 8.5 & 0.5 \\
\hline 25.5 & 25.5 & 25.5 & 25.5 \\
\hline 8.5 & 8.5 & 0.5 & 0.5 \\
\hline 1.2 & 4.2 & 4.2 & 4.2 \\
\hline 12.7 & 12.7 & 12.7 & 12.7 \\
\hline 0.7 & 0.7 & 0.7 & 0.7 \\
\hline 0.0 & 0.0 & 0.0 & 0.0 \\
\hline 0.7 & 0.7 & 0.7 & 0.7 \\
\hline 1.4 & 1.1 & 1.4 & 1.4 \\
\hline 0.7 & 0.7 & 0.7 & 0.7 \\
\hline 1.1 & 1.1 & 1.1 & 1.4 \\
\hline 1.1 & 1.1 & 1.1 & 1.1 \\
\hline 0.0 & 0.0 & 0.0 & 0.0 \\
\hline 1.1 & 1.1 & 1.1 & 1.1 \\
\hline 2.6 & 2.6 & 2.6 & 0.0 \\
\hline 0.0 & 0.0 & 0.0 & 0.0 \\
\hline 3.1 & 3.2 & $3.1^{\circ}$ & 0.0 \\
\hline 0.0 & 0.0 & 0.0 & 0.0 \\
\hline 0.0 & 0.0 & 0.0 & 0.0 \\
\hline 0.0 & 0.0 & 0.0 & 0.0 \\
\hline
\end{tabular}

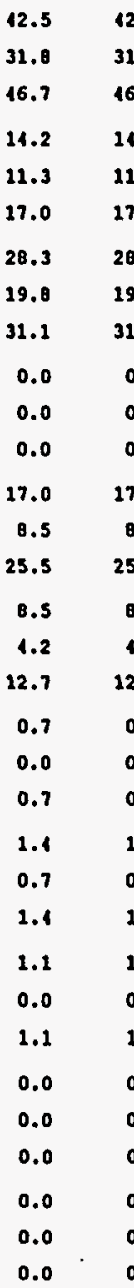

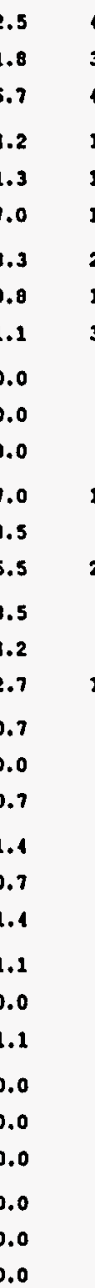

\begin{tabular}{l}
12.5 \\
31.8 \\
16.7 \\
11.2 \\
11.3 \\
17.0 \\
28.3 \\
19.8 \\
31.1 \\
0.0 \\
0.0 \\
0.0 \\
17.0 \\
0.5 \\
25.5 \\
8.5 \\
1.2 \\
22.7 \\
0.7 \\
0.0 \\
0.7 \\
1.1 \\
0.1 \\
1.1 \\
1.1 \\
0.0 \\
1.1 \\
0.0 \\
0.0 \\
0.0 \\
0.0 \\
0.0 \\
0.0 \\
\hline
\end{tabular}

$$
\begin{array}{r}
12.5 \\
31.8 \\
16.7 \\
11.2 \\
11.3 \\
17.0 \\
29.3 \\
19.8 \\
31.1 \\
0.0 \\
0.0 \\
0.0 \\
17.0 \\
8.5 \\
25.5 \\
0.5 \\
1.2 \\
12.7 \\
0.7 \\
0.0 \\
0.7 \\
1.1 \\
0.7 \\
1.1 \\
1.1 \\
0.0 \\
1.1 \\
0.0 \\
0.0 \\
0.0 \\
0.0 \\
0.0 \\
\hline
\end{array}
$$$$
11.2 \quad 14.2
$$$$
11.3
$$$$
27.0
$$$$
\begin{array}{r}
0.0 \\
17.0
\end{array}
$$$$
\begin{array}{r}
8.5 \\
25.5
\end{array}
$$$$
1.2 \quad 0.5
$$$$
1.2 \quad 1.2
$$$$
\begin{array}{r}
12.7 \\
0.7
\end{array}
$$$$
\begin{aligned}
& 0.0 \\
& 0.7
\end{aligned}
$$$$
1.1
$$$$
1.1 \quad 1.1
$$$$
1.1
$$$$
\begin{aligned}
& 0.0 \\
& 1.1
\end{aligned}
$$$$
1.1
$$$$
0.0
$$

\section{1}




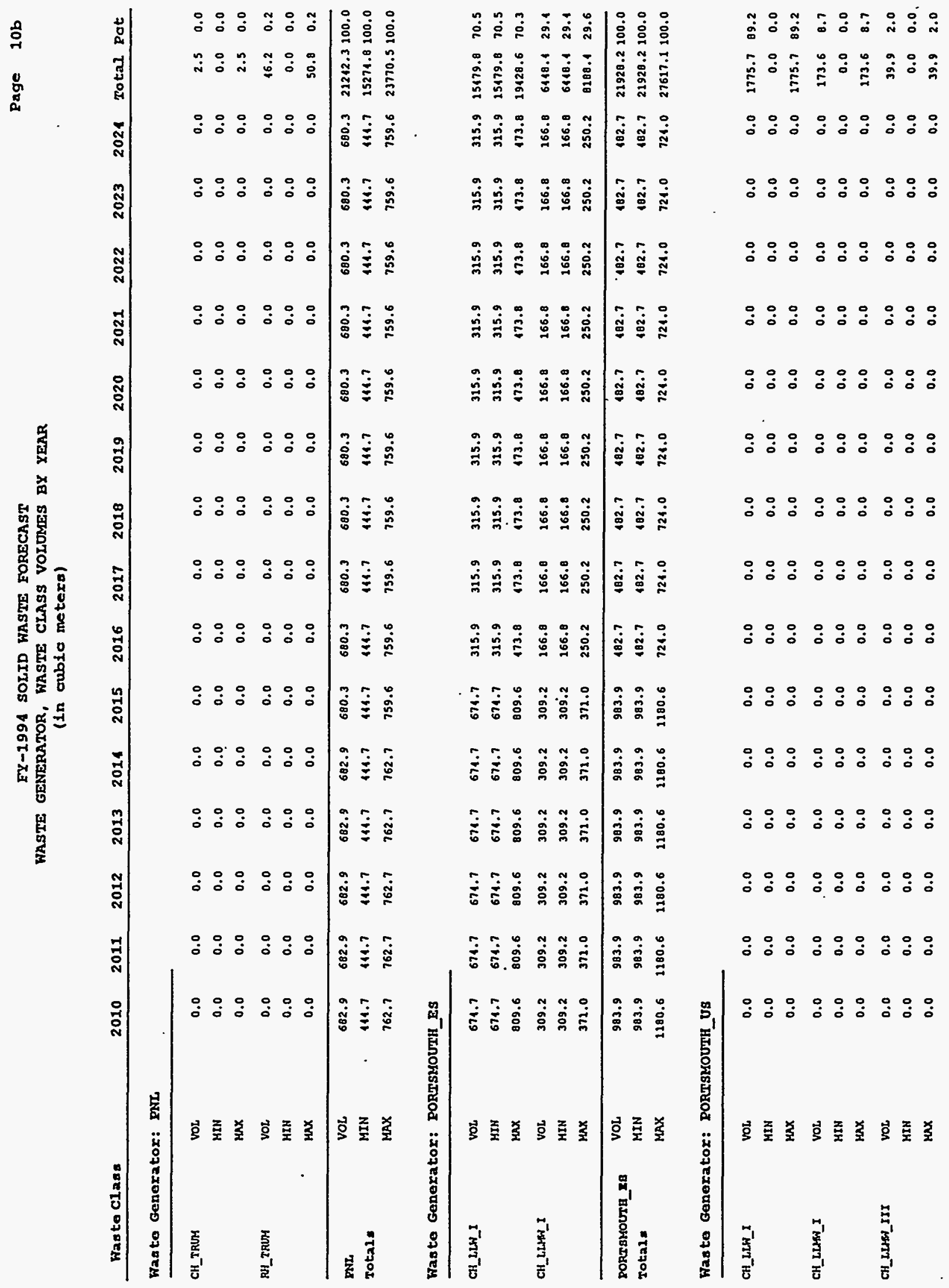


FY-1994 SOLID KASTE FORECAST

HASTE GERERATOR, HASTE CLASS VOLOMES BY YEAR

(in cublo metera)

\begin{tabular}{|c|c|c|c|c|c|c|c|c|c|c|c|c|c|c|c|c|c|}
\hline Hasto Class & & 2010 & 2011 & 2012 & 2013 & 2014 & 2015 & 2016 & 2017 & 2018 & 2019 & 2020 & 2021 & 2022 & 2023 & 2024 & Total Pot \\
\hline & vot, & 0.0 & 0.0 & 0.0 & 0.0 & 0.0 & 0.0 & 0.0 & 0.0 & 0.0 & 0.0 & 0.0 & 0.0 & 0.0 & 0.0 & 0.0 & 2989.2100 .0 \\
\hline Tota2= & MIN & 0.0 & 0.0 & 0.0 & 0.0 & 0.0 & 0.0 & 0.0 & 0.0 & 0.0 & 0.0 & 0.0 & 0.0 & 0.0 & 0.0 & 0.0 & 0.0100 .0 \\
\hline & $\operatorname{MAX}$ & 0.0 & 0.0 & 0.0 & 0.0 & 0.0 & 0.0 & 0.0 & 0.0 & 0.0 & 0.0 & 0.0 & 0.0 & 0.0 & 0.0 & 0.0 & 1989.2100 .0 \\
\hline
\end{tabular}

Faste Generator: PRnNCETON

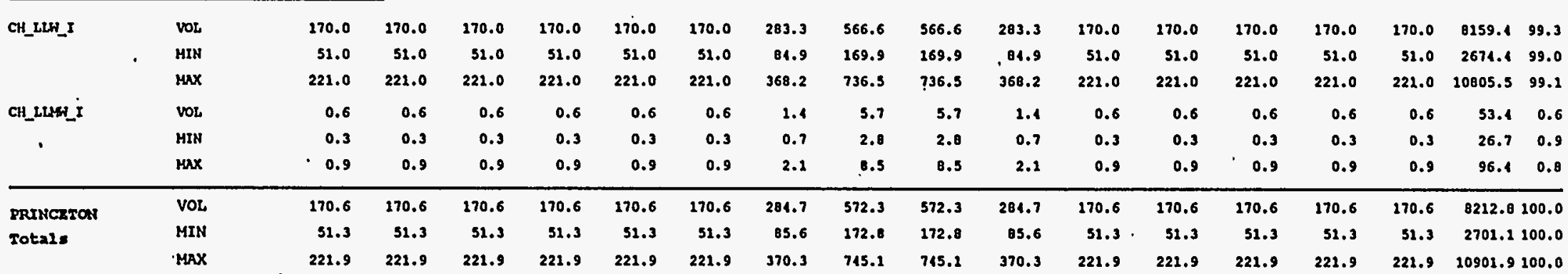

Hasto Generator: RKG_CRivoen

\begin{tabular}{|c|c|c|c|c|c|c|c|c|c|c|c|c|c|c|c|c|c|c|}
\hline \multirow[t]{3}{*}{ CH_LLW_I } & VoL & 0.0 & 0.0 & 0.0 & 0.0 & 0.0 & 0.0 & 0.0 & 0.0 & 0.0 & 0.0 & 0.0 & 0.0 & 0.0 & 0.0 & 0.0 & 1274.3 & 98.7 \\
\hline & HIM & 0.0 & 0.0 & 0.0 & 0.0 & 0.0 & 0.0 & 0.0 & 0.0 & 0.0 & 0.0 & 0.0 & 0.0 & 0.0 & 0.0 & 0.0 & 1038.9 & 100.0 \\
\hline & $\max$ & 0.0 & 0.0 & 0.0 & 0.0 & 0.0 & 0.0 & 0.0 & 0.0 & 0.0 & 0.0 & 0.0 & 0.0 & 0.0 & 0.0 & 0.0 & 1451.5 & 98.9 \\
\hline \multirow[t]{3}{*}{ CH_LLEA_I } & VoL & 0.0 & 0.0 & 0.0 & 0.0 & 0.0 & 0.0 & 0.0 & 0.0 & 0.0 & 0.0 & 0.0 & 0.0 & 0.0 & 0.0 & 0.0 & 25.8 & 1.2 \\
\hline & MIN & 0.0 & 0.0 & 0.0 & 0.0 & 0.0 & 0.0 & 0.0 & 0.0 & 0.0 & 0.0 & 0.0 & 0.0 & 0.0 & 0.0 & 0.0 & 0.0 & 0.0 \\
\hline & $\max$ & 0.0 & 0.0 & 0.0 & 0.0 & 0.0 & 0.0 & 0.0 & 0.0 & 0.0 & 0.0 & 0.0 & 0.0 & 0.0 & 0.0 & 0.0 & 15.8 & 1.0 \\
\hline \multirow{2}{*}{$\begin{array}{l}\text { Rxy_CANoan } \\
\text { Total. }\end{array}$} & vor & 0.0 & 0.0 & 0.0 & 0.0 & 0.0 & 0.0 & 0.0 & 0.0 & 0.0 & 0.0 & 0.0 & 0.0 & 0.0 & 0.0 & 0.0 & 1290.1 & 300.0 \\
\hline & MIN & 0.0 & 0.0 & 0.0 & 0.0 & 0.0 & 0.0 & 0.0 & 0.0 & 0.0 & 0.0 & 0.0 & 0.0 & 0.0 & 0.0 & 0.0 & 1038.9 & 200.0 \\
\hline
\end{tabular}

Fasto Genorator: BTANFORD

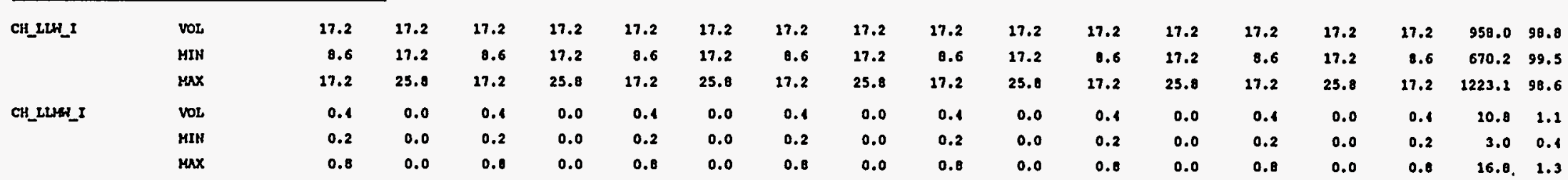


FY-1994 SOLID WASTE FORECAST

WASTE GENERATOR, WASTE CLASS VOLOMES BY YEAR

(in cublo metera)

\begin{tabular}{|c|c|c|c|c|c|c|c|c|c|c|c|c|c|c|c|c|c|}
\hline Wasto & & 2010 & 2011 & 2012 & 2013 & 2014 & 2015 & 2016 & 2017 & 2018 & 2019 & 2020 & 2021 & 2022 & 2023 & 2024 & Total Pot \\
\hline \multirow{3}{*}{$\begin{array}{l}\text { BTANEORD } \\
\text { Total. }\end{array}$} & VOL & 17.6 & 17.2 & 27.6 & 17.2 & 17.6 & 17.2 & 17.6 & 17.2 & 17.6 & 17.2 & 17.6 & 27.2 & 27.6 & 17.2 & 17.6 & 968.8100 .0 \\
\hline & MIN & 8.8 & 17.2 & B. $\theta$ & 17.2 & 8.8 & 17.2 & 0.8 & 17.2 & $\theta .0$ & 27.2 & 8.8 & 27.2 & 8.8 & 17.2 & 8.8 & 673.2100 .0 \\
\hline & $\max$ & 10.0 & 25.0 & 18.0 & 25.8 & 18.0 & 25.8 & 18.0 & 25.0 & 10.0 & 25.8 & 18.0 & 25.0 & 10.0 & 25.8 & 18.0 & $1239,9100.0$ \\
\hline
\end{tabular}

Was to Generator: TPNR_2312

\begin{tabular}{|c|c|c|c|c|c|c|c|c|c|c|c|c|c|c|c|c|c|c|c|}
\hline & CH_LLW_I & Vot & 0.0 & 0.0 & 0.0 & 0.3 & 0.3 & 0.3 & 0.3 & 0.3 & 0.3 & 0.3 & 0.3 & 0.3 & 0.3 & 0.0 & 0.0 & 3.8 & 10.0 \\
\hline & & MIN & 0.0 & 0.0 & 0.0 & 0.3 & 0.3 & 0.3 & 0.3 & 0.3 & 0.3 & 0.3 & 0.3 & 0.3 & 0.3 & 0.0 & 0.0 & 3.8 & 10.0 \\
\hline & & $\max$ & 0.0 & 0.0 & 0.0 & 0.3 & 0.3 & 0.3 & 0.3 & 0.3 & 0.3 & 0.3 & 0.3 & 0.3 & 0.3 & 0.0 & 0.0 & 3.8 & 10.0 \\
\hline & $\mathrm{CH}_{\text {_TRU }}$ & VoL & 0.0 & 0.0 & 0.0 & 3.2 & 3.2 & 3.2 & 3.2 & 3.2 & 3.2 & 3.2 & 3.2 & 3.2 & 3.2 & 0.0 & 0.0 & 32.0 & 84.6 \\
\hline & ' & HIN & 0.0 & 0.0 & 0.0 & 3.2 & 3.2 & 3.2 & 3.2 & 3.2 & 3.2 & 3.2 & 3.2 & .3 .2 & 3.2 & 0.0 & 0.0 & 32,0 & 84.6 \\
\hline & & $\max$ & 0.0 & 0.0 & 0.0 & 3.2 & 3.2 & 3.2 & 3.2 & 3.2 & 3.2 & 3.2 & 3.2 & 3.2 & 3.2 & 0.0 & 0.0 & 32,0 & 81.6 \\
\hline$i$ & $\mathrm{CH}_{-}$TRUH & SOL & 0.0 & 0.0 & 0.0 & 0.2 & 0.2 & 0.2 & 0.2 & 0.2 & 0.2 & 0.2 & 0.2 & 0.2 & 0.2 & 0.0 & 0.0 & 2.0 & 5.2 \\
\hline & & MIN & 0.0 & 0.0 & 0.0 & 0.2 & 0.2 & 0.2 & 0.2 & 0.2 & 0.2 & 0.2 & 0.2 & 0.2 & 0.2 & 0.0 & 0.0 & 2.0 & 5.2 \\
\hline & & $\max$ & 0.0 & 0.0 & 0.0 & 0.2 & 0.2 & 0.2 & 0.2 & 0.2 & 0.2 & 0.2 & 0.2 & 0.2 & 0.2 & 0.0 & 0.0 & 2.0 & 5.2 \\
\hline & $\operatorname{TrnL} 2322$ & VOL & 0.0 & 0.0 & 0.0 & 3.7 & 3.7 & 3.7 & 3.7 & 3.7 & 3.7 & 3.7 & $3 . ?$ & 3.7 & 3.7 & 0.0 & 0.0 & 37.8 & 100,0 \\
\hline & Total= & MIN & 0.0 & 0.0 & 0.0 & 3.7 & 3.7 & 3.7 & 3.7 & 3.7 & 3.7 & 3.7 & 3.7 & 3.7 & 3.7 & 0.0 & 0.0 & 37.8 & 100.0 \\
\hline & & $\max$ & 0.0 & 0.0 & 0.0 & 3.7 & 3.7 & 3.7 & 3.7 & 3.7 & 3.7 & 3.7 & 3.7 & 3.7 & 3.7 & 0.0 & 0.0 & 37.8 & 100.0 \\
\hline
\end{tabular}

Waste Generator: TPNL_242B/BL

\begin{tabular}{|c|c|c|c|c|c|c|c|c|c|c|c|c|c|c|c|c|c|}
\hline \multirow[t]{3}{*}{ CH_LLW_I } & vor & 0.0 & 0.0 & 0.0 & 9.6 & 9.6 & 9.6 & 9.6 & 9.6 & 9.6 & 9.6 & 9.6 & 9.6 & 9.6 & 0.0 & 0.0 & 96.0100 .0 \\
\hline & MIN & 0.0 & 0.0 & 0.0 & 9.6 & 9.6 & 9.6 & 9.6 & 9.6 & 9.6 & 9.6 & 9.6 & 9.6 & 9.6 & 0.0 & 0.0 & $96.0 \quad 100.0$ \\
\hline & $\max$ & 0.0 & 0.0 & 0.0 & 9.6 & 9.6 & 9.6 & 9.6 & 9.6 & 9.6 & 9.6 & 9.6 & 9.6 & 9.6 & 0.0 & 0.0 & 96.0100 .0 \\
\hline \multirow{3}{*}{$\begin{array}{l}\text { TPNI_2128/BI } \\
\text { 2otal. }\end{array}$} & VOL & 0.0 & 0.0 & 0.0 & 9.6 & 9.6 & 9.6 & 9.6 & 9.6 & 9.6 & 9.6 & 9.6 & 9.6 & 9.6 & 0.0 & 0.0 & 96.0100 .0 \\
\hline & MIN & 0.0 & 0.0 & 0.0 & 9.6 & 9.6 & 9.6 & 9.6 & 9.6 & 9.6 & 9.6 & 9.6 & 9.6 & 9.6 & 0.0 & 0.0 & 96.0100 .0 \\
\hline & $\operatorname{MAX}$ & 0.0 & 0.0 & 0.0 & 9.6 & 9.6 & 9.6 & 9.6 & 9.6 & 9.6 & 9.6 & 9.6 & 9.6 & 9.6 & 0.0 & 0.0 & 96.0100 .0 \\
\hline
\end{tabular}

Waste Generztor: TPNL_30

\begin{tabular}{|c|c|c|c|c|c|c|c|c|c|c|c|c|c|c|c|c|c|}
\hline \multirow[t]{3}{*}{$\mathrm{CH}_{-} \mathrm{LLH}_{-} \mathrm{I}$} & voL & 0.0 & 0.0 & 0.0 & 5.1 & 5.1 & 5.1 & 5.1 & 5.1 & 5.1 & 5.1 & 3.1 & 5.1 & 5.1 & 0.0 & 0.0 & $51.0 \quad 100.0$ \\
\hline & HIN & 0.0 & 0.0 & 0.0 & 5.1 & 5.1 & 5.1 & 5.1 & 3.1 & 5.1 & 5.1 & 5.1 & 5.1 & 3.1 & 0.0 & 0.0 & 51.0100 .0 \\
\hline & $\max$ & 0.0 & 0.0 & 0.0 & 5.1 & 5.1 & 5.1 & 5.1 & 5.2 & 5.1 & 5.1 & 5.1 & 5.1 & 5.1 & 0.0 & 0.0 & 51.0100 .0 \\
\hline
\end{tabular}


FY-1994 SOLID WASTE FORECAST

WASTE GENERATOR, WASTE CLASS VOLUMES BY YEAR

$$
\text { (in cubic meters) }
$$

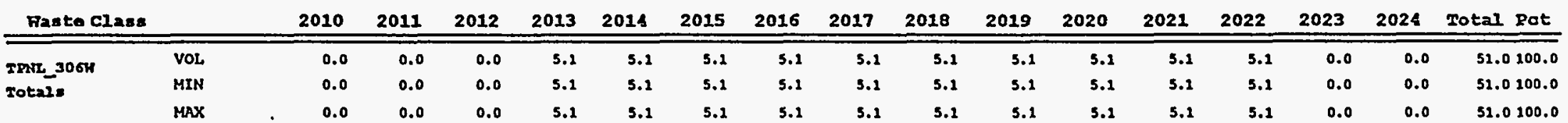

Wasto Genorator: TPNL_314

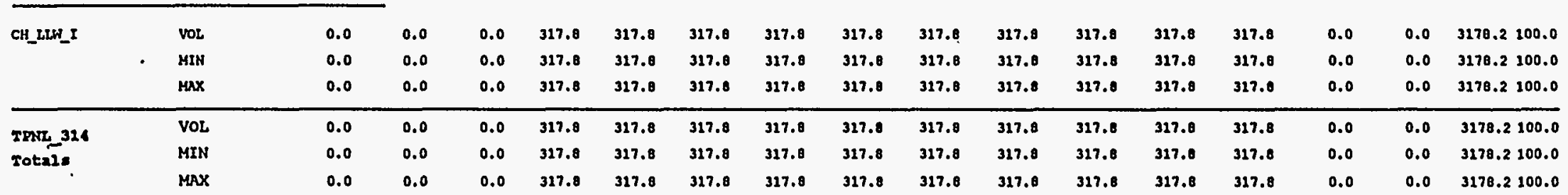

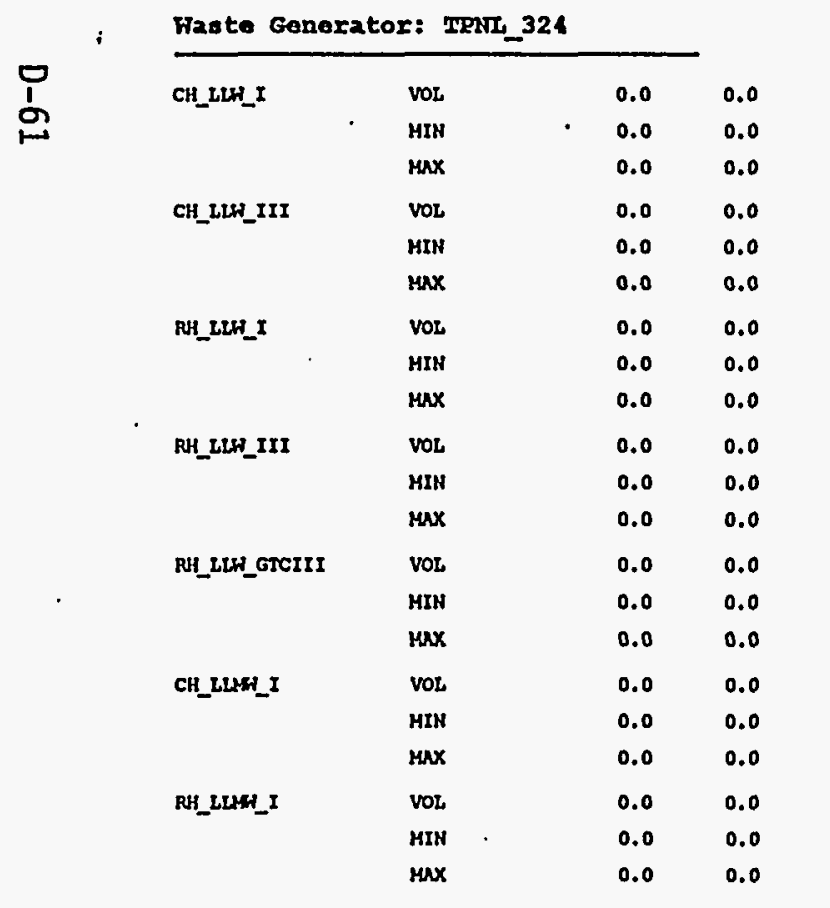

$\begin{array}{rrrrrrrrrrrrrrr}0.0 & 35.0 & 35.0 & 35.0 & 35.0 & 35.0 & 35.0 & 35.0 & 35.0 & 35.0 & 35.0 & 0.0 & 0.0 & 350.1 & 12.9 \\ 0.0 & 35.0 & 35.0 & 35.0 & 35.0 & 35.0 & 35.0 & 35.0 & 35.0 & 35.0 & 35.0 & 0.0 & 0.0 & 350.1 & 12.9 \\ 0.0 & 35.0 & 35.0 & 35.0 & 35.0 & 35.0 & 35.0 & 35.0 & 35.0 & 35.0 & 35.0 & 0.0 & 0.0 & 350.1 & 12.9 \\ 0.0 & 0.9 & 0.9 & 0.9 & 0.9 & 0.9 & 0.9 & 0.9 & 0.9 & 0.9 & 0.9 & 0.0 & 0.0 & 9.7 & 1.1 \\ 0.0 & 0.9 & 0.9 & 0.9 & 0.9 & 0.9 & 0.9 & 0.9 & 0.9 & 0.9 & 0.9 & 0.0 & 0.0 & 9.7 & 1.1 \\ 0.0 & 0.9 & 0.9 & 0.9 & 0.9 & 0.9 & 0.9 & 0.9 & 0.9 & 0.9 & 0.9 & 0.0 & 0.0 & 9.7 & 1.1 \\ 0.0 & 1.1 & 1.1 & 1.1 & 1.2 & 1.1 & 1.1 & 1.1 & 1.1 & 1.1 & 1.1 & 0.0 & 0.0 & 11.0 & 1.4 \\ 0.0 & 1.1 & 1.1 & 1.1 & 1.1 & 1.1 & 1.1 & 1.1 & 1.1 & 1.1 & 1.1 & 0.0 & 0.0 & 11.0 & 1.1 \\ 0.0 & 1.1 & 1.1 & 1.1 & 1.1 & 1.1 & 1.1 & 1.1 & 1.2 & 1.1 & 1.1 & 0.0 & 0.0 & 11.0 & 1.1 \\ 0.0 & 1.3 & 1.3 & 1.3 & 1.3 & 1.3 & 1.3 & 1.3 & 1.3 & 1.3 & 1.3 & 0.0 & 0.0 & 13.0 & 1.6 \\ 0.0 & 1.3 & 1.3 & 1.3 & 1.3 & 1.3 & 1.3 & 1.3 & 1.3 & 1.3 & 1.3 & 0.0 & 0.0 & 13.8 & 1.6 \\ 0.0 & 1.3 & 1.3 & 1.3 & 1.3 & 1.3 & 1.3 & 1.3 & 1.3 & 1.3 & 1.3 & 0.0 & 0.0 & 13.0 & 1.6 \\ 0.0 & 0.1 & 0.1 & 0.1 & 0.1 & 0.1 & 0.1 & 0.1 & 0.1 & 0.1 & 0.1 & 0.0 & 0.0 & 1.0 & 0.1 \\ 0.0 & 0.1 & 0.1 & 0.1 & 0.1 & 0.1 & 0.1 & 0.1 & 0.1 & 0.1 & 0.1 & 0.0 & 0.0 & 1.0 & 0.1 \\ 0.0 & 0.1 & 0.1 & 0.1 & 0.1 & 0.4 & 0.1 & 0.4 & 0.1 & 0.1 & 0.1 & 0.0 & 0.0 & 1.0 & 0.1 \\ 0.0 & 0.3 & 0.3 & 0.3 & 0.3 & 0.3 & 0.3 & 0.3 & 0.3 & 0.3 & 0.3 & 0.0 & 0.0 & 3.0 & 0.3 \\ 0.0 & 0.3 & 0.3 & 0.3 & 0.3 & 0.3 & 0.3 & 0.3 & 0.3 & 0.3 & 0.3 & 0.0 & 0.0 & 3.0 & 0.3 \\ 0.0 & 0.3 & 0.3 & 0.3 & 0.3 & 0.3 & 0.3 & 0.3 & 0.3 & 0.3 & 0.3 & 0.0 & 0.0 & 3.0 & 0.3 \\ 0.0 & 21.2 & 21.2 & 21.2 & 21.2 & 21.2 & 21.2 & 21.2 & 21.2 & 21.2 & 21.2 & 0.0 & 0.0 & 212.0 & 25.9 \\ 0.0 & 21.2 & 21.2 & 21.2 & 21.2 & 21.2 & 21.2 & 21.2 & 21.2 & 21.2 & 21.2 & 0.0 & 0.0 & 212.0 & 25.9 \\ 0.0 & 21.2 & 21.2 & 21.2 & 21.2 & 21.2 & 21.2 & 21.2 & 21.2 & 21.2 & 21.2 & 0.0 & 0.0 & 212.0 & 25.9\end{array}$




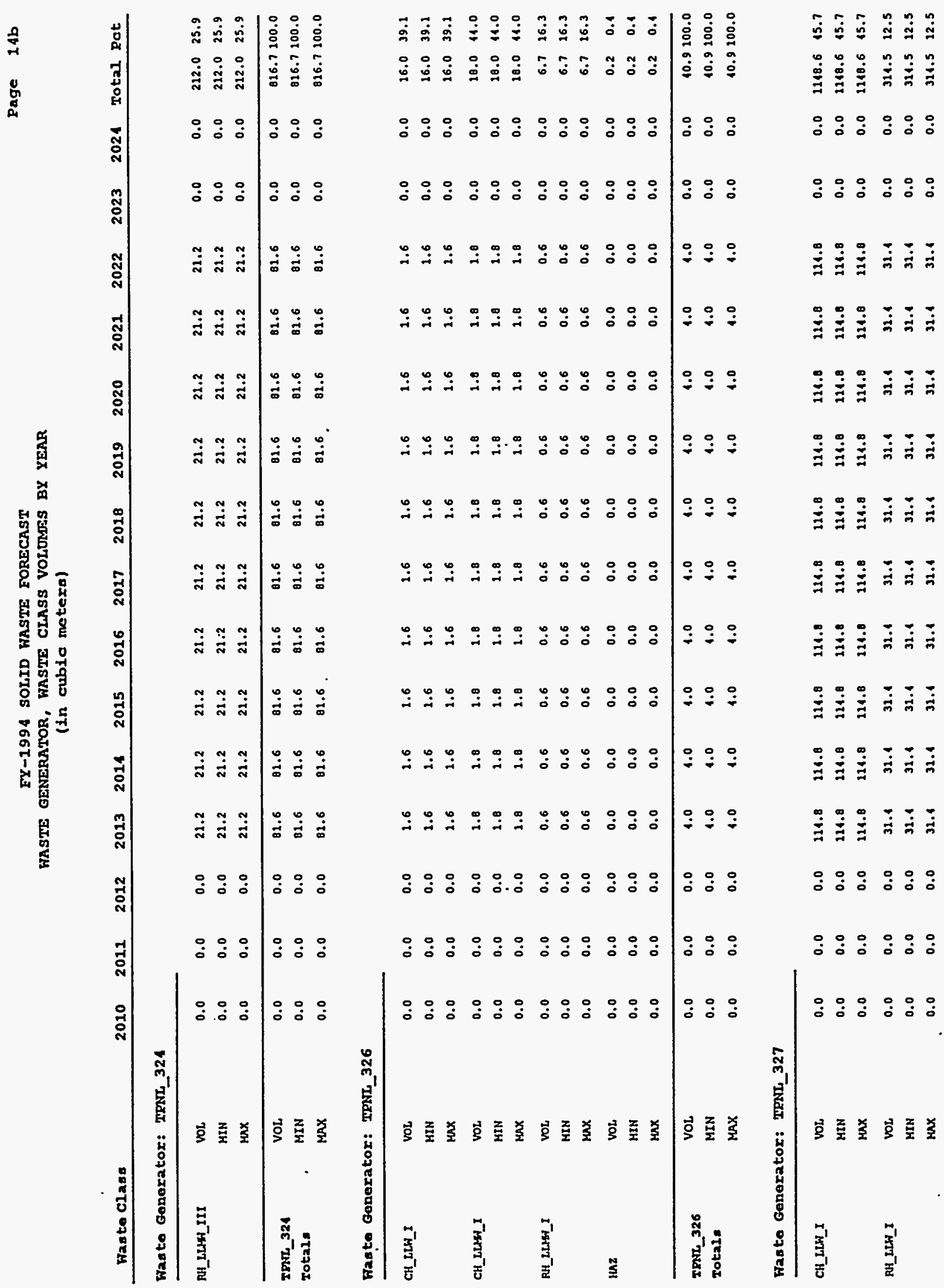


FY-1994 SOLID WASTE FORECAST

WASTE GENERATOR, HASTE CLASS VOLOMES BY YEAR

(in cubic metera)

Waste class

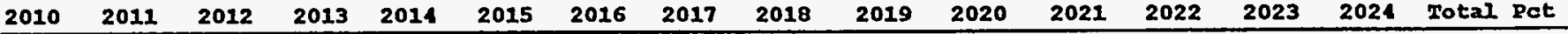

Wasto Generator: TP2 327

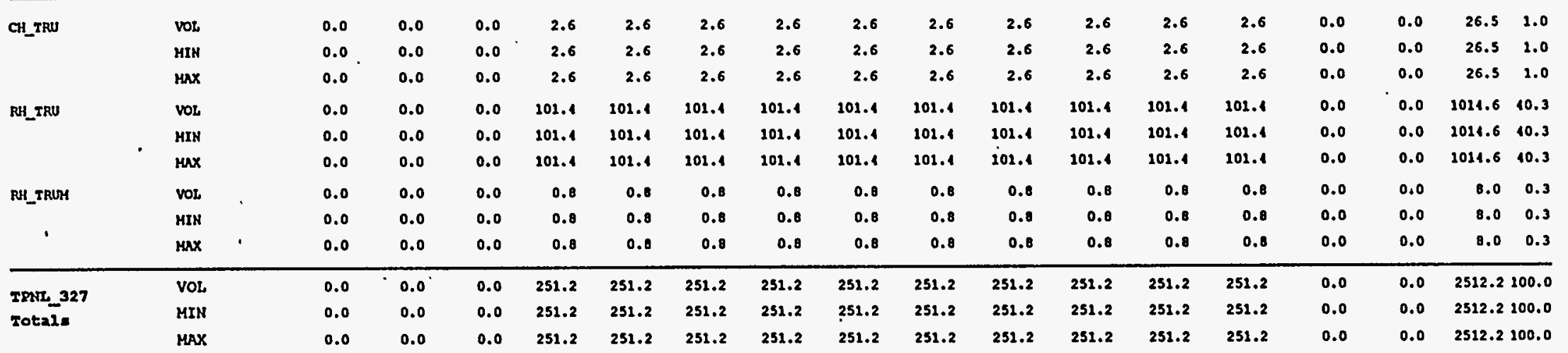

Was te Generator: MPNL_331

\begin{tabular}{|c|c|c|c|c|c|c|c|c|c|c|c|c|c|c|c|c|c|}
\hline \multirow[t]{3}{*}{ CH_LLH_I } & vor & 0.0 & 0.0 & 0.0 & 190.8 & 190.8 & 290.8 & 190.8 & 290.8 & 290.0 & 290.0 & 290.8 & 190.8 & 190.8 & 0.0 & 0.0 & 1908.0100 .0 \\
\hline & HIN & 0.0 & 0.0 & 0.0 & 190.0 & 290.0 & 190.8 & 190.0 & 190.8 & 190.8 & 190.8 & 190.8 & 190.8 & 290.8 & 0.0 & 0.0 & 1908.0100 .0 \\
\hline & $\max$ & 0.0 & 0.0 & 0.0 & 190.8 & 190.8 & 190.8 & 290.0 & 190:0 & 190.8 & 190.8 & 190.8 & 190.8 & 190.8 & 0.0 & 0.0 & 1908.0100 .0 \\
\hline \multirow{3}{*}{$\begin{array}{l}\text { TRIr__331 } \\
\text { Totald }\end{array}$} & VOL & 0.0 & 0.0 & 0.0 & 290.0 & 190.0 & 190.8 & 190.8 & 190.8 & 190.0 & 190.8 & 190.0 & 190.8 & 290.8 & 0.0 & 0.0 & 1908.0100 .0 \\
\hline & MIN & 0.0 & 0.0 & 0.0 & 190.0 & 190.8 & 190.8 & 190.0 & 190.8 & 190.8 & 190.8 & 190.8 & 190.8 & 190.8 & 0.0 & .0 .0 & 1908.0100 .0 \\
\hline & $\operatorname{Max}$ & 0.0 & 0.0 & 0.0 & 190.0 & 190.8 & 190.8 & 190.8 & 190.8 & 190.8 & 190.8 & 190.8 & 190.8 & 190.8 & 0.0 & 0.0 & 1908.0100 .0 \\
\hline
\end{tabular}

Fasto Generator: IFHC_100N

\begin{tabular}{|c|c|c|c|c|c|c|c|c|c|c|c|c|c|c|c|c|c|c|}
\hline \multirow[t]{3}{*}{ CH_LLH_I } & VOL & 0.0 & 0.0 & 0.0 & 832.6 & 832.6 & 832.6 & 832.6 & 832.6 & 832.6 & 832.6 & 832.6 & 832.6 & 832.6 & 0.0 & 0.0 & 8326.1 & 87.5 \\
\hline & HIN & 0.0 & 0.0 & 0.0 & 832.6 & 832.6 & 032.6 & 832.6 & 832.6 & 832.6 & 832.6 & 832.6 & 032.6 & 832.6 & 0.0 & 0.0 & 0326.1 & 07.5 \\
\hline & HNX & 0.0 & 0.0 & 0.0 & 832.6 & 832.6 & 832.6 & 832.6 & 832.6 & 832.6 & B32.6 & 832.6 & 832.6 & 832.6 & 0.0 & 0.0 & 0326.1 & 87.5 \\
\hline \multirow[t]{3}{*}{ RH_TRU } & VoL & 0.0 & 0.0 & 0.0 & 118.5 & 118.5 & 118.5 & 110.5 & 118.5 & 118.5 & 118.5 & 118.5 & 118.5 & 118.5 & 0.0 & 0.0 & 1185.0 & 12.4 \\
\hline & MIN & 0.0 & 0.0 & 0.0 & 120.5 & 118.5 & 118.5 & 218.5 & 118.5 & 218.5 & 118.5 & 118.5 & 116.5 & 218.5 & 0.0 & 0.0 & 1185.8 & 12.4 \\
\hline & $\max$ & 0.0 & 0.0 & 0.0 & 128.5 & 118.5 & 210.5 & 218.5 & 128.5 & 118.5 & 118.5 & 118.5 & 218.5 & 118.5 & 0.0 & 0.0 & 1385.8 & 12.4 \\
\hline \multirow{3}{*}{$\begin{array}{l}\text { THEC_10ON } \\
\text { Total. }\end{array}$} & VOL & 0.0 & 0.0 & 0.0 & 951.1 & 951.1 & 951.1 & 951.1 & 951.1 & 951.1 & 951.1 & 951.1 & 951.1 & 951.1 & 0.0 & 0.0 & 9311.9 & 100.0 \\
\hline & MIN & 0.0 & 0.0 & 0.0 & 951.2 & 951.1 & 951.1 & 951.1 & 951.1 & 951.1 & 951.1 & 951.1 & 951.1 & 951.1 & 0.0 & 0.0 & 9511.9 & 100.0 \\
\hline & $\operatorname{MAX}$ & 0.0 & 0.0 & 0.0 & 951.1 & 951.1 & 951.1 & 951.1 & 951.1 & 951.1 & 951.1 & 951.1 & 931.1 & 951.1 & 0.0 & 0.0 & 9511.9 & 100.0 \\
\hline
\end{tabular}


WASTE GENERATOR, WASTE CLASS VOLUMES BY YEAR

(in cubio meters)

Haste Class

\begin{tabular}{llllllllllllllll}
2010 & 2011 & 2012 & 2013 & 2014 & 2015 & 2016 & 2017 & 2018 & 2019 & 2020 & 2021 & 2022 & 2023 & 2024 & Total Pct \\
\hline
\end{tabular} Wasto Genorator: IFHC_2228

\begin{tabular}{|c|c|c|c|c|c|c|c|c|c|c|c|c|c|c|c|c|c|c|}
\hline \multirow[t]{3}{*}{ CH_LLW_I } & Vow & 0.0 & 0.0 & 0.0 & 1280.2 & $12 \theta 0.2$ & 1280.2 & 1280.2 & 1280.2 & 1280.2 & 1280.2 & 1280.2 & 1200.2 & 1280.2 & 0.0 & 0.0 & 12802.7 & 98.8 \\
\hline & HIN & 0.0 & 0.0 & 0.0 & 1280.2 & 1280.2 & 1200.2 & 1280.2 & 1280.2 & 1280.2 & 1200.2 & 1280.2 & 2200.2 & 1280.2 & 0.0 & 0.0 & 12802.7 & 98.8 \\
\hline & $\operatorname{mAX}$ & 0.0 & 0.0 & 0.0 & 1280.2 & 1280.2 & 1280.2 & $12 \theta 0.2$ & 1280.2 & 1280.2 & 1280.2 & 1280.2 & 1280.2 & 1280.2 & 0.0 & 0.0 & 12802.7 & 98.8 \\
\hline \multirow[t]{3}{*}{ CH_LLNA_I } & vol. & 0.0 & 0.0 & 0.0 & 3.6 & 3.6 & 3.6 & 3.6 & 3.6 & 3.6 & 3.6 & 3.6 & 3.6 & 3.6 & 0.0 & 0.0 & 36.3 & 0.2 \\
\hline & HIN & 0.0 & 0.0 & 0.0 & 3.6 & 3.6 & 3.6 & 3.6 & 3.6 & 3.6 & 3.6 & 3.6 & 3.6 & 3.6 & 0.0 & 0.0 & 36.3 & 0.2 \\
\hline & $\max$ & 0.0 & 0.0 & 0.0 & 3.6 & 3.6 & 3.6 & 3.6 & 3.6 & 3.6 & 3.6 & 3.6 & 3.6 & 3.6 & 0.0 & 0.0 & 36.3 & 0.2 \\
\hline \multirow[t]{3}{*}{ CH_LLHE_GTCTII } & VoL & 0.0 & 0.0 & 0.0 & 0.5 & 0.5 & 0.5 & 0.5 & 0.5 & 0.5 & 0.5 & 0.5 & 0.5 & 0.5 & 0.0 & 0.0 & 5.0 & 0.0 \\
\hline & HIN & 0.0 & 0.0 & 0.0 & 0.5 & 0.5 & 0.5 & 0.5 & 0.5 & 0.5 & 0.5 & 0.5 & 0.5 & 0.5 & 0.0 & 0.0 & 5.0 & 0.0 \\
\hline & $\max$ & 0.0 & 0.0 & 0.0 & 0.5 & 0.5 & 0.5 & 0.5 & 0.5 & 0.5 & 0.5 & 0.5 & 0.5 & 0.5 & 0.0 & 0.0 & 5.0 & 0.0 \\
\hline \multirow[t]{3}{*}{ RH_LLWH_GTCIII } & vor & 0.0 & 0.0 & 0.0 & 10.7 & 10.7 & 10.7 & 10.7 & 10.7 & 10.7 & 10.7 & 10.7 & 10.7 & 10.7 & 0.0 & 0.0 & 107.1 & 0.0 \\
\hline & MIM & 0.0 & 0.0 & 0.0 & 10.7 & 10.7 & 10.7 & 10.7 & 10.7 & 10.7 & 10.7 & 10.7 & 10.7 & 10.7 & 0.0 & 0.0 & 107.4 & 0.8 \\
\hline & $\max$ & 0.0 & 0.0 & 0.0 & 10.7 & 10.7 & 10.7 & 10.7 & 10.7 & 10.7 & 10.7 & 20.7 & 10.7 & 20.7 & 0.0 & 0.0 & 107.4 & 0.8 \\
\hline \multirow{3}{*}{$\begin{array}{l}\text { THIL_222B } \\
\text { Totals }\end{array}$} & VOL & 0.0 & 0.0 & 0.0 & 1295.1 & 1295.1 & 2295.1 & 1295.1 & 1295.1 & 1295.1 & 1295.1 & 1293.1 & 1295.1 & 1295.1 & 0.0 & 0.0 & 12951.4 & 100.0 \\
\hline & MIN & 0.0 & 0.0 & 0.0 & 1295.1 & 1295.1 & 1295.1 & 1295.1 & 1295.1 & 1295.1 & 1295.1 & 1295.1 & 1295.1 & 1295.1 & 0.0 & 0.0 & 12951.4 & 100.0 \\
\hline & $\operatorname{MAX}$ & 0.0 & 0.0 & 0.0 & 1295.1 & 1295.1 & 1295.1 & 2295.1 & 1295.1 & 1295.1 & 1295.2 & 1295.1 & 1295.1 & 1295.1 & 0.0 & 0.0 & 12951.4 & 200.0 \\
\hline
\end{tabular}

Waste Gonerator: Twitc_242X

\begin{tabular}{|c|c|c|c|c|c|c|c|c|c|c|c|c|c|c|c|c|c|c|c|}
\hline \multirow[t]{3}{*}{ CH_LLAH_I } & vor & & 0.0 & 0.0 & 0.0 & 20.3 & 20.3 & 20.3 & 20.3 & 20.3 & 20.3 & 20.3 & 20.3 & 20.3 & 20.3 & 0.0 & 0.0 & 203.7 & 58.5 \\
\hline & MIN & & 0.0 & 0.0 & 0.0 & 20.3 & 20.3 & 20.3 & 20.3 & 20.3 & 20.3 & 20.3 & 20.3 & 20.3 & 20.3 & 0.0 & 0.0 & 203.7 & 50.5 \\
\hline & $\boldsymbol{m a x}$ & & 0.0 & 0.0 & 0.0 & 20.3 & 20.3 & 20.3 & $20.3^{\prime}$ & 20.3 & 20.3 & 20.3 & 20.3 & 20.3 & 20.3 & 0.0 & 0.0 & 203.7 & 58.5 \\
\hline \multirow[t]{2}{*}{ CH_LLWW_III } & VOL & & 0.0 & 0.0 & 0.0 & 12.2 & 12.2 & 22.2 & 12.2 & 12.2 & 12.2 & 12.2 & 12.2 & 12.2 & 12.2 & 0.0 & 0.0 & 122.4 & 35.8 \\
\hline & $\operatorname{man}$ & $\cdot$ & 0.0 & 0.0 & 0.0 & 12.2 & 12.2 & 12.2 & 12.2 & 12.2 & 12.2 & 12.2 & 12.2 & 12.2 & 12.2 & 0.0 & 0.0 & 222.4 & 35.1 \\
\hline \multirow[t]{3}{*}{ RH_LLHW_GTCIII } & vol & & 0.0 & 0.0 & 0.0 & 2.2 & 2.2 & 2.2 & 2.2 & 2.2 & 2.2 & 2.2 & 2.2 & 2.2 & 2.2 & 0.0 & 0.0 & 22.0 & 6.3 \\
\hline & MIN & & 0.0 & 0.0 & 0.0 & 2.2 & 2.2 & 2.2 & 2.2 & 2.2 & 2.2 & 2.2 & 2.2 & 2.2 & 2.2 & 0.0 & 0.0 & 22.0 & 6.3 \\
\hline & $\max$ & & 0.0 & 0.0 & 0.0 & 2.2 & 2.2 & 2.2 & 2.2 & 2.2 & 2.2 & 2.2 & 2.2 & 2.2 & 2.2 & 0.0 & 0.0 & 22.0 & 6. \\
\hline \multirow{3}{*}{$\begin{array}{l}\text { IFRC_242A } \\
\text { Jotal. }\end{array}$} & vol & & 0.0 & 0.0 & 0.0 & 34.8 & 34.8 & 34.8 & 34.0 & 34.0 & 34.8 & 34.8 & 34.8 & 34.8 & 34.8 & 0.0 & 0.0 & 348.1 & 100.0 \\
\hline & MIN & & 0.0 & 0.0 & 0.0 & 34.8 & 34.0 & 34.8 & 34.0 & 31.8 & 34.8 & 34.8 & 34.8 & 34.8 & 34.8 & 0.0 & 0.0 & 348.1 & 100.0 \\
\hline & $\operatorname{MAX}$ & & 0.0 & 0.0 & 0.0 & 34.8 & 34.8 & 34.8 & 31.8 & 34.8 & 31.8 & 34.8 & 36.8 & 34.0 & 34.8 & 0.0 & 0.0 & 348.1 & 100. \\
\hline
\end{tabular}


Faste Clase

$\begin{array}{lllllllllllllllll}2010 & 2011 & 2012 & 2013 & 2014 & 2015 & 2016 & 2017 & 2018 & 2019 & 2020 & 2021 & 2022 & 2023 & 2024 & \text { Total Pct }\end{array}$

\begin{tabular}{|c|c|c|c|c|c|c|c|c|c|c|c|c|c|c|c|c|c|c|c|}
\hline \multirow[t]{3}{*}{ CH_LLWE_I } & vor & & 0.0 & 0.0 & 0.0 & 20.3 & 20.3 & 20.3 & 20.3 & 20.3 & 20.3 & 20.3 & 20.3 & 20.3 & 20.3 & 0.0 & 0.0 & 203.7 & 38.5 \\
\hline & MIN & & 0.0 & 0.0 & 0.0 & 20.3 & 20.3 & 20.3 & 20.3 & 20.3 & 20.3 & 20.3 & 20.3 & 20.3 & 20.3 & 0.0 & 0.0 & 203.7 & 58.5 \\
\hline & $\max$ & & 0.0 & 0.0 & 0.0 & 20.3 & 20.3 & 20.3 & 20.3 & 20.3 & 20.3 & 20.3 & 20.3 & 20.3 & 20.3 & 0.0 & 0.0 & 203.7 & 58.5 \\
\hline \multirow[t]{3}{*}{ CH_LLWE_III } & vor & & 0.0 & 0.0 & 0.0 & 12.2 & 12.2 & 12.2 & 12.2 & 22.2 & 12.2 & 12.2 & 12.2 & 12.2 & 12.2 & 0.0 & 0.0 & 122.4 & 35.1 \\
\hline & MIN & & 0.0 & 0.0 & 0.0 & 12.2 & 22.2 & 12.2 & 12.2 & 12.2 & 12.2 & 12.2 & 12.2 & 12.2 & 12.2 & 0.0 & 0.0 & 122.4 & 35.1 \\
\hline & $\operatorname{Max}$ & & 0.0 & 0.0 & 0.0 & 12.2 & 22.2 & 12.2 & 12.2 & 12.2 & 12.2 & 12.2 & 12.2 & 12.2 & 12.2 & 0.0 & 0.0 & 122.4 & 35.1 \\
\hline \multirow[t]{2}{*}{ RH_LLAS_GTCIII } & vol & & 0.0 & 0.0 & 0.0 & 2.2 & 2.2 & 2.2 & 2.2 & 2.2 & 2.2 & 2.2 & 2.2 & 2.2 & 2.2 & 0.0 & 0.0 & 22.0 & 6.3 \\
\hline & $\operatorname{Mxx}$ & . & 0.0 & 0.0 & 0.0 & 2.2 & 2.2 & 2.2 & 2.2 & 2.2 & 2.2 & 2.2 & 2.2 & 2.2 & 2.2 & 0.0 & 0.0 & 22.0 & 6.3 \\
\hline \multirow{3}{*}{$\begin{array}{l}\text { TWBC } 2128 \\
\text { Tot:21: }\end{array}$} & VoL & & 0.0 & 0.0 & 0.0 & 34.0 & 34.8 & 34.8 & 34.0 & 34.8 & 34.8 & 34.8 & 34.8 & 31.0 & 34.8 & 0.0 & 0.0 & 318.1 & \\
\hline & MIN & & 0.0 & 0.0 & 0.0 & 34.8 & 34.8 & 34.8 & 34.0 & 36.8 & 34.8 & 34.8 & 34.8 & 34.8 & 34.8 & 0.0 & 0.0 & 348.1 & \\
\hline & $\operatorname{MAX}$ & & 0.0 & 0.0 & 0.0 & 34.0 & 34.8 & 36.8 & 34.8 & 34.8 & 34.8 & 34.8 & 34.8 & 34.8 & 31.8 & 0.0 & 0.0 & 318.1 & 100. \\
\hline
\end{tabular}

\section{Waste Gonerator: TwhC_242T}

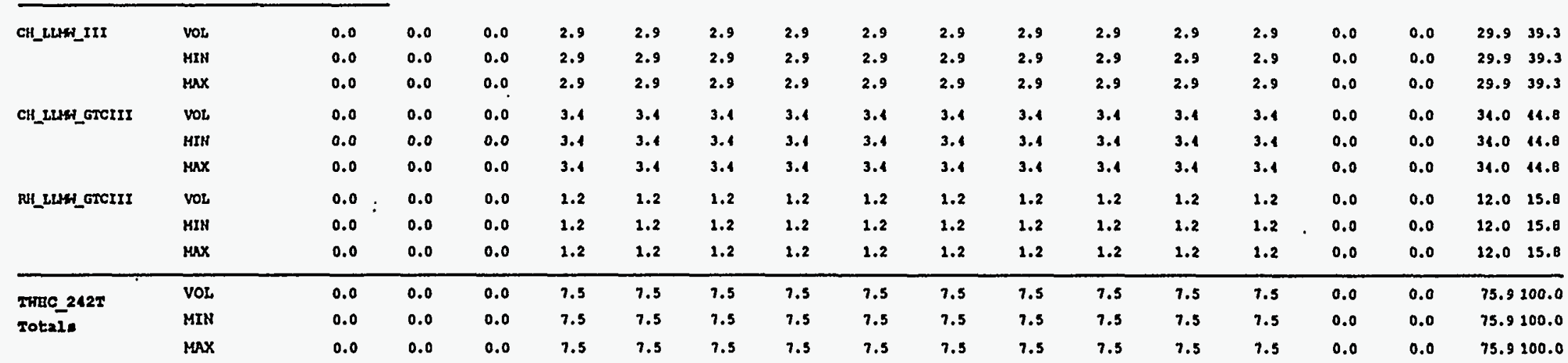

Wasto Generator: MFHC_306

\begin{tabular}{|c|c|c|c|c|c|c|c|c|c|c|c|c|c|c|c|c|c|}
\hline \multirow{3}{*}{ CH_LLW_I } & VoL & 0.0 & 0.0 & 0.0 & 0.5 & 0.5 & 0.5 & 0.5 & 0.5 & 0.5 & 0.5 & 0.5 & 0.5 & 0.5 & 0.0 & 0.0 & 3.1 \\
\hline & MIN & 0.0 & 0.0 & 0.0 & 0.5 & 0.5 & 0.5 & 0.5 & 0.5 & 0.5 & 0.5 & 0.5 & 0.5 & 0.5 & 0.0 & 0.0 & 3.1 \\
\hline & $\max$ & 0.0 & 0.0 & 0.0 & 0.5 & 0.5 & 0.5 & 0.5 & 0.5 & 0.5 & 0.5 & 0.5 & 0.5 & 0.5 & 0.0 & 0.0 & 5.4 \\
\hline
\end{tabular}


Fasto Class

$\begin{array}{lllllllllllllllll}2010 & 2011 & 2012 & 2013 & 2014 & 2015 & 2016 & 2017 & 2018 & 2019 & 2020 & 2021 & 2022 & 2023 & 2024 & \text { Total Pot }\end{array}$ Waste Generator: THHC_306

\begin{tabular}{|c|c|c|c|c|c|c|c|c|c|c|c|c|c|c|c|c|c|c|}
\hline \multirow[t]{3}{*}{ HAZ } & VoL & 0.0 & 0.0 & 0.0 & 0.1 & 0.4 & 0.4 & 0.4 & 0.1 & 0.4 & 0.1 & 0.4 & 0.1 & 0.1 & 0.0 & 0.0 & 1.0 & 12.5 \\
\hline & MIN & 0.0 & 0.0 & 0.0 & 0.1 & 0.1 & 0.1 & 0.1 & 0.1 & 0.1 & 0.1 & 0.1 & 0.1 & 0.1 & 0.0 & 0.0 & 1.0 & 12.5 \\
\hline & $\max$ & 0.0 & 0.0 & 0.0 & 0.1 & 0.1 & 0.4 & 0.1 & 0.4 & 0.1 & 0.4 & 0.4 & 0.4 & 0.4 & 0.0 & 0.0 & 1.0 & 42.5 \\
\hline \multirow{3}{*}{$\begin{array}{l}\text { TFBC_306 } \\
\text { Totals }\end{array}$} & VOL. & 0.0 & 0.0 & 0.0 & 0.9 & 0.9 & 0.9 & 0.9 & 0.9 & 0.9 & 0.9 & 0.9 & 0.9 & 0.9 & 0.0 & 0.0 & & 100.0 \\
\hline & MIN & 0.0 & 0.0 & 0.0 & 0.9 & 0.9 & 0.9 & 0.9 & 0.9 & 0.9 & 0.9 & 0.9 & 0.9 & 0.9 & 0.0 & 0.0 & 9.1 & 100.0 \\
\hline & MAX & 0.0 & 0.0 & 0.0 & 0.9 & 0.9 & 0.9 & $0 . \dot{9}$ & 0.9 & 0.9 & 0.9 & 0.9 & 0.9 & 0.9 & 0.0 & 0.0 & 9.1 & 200.0 \\
\hline
\end{tabular}

Waste Generator: THIC_308

\begin{tabular}{|c|c|c|c|c|c|c|c|c|c|c|c|c|c|c|c|c|c|c|c|}
\hline \multirow{6}{*}{$\begin{array}{l}\text { p } \\
\text { ó }\end{array}$} & \multirow[t]{3}{*}{ CH_LLH_I } & vor. & 0.0 & 0.0 & 0.0 & 12.0 & 12.8 & 12.8 & 12.8 & 12.8 & i2.8 & 12.8 & 12.8 & 12.8 & 12.8 & 0.0 & 0.0 & 128.7 & 41.5 \\
\hline & & MIH & 0.0 & 0.0 & 0.0 & 12.8 & 12.0 & 12.0 & 12.0 & 12.8 & 12.8 & 12.8 & 12.8 & 12.8 & 12.8 & 0.0 & 0.0 & 128.7 & 11.5 \\
\hline & & $\max$ & 0.0 & 0.0 & 0.0 & 12.8 & 12.8 & 12.0 & 12.8 & 12.8 & 12.0 & 22.8 & 12.8 & 12.8 & 12.8 & 0.0 & 0.0 & 128.7 & 11.5 \\
\hline & \multirow[t]{3}{*}{ CH_IRU } & Vor & 0.0 & 0.0 & 0.0 & 9.0 & 9.0 & 9.0 & 9.0 & 9.0 & 9.0 & 9.0 & 9.0 & 9.0 & 9.0 & 0.0 & 0.0 & 90.0 & 31.1 \\
\hline & & MIN & 0.0 & 0.0 & 0.0 & 9.0 & 9.0 & 9.0 & 9.0 & 9.0 & 9.0 & 9.0 & 9.0 & 9.0 & 9.0 & 0.0 & 0.0 & 90.0 & 31.1 \\
\hline & & $\max$ & 0.0 & 0.0 & 0.0 & 9.0 & 9.0 & 9.0 & 9.0 & 9.0 & 9.0 & 9.0 & 9.0 & 9.0 & 9.0 & 0.0 & 0.0 & 90.0 & 31.1 \\
\hline & \multirow[t]{3}{*}{ HAZ } & vor & 0.0 & 0.0 & 0.0 & 7.0 & 7.0 & 7.0 & 7.0 & 7.0 & 7.0 & 7.0 & 7.0 & 7.0 & 7.0 & 0.0 & 0.0 & 70.0 & 24.2 \\
\hline & & MIN & 0.0 & 0.0 & 0.0 & 7.0 & 7.0 & 7.0 & 7.0 & 7.0 & 7.0 & 7.0 & 7.0 & 7.0 & 7.0 & 0.0 & 0.0 & 70.0 & 21.2 \\
\hline & & $\max$ & 0.0 & 0.0 & 0.0 & 7.0 & 7.0 & 7.0 & 7.0 & 7.0 & 1.0 & 7.0 & 7.0 & 7.0 & 1.0 & 0.0 & 0.0 & 70.0 & 21.2 \\
\hline & \multirow{3}{*}{$\begin{array}{l}\text { THBC_308 } \\
\text { Total. }\end{array}$} & VoL & 0.0 & 0.0 & 0.0 & 28.8 & 28.8 & 28.8 & 28.8 & 28.8 & 28.8 & 28.8 & 28.8 & 28.8 & 28.8 & 0.0 & 0.0 & 288.7 & 100.0 \\
\hline & & MIN & 0.0 & 0.0 & 0.0 & 28.8 & 28.8 & 28.8 & 28.8 & 28.8 & 28.8 & 28.8 & 28.8 & 28.8 & 28.0 & 0.0 & 0.0 & 288.7 & 100.0 \\
\hline & & $\operatorname{Max}$ & 0.0 & 0.0 & 0.0 & 28.8 & 28.8 & 28.8 & 28.8 & 28.0 & 28.8 & 28.8 & 28.8 & 28.8 & 28.8 & 0.0 & 0.0 & 288.7 & 200.0 \\
\hline
\end{tabular}

Fas te Gonerator: ThHC_310

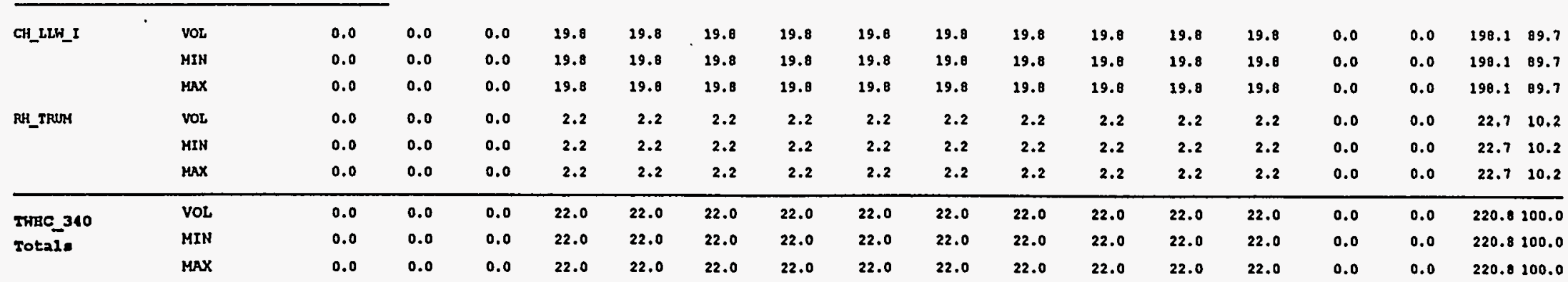


FY-1994 SOLID WASTE FORECAST

WASTE GENERATOR, FASTE CLASS VOLUMES BY YEAR

(In cubio meters)

Fas to Class 2010

\section{Waste Generator: TWHC_BPLANT}

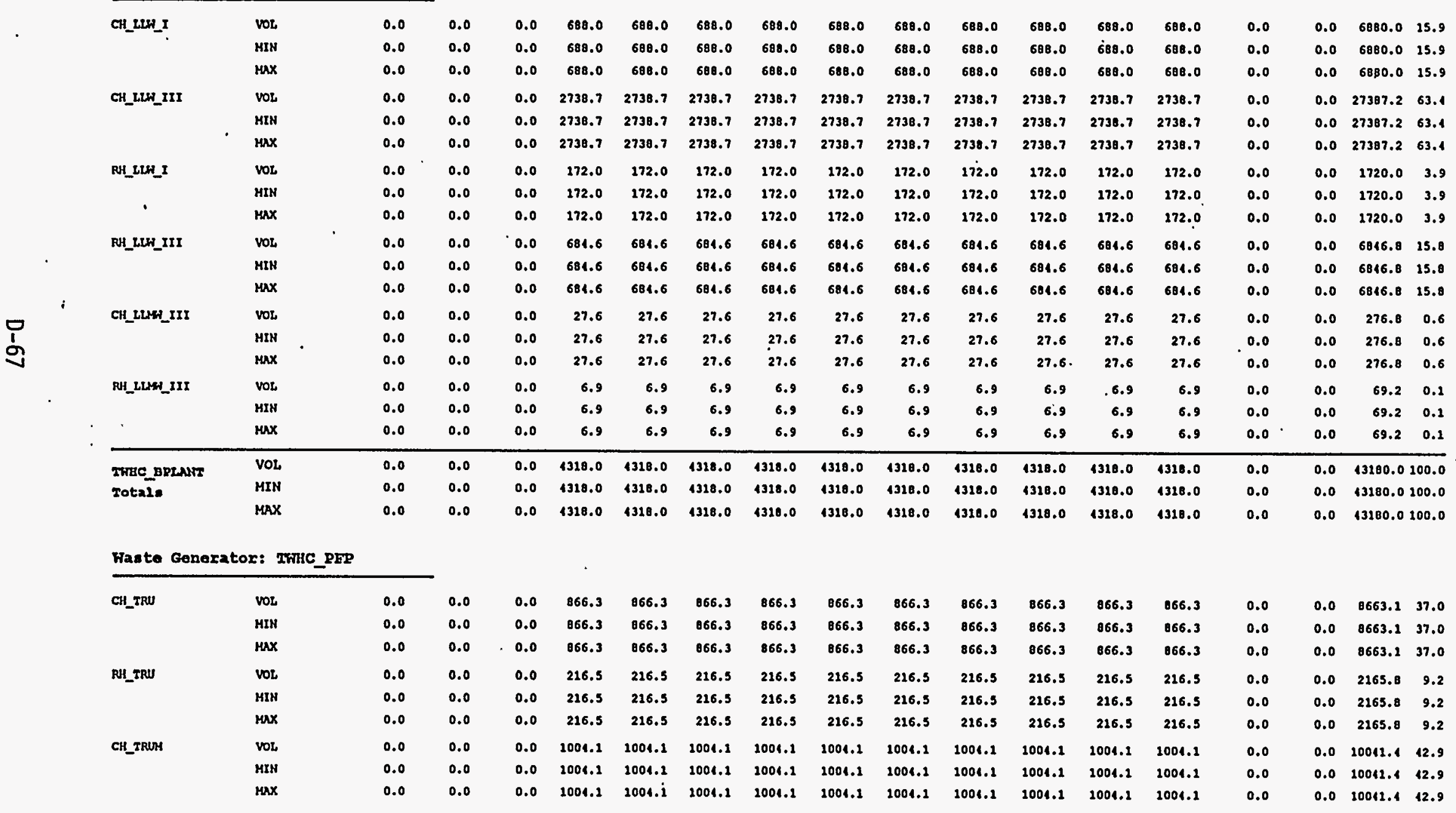


FY-1994 SOLID WASTE EORECAST

Wasto Gonerator: TWHC_PFP

\begin{tabular}{|c|c|c|c|c|c|c|c|c|c|c|c|c|c|c|c|c|c|c|}
\hline \multirow[t]{3}{*}{ RH_TRUM } & Vor & 0.0 & 0.0 & 0.0 & 251.0 & 251.0 & 251.0 & 251.0 & 251.0 & 252.0 & 251.0 & 251.0 & 251.0 & 251.0 & 0.0 & 0.0 & 2510.4 & 10.7 \\
\hline & MIN & 0.0 & 0.0 & 0.0 & 251.0 & 251.0 & 251.0 & 251.0 & 251.0 & 251.0 & 251.0 & 251.0 & 251.0 & 251.0 & 0.0 & 0.0 & 2510.4 & 20.7 \\
\hline & $\operatorname{Hax}$ & 0.0 & 0.0 & 0.0 & 251.0 & 251.0 & 251.0 & 251.0 & 251.0 & 231.0 & 251.0 & 251.0 & 251.0 & 251.0 & 0.0 & 0.0 & 2510.4 & 10.7 \\
\hline \multirow{3}{*}{$\begin{array}{l}\text { TWRE_PFP } \\
\text { Totals }\end{array}$} & VOL & 0.0 & 0.0 & 0.0 & 2338.0 & 2338.0 & 2338.0 & 2338.0 & 2338.0 & 2338.0 & 2338.0 & 2338.0 & 2338.0 & 2338.0 & 0.0 & 0.0 & 23380.7 & 100.0 \\
\hline & MIN & 0.0 & 0.0 & 0.0 & 2338.0 & 2338.0 & 2338.0 & 2338.0 & 2339.0 & 2338.0 & 2338.0 & 2338.0 & 2338.0 & 2338.0 & 0.0 & 0.0 & 23380.7 & 100.0 \\
\hline & $\operatorname{MAX}$ & 0.0 & 0.0 & 0.0 & 2338.0 & 2338.0 & 2338.0 & 2338.0 & 2338.0 & 2338.0 & 2338.0 & 2338.0 & 2338.0 & 2330.0 & 0.0 & 0.0 & 23380.7 & 100.0 \\
\hline
\end{tabular}

Waste Generator: TWHC_PUREX

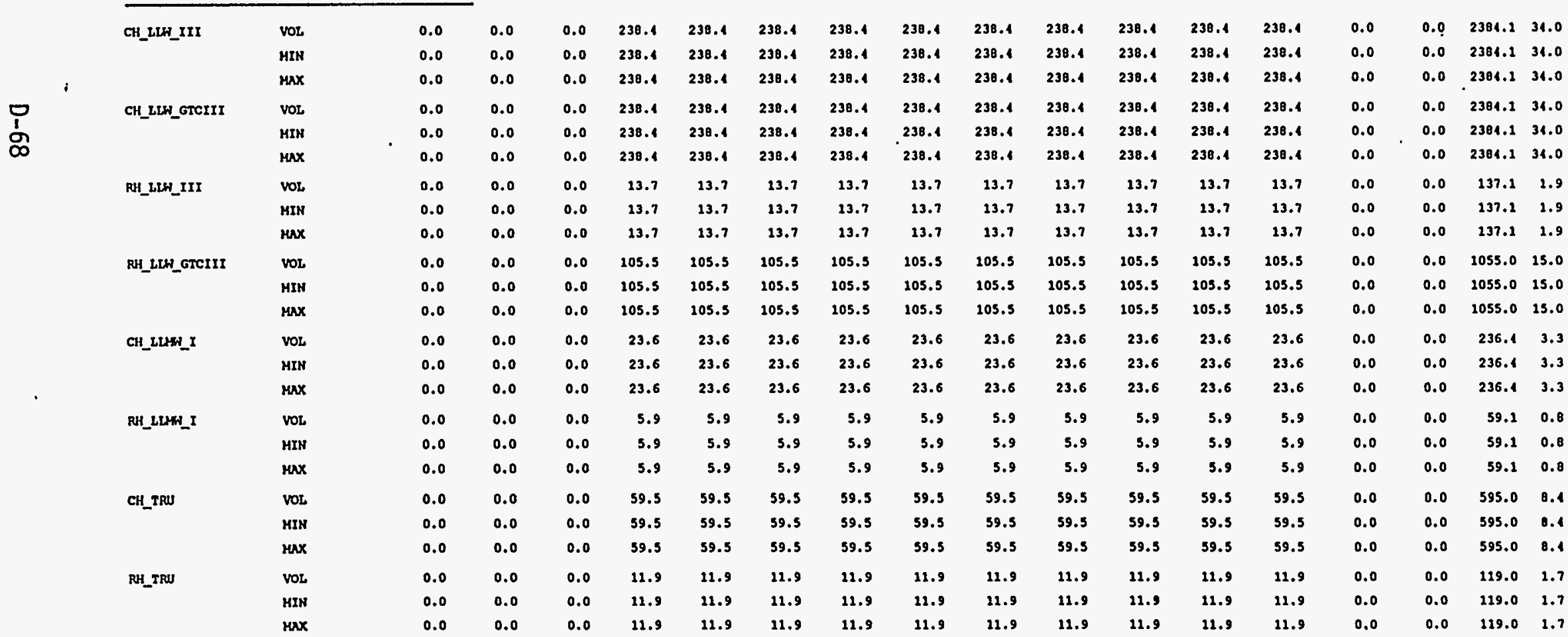




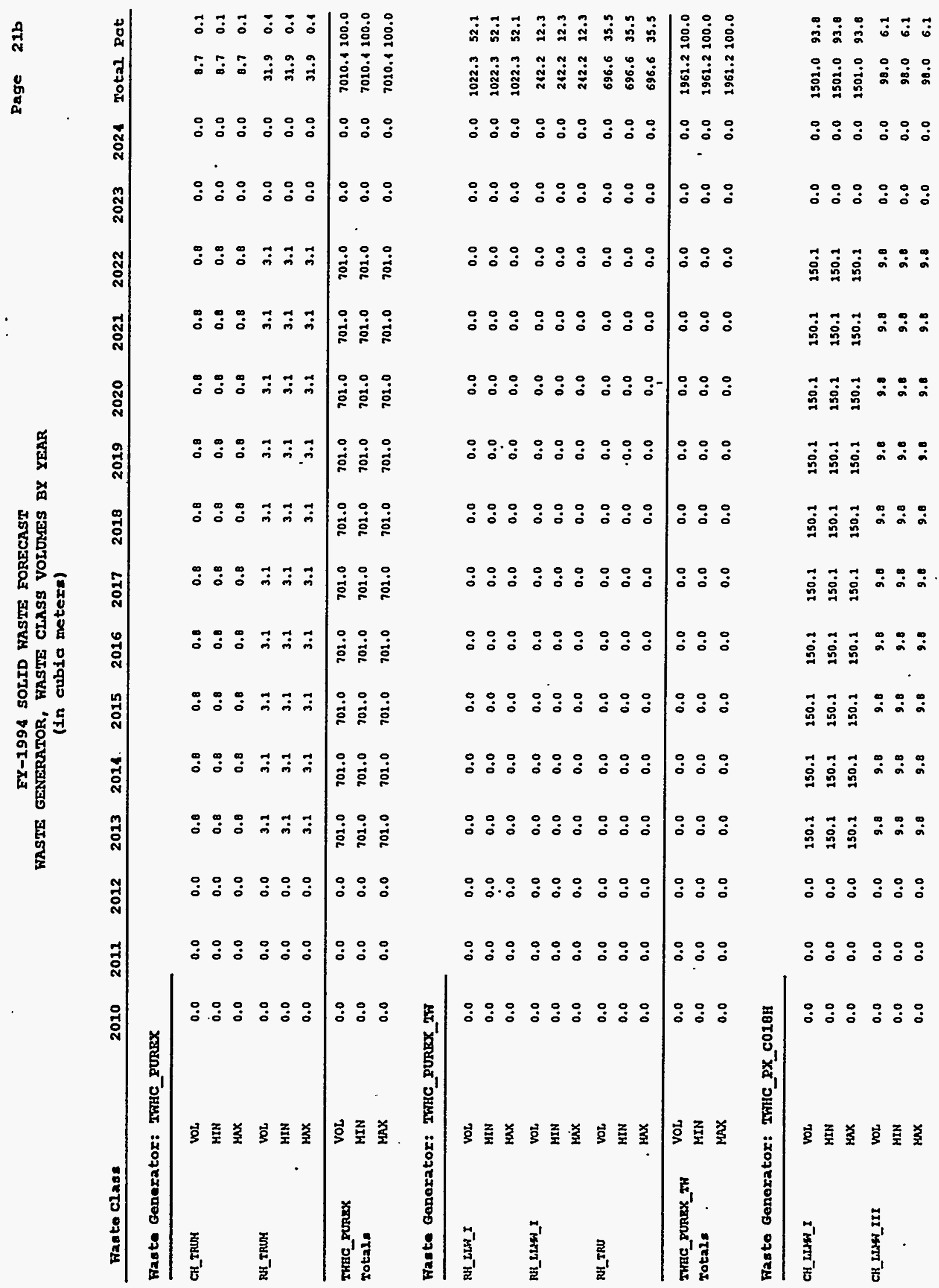


FY-1994 SOLID WASTE FORECAST

WASTE GENERATOR, WASTE CLASS VOLUMES BY YEAR

(in cublo meters)

Waste Class

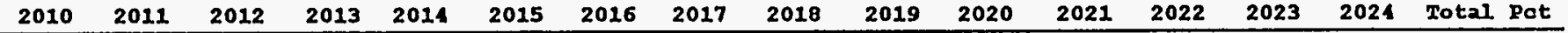

\begin{tabular}{|c|c|c|c|c|c|c|c|c|c|c|c|c|c|c|c|c|c|}
\hline \multirow{3}{*}{$\begin{array}{l}\text { THRC_PX_CO20B } \\
\text { Tot21: }\end{array}$} & VOL & 0.0 & 0.0 & 0.0 & 159.9 & 159.9 & 159.9 & 159.9 & 159.9 & 159.9 & 159.9 & 159.9 & 159.9 & 159.9 & 0.0 & 0.0 & 1599.0100 .0 \\
\hline & MIN & 0.0 & 0.0 & 0.0 & 159.9 & 159.9 & 159.9 & 139.9 & 159.9 & 159.9 & 159.9 & 159.9 & 159.9 & 139.9 & 0.0 & 0.0 & 1599.0100 .0 \\
\hline & $\operatorname{MAX}$ & 0.0 & 0.0 & 0.0 & 159.9 & 159.9 & 159.9 & 159.9 & 159.9 & 159.9 & 159.9 & 159.9 & 159.9 & 159.9 & 0.0 & 0.0 & 1599.0100 .0 \\
\hline
\end{tabular}

Waste Generator: TWHC_TPLANT

\begin{tabular}{|c|c|c|c|c|c|c|c|c|c|c|c|c|c|c|c|c|c|c|}
\hline \multirow[t]{3}{*}{ CH_LLW_I } & voL & 0.0 & 0.0 & 0.0 & 714.4 & 714.4 & 714.4 & 711.1 & 714.4 & 714.4 & 714.1 & 711.4 & 714.4 & 714.4 & 0.0 & 0.0 & 7114.1 & 35.3 \\
\hline & HIN & 0.0 & 0.0 & 0.0 & 711.1 & 714.4 & 711.4 & 714.1 & 714.1 & 714.1 & 714.1 & 721.1 & 734.1 & 714.1 & 0.0 & 0.0 & 7114.1 & 35.3 \\
\hline & $\max$ & 0.0 & 0.0 & 0.0 & 714.4 & 714.4 & 714.4 & 714.1 & 714.4 & 714.1 & 714.4 & 714.4 & 734.1 & 714.4 & 0.0 & 0.0 & 7144.1 & 35.3 \\
\hline \multirow{2}{*}{ PH_LLK_I } & vor & 0.0 & 0.0 & 0.0 & 178.6 & 178.6 & 278.6 & 170.6 & 178.6 & 178.6 & 170.6 & 178.6 & 278.6 & 178.6 & 0.0 & 0.0 & 2786.0 & 8.8 \\
\hline & $\max$ & 0.0 & 0.0 & 0.0 & 178.6 & 178.6 & 178.6 & 270.6 & 278.6 & 278.6 & 178.6 & 178.6 & 278.6 & 178.6 & 0.0 & 0.0 & 1786.0 & 0.8 \\
\hline \multirow[t]{3}{*}{ CH_LLHE__III } & vot & 0.0 & 0.0 & 0.0 & 362.5 & 362.5 & 362.5 & 362.5 & 362.5 & 362.5 & 362.5 & 362.5 & 362.5 & 362.5 & 0.0 & 0.0 & 3625.6 & 17.9 \\
\hline & HIN & 0.0 & 0.0 & 0.0 & 362.5 & 362.5 & 362.5 & 362.5 & 362.5 & 362.5 & 362.5 & 362.5 & 362.3 & 362.5 & 0.0 & 0.0 & 3625.6 & 17.9 \\
\hline & $\max$ & .0 .0 & 0.0 & 0.0 & 362.5 & 362.5 & 362.5 & 362.5 & 362.5 & 362.5 & 362.5 & 362.5 & 362.5 & 362.5 & 0.0 & 0.0 & 3625.6 & 17.9 \\
\hline \multirow[t]{3}{*}{ CH_LLWW_GTCTII } & vor & 0.0 & 0.0 & 0.0 & 101.2 & 181.2 & 181.2 & 101.2 & 181.2 & 181.2 & 181.2 & 181.2 & 181.2 & 181.2 & 0.0 & 0.0 & 1012.8 & 0.9 \\
\hline & MIN & $0.0^{\circ}$ & 0.0 & 0.0 & 182.2 & 101.2 & 181.2 & 181.2 & 101.2 & 181.2 & 181.2 & 101.2 & 181.2 & 181.2 & 0.0 & 0.0 & 1812.8 & 8.9 \\
\hline & $\operatorname{mux}$ & 0.0 & 0.0 & 0.0 & 181.2 & 181.2 & 183.2 & 181.2 & 181.2 & 101.2 & 101.2 & 101.2 & 181.2 & 101.2 & 0.0 & 0.0 & 1812.8 & 8.9 \\
\hline \multirow[t]{3}{*}{ RH_LLAW_III } & vol. & 0.0 & 0.0 & 0.0 & 90.6 & 90.6 & 90.6 & 90.6 & 90.6 & 90.6 & 90.6 & 90.6 & 90.6 & 90.6 & 0.0 & 0.0 & 906.4 & 4.1 \\
\hline & MrN & 0.0 & 0.0 & 0.0 & 90.6 & 90.6 & 90.6 & 90.6 & 90.6 & 90.6 & 90.6 & 90.6 & 90.6 & 90.6 & 0.0 & 0.0 & 906.4 & 4.1 \\
\hline & $\max$ & 0.0 & 0.0 & .0 .0 & 90.6 & 90.6 & 90.6 & 90.6 & 90.6 & 90.6 & 90.6 & 90.6 & 90.6 & 90.6 & 0.0 & 0.0 & 906.4 & 4.4 \\
\hline \multirow[t]{3}{*}{ PH_LLHA_GTCIII } & vol & 0.0 & 0.0 & 0.0 & 45.3 & 45.3 & 45.3 & 45.3 & 45.3 & 45.3 & 15.3 & 15.3 & 15.3 & 15.3 & 0.0 & 0.0 & 453.2 & 2.2 \\
\hline & MIN & 0.0 & 0.0 & 0.0 & 15.3 & 45.3 & 15.3 & 15.3 & 15.3 & 15.3 & 15.3 & 15.3 & 45.3 & 15.3 & 0.0 & 0.0 & 453.2 & 2.2 \\
\hline & $\max$ & 0.0 & 0.0 & 0.0 & 15.3 & 45.3 & 45.3 & 13.3 & 45.3 & 15.3 & 15.3 & 45.3 & 15.3 & 45.3 & 0.0 & 0.0 & 453.2 & 2.2 \\
\hline \multirow{2}{*}{$\mathrm{CH}_{\text {IIRU }}$} & MIN & 0.0 & 0.0 & 0.0 & 04.9 & 84.9 & 84.9 & 84.9 & 81.9 & 84.9 & 84.9 & 81.9 & 84.9 & 84.9 & 0.0 & 0.0 & 019.6 & 1.2 \\
\hline & $\max$ & 0.0 & 0.0 & 0.0 & 81.9 & 84.9 & 84.9 & 81.9 & 81.9 & 84.9 & 84.9 & 84.9 & 84.9 & 04.9 & 0.0 & 0.0 & 049.6 & 1.2 \\
\hline \multirow[t]{3}{*}{ RH_IRU } & vor & 0.0 & 0.0 & 0.0 & 21.2 & 21.2 & 21.2 & 21.2 & 21.2 & 21.2 & 21.2 & 21.2 & 21.2 & 21.2 & 0.0 & 0.0 & 212.4 & 1.0 \\
\hline & MIN & 0.0 & 0.0 & 0.0 & 21.2 & 21.2 & 21.2 & 21.2 & 21.2 & 21.2 & 21.2 & 21.2 & 21.2 & 21.2 & 0.0 & 0.0 & 212.1 & 1.0 \\
\hline & $\max$ & 0.0 & 0.0 & 0.0 & 21.2 & 21.2 & 21.2 & 21.2 & 21.2 & 21.2 & 21.2 & 21.2 & 21.2 & 21.2 & 0.0 & 0.0 & 212.1 & 1.0 \\
\hline \multirow[t]{3}{*}{ CH_IRUS } & vot & 0.0 & 0.0 & 0.0 & 272.0 & 272.0 & 272.0 & 272:0 & 272.0 & 272.0 & 272.0 & 272.0 & 272.0 & 272.0 & 0.0 & 0.0 & 2720.0 & 23.4 \\
\hline & HIN & 0.0 & 0.0 & 0.0 & 272.0 & 272.0 & 272.0 & 272.0 & 272.0 & 272.0 & 272.0 & 272.0 & 272.0 & 272.0 & 0.0 & 0.0 & 2720.0 & 13.1 \\
\hline & $\max$ & 0.0 & 0.0 & 0.0 & 272.0 & 272.0 & 272.0 & 272.0 & 272.0 & 272.0 & 272.0 & 272.0 & 212.0 & 272.0 & 0.0 & 0.0 & 2720.0 & 13.1 \\
\hline \multirow[t]{3}{*}{ RH_TRUM } & vor & 0.0 & 0.0 & 0.0 & 68.0 & 68.0 & 68.0 & 68.0 & 68.0 & 68.0 & 68.0 & 68.0 & 60.0 & 68.0 & 0.0 & 0.0 & 680.0 & 3.3 \\
\hline & MIN & 0.0 & 0.0 & 0.0 & 68.0 & 68.0 & 60.0 & 68.0 & 68.0 & 68.0 & 60.0 & 60.0 & 68.0 & 68.0 & 0.0 & 0.0 & 680.0 & 3.3 \\
\hline & $\max$ & 0.0 & 0.0 & 0.0 & 68.0 & 60.0 & 68.0 & 60.0 & 68.0 & 68.0 & 68.0 & 68.0 & 68.0 & 68.0 & 0.0 & 0.0 & 680.0 & 3.3 \\
\hline
\end{tabular}




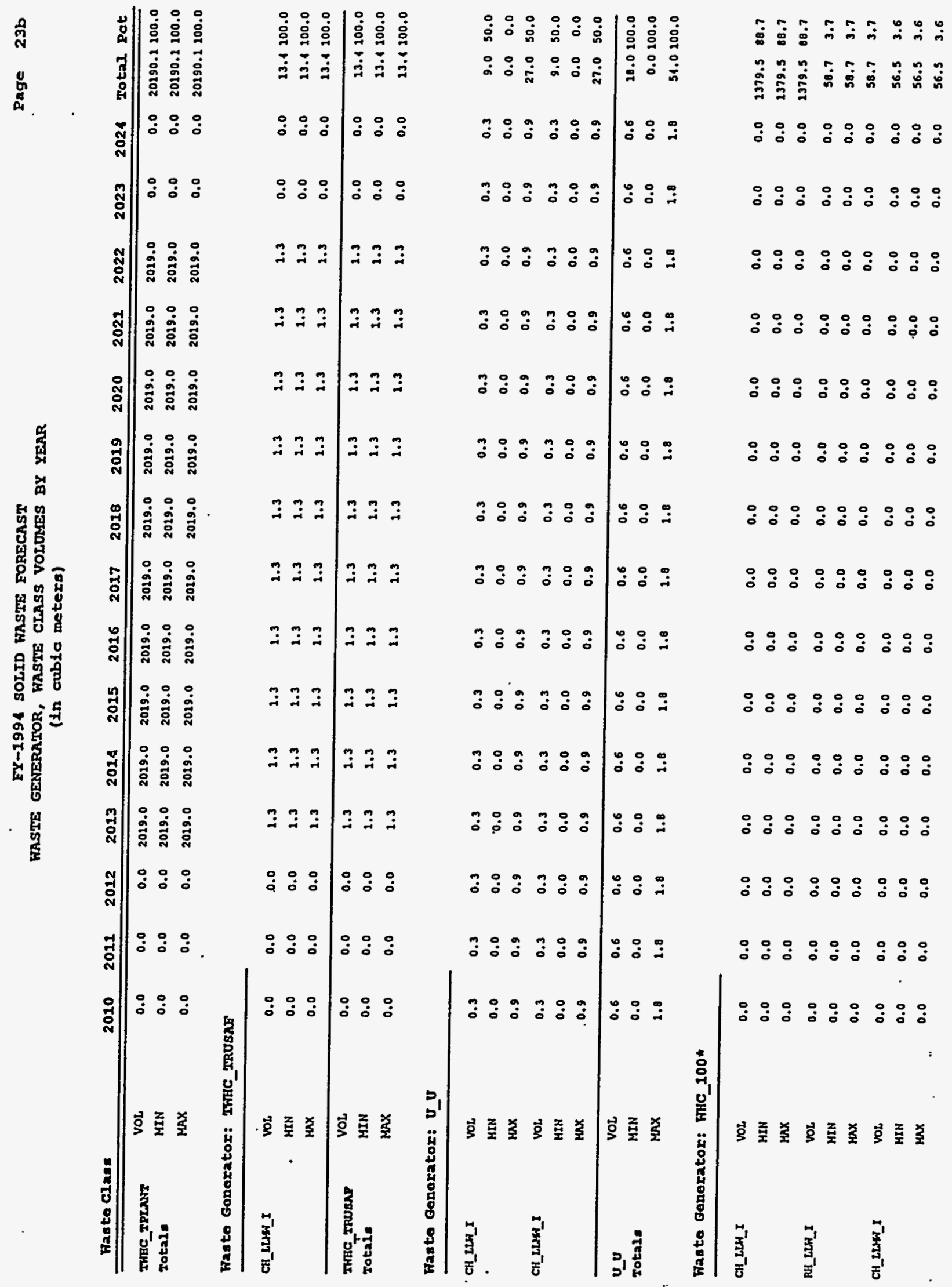


FY-1994 SOLID WASTE FORECAST

WASTE GENERATOR, WASTE CLASS VOLOMES BY YEAR

(In cubla meters)

Waste class

$\begin{array}{llllllllllllllll}2010 & 2011 & 2012 & 2013 & 2014 & 2015 & 2016 & 2017 & 2018 & 2019 & 2020 & 2021 & 2022 & 2023 & 2021 & \text { Total Pot }\end{array}$

Faste Generator: FHC_100*

\begin{tabular}{|c|c|c|c|c|c|c|c|c|c|c|c|c|c|c|c|c|c|c|}
\hline \multirow{3}{*}{ HNZ } & Vor & 0.0 & 0.0 & 0.0 & 0.0 & 0.0 & 0.0 & 0.0 & 0.0 & 0.0 & 0.0 & 0.0 & 0.0 & 0.0 & 0.0 & 0.0 & 60.0 & 3.8 \\
\hline & HIN & 0.0 & 0.0 & 0.0 & 0.0 & 0.0 & 0.0 & 0.0 & 0.0 & 0.0 & 0.0 & 0.0 & 0.0 & 0.0 & 0.0 & 0.0 & 60.0 & 3.8 \\
\hline & $\max$ & 0.0 & 0.0 & 0.0 & 0.0 & 0.0 & 0.0 & 0.0 & 0.0 & 0.0 & 0.0 & 0.0 & 0.0 & 0.0 & 0.0 & 0.0 & 60.0 & 3.8 \\
\hline \multirow{3}{*}{$\begin{array}{l}\text { หнс_100* } \\
\text { Total: }\end{array}$} & VOL & 0.0 & 0.0 & 0.0 & 0.0 & 0.0 & 0.0 & 0.0 & 0.0 & 0.0 & 0.0 & 0.0 & 0.0 & 0.0 & 0.0 & 0.0 & 1554.7 & 100.0 \\
\hline & MIN & 0.0 & 0.0 & 0.0 & 0.0 & 0.0 & 0.0 & 0.0 & 0.0 & 0.0 & 0.0 & 0.0 & 0.0 & 0.0 & 0.0 & 0.0 & 2554.7 & 100.0 \\
\hline & $\operatorname{MAX}$ & 0.0 & 0.0 & 0.0 & 0.0 & 0.0 & 0.0 & 0.0 & 0.0 & 0.0 & 0.0 & 0.0 & 0.0 & 0.0 & 0.0 & 0.0 & 1554.7 & 100.0 \\
\hline
\end{tabular}

Faste Generatox: WHC_1154_3000

\begin{tabular}{|c|c|c|c|c|c|c|c|c|c|c|c|c|c|c|c|c|c|}
\hline \multirow[t]{3}{*}{ HAZ } & Vot & 0.0 & 0.0 & 0.0 & 0.0 & 0.0 & 0.0 & 0.0 & 0.0 & 0.0 & 0.0 & 0.0 & 0.0 & 0.0 & 0.0 & 0.0 & 0.1100 .0 \\
\hline & MIN & 0.0 & 0.0 & 0.0 & 0.0 & 0.0 & 0.0 & 0.0 & 0.0 & 0.0 & 0.0 & 0.0 & 0.0 & 0.0 & 0.0 & 0.0 & 0.1200 .0 \\
\hline & $\max$ & 0.0 & 0.0 & 0.0 & 0.0 & 0.0 & 0.0 & 0.0 & 0.0 & 0.0 & 0.0 & 0.0 & 0.0 & 0.0 & 0.0 & 0.0 & 0.1100 .0 \\
\hline \multirow{3}{*}{$\begin{array}{l}\text { NaC_1154_3000 } \\
\text { 2otal. }\end{array}$} & VOL & 0.0 & 0.0 & 0.0 & 0.0 & 0.0 & 0.0 & 0.0 & 0.0 & 0.0 & 0.0 & 0.0 & 0.0 & 0.0 & 0.0 & 0.0 & 0.1100 .0 \\
\hline & MIN & 0.0 & 0.0 & 0.0 & 0.0 & 0.0 & 0.0 & 0.0 & 0.0 & 0.0 & 0.0 & 0.0 & 0.0 & 0.0 & 0.0 & 0.0 & 0.1100 .0 \\
\hline & $\operatorname{MAX}$ & 0.0 & 0.0 & 0.0 & 0.0 & 0.0 & 0.0 & 0.0 & 0.0 & 0.0 & 0.0 & 0.0 & 0.0 & 0.0 & 0.0 & 0.0 & 0.1100 .0 \\
\hline
\end{tabular}

Faa te Generatox: WHC 1163

\begin{tabular}{|c|c|c|c|c|c|c|c|c|c|c|c|c|c|c|c|c|c|}
\hline \multirow[t]{3}{*}{ Haz } & vol & 1.4 & 1.4 & 1.1 & 1.1 & 1.4 & 1.4 & 2.1 & 1.4 & 1.4 & 1.1 & 1.4 & 1.1 & 1.4 & 1.1 & 1.4 & 13.8100 .0 \\
\hline & HIN & 1.4 & 1.1 & 1.1 & 1.4 & 1.4 & 1.1 & 1.1 & 1.1 & 1.1 & 1.4 & 1.1 & 1.1 & 1.1 & 1.1 & 1.4 & $13.8 \quad 100.0$ \\
\hline & $\max$ & 1.4 & 1.1 & 1.1 & 1.1 & 1.4 & 1.4 & 1.4 & 1.1 & 1.4 & 1.4 & 1.1 & 1.1 & 2.4 & 1.4 & 1.1 & 43.6100 .0 \\
\hline \multirow{3}{*}{$\begin{array}{l}\text { HaC_1163 } \\
\text { Totals }\end{array}$} & VOL & 1.4 & 1.1 & 1.1 & 1.4 & 1.4 & 1.1 & 1.1 & 1.4 & 1.4 & 1.1 & 1.4 & 1.1 & 1.1 & 1.4 & 1.1 & 13.8100 .0 \\
\hline & MIN & 1.4 & 1.4 & 2.1 & 1.1 & 1.4 & 1.1 & 1.6 & 1.1 & 1.1 & 1.1 & 1.4 & 1.1 & 1.4 & 1.4 & 1.1 & 13.8100 .0 \\
\hline & $\operatorname{Max}$ & 1.4 & 1.4 & 2.4 & 1.4 & 1.4 & 1.4 & 1.1 & 2.1 & 1.1 & 2.1 & 1.4 & 1.4 & 1.4 & 1.1 & 1.4 & 43.8100 .0 \\
\hline
\end{tabular}

Waste Generator: FHC_1171M

\begin{tabular}{|c|c|c|c|c|c|c|c|c|c|c|c|c|c|c|c|c|c|}
\hline \multirow[t]{3}{*}{ Haz } & vos & 6.1 & 6.1 & .6 .1 & 6.1 & 6.1 & 6.1 & 6.4 & 6.1 & 6.4 & 6.4 & 6.4 & 6.1 & 6.1 & 6.1 & 6.1 & 292.0100 .0 \\
\hline & HIN & 5.1 & 5.7 & 5.7 & 5.7 & 3.7 & 5.7 & 5.7 & 5.7 & 5.7 & 5.7 & 5.7 & 5.7 & 5.7 & 5.7 & 3.7 & 172.0100 .0 \\
\hline & $\sin x$ & 7.0 & 7.0 & 7.0 & 7.0 & 7.0 & 7.0 & 7.0 & 7.0 & 1.0 & 7.0 & 7.0 & 7.0 & 7.0 & 7.0 & 7.0 & 211.2100 .0 \\
\hline \multirow{3}{*}{$\begin{array}{l}\text { WBC_11721 } \\
\text { Iotal: }\end{array}$} & VoL, & 6.1 & 6.1 & 6.1 & 6.1 & 6.1 & 6.1 & 6.1 & 6.1 & 6.1 & 6.1 & 6.1 & 6.1 & 6.1 & 6.1 & 6.1 & 192.0100 .0 \\
\hline & MIN & 5.7 & 5.7 & 5.7 & 3.7 & 5.7 & 5.7 & 5.7 & 5.7 & 3.7 & 5.7 & 5.7 & 5.7 & 3.7 & 5.7 & 5.7 & 172.0100 .0 \\
\hline & $\max$ & 7.0 & 7.0 & 7.0 & 7.0 & 7.0 & 7.0 & 7.0 & 7.0 & 1.0 & 7.0 & 7.0 & 7.0 & 7.0 & 7.0 & 9.0 & 211.2100 .0 \\
\hline
\end{tabular}




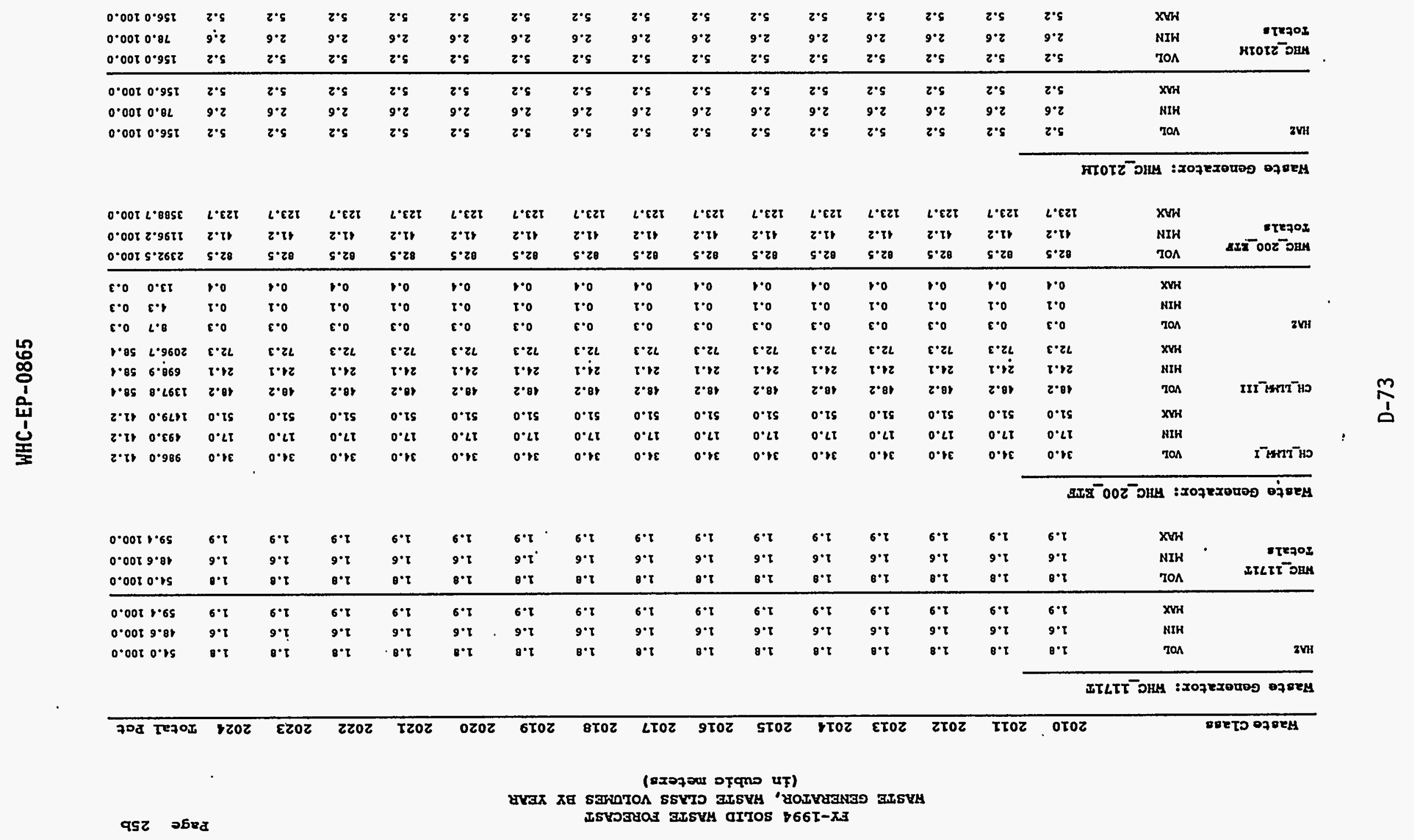




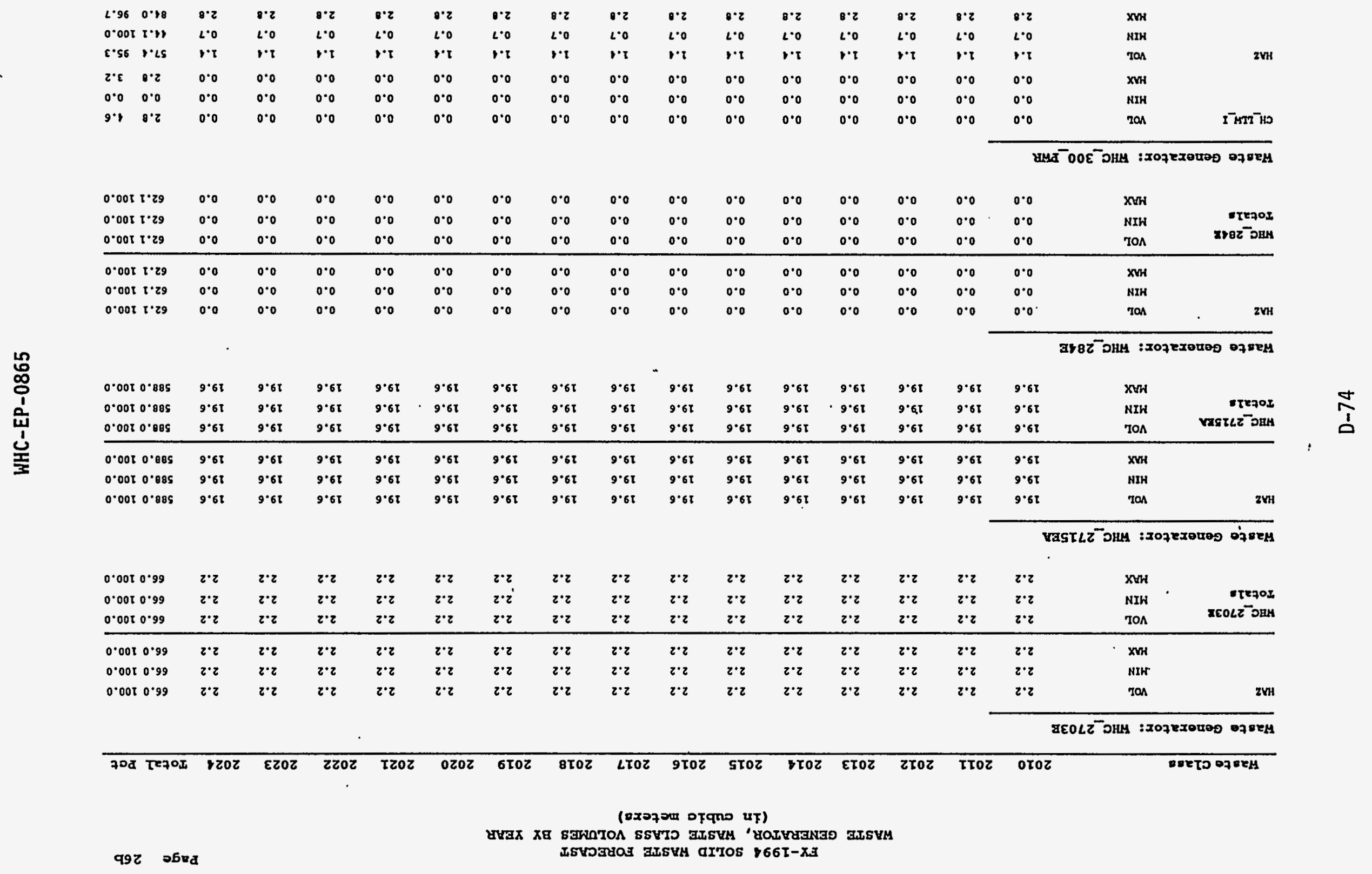


FY-1994 SOLID WASTE FORECAST

WASTE GENERATOR, WASTE CLASS VOLUNES BY YEAR

(in cubic metera)

Fasto Class

$\begin{array}{llllllllllllllll}2010 & 2011 & 2012 & 2013 & 2014 & 2015 & 2016 & 2017 & 2018 & 2019 & 2020 & 2021 & 2022 & 2023 & 2024 & \text { Total Pot }\end{array}$

\begin{tabular}{|c|c|c|c|c|c|c|c|c|c|c|c|c|c|c|c|c|c|}
\hline \multirow{3}{*}{$\begin{array}{l}\text { HEG_300_PRR } \\
\text { Totale }\end{array}$} & VoL & 1.4 & 1.1 & 1.4 & 1.4 & 1.4 & 2.1 & 1.1 & 1.4 & 1.1 & 1.1 & 1.1 & 1.4 & 1.4 & 1.4 & 1.4 & 60.2100 .0 \\
\hline & MIN & 0.7 & 0.7 & 0.7 & 0.7 & 0.7 & 0.7 & 0.7 & 0.7 & 0.7 & 0.7 & 0.7 & 0.7 & 0.7 & 0.7 & 0.7 & 11.2100 .0 \\
\hline & $\max$ & 2.8 & 2.8 & 2.8 & 2.8 & 2.8 & 2.8 & 2.8 & 2.8 & 2.8 & 2.8 & 2.8 & 2.8 & 2.0 & 2.8 & 2.8 & 06.0100 .0 \\
\hline
\end{tabular}

Fas te Generator: FHC_300_TEDE

\begin{tabular}{|c|c|c|c|c|c|c|c|c|c|c|c|c|c|c|c|c|c|c|}
\hline \multirow[t]{3}{*}{$\mathrm{CH}_{-} \mathrm{LLH}_{-} \mathrm{I}$} & vor & 360.3 & 368.3 & 368.3 & 368.3 & 368.3 & 368.3 & 360.3 & 368.3 & 368.3 & 368.3 & 368.3 & 368.3 & 368.3 & 368.3 & 360.3 & 11049.0 & 98.9 \\
\hline & HIN & 384.1 & 104.1 & 184.1. & 184.1 & 284.1 & 284.1 & 184.2 & 284.1 & 184.1 & 184.1 & 194.1 & 184.1 & 184.1 & 184.1 & 184.1 & 5524.5 & 97.8 \\
\hline & $\max$ & 552.4 & 552.4 & 552.4 & 552.4 & 552.4 & 552.1 & 552.4 & 552.4 & 552.4 & 552.1 & 552.4 & 352.4 & 552.1 & 552.4 & 352.1 & 16573.5 & 98.5 \\
\hline \multirow[t]{3}{*}{ CH_LLWW_I } & VoL & 0.0 & 0.5 & 0.0 & 0.5 & 0.0 & 8.5 & 0.0 & 8.5 & 0.0 & 8.5 & 0.0 & 8.5 & 0.0 & 8.5 & 0.0 & 119.0 & 1.0 \\
\hline & HIH & 0.0 & 8.5 & 0.0 & 8.5 & 0.0 & 6.5 & 0.0 & 0.5 & 0.0 & 0.5 & 0.0 & 8.5 & 0.0 & 8.5 & 0.0 & 119.0 & 2.1 \\
\hline & $\operatorname{HAX}$ & 0.0 & 17.0 & 0.0 & 17.0 & 0.0 & 27.0 & 0.0 & 17.0 & 0.0 & 17.0 & 0.0 & 27.0 & 0.0 & 17.0 & 0.0 & 238.0 & 1.1 \\
\hline \multirow{2}{*}{$\begin{array}{l}\text { WRC_300_FPDY } \\
\text { Total= }\end{array}$} & VoL & 360.3 & 376.8 & 360.3 & 376.8 & 368.3 & 376.8 & 368.3 & 376.8 & 360.3 & 376.8 & 368.3 & 376.8 & 368.3 & 376.8 & 368.3 & 11168.0 & 100.0 \\
\hline & MIN & 184.1 & 192.6 & 184.1 & 192.6 & 184.1 & 192.6 & 184.1 & 192.6 & 184.1 & 192.6 & 184.1 & 192.6 & 184.1 & 192.6 & 184.1 & 5643.5 & 100.0 \\
\hline
\end{tabular}

Haste Generatox: FHC 305

\begin{tabular}{|c|c|c|c|c|c|c|c|c|c|c|c|c|c|c|c|c|c|}
\hline \multirow[t]{3}{*}{ HA2 } & vot & 0.2 & 0.2 & 0.2 & 0.2 & 0.2 & 0.2 & 0.2 & 0.2 & 0.2 & 0.2 & 0.2 & 0.2 & 0.2 & 0.2 & 0.2 & 6.5100 .0 \\
\hline & MIN & 0.2 & 0.2 & 0.2 & 0.2 & 0.2 & 0.2 & 0.2 & 0.2 & 0.2 & 0.2 & 0.2 & 0.2 & 0.2 & 0.2 & 0.2 & 6.5100 .0 \\
\hline & $\max$ & 0.2 & 0.2 & 0.2 & 0.2 & 0.2 & 0.2 & 0.2 & 0.2 & 0.2 & 0.2 & 0.2 & 0.2 & 0.2 & 0.2 & 0.2 & 6.5100 .0 \\
\hline \multirow{3}{*}{$\begin{array}{l}\text { HBC_305 } \\
\text { Total. }\end{array}$} & VoL & 0.2 & 0.2 & 0.2 & 0.2 & 0.2 & 0.2 & 0.2 & 0.2 & 0.2 & 0.2 & 0.2 & 0.2 & 0.2 & 0.2 & 0.2 & 6.5100 .0 \\
\hline & MIN & 0.2 & 0.2 & 0.2 & 0.2 & 0.2 & 0.2 & 0.2 & 0.2 & 0.2 & 0.2 & 0.2 & 0.2 & 0.2 & 0.2 & 0.2 & 6.5100 .0 \\
\hline & MAX & 0.2 & 0.2 & 0.2 & 0.2 & 0.2 & 0.2 & 0.2 & 0.2 & 0.2 & 0.2 & 0.2 & 0.2 & 0.2 & 0.2 & 0.2 & 6.5100 .0 \\
\hline
\end{tabular}

Waste Generator: WHC_306

\begin{tabular}{|c|c|c|c|c|c|c|c|c|c|c|c|c|c|c|c|c|c|}
\hline \multirow[t]{3}{*}{$\operatorname{Haz}$} & VoL & 2.7 & 2.7 & 2.7 & 2.7 & 2.7 & 2.7 & 2.7 & 2.7 & 2.7 & 2.7 & 2.7 & 2.7 & 2.7 & 2.7 & 2.7 & 04.6100 .0 \\
\hline & MIN & 2.5 & 2.5 & 2.5 & 2.3 & 2.3 & 2.5 & 2.5 & 2.5 & 2.5 & 2.5 & 2.5 & 2.5 & 2.5 & 2.5 & 2.5 & 80.5100 .0 \\
\hline & $\max$ & 2.8 & 2.8 & 2.8 & 2.8 & 2.8 & 2.8 & 2.8 & 2.8 & 2.8 & 2.8 & 2.8 & 2.0 & 2.8 & 2.8 & 2.8 & 80.9100 .0 \\
\hline \multirow{3}{*}{$\begin{array}{l}\text { Hac_306 } \\
\text { Tot21: }\end{array}$} & VOL & 2.7 & 2.7 & 2.7 & 2.7 & 2.7 & 2.7 & 2.7 & 2.7 & 2.7 & 2.7 & 2.7 & 2.7 & 2.7 & 2.7 & 2.7 & 01.6100 .0 \\
\hline & MIN & 2.5 & 2.5 & 2.5 & 2.5 & 2.3 & 2.5 & 2.5 & 2.5 & 2.5 & 2.5 & 2.5 & 2.5 & 2.5 & 2.5 & 2.5 & 80.5100 .0 \\
\hline & $\max$ & 2.8 & 2.0 & 2.8 & 2.8 & 2.8 & 2.8 & 2.8 & 2.0 & 2.0 & 2.8 & 2.8 & 2.8 & 2.0 & 2.8 & 2.8 & 80.9100 .0 \\
\hline
\end{tabular}




\begin{tabular}{|c|c|c|c|c|c|c|c|c|c|c|c|c|c|c|c|c|c|}
\hline $\begin{array}{l}0.0010 .12 \\
0.0010 .12 \\
0.0010 .12\end{array}$ & $\begin{array}{l}0.0 \\
800 \\
0 \div 0\end{array}$ & $\begin{array}{l}0.0 \\
800 \\
800\end{array}$ & $\begin{array}{l}8.0 \\
0.0 \\
0.0\end{array}$ & $\begin{array}{l}8.0 \\
8.0 \\
8.0\end{array}$ & $\begin{array}{l}8.0 \\
8.0 \\
0.0\end{array}$ & $\begin{array}{l}9 \times 0 \\
8 \times 0 \\
9 \div 0\end{array}$ & $\begin{array}{l}8.0 \\
8.0 \\
8.0\end{array}$ & $\begin{array}{l}8.0 \\
8.0 \\
0.0\end{array}$ & $\begin{array}{l}8 \cdot 0 \\
8.0 \\
8.0\end{array}$ & $\begin{array}{l}0.0 \\
8.0 \\
0.0\end{array}$ & $\begin{array}{l}8 \cdot 0 \\
8.0 \\
8.0\end{array}$ & $\begin{array}{l}0.0 \\
0.0 \\
0.0\end{array}$ & $\begin{array}{l}0 \cdot 0 \\
\because \bullet 0 \\
0 \times 0\end{array}$ & $\begin{array}{l}8 \div 0 \\
8 \div 0 \\
0 \div 0\end{array}$ & $\begin{array}{l}8 \cdot 0 \\
8.0 \\
8.0\end{array}$ & $\begin{array}{l}X W \\
\text { NIK } \\
\text { TOA }\end{array}$ & 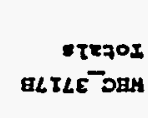 \\
\hline $0.00 \tau 0.02$ & 8.0 & $8 \cdot 0$ & $8 \cdot 0$ & $P \cdot 0$ & 0.0 & $9 \cdot 0$ & $0 \cdot 0$ & $\theta \cdot 0$ & 0.0 & $8 \cdot 0$ & 8.0 & $8 \cdot 0$ & D.o & 8.0 & $8 \cdot 0$ & $\mathbf{x W H}$ & \\
\hline 0.0010 .32 & $0 \cdot 0$ & 00 & 0.0 & $9 \cdot 0$ & $0 \cdot 0$ & 0.0 & B.0 & $0 \cdot 0$ & 8.0 & 8.0 & 0.0 & 0.0 & $8 \cdot 0$ & $8 \cdot 0$ & $8 \cdot 0$ & NIH & \\
\hline $0.00 \tau 0.12$ & $8 \cdot 0$ & 0.0 & B.0 & 0.0 & $8 \cdot 0$ & B.0 & 0.0 & 0.0 & 8.0 & $8 \cdot 0$ & 8.0 & 0.0 & 0.0 & 8.0 & 8.0 & Tas & 2VH \\
\hline
\end{tabular}

\begin{tabular}{|c|c|c|c|c|c|c|c|c|c|c|c|c|c|c|c|c|c|}
\hline $\begin{array}{l}0.00 t 0.5 t \\
0.00 t 0.5 t \\
0.0010 .9 t\end{array}$ & $\begin{array}{l}s \cdot 0 \\
s \cdot 0 \\
s \cdot 0\end{array}$ & $\begin{array}{l}5.0 \\
5 \cdot 0 \\
5 \cdot 0\end{array}$ & $\begin{array}{l}S \cdot 0 \\
\text { S.0 } \\
\text { S.0 }\end{array}$ & $\begin{array}{l}5 \cdot 0 \\
5 \cdot 0 \\
5.0\end{array}$ & $\begin{array}{l}5.0 \\
5.0 \\
s .0\end{array}$ & $\begin{array}{l}s .0 \\
5 \cdot 0 \\
s: 0\end{array}$ & $\begin{array}{l}5: 0 \\
5 \cdot 0 \\
5.0\end{array}$ & $\begin{array}{l}s: 0 \\
s \cdot 0 \\
s: 0\end{array}$ & $\begin{array}{l}5.0 \\
5 \cdot 0 \\
5 \cdot 0\end{array}$ & $\begin{array}{l}S \cdot 0 \\
S \cdot 0 \\
S \cdot 0\end{array}$ & $\begin{array}{l}5 \cdot 0 \\
5 \cdot 0 \\
5 \cdot 0\end{array}$ & $\begin{array}{l}\text { s.0 } \\
\text { s.0 } \\
\text { s:0 }\end{array}$ & $\begin{array}{l}s \cdot 0 \\
5 \cdot 0 \\
s \cdot 0\end{array}$ & $\begin{array}{l}5 \cdot 0 \\
5 \cdot 0 \\
5 \cdot 0\end{array}$ & $\begin{array}{l}5 \cdot 0 \\
s \cdot 0 \\
5 \cdot 0\end{array}$ & $\begin{array}{l}\text { XWW } \\
\text { NIW } \\
\text { TON }\end{array}$ & $\begin{array}{r}\text { TEZOI } \\
\text { OLOLE }\end{array}$ \\
\hline $0.0010 .5 T$ & s.o & $s \cdot 0$ & s.o & s.o & $s: 0$ & 5.0 & $s \cdot 0$ & $5 \cdot 0$ & s.o & $s \cdot 0$ & $5 \cdot 0$ & s.o & s.o & 5.0 & s.0 & $x w+1$ & \\
\hline זפת & s.o & $s: 0$ & 5.0 & $s \cdot 0$ & $5 \cdot 0$ & $5 \cdot 0$ & s.o & $5 \cdot 0$ & $s \cdot 0$ & s.o & s.o & s.o & $s=0$ & $5 \% 0$ & s.0 & $\operatorname{Tan}$ & $2 \mathbf{V H}$ \\
\hline
\end{tabular}

OLOLET DHM : X072X0U09 078EM

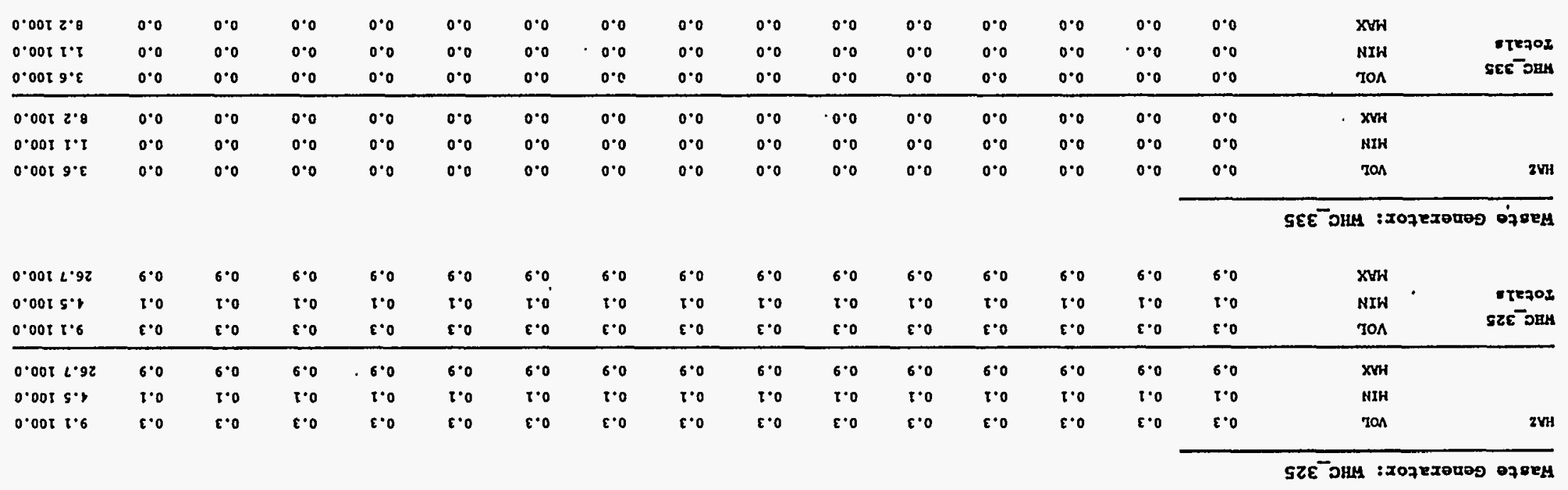

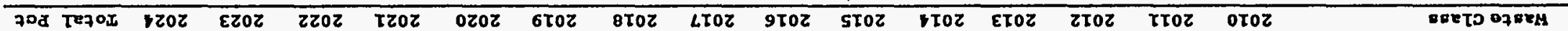

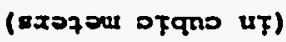

982 ग68d

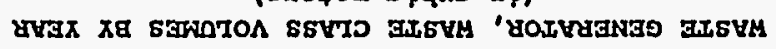

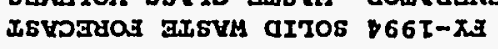


FY-1994 SOLID HASTE FORECAST

WASTE GENERATOR, WASTE CLASS VOLUMES BY YEAR

\author{
(in cublo meters)
}

Faste Class

$\begin{array}{lllllllll}2010 & 2011 & 2012 & 2013 & 2014 & 2015 & 2016 & 2017 & 2018\end{array}$

20232021 Total Pet

Waste Gonorator: FHC_ANALYT_LAB

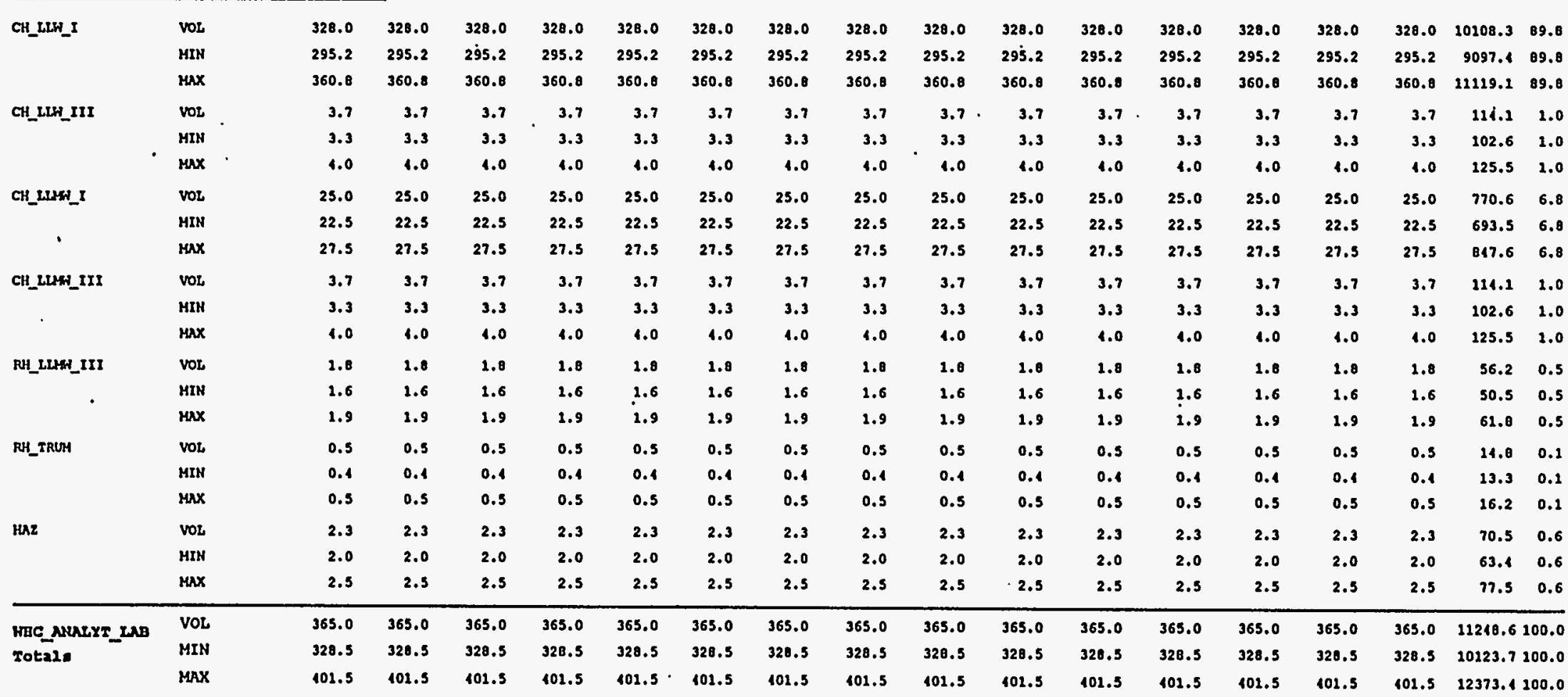

Wasto Generatox: FHC_BQUTP

\begin{tabular}{|c|c|c|c|c|c|c|c|c|c|c|c|c|c|c|c|c|c|}
\hline \multirow[t]{3}{*}{ CH_LLH_I } & VOL & 170.0 & 170.0 & 226.6 & 226.6 & 255.0 & 203.3 & 339.9 & 198.3 & 198.3 & 226.6 & 283.3 & 339.9 & 170.0 & 0.0 & 0.0 & $7102.7 \quad 100.0$ \\
\hline & MIN & 170.0 & 170.0 & 226.6 & 226.6 & 255.0 & 283.3 & 339.9 & 198.3 & 198.3 & 226.6 & 283.3 & 339.9 & 170.0 & 0.0 & 0.0 & 7102.7100 .0 \\
\hline & $\max$ & 270.0 & 170.0 & 226.6 & 226.6 & 233.0 & 283.3 & 339.9 & 198.3 & 198.3 & 226.6 & 283.3 & 339.9 & 170.0 & 0.0 & 0.0 & 1402.7100 .0 \\
\hline \multirow{3}{*}{$\begin{array}{l}\text { HBC_BQUIP } \\
\text { Total. }\end{array}$} & VoL & 170.0 & 270.0 & 226.6 & 226.6 & 255.0 & 283.3 & 339.9 & 198.3 & 198.3 & 226.6 & 283.3 & 339.9 & 170.0 & 0.0 & 0.0 & 7402.7100 .0 \\
\hline & MIN & 170.0 & 170.0 & 226.6 & 226.6 & 255.0 & 203.3 & 339.9 & 198.3 & 198.3 & 226.6 & 203.3 & 339.9 & 270.0 & 0.0 & 0.0 & 7102.7100 .0 \\
\hline & $\operatorname{MAX}$ & 270.0 & 170.0 & 226.6 & 226.6 & 255.0 & 203.3 & 339.9 & 198.3 & 298.3 & 226.6 & 283.3 & 339.9 & 270.0 & 0.0 & 0.0 & 7102.7100 .0 \\
\hline
\end{tabular}


WASTE GENERATOR, WASTE CLASS VOLOMES BY YEAR (in cublo meters)

Haste class

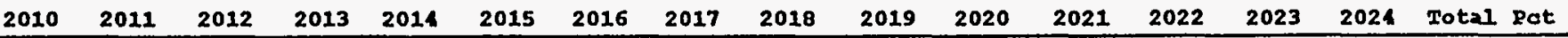

\section{Was to Genorator: WHC_B_pLANT}

\begin{tabular}{|c|c|c|c|c|c|c|c|c|c|c|c|c|c|c|c|c|c|c|}
\hline CH_LLH_I & $\begin{array}{l}\text { Vor } \\
\text { HIN } \\
\max \end{array}$ & $\begin{array}{r}113.3 \\
84.9 \\
111.6\end{array}$ & $\begin{array}{r}113.3 \\
04.9 \\
111.6\end{array}$ & $\begin{array}{r}113.3 \\
81.9 \\
111.6\end{array}$ & $\begin{array}{r}113.3 \\
84.9 \\
111.6\end{array}$ & $\begin{array}{r}113.3 \\
81.9 \\
211.6\end{array}$ & $\begin{array}{r}123.3 \\
84.9 \\
242.6\end{array}$ & $\begin{array}{r}113.3 \\
84.9 \\
111.6\end{array}$ & $\begin{array}{r}113.3 \\
81.9 \\
111.6\end{array}$ & $\begin{array}{r}113.3 \\
84.9 \\
111.6\end{array}$ & $\begin{array}{r}313.3 \\
84.9 \\
111.6\end{array}$ & $\begin{array}{r}113.3 \\
81.9 \\
141.6\end{array}$ & $\begin{array}{r}113.3 \\
81.9 \\
141.6\end{array}$ & $\begin{array}{r}113.3 \\
01.9 \\
111.6\end{array}$ & $\begin{array}{r}113.3 \\
84.9 \\
141.6\end{array}$ & $\begin{array}{r}113.3 \\
81.9 \\
141.6\end{array}$ & $\begin{array}{l}4081.1 \\
3017.0 \\
5119.2\end{array}$ & $\begin{array}{l}77.8 \\
01.1 \\
76.3\end{array}$ \\
\hline \multirow[t]{3}{*}{ CH_LLH_III } & vol & 9.9 & 9.9 & 9.9 & 9.9 & 9.9 & 9.9 & 9.9 & 9.9 & 9.9 & 9.9 & 9.9 & 9.9 & 9.9 & 9.9 & 9.9 & 314.0 & 6.5 \\
\hline & HIM & 7.1 & 7.4 & 7.4 & 7.1 & 7.4 & 7.1 & 7.1 & 7.4 & 7.4 & $? .4$ & 7.4 & 7.4 & 7.4 & 7.1 & 1.4 & 258.1 & 6.9 \\
\hline & $\operatorname{MAX}$ & 12.3 & 12.3 & 12.3 & 12.3 & 12.3 & 12.3 & 12.3 & 12.3 & 12.3 & 12.3 & 22.3 & 12.3 & 12.3 & 12.3 & 22.3 & 430.1 & 6.1 \\
\hline \multirow{3}{*}{ PH_LLH_III } & vor & 3.5 & 3.5 & 3.5 & 3.5 & 3.5 & 3.5 & 3.5 & 3.5 & 3.5 & 3.5 & 3.3 & 3.5 & 3.5 & 3.5 & 3.5 & 611.6 & 11.6 \\
\hline & HIN & 2.6 & 2.6 & 2.6 & 2.6 & 2.6 & 2.6 & 2.6 & 2.6 & 2.6 & 2.6 & 2.6 & 2.6 & 2.6 & 2.6 & 2.6 & 278.9 & 7.5 \\
\hline & $\max$ & 4.3 & 1.3 & 4.3 & 1.3 & 1.3 & 4.3 & 4.3 & 1.3 & 4.3 & 4.3 & 4.3 & 1.3 & 4.3 & 4.3 & 1.3 & 894.9 & 13.3 \\
\hline \multirow{2}{*}{ CH_LLWA_I } & MIN & 1.0 & 1.0 & 1.0 & 1.0 & 1.0 & 1.0 & 1.0 & 1.0 & 1.0 & 1.0 & 2.0 & 1.0 & 1.0 & 1.0 & 1.0 & 37.4 & 1.0 \\
\hline & $\max$ & 1.7 & 1.7 & 1.7 & 1.7 & 2.7 & 1.7 & 1.7 & 1.7 & 1.7 & 1.7 & 1.7 & 1.7 & 1.7 & 1.7 & 1.7 & 62.7 & 0.9 \\
\hline \multirow[t]{3}{*}{ RH_LLAN_III } & vor & 0.0 & 0.0 & 0.0 & 0.0 & 0.0 & 0.0 & 0.0 & 0.0 & 0.0 & 0.0 & 0.0 & 0.0 & 0.0 & 0.0 & 0.0 & 5.5 & 0.1 \\
\hline & MIN & 0.0 & 0.0 & 0.0 & 0.0 & 0.0 & 0.0 & 0.0 & 0.0 & 0.0 & 0.0 & 0.0 & 0.0 & 0.0 & 0.0 & 0.0 & 2.2 & 0.0 \\
\hline & $\max$ & 0.0 & 0.0 & 0.0 & 0.0 & 0.0 & 0.0 & 0.0 & 0.0 & 0.0 & 0.0 & 0.0 & 0.0 & 0.0 & 0.0 & 0.0 & 9.3 & 0.2 \\
\hline \multirow[t]{3}{*}{ HAz } & vor & 4.2 & 1.2 & 1.2 & 4.2 & 1.2 & 4.2 & 1.2 & 1.2 & 1.2 & 1.2 & 1.2 & 1.2 & 4.2 & 1.2 & 1.2 & 152.5 & 2.9 \\
\hline & HIN & 3.1 & 3.1 & 3.2 & 3.1 & 3.1 & 3.1 & 3.1 & 3.1 & 3.1 & 3.1 & 3.1 & 3.1 & 3.1 & 3.1 & 3.1 & 112.3 & 3.0 \\
\hline & $\max$ & 3.2 & 3.2 & 3.2 & 5.2 & 5.2 & 5.2 & 5.2 & 5.2 & 5.2 & 5.2 & 5.2 & 5.2 & 5.2 & 5.2 & 3.2 & 189.9 & 2.8 \\
\hline rotal: & MIN & 99.2 & 99.2 & 99.2 & 99.2 & 99.2 & 99.2 & 99.2 & 99.2 & 99.2 & 99.2 & 99.2 & 99.2 & 99.2 & 99.2 & 99.2 & 3706.1 & 100.0 \\
\hline & $\operatorname{MAX}$ & 165.3 & 265.3 & 165.3 & 165.3 & 265.3 & 165.3 & 165.3 & 265.3 & 265.3 & 165.3 & 265.3 & 165.3 & 165.3 & 165.3 & 265.3 & 6706.2 & 200.0 \\
\hline
\end{tabular}

Hasto Generator: HHC_DSI_PSRET

\begin{tabular}{|c|c|c|c|c|c|c|c|c|c|c|c|c|c|c|c|c|c|}
\hline \multirow[t]{3}{*}{$\mathrm{CH}_{-} \mathrm{LLH}_{-} \mathrm{I}$} & VOL & 0.0 & 0.0 & 0.0 & 0.0 & 0.0 & 0.6 & 0.0 & 0.0 & 0.0 & 0.0 & 0.0 & 0.0 & 0.0 & 0.0 & 0.0 & 1.2100 .0 \\
\hline & MIN & 0.0 & 0.0 & 0.0 & 0.0 & 0.0 & 0.6 & 0.0 & 0.0 & 0.0 & 0.0 & 0.0 & 0.0 & 0.0 & 0.0 & 0.0 & 1.2100 .0 \\
\hline & $\max$ & 0.0 & 0.0 & 0.0 & 0.0 & 0.0 & 0.6 & 0.0 & 0.0 & 0.0 & 0.0 & 0.0 & 0.0 & 0.0 & 0.0 & 0.0 & 1.2100 .0 \\
\hline \multirow{3}{*}{$\begin{array}{l}\text { KBC_DST_PBRXT } \\
\text { Total. }\end{array}$} & VOL & 0.0 & 0.0 & 0.0 & 0.0 & 0.0 & 0.6 & 0.0 & 0.0 & 0.0 & 0.0 & 0.0 & 0.0 & 0.0 & 0.0 & 0.0 & 1.2100 .0 \\
\hline & MIN & 0.0 & 0.0 & 0.0 & 0.0 & 0.0 & 0.6 & 0.0 & 0.0 & 0.0 & 0.0 & 0.0 & 0.0 & 0.0 & 0.0 & 0.0 & 1.2100 .0 \\
\hline & MAX & 0.0 & 0.0 & 0.0 & 0.0 & 0.0 & 0.6 & 0.0 & 0.0 & 0.0 & 0.0 & 0.0 & 0.0 & 0.0 & 0.0 & 0.0 & 1.2100 .0 \\
\hline
\end{tabular}




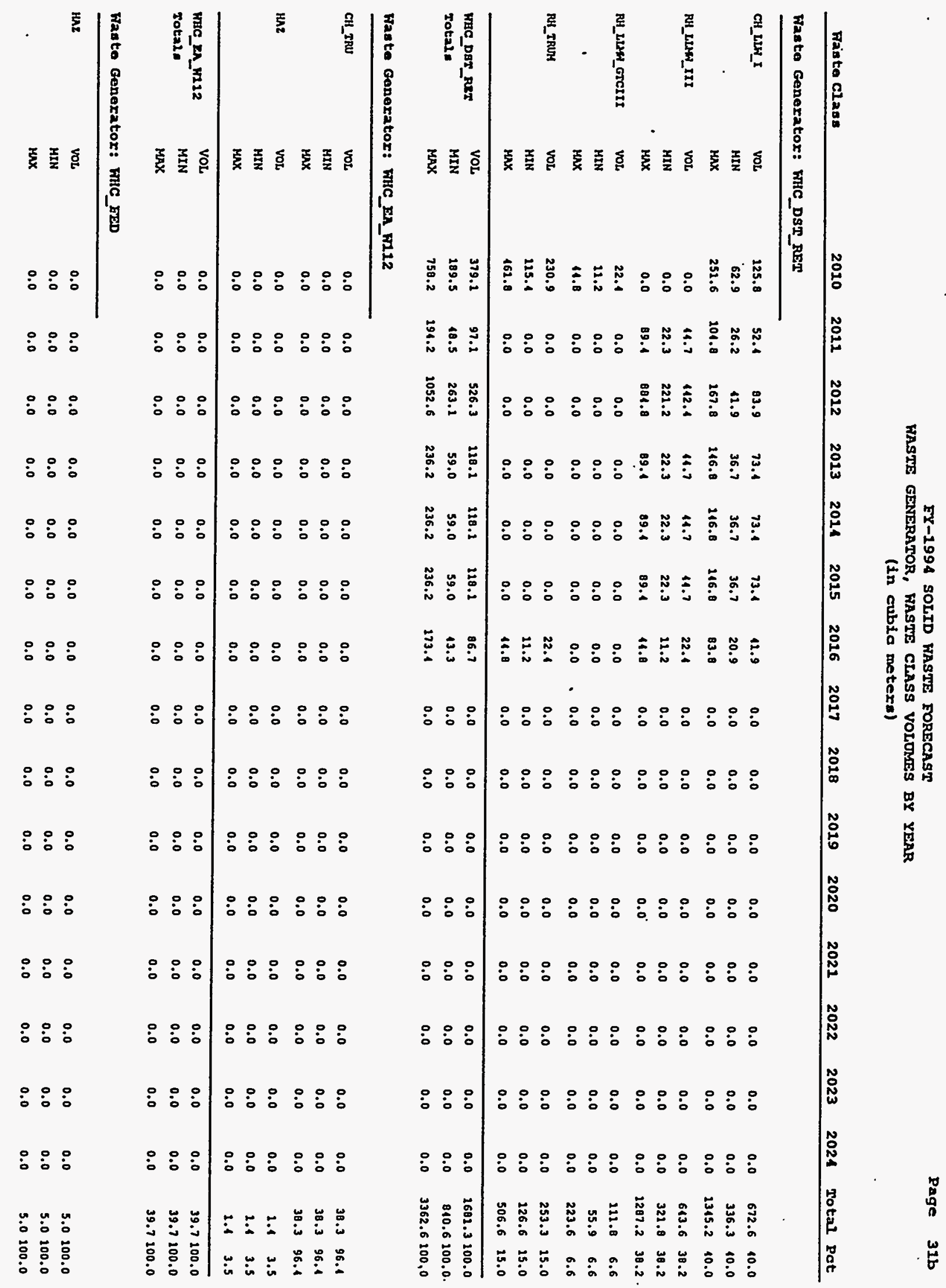


WHC-EP-0865

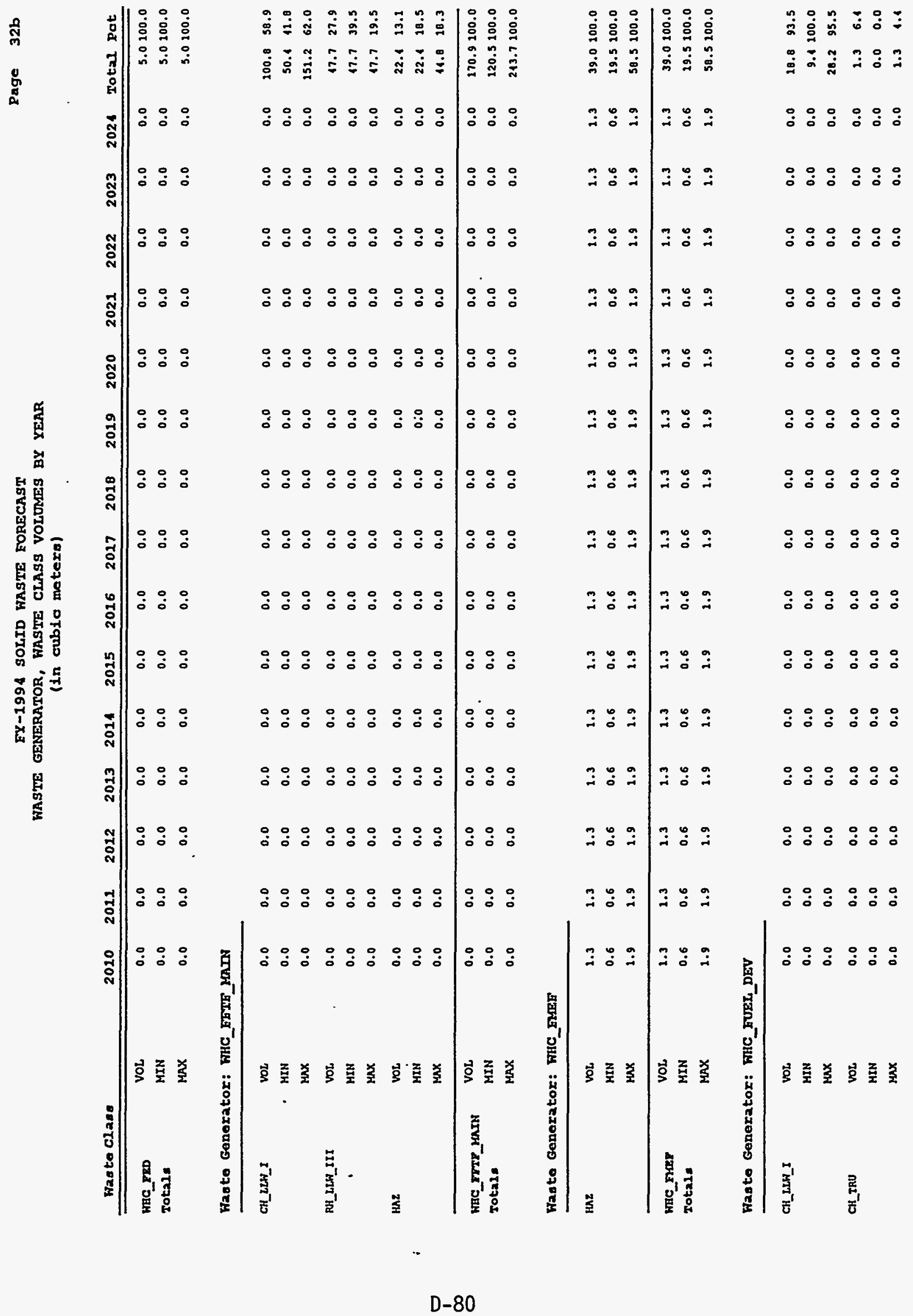




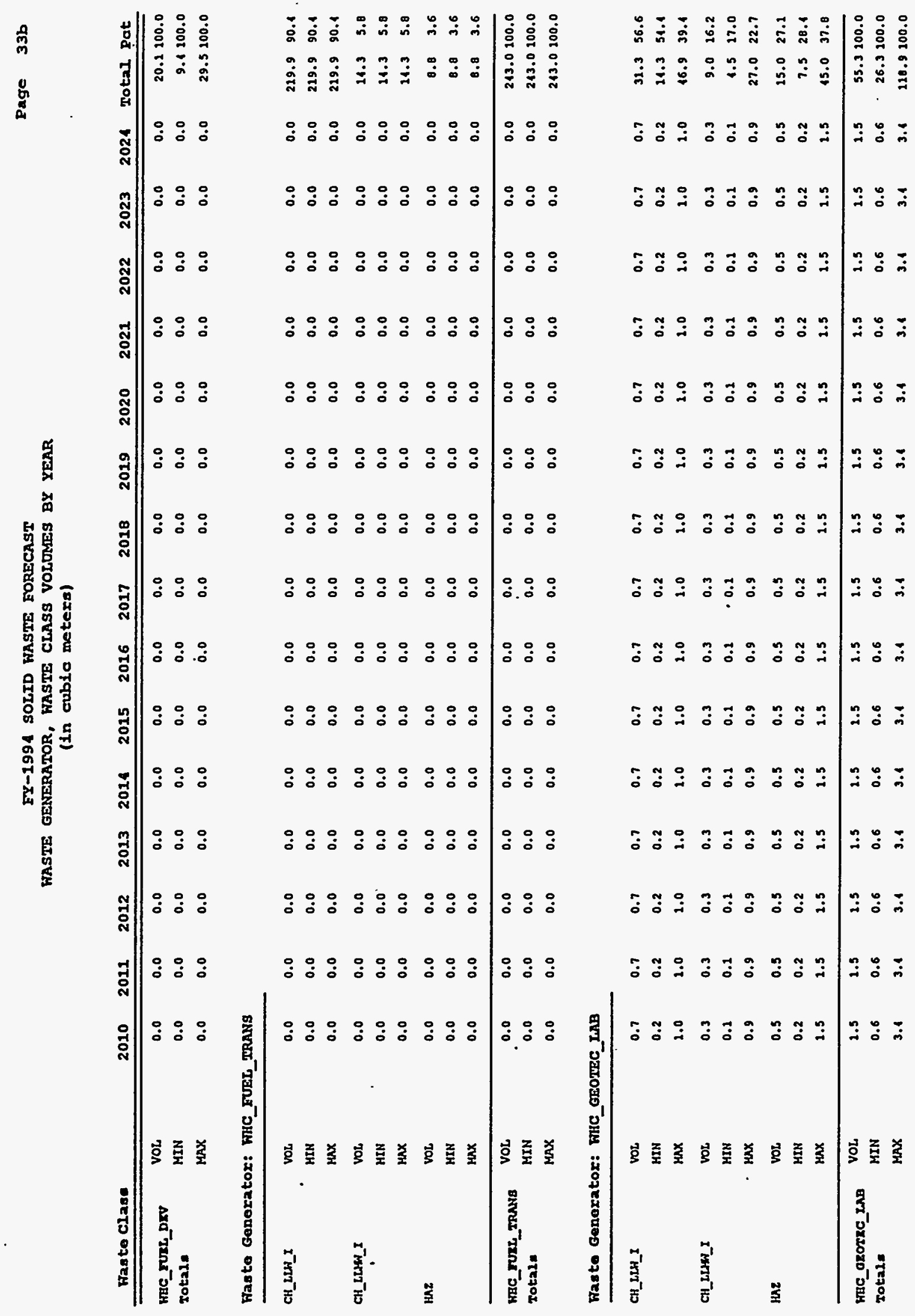


WASTE GENERATOR, HASTE CLASS VOLUMES BY YEAR

(in cubic metera)

Wasto class

$\begin{array}{llllllllllllllll}2010 & 2011 & 2012 & 2013 & 2014 & 2015 & 2016 & 2017 & 2018 & 2019 & 2020 & 2021 & 2022 & 2023 & 2024 & \text { Total Pot }\end{array}$

\section{Wasto Gonorator: WHC_HLVP}

\begin{tabular}{|c|c|c|c|c|c|c|c|c|c|c|c|c|c|c|c|c|c|c|}
\hline \multirow[t]{3}{*}{ CH_LLH_I } & VOL & 149.7 & 149.7 & 119.7 & 119.7 & 149.7 & 119.7 & 119.7 & 419.7 & 149.7 & 119.7 & 419.7 & 149.7 & 119.7 & 419.7 & 419.7 & 7195.2 & 27.1 \\
\hline & MIN & 224.8 & 224.8 & 224.8 & 224.8 & 224.8 & 224.8 & 221.0 & 224.0 & 224.8 & 226.8 & 224.0 & 224.8 & 224.8 & 224.8 & 224.8 & 3372.7 & 27.1 \\
\hline & $\max$ & 899.1 & 899.1 & 899.1 & 899.1 & 899.1 & 899.1 & 899.1 & 899.1 & 899.4 & 899.1 & 899.1 & 899.4 & 899.4 & 099.1 & 899.1 & 11390.4 & 27.1 \\
\hline \multirow[t]{3}{*}{ CH_LLW_III } & vor & 810.2 & 810.2 & 810.2 & 810.2 & 010.2 & 810.2 & 810.2 & 810.2 & 810.2 & 810.2 & 810.2 & 830.2 & 810.2 & 810.2 & 810.2 & 12963.2 & 18.8 \\
\hline & HIN & 105.1 & 105.1 & 105.1 & 405.1 & 405.1 & 105.1 & 405.1 & 105.1 & 105.1 & 105.1 & 405.1 & 103.1 & 105.1 & 105.1 & 105.1 & 6076.5 & 40.8 \\
\hline & $\max$ & 1620.4 & 1620.1 & 2620.4 & 1620.1 & 1620.4 & 1620.4 & 1620.1 & 1620.4 & 1620.4 & 1620.1 & 1620.1 & 1620.4 & 1620.1 & 1620.1 & 1620.1 & 25926.4 & 18.0 \\
\hline \multirow[t]{3}{*}{ RH_LLH_III } & VOL & 126.9 & 126.9 & 126.9 & 126.9 & 126.9 & 126.9 & 126.9 & 126.9 & 126.9 & 126.9 & 126.9 & 126.9 & 126.9 & 126.9 & 126.9 & 2030.4 & 7.6 \\
\hline & MIN & 63.1 & 63.1 & 63.4 & 63.1 & 63.1 & 63.4 & 63.1 & 63.4 & 63.4 & 63.1 & 63.4 & 63.4 & 63.4 & 63.4 & 63.1 & 951.7 & 7.6 \\
\hline & $\max$ & 253.8 & 253.8 & 253.8 & 253.8 & 253.8 & 253.0 & 253.8 & 253.8 & 253.8 & 253.8 & 253.8 & 253.0 & 253.8 & 253.8 & 253.8 & 1060.8 & 7.6 \\
\hline \multirow[t]{3}{*}{ CH_LLHF_I } & vor & 18.2 & 18.2 & 28.2 & 18.2 & 18.2 & 18.2 & 18.2 & 18.2 & 18.2 & 10.2 & 18.2 & 18.2 & 18.2 & 18.2 & 18.2 & 291.2 & 1.1 \\
\hline & MIM & 9.1 & 9.1 & 9.1 & 9.1 & 9.1 & 9.1 & 9.1 & 9.1 & 9.1 & 9.1 & 9.1 & 9.1 & 9.1 & 9.1 & 9.1 & 136.5 & 1.1 \\
\hline & $\operatorname{man} x$ & 36.4 & 36.4 & 36.4 & 36.4 & 36.4 & 36.4 & 36.4 & 36.4 & 36.4 & 36.4 & 36.4 & 36.4 & 36.4 & 36.4 & 36.4 & 582.4 & 1.2 \\
\hline \multirow[t]{3}{*}{ CH_LLW_III } & vor. & 24.8 & 24.8 & 24.8 & 21.8 & 21.8 & 21.8 & 21.8 & 21.8 & 21.8 & 21.8 & 24.8 & 21.8 & 21.8 & 24.8 & 24.8 & 396.8 & 1.5 \\
\hline & MIN & 22.4 & 12.4 & 12.4 & 12.4 & 12.1 & 12.4 & 12.1 & 12.4 & .12 .4 & 12.4 & 12.4 & 12.4 & 22.4 & 12.4 & 12.4 & 186.0 & 1.5 \\
\hline & $\max$ & 49.6 & 49.6 & 49.6 & 19.6 & 49.6 & 19.6 & 19.6 & 19.6 & 49.6 & 49.6 & 19.6 & 19.6 & 49.6 & 19.6 & 49.6 & 793.6 & 1.5 \\
\hline \multirow[t]{3}{*}{ CH_TRU } & VOL & 50.6 & 58.6 & 58.6 & 38.6 & 58.6 & 58.6 & 58.6 & 58.6 & 58.6 & 38.6 & 58.6 & 58.6 & 50.6 & 58.6 & 50.6 & 937.6 & 3.5 \\
\hline & MIN & 29.3 & 29.3 & 29.3 & 29.3 & 29.3 & 29.3 & 29.3 & 29.3 & 29.3 & 29.3 & 29.3 & 29.3 & 29.3 & 29.3 & 29.3 & 139.5 & 3.5 \\
\hline & $\max$ & 117.2 & 217.2 & 127.2 & 137.2 & 217.2 & 117.2 & 117.2 & 217.2 & 117.2 & 117.2 & 117.2 & 117.2 & 117.2 & 117.2 & 217.2 & 2875.2 & 3.5 \\
\hline \multirow[t]{3}{*}{ PH_TRU } & vor & 19.9 & 19.9 & 19.9 & 19.9 & 19.9 & 19.9 & 19.9 & 19.9 & 49.9 & 19.9 & 19.9 & 19.9 & 49.9 & 19.9 & 19.9 & 798.1 & 3.0 \\
\hline & MIN & 24.9 & 24.9 & 24.9 & 21.9 & 24.9 & 21.9 & 24.9 & 24.9 & 24.9 & 24.9 & 21.9 & 24.9 & 24.9 & 24.9 & 24.9 & 374.2 & 3.0 \\
\hline & $\max$ & 99.8 & 99.8 & 99.8 & 99.8 & 99.8 & 99.8 & 99.8 & 99.8 & 99.8 & 99.8 & 99.8 & 99.8 & 99.8 & 99.8 & 99.8 & 1596.8 & 3.0 \\
\hline \multirow[t]{3}{*}{ CH_IRUM } & vor & 58.6 & 58.6 & 58.6 & 58.6 & 58.6 & 58.6 & 38.6 & 58.6 & 58.6 & 58.6 & 58.6 & 58.6 & 38.6 & 58.6 & 58.6 & 937.6 & 3.5 \\
\hline & MIN & 29.3 & 29.3 & 29.3 & 29.3 & 29.3 & 29.3 & 29.3 & 29.3 & 29.3 & 29.3 & 29.3 & 29.3 & 29.3 & 29.3 & 29.3 & 139.5 & 3.5 \\
\hline & $\max$ & 117.2 & 117.2 & 117.2 & 117.2 & 217.2 & 117.2 & 117.2 & 217.2 & 117.2 & 117.2 & 117.2 & 117.2 & 117.2 & 117.2 & 117.2 & 1875.2 & 3.5 \\
\hline \multirow[t]{3}{*}{ RH_TRUSY } & vor & 56.1 & 56.1 & 56.1 & 56.1 & 56.1 & 56.1 & 56.1 & 56.1 & 56.1 & 56.1 & 56.1 & 56.1 & 56.1 & 36.1 & 56.1 & 097.6 & 3.3 \\
\hline & MIN & 28.0 & 28.0 & 28.0 & 28.0 & 28.0 & 28.0 & 28.0 & 28.0 & 28.0 & 28.0 & 28.0 & 28.0 & 28.0 & 28.0 & 28.0 & 420.7 & 3.3 \\
\hline & $\max$ & 112.2 & 112.2 & 112.2 & 112.2 & 112.2 & 112.2 & 112.2 & 112.2 & 112.2 & 112.2 & 112.2 & 112.2 & 122.2 & 112.2 & 112.2 & 1795.2 & 3.3 \\
\hline \multirow[t]{3}{*}{ HAZ } & vor & 4.7 & 1.7 & 1.7 & 4.7 & 1.7 & 8.7 & 4.7 & 1.7 & 1.7 & 6.7 & 6.7 & 1.7 & 4.7 & 4.7 & 1.7 & 75.2 & 0.2 \\
\hline & MIN & 2.3 & 2.3 & 2.3 & 2.3 & 2.3 & 2.3 & 2.3 & 2.3 & 2.3 & 2.3 & 2.3 & 2.3 & 2.3 & 2.3 & 2.3 & 35.2 & 0.2 \\
\hline & $\min$ & 9.4 & 9.1 & 9.4 & 9.4 & 9.4 & 9.1 & 9.1 & 9.1 & 9.1 & 9.1 & 9.1 & 9.1 & 9.1 & 9.4 & 9.4 & 150.4 & 0.2 \\
\hline \multirow{3}{*}{$\begin{array}{l}\text { NBC_ELYP } \\
\text { Total: }\end{array}$} & VoL & 1657.7 & 1657.7 & 1657.7 & 2657.7 & 2657.7 & 1657.7 & 1657.7 & 2657.7 & 1657.7 & 1657.7 & 1657.7 & 1657.7 & 1657.7 & 1657.7 & 1657.7 & 26523.2 & 100.0 \\
\hline & MIN & 028.8 & 828.8 & 828.0 & $028 . \theta$ & 828.8 & B28.8 & 820.8 & 028.8 & 828.8 & 828.8 & 828.8 & 828.0 & 028.8 & 028.8 & B28.8 & 12432.7 & 100.0 \\
\hline & $\max$ & 3313.4 & 3315.4 & 3315.4 & 3315.4 & 3315.4 & 3315.4 & 3315.6 & 3315.1 & 3315.4 & 3315.4 & 3315.1 & 3315.4 & 3315.4 & 3315.4 & 3315.4 & 53046.4 & 100.0 \\
\hline
\end{tabular}


EX-1994 SOLID WASTE FORECAST

WASTE GENERATOR, HASTE CLASS VOLOMES BY YEAR

(in cubic meters)

Fasto Clase

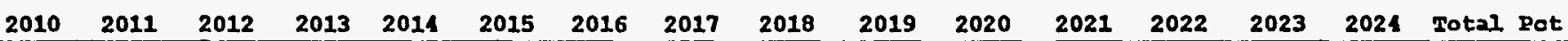

Waste Generator: FHC_ $\mathrm{K}$ BASIN

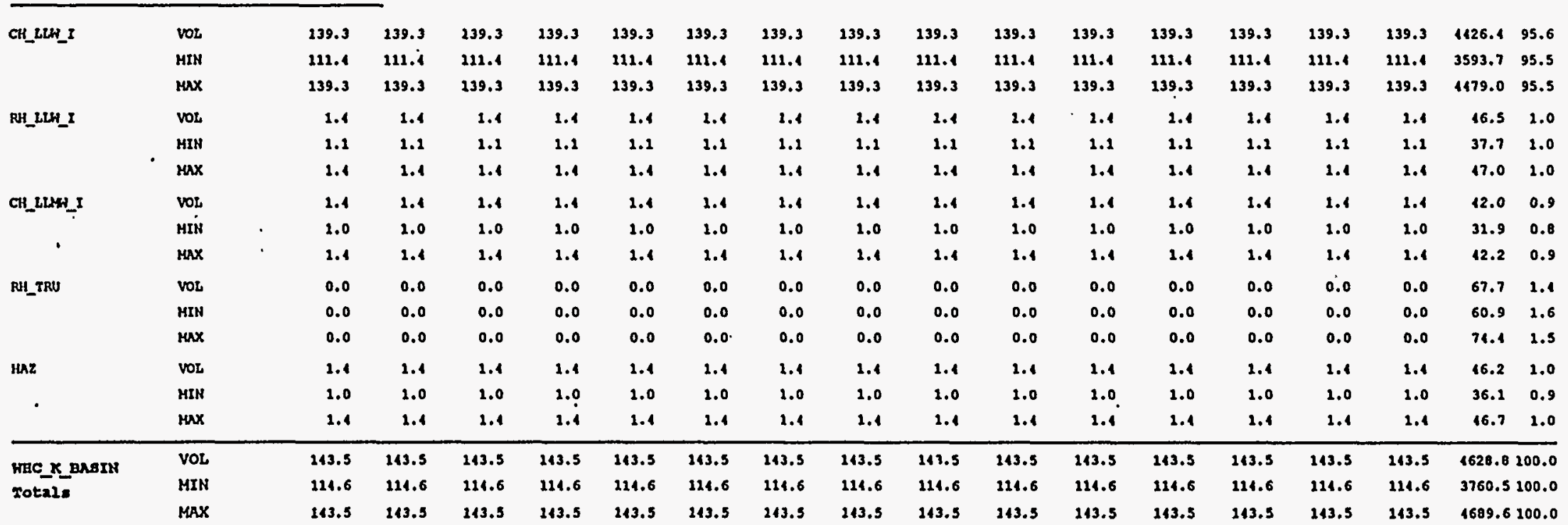

Wasto Generator: FHC_IAUNDRX

\begin{tabular}{|c|c|c|c|c|c|c|c|c|c|c|c|c|c|c|c|c|c|c|}
\hline \multirow[t]{3}{*}{$\mathrm{CH}_{-} \mathrm{LLH}-\mathrm{I}$} & Vol & 213.3 & 213.3 & 113.3 & 113.3 & 113.3 & 113.3 & 113.3 & 113.3 & 113.3 & 113.3 & 213.3 & 113.3 & 213.3 & 113.3 & 113.3 & 3399.0 & 99.7 \\
\hline & MIN & 113.3 & 113.3 & 113.3 & 113.3 & 113.3 & 113.3 & 113.3 & 113.3 & 113.3 & 113.3 & 113.3 & 113.3 & 113.3 & 113.3 & 113.3 & 3399.0 & 99.7 \\
\hline & $M X X$ & 224.6 & 224.6 & 224.6 & 124.6 & 124.6 & 121.6 & 124.6 & 124.6 & 124.6 & 121.6 & 121.6 & 124.6 & 124.6 & 124.6 & 121.6 & 3730.9 & 99.7 \\
\hline \multirow[t]{3}{*}{ HAZ } & vor & 0.3 & 0.3 & 0.3 & 0.3 & 0.3 & 0.3 & 0.3 & 0.3 & 0.3 & 0.3 & 0.3 & 0.3 & 0.3 & 0.3 & 0.3 & 9.0 & 0.2 \\
\hline & MNN & 0.3 & 0.3 & 0.3 & 0.3 & 0.3 & 0.3 & 0.3 & 0.3 & 0,3 & 0.3 & 0.3 & 0.3 & 0.3 & 0.3 & 0.3 & 9.0 & 0.2 \\
\hline & $\operatorname{MAX}$ & 0.3 & 0.3 & 0.3 & 0.3 & 0.3 & 0.3 & 0.3 & 0.3 & 0.3 & 0.3 & 0.3 & 0.3 & 0.3 & 0.3 & 0.3 & 9.0 & 0.2 \\
\hline \multirow{3}{*}{$\begin{array}{l}\text { HHC_LAURDRY } \\
\text { Total. }\end{array}$} & VOL & 113.6 & 113.6 & 213.6 & 113.6 & 113.6 & 113.6 & 113.6 & 113.6 & 113.6 & 113.6 & 113.6 & 113.6 & 213.6 & 113.6 & 213.6 & 3108.0 & 100.0 \\
\hline & MIN & 113.6 & 113.6 & 113.6 & 113.6 & 213.6 & 113.6 & 113.6 & 113.6 & 113.6 & 213.6 & 113.6 & 213.6 & 113.6 & 213.6 & 113.6 & 3408.0 & 100.0 \\
\hline & MAX & 124.9 & 124.9 & 124.9 & 124.9 & 124.9 & 124.9 & 124.9 & 124.9 & 124.9 & 121.9 & 124.9 & 124.9 & 124.9 & 124.9 & 221.9 & 3747.9 & 100.0 \\
\hline
\end{tabular}


FY-1994 SOLID HASTE FORECAST

WASTE GENERATOR, WASTE CLASS VOLOMES BY YEAR

(in cubic meters)

Wasto Clasa $2010 \quad 2011 \quad 2012$

$12 \quad 2013 \quad 2014 \quad 2015 \quad 2016 \quad 2017$

$\begin{array}{llllllll}2018 & 2019 & 2020 & 2021 & 2022 & 2023 & 2024 & \text { Total Pot }\end{array}$

Waste Generator: FHC_LIBG

\begin{tabular}{|c|c|c|c|c|c|c|c|c|c|c|c|c|c|c|c|c|c|c|}
\hline \multirow[t]{3}{*}{$\mathrm{CH}_{2} \mathrm{LLH}$-I } & vor & 6.3 & 6.3 & 6.3 & 6.3 & 6.3 & 6.3 & 6.3 & 6.3 & 6.3 & 6.3 & 6.3 & 6.3 & 6.3 & 6.3 & 6.3 & 189.0 & 86.0 \\
\hline & HIN & 6.3 & 6.3 & 6.3 & 6.3 & 6.3 & 6.3 & 6.3 & 6.3 & 6.3 & 6.3 & 6.3 & 6.3 & 6.3 & 6.3 & 6.3 & 189.0 & 06.0 \\
\hline & $\operatorname{HAX}$ & 6.3 & 6.3 & 6.3 & 6.3 & 6.3 & 0.3 & 6.3 & 6.3 & 6.3 & 6.3 & 6.3 & 6.3 & 6.3 & 6.3 & 6.3 & 189.0 & 86.0 \\
\hline \multirow[t]{3}{*}{ CH_LLWH_I } & vol & 0.0 & 0.0 & 3.4 & 0.0 & 0.0 & 3.1 & 0.0 & 0.0 & 3.1 & 0.0 & 0.0 & 3.4 & 0.0 & 0.0 & 0.0 & 30.6 & 13.9 \\
\hline & MIN & 0.0 & 0.0 & 3.4 & 0.0 & 0.0 & 3.1 & 0.0 & 0.0 & 3.1 & 0.0 & 0.0 & 3.4 & 0.0 & 0.0 & 0.0 & 30.6 & 13.9 \\
\hline & $\max$ & 0.0 & 0.0 & 3.4 & 0.0 & 0.0 & 3.1 & 0.0 & 0.0 & 3.4 & 0.0 & 0.0 & 3.4 & 0.0 & 0.0 & 0.0 & 30.6 & 13.9 \\
\hline \multirow{3}{*}{$\begin{array}{l}\text { KHC_InBo } \\
\text { Totail: }\end{array}$} & VOL & 6.3 & 6.3 & 9.7 & 6.3 & 6.3 & 9.7 & 6.3 & 6.3 & 9.7 & 6.3 & 6.3 & 9.7 & 6.3 & 6.3 & 6.3 & 219.6 & 100.0 \\
\hline & MIN & 6.3 & 6.3 & 9.7 & 6.3 & 6.3 & 9.7 & 6.3 & 6.3 & 9.7 & 6.3 & 6.3 & 9.7 & 6.3 & 6.3 & 6.3 & 219.6 & 100.0 \\
\hline & $\operatorname{MAX}$ & 6.3 & 6.3 & 9.7 & 6.3 & 6.3 & 9.7 & 6.3 & 6.3 & 9.7 & 6.3 & 6.3 & 9.7 & 6.3 & 6.3 & 6.3 & 219.6 & 100.0 \\
\hline
\end{tabular}

Wasto Generator: WHC_LIVP

\begin{tabular}{|c|c|c|c|c|c|c|c|c|c|c|c|c|c|c|c|c|c|c|c|}
\hline \multirow{21}{*}{$\begin{array}{l}0 \\
1 \\
\infty \\
\infty\end{array}$} & CH_LLW_I & Vot & 125.1 & 125.4 & 125.4 & 125.4 & 425.1 & 425.4 & 125.4 & 125.4 & 125.4 & 125.1 & 125.4 & 125.1 & $\$ 25.4$ & 125.4 & 425.4 & 8508.0 & 71.5 \\
\hline & & MXN & 212.7 & 212.7 & 212.7 & 212.7 & 212.7 & 212.7 & 212.7 & 212.7 & 212.7 & 212.7 & 212.7 & 212.7 & 212.7 & 212.7 & 212.7 & 1041.3 & 71.5 \\
\hline & & $\max$ & 850.8 & 850.8 & 850.8 & 850.0 & 850.8 & 850.0 & 850.0 & 850.8 & 050.8 & 850.8 & 850.8 & 850.0 & O50.8 & 850.8 & 850.8 & 27016.0 & 71.5 \\
\hline & CH_LLW_III & vor & 111.8 & 111.0 & 141.0 & 141.8 & 111.8 & 111.8 & 141.8 & 161.8 & 141.8 & 141.8 & 141.8 & 141.8 & 141.8 & 141.0 & 141.8 & 2836.0 & 23.8 \\
\hline & & MIN & 20.9 & 70.9 & 70.9 & 70.9 & 70.9 & 70.9 & 70.9 & 70.9 & 70.9 & 70.9 & 70.9 & 70.9 & 70.9 & 70.9 & 70.9 & 1347.1 & 23.8 \\
\hline & & $\operatorname{mAx}$ & 203.6 & 203.6 & 283.6 & 203.6 & 283.6 & 283.6 & 283.6 & 283.6 & 283.6 & 283.6 & 283.6 & 283.6 & 283.6 & 283.6 & 283.6 & 5672.0 & 23.0 \\
\hline & PH_LLH_III & vor & 21.8 & 21.8 & 21.8 & 21.8 & 21.0 & 21.6 & 21.8 & 21.8 & 21.8 & 21.8 & 21.0 & 21.8 & 21.0 & 21.8 & 21.8 & 136.0 & 3.6 \\
\hline & & HYM & 10.9 & 10.9 & 10.9 & 10.9 & 10.9 & 10.9 & 10.9 & 10.9 & 10.9 & 10.9 & 20.9 & 20.9 & 10.9 & 10.9 & 10.9 & 207.1 & 3.6 \\
\hline & & $\max$ & 13.6 & 43.6 & 43.6 & 13.6 & 13.6 & 13.6 & 43.6 & \$3.6 & 13.6 & 43.6 & , 13.6 & 43.6 & 13.6 & 43.6 & 13.6 & 872.0 & 3.6 \\
\hline & CH_LLWW_I & vot & 1.6 & 1.6 & 1.6 & 1.6 & 1.6 & 1.6 & 1.6 & 1.6 & 1.6 & 1.6 & 1.6 & 1.6 & 1.6 & 1.6 & 1.6 & 32.0 & 0.2 \\
\hline & & HIN & 0.8 & 0.8 & 0.0 & 0.8 & 0.8 & 0.8 & 0.8 & 0.8 & 0.8 & 0.8 & 0.0 & 0.8 & 0.8 & $0.8^{\prime}$ & 0.8 & 15.2 & 0.2 \\
\hline & & $\max$ & 3.2 & 3.2 & 3.2 & 3.2 & 3.2 & 3.2 & 3.2 & 3.2 & 3.2 & 3.2 & 3.2 & 3.2 & 3.2 & 3.2 & 3.2 & 64.0 & 0.2 \\
\hline & CH_ILWN_III & Vol & 1.0 & 1.0 & 1.0 & 1.0 & 1.0 & 1.0 & 1.0 & 1.0 & 1.0 & 1.0 & 1.0 & 1.0 & 1.0 & 1.0 & 1.0 & 20.0 & 0.1 \\
\hline & & HIN & 0.5 & 0.5 & 0.5 & 0.5 & 0.5 & 0.5 & 0.5 & 0.5 & 0.5 & 0.5 & 0.5 & 0.5 & 0.5 & 0.5 & 0.5 & 9.5 & 0.1 \\
\hline & & $\operatorname{mAx}$ & 2.0 & 2.0 & 2.0 & 2.0 & 2.0 & 2.0 & 2,0 & 2.0 & 2.0 & 2.0 & 2.0 & 2.0 & 2.0 & 2.0 & 2.0 & 10.0 & 0.1 \\
\hline & HAZ & vor & 3.1 & 3.4 & 3.1 & 3.1 & 3.4 & 3.4 & 3.4 & 3.4 & 3.4 & 3.4 & 3.4 & 3.4 & 3.1 & 3.4 & 3.4 & 68.0 & 0.5 \\
\hline & & MIN & 1.7 & 1.7 & 1.7 & 1.7 & 1.7 & 1.7 & 1.7 & 1.7 & 1.7 & 1.7 & 2.7 & 1.7 & 1.7 & 1.7 & 1.7 & 32.3 & 0.5 \\
\hline & & $\max$ & 6.8 & 6.8 & 6.8 & 6.8 & 6.8 & 6.0 & 6.8 & .6 .8 & 6.8 & 6.8 & 6.8 & 6.8 & 6.8 & 6.8 & 6.0 & 136.0 & 0.5 \\
\hline & भвС นLVP & VOL & 595.0 & 595.0 & 595.0 & 595.0 & 595.0 & 595.0 & 595.0 & 595.0 & 595.0 & 395.0 & 395.0 & 595.0 & 595.0 & 395.0 & 595.0 & 11900.0 & 100.0 \\
\hline & Total= & MIN & 297.5 & 297.5 & 297.5 & 297.5 & 297.5 & 297.5 & 297.5 & 297.5 & 297.3 & 297.5 & 297.5 & 297.5 & 297.5 & 297.5 & 297.5 & 5652.5 & 100.0 \\
\hline & & $\max$ & 1190.0 & 1190.0 & 1190.0 & 1190.0 & 1190.0 & 1190.0 & 1190.0 & 1190.0 & 1190.0 & 1290.0 & 2190.0 & 1190.0 & 1190.0 & 1190.0 & 1190.0 & 23800.0 & 100.0 \\
\hline
\end{tabular}


FY-1994 SOLID WASTE FORECAST

Page $37 b$

HASTE GENERATOR, WASTE CLASS VOLOMES BY YEAR

(in cubic meters)

Fasto Class

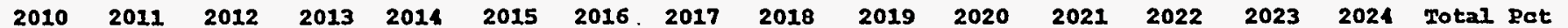

Haste Gonorator: FHC_PEP

\begin{tabular}{lllllllllllllllllllll}
\hline CH_LL_I & VOI & 41.6 & 41.6 & 11.6 & 13.6 & 11.6 & 27.6 & 27.6 & 27.6 & 27.6 & 27.6 & 27.6 & 27.6 & 27.6 & 27.6 & 27.6 & 1694.5 & 12.4
\end{tabular}

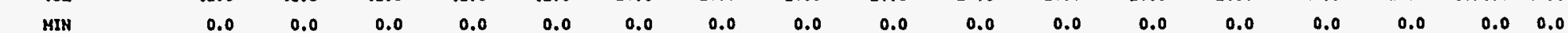

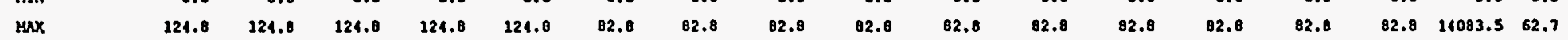

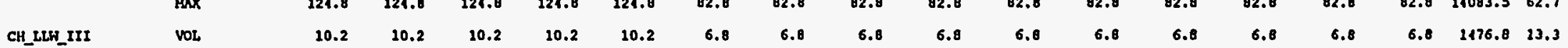

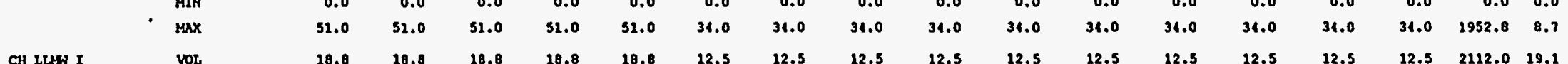

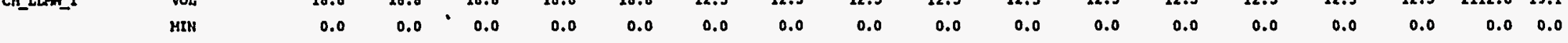

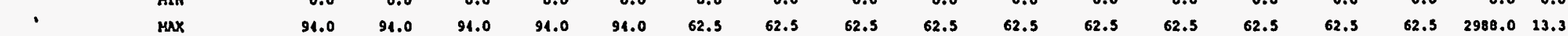

CH_LLE_III NOF

\begin{tabular}{|c|c|c|c|c|c|c|c|c|c|c|c|c|c|c|c|c|c|c|}
\hline & MIN & 0.0 & 0.0 & 0.0 & 0.0 & 0.0 & 0.0 & 0.0 & 0.0 & 0.0 & 0.0 & 0.0 & 0.0 & 0.0 & 0.0 & 0.0 & 0.0 & 0.0 \\
\hline & $\max$ & 3.0 & 5.0 & 5.0 & 5.0 & 5.0 & 3.0 & 3.0 & 3.0 & 3.0 & 3.0 & 3.0 & 3.0 & 3.0 & 3.0 & 3.0 & $101.0^{\circ}$ & 1.7 \\
\hline \multirow[t]{3}{*}{ CH_IRW } & Vor & 7.3 & 7.3 & 7.3 & 7.3 & 7.3 & 1.3 & 3.7 & 3.7 & 3.7 & 3.7 & 3.7 & 3.7 & 3.7 & 3.7 & 3.7 & 2651.4 & 15.0 \\
\hline & MIN & 0.0 & 0.0 & 0.0 & 0.0 & 0.0 & 0.0 & 0.0 & 0.0 & 0.0 & 0.0 & 0.0 & 0.0 & 0.0 & 0.0 & 0.0 & 0.0 & 0.0 \\
\hline & $\max$ & 36.5 & 36.5 & 36.5 & 36.5 & 36.5 & 36.5 & 18.5 & 18.5 & 18.5 & 18.5 & 18.5 & 18.5 & 18.5 & 10.5 & 18.5 & 1969.8 & 9.7 \\
\hline \multirow[t]{3}{*}{ CH_IRUM } & Vor & 0.0 & 0.0 & 0.0 & 0.0 & 0.0 & 0.0 & 0.0 & 0.0 & 0.0 & 0.0 & 0.0 & 0.0 & 0.0 & 0.0 & 0.0 & 365.8 & 3.3 \\
\hline & MIH & 0.0 & 0.0 & 0.0 . & 0.0 & 0.0 & 0.0 & 0.0 & 0.0 & 0.0 & 0.0 & 0.0 & 0.0 & 0.0 & 0.0 & 0.0 & 0.0 & 0.0 \\
\hline & $\operatorname{mux}$ & 0.0 & 0.0 & 0.0 & 0.0 & 0.0 & 0.0 & 0.0 & 0.0 & 0.0 & 0.0 & 0.0 & 0.0 & 0.0 & 0.0 & 0.0 & 365.0 & 1.6 \\
\hline \multirow[t]{3}{*}{ HAZ } & Vor & 0.1 & 0.1 & 0.1 & 0.1 & 0.1 & 0.1 & 0.1 & 0.1 & 0.1 & 0.1 & 0.1 & 0.1 & 0.1 & 0.1 & 0.1 & 675.0 & 6.1 \\
\hline & MIN & 0.0 & 0.0 & 0.0 & 0.0 & 0.0 & 0.0 & 0.0 & 0.0 & 0.0 & 0.0 & 0.0 & 0.0 & 0.0 & 0.0 & 0.0 & 0.0 & 0.0 \\
\hline & $\max$ & 0.5 & 0.5 & 0.5 & 0.5 & 0.5 & 0.5 & 0.5 & 0.5 & 0.5 & 0.5 & 0.5 & 0.5 & 0.5 & 0.5 & 0.5 & 681.0 & 3.0 \\
\hline \multirow{3}{*}{$\begin{array}{l}\text { HBC_PFP } \\
\text { Totals }\end{array}$} & VoL & 78.3 & 78.5 & 78.5 & 78.5 & 78.5 & 51.6 & 51.0 & 52.0 & 51.0 & 51.0 & 51.0 & 51.0 & 51.0 & 51.0 & 51.0 & 21060.2 & 100.0 \\
\hline & MIN & 0.0 & 0.0 & 0.0 & 0.0 & 0.0 & 0.0 & 0.0 & 0.0 & 0.0 & 0.0 & 0.0 & 0.0 & 0.0 & 0.0 & 0.0 & 0.0 & 100.0 \\
\hline & $\operatorname{Max}$ & 311.0 & 311.8 & 311.0 & 311.0 & 311.0 & 219.3 & 201.3 & 201.3 & 201.3 & 201.3 & 201.3 & 201.3 & 201.3 & 201.3 & 201.3 & 22441.9 & 00.0 \\
\hline
\end{tabular}

Waste Generator: HHC_PRETRT_TW

\begin{tabular}{|c|c|c|c|c|c|c|c|c|c|c|c|c|c|c|c|c|c|c|}
\hline \multirow[t]{3}{*}{ CH_LWWII } & VOI & 111.8 & 141.8 & 111.8 & 111.8 & 111.8 & 141.8 & 111.0 & 141.8 & 111.0 & 111.8 & 141.8 & 111.8 & 141.8 & 111.8 & 112.8 & 2977.8 & 17.1 \\
\hline & MIN & 70.9 & 70.9 & 70.9 & 70.9 & 10.9 & 70.9 & 70.9 & 70.9 & 70.9 & 70.9 & 70.9 & 70.9 & 70.9 & 70.9 & 70.9 & 2110.0 & 17.1 \\
\hline & $\max$ & 283.6 & 283.6 & 283.6 & 283.6 & 203.6 & 283.6 & 283.6 & 283.6 & 203.6 & 283.6 & 203.6 & 203.6 & 283.6 & 283.6 & 283.6 & 5955.6 & 47.1 \\
\hline \multirow[t]{2}{*}{ CH_LIW_III } & Vor & 141.8 & 111.0 & 141.0 & 111.8 & 111.8 & 141.0 & 141.8 & 141.8 & 141.0 & 141.0 & 141.8 & 142.0 & 141.8 & 141.0 & 141.8 & 2977.0 & 17.1 \\
\hline & $\max$ & 283.6 & 283.6 & 283.6 & 283.6 & 283.6 & 283.6 & 283.6 & 283.6 & 283.6 & 283.6 & 283.6 & 203.6 & 283.6 & 283.6 & 283.6 & 5955.6 & 67.2 \\
\hline
\end{tabular}




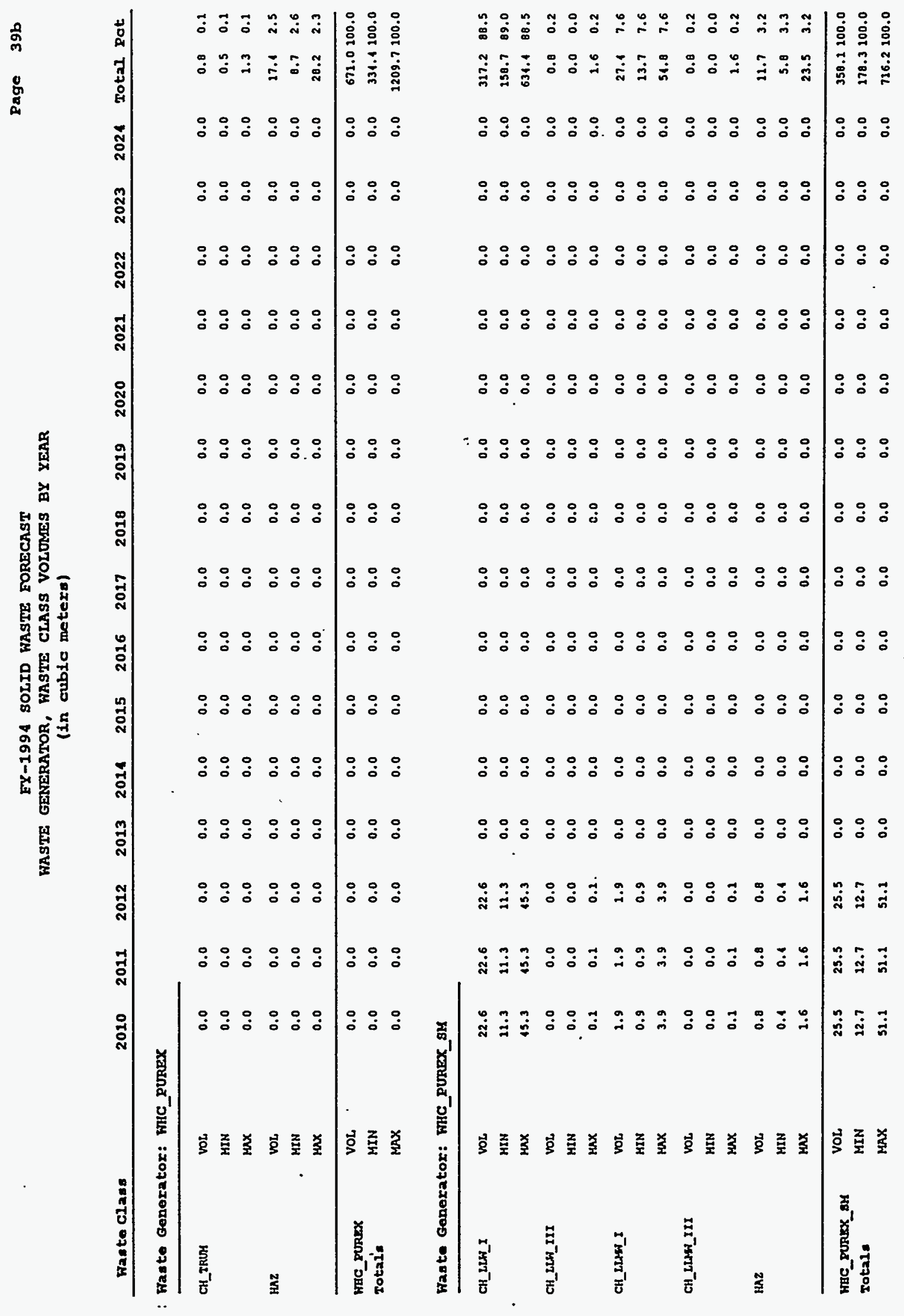




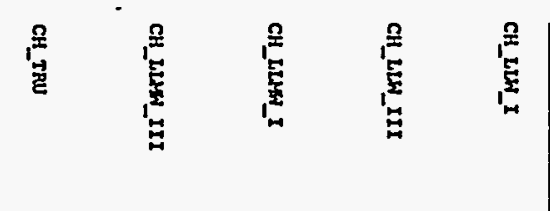

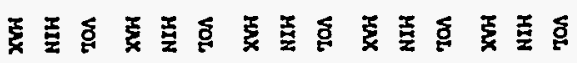

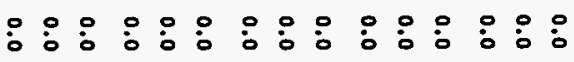

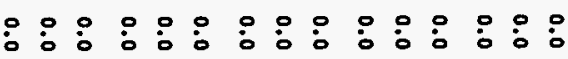

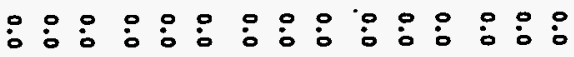

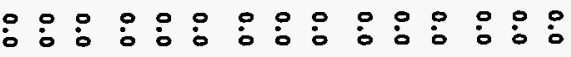

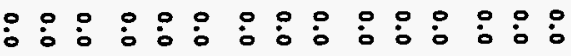

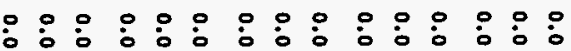

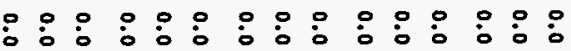

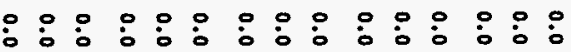

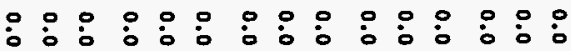

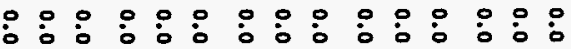

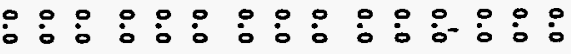

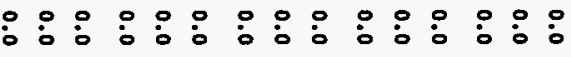

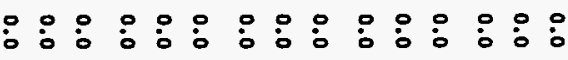

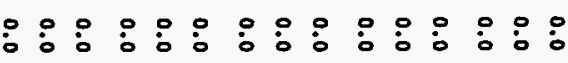

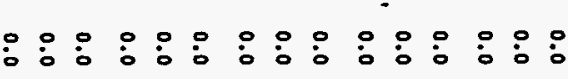

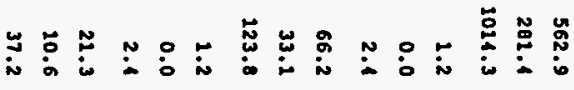

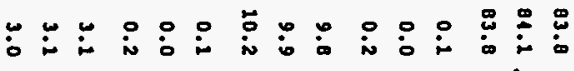

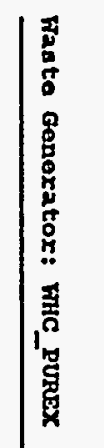

要要

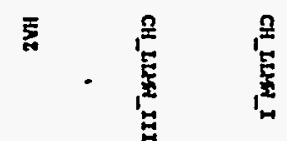

焉

8

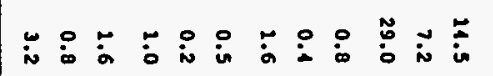

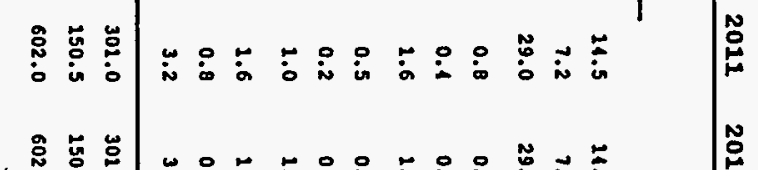

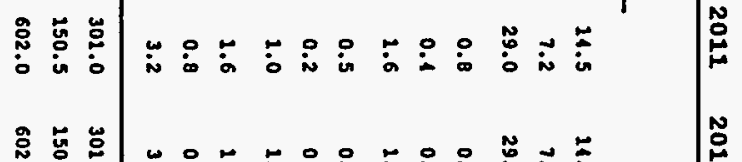

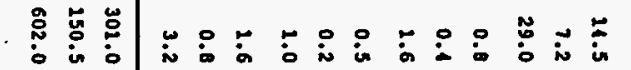

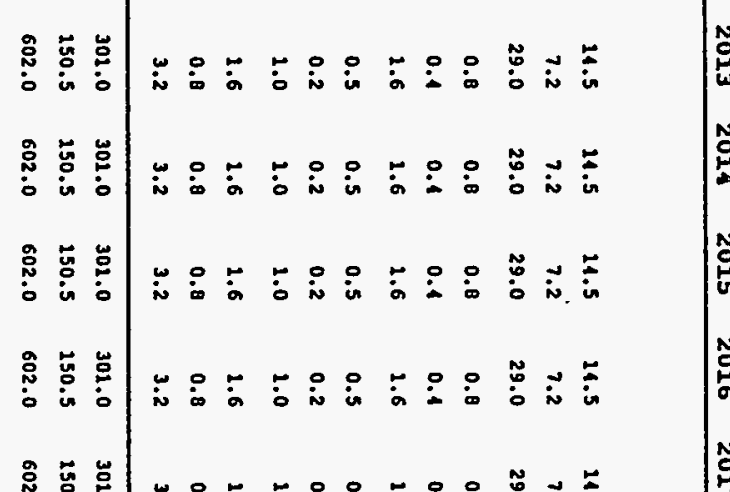

隶

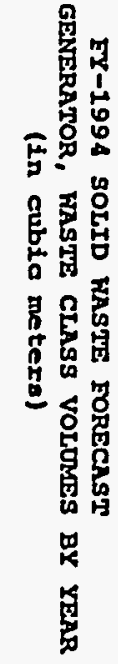

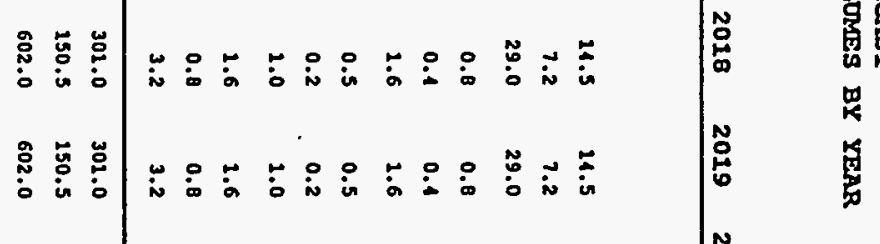

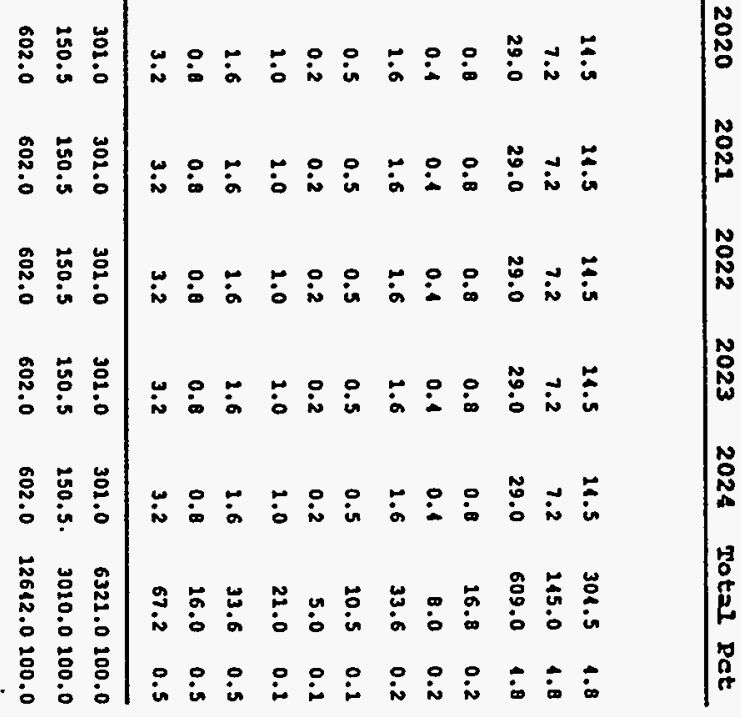

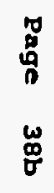


WHC-EP-0865

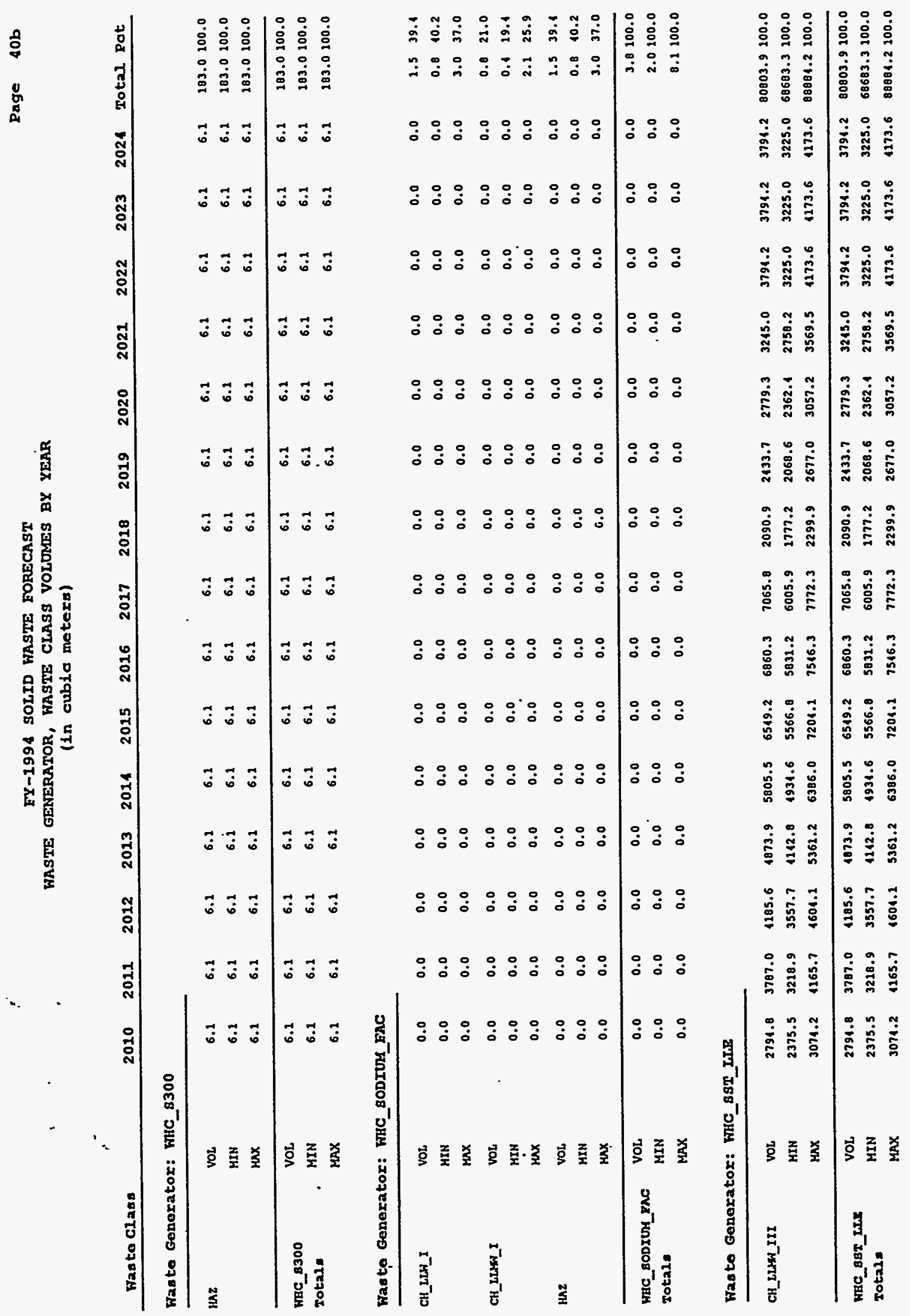


WHC-EP-0865

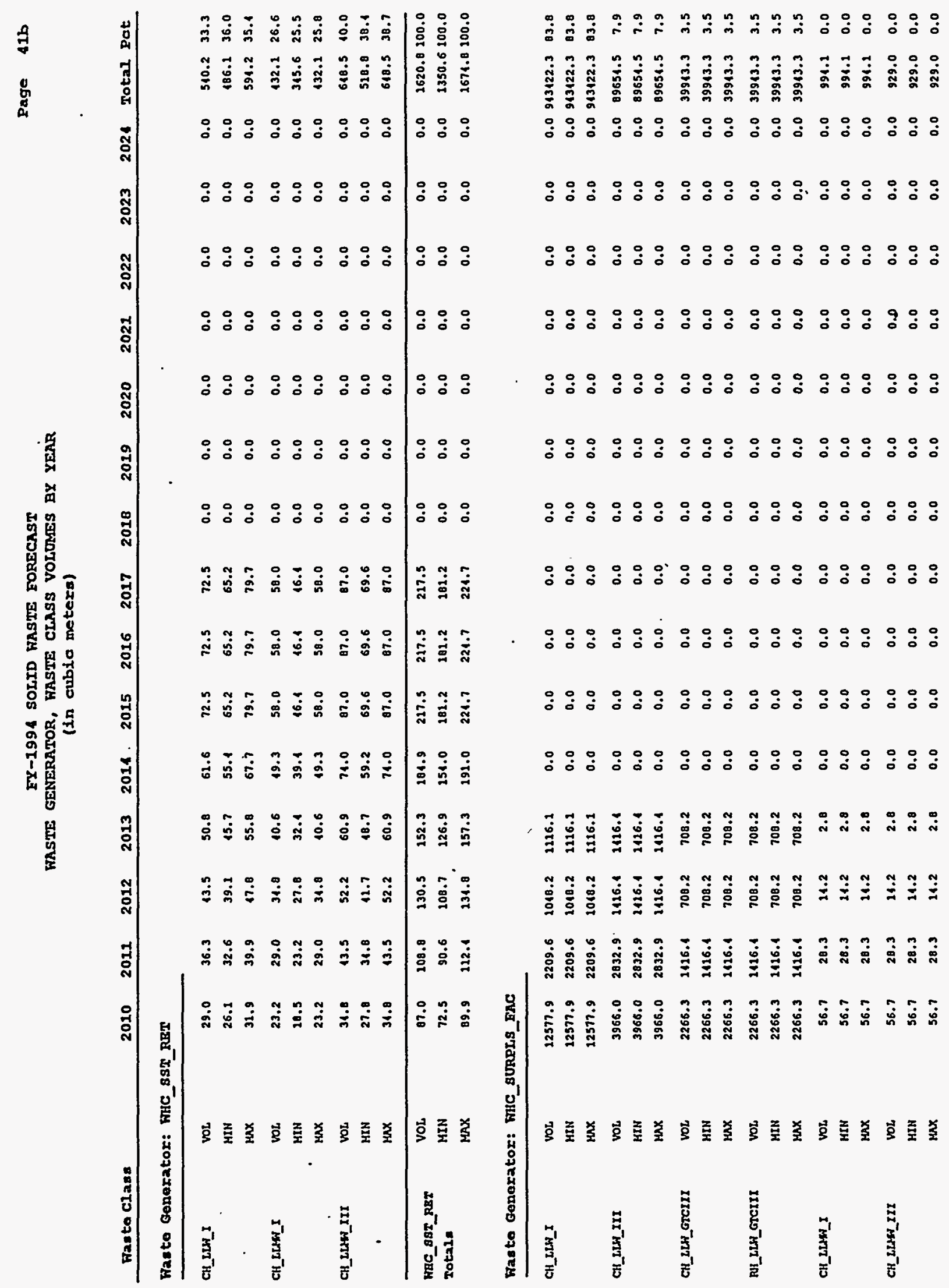


EY-1994 SOLID WASTE FORECAST

WASTE GENERATOR, WASTE CLASS VOLUMES BY YEAR

(in cubia meters)

Haste Class

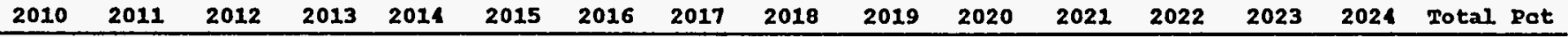

\begin{tabular}{|c|c|c|c|c|c|c|c|c|c|c|c|c|c|c|c|c|c|c|}
\hline \multirow[t]{3}{*}{ CH_LLWW_GTCIII } & Vor & 56.7 & 28.3 & 14.2 & 2.8 & 0.0 & 0.0 & 0.0 & 0.0 & 0.0 & 0.0 & 0.0 & 0.0 & 0.0 & 0.0 & 0.0 & 923.3 & 0.0 \\
\hline & MIN & 56.7 & 28.3 & 11.2 & 2.8 & 0.0 & 0.0 & 0.0 & 0.0 & 0.0 & 0.0 & 0.0 & 0.0 & 0.0 & 0.0 & 0.0 & 923.3 & 0.0 \\
\hline & $\max$ & 56.7 & 28.3 & 14.2 & 2.8 & 0.0 & 0.0 & 0.0 & 0.0 & 0.0 & 0.0 & 0.0 & 0.0 & 0.0 & 0.0 & 0.0 & 923.3 & 0.0 \\
\hline \multirow[t]{3}{*}{ RH_LLMH_I } & VoL & 56.7 & 28.3 & 24.2 & 2.8 & 0.0 & 0.0 & 0.0 & 0.0 & 0.0 & 0.0 & 0.0 & 0.0 & 0.0 & 0.0 & 0.0 & 923.3 & 0.0 \\
\hline & HIN & 56.7 & 28.3 & 14.2 & 2.8 & 0.0 & 0.0 & 0.0 & 0.0 & 0.0 & 0.0 & 0.0 & 0.0 & 0.0 & 0.0 & 0.0 & 923.3 & 0.0 \\
\hline & $\max$ & 56.7 & 28.3 & 21.2 & 2.8 & 0.0 & 0.0 & 0.0 & 0.0 & 0.0 & 0.0 & 0.0 & 0.0 & 0.0 & 0.0 & 0.0 & 923.3 & 0.0 \\
\hline \multirow[t]{3}{*}{ PH_LLWF_IIII } & vor & 36.7 & 28.3 & 14.2 & 2.8 & 0.0 & 0.0 & 0.0 & 0.0 & 0.0 & 0.0 & 0.0 & 0.0 & 0.0 & 0.0 & 0.0 & 923.3 & 0.0 \\
\hline & MIN & 36.7 & 28.3 & 14.2 & 2.8 & 0.0 & 0.0 & 0.0 & 0.0 & 0.0 & 0.0 & 0.0 & 0.0 & 0.0 & 0.0 & 0.0 & 923.3 & 0.0 \\
\hline & $\sin x$ & 56.7 & 28.3 & 14.2 & 2.8 & 0.0 & 0.0 & 0.0 & 0.0 & 0.0 & 0.0 & 0.0 & 0.0 & 0.0 & 0.0 & 0.0 & 923.3 & 0.0 \\
\hline \multirow[t]{3}{*}{ RH_LLW_GTCIII } & vor & 56.7 & 28.3 & 14.2 & 2.0 & 0.0 & 0.0 & 0.0 & 0.0 & 0.0 & 0.0 & 0.0 & 0.0 & 0.0 & 0.0 & 0.0 & 923.3 & 0.0 \\
\hline & MIN & 56.7 & 28.3 & 11.2 & 2.8 & 0.0 & 0.0 & 0.0 & 0.0 & 0.0 & 0.0 & 0.0 & 0.0 & 0.0 & 0.0 & 0.0 & 923.3 & 0.0 \\
\hline & $\max$ & 56.7 & 28,3 & 14.2 & 2.8 & 0.0 & 0.0 & 0.0 & 0.0 & 0.0 & 0.0 & 0.0 & 0.0 & 0.0 & 0.0 & 0.0 & 923.3 & 0.0 \\
\hline \multirow[t]{3}{*}{ CH_IRU } & vor & 28.3 & 28.3 & 28.3 & 28.3 & 0.0 & 0.0 & 0.0 & 0.0 & 0.0 & 0.0 & 0.0 & 0.0 & 0.0 & 0.0 & 0.0 & 1132.8 & 0.1 \\
\hline & MIN & 28.3 & 28.3 & 28.3 & 28.3 & 0.0 & 0.0 & 0.0 & 0.0 & 0.0 & 0.0 & 0.0 & 0.0 & 0.0 & 0.0 & 0.0 & 2132.8 & 0.1 \\
\hline & $\max$ & 28.3 & 28.3 & 20.3 & 28.3 & 0.0 & 0.0 & 0.0 & 0.0 & 0.0 & 0.0 & 0.0 & 0.0 & 0.0 & 0.0 & 0.0 & $2152 . \theta$ & 0.1 \\
\hline \multirow[t]{3}{*}{ RH_TRU } & vor & 0.0 & 0.0 & 0.0 & 0.0 & 0.0 & 0.0 & 0.0 & 0.0 & 0.0 & 0.0 & 0.0 & 0.0 & 0.0 & 0.0 & 0.0 & 2.8 & 0.0 \\
\hline & MIM & 0.0 & 0.0 & 0.0 & 0.0 & 0.0 & 0.0 & 0.0 & 0.0 & 0.0 & 0.0 & 0.0 & 0.0 & 0.0 & 0.0 & 0.0 & 2.8 & 0.0 \\
\hline & $\max$ & 0.0 & 0.0 & 0.0 & 0.0 & 0.0 & 0.0 & 0.0 & 0.0 & 0.0 & 0.0 & 0.0 & 0.0 & 0.0 & 0.0 & 0.0 & 2.8 & 0.0 \\
\hline \multirow[t]{3}{*}{ CH_TRUM } & vol & 20.3 & 28.3 & 28.3 & 28.3 & 0.0 & 0.0 & 0.0 & 0.0 & 0.0 & 0.0 & 0.0 & 0.0 & 0.0 & 0.0 & 0.0 & 673.9 & 0.0 \\
\hline & MIN & 28.3 & 28.3 & 28.3 & 28.3 & 0.0 & 0.0 & 0.0 & 0.0 & 0.0 & 0.0 & 0.0 & 0.0 & 0.0 & 0.0 & 0.0 & 673.9 & 0.0 \\
\hline & $\max$ & 28.3 & 28.3 & 28.3 & 28.3 & 0.0 & 0.0 & 0.0 & 0.0 & 0.0 & 0.0 & 0.0 & 0.0 & 0.0 & 0.0 & 0.0 & 673.9 & 0.0 \\
\hline \multirow[t]{3}{*}{ RH_TRUH } & vor & 28.3 & 28.3 & 28.3 & 28.3 & 0.0 & 0.0 & 0.0 & 0.0 & 0.0 & 0.0 & 0.0 & 0.0 & 0.0 & 0.0 & 0.0 & 708.1 & 0.0 \\
\hline & MIN & 28.3 & 28.3 & 28.3 & 28.3 & 0.0 & 0.0 & 0.0 & 0.0 & 0.0 & 0.0 & 0.0 & 0.0 & 0.0 & 0.0 & 0.0 & 708.1 & 0.0 \\
\hline & MNX & 28.3 & 28.3 & 28.3 & 28.3 & 0.0 & 0.0 & 0.0 & 0.0 & 0.0 & 0.0 & 0.0 & 0.0 & 0.0 & 0.0 & 0.0 & 708.1 & 0.0 \\
\hline \multirow[t]{3}{*}{ Haz } & VOL & 28.3 & 28.3 & 28.3 & 28.3 & 0.0 & 0.0 & 0.0 & 0.0 & 0.0 & 0.0 & 0.0 & 0.0 & 0.0 & 0.0 & 0.0 & 3116.2 & 0.3 \\
\hline & HIN & 28,3 & 28,3 & 28,3 & 28.3 & 0.0 & 0.0 & 0.0 & 0.0 & 0.0 & 0.0 & 0.0 & 0.0 & 0.0 & 0.0 & 0.0 & 3416.2 & 0.3 \\
\hline & $\operatorname{mAx}$ & 28.3 & 28.3 & 28.3 & 28.3 & 0.0 & 0.0 & 0.0 & 0.0 & 0.0 & 0.0 & 0.0 & 0.0 & 0.0 & 0.0 & 0.0 & 3416.2 & 0.3 \\
\hline \multirow{3}{*}{$\begin{array}{l}\text { KaC_BURPLS_FAC } \\
\text { Totals }\end{array}$} & Vor & 21529.9 & 8158.3 & 4079.1 & 1078.9 & 0.0 & 0.0 & 0.0 & 0.0 & 0.0 & 0.0 & 0.0 & 0.0 & 0.0 & 0.0 & $0.0 \mathrm{~J}$ & 124533.5 & 100.0 \\
\hline & MIN & 21529.9 & 8158.3 & 4079.4 & 1078.9 & 0.0 & 0.0 & 0.0 & 0.0 & 0.0 & 0.0 & 0.0 & 0.0 & 0.0 & 0.0 & 0.0 & 124533.5 & 100.0 \\
\hline & $\operatorname{MAX}$ & 21529.9 & 8158.3 & 1079.4 & 1078.9 & 0.0 & 0.0 & 0.0 & 0.0 & 0.0 & 0.0 . & 0.0 & 0.0 & 0.0 & 0.0 & 0.0 & 124533.5 & 100.0 \\
\hline
\end{tabular}




\section{Was te Genorator: WHC_TR_OPER}

\begin{tabular}{|c|c|c|c|c|c|c|c|c|c|c|c|c|c|c|c|c|c|c|}
\hline \multirow[t]{3}{*}{ CH_ILH_I } & VoL & 290.9 & 298.9 & 298.9 & 298.9 & 298.9 & 298.9 & 298.9 & 298.9 & 298.9 & 298.9 & 298.9 & 298.9 & 298.9 & 298.9 & 298.9 & 8939.8 & 57.0 \\
\hline & MIN & 179.3 & 279.3 & 279.3 & 179.3 & 179.3 & 179.3 & 279.3 & 179.3 & 279.3 & 179.3 & 279.3 & 279.3 & 279.3 & 179.3 & 179.3 & 5363.8 & 19.9 \\
\hline & $\max$ & 1246.4 & 1246.4 & 1246.4 & 1246.1 & 1246.1 & 1246.1 & 1216.1 & 1216.1 & 1246.4 & 1216.4 & 1246.4 & 1246.4 & 1246.1 & 1246.1 & 1246.1 & 37278.8 & 84.7 \\
\hline \multirow[t]{2}{*}{ CH_LLH_I } & VoL & 130.9 & 130.9 & 130.9 & 230.9 & 230.9 & 130.9 & 130.9 & 130.9 & 130.9 & 130.9 & 130.9 & 130.9 & 230.9 & 130.9 & 130.9 & 3915.1 & 25.0 \\
\hline & $\max$ & 130.9 & 130.9 & 130.9 & 130.9 & 130.9 & 130.9 & 230.9 & 130.9 & 230.9 & 130.9 & 130.9 & 230.9 & 130.9 & 130.9 & 130.9 & 3915.1 & 8.9 \\
\hline \multirow[t]{2}{*}{ CH_LLH_IIII } & vol & 56.1 & 36.1 & 56.1 & 56.1 & 56.2 & 56.1 & 36.1 & 56.1 & 56.1 & 56.1 & 36.1 & 36.1 & 36.1 & 56.1 & 36.1 & 1677.9 & 10.7 \\
\hline & HIN & 41.8 & 14.8 & 11.8 & 11.8 & 44.8 & 14.0 & 14.8 & 14.8 & 11.8 & 11.8 & 14.8 & 11.8 & 11.0 & 11.8 & 14.8 & 1342.3 & 12.5 \\
\hline - & $\max$ & 56.1 & 36.2 & 56.1 & 36.2 & 56.1 & 56.1 & 56.2 & 56.1 & 56.1 & 56.1 & 36.1 & 36.1 & 56.1 & 56.1 & 56.1 & 1677.9 & 3.8 \\
\hline \multirow[t]{3}{*}{$\operatorname{maz}$} & Vor & 37.8 & 37.8 & 37.8 & 37.8 & 37.0 & 37.0 & 37.0 & 37.8 & 37.8 & 37.0 & 37.8 & 37.8 & 37.0 & 37.8 & 37.8 & 1130.5 & 7.2 \\
\hline & MIN & 30.2 & 30.2 & 30.2 & 30.2 & 30.2 & 30.2 & 30.2 & 30.2 & 30.2 & 30.2 & 30.2 & 30.2 & 30.2 & 30.2 & 30.2 & 901.4 & 8.4 \\
\hline & $\max$ & 37.8 & 37.0 & 37.8 & 37.8 & 37.8 & 37.8 & 37.8 & 37.8 & 37.8 & 37.8 & 37.6 & 37.8 & 37.0 & 37.0 & 37.8 & 1130.5 & 2.5 \\
\hline \multirow{3}{*}{$\begin{array}{l}\text { WBC_IF_OPER } \\
\text { Total. }\end{array}$} & VOL & 523.7 & 523.7 & 523.7 & 323.7 & 323.7 & 323.7 & 523.7 & 523.7 & 323.7 & 523.7 & 523.7 & 523.7 & 523.7 & 523.7 & 523.7 & 25663.3 & 100.0 \\
\hline & MIN & 359.1 & 359.1 & 359.2 & 359.1 & 359.1 & 359.1 & 359.1 & 359.1 & 359.1 & 359.1 . & 359.1 & 359.1 & 359.1 & 359.1 & 359.1 & 20712.6 & 200.0 \\
\hline & $\operatorname{MAX}$ & 1471.2 & 1471.2 & 1171.2 & 1471.2 & 1471.2 & 1471.2 & 1471.2 & 1471.2 & 1171.2 & 1471.2 & 2471.2 & 1471.2 & 1471.2 & 1471.2 & 2171.2 & 11002.3 & 200.0 \\
\hline
\end{tabular}

Was to Generator: HHC__RUSAS

\begin{tabular}{|c|c|c|c|c|c|c|c|c|c|c|c|c|c|c|c|c|c|c|}
\hline \multirow[t]{3}{*}{$\mathrm{CH}_{-} \mathrm{LLH} \mathrm{I}$} & vols & 0.0 & 0.0 & 0.0 & 0.0 & 0.0 & 0.0 & 0.0 & 0.0 & 0.0 & 0.0 & 0.0 & 0.0 & 0.0 & 0.0 & 0.0 & 10.6 & 78.5 \\
\hline & HIM & 0.0 & 0.0 & 0.0 & 0.0 & 0.0 & 0.0 & 0.0 & 0.0 & 0.0 & 0.0 & 0.0 & 0.0 & 0.0 & 0.0 & 0.0 & 10.6 & 70.5 \\
\hline & $\max$ & 0.0 & 0.0 & 0.0 & 0.0 & 0.0 & 0.0 & 0.0 & 0.0 & 0.0 & 0.0 & 0.0 & 0.0 & 0.0 & 0.0 & 0.0 & 10.6 & 78.5 \\
\hline \multirow[t]{3}{*}{ CH_LLW_I } & vol & 0.0 & 0.0 & 0.0 & 0.0 & 0.0 & 0.0 & 0.0 & 0.0 & 0.0 & 0.0 & 0.0 & 0.0 & 0.0 & 0.0 & 0.0 & 2.9 & 21.1 \\
\hline & HIN & 0.0 & 0.0 & 0.0 & 0.0 & 0.0 & 0.0 & 0.0 & 0.0 & 0.0 & 0.0 & 0.0 & 0.0 & 0.0 & 0.0 & 0.0 & 2.9 & 21.1 \\
\hline & $\max$ & 0.0 & 0.0 & 0.0 & 0.0 & 0.0 & 0.0 & 0.0 & 0.0 & 0.0 & 0.0 & 0.0 & 0.0 & 0.0 & 0.0 & 0.0 & 2.9 & 21.1 \\
\hline \multirow{2}{*}{$\begin{array}{l}\text { भвс_тRugar } \\
\text { Totals }\end{array}$} & VoL & 0.0 & 0.0 & 0.0 & 0.0 & 0.0 & 0.0 & 0.0 & 0.0 & 0.0 & 0.0 & 0.0 & 0.0 & 0.0 & 0.0 & 0.0 & 13.5 & 100.0 \\
\hline & MIN & 0.0 & 0.0 & 0.0 & 0.0 & 0.0 & 0.0 & 0.0 & 0.0 & 0.0 & 0.0 & 0.0 & 0.0 & 0.0 & 0.0 & 0.0 & 13.5 & 200.0 \\
\hline
\end{tabular}

Waste Genoratox: FHC_IFP_FO2B

\begin{tabular}{|c|c|c|c|c|c|c|c|c|c|c|c|c|c|c|c|c|c|}
\hline \multirow[t]{3}{*}{$\mathrm{CH}_{-} \mathrm{LLH}+\mathrm{I}$} & Vor & 0.0 & 0.0 & 0.0 & 0.0 & 0.0 & 0.0 & 0.0 & 0.0 & 0.0 & 0.0 & 0.0 & 0.0 & 0.0 & 0.0 & 0.0 & 1.1200 .0 \\
\hline & MIN & 0.0 & 0.0 & 0.0 & 0.0 & 0.0 & 0.0 & 0.0 & 0.0 & 0.0 & 0.0 & 0.0 & 0.0 & 0.0 & 0.0 & 0.0 & 7.4100 .0 \\
\hline & $\operatorname{mx}$ & 0.0 & 0.0 & 0.0 & 0.0 & 0.0 & 0.0 & 0.0 & 0.0 & 0.0 & 0.0 & 0.0 & 0.0 & 0.0 & 0.0 & .0 .0 & $55.5 \quad 200.0$ \\
\hline
\end{tabular}


WHC-EP-0865

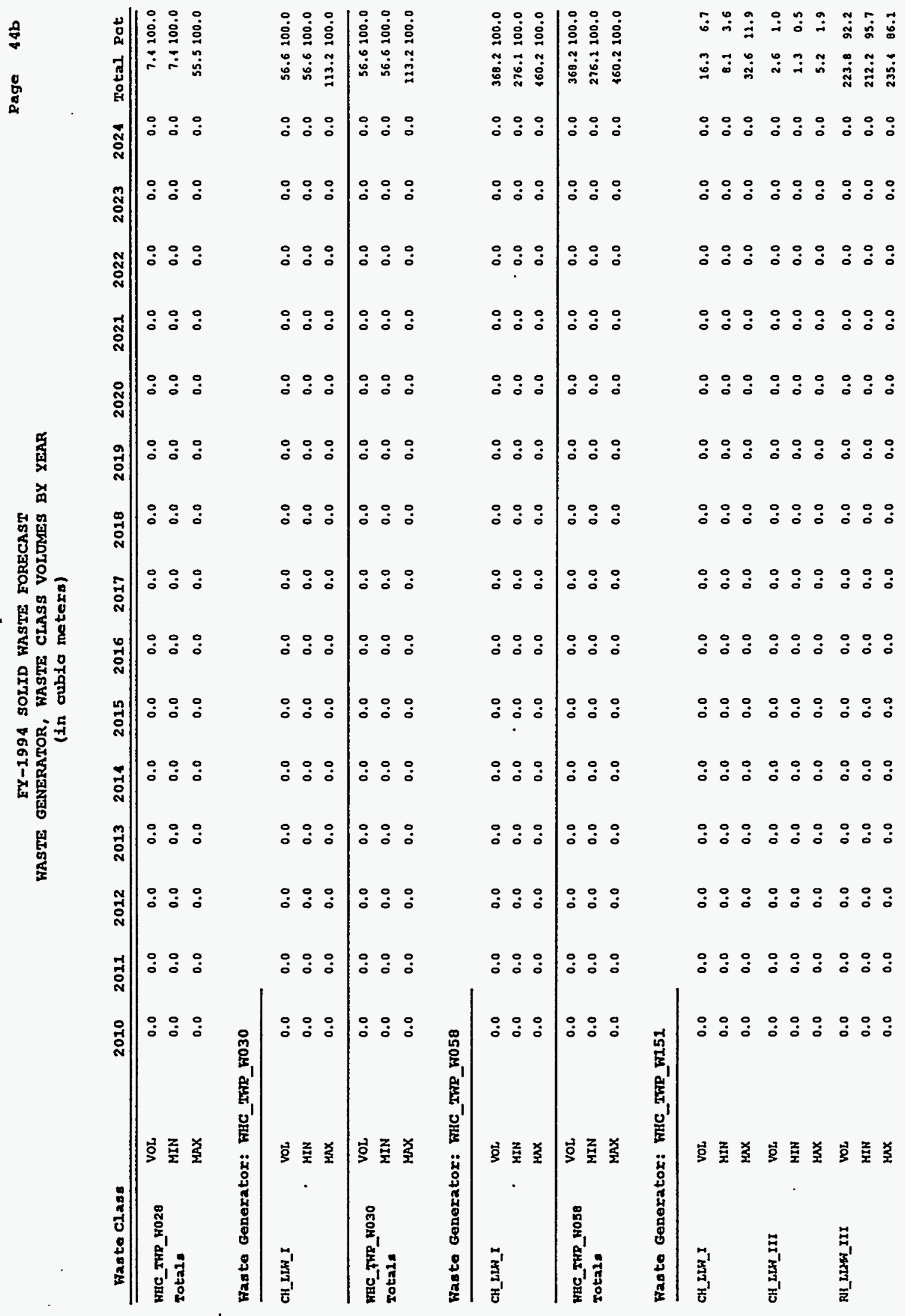




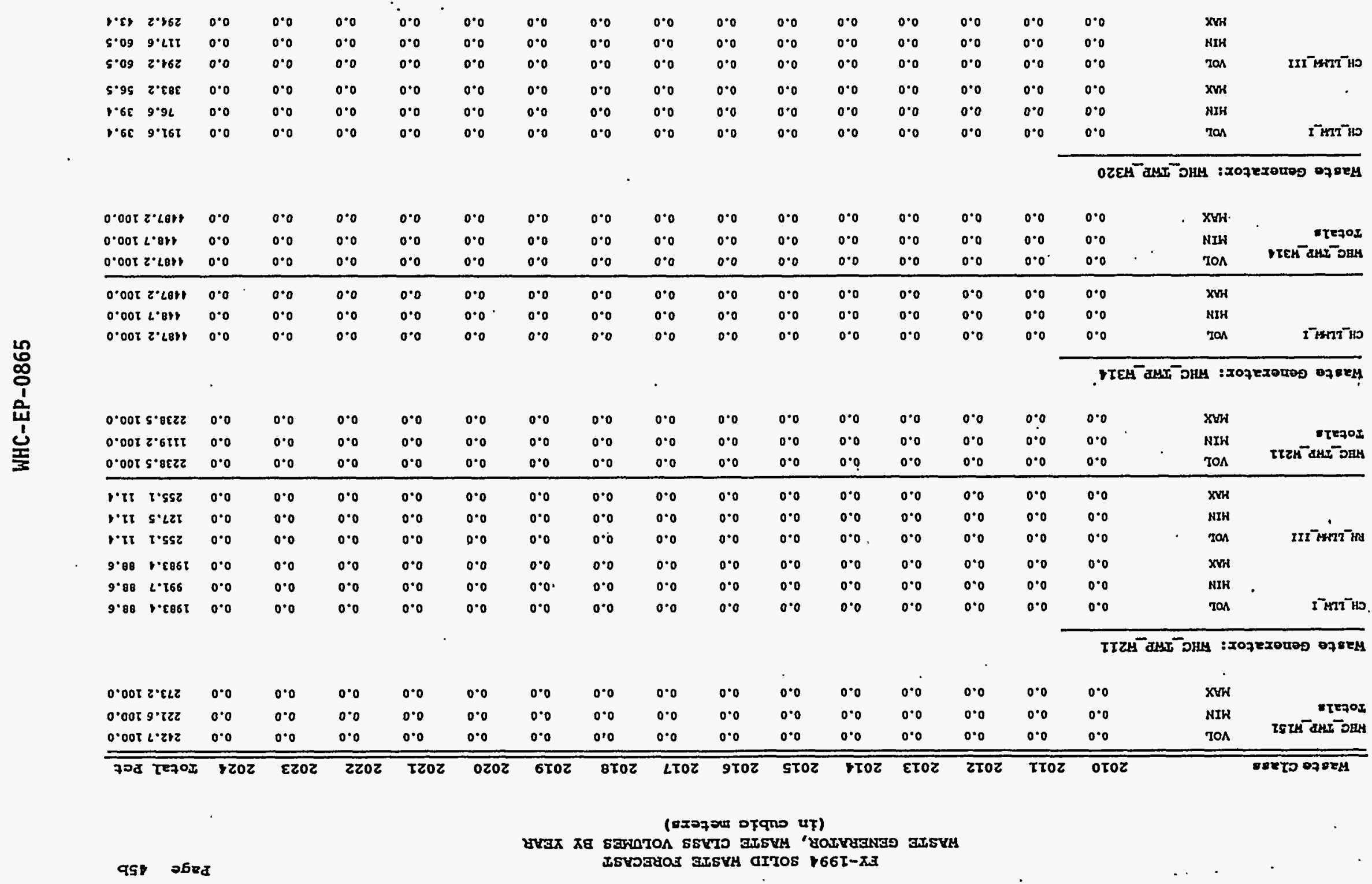


FY-1994 SOLID HASTE FORECAST

WASTE GENERATOR, WASTE CLASS VOLTMES BY YEAR (in cubic meters)

Wasto Class

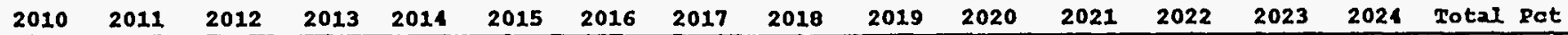

\begin{tabular}{|c|c|c|c|c|c|c|c|c|c|c|c|c|c|c|c|c|c|}
\hline \multirow{3}{*}{$\begin{array}{l}\text { HAC_TWP_Y320 } \\
\text { Total. }\end{array}$} & VoL & 0.0 & 0.0 & 0.0 & 0.0 & 0.0 & 0.0 & 0.0 & 0.0 & 0.0 & 0.0 & 0.0 & 0.0 & 0.0 & 0.0 & 0.0 & 185.8100 .0 \\
\hline & MIN & 0.0 & 0.0 & 0.0 & 0.0 & 0.0 & 0.0 & 0.0 & 0.0 & 0.0 & 0.0 & 0.0 & 0.0 & 0.0 & 0.0 & 0.0 & 294.3100 .0 \\
\hline & $\operatorname{Max}$ & 0.0 & 0.0 & 0.0 & 0.0 & 0.0 & 0.0 & 0.0 & 0.0 & 0.0 & 0.0 & 0.0 & 0.0 & 0.0 & 0.0 & 0.0 & 677.1100 .0 \\
\hline
\end{tabular}

พaste Generator: WHC IFP W310

\begin{tabular}{|c|c|c|c|c|c|c|c|c|c|c|c|c|c|c|c|c|c|c|}
\hline \multirow{3}{*}{$\mathrm{CH}_{-} \mathrm{LLW}-\mathrm{I}$} & VoL & 0.0 & 0.0 & 0.0 & 0.0 & 0.0 & 0.0 & 0.0 & 0.0 & 0.0 & 0.0 & 0.0 & 0.0 & 0.0 & 0.0 & 0.0 & 7.8 & 56.1 \\
\hline & MIN & 0.0 & 0.0 & 0.0 & 0.0 & 0.0 & 0.0 & 0.0 & 0.0 & 0.0 & .0 .0 & 0.0 & 0.0 & 0.0 & 0.0 & 0.0 & 0.0 & 0.0 \\
\hline & $\max$ & 0.0 & 0.0 & 0.0 & 0.0 & 0.0 & 0.0 & 0.0 & 0.0 & 0.0 & 0.0 & 0.0 & 0.0 & 0.0 & 0.0 & 0.0 & 15.6 & 67.1 \\
\hline \multirow{2}{*}{ RH_LLH_I } & Vor. & 0.0 & 0.0 & 0.0 & 0.0 & 0.0 & 0.0 & 0.0 & 0.0 & 0.0 & 0.0 & 0.0 & 0.0 & 0.0 & 0.0 & 0.0 & 6.1 & 13.8 \\
\hline & $\max$ & 0.0 & 0.0 & 0.0 & 0.0 & 0.0 & 0.0 & 0.0 & 0.0 & 0.0 & 0.0 & 0.0 & 0.0 & 0.0 & 0.0 & 0.0 & 7.6 & 32.0 \\
\hline \multirow{3}{*}{$\begin{array}{l}\text { HBC_2WP_H340 } \\
\text { Total: }\end{array}$} & VoL. & 0.0 & 0.0 & 0.0 & 0.0 & 0.0 & 0.0 & 0.0 & 0.0 & 0.0 & 0.0 & 0.0 & 0.0 & 0.0 & 0.0 & 0.0 & 83.9 & 200.0 \\
\hline & MIN & 0.0 & 0.0 & 0.0 & 0.0 & 0.0 & 0.0 & 0.0 & 0.0 & 0.0 & 0.0 & 0.0 & 0.0 & 0.0 & 0.0 & 0.0 & 4.5 & 100.0 \\
\hline & $\max$ & 0.0 & 0.0 & 0.0 & 0.0 & 0.0 & 0.0 & 0.0 & 0.0 & 0.0 & 0.0 & 0.0 & 0.0 & 0.0 & 0.0 & 0.0 & 23.2 & 100.0 \\
\hline \multicolumn{19}{|c|}{ Waste Generator: FHC_I_PLANT } \\
\hline \multirow[t]{3}{*}{ CH_LLH_I } & vor & 14.2 & 14.2 & 14.2 & 21.2 & 14.2 & 14.2 & 24.2 & 14.2 & 14.2 & 34.2 & 24.2 & 14.2 & 24.2 & 31.2 & 21.2 & 126.0 & 47.2 \\
\hline & MIN & 11.3 & 11.3 & 11.3 & 11.3 & 11.3 & 11.3 & 11.3 & 11.3 & 11.3 & 11.3 & 21.3 & 21.3 & 11.3 & 11.3 & 11.3 & 310.8 & 69.6 \\
\hline & $\max$ & 28.4 & 20.4 & 28.4 & 28.4 & 28.4 & 28.4 & 28.4 & 28.4 & 28.4 & 28.4 & 28.4 & 28.4 & 28.4 & 28.4 & 28.4 & 852.0 & 53.6 \\
\hline \multirow[t]{3}{*}{ RH_LIW_I } & vor & 0.0 & 0.0 & 0.0 & 0.0 & 0.0 & 0.0 & 0.0 & 0.0 & 0.0 & 0.0 & 0.0 & 0.0 & 0.0 & 0.0 & 0.0 & 85.2 & 9.4 \\
\hline & HIN & 0.0 & 0.0 & 0.0 & 0.0 & 0.0 & 0.0 & 0.0 & 0.0 & 0.0 & 0.0 & 0.0 & 0.0 & 0.0 & 0.0 & 0.0 & 0.0 & 0.0 \\
\hline & $\max$ & 0.0 & 0.0 & 0.0 & 0.0 & 0.0 & 0.0 & 0.0 & 0.0 & 0.0 & 0.0 & ' 0.0 & 0.0 & 0.0 & 0.0 & 0.0 & 85.2 & 5.3 \\
\hline \multirow{2}{*}{ CH_LLPAN_I } & MIN & 3.5 & 3.5 & 3.5 & 3.5 & 3.5 & 3.5 & 3.5 & 3.5 & 3.5 & 3.5 & 3.5 & 3.5 & 3.5 & $3.5^{\circ}$ & 3.5 & 106.5 & 21.7 \\
\hline & $\max$ & 24.2 & 14.2 & 14.2 & 14.2 & 11.2 & 14.2 & 14.2 & 14.2 & 14.2 & 14.2 & 14.2 & 14.2 & 11.2 & 14.2 & 14.2 & $\$ 26.0$ & 26.8 \\
\hline \multirow[t]{3}{*}{ PH_LIHW_I } & vor & 0.0 & 0.0 & 0.0 & 0.0 & 0,0 & 0.0 & 0.0 & 0.0 & 0.0 & 0.0 & 0.0 & 0.0 & 0.0 & 0.0 & 0.0 & 85.2 & 9.1 \\
\hline & MIN & 0.0 & 0.0 & 0.0 & 0.0 & 0.0 & 0.0 & 0.0 & 0.0 & 0.0 & 0.0 & 0.0 & 0.0 & 0.0 & 0.0 & 0.0 & 0.0 & 0.0 \\
\hline & $\max$ & 0.0 & 0.0 & 0.0 & 0.0 & 0.0 & 0.0 & 0.0 & 0.0 & 0.0 & 0.0 & 0.0 & 0.0 & 0.0 & 0.0 & 0,0 & 85.2 & 5.3 \\
\hline \multirow[t]{3}{*}{ RH__RUS } & vor & 0.0 & 0.0 & 0.0 & 0.0 & 0.0 & 0.0 & 0.0 & 0.0 & 0.0 & 0.0 & 0.0 & 0.0 & 0.0 & 0.0 & 0.0 & 28.1 & 3.1 \\
\hline & MIN & 0.0 & 0.0 & 0.0 & 0.0 & 0.0 & 0.0 & 0.0 & 0.0 & 0.0 & 0.0 & 0.0 & 0.0 & 0.0 & 0.0 & 0.0 & 0.0 & 0.0 \\
\hline & $\max$ & 0.0 & 0.0 & 0.0 & 0.0 & 0.0 & 0.0 & 0.0 & 0.0 & 0.0 & 0.0 & 0.0 & 0.0 & 0.0 & 0.0 & 0.0 & 56.8 & 3.5 \\
\hline \multirow[t]{3}{*}{ HAZ } & voL & 2.8 & 2.0 & 2.8 & 2.8 & 2.8 & 2.8 & 2.8 & 2.8 & 2.0 & 2.8 & 2.8 & 2.0 & 2.8 & 2.8 & 2.8 & 63.0 & 6.9 \\
\hline & MIH & 2.1 & 2.1 & 2.1 & 2.1 & 2.1 & 2.1 & 2.2 & 2.1 & 2.1 & 2.1 & 2.1 & 2.1 & 2.1 & 2.1 & 2.2 & 12.0 & 8.5 \\
\hline & $\max$ & 3.5 & 3,5 & 3.5 & 3.5 & 3.5 & 3.5 & 3.5 & 3.3 & 3.5 & 3.5 & 3.5 & 3.5 & 3.5 & 3.5 & 3.5 & 81.0 & 5.2 \\
\hline
\end{tabular}


EY-1994 SOLID WASTE FORECAST

WASTE GENERATOR, WASTE CLASS VOLUMES BY YEAR

(in cubic metera)

\begin{tabular}{|c|c|c|c|c|c|c|c|c|c|c|c|c|c|c|c|c|c|}
\hline Haste class & & 2010 & 2011 & 2012 & 2013 & 2014 & 2015 & 2016 & 2017 & 2018 & 2019 & 2020 & 2021 & 2022 & 2023 & 2024. & Total Pct \\
\hline \multirow{3}{*}{$\begin{array}{l}\text { FHC_2_pLANT } \\
\text { rotai: }\end{array}$} & VoL & 24.1 & 21.1 & 24.1 & 24.1 & 24.1 & 24.1 & 24.1 & 24.1 & 24.1 & 24.1 & 24.1 & 24.1 & 24.1 & 24.1 & 24.1 & 900.8100 .0 \\
\hline & MIN & 17.0 & 27.0 & 17.0 & 17.0 & 17.0 & 17.0 & 17.0 & 17.0 . & 17.0 & 17.0 & 17.0 & 17.0 & 27.0 & 17.0 & 17.0 & 489.3100 .0 \\
\hline & $\max$ & 46.1 & 18.1 & 46.2 & 16.1 & 16.1 & 16.1 & 16.1 & 16.1 & 66.1 & 16.1 & 16.1 & 16.1 & 16.1 & 16.1 & 46.1 & 1589.2100 .0 \\
\hline
\end{tabular}

Wasto Generator: KHC_FASTR__REUT

\begin{tabular}{|c|c|c|c|c|c|c|c|c|c|c|c|c|c|c|c|c|c|c|}
\hline CH_LLW_I & $\begin{array}{l}\text { VOL } \\
\text { MIN } \\
\text { HAX }\end{array}$ & $\begin{array}{r}19.7 \\
9.8 \\
39.4\end{array}$ & $\begin{array}{r}19.7 \\
9.8 \\
39.4\end{array}$ & $\begin{array}{r}19.7 \\
9.8 \\
39.4\end{array}$ & $\begin{array}{r}19.7 \\
9.8 \\
39.4\end{array}$ & $\begin{array}{r}19.7 \\
9.8 \\
39.1\end{array}$ & $\begin{array}{r}19.7 \\
9.8 \\
39.4\end{array}$ & $\begin{array}{r}19.7 \\
9.8 \\
39.4\end{array}$ & $\begin{array}{r}29.7 \\
9.0 \\
39.1\end{array}$ & $\begin{array}{r}29.7 \\
9.8 \\
39.1\end{array}$ & $\begin{array}{l}19.7 \\
.9 .8 \\
39.4\end{array}$ & $\begin{array}{r}29.7 \\
9.8 \\
39.1\end{array}$ & $\begin{array}{r}29.7 \\
9.0 \\
39.4\end{array}$ & $\begin{array}{r}29.7 \\
9.8 \\
39.4\end{array}$ & $\begin{array}{r}19.7 \\
9.8 \\
39.1\end{array}$ & $\begin{array}{r}19.7 \\
9.0 \\
39.1\end{array}$ & $\begin{array}{r}604.5 \\
295.5 \\
1195.5\end{array}$ & $\begin{array}{l}91.8 \\
93.8 \\
91.7\end{array}$ \\
\hline \multirow{3}{*}{ CH_LL_III } & vor & 0.5 & 0.5 & 0.5 & 0.5 & 0.5 & 0.5 & 0.5 & 0.5 & 0.5 & 0.5 & 0.5 & 0.5 & 0.5 & 0.5 & 0.5 & 15.0 & 2.2 \\
\hline & MIN & 0.0 & 0.0 & 0.0 & 0.0 & 0.0 & 0.0 & 0.0 & 0.0 & 0.0 & 0.0 & 0.0 & 0.0 & 0.0 & 0.0 & 0.0 & 0.0 & 0.0 \\
\hline & MAX & 1.0 & 1.0 & 1.0 & 2.0 & 1.0 & 2,0 & 1.0 & 1.0 & 1.0 & 1.0 & 1.0 & 1.0 & 1.0 & 1.0 & 1.0 & 30.0 & 2.3 \\
\hline \multirow[t]{3}{*}{ CH_LLHE_I } & vor & 1.3 & 2.3 & 1.3 & 1.3 & 2.3 & 1.3 & 1.3 & 1.3 & 1.3 & 1.3 & 1.3 & 1.3 & 1.3 & 1.3 & 1.3 & 39.0 & 5.9 \\
\hline & HIN & 0.6 & 0.6 & 0.6 & 0.6 & 0.6 & 0.6 & 0.6 & 0.6 & 0.6 & 0.6 & 0.6 & 0.6 & 0.6 & 0.6 & 0.6 & 19.5 & 6.1 \\
\hline & $\max$ & 2.6 & 2.6 & 2.6 & 2.6 & 2.6 & 2.6 & 2.6 & 2.6 & 2.6 & 2.6 & 2.6 & 2.6 & 2.6 & 2.6 & 2.6 & 78.0 & 5.9 \\
\hline \multirow{3}{*}{$\begin{array}{l}\text { RBC_HASTI_KBUT } \\
\text { Total. }\end{array}$} & vol & 21.5 & 21.5 & 21.3 & 21.5 & 21.5 & 21.5 & 21.5 & 21.5 & 21.5 & 21.5 & 21.5 & 21.5 & 21.5 & 21.5 & 21.5 & \multicolumn{2}{|c|}{658.5100 .0} \\
\hline & MIN & 10.5 & 10.5 & 20.5 & 20.5 & 10.5 & 20.5 & 10.5 & 10.5 & 10.5 & 10.5 & 10.5 & 10.5 & 10.5 & 20.5 & 10.5 & \multicolumn{2}{|c|}{315.0100 .0} \\
\hline & MAX & 43.0 & 13.0 & 13.0 & 13.0 & 43.0 & 43.0 & 13.0 & 43.0 & 13.0 & 13.0 & 13.0 & 13.0 & 43.0 & 13.0 & 13.0 & 2303.5 & 100.0 \\
\hline
\end{tabular}

Waste Gonorator: WHC_FELI_DRI

\begin{tabular}{|c|c|c|c|c|c|c|c|c|c|c|c|c|c|c|c|c|c|c|}
\hline \multirow[t]{3}{*}{ CH_LLW_I } & vor & 1.3 & 1.3 & 1.3 & 2.3 & 1.3 & 1.3 & 1.3 & 1.3 & 1.3 & 1.3 & 1.3 & 1.3 & 1.3 & 1.3 & 1.3 & 39.0 & 15.2 \\
\hline & MIN & 0.0 & 0.0 & 0.0 & 0.0 & 0.0 & 0.0 & 0.0 & 0.0 & 0.0 & 0.0 & 0.0 & 0.0 & 0.0 & 0.0 & 0.0 & 0.0 & 0.0 \\
\hline & $\max$ & 3.9 & 3.9 & 3.9 & 3.9 & 3.9 & 3.9 & 3.9 & 3.9 & 3.9 & 3.9 & 3.9 & 3.9 & 3.9 & 3.9 & 3.9 & 117.0 & 21.0 \\
\hline \multirow[t]{2}{*}{ CH_LLHE_I } & vor & 0.8 & 0.8 & 0.8 & 0.8 & 0.8 & 0.8 & 0.8 & 0.8 & 0.8 & 0.8 & 0.8 & 0.0 & 0.8 & 0.8 & 0.8 & 24.0 & 9.3 \\
\hline & $\max$ & 1.6 & 1.6 & 1.6 & 1.6 & 1.6 & 1.6 & .2 .6 & 1.6 & 1.6 & 1.6 & 1.6 & 1.6 & 1.6 & 1.6 & 1.6 & 48.0 & 8.6 \\
\hline \multirow[t]{3}{*}{ HAz } & VoL & 6.5 & 6.5 & 6.5 & 6.5 & 6.5 & 6.5 & 6.5 & 6.5 & 6.5 & 6.5 & 6.5 & 6.5 & 6,5 & 6.5 & 6.5 & 195.0 & 75.5 \\
\hline & MIH & 0.0 & 0.0 & 0.0 & 0.0 & 0.0 & 0.0 & 0.0 & 0.0 & 0.0 & 0.0 & 0.0 & 0.0 & 0.0 & 0.0 & 0.0 & 0.0 & 0.0 \\
\hline & $\max$ & 13.0 & 13.0 & 13.0 & 13.0 & 13.0 & 13.0 & 13.0 & 13.0 & 13.0 & 13.0 & 13.0 & 23.0 & 13.0 & 13.0 & 13.0 & 390.0 & 70.2 \\
\hline \multirow{3}{*}{$\begin{array}{l}\text { HEC_HEIX, DRS } \\
\text { Tot22. }\end{array}$} & VOL & 8.6 & 0.6 & 8.6 & 8.6 & 8.6 & 8.6 & 8.6 & 8.6 & 8.6 & 8.6 & 0.6 & 8.6 & B.6 & 8.6 & 8.6 & 258.0 & 100.0 \\
\hline & MIN & 0.0 & 0.0 & 0.0 & 0.0 & 0.0 & 0.0 & 0.0 & 0.0 & 0.0 & 0.0 & 0.0 & 0.0 & 0.0 & 0.0 & 0.0 & 0.0 & 100.0 \\
\hline & $\operatorname{MAX}$ & 18.5 & 10.5 & 18.5 & 18.5 & 10.5 & 28.5 & 18.5 & 18.5 & 28.5 & 18.5 & 20.5 & 28.5 & 18.5 & 118.5 & 28.5 & 555.0 & 100.0 \\
\hline
\end{tabular}


FY-1994 SOLID HASTE FORECAST

WASTE GENERATOR, WASTE CLASS VOLUMES BY YEAR

(in cubio meters)

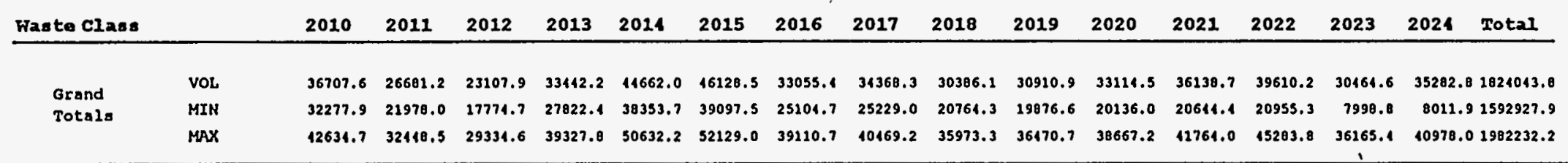


WHC-EP-0865

APPENDIX E

WASTE GENERATORS BY HANFORD PROGRAM AREA 
WHC-EP-0865

Intentionally Left Blank

$E-j i$ 
WHC-EP-0865

\section{APPENDIX E}

WASTE GENERATOR LIST BY HANFORD PROGRAM AREA

Table E-1. Waste Generator List by Hanford Program Area

\begin{tabular}{|c|c|c|c|}
\hline Program Area & Waste Generator Name & $\begin{array}{c}1994 \\
\text { Abbreviation }\end{array}$ & $\begin{array}{c}1993 \\
\text { Abbreviation }\end{array}$ \\
\hline \multirow{2}{*}{$\begin{array}{l}\text { Analytical } \\
\text { Services }\end{array}$} & 222-S Analytical Laboratory & WHC_ANALYT_LAB & WHC_222S \\
\hline & 325 Radiochemi stry Laboratory & WHC_325 & WHC_325 \\
\hline \multirow{21}{*}{$\begin{array}{l}\text { Environmental } \\
\text { Restoration }\end{array}$} & 100 Area Transition (BHI) & WHC_ $100^{*}$ & WHC_100 \\
\hline & $\begin{array}{l}\text { D\&D of } 200 \text { Area Effluent } \\
\text { Treatment (Project C018H) }\end{array}$ & TWHC_PX_CO18H & TWHC_PX_CO18H \\
\hline & $\begin{array}{l}\text { D\&D of 222-S Analytical } \\
\text { Laboratory }\end{array}$ & TWHC_222S & TWHC_222S \\
\hline & $\begin{array}{l}\text { D\&D of 224T TRU Storage \& Assay } \\
\text { Facility (TRUSAF) }\end{array}$ & TWHC_TRUSAF & TWHC_TRUSAF \\
\hline & D\&D of $242-A$ Evaporator & TWHC_242A & TWHC_242A \\
\hline & D\&D of 242-S Evaporator & TWHC_242S & TWHC_242S \\
\hline & D\&D of 242-T Evaporator & TWHC_242T & TWHC_242T \\
\hline & $\begin{array}{l}\text { D\&D of } 306 \text { DeveTopment/ } \\
\text { Fabrication/Test Lab. }\end{array}$ & TWHC_306 & TWHC_306 \\
\hline & $\begin{array}{l}\text { D\&D of } 308 \text { Fuels Development } \\
\text { Laboratory }\end{array}$ & TWHC_308 & TWHC_308 \\
\hline & $\begin{array}{l}\text { D\&D of } 340 \text { Waste Neutralization } \\
\text { Facility }\end{array}$ & TWHC_340 & TWHC_340 \\
\hline & D\&D of B PTant & TWHC_BPLANT & TWHC_225B \\
\hline & D\&D of $N$ Reactor & TWHC_100N & TWHC_100N \\
\hline & D\&D of PFP & TWHC_PFP & TWHC_2345Z \\
\hline & D\&D of PNL 231-Z Building & TPNL_231Z & TPNL_231Z \\
\hline & D\&D of PNL 242 Building & TPNL_242B/BL & TPNL_242B/BL \\
\hline & D\&D of PNL 306W Building & TPNL_306W & TPNL_306W \\
\hline & D\&D of PNL 314 Building & TPNL_314 & TPNL_314 \\
\hline & D\&D of PNL 326 Building & TPNL_326 & TPNL_326 \\
\hline & D\&D of PNL 327 Building & TPNL_327 & TPNL_327 \\
\hline & D\&D of PNL 331 Building & TPNL_331 & TPNL_331 \\
\hline & D\&D of PNL 324 Building & TPNL_324 & TPNL_324 \\
\hline
\end{tabular}


Table E-1. Waste Generator List by Hanford Program Area

\begin{tabular}{|c|c|c|c|}
\hline Program Area & Waste Generator Name & $\begin{array}{c}1994 \\
\text { Abbreviation }\end{array}$ & $\begin{array}{c}1993 \\
\text { Abbreviation }\end{array}$ \\
\hline \multirow{6}{*}{$\begin{array}{l}\text { Environmental } \\
\text { Restoration } \\
\text { (cont.) }\end{array}$} & D\&D of PUREX & TWHC_PUREX & TWHC_202A \\
\hline & D\&D of T Plant & TWHC_TPLANT & TWHC_221T \\
\hline & Past Practice Remediation & PAST_PRAC_REM & PAST_PRAC_REM \\
\hline & PUREX Tunnel Waste & TWHC_PUREX_TW & TWHC_202A_TW \\
\hline & Surplus Facilities & WHC_SURPLS_FAC & $\begin{array}{l}\text { WHC_SURPLS_FA } \\
\text { C }\end{array}$ \\
\hline & $\begin{array}{l}\text { We11 Drilling - Environmental } \\
\text { Projects }\end{array}$ & WHC_WELL_DRL & WHC_WELL_DRL \\
\hline \multirow[t]{3}{*}{ Liquid Effluent } & $\begin{array}{l}200 \text { Area Effluent Treatment } \\
\text { Facility (Project C018H) }\end{array}$ & WHC_200_ETF & WHC_PX_CO18H \\
\hline & $\begin{array}{l}300 \text { Area Treated Effluent } \\
\text { Disposal Facility } \\
\text { (Project L045H) }\end{array}$ & WHC_300_TEDF & WHC_LO45H \\
\hline & $\begin{array}{l}\text { Waste Neutralization Facility } \\
\text { (340/307 Buildings) }\end{array}$ & WHC_WASTE_NEUT & WHC_340 \\
\hline \multirow[t]{13}{*}{ Non-Programmatic } & 105DR Large Sodium Fire Facility & WHC_SODIUM_FAC & WHC_105DR \\
\hline & 1154 Radio Maintenance- 3000 Area & WHC_RADIO_MAIN & WHC_1154_3000 \\
\hline & $\begin{array}{l}1163 \text { Procurement \& Material } \\
\text { Management }\end{array}$ & WHC_PROCURMENT & WHC_1163 \\
\hline & 1171 FSS Fleet Maintenance & WHC_FLEET_MAIN & WHC_1171M \\
\hline & 1171 FSS Transportation & WHC_TRANSPORT & WHC_1171T \\
\hline & 2101M FSS Electric Utilities & WHC_ELEC_UTIL & WHC_2101M \\
\hline & $\begin{array}{l}\text { 2703E Chemical Engineering } \\
\text { Laboratory }\end{array}$ & WHC_CHEM_E_LAB & WHC_2703E \\
\hline & $\begin{array}{l}\text { 2715EA General Area Services } \\
\text { North }\end{array}$ & WHC_GAS_NORTH & WHC_2715EA \\
\hline & $\begin{array}{l}\text { 2724W Laundry/Equipment } \\
\text { Decontamination }\end{array}$ & WHC_LAUNDRY & WHC_2724W \\
\hline & $\begin{array}{l}\text { 284E/284W East \& West } \\
\text { Powerhouses }\end{array}$ & WHC_200_PWR & WHC_284E \\
\hline & 305 Engineering Testing Facility & WHC_ENG_TEST & WHC_305 \\
\hline & $\begin{array}{l}306 \text { Development/Fabrication/Test } \\
\text { Lab. }\end{array}$ & WHC_ENG_DEV & WHC_306 \\
\hline & 308 Fuels Development Laboratory & WHC_FUEL_DEV & WHC_308 \\
\hline
\end{tabular}


Table E-1. Waste Generator List by Hanford Program Area

\begin{tabular}{|c|c|c|c|}
\hline Program Area & Waste Generator Name & $\begin{array}{c}1994 \\
\text { Abbreviation }\end{array}$ & $\begin{array}{c}1993 \\
\text { Abbreviation }\end{array}$ \\
\hline \multirow{12}{*}{$\begin{array}{l}\text { Non-Programmatic } \\
\text { (cont.) }\end{array}$} & 335 Sodium Test Facility & WHC_SODIUM_TST & T/WHC_335 \\
\hline & $\begin{array}{l}\text { 3707C Safeguards/Security } \\
\text { Maintenance }\end{array}$ & WHC_SS_MAIN & WHC_3707C \\
\hline & 3717B Standards Laboratory & WHC_STAND_LAB & WHC_3717B \\
\hline & $\begin{array}{l}377 \text { Geotechnical Engineering } \\
\text { Laboratory }\end{array}$ & WHC_GEOTEC_LAB & WHC_377 \\
\hline & 384 Power House, 300 Area & WHC_300_PWR & WHC 384 \\
\hline & $\begin{array}{l}\text { BCSR Computer Service Federal } \\
\text { Bldg. }\end{array}$ & WHC_FED & WHC_FED \\
\hline & Buried Equipment (OSS/TFS) 1992 & WHC_BQUIP & WHC_BQUIP \\
\hline & FFTF/FMEF/MASF Maintenance & WHC_FFTF_MAIN & WHC_FFTF_MAIN \\
\hline & Fuel \& Materials Examination & WHC_FMEF & WHC FMEF \\
\hline & General Area Services South & WHC_GAS_SOUTH & WHC S300 \\
\hline & $\begin{array}{l}\text { Hanford Environmental Health } \\
\text { Foundation }\end{array}$ & HEHF & HEHF \\
\hline & $\begin{array}{l}\text { Kaiser Construction Services }-100 \\
\text { Area }\end{array}$ & KEH_100 & KEH_100 \\
\hline \multirow[t]{16}{*}{ Offsite } & Ames Laboratory-Ames, Iowa & AMES & AMES \\
\hline & Argonne National Laboratory-East & ANL_E & ANL_E \\
\hline & Bates Accelerator-Massachusetts & MIT_BATES & MIT_BATES \\
\hline & Battelle Columbus Laboratories & BAT_CLBS_LAB & BAT_CLBS_LAB \\
\hline & Bettis Atomic Power-Laboratory & BAPL & BAPL \\
\hline & Bettis Atomic Power-Shipyards & BAPL_SHIPYDS & BAPL_SHIPYDS \\
\hline & Brookhaven National Laboratory & BRKHVN & BRKHVN \\
\hline & EG\&G Rocky Flats Plant & $E G \& G$ & $E G \& G$ \\
\hline & $\begin{array}{l}\text { FERMI National Accelerator } \\
\text { Laboratory }\end{array}$ & FERMI & FERMI \\
\hline & $\begin{array}{l}\text { Formerly Utilized Sites Remedial } \\
\text { Action Program }\end{array}$ & FUSRAP & FUSRAP \\
\hline & General Atomics & GEN_ATOM & GEN_ATOM \\
\hline & Knolls Atomic Power-Laboratory. & KAPL & KAPL \\
\hline & Knolls Atomic Power-Shipyards & KAPL_SHIPYDS & KAPL_SHIPYDS \\
\hline & Lawrence Berkeley Laboratory & $L B L$ & LBL \\
\hline & Paducah Energy Systems & PADUCAH_ES & PADUCAH_ES \\
\hline & Paducah Utility Services & PADUCAH_US & PADUCAH_US \\
\hline
\end{tabular}


Table E-1. Waste Generator List by Hanford Program Area

\begin{tabular}{|c|c|c|c|}
\hline Program Area & Waste Generator Name & $\begin{array}{c}1994 \\
\text { Abbreviation }\end{array}$ & $\begin{array}{c}1993 \\
\text { Abbreviation }\end{array}$ \\
\hline \multirow[t]{7}{*}{ Offsite (cont.) } & $\begin{array}{l}\text { Princeton Plasma Physics } \\
\text { Laboratory }\end{array}$ & PRINCETON & PRINCETON \\
\hline & Portsmouth Energy Systems & PORTSMOUTH_ES & PORTSMOUTH_ES \\
\hline & Portsmouth Utility Services & PORTSMOUTH_US & PORTSMOUTH_US \\
\hline & Rockwell-Canoga Park & RKW_CANOGA & RKW_CANOGA \\
\hline & $\begin{array}{l}\text { Stanford Linear Accelerator } \\
\text { Center }\end{array}$ & STANFORD & STANFORD \\
\hline & University of California-Davis & B_LEHR_DAV & B_LEHR_DAV \\
\hline & University of Utah & U_U & U U \\
\hline PNL & Pacific Northwest Laboratories & PNL & PNL \\
\hline SNF & 100K K-Basin Operations & WHC_K_BASIN & WHC_100K \\
\hline \multirow[t]{4}{*}{ Solid Waste } & 218E/W Low Level Burial Grounds & WHC_LLBG & WHC_LLBG \\
\hline & $221 \mathrm{~T} / 2706 \mathrm{~T}$ T-PTant Operations & WHC_T_PLANT & WHC_221T \\
\hline & $\begin{array}{l}224 T \text { TRU Storage \& Assay } \\
\text { Facility (TRUSAF) }\end{array}$ & WHC_TRUSAF & WHC_TRUSAF \\
\hline & Mixed Waste Storage Facility & WHC_EA_W112 & WHC_EA_W112 \\
\hline \multirow{5}{*}{$\begin{array}{l}\text { Transition } \\
\text { Facilities }\end{array}$} & Purex (202A) Operations & WHC_PUREX & WHC_202A \\
\hline & $\begin{array}{l}\text { Purex (202A) Surveillance \& } \\
\text { Maintenance }\end{array}$ & WHC_PUREX_SM & $\begin{array}{l}\text { Not } \\
\text { Appl icable }\end{array}$ \\
\hline & B-Plant & WHC_B_PLANT & WHC_225B \\
\hline & Plutonium Finishing Plant & WHC_PFP & WHC_2345Z \\
\hline & 300 Area Fuel Supply & WHC_FUEL_TRANS & WHC_303K \\
\hline \multirow[t]{11}{*}{ TWRS } & Aging Waste Transfer Lines & WHC_TWP_W028 & WHC_TWP_W028 \\
\hline & Cross-Site Transfer System & WHC_TWP_W058 & WHC_TWP_W058 \\
\hline & DST I01AZ Retrieval (1 tank) & WHC_TWP_W151 & WHC_TWP_W151 \\
\hline & DST Retrieval Systems (10 tanks) & WHC_TWP_W211 & WHC_TWP_W211 \\
\hline & Tank Farm Restoration & WHC_TWP_W314 & WHC_TWP_W314 \\
\hline & High Level Vitrification Project & WHC_HLVP & WHC_HLVP \\
\hline & Low Level Vitrification Project & WHC_LLVP & WHC_LLVP \\
\hline & SST Long Length Equipment & WHC_SST_LLE & WHC_SSTLLE \\
\hline & SST Retrieval (149 tanks) & WHC_SST_RET & WHC_SSTRET \\
\hline & SST Tank 106C Manipulator & WHC_TWP_W340 & WHC_TWP_W340 \\
\hline & SST Tank 106C Sluicing & WHC_TWP_W320 & WHC_TWP_W320 \\
\hline
\end{tabular}


Table E-1. Waste Generator List by Hanford Program Area

\begin{tabular}{||l|l|l|l||}
\hline \hline Program Area & \multicolumn{1}{|c|}{ Waste Generator Name } & $\begin{array}{c}1994 \\
\text { Abbreviation }\end{array}$ & $\begin{array}{c}1993 \\
\text { Abbreviation }\end{array}$ \\
\hline \hline \multirow{5}{*}{ TWRS (cont.) } & SST/DST Tank Farm Operations & WHC_TF_OPER & WHC_2ETF \\
\cline { 2 - 5 } & Tank Farm Ventilation Upgrade & WHC_TWP_W030 & WHC_TWP_W030 \\
\cline { 2 - 5 } & Tank Waste Pretreatment Facility & WHC_PRETRT_TW & WHC_PRETRT_TW \\
\cline { 2 - 4 } & W315 Pilot Scale Retrieval & WHC_DST_PSRET & WHC_DSTPSRET \\
\cline { 2 - 4 } & W343 DST Retrieval (17 Tanks) & WHC_DST_RET & WHC_DST_RET \\
\hline
\end{tabular}


WHC-EP-0865

Intentionally Left $B$ lank

E-6 